\title{
Transition Projects
}

Fiscal Year 1996 Multi-Year

Program Plan (MYPP) for

WBS 1.3.1, 7.1, and 6.12

\author{
D. B. Cartmell
}

Date Published

September 1995

Prepared for the U.S. Department of Energy

Assistant Secretary for Environmental Management

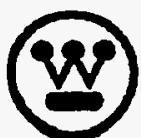

Approved for public release; distribution is unlimited 


\section{DISCLAIMER}

Portions of this document may be illegible in electronic image products. Images are produced from the best available original document. 
Mr. J. E. Mecca, Director

Transition Program Division

U.S. Department of Energy

Richland Operations Office

Richland, Washington 99352

Dear Mr. Mecca:

\section{TRANSITION PROJECTS 1996 MULTI-YEAR PROGRAM PLAN}

Based on U.S. Department of Energy (DOE), Richland Operations Office (RL) review, specific areas of Westinghouse Hanford Company (WHC), Transition Projects "Draft" Multi-Year Program Plan (MYPP) were revised in preparation for the RL approval ceremony on September 26, 1995. These changes were reviewed with the appropriate RL Project Manager. The changes have been incorporated to the MYPP electronic file, and hard copies replacing the "Draft" MYPP will be distributed after the formal signing.

In addition to the comments received, a summary level schedule and outyear estimates for the $K$ Basin deactivation beginning in FY 2001 have been included. The $K$ Basin outyear waste data is nearing completion this week and will be incorporated. This exclusion was discussed with Mr. N. D. Moorer, RL, Facility Transition Program Support/Integration.

The attached MYPP scope/schedule reflects the Integrated Target Case submitted in the April 1995 Activity Data Sheets (ADS) with the exception of $B$ Plant and the Plutonium Finishing Plant (PFP). The B PIant assumption in FY 1997 reflects the planning case in the FY 1997 ADS with a shortfall of $\$ 5$ million. PFP assumptions have been revised from the FY 1997 ADS based on the direction provided this past summer by DOE-Headquarters. This includes the acceleration of the polycube stabilization back to its originally planned completion date. Although the overall program repricing in FY 1996 allowed the scheduled acceleration to fall with the funding allocation, the FY 1997 total reflects a shortfall of $\$ 6$ million.

Rather than negotiating a schedule deferral as a result of the shortfall, WHC feels that through the use of final FY 1995 carryover dollars, redeployment of resources resulting from Activity Based Cost Estimating critical analysis and implementation of re-engineering processes, activities 
Mr. J. E. Mecca

9555161

Page 2

September 26, 1995

can be accelerated into FY 1996 and total estimates for FY 1997 reduced. The actions to align to the funding allocation in FY 1997 will be documented through change control.

If you have any questions regarding the revisions, please call Ms. M. A. Baumann on 372-3672.

Very truiy yours,

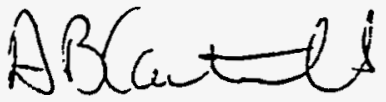

D. B. Cartme17, Director

Transition Programs

Transition Projects

dm

Attachment

RL - K. D. Cameron (w/attachment)

D. T. Evans*

E. W. Higgins*

A. B. Joy*

N. D. Moorer*

L. L. Piper*

L. D. Romine*

D. W. Templeton*

A. H. Hirkkala (w/o attachment)

*attachment sent electronically 


\section{RELEASE AUTHORIZATION}

Document Number: WHC-SP-1126, Rev. 1

Document Title: $\quad$ Transition Projects Fiscal Year 1996 Multi-Year

Program Plan (MYPP) for WBS 1.3.1, 7.1, and 6.12

Release Date:

$9 / 28 / 95$

This document was reviewed following the procedures described in WHC-CM-3-4 and is:

APPROVED FOR PUBLIC RELEASE

WHC Information Release Administration Specialist:

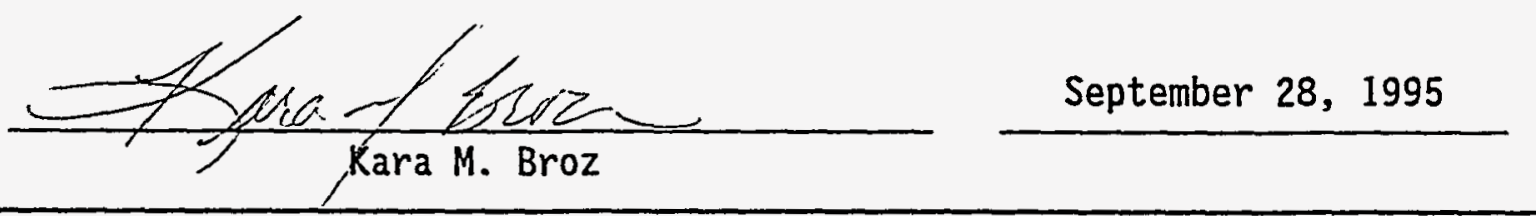




\section{MYPP Approval Sheet}

Transition Program Division Program Office (7.1/1.3.1/6.12)

U. S. Department of Energy

Richland Operations office

\section{Assistant Manager-Contracting Officer's Representative} Faciitity Transition
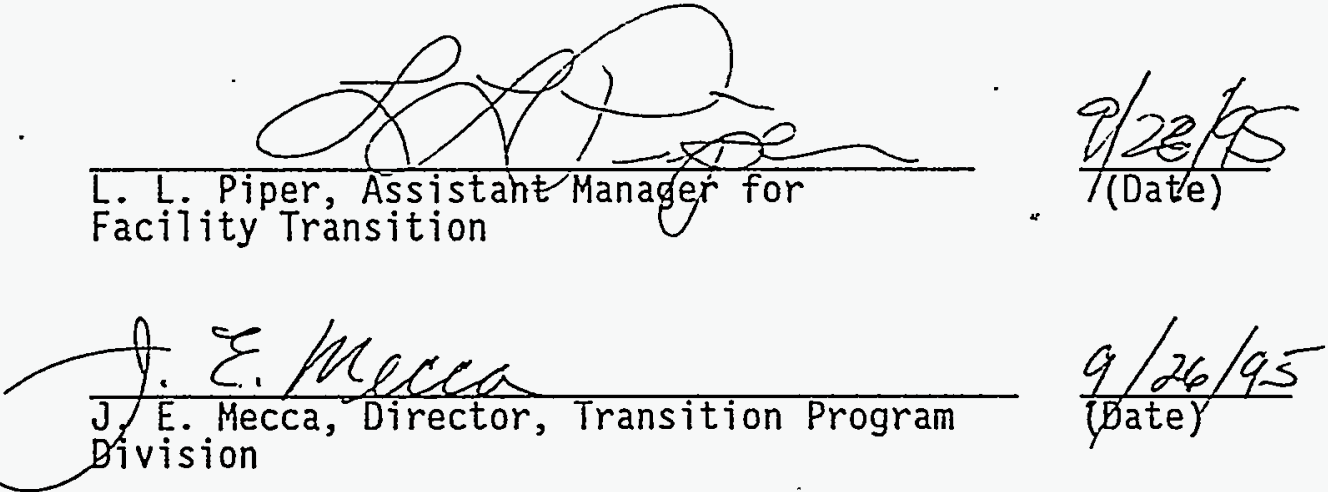

Westinghouse Hanford Program Management

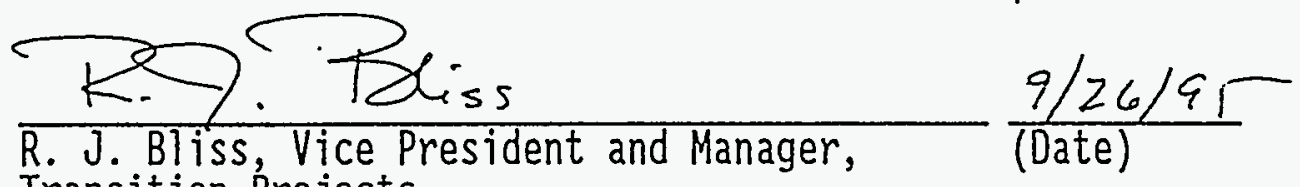

Transition Projects

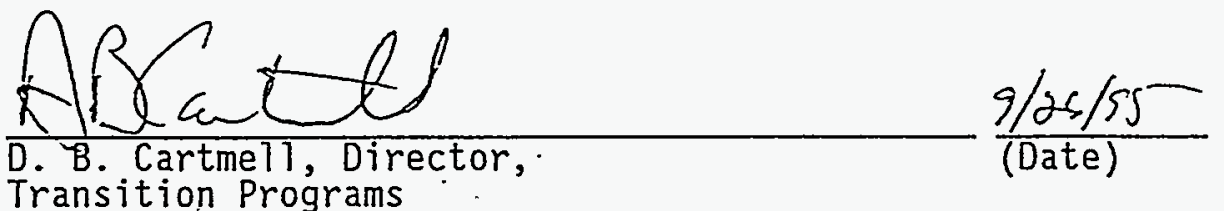


TABLE OF CONTENTS

TRANSITION PROJECTS $(7.1 / 1.3 .1)$

FY 1996 MYPP

1.0 PROGRAM OVERVIEW

1.A Program Mission

- 1.B Program Strategy

1.B.1 Technical Functions/Requirements

1.B.2 Program Objectives

1.B:3 Planning Assumptions

1.B.4 Program Constraints

2.0 PROGRAM BASELINES

2.A Technical Requirements Baseline

2.A.1 Technical Documents Tree \& Products Structure

2.A.2 Work Breakdown Structure (WBS)

2.A.3 WBS Dictionary

2.A.4 Responsibility Assignment Matrix (RAM)

2.A.5 Technology Requirements

2.A.6 Waste Type Data

2.B Schedule Baseline

2.B.1 Program Master Baseline Schedule (PMBS)

2.B.2 Milestone List

2.B.3 Milestone Description Sheets

2.C Cost Baseline

2.C.1 Cost Baseline Summary By Year

2.C.2 Basis of Estimate (BOE)

2.C.3 Planned Staffing Profiles

3.0 EXECUTION YEAR

3.A Technical Objectives

3.B Program Performance Baseline Schedule (PPBS)

3.C Cost Baseline by Month

3.C.1 Cost Baseline by Month - Operating Expense

3.C.2 Cost Baseline by Month - CENRTC

3.C.3 Cost Baseline by Month - GPP

3.C. 4 Cost Baseline by Month - Line Items

3.D Program Funding Required

3.E Performance Measures

3.E.1 Performance Based Incentives (PBI)

3.E.2 Other Performance Measures

4.0 DEPARTMENT OVERHEAD (DOH)

4.A DOH Program Objectives

4.B DOH Program Planning Assumptions

4.C DOH WBS

4.D DOH RAM

4.E DOH WBS Dictionary

4.F DOH Planned Staffing Profile

4.G DOH Liquidation Base Analysis 
1996 MYPP Acronym List

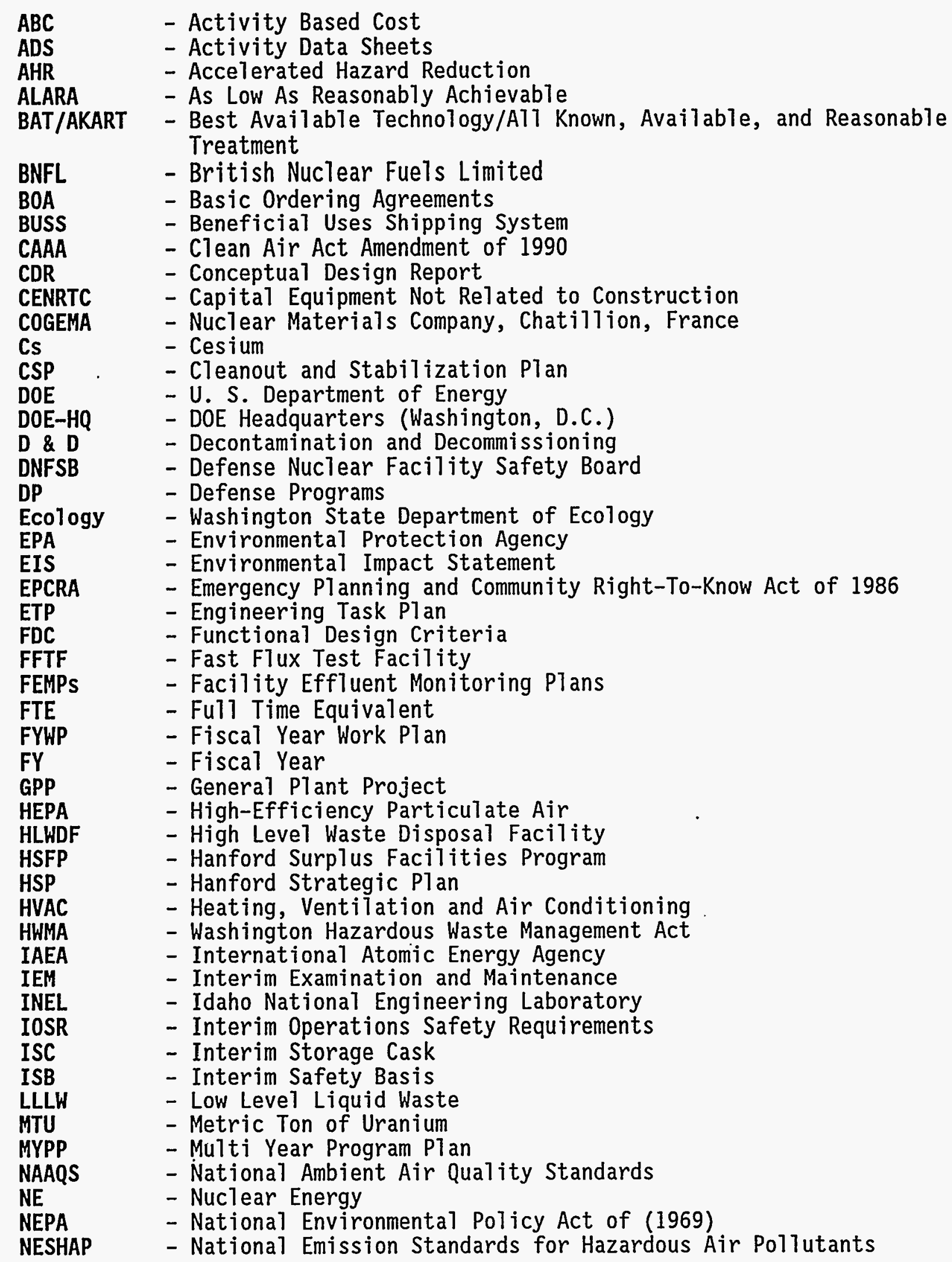




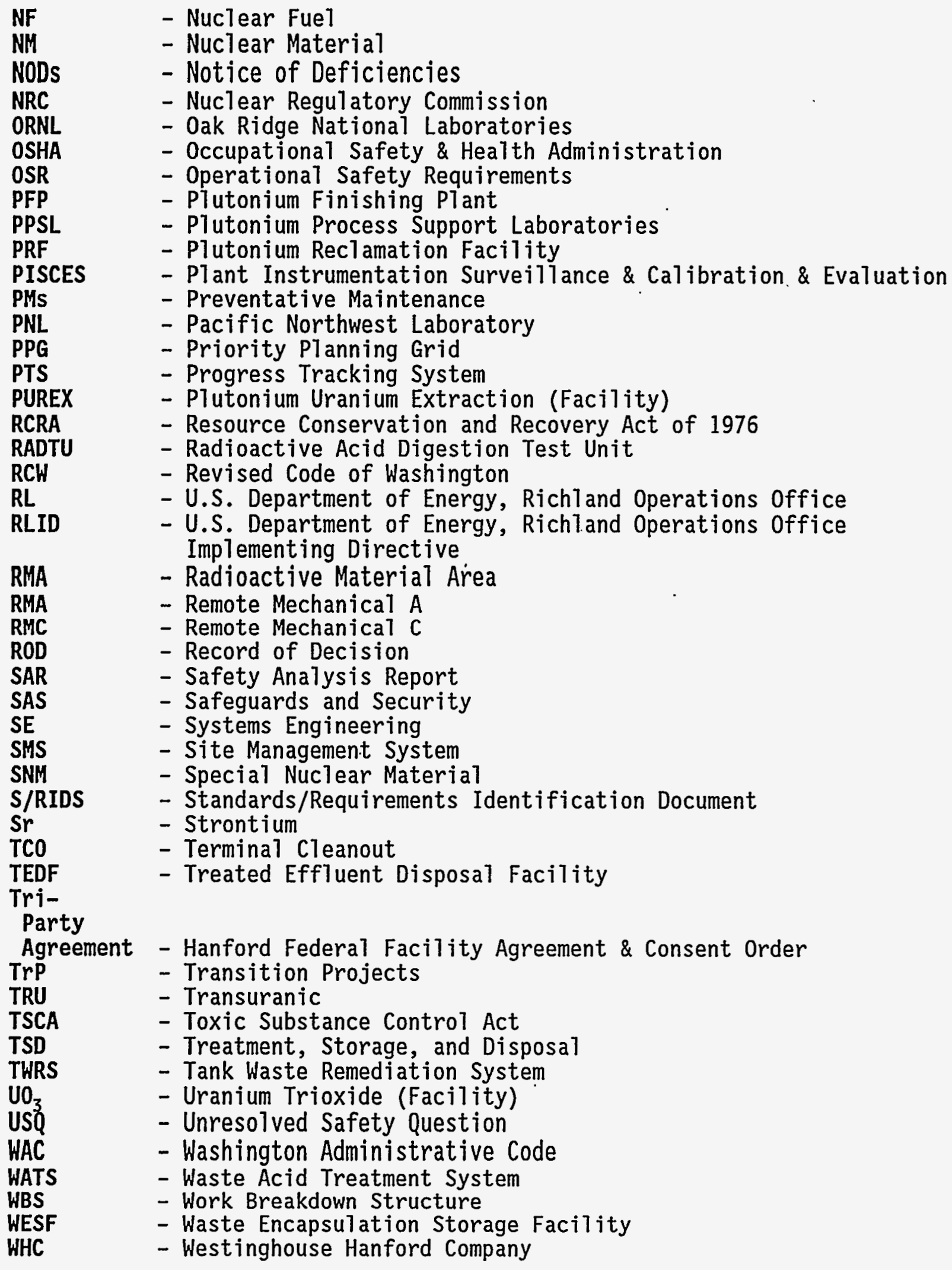




1.A Program Mission

The primary Transition Projects mission is to deactivate facilities on the Hanford Site, in preparation for Decontamination and Decommissioning (D\&D), and to provide safe and secure storage of special nuclear materials, nuclear materials, and nuclear fuel (SNM/NM/NF). Transition Projects will protect the health and safety of the public and our workers, protect the environment, and provide beneficial use of the facilities and other resources. Work will be in accordance with the Hanford Federal Facility Agreement and Consent Order (Tri-Party Agreement), loca1, national, international and other agreements, and in compliance with all applicable Federal, state, and local laws. The stakeholders will be active participants in the decision processes including establishing priorities, and in developing a consistent set of rules, regulations, and laws. The work will be leveraged with a view of providing positive, lasting economic impact in the region. Effectiveness, efficiency, and discipline in all mission activities will enable us to achieve our mission in a continuous and substantive manner.

As the mission for Transition Projects has shifted from production to support of environmental restoration, each facility is making a transition to support the Site mission. The mission goals include the following:

- Achieve deactivation of facilities for transfer to the Hanford Surplus Facility Program (EM40), using Plutonium/Uranium Extraction (PUREX), plant deactivation as a model for future facility deactivation.

- Manage nuclear materials in a safe and secure condition and where appropriate, in accordance with International Atomic Energy Agency (IAEA) safeguards rules.

- Treat nuclear materials as necessary, and store onsite in long-term interim safe storage awaiting a final disposition decision by U.S. Department of Energy (DOE).

- Implement nuclear materials disposition directives. In the near term these are anticipated to mostly involve transferring uranium to other location for beneficial use.

- Work will be in accordance with the Tri-Party Agreement, and other agreements and in compliance with all applicable Federal, state and local laws. 
1. B Program Strategy sus

The overal1 Transition Projects strategy is to transition former Defense Production facilities to a deactivated condition ready for transfer to the EM-40, while maintaining the facilities in a safe and compliant configuration. The transition to deactivation will be accomplished through a phased approach. In addition, Transition Projects will continue to maintain safe long-term storage facilities for Special Nuclear Material (SNM), Nuclear Material (NM), and Nuclear Fuel (NF).:

The Transition Projects strategy aligns with the deactivate facilities mission outlined in the Hanford Site Systems Engineering document and the Hanford Strategic PIan (HSP). Inherent to the Transition Projects strategies are specific HSP goals such as (1) managing and reducing hazards, (2) enhancing worker safety and health, (3) managing cleanup as a project, and (4) building partnerships.

- Facilities having no confirmed future use will be rapidly. deactivated to the point where a stable configuration exists which poses a low risk to follow-on Decontamination and Decommissioning (D\&D) activities.

- Facility deactivation plans will be developed to manage deactivation and will include a defined turnover endpoint where deactivation activities end and long term surveillance and maintenance and D\&D begin.

- Radioactive and hazardous materials will be identified and removed or stabilized in place and SNM/NM/NF will be removed from the facility. Utilities and facility inter-ties will be deactivated except as required for facility surveillance and security.

- Oniy those systems and portions of a facility involved with the active safety and compliance envelope will be maintained and operated at the completion of deactivation. All aspects of the safety envelope will be challenged during the deactivation process to reflect the continual reduction in the safety and compliance envelope. All facility reconfiguration will be performed under Occupational Safety and Health Administration (OSHA) construction requirements.

- Because long time periods may be expected between deactivation and final disposition, full characterization of the facility will be performed while knowledgeable facility staff are available and records are fresh. A graded approach to characterization of the facility will be employed to focus on high risk areas. 


\section{TRANSITION PROJECTS}

1.3.1/7.1/6.12

1 B Program Strategy

- SNM/NM/NF materials will be consolidated early in the facility deactivation process to minimize the safety and compliance envelope. Where feasible, these materials should be removed from the facility early in the deactivation process. When deactivation is complete a facility will no longer contain SNM/NM/NF.

- Stakeholders will be actively involved during the development of deactivation plans and their execution

The long term storage mission within Transition Projects aligns with the storage, treatment and disposition function from the Hanford Site Systems Engineering document and the Hanford Strategic Plan:

- SNM/NM/NF will continued to be safely and compliantly stored until a national policy on their disposition is formulated. SNM/NM/NF will be transferred to other beneficial uses where possible.

- The current safety and security approach, with necessary interim upgrades, will be continued until a preferred longer term approach is selected.

- The criteria for the receipt, storage, treatment, and disposition of SNM/NM/NF will continue to be developed, negotiated, and implemented.

- SNM/NM/NF will be stored in a safe storage mode, and treated where necessary to provide safe storage. Studies to determine the best alternatives for onsite material storage will continue.

- Stakeholders will be actively involved in the development of long term treatment, storage and disposition plans through the National Environmental Polity Act (NEPA) process.

- Treatment for ultimate disposition will await determination of the final disposition.

To support the capability to manage the site cleanup as a project and the overall Transition Projects mission, Systems Engineering is being utilized in each program element to aid in the enhancement of the technical functions and requirements. As a part of this systems engineering effort, each program element is currently developing the following documents: Mission Analysis, Functions \& Analysis and a Product Breakdown structure. These documents will form a portion of the technical baseline and provide guidance for work to be performed within each program element. Upon approval of the Transition Projects Systems 


\section{B Program Strategy}

Engineering effort, change control will be utilized to incorporate revisions to the current baseline as contained within this Program PTan.

The following are the specific program element mission strategies:

\section{B Plant/WESF (1.3.1.7)}

The B Plant/Waste Encapsulation Storage Facility (WESF) Transition Project is moving rapidly towards a minimum cost safe storage mode pending transfer to EM-40 through accelerating the reduction of known hazards and aggressive transition activities. During the accelerated hazard reduction and transition activities, the facilities will be maintained in a safe, secure, and environmentally compliant status.

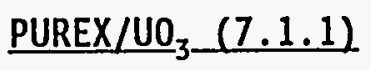

The Plutonium Uranium Extraction Facility (PUREX)/Uranium Trioxide $\left(\mathrm{UO}_{3}\right)$ Deactivation Project will demonstrate a safe, cost-effective model for facility transition of the PUREX and $\mathrm{UO}_{3}$ Plants and completing the transfer to the Richland Environmental Restoration Program (EM-40). The project's chief emphasis is on the transition activities that remove, reduce, and/or stabilize the major remaining radioactive sources and hazardous sources within the confinement structure. During transition the Project is responsible for the safe, secure, and environmentally sound management of the facilities. The surveillance and maintenance requirements will be maintained at the minimum safe compliance level. ABC Estimating, Re-engineering, and Breakthrough should lead to the cost effective model for future deactivation projects to improve upon.

\section{Area Fuel Supply $(7.1 .2)$}

The Fuel Supply Shutdown Facility will be maintained in a regulatory-compliant state until turnover to EM-40 is completed. Shutdown activities will be performed as described in the "Shutdown Plan for the 300 Area Fuel Supply Facilities". In addition, compliant storage of 1,900 metric tons (MT) of SNM is being maintained until relocation of material to the 400 Area (or alternate location) in Fiscal Year (FY) 1998. Compliant storage will be provided in the alternate location.

Resource Conservation and Recovery Act of 1976 (RCRA) implementation activities are also being carried out per the following RCRA Closure PTans: 304 Concretion, 303K Facility, and Waste Acid Treatment System (WATS). 


\section{TRANSITION PROJECTS}

\section{B Prögram Strategy}

New planning does not include 313 Building Demolition being performed in this planning period. As an alternate, the 313 Building south portion will be isolated with no access or utilities until turnover.

Upon completion of shutdown, RCRA activities, and SNM relocation (and/or disposition) of all Fuel Supply buildings will be ready to be transferred to EM-40.

\section{PFP $(7.1 .3)$}

The mission strategy for the Plutonium Finishing Plant (PFP) is to prepare the PFP Complex to implement the Record of Decision (ROD) resulting from the PFP Environmental Impact Statement (EIS). This currently entails the following activities:

- Implementation of Defense Nuclear Facility Safety Board (DNFSB) Recommendation 94-1 by safely stabilizing reactive scrap inventories, initiating terminal cleanout activities, implementing required capital upgrades (New Packaging System, Continuous Calciner, Vault Modifications, etc.) and stabilizing vault material by calcination or other methods as appropriate in order to complete these activities by May 2002;

- Transition parts of the PFP Complex into a D\&D ready state, including the implementation of ductwork remediation and radiation zone reduction activities as necessary;

- Continue to maintain the safety envelope for the PFP Complex as defined in approved Operational Safety Requirement documentation;

- Safely and securely operate the PFP Vault complex to receive, store and ship SNM consistent with applicable Federal, State and International regulations and requirements; and,

- Continue to complete scheduled environmental upgrades and perform activities necessary to maintain compliance with environmental regulations and compliance agreements. 


\title{
TRANSITION PROJECTS
}

FY 1996 MYPP

\author{
1.3.1/7.1/6.12
}

1. B. Program Strategy

Program and Environmental Management (7.1.6)

The Program and Environmental Management program element provides centralized program/project management to plan, execute and control the Transition Projects baseline. This program element also provides for common safeguard and security support, centralized coordination of environmental compliance activities, systems engineering, communications support, management of SNM, and conduct of operations support.

范 


\section{B.1 Technical Functions/Requirements}

\section{B Plant/WESF $(1.3 .1 .7)$}

Technical Functions and Requirements for the operation and transition of B Plant and WESF were established during the fall of 1994 and a report prepared for each facility. The WESF report is documented as WHC-SD-WM-MAR-007, Rev 0, dated ApriT 1995 while the B Plant report, dated September 26, 1994 is in final draft form.

B Plant Technical Functions/Requirements

The B Plant Technical Functions and Requirements work structure as defined to the second level of detail in the September 26, 1994 draft B Plant Functions and Requirements Report and further refined is as follows:

- Reduce the outyear costs for maintaining B Plant/WESF in safe storage via reducing the hazards and residual inventories as quickly as possible

- Mitigate significant outdoor, indoor and canyon radiological risks

- Remove organics from the canyon

- ETiminate the requirements for steam

I solate retired HEPA filter systems from the environment

El iminate WESF's dependency on B PTant utilities and systems

- Reconfigure B Plant for minimum surveillance

Update and implement the safety basis for B PI ant to reflect current hazards

- Modify B Plant for minimum maintenance and operations cost

- Deactivate non-essential plant systems, components, and structures

- Disposition plant resources and waste

- Transfer stabilized B Plant facilities

- Determine the final B Plant deactivation plan and negotiate the turnover endpoint

- Characterize the B Plant initial state

- Develop B Plant deactivation plans (see B Plant Cleanout and Stabilization Plan (CSP), WHCSD-WM-AP-023, Rev 3.)

- Negotiate and maintain B Plant turnover criteria, specifications, and documentation - Design the B Plant turnover state

- Maintain the B Plant safety and compliance envelope

- Maintain safe and compliant B Plant operations

- Maintain safe and compliant B Plant systems, equipment, and structures

- Maintain B Plant safety and compliance documentation 


\section{TRANSITION PROJECTS}

\section{B.1 Technical Functions/Requirements}

- Maintain qualified B Plant staff

- Assess B Plant safety and compliance states

- $\quad$ Operate and maintain B PI ant systems supporting WESF

WESF Technical Functions/Requirements

The WESF Technical Functions and Requirements work structure as defined to the second level of detail is as follows:

- Maintain safety envelope for Cesium(Cs)137 and Strontium(Sr)90 capsule storage

- Maintain safe and compliant operations

- Maintain safe and compliant systems, equipment, and structures

- Maintain safety and compliant documentation

- Maintain qualified facility staff

Upon completion of the transfer of $C S$ and $S r$ capsules to final disposal, the transition of WESF to EM-40 will be performed in a similar manner as B Plant, with virtually identical Functions and Requirements. 
1.B.1 Technical Functions/Requirements

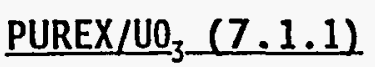

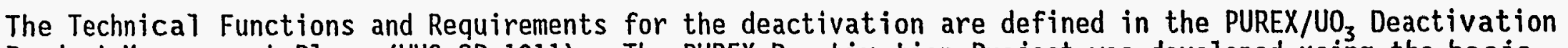
Project Management Plan, (WHC-SP-1011). The PUREX Deactivation Project was developed using the basic principles of a system engineering approach. This entailed continued operation of those parts of the plant needed for safe operation including the required safety and operating documentation, as well as ongoing surveiliance requirements. Before the draft Project Management Plan, the facility was informally characterized and technical alternatives were outlined for major deactivation activities.

A11 deactivation activities, including assumptions, were reviewed by an Independent Technicai Review Team commissioned by the U.S. Department of Energy (DOE) Headquarters (DOE-HQ). The results of these reviews were documented as an action plan in the Project Management ScheduTes for deactivation. The deactivation schedules 1 ist all activities, including appropriate engineering studies where alternatives need to be defined for decision making, as well as field actions, including interfaces and field end point criteria development/negotiation for plant transfer to EM-40. The following sections in the Project Management Plan contain the key information in defining the Technical Functions and Requirements:

\begin{tabular}{l} 
Section \\
\hline 2.2 .1 \\
2.2 .2 \\
2.2 .3 \\
2.2 .4 \\
2.2 .5 \\
2.2 .6 \\
2.2 .7 \\
APPENDICES \\
A \\
B \\
C \\
D \\
E \\
F \\
G \\
H
\end{tabular}

\section{Tit7e}

General Technical Requirements Configuration Requirements

Workforce Restructure Requirements

Hazardous and Radioactive Materials Removal/Stabilization Requirements Surveillance Requirements

Operational Readiness Reviews

Systems Engineering

Title

REGULATORY COMPLIANCE PLAN

PUREX/UO 3 DEACTIVATION STANDARDS PROGRAM

END STATE CRITERIA

STAKEHOLDER INVOLVEMENT PLAN

SURVEILLANCE AND MAINTENANCE PLAN

SAFETY DOCUMENTATION PLAN

TECHNICAL PLANNING BASES

DOE ORDER 4700. I COMPLIANCE MATRIX 
1.B.1 Technical Functions/Requirements

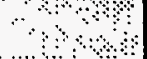

Technical functions to transition PUREX $/ \mathrm{UO}_{3}$ and transfer the facilities to Richland Environmental Restoration Program (EM-40) include:

- Organic disposal, recovered acid removal, cold chemical disposal, radionuclides removal from waste concentrator systems, disposal of plutonium nitrate/uranium nitrate solution.

- Contamination reduction of $\mathrm{N}-\mathrm{Ce} \mathrm{ll}, \mathrm{Q}-\mathrm{Ce} 11$, and product recovery room, flushing of radionuclides and chemicals, disposition/disposal of silver reactors, disposition of fuel elements in $A, B$, and CCelis, disposition of aluminum clad fuel in the slug storage basin, and transfer of solutions within the plant to a permitted waste storage/treatment facility.

Surveillance and maintenance require the following technical functions:

- Provide direct facility supervision and support personnel for activities including but not limited to the following: Health and safety surveillance; radiological, routine and special surveillance; facility maintenance; DOE Rule Compliance; facility ventilation and power operation; solid waste handling; alarm response; and general facility housekeeping per the following DOE Orders: 4330.4B, $5400.5,5480.11,5480.19$, and 5482.1B.

- Surveillance and maintenance activities will be phased out throughout the deactivation period.

Environmental management requires the following technical functions:

- Support Washington State Department of Ecology (Ecology) and the Environmental Protection Agency (EPA) in conducting hazardous waste, and mixed waste inspections per the following drivers: DOE Order 5400.1, Washington Hazardous Waste Management Act (HWMA), Revised Code of Washington (RCW) 70.105.130 and Washington Administrative Code (WAC) chapter 173-303.

- Prepare or update waste minimization plans and reports, dangerous waste reports, Emergency Planning and Community Right-to-Know Act of 1986 (EPCRA) emergency/hazardous chemical inventories, and waste reduction reports when required per the following drivers: DOE Order 5400.1, HWMA, RCW 70.105.130 and WAC chapter 173-303.

- Update Facility Effluent Monitor Plans (FEMPs) annualiy, issue annual air emission reports, provide facility-specific air emission information, and conduct air emission inventories. 


\section{B. 1 Technical Functions/Reqüirements}

\section{Area Fuel Supply (7.1.2)}

Technical functions that are required to complete shutdown and turnover of the Fuel Supply complex to EM40, while maintaining the facility in a compliant state include the following:

- Provide direct facility supervision and support personnel for activities including, but not 1 imited to, the following: Health and safety surveillances; radiological, routine and special

surveillances; facility maintenance; DOE Rule Compliance; facility ventilation and power operation; solid waste handling; alarm response; and general facility housekeeping per the following DOE Orders: $4330.4 \mathrm{~B}, 5400.5,5480.11,5480.7,5480.19,5482.1 \mathrm{~B}, 10 \mathrm{CFR} \# 830.120,10 \mathrm{CFR} 835$.

- Support Ecology and the EPA in conducting hazardous waste and mixed waste inspections per the following drivers: DOE Order 5400.1, HWMA, RCW 70.105.130, and WAC chapter 173-303.

- Prepare or update waste minimization plans and reports, dangerous waste reports, EPCRA emergency/hazardous chemical inventories, and waste reduction reports as required per the following drivers: DOE Order 5400.1, HWMA, RCW 70.105.130, and WAC chapter 173-303.

- Update Annual Waste Water Discharge Report, issue annual air emission reports, provide facilityspecific air emission information, and conduct air emission inventories per the following drivers: Clean Air Act Amendments of 1990 (CAAA), National Emission Standards for Hazardous Air Pollutants (NESHAP) (40 CFR 61), and National Ambient Air Quality Standards (NAAQS) (40 CFR 50).

- Compliance with RCRA, OSHA, and Toxic Substance Control Act (TSCA) requirements.

- Provide required operations, maintenance, and surveillance necessary to continue safe storage and transfer of 1900 MT of SNM per the following DOE Orders: 5630.11B, 5630.15, 5633.3B, (RLID 5633.3), $5480.1 B$, and 5632.1C.

- Isolation of 313 South Building: remove all 313 South utilities, roof loadings, (i.e. Heating, Ventilation, and Air Conditioning (HVAC) units, etc) complete NEPA, historical studies, complete sampling/characterization activities, remove (or stabilize) unattached hazardous materials (or stabilize) and unattached radiologically contaminated materials. 


\section{B. 1 Technical Functions/Réquirements}

- Complete all activities identified in the "Shutdown Plan for the 300 Area Fuel Supply Facilities," WHC-SD-FL-SSP-002 and revise planning efforts to meet criteria for transfer to EM-40. Listed below is a partial summarization of these activities:

- Removal of alt contaminated liquids.

- Removal of all possible low-level, mixed, and hazardous wastes per criteria agreed upon with EM-40.

- Removal of all possible hazardous materials per criteria agreed upon with EM-40.

- Deactivate remaining fire suppression systems.

- Deactivate and isolate utility systems where appropriate.

- Determine that facilities are structuraliy sound for deferred demolition or take corrective action required to meet $E M-40$ criteria.

- Eliminate all temporary surface contamination zones.

- Eliminate or stabilize surface contamination zones.

- Disposal of assets per DOE requirements.

- Attempt Closure of three remaining RCRA Permitted Treatment, Storage, and Disposal (TSD) systems.

- Preparation of facility documentation required to support transfer to EM-40.

- Relocation of SNM to 400 Area or alternate storage facility. 
1.B.1 Technical Functions/Requirements $\quad \cdots \quad \because \quad, \quad$

PFP (7.1.3)

The technical functions to be accomplished at PFP to support the Transition Projects mission of transitioning former production facilities to deactivation include:

- Operate and maintain PFP in a formal, safe and secure manner in compliance with applicable regulations and DOE Orders.

- Materials that remain in plant ducting will begin to be removed and dispositioned as a number of sections are removed. This will contribute to the ultimate decrease for the continuing 1 evel of accountability, surveillance, and maintenance support required.

- Storage activities to include surveillance, occasional container handling required for stabilization of materials to the specifications in the DOE Standard - Criteria for Safe Storage of Plutonium Metals and Oxides (DOE-STD-3013-94), and shipping and receiving of SNM as directed by the DOE.

- Operate and maintain the following buildings to support the material management, material dispositioning, material stabilization and/or deactivation activities:

- Major former operations buildings currently being utilized to support the material management and material dispositioning activity are 234-5Z, 236-Z, 241-Z, 242-Z, 243-Z, 291-Z, 2736-Z, and 2736-ZB.

- The 234-5Z building houses the standby Remote Mechanical C (RMC) and inactive Remote Mechanical A (RMA) Lines, Analytical Lab and Plutonium Process Support Laboratories (PPSL), various workshops, store rooms, offices, SNM vault storage and locker rooms.

- The 236-Z building houses the standby Plutonium Reclamation Facility (PRF).

- The 241-Z building houses tanks which receive and act as intermediate storage for high and low salt chemical wastes formerly produced by the RMC and PRF production processes and the laboratories prior to transfer to the tank farms.

- The $243-Z$ building is the low level waste treatment facility which treats liquid effluents: 


1. B I Techn cal Funct ons /Requirements

- The 291-Z building contains equipment to provide the exhaust ventilation and other support services, such as instrument air, emergency exhaust steam turbine ventilation, and cooling water support to the 234-5Z, 236-Z, and $242-Z$ buildings.

- The 242-Z building is currently in layaway status awaiting final decontamination.

- The 2736-ZB building is the central point for al1 SNM product handling operations pertaining to shipments, receipts and storage of SNM. Vauits for plutonium storage are located in the $2736-Z$ and $2736-Z B$ buildings.

- Technical training support is included which consists of development of training certification packages and performance of training and testing, as required by DOE Order 5480.20.

- Perform engineering studies, prepare functional design criteria, manage conceptual design, and provide expense support for capital projects necessary to correct identified deficiencies, as required by RCRA and WAC $173-303$.

- Develop and submit RCRA Part A and B permit applications and closure plans for TSD units at PFP (M20-48) and respond to Notices of Deficiencies (NODs). Prepare waste handling and minimization plans and reports, dangerous waste and chemical inventory reports, process waste assessments, etc. per DOE Order 5480.4. Conduct and report on integrity assessments of dangerous waste tank systems and perform required environmental evaluations.

- Perform evaluations and engineering studies utilizing Best Available Technology/A11 Known, Available, and Reasonable Treatment (BAT/AKART) for air and liquid effluents.

- Conduct air emission inventories, update FEMPs, assess compliance with NESHAPs (40 CFR 61) monitoring requirements, and support preparation of annual Hanford Site air emissions reports.

- As a material management responsibility PFP will continue to store most of the remaining SNM at the Hanford Site in safe, secure vaults within the PFP protected area per DOE Orders 5630.11A, 5633.2A and 5480.1B. Operation of storage vaults, shipping/receiving capability and associated security and support requirements will continue indefinitely until the SNM is dispositioned for disposal or transferred to another onsite or offsite facility. 


\section{TRANSITION PROJECTS}

1 B. Technical Functions/Requirements

- As part of the facilities transition to deactivation a review of the present status of essential PFP building support services and equipment to indicate which enhancements are necessary will be performed. It is necessary to determine the support required for stabilization, terminal clean out, and vault storage activities, as requested by DOE. It.is anticipated that the maintenance or installation of support systems will be needed for laboratory activities, vault storage, and other long-term plant activities per the Hanford Site Integrated Stabilization Management Plan. This includes the following:

- Installation of the packaging and load-out system and associated vault modifications to allow for the implementation of DOE Standard DOE-STD-3013-94 as part of the material dispositioning responsibility. Vault modifications refers to the changes necessary to handle the to-bedetermined standardized Pu storage container. Expected vault changes are the container storage racks, security instrumentation, container detectors, security bars, shielding, and any ancillary modifications once the Pu storage container configuration is available from the DOE.

- Upgrades to the existing PFP laboratories to enable complete characterization of plutonium-bearing materials and waste, part of the material dispositioning responsibility.

- Upgrades to the existing facility infrastructure to enable the facility to support plutonium handling in a cost-effective manner. These upgrades are not fully defined but include replacement of the steam system with electric service, replacement of old ventilation control equipment with more reliable and maintainable systems, and modification of facilities as. required to support the general transition and deactivation of the PFP complex.

- Modernization of the existing Safeguards and Security (SAS) systems to provide maintainable remote inventory and surveillance capabilities, part of the material management responsibility.

- Maintain facility Safety Analysis Report (SAR) and safety documentation per DOE Orders, particularly 5480.23 .

- Provide technical support to the preparation of the EIS to determine cleanup options, including analysis of any future environmental and waste management missions at PFP. The EIS will: 
1.B.1 Technical Functions/Requirethents $\quad \therefore \quad$ s.

- Evaluate storage and treatment of all materials remaining within the PFP.

- Identify the preferred disposition of chemically reactive plutonium bearing materials remaining after interim actions including the bulk of the nitrate solutions, and other plutonium bearing materials not suitable for long term vautt storage.

- Determine the most reasonable method to handle remaining un-stabilized Pu-bearing materials and to determine the future mission of the. PFP.

- Support for all general plant and small plant projects as well as support for two line-item-funded projects envisioned as part of DNFSB 94-1: one for the new packaging system and associated vault modifications, and one for the thermal treatment system. The SAS system modernization is expected to be expense-funded replacement-in-kind. The laboratory upgrades are expected to be within the constraints of a general plant project.

- A dose evaluation engineering study on the overall furnace stabilization system will be performed to ascertain if individual dose rates. for stabilization and cleanup activities can be held within the administrative limit of 1,500 millirem per year with all systems operational and at projected staff levels. The study will map the existing dose rates in the processing gloveboxes areas, model the individual processing operations and calculate worker dose. Based on the above evaluations the total dose commitment will be calculated for operation of the entire system. If radiation doses cannot be maintained within acceptable limits, a continuous calciner will be designed to replace the additional furnace operations.

- Containers of plutonium-bearing residues shall be characterized and classified according to their contents and container integrity into categories for indefinite storage as is, indefinite storage after repackaging, needs thermal stabilization and packaging for indefinite storage, needs denitration, thermal stabilization, and packaging for indefinite storage, needs pyrolysis, thermal stabilization, and packaging for indefinite storage, or packaging and disposing of as waste.

- Vault \#3 will remain under the custody of the IAEA and once vault modifications for the new DOE packaging standard are effected other vaults may be turned over. 
1.B.1 Technical Functions/Requirements

\section{Program and Environmental Management (7.1.6)}

The Technical Functions and Requirements of the Program and Environmental Management program element are to:

- Provide management support for resources and activities that are common to all Transition Projects plants to include program and financial management, commitment planning, systems engineering, nuclear materials management, and conduct of operations.

- Provide the non-plant specific 200 Area Transition Projects safeguards, security, and accountability functions required to meet DOE Order 5633.3 .

- Provide program management and direction, oversight, and a centralized point-of-contact for Transition Projects environmental and historical preservation activities.

- Develop and implement the policies and procedures for the use, control and disposition of nuclear material to ensure compliance with DOE Order 5660.1B.

- Provide company wide Nuciear Material Management functions in accordance with D0E Order 5660.1B.

- Administer the Site Management System (SMS) and Progress Tracking System (PTS) reports, and develop required planning documents to include Five Year Plan Activity Data Sheets (ADS), and a Multi-Year Program PIan (MYPP) as required by DOE Order 4700.1, DOE, Richland Operations Office (RL) Policy Directive (RLPD) 5000.1, and RL Implementing Directives (RLID) 5000.1 and 5000.2 .

- Develop the Hanford Material Management Plan and all other reports mandated by DOE Order 5660.1B.

- Manage the Lessons Learned Program, and Conduct of Operation Assessment Program across the site, in accordance with DOE Order 5480.19.

- Provide special analysis in response to DOE requests on issues related to storage, stabilization, disposition, and transfer of nuclear materials.

- Provide technical support to DOE initiatives associated with the storage and disposition of plutonium. 


\section{TRANSITION PROJECTS}

1.B. 1 Technical Functions/Requilrements

- Provide long range strategic planning to define Hanford's future plutonium mission(s) and options for implementation.

- Perform systems engineering at program and project levels.

- Provide communications support for Transition Projects. 


1. B.2Program objectives $3+1$

\section{B Plant/WESF (1.3.1.7)}

The top leve1 B PIant/WESF Program Mission objectives are as follows:

- Transition B P1 ant and its auxiliary facilities to a safe and cost effective surveillance and maintenance configuration, and effect an orderly turnover to EM-40 by the year 2002.

- $\quad P 7$ an for and disposition the cesium and strontium capsule inventory to productive uses by others and/or effect an orderly turnover of the capsules to the High Level Waste Disposal Program by the year 2011 .

- Transition WESF and its auxiliary facilities to a safe and cost-effective surveillance and maintenance configuration and effect an orderly turnover EM-40 by the year 2013.

- Maintain 75 million curies of cesium and strontium capsules in safe, environmentally sound, and cost effective storage.

- Maintain the B Plant/WESF

environmentaliy sound, cost-effective condition.

Key near term objectives have been selected that are designed to aid in the fulfillment of the top level objectives. These objectives, organized by Work Breakdown Element, are as follows:

\section{PROGRAM MANAGEMENT AND ADMINISTRATION}

- Prepare and submit an annual budget request in the form of Activity Data Sheets and comprehensive Multi-Year Program P1ans which establish the scope, schedule, and cost baseline for the program.

- Maintain program baseline management including schedules, budgets, and reports.

- Define and maintain a training baseline and provide schedule and reporting services to the facility.

\section{B PLANT SURVEILLANCE AND MAINTENANCE}

Implement the B Plant Interim Safety Basis documentation.

Manage the nuclear, chemical, and industrial hazards present in the facility pending their elimination.

- Develop, implement, and maintain safety basis documentation for B Plant.

- Coordinate implementation of disciplined Conduct of Operations, maintenance, and training throughout B Plant.

- Complete required surveillance and maintenance of essential facility systems.

- Develop and maintain an effective Emergency Preparedness program. 
1.B.2 Program objectives

- Develop new and update current operational and maintenance procedures to support facility evolutions.

\section{TRANSITION}

Complete surface decontamination of B Plant and WESF areas external to the canyons.

Complete the radiological hazard reduction for the 221-B canyon.

Isolate and stabilize the retired exhaust air filter systems.

Remove and dispose organic inventory in B Plant.

Eliminate the need for steam in B PI ant and WESF by switching to alternative methods for heating and providing motive power.

- Complete engineering, design, and construction necessary to make the operation of WESF independent of B Plant.

WESF OPERATIONS, MAINTENANCE, AND UPGRADES

- Prepare and issue Life cycle alternative study(s) for disposition of cesium and strontium capsules. Develop, implement, and maintain the safety basis documentation for WESF.

Develop an effective capsule inspection, leak detection and recovery system.

\section{REGULATORY COMPLIANCE}

- Prepare a Standards and Requirements Identification Document (S/RID) for B PIant and WESF. Assess compliance with the B Plant / WESF S/RID.

Maintain and develop environmental permitting, compliance, and management documentation.

\section{CESIUM RECOVERY PROGRAM}

- Return 25 capsules from ARECO in Lynchburg, Virginia to WESF for storage and exit the facility without incident.

- Reencapsulate failed and suspect cesium capsules into a configuration acceptable for WESF pool cell storage.

- Return all cesium and strontium capsules from the Pacific Northwest Laboratory (PNL) including those shipped to PNL for reencapsulation.

- Maintain the NuClear Regulatory Commission. (NRC) and DOE Certificates of Compliance for the Beneficial Uses Shipping System (BUSS) cask. Maintain the cask in a state of readiness for use. 


\section{TRANSITION PROJECTS}

\section{B.2 Program objectives}

\section{PUREX $/ \mathrm{UO}_{3}(7.1 .1)$}

The PUREX/UO $\mathrm{O}_{3}$ Deactivation Project objectives support the DOE Office of Nuclear Materials and Facility Stabilization (EM-60) of developing swift, uniform methods for deactivating similar facilities. The Project objectives are as follows:

- Establish a safe and environmentaliy secure configuration of the plant (no active internal functions or equipment with in confinement), and retain that configuration for a 10-year horizon.

- Ensure worker health and safety throughout the life of the Project. Maintain a high degree of emphasis toward worker health and safety by applying a graded approach to implementing safety controls, providing adequate worker training, and a strong emphasis on conduct of operations.

- Achieve a total yearly cost target of less than $\$ 2$ million/year at turnover.

- Implement cost-effective, industry standards, innovative approaches to ensure the required safety envelope is defined and maintained during transition.

- Achieve compliance with environmental, safety, and health codes and standards during transition.

- Involve stakeholders in the development of the PUREX/UO $\mathrm{O}_{3}$ Deactivation Project Management Plan and ensure continued involvement through the life of the Project.

- Transition the workforce out of the PUREX and $\mathrm{UO}_{3}$ plants through redeployment or outplacement.

- Apply lessons learned from commercial deactivation experience.

- Establish the PUREX/UO $\mathrm{O}_{3}$ Deactivation Project as a model for canyon facilities.

300 Area Fue1 Supply (7.1.2)

The overall' objective of the Fuel Supply Shutdown project is to perform shutdown activities and transfer facilities to EM-40, while maintaining compliance with applicable DOE, state, and federal requirements. 


\section{B.2 Program objectives}

- Provide required administrative, safeguard, maintenance, and surveillances necessary to continue safe storage of 1900 MT of Special Nuclear Materials (SNM).

- Provide réquired surveillance to maintain RCRA compliance until closure, including safety, quality, environmental, and security requirements. Provide maintenance to meet Interim Operations Safety Requirements (IOSR), and code requirements, and to maintain a safe work place.

- Maintain the following programs for Fuel Supply: Conduct of Operations, Lessons Learned, Systems Engineering, Self-Assessment, Radiological Control, Fissile Material Facilities, Safety Documentation, Unresolved Safety Question (USQ), Priority Planning Grid (PPG) System, Occurrence Reporting, etc..

- Specific objectives relating to RCRA permitted TSD Closure Activities for 304, 303K, and WATS are as follows:

- Continue required closure plan reviews and support monthiy Unit Manager Meetings with Ecology.

- Complete closure of 304 Concretion and $303 \mathrm{~K}$.

- Complete closure of WATS.

- Specific objectives relating to the isolation of the 313 South building (to decrease a serious safety hazard caused by an unsafe roof) are as follows:

- Complete 313 isolation of South Building access.

- Remove all 313 South utilities.

- Remove 313 South roof Toadings (i.e. HVAC units, etc.)

- Complete NEPA, historical studies, and sampling/characterization activities.

- Stabilize or remove unattached hazardous materials.

- Stabilize or remove unattached radiologically contaminated materials.

- Transfer Fuel Supply to EM-40 by completing the following shutdown activities:

- Clean and stabilize each building per criteria agreed upon with EM-40.

- Clean and stabilize trenches from interconnecting buildings.

- Complete phase I building shutdown (303M \& East Storage Pad, 304/304A, and 311 Tank Farm). 


\section{TRANSITION PROJECTS}

\subsection{1/7.1/6.12}

\section{B.2 Progran objectives}

- Complete phase II building shutdown (303B, 303G, 303F, 313S, 334, 334 Tank Farm).

- Relocation of SNM.

- Complete phase III building shutdown (3712,3716,303A, 303E, 303K/3707G, 313N, 333, 334A, FSS Trenches, and M0-52).

- Turnover Fuel Supply Shutdown Complex to EM-40.

PFP (7.1.3)

The overall objective of the Plutonium Finishing PIant (PFP) Transition Program is to manage the PFP complex to a deactivated condition through rigorous stabilization activities while maintaining the facilities in a safe and environmentally sound condition, and while performing Material Management and Material Dispositioning activities as part of deactivation process. As part of the overall objective plutonium vulnerability corrective actions will be undertaken. Many of the identified vulnerabilitjes will be corrected through the stabilization and packaging activities required by the WHC response to the DNFSB Recommendation 94-1 (WHC-EP-0853), while others will be corrected as a part of plutonium hand1 ing facilities transition (deactivation) to the Environmental Restoration Program. It is expected that the result of the PFP EIS ROD will be consistent with these actions. Specific activities to be performed are as follows:

- Perform material stabilization and dispositioning in order to meet the DNFSB 94-1 May 2002 completion and facility stabilization by the 2007 schedule. This will result in the stabilizing and/or dispositioning of approximately 4 metric tons, net weight, of plutonium distributed among approximately 8,300 items. These items constitute a wide range of chemical and physical properties, including metals, oxides, ash, sludges, solutions, combustibles, and other residues. The material stabilization and/or disposition objectives include the following;

- 4,600 liters of plutonium-bearing solutions;

- $\quad 1,893$ bulk kilograms of plutonium-bearing reactive solids;

- 2,700 pieces of plutonium-bearing polystyrene cubes;

- $\quad 7,560$ bulk kilograms of interim stable plutonium-bearing solids; and 


\section{TRANSITION PROJECTS}

1.B.2 Program objectives

- $\quad 340$ bulk kilograms of interim stable plutonium-bearing solids that will be repackaged in the Hanford interim storage container.

- Approximately half of PFP's current inventory of plutonium-bearing material is expected to be covered by DOE Standard DOE-STD-3013-94. To implement this standard, PFP will need to: (1) install a new packaging system; (2) modify the existing vault storage arrays to accept the new package; and (3) develop a new material container meeting the requirements of DOE-STD-3013-94. The new material container will be designed to be placed directly into a boundary container. The seal on the material containers will ensure that the material has not been re-exposed to any glovebox atmosphere, and that it has maintained the necessary characteristics established during the stabilization processing. The following are scheduled activities for container development:

- Development of the Hanford interim storage container started in May 1995 with a functional - container being available by September 1996.

- The DOE standard packaging system is currently scheduled to be ready in June 1998.

- Facility modifications needed for packaging and storage of plutonium containers to the DOE standard (DOE-STD-3013-94) are scheduled to be operational by July 1999.

- The existing safeguards and security systems will need to be modified as well to support the new package and/or to maintain reliability for the next 30 to 50 years of storage.

- PFP Analytical Laboratory upgrade is needed to facilitate characterization of the plutonium-bearing material for processing or disposal, to provide better process control of the various stabilization operations, and to meet Waste Isolation Pilot Plant and RCRA criteria for transuranic (TRU) waste. Additional instrumentation wil1 be provided to support necessary analyses in characterization of miscellaneous solid residues and process support of the stabilization operations. The laboratory upgrade is expected to be complete by September 1997.

- By the end of March 1996 develop and demonstrate the technology to be used at PFP for stabilization of aqueous plutonium solutions work in the PFP Process Support Laboratory.

- Vulnerabilities associated with residual contamination in facilities will be mitigated through facility transition and eventual deactivation. The end result of this effort will be facilities 


\section{TRANSITION PROJECTS}

1.3.1/7.1/6.12

1 B.2 Program Objectives.

that are acceptable for turnover to the Environmental Restoration Program. Terminal cleanout and cleanup planning has been developed through the year 2006 and these activities include the following general activities:

- Glovebox TCO

- Duct Remediation

- 241-Z Cleanout

- $\quad 236-Z$ (PRF) Canyon TCO

- $\quad$ Tank 241-Z-361 Cleanout

- $\quad 231-Z$ Cel1 Cleanout and 231-Z Cleanout (E-4 Ducts)

- 242-Z Cleanout

- 291-Z Holdup Remediation

- Drain Line Cleanout

- General PIant TCO

- 216-Z-9 Cleanout

- Current infrastructure (basic facility services such as power, ventilation, heat, etc.) at the PFP are in need of upgrades to support facility operations for the next 8 to 15 years of stabilization, cleanout, and transition to a deactivated state.

- Transitioning of plutonium handling facilities will result in facilities that are acceptable for turnover to the Environmental Restoration Program.

- $\quad$ Prepare the PFP TSD Units' RCRA Part 8 permit application.

- Receive Fast Flux Test Facility fuel assemblies for storage at PFP. Funding for modifications and transfer are included in the Advanced Reactor program plan.

- Complete annual updates of the PFP SAR. 


\section{TRANSITION PROJECTS}

\section{B. 2 Program objectives}

\section{Program and Environmental Management (7.1.6)}

The overall objective of the Program and Environmental Management program eiement is to provide management support for resources and activities that are common to all Transition Projects plants. The Program and Environmental Management program element also includes the following general objectives.

- Provide management support for resources and activities that are common to al1 Transition Projects plants.

- Interact and provide integration weekly with $\mathrm{RL}$, DOE-HQ, regulators, other Hanford Site contractors, and other contractors within the DOE complex.

- Ensure consistent application and administration of accounting and business management principles for the Transition Projects program.

- Submit Five Year PIan ADSs to RL.

- Prepare and submit the Transition Projects monthly SMS and PTS reports per schedules agreed to with RL.

- Maintain and status the detailed Performance Baseline schedules, monthiy.

- Conduct monthly performance reviews.

- Support four Nuclear Materials and Facilities Stabilization (EM-60) reviews/visits.

- Prepare and submit the annual update to the Transition Projects MYPP.

- Provide 200 Area Transition Projects Safeguards and Security functions which are not plant specific.

- Provide security maintenance for the Patrol Headquarters facility.

- Provide security training for the Transition Projects 200 Area personnel. 


1. B.2 Program objectives $\quad$ a

- Provide coordination of visits by foreign nationals.

- Provide communications support for Transition Projects.

- Provide overall guidance for management and disposition of the Westinghouse Hanford Company (WHC) SNM inventory, and provide technical support to DOE with respect to defining Hanford's role in the disposition and long term storage of DOE surplus plutonium.

- Provide company wide Nuclear Material Management in accordance with DOE Orders.

- Develop the Hanford Site Plutonium Strategic Plan to establish an integrated approach to both near and long term planning of plutonium related activities.

- Provide coordination of eight to ten SNM shipments to, and within, the Hanford Site. (This effort is limited to approximately three fult time equivalents (FTE) of management, packaging, environmental, and quality assurance staff support to facilitate small shipments.)

- Perform three to four studies to determine methods for SNM disposition, stabilization, treatment, storage, and shipping.

- Participate in four to six DOE complex-wide committees and represent Hanford Site interests regarding SNM disposition, stabilization, treatment, storage, and shipping.

- Provide centralized support for Transition Projects by integrating, coordinating, negotiating, standardizing, and completing environmental and historical preservation compliance activities.

- Provide a central point of contact for integration of Transition Projects' environmental compliance activities with $\mathrm{RL}$, DOE-HQ, regulators, other Hanford Site contractors, and other contractors within the DOE complex.

- Coordinate Transition Projects responses to site-wide issues to ensure consistent application and administration of environmental "best management practices" for the Transition Projects. 
1.B 2 Program objectives

- Coordinate negotiations with regulators to achieve compliance agreements and monitor compliance agreement schedules to ensure Transition Projects achieve compliance with state and federal environmental 7 aws and regulations.

- Develop and implement site-wide plans for identifying and evaluating potentially historic buildings, and preparing appropriate historical documentation, in accordance with the National Historic Preservation Act.

- Represent Transition Projects on site-wide multi-contractor teams, such as the Central Environmental Committee's Executive Committee and related subcommittees, the Value Engineering Studies, the Historic Buildings Task Force, the RCRA, liquid effluent, and air operating permit application review teams and other various teams to implement the permits.

- Improve Conduct of Operations with the following site-wide program activities:

- Perform Assessments in sixteen areas of Conducts of Operations.

- Publish forty Lessons Learned.

- Provide for the centralized integration, coordination, negotiating, and completion of Transition Projects Systems Engineering activities to ensure:

- Program and project scope and interfaces are defined, understood and integrated to ensure cleanup at Hanford can be managed as a single large project.

- Oniy work necessary to ensure the Transition Projects mission is performed. 


1.B.3 Program Planning Assumptions :

\section{General Assumptions}

- The FY 1996 MYPP is based on the target case of the 1997 Five Year Plan ADS with the following exception:

- PFP will plan to the schedule outlined by the DNFSB Recommendation 94-1 Implementation Plan.

- Completion of 300 Area Fuel Supply Shutdown Program in FY 1998.

- Activity based cost ( $A B C$ ) estimates will be the basis for the detailed FY 1996 and outyear budget. Adjustments resulting from an $A B C$ estimate critical analysis will be incorporated through change controt.

- $\quad A B C$ estimate will be updated annually and upon determination by the DOE Project Manager for significant technical workscope changes documented in Class I Change Requests. Funding will be budgeted for an annual update; however, funding for estimate updates due to significant technical scope changes will be identified within the Class I Change Request documenting the baseline revision.

- Defense Programs (DP) will continue to provide funding of $\$ 20,679,000$ annually. Additional workscope ,previously funded by the Defense Program (DP) will be funded by EM-60.

- Prior year uncommitted capital equipment not related to construction (CENRTC) funding will be available to support capital equipment needs.

- Fy 1995 uncosted balances and the associated workscope and schedule will be incorporated into the MYPP through change control. In addition to FY 1995 workscope which has not been completed, FY 1995 uncosted balances will be utilized to support current unfunded FY 1996 activities.

- High level endpoint negotiations and deactivation planning for the $K$ Basins will be initiated in FY 1996. A Program Master Baseline Schedule, Waste Type Data and a Cost Estimate by Year are included in this MYPP. Additional planning and MYPP data will be developed upon receipt of formal direction to transfer $K$ Basins from the Spent Nuclear Fuel Program to Transition Projects. 


\section{TRANSITION PROJECTS}

\section{B.3 Program Pianning Assumptions}

- The FY 1996 budget has been prepared without any resources available to absorb emergent work. Any new requirements, requests for studies requests for documents, etc. will impact the scope of this plan and will require approval via the formal change control process prior to initiation.

- An active public involvement process will be maintained throughout the project to insure that stakeholder values are incorporated into program goals.

- Early involvement of regulators will allow regulatory issues to be resolved without impacting the program schedule.

- Additional requirements needed to comply with the Hanford Site-wide RCRA Part B Permit will be assessed after the permit is issued (e.g. underground piping mapping and marking, operating and monitoring records, training, contingency plans, emergency procedures, post closure monitoring, inspections, facility closure cost estimating, manifest discrepancy reports, etc.). Of these, the mapping and marking requirements will have the greatest impact.

\section{B Plant/WESF (1.3.1.7)}

The B Plant / WESF Multi-Year Program Plan and Fiscal Year Work Plan will be revised and reissued by the end of January 1996 to reflect the change of mission from safe standby to transitioning to a mode suitable for transfer to EM-40.

The Accelerated Hazard Reduction (AHR) activities will invest near-term funding in accelerated completion of deactivation activities which provide significant risk reduction and substantial, timely economic payback. The projected net results will be a recategorization of B Plant to a low hazard facility and a $80 \%$ reduction in annual surveillance and maintenance costs, resulting in a savings of approximate1y $\$ 20$ million per year starting in FY 1999. The ability to adequately isolate the radiological inventory in the high efficiency filters and effect a recategorization of the facility to a low hazard facility is not certain but is used as a reasonable planning assumption.

Characterization of the equipment and inventories remaining after the completion of AHR activities will proceed to aid shutdown planning and to facilitate turnover to EM-40. DOE/EP-0100, "A Guide for Radiological Characterization and Measurements for Decommissioning of U.S. Department of Energy Surplus Facilities," will be used to guide the planning and implementation of the characterization. 


1.B.3 Program Planniong Assumptions

A new or revised Safety Analysis Report will not be required for the deactivated B Plant. The Interim Safety Basis (ISB) documentation will be utilized to establish the safety boundaries and set Operational Safety Requirements (OSR).

The hazardous material. currently in the facility as a consequence of past operations will continue to be stored under a RCRA Part A Interim Status permit until removed as part of facility shutdown or decommissioning activities. No Part B RCRA permitting will be required.

The WESF structures and systems are capable of safely storing the cesium and strontium capsules until their transfer to the High Level Waste Disposal Program (HLWDP). Structure and system components wil1 be repaired as necessary to maintain their safe operability and function. The replacement of structure and system components will be accomplished as required based upon evaluations of the facility lifecycle and shutdown date.

Cesium and Strontium capsules will be transferred to the HLWDP by 2011 and WESF transitioned to EM-40 by 2013 and long range schedules will reflect this strategy. However, until. negotiations have been completed for the transfer of WESF to EM-40, the surveiliance and maintenance of WESF will continue to be funded in this MYPP.

${\underline{\text { PUREX } / U 0_{3}}}_{3}-(7.1 .1)$

- The project will be managed using DOE Order 4700.1 methodology using approved cost, schedule, and technical baselines and formal change control.

- The PUREX/U03 Deactivation Project Management Plan and Project Schedules are the basis for the scope of work. Changes to work scope will be incorporated by change control.

- The initial transition project cost estimate was based on the Activity Based Cost Estimate. Several change control packages have been approved and incorporated into the baseline. The program plan for the transition activities is the currently approved project baseline. A Change Request will be prepared to incorporate the "Phase 2" schedule changes into the project baseline.

- The draft Surveillance and Maintenance $A B C$ estimate is the basis for the S\&M cost estimate. However, since the $A B C$ estimate has not been finalized, an estimation of the final outcome has been 


\section{TRANSITION PROJECTS}

1.3.1/7.1/6.1.2

\section{B.3 Program Planining Assumptions}

made. When the $A B C$ estimate has been completed and approved, change control will be used to incorporate that estimate into the program $\mathrm{plan}$.

\section{Area Fuel Supply (7.1.2)}

- Completion of the 300 Area Fuel Supply Shutdown Program will be accelerated to mid FY 1999.

- The north portion of the 313 Building will be leased for a duration of 3 years, from August 1994 to August 1997. Planning assumes the building will be shutdown in FY 1998.

- Whenever SNM is referenced as 1900 MT, it indicates amount of remaining inventory. Cost estimates and scheduTes are based on 1900 MT of Uranium (MTU). They may be reduced as inventories are reduced.

- DP provides $\$ 612 \mathrm{~K} /$ year at current inventory levels and EM provides the balance until shutdown activities are completed in FY 1998. After FY 1998, DP provides full funding for surveillance and maintenance of the SNM material until final disposition. Reductions to surveillance and maintenance costs could occur when inventory levels are reduced.*

- EM-60 provides full funding for the packaging, shipping, and relocation of the SNM to another storage area within the Hanford Site. This will allow turnover of the six SNM buildings.

- In FY 1996, 706 of SNM will be shipped to the United Kingdom. This activity will be supported by FY 1995 carryover funding from the Environment Management Program and will be incorporated by Change Control.

- Any further sale/disposal of SNM will be funded separately and incorporated by Change Control at that time.

- Soil cleanup associated with RCRA Closure Plans will be deferred to CERCLA cleanup activities.

- If clean closure of RCRA units is not successful, further remediation will be deferred to EM-40.

- No public comments from public review process will be received on RCRA Closure Plans. If comments are received, a schedule slip may occur. 


\section{TRANSITION PROJECTS .}

\section{B.3 Program Planning Assumptions}

- Discussion will continue with EM-40 to decide 313 Building transfer and demolition. No funding for 313 Building demolition has been identified in this program plan. EM-60 will provide FY 1996 and FY 1997 funds for isolation of the South portion of 313 Building.

- Funding provided by EM-60 for minimum safe surveillance and maintenance after deactivation is complete through the end of this planning period.

- The Waste Type Data Projections assume deactivation scheduled for 3-31-99.

*NOTE: Current assumption is that DP will continue funding surveillance and maintenance of SNM inventory, . however the program is aware of a current Memorandum of Agreement between EM-60 and DP, that EM-60 may pick up funding sometime in the future.

$\quad \quad \quad P F P(7.1 .3)$

- Hanford has 35 identified Plutonium Environmental, Safety, and Health Vuinerabilities. Many of the identified vulnerabilities will be corrected through the stabilization and packaging activities required by the DNFSB Recommendation 94-1 Implementation P1an, others will be corrected as a part of plutonium handling facilities transition (deactivation) to the Environmental Restoration Program, the remaining can not be fully corrected until environmental restoration is complete.

Vulnerabilities associated with stabilization and packaging of plutonium bearing materials will be mitigated by May 2002.

- Operations on sludge stabilization, in connection with terminal cleanout, will continue until the end of the program in January 2005.

- A variety of dissimilar fuel types are or will be stored at PFP, including LAMPRE Fuel and Fast Flux Test Facility (FFTF) SNF. The planning basis assumes that these SNF inventories will remain at PFP at least until final disposition is determined through the SNF EIS.

- Modifications to receive and store FFTF fuel assemblies will be funded from the Nuclear Energy (NE) Program. During FY 1996 and 1997 PFP will be modified to receive FFTF fuel assemblies. The material receipt completion date is October 1998. The Interim Storage Casks (ISC) containing FFTF SNF designated for storage at PFP will be transported to an outdoor location inside PFP's protected area for storage on a precast concrete pad. This transfer is expected to take place by November 


1. B.3 Program pianning Assumptions

1998, but may be sooner depending on the staging of fuel for cleaning in the FFTF Interim Examination and Maintenance (IEM) Cell and the completion and approval of the onsite Safety Analysis Report for Packaging. The unirradiated FFTF fuel pins and assemblies at PFP are considered acceptable as-is for long term storage and no further actions are envisioned for these materials.

- The PFP can stabilize and store or dispose of as transuranic waste all of the solutions, residues and oxides (less than $50 \mathrm{wt} \% \mathrm{pu}$ ), and metals and oxides in the current inventory, and from the Hanford Site's terminal cleanout operations, with additions of glovebox-scale processing capability. Some development work will be required by the PFP Analytical laboratory, depending on the final selection of stabilization technology. A radiological dose study will confirm that the use of glovebox processes will keep doses to personnel associated with the work as low as reasonably achievable.

- Materials are currently being stabilized and packaged to specifications in the DOE Standard Criteria for Safe Storage of Plutonium Metals and 0xides (DOE-STD-3013-94). Plutonium-bearing residues will continue to be stabilized at $1000^{\circ} \mathrm{C}$ and stored in vaults to the existing Hanford vault storage criteria. When a formal DOE criteria for residues is approved, material will be packaged to that criteria with restabilization of the material if necessary.

- Stabilization processes will be selected based on the assumption that the contained plutonium has no asset value.

- There is no container existing today which meets the DOE Standard, DOE-STD-3013-94, storage standard and material stabilization activities must proceed on an aggressive schedule in order to accomplish 94-1 programmatic goals. A new Hanford material container will be designed to store stabilized materials for an interim period in order tó move forward with stabilization activities. Since the development of a container and storage method must be performed an interim packaging capability needs to be established such that stabilized material can be eventually placed and sealed inside a final boundary container.

- Facility modifications needed for packaging and storage of plutonium containers to the DOE Standard, DOE-STD-3013-94, are scheduled to be operational, with special capital funding dispensation, by July 1999. The Hanford container, suitable for overpacking to the DOE Standard, will be availabie by September 1996. Stabilized high-assay (>50 wt\% plutonium) will be retrieved from storage and overpacked for 50-year storage. No further processing or repackaging will be required. Vault 


\section{1.: 3 Program Planning Assumptions}

modifications will be required to store the DOE standard container. Vault modifications necessary to handle the to-be-determined standardized Pu storage container are expected to the container storage racks, security instrumentation, container detectors, security bars, shielding, and any ancillary modifications once the Pu storage container configuration is available from the DOE.

- The current laboratory development program will identify and resolve any technology and equipment development issues and will serve as an adequate basis for design of the selected stabilization process systems. The PFP Analytical Laboratory is expected to be upgraded. Additional

instrumentation wi11 be provided to support necessary analyses in characterization of miscellaneous solid residues and process support of the stabilization operations. The laboratory upgrade is expected to be complete by September 1997.

- Operations will be on a 24-hour-per-day, 5-day-per-week basis, and personnel and funding will be provided to support this level of effort.

- Storage of SNM within existing PFP secure vault areas will continue throughout the foreseeable future. As permitted by the State of Washington and required by site support needs, SNM will be consolidated at PFP to permit the reduction in the number of protected areas throughout the Hanford Site.

- An activity based cost estimate will be the basis for the detailed fiscal year 1996 budget, and for all outyear workscope, budget, and schedule requests. This estimate incorporates all safe compliance issues into a systems engineering/projectization management approach.

- Vault \#3 wil1 remain under the custody of the IAEA during DNFSB 94-1 activities. No more vaults will be made available for IAEA custodianship until after all packaging system and vault upgrades have been completed through the year 2002. There is a significant potential for schedule delays to DNFSB 94-1 commitments if additional vaults are turned over to IAEA.

- DP will continue to provide approximately $\$ 19$ million per year for continued storage and management of SNM fuels/materials inventories pending Department decisions on ultimate disposition or storage. The workscope associated with vault operations and maintenance, safeguards and security, nuclear material accountability, patrol, etc. exceeds the DP funding provided. The workscope previously funded by DP will be funded by EM-60 and any changes to DP funding level witl be associated with an equal and opposite change in EM-60 funding level. 
1.B.3 Program Planning Assumptions

- NEPA analysis will be directed in accordance with the March 8, 1994, Tetter from RL, Comprehensive NEPA Strategy for Deactivation of PFP. The PFP Stabilization EIS is in preparation to provide a basis under NEPA for actions at the PFP including stabilization of reactive residual SNM, continuing SNM storage and stabilization of various areas of the facility as deactivation is begun. The EIS Record of Decision will be issued in June, 1996. The determinations to be made are:

- implementation of alternatives for stabilization of reactive residual Pu-bearing materials, - implementation of facility stabilization actions.

- The PFP 241-Z Part B Permit Application will continue in FY 1996 towards the FY 1996 Hanford Federal Facility Agreement Consent Order (Tri-Party Agreement) milestone.

- The PFP dangerous waste tank integrity assessments and related reports will be completed in FY 1998 , but no related upgrades other than those already $\mathrm{planned}$ will be required by the assessments.

- Facility modifications for minimum surveillance and maintenance will continue.

- The facility will be maintained with a minimum safety compliant configuration.

- Workscope contained in the New Facility Planning ADS (6625) is included within this program element for program planning purposes.

- The PFP Transition Project End Point is anticipated in FY 2008. This end point is NOT the cessation of all activities as with other Hanford facilities. Instead, this End Point assumes the continued operation of all Special Nuclear Material (SNM) shipping, receiving, storage and vault systems, including all safeguards \& security (SAS) systems plus laboratory capabilities as needed, for an indefinite period. This end point condition is also based on the following:

- full implementation of DNFSB Recommendation 94-1 was completed in FY 2002 and applicable plutonium vulnerabilities have been mitigated;

- remaining facility deactivation and demolition was initiated immediately following completion of DNFSB Recommendation 94-1 activities; and, 


\section{B.3 Program Pjanning Assumptions}

- deactivation and demolition occurs over fiscal years 2003 through 2007, or over a five year period.

The staffing profile upon completion of DNFSB Recommendation 94-1 implementation has been estimated at approximately 254 persons. However, a minimum additional 72 persons is anticipated to be required to pursue deactivation and demolition of PFP facilities over the five period. Once the deactivation/demolition activity on remaining facilities has been completed, the remaining shipping, receiving, storage and vault systems, plus the required SAS and laboratory support, would require approximately 254 persons to operate in a safe and secure manner.

\section{Program and Environmental Management $(7.1 .6)$}

- WHC will continue to receive SNM from other sites to support the DOE national mission. Quantities received will not exceed current storage capacity.

- A.portion of the SAS budget will be funded by DP.

- Improvement in Conduct of Operations will continue to be a Hanford Site goal.

- Hanford will continue to use the SMS to manage the program/project. 


\section{B 4 Program Constraints,}

\section{B P1ant/WESF (1.3.1.7)}

The acceptance criteria for transfer of B Plant to EM-40 have not been completely defined. The determination of responsibilities, the end-point criteria, the activities to be performed and the interfaces are not clear.

The date for dispositioning the Cs and Sr capsules being stored in WESF has not been established. This makes the determination of the requirements for long term maintenance of WESF difficult to determine and often leads to "band-aid" fixes or incremental upgrades which may cost more than one time expenditures.

The WESF facility was constructed in 1974. The original design lifetime of that facility is presumed to be thirty years. Nine years from now projects must be established to upgrade building infrastructure subsystems (e.g., wiring, piping, controls) before degradation jeopardizes the safety envelope. Given the seven-year typical lead time required to complete the National Environmental Policy Act (NEPA) process, the decisions to plan for these upgrades or alternative interim storage capability must be made no later than 1997.

The B Plant / WESF staffing strategy includes limiting hiring and pursuing alternative assignment opportunities for the staff to minimize the impacts of shut-down and transition to a deactivated state. This strategy, while causing the least amount of staff impact, causes significant difficulty in the ability to perform the work scope required to achieve a shut-down state and transition to EM-40.

\section{PUREX/U0 ${ }_{3}(7.1 .1)$}

- none

\section{Area Fuel Supply (7.1.2)}

- SNM is to be relocated to outside the 300 Area in FY 1998. Approximately $\$ 612 K$ yearly of DP funding will be required to support compliant storage until relocation and $\$ 950 \mathrm{~K}$ once no longer mutually supported by EM resources.

- Separate funding be provided for possible uranium shipments. 


1:B.4 Program Constraints

PFP (7.1.3)

The following issues courd limit the ability to achieve the PFP mission objectives:

- Until the processing activities are defined and an integrated system dose evaluation is completed in June 1996, worker exposure cannot be accurately determined. Results of the dose evaluation may identify a need for additional shielding, automation, or an entirely new system to meet the WHC/DOE administrative guideline of 1,500 millirem per year.

- The Hanford Site lacks the necessary laboratory instrumentation to adequately characterize plutonium-bearing material. An upgrade of the PFP Analytical Laboratory is needed to facilitate characterization of the plutonium-bearing material for processing or disposal, to provide better process control of the various stabilization operations, and to meet Waste Isolation Pilot Plant (WIPP) and Resource Conservation and Recovery Act (RCRA) criteria for TRU waste.

- To expedite the disposal process, approval authority for designating items as waste needs to be transferred to the Field offices. Additionally, to avoid problems in the future associated with the ultimate disposal of the TRU waste, the Field Offices must be given a space allocation in WIPP.

- Public and Worker Health and Safety concern will remain high until all safety items have been characterized and mitigated.

- The PFP EIS is being prepared and the Record of Decision (ROD) will be issued in June, 1996. The new PFP planning scenario is based on preparing for Facility deactivation and the processes selected should be consistent with the ROD.

- If any significant shift occurs in the Mission Environment, such as public distrust grows instead of subsides or the funding is not sufficient, the PFP Mission will be affected.

- The duration of the temporary SNM storage period at PFP is unknown, but is expected to be in excess of 20 years. Draft documents by DOE indicate that long-term storage prior to final disposition is potentially greater than 50 years. PFP is an option for this long-term storage. 
1. B. 4 Program Constraints

- The DOE is in the process of developing guidance for the final disposition of SNM/NM/NF materials. Until this guidance is provided, the final disposition of SNM is undefined. Therefore, the role PFP may plan in final disposition of SNM cannot be determined at this time.

- The acceptance criteria for transfer of PFP facilities to the Richland Environmental Restoration (EM-40) is still being developed. The determination of responsibilities, the end-point criteria, and the activities to be performed and the interfaces are not clear.

- The PFP mission includes the receipt and temporary storage of SNM from other Hanford cleanup mission areas. DOE may direct that the scope be expanded to inciude SNM receipt from other (off-site) facilities within the DOE complex.

- The role of the PFP laboratories, if any, to support other Hanford site cleanup activities is not yet determined.

- Additional activities, such as FFTF fuel assembly storage will continue as scheduled.

- IAEA Support involving Vault \#3 will continue consistent with the FY 1996, budget year.

Program and Environmental Management (7.1.6)

- The 1,618 metric tons of unirradiated uranium which was anticipated to be sold to COGEMA will remain at Hanford and require continued safe storage while a new disposition is determined and implemented. Funding is not available to implement agreements for uranium sales and are not included in the MYPP. Carryover scope and funding for AlliedSignal and United Kingdom sale will be incorporated into the baseline through change control.

- The Transition Projects' Systems Engineering function is not fully funded in FY 1996 and FY 1997 . A graded approach will be taken such that Systems Engineering support is provided at the program level for development of program level Systems Engineering documentation in FY 1996 and FY 1997, but there will be no site level or project level Systems Engineering documentation supported in FY 1996 and FY 1997. 


\section{B. 4 . Program Constraints}

- The majority of nuclear materials currently in inventory will remain at Hanford and require current levels of control and accountability. Any significant receipt and storage of SNM from other sites will be considered as new work scope and will be addressed through change control.

- Funding has not been provided to conduct special studies which may be associated with the storage of material under the International Atomic Energy Agency program. Additional workscope will be incorporated into the baseline through change control upon receipt of incremental funding.

- The 200 Area Canyon Entombment study was initiated to assess the potential of using this concept. Carryover scope and funding for the 200 Area Canyon Entombment will be incorporated into the baseline through change control.

\section{DEPARTMENT OVERHEAD $(6.12 .1)$}

- The FY 1996 Transition Projects Department Overhead budget is built based upon the current organizational structure effective 7/31/95. Any significant change in this structure would have a corresponding effect on pool contents and potentialiy on pool liquidations.

- The FY 1996 Transition Projects Department Overhead rate structure submittal is based upon headcount. Changes to this headcount could impact the proposed rate. 


\section{TRANSITION PROJECTS}

FY 1996 MYPP

1.3.1/7.1/6.12

\section{A.1 Technical Documents Tree \& products structure}

The Technical Documents Tree \& Product Structure table was created to display the products produced by WBS elements and show the linkage between WBS elements and Systems Engineering (SE) functions. Showing this linkage is important because comparison of SE functions that define what needs to be done, with WBS elements that describe work that is going to be performed is the key to ensuring that planned work consists only of those activities necessary to support the facility, program and site missions. Identification of the product or result of performing WBS identified work should facilitate the development of activity-based cost estimates and schedules.

Onty the SE function numbers are referenced in the SE Function field of the Technical Documents Tree \& Product Structure table to save space. Function titles, definitions and products produced by those functions are contained in the following facility SE documents, expected to be released by the end of FY 1995:

308 Building Deactivation Function Analys is Report

WHC-SD-FL-FAR-001, RO

309 Building Deactivation Function Analys is Report WHC-SD-SP-FAR-001, RO

300 Area Fuel Supply Facilities Deactivation Function Analys is Report

WHC-SD-FL-FAR-002, RO

FFTF Plant Transition Function Analys is Report

WHC-SD-FF-FAR-001, RO

B Plant Function Analys is Report.

WESF Function Analys is Report

WHC-SD-WM-FAR-001, RO

WHC-SD-WM-FAR-002, RO

PFP Transition Project Function Analys is Report

WHC-SD-CP-FAR-001, RO

PUREX Plant Deactivation Function Analys is Report

WHC-SD-CP-FAR-002, RO 


\section{TRANSITION PROJECTS}

FY 1996 MYPP

1.3.1/7.1/6.12

\section{A.1 Technical Documents Tree \& Products Structure}

\begin{tabular}{|c|c|c|c|c|c|}
\hline $\begin{array}{l}\text { PROGRAM } \\
\text { ELEMENT }\end{array}$ & ACtIVITY & Cost ACcount & 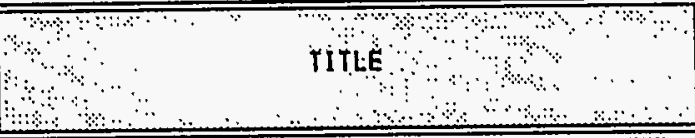 & 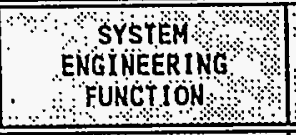 & 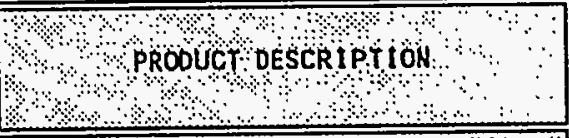 \\
\hline \multirow[t]{4}{*}{$1: 3.1: 7$} & 4 & 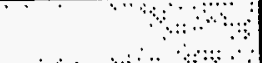 & $\because \quad \operatorname{s} \quad 4 \quad \cdots$ & २े & - गै। \\
\hline & $\begin{array}{l}133.1 .7 .1 \\
\times B 1\end{array}$ & & Progran Management and Adninis stration & & 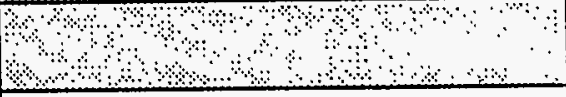 \\
\hline & & $\begin{array}{l}1.3 .1 .7 .1 .01 \\
\text { KB101 }\end{array}$ & Administration & $\begin{array}{l}4.1 .2 \cdot 1 \cdot 1.1 .5 \\
4.1 .2 .1=1.5 \cdot 1 \\
4.1 .2 .1=1.5 .2 \\
4.7 .3=1.1 .5 \\
4.7 .3 \cdot 1.2 .3 \\
4.7 .3 .4 .3\end{array}$ & $\begin{array}{l}\text { Activity Data Sheets, Multi-Year } \\
\text { Program Plans, tours, } \\
\text { presentations, personnel } \\
\text { management and mentoring, site } \\
\text { generic training, self } \\
\text { assessments, office supplies, } \\
\text { property management, HATS database } \\
\text { management }\end{array}$ \\
\hline & & $\begin{array}{l}1.3 .1 .7 .1 .02 \\
\text { KB102 }\end{array}$ & Site Service Assessments & 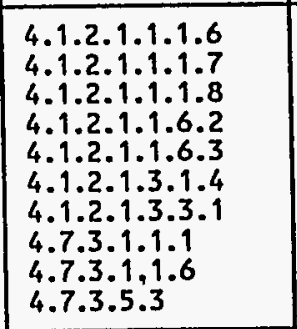 & $\begin{array}{l}\text { Steam, Hater, electricity, waste } \\
\text { disposal, and laundry provided. } \\
\text { Computer network access provided. } \\
\text { Fire prevention and rail service } \\
\text { provided. }\end{array}$ \\
\hline & $\cdot$ & $\begin{array}{l}1.3 .1 .7 .1 .03 \\
\mathrm{~KB} 103\end{array}$ & Training Administration & $\begin{array}{l}4 \cdot 1 \cdot 2 \cdot 1 \cdot 1 \cdot 4 \cdot 1 \\
4 \cdot 1 \cdot 2 \cdot 1 \cdot 1 \cdot 4 \cdot 2 \\
4 \cdot 1 \cdot 2 \cdot 1 \cdot 1 \cdot 4 \cdot 3 \\
4 \cdot 7 \cdot 3 \cdot 1 \cdot 4 \cdot 1 \\
4.7 \cdot 3 \cdot 1.4 .2 \\
4.7 .3 \cdot 1.4 .3\end{array}$ & $\begin{array}{l}\text { Staff training, and documentation } \\
\text { provided. Training materials and } \\
\text { aids developed. Training records } \\
\text { and requirements maintained. }\end{array}$ \\
\hline & $\begin{array}{l}1.3 .1 .7 .2 \\
\mathrm{KB2}\end{array}$ & & 8 pilant Survellitance and Maintenance & ओ। & की \\
\hline
\end{tabular}




\section{A. 1 Technical Documents Tree \& Products structure}

\begin{tabular}{|c|c|c|c|c|c|}
\hline $\begin{array}{l}\text { PROGRAM } \\
\text { ELEMENT } \\
\end{array}$ & Activity & cost account & 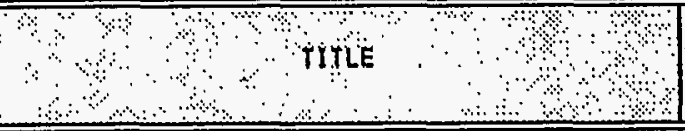 & $\begin{array}{l}\text { SYSTEMY } \\
\text { ENGINEER ING } \\
\text { HEUNCTION } \\
\end{array}$ & 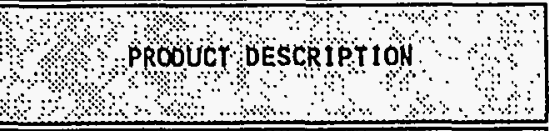 \\
\hline \multirow[t]{6}{*}{$1+31.7$} & $\therefore \therefore \quad \therefore \quad$ & +1: & B plant tomplex & कासे & अ४४ \\
\hline & & $\begin{array}{l}1.3 .1 \cdot 7.2 .01 \\
\text { KB201 }\end{array}$ & B Plant Safe and Compliant Operations & 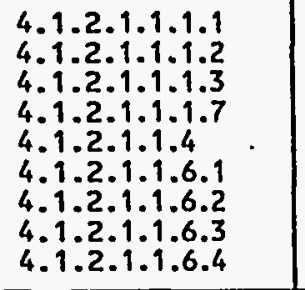 & $\begin{array}{l}\text { Surveillance data which is } \\
\text { utilized to ensure the facility is } \\
\text { maintained safe. Emergency } \\
\text { drills. Safe storage, treatment, } \\
\text { and disposal of radiological } \\
\text { wastes. Liquid and gaseous } \\
\text { effluent emissions data. Safe } \\
\text { handl ing of hazardous and mixed } \\
\text { solid wastes. }\end{array}$ \\
\hline & & $\begin{array}{l}1.3 .1 .7 .2 .02 \\
\mathrm{~KB} 202\end{array}$ & $\begin{array}{l}\text { Maintain Plant Systems, Equipment, and } \\
\text { Structure }\end{array}$ & $\begin{array}{l}4.1 .2 .1 .1 .1 .4 \\
4.1 .2 .1 .1 .2 .1 \\
4.1 .2 .1 .1 .2 .2 \\
4.1 .2 .1 .1 .2 .3 \\
4.1 .2 .1 .1 .2 .4\end{array}$ & $\begin{array}{l}\text { Utility and material handling } \\
\text { systems maintained within safe } \\
\text { configuration limits resulting in } \\
\text { the plant staying within the } \\
\text { safety envelop as defined in the } \\
\text { Safety Analysis Report. }\end{array}$ \\
\hline & & $\begin{array}{l}1.3 .1 \cdot 7.2 .03 \\
\mathrm{~KB} 203\end{array}$ & $\begin{array}{l}\text { Maintain Facility Operating and Safety } \\
\text { Documentation }\end{array}$ & $\begin{array}{l}4 \cdot 1 \cdot 2 \cdot 1 \cdot 1 \cdot 3.1 \\
4 \cdot 1 \cdot 2 \cdot 1 \cdot 1 \cdot 3.2 \\
4 \cdot 1 \cdot 2 \cdot 1 \cdot 1 \cdot 3.3 \\
4.1 .2 \cdot 1.1 .3 .5\end{array}$ & $\begin{array}{l}\text { Procedures, designs, and } \\
\text { documentation required to maintain } \\
\text { the facility in a safe } \\
\text { configuration. }\end{array}$ \\
\hline & $1.3 .1 .7 \% 3$ & $\because \because$ & Transition $\quad$ o $\quad \because$ & @ & \%ै। \\
\hline & $\cdot$ & ${ }_{K B 301}^{1.3 .1 \cdot 7.3 .01}$ & B Plant Accelerated Hazard Reduction & 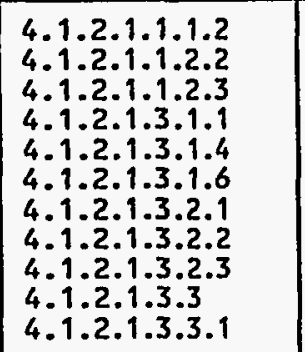 & $\begin{array}{l}\text { Implemented Interim Safety Basis } \\
\text { documentation. Organics } \\
\text { transferred outside of the Plant } \\
\text { canyon. HESF isolated from B } \\
\text { Plant. Deactivated B Plant } \\
\text { ventilation system filters } \\
\text { isolated and stabilized. Surface } \\
\text { contamination areas reduced or } \\
\text { el iminated. }\end{array}$ \\
\hline
\end{tabular}




\section{TRANSITION PROJECTS}

\section{A.1 Technical Documents Tree \& Products Structure}

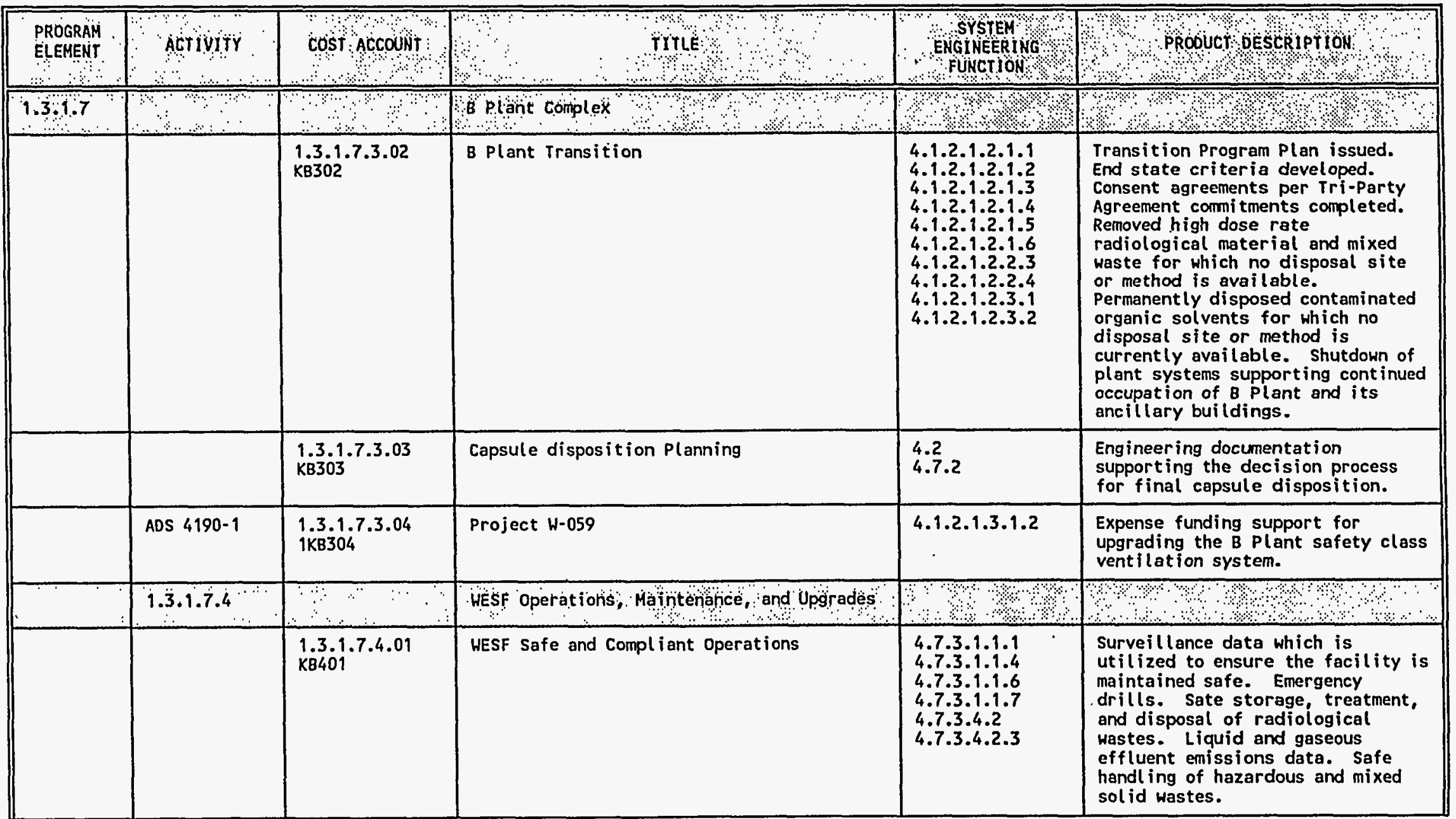


TRANSITION PROJECTS

FY 1996 MYPP

1.3.1/7.1/6.12

\section{A.1 Technica 1 Documents Tree \& Products Structure}

\begin{tabular}{|c|c|c|c|c|c|}
\hline $\begin{array}{l}\text { PROGRAM } \\
\text { ELEMENT }\end{array}$ & Actiôitri & cóst Account & $\therefore$ औItLE & $\begin{array}{r}\text { SYSTEM } \\
\text { ENGINERING } \\
\text { FUNCTION }\end{array}$ & 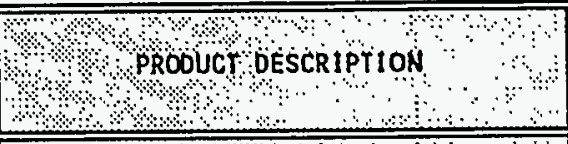 \\
\hline \multirow[t]{7}{*}{1.3 .1 .7} & & : & B.plant Complex & 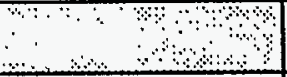 & 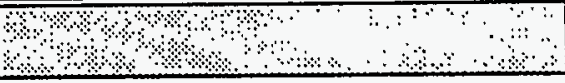 \\
\hline & & $\begin{array}{l}1.3 .1 .7 .4 .02 \\
K B 402\end{array}$ & $\begin{array}{l}\text { Maintain Plant Systems, Equipment, and } \\
\text { structure }\end{array}$ & $\begin{array}{l}4.7 .3 \cdot 1 \cdot 2.1 \\
4.7 .3 \cdot 1 \cdot 2.2 \\
4.7 .3 \cdot 1 \cdot 2.3 \\
4.7 .3 \cdot 1.2 .4 \\
4\end{array}$ & $\begin{array}{l}\text { Utility and material handl ing } \\
\text { systems maintained within safe } \\
\text { conf iguration limits result ing in } \\
\text { the plant staying within the } \\
\text { safety envelop as defined in. the } \\
\text { Safety Analysis Report. }\end{array}$ \\
\hline & & $\begin{array}{l}1.3 .1 .7 .4 .03 \\
\mathrm{~KB} 403\end{array}$ & $\begin{array}{l}\text { Maintain Facility Operating and Safety } \\
\text { Documentation }\end{array}$ & $\begin{array}{l}4 \cdot 7 \cdot 3 \cdot 1 \cdot 3 \cdot 1 \\
4 \cdot 7 \cdot 3 \cdot 1 \cdot 3 \cdot 2 \\
4 \cdot 7 \cdot 3 \cdot 1 \cdot 3 \cdot 3 \\
4 \cdot 7 \cdot 3 \cdot 1 \cdot 3 \cdot 4 \\
4 \cdot 7 \cdot 3 \cdot 1 \cdot 3 \cdot 6\end{array}$ & $\begin{array}{l}\text { Procedures, designs, and } \\
\text { documentation required to maintain } \\
\text { the facility in a safe } \\
\text { configuration. }\end{array}$ \\
\hline & ' & $\begin{array}{l}1.3 .1 .7 .4 .04 \\
1 \times B 404\end{array}$ & HESF Near Term Upgrades & $\begin{array}{l}4.7 .3 \cdot 1 \cdot 2 \cdot 4 \cdot 2.1 \\
4.7 .3 \cdot 1 \cdot 2 \cdot 4 \cdot 2.2 \\
4.7 \cdot 3 \cdot 1 \cdot 2 \cdot 4 \cdot 2.3\end{array}$ & $\begin{array}{l}\text { Upgrades Project Management Plan. } \\
\text { Capsule surveillance upgrades to } \\
\text { improve leak detection, isolation } \\
\text { and failure recovery capabilities. } \\
\text { Facility viability upgrades to } \\
\text { assure utility and confinement } \\
\text { systems are reliable through final } \\
\text { capsule disposition. }\end{array}$ \\
\hline & $1.3 .1 .7: 5$ & $\because \because$ & Regul atory complitance & un & $\begin{array}{ll}\text { +ै। } \\
\end{array}$ \\
\hline & & $\begin{array}{l}1.3 .1 .7 .5 .01 \\
\text { KB501 }\end{array}$ & Environmental Compliance & $\begin{array}{l}4 \cdot 1 \cdot 2 \cdot 1 \cdot 1 \cdot 1.2 \\
4.1 \cdot 2 \cdot 1 \cdot 1 \cdot 3.4 \\
4 \cdot 7 \cdot 3 \cdot 1 \cdot 1 \cdot 1 \\
4.7 .3 \cdot 1.3 .5\end{array}$ & $\begin{array}{l}\text { Revised RCRA Part A Permit } \\
\text { application. Updated air } \\
\text { emissions permit. Updated air } \\
\text { emissions database. Revised RCRA } \\
\text { TSD inspection plan. Revised } \\
\text { Haste analysis plan. Tank } \\
\text { integrity report. Annual } \\
\text { dangerous waste reports. Process } \\
\text { standdown history document. }\end{array}$ \\
\hline & & 1.3 .1 .7 .5 .02 & Compl iance Support & $\begin{array}{l}4 \cdot 1 \cdot 2 \cdot 1 \cdot 1 \cdot 3 \cdot 4 \\
4 \cdot 1 \cdot 2 \cdot 1 \cdot 1 \cdot 3.5 \\
4 \cdot 1 \cdot 2 \cdot 1 \cdot 1 \cdot 5.1 \\
4.1 \cdot 2 \cdot 1 \cdot 1 \cdot 5.2 \\
4.1 \cdot 2 \cdot 1.2 .4 .2 \\
4.7 \cdot 3 \cdot 1.5\end{array}$ & $\begin{array}{l}\text { Standards and Requirements } \\
\text { Implementation Documentat ion and } \\
\text { assessments. Compliance } \\
\text { implementation plans. }\end{array}$ \\
\hline
\end{tabular}




\section{TRANSITION PROJECTS}

FY 1996 MYPP

1.3.1/7.1/6.12

\section{A.1 Technical Documents Tree \& Products Structure}

\begin{tabular}{|c|c|c|c|c|c|}
\hline $\begin{array}{l}\text { PROGRAM } \\
\text { ELEMENT } \\
\end{array}$ & \begin{tabular}{c} 
ACTIVITY \\
\hdashline
\end{tabular} & Cost Account & $\begin{array}{ll}0 \\
\vdots \\
\vdots\end{array}$ & $\begin{array}{r}\text { SYSTEM } \\
\text { ENGINERTHG } \\
\text { FUNCTION }\end{array}$ & 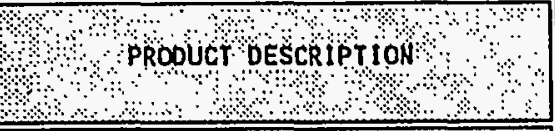 \\
\hline $1,3,1,7$ & $\because$ & " & B plant Complex & - & अम \\
\hline $\begin{array}{lll} & \ddots \\
\ddots & \ddots\end{array}$ & $\begin{array}{l}13.1976 \\
A D S: 4195\end{array}$ & $\therefore \quad \therefore \quad$ & Ces pun Recovery proprat & $\mathrm{OH}+\mathrm{P}$ & 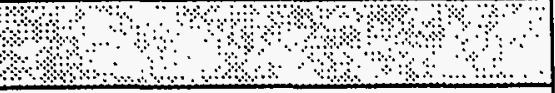 \\
\hline & & $\begin{array}{l}1.3 .1 .7 .6 .01 \\
1 \mathrm{~KB} 601\end{array}$ & Cesium Capsule Return & 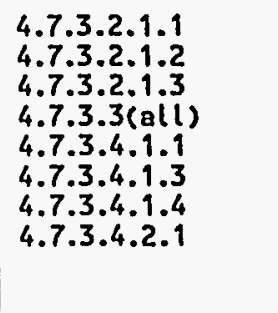 & $\begin{array}{l}\text { Return of all capsules leased to } \\
\text { private companies to WESF for } \\
\text { long-term storage. Reencapsulated } \\
\text { failed and suspect capsules } \\
\text { currently located at WESF and the } \\
\text { Pacific Northwest Laboratories. } \\
\text { Capability to ship capsules from } \\
\text { offsite and onsite locations. } \\
\text { Surveillance of capsules at PNL } \\
\text { until removal to WESF. }\end{array}$ \\
\hline
\end{tabular}


TRANSITION PROJECTS

1.3.1/7.1/6.12

\section{A.1 Technical Documents Tree \& Products structure}

\begin{tabular}{|c|c|c|c|c|c|}
\hline $\begin{array}{l}\text { PROGRAM } \\
\text { ELEMENT }\end{array}$ & ACTIVITY & COST ACCOUNT & TITLE & $\begin{array}{l}\text { SYSTEM ENGINEERING } \\
\text { FUNCTION }\end{array}$ & PROOUCT DESCRIPTION \\
\hline \multirow[t]{2}{*}{7.1 .1} & $\begin{aligned} \therefore \\
\therefore\end{aligned}$ & 亦 & PUREX Uo3 beactivation & a & 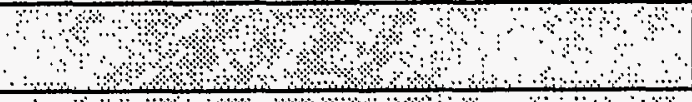 \\
\hline & $\begin{array}{l}7.1 .1 .1 \\
1 \mathrm{KP1}\end{array}$ & & PUREX SUrveillance \& Maintenance & $\because 4$ & ) \\
\hline & & $\begin{array}{l}7.1 .1 .1 .01 \\
\text { 1KP101 }\end{array}$ & PUREX SUrveillance & 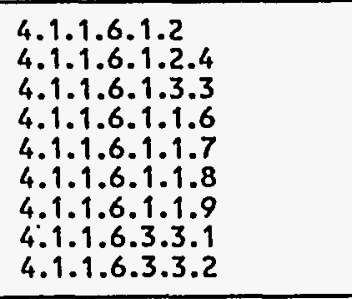 & $\begin{array}{l}\text { OSR equipment/systems operationa! condition } \\
\text { verified \& associated data sheets } \\
\text { completed. Training of operations personnel } \\
\text { performed \& qual ifications verified. Sol id } \\
\text { wastes disposed. Plant engineering } \\
\text { documents (ECNs, drawings, design } \\
\text { documents, etc.) prepared, distributed and } \\
\text { maintained. }\end{array}$ \\
\hline & . & $\begin{array}{l}7.1 .1 .1 .02 \\
1 \mathrm{KPP} 102\end{array}$ & PUREX Maintenance & $\begin{array}{l}4 \cdot 1 \cdot 1 \cdot 6 \cdot 1 \cdot 2 \\
4 \cdot 1 \cdot 1 \cdot 6 \cdot 1 \cdot 1 \cdot 4 \\
4.1 \cdot 1 \cdot 6 \cdot 1 \cdot 3 \cdot 3 \\
4.1 .1 \cdot 6 \cdot 1.3 .6\end{array}$ & $\begin{array}{l}\text { Critical utility systems, environmental } \\
\text { monitoring, \& OSR related equipment } \\
\text { repaired/replaced/maintained and } \\
\text { operational status/condition verified. } \\
\text { Work packages developed, scheduled, } \\
\text { processed, \& archived. }\end{array}$ \\
\hline & & $\begin{array}{l}7.1 .1 .1 .03 \\
1 \mathrm{KP} 103\end{array}$ & PUREX Assessments & $\begin{array}{l}5.1 \cdot 1 \cdot 1 \\
5.1 \cdot 1 \cdot 2 \\
5.3 .4 .1 \\
5.1 .6 \\
5.1 .4 \\
4.3 \\
4.1 \cdot 1 \cdot 6 \cdot 3.3 \\
4.1 \cdot 1 \cdot 6 \cdot 1 \cdot 1.4\end{array}$ & $\begin{array}{l}\text { Steam, water, electricity, and laundry } \\
\text { provided. Fire suppression and detection } \\
\text { systems maintained. Solid waste disposed } \\
\text { of. Maintenance of JCS system sof tware, } \\
\text { HLAN, computers, office equipment provided. } \\
\text {. }\end{array}$ \\
\hline & & $\begin{array}{l}7.1 .1 .1 .04 \\
1 \mathrm{KP} 104\end{array}$ & Radiological Control & $\begin{array}{l}4 \cdot 1 \cdot 1 \cdot 6 \cdot 1 \cdot 1 \cdot 3 \\
4 \cdot 1 \cdot 1 \cdot 6 \cdot 1 \cdot 1 \cdot 8 \\
4.1 .1 \cdot 6 \cdot 1 \cdot 1.2\end{array}$ & $\begin{array}{l}\text { CAH operations verified, radiation } \\
\text { equipment calibrated, radiation surveys } \\
\text { performed, and associated data sheets } \\
\text { verified. }\end{array}$ \\
\hline
\end{tabular}




\section{TRANSITION PROJECTS}

\section{FY 1996 MYPP}

\subsection{1/7.1/6.12}

\section{A.I Technicäl Documents Tree \& Products Structure}

\begin{tabular}{|c|c|c|c|c|c|}
\hline $\begin{array}{l}\text { PROGRAM } \\
\text { ELEMENT }\end{array}$ & ACTIVITY & COST ACCOUNT & TITLE & $\begin{array}{l}\text { SYSTEM ENGINEERING } \\
\text { FUNCTION }\end{array}$ & PRODUCT DESCRIPTION \\
\hline \multirow[t]{2}{*}{$i_{1 K P}{ }^{1.1}$} & $\vdots$ & & PUREX/UÖ3 beact ivation & 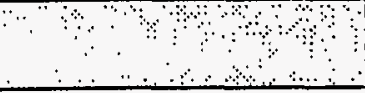 & $\therefore$ का \\
\hline & & $\begin{array}{l}7.1 .1 .1 .05 \\
1 \mathrm{KP} 105\end{array}$ & PUREX Support & $\begin{array}{l}4 \cdot 1 \cdot 1 \cdot 6 \cdot 1 \cdot 3 \cdot 6 \\
4.1 \cdot 1 \cdot 6 \cdot 1 \cdot 3 \cdot 1 \\
4.1 \cdot 1 \cdot 6 \cdot 1 \cdot 3 \cdot 2 \\
4.1 \cdot 1 \cdot 6 \cdot 1 \cdot 3 \cdot 4 \\
4.1 \cdot 1 \cdot 6 \cdot 1 \cdot 5 \cdot 1 \\
4.1 \cdot 1 \cdot 6 \cdot 1 \cdot 5 \cdot 2 \\
4.1 \cdot 1 \cdot 6 \cdot 1 \cdot 1 \cdot 2 \\
4.1 \cdot 1 \cdot 6 \cdot 1 \cdot 1.5\end{array}$ & $\begin{array}{l}\text { Operations, Heal th Physics, \& } \\
\text { administrative procedures maintained. SHM } \\
\text { inventory books maintained. Root Cause } \\
\text { Analysis of unusual occurrences, emergency } \\
\text { preparedness drills performed. Management \& } \\
\text { internal assessments performed. }\end{array}$ \\
\hline$\cdot$ & · & $\begin{array}{l}7.1 .1 .1 .06 \\
1 \mathrm{KP} 106\end{array}$ & Purchased Support & $\begin{array}{l}4.1 .1 .6 .1 .2 \\
5.2 \\
5.3 \\
5.1 .1 .2\end{array}$ & $\begin{array}{l}\text { Materials (PPE's, safety shoes, plastics, } \\
\text { etc.), plant equipment, and spares } \\
\text { inventoried, ordered, received, stocked and } \\
\text { distributed. Transportation \& preventative } \\
\text { maintenance of diesel generators performed. }\end{array}$ \\
\hline & & $\begin{array}{l}7.1 .1 .1 .07 \\
1 \mathrm{KP} 107\end{array}$ & PUREX Training & $\begin{array}{l}4 \cdot 1 \cdot 1 \cdot 6 \cdot 1 \cdot 4 \cdot 1 \\
4 \cdot 1 \cdot 1 \cdot 6 \cdot 1 \cdot 4 \cdot 2 \\
4 \cdot 1 \cdot 1 \cdot 6 \cdot 1 \cdot 4 \cdot 3\end{array}$ & $\begin{array}{l}\text { Plant personnel trained and qualifications } \\
\text { verified. Records, documentation, } \\
\text { schedul ing and testing administered. }\end{array}$ \\
\hline & & 7.1.1.1.12 & 200 Area Support Services & $\begin{array}{l}4 \cdot 1 \cdot 1 \cdot 6 \cdot 1 \cdot 2 \\
4 \cdot 1 \cdot 1 \cdot 6 \cdot 1 \cdot 1.2 \\
5.3\end{array}$ & Plant infrastructure maintained. \\
\hline & & $\begin{array}{l}7.1 .1 .1 .14 \\
1 \mathrm{KP} 114\end{array}$ & Inventory Change & $n / a$ & $n / a$ \\
\hline & $\begin{array}{l}7.1 .1 .4 \\
1 K 4^{4}\end{array}$ & $\therefore$ & beạctivation & $\therefore$ औ & ablo \\
\hline & & $\begin{array}{l}7.1 .1 .4 .01 \\
1 \mathrm{KP} 402\end{array}$ & PUREX Deactivation & $\begin{array}{l}4 \cdot 1 \cdot 1 \cdot 6 \cdot 3 \\
4 \cdot 1 \cdot 1 \cdot 6 \cdot 4 \\
4.1 \cdot 1 \cdot 6.2 .4\end{array}$ & $\begin{array}{l}\text { PUREX transitioned to the Hanford Surplus } \\
\text { Facility Program. }\end{array}$ \\
\hline & & $\begin{array}{l}7.1 .1 .4 .04 \\
1 \mathrm{KP} 404\end{array}$ & Project Management & $\begin{array}{l}4 \cdot 1 \cdot 1 \cdot 6 \cdot 3 \cdot 4 \\
4 \cdot 1 \cdot 1 \cdot 6 \cdot 2 \cdot 4 \\
4 \cdot 1 \cdot 1 \cdot 6 \cdot 1 \cdot 3 \cdot 1 \\
4 \cdot 1 \cdot 1 \cdot 6 \cdot 2 \cdot 2 \cdot 3 \\
4 \cdot 1 \cdot 1 \cdot 6 \cdot 2 \cdot 2.4\end{array}$ & $\begin{array}{l}\text { Project turnover criteria and end points } \\
\text { developed and negotiated. Transition } \\
\text { safety basis maintained. Safety } \\
\text { documentation for turnover developed. } \\
\text { Project schedules developed, maintained and } \\
\text { statused. }\end{array}$ \\
\hline
\end{tabular}




\section{TRANSITION PROJECTS}

FY 1996 MYPP

1.3.1/7.1/6.12

\section{A 1 Technical Documents Tree \& Products structure}

\begin{tabular}{|c|c|c|c|c|c|c|}
\hline $\begin{array}{c}\text { PROGRAM } \\
\text { ELEMENT }\end{array}$ & ACTIVITY & COST ACCOUNT & & SYSTEM ENGINEERING \\
\hline FUNCTION
\end{tabular}




\section{TRANSITION PROJECTS}

FY 1996 MYPP

1.3.1/7.1/6.12

\section{A.1 TechnicaT Documents Tree \& Products Structure}

\begin{tabular}{|c|c|c|c|c|c|}
\hline $\begin{array}{l}\text { PROGRAM } \\
\text { ELEMENT }\end{array}$ & ACTIVITY & COST ACCOUNT & TITLE & $\begin{array}{l}\text { SYSTEM ENGINEERING } \\
\text { FUNCTION }\end{array}$ & PROOUCT DESCRIPTION \\
\hline 71.2 & & और & 300 Area fuel supply shitdonn & $\because$ & Pै \\
\hline & 7.1 .2 .1 & & Fuel Supply Shutdown EM Activities & $\therefore$ ४खे & 3 \\
\hline & & $\begin{array}{l}7.1 .2 .1 .01 \\
\text { K3A01 }\end{array}$ & $\begin{array}{l}\text { Fuel Supply Shutdown Surveillance } \\
\text { \& Maintenance }\end{array}$ & 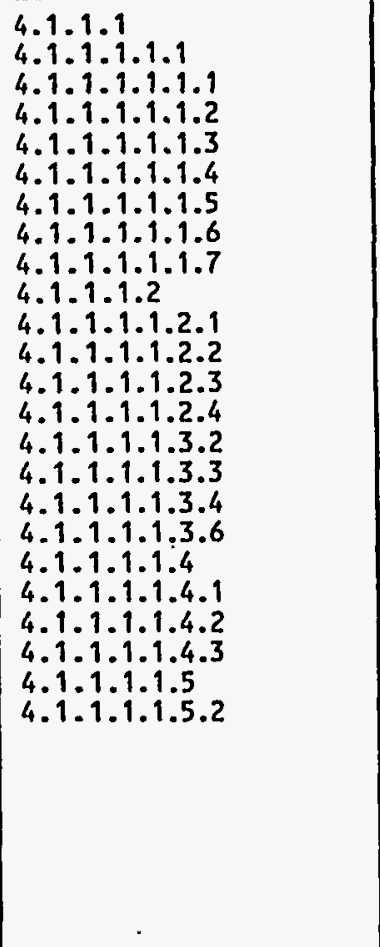 & $\begin{array}{l}\text { Surveillance and Maintenance for compl iance } \\
\text { with DOE and Regulatory requirements and to } \\
\text { maintain safe operation of the facility. } \\
\text { Surveillances to detect potential spread of } \\
\text { contamination and early response to } \\
\text { problems. Assessments necessary to } \\
\text { maintain facility including fire systems } \\
\text { maintenance. Projects, Hork task, etc } \\
\text { managed; status reports issued, administer } \\
\text { personnel training, plant coordination of } \\
\text { all maintenance \& operations activities, } \\
\text { plan and schedule plant tours per DOE } \\
\text { request, corrective action management, } \\
\text { administer occurrence reporting and perform } \\
\text { management self assessments. Unreviewed } \\
\text { Safety Question (UsQ) screening performed } \\
\text { and documented (including resolution of any } \\
\text { identified usas); emergency plans and } \\
\text { documentation maintained and emergency } \\
\text { drills performed. Facility modificat ions } \\
\text { necessary to stabilize and reconfigure the } \\
\text { facility for minimum surveillance while } \\
\text { continuing with the safe deactivation of } \\
\text { nonessential systems, components and } \\
\text { physical structures. Project management, } \\
\text { administration supports (such as } \\
\text { secretaries and clerks) and training } \\
\text { necessary. Includes formal and informal } \\
\text { training. Includes program Integration for } \\
\text { Basel ine control, ADS, and MYpp } \\
\text { preparation. }\end{array}$ \\
\hline
\end{tabular}


TRANSITION PROJECTS

FY 1996 MYPP

1.3.1/7.1/6.12

2.A.1 Technical Documents Tree \& products Structure

\begin{tabular}{|c|c|c|c|c|c|}
\hline $\begin{array}{l}\text { PROGRAM } \\
\text { ELEMENT }\end{array}$ & ACTIVITY & COST ACCOUNT & TITLE & $\begin{array}{l}\text { SYSTEM ENGINEERING } \\
\text { FUNCTION }\end{array}$ & PRODUCT DESCRIPTION \\
\hline 7.1 .2 & 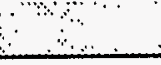 & $\therefore \quad \because$ & 300 Area Fuel Supply shutdown & 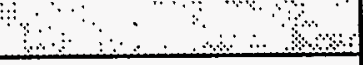 & 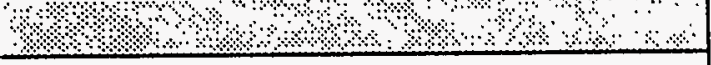 \\
\hline & • & $\begin{array}{l}7.1 .2 .1 .02 \\
K 3 \mathrm{~A} 02\end{array}$ & $\begin{array}{l}\text { Fuel supply Shutdown RCRA } \\
\text { Activities }\end{array}$ & 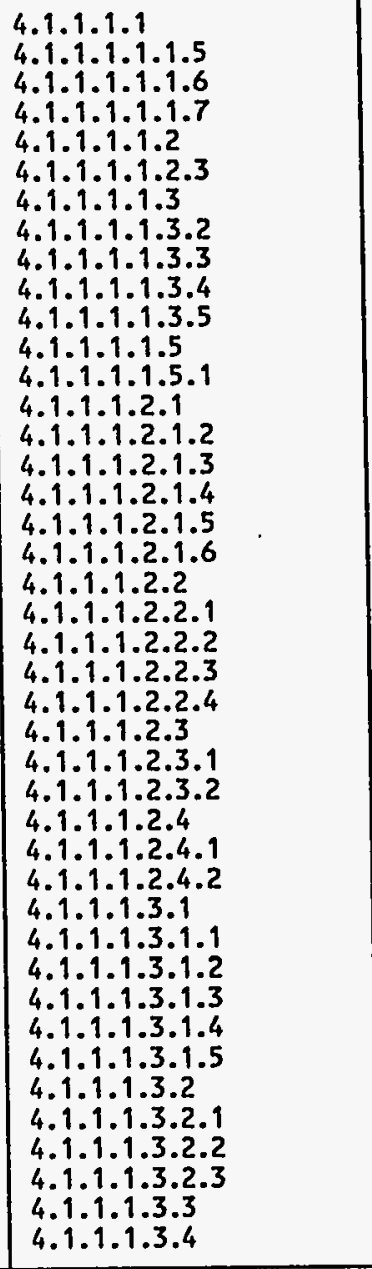 & $\begin{array}{l}\text { Includes environmental compliance, } \\
\text { environimental reporting, independent and } \\
\text { management assessments. Includes RCRA } \\
\text { Closures which contributes to the } \\
\text { deactivation project, including sampl ing } \\
\text { and analysis, validation of data, packaging } \\
\text { and shipping of waste. } \\
\qquad\end{array}$ \\
\hline
\end{tabular}


TRANSITION PROJECTS

FY 1996 MYPP

1.3.1/7.1/6.12

2.A.1 Techinical Documents Tree \& Products Structure

\begin{tabular}{|c|c|c|c|c|c|}
\hline $\begin{array}{l}\text { PROGRAM } \\
\text { ELEMENT }\end{array}$ & ACtivity & COST ACCOUNT & TITLE & $\begin{array}{l}\text { SYSTEM ENGINEERING } \\
\text { FUNCTION }\end{array}$ & PRODUCT DESCRIPTION \\
\hline 7.1 .2 & & & 300 Area fuel supply shutdounn & 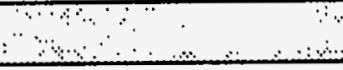 & ? \\
\hline & & $\underset{\mathrm{K} 3 \mathrm{~A} 03}{7.1 .2 .1 .03}$ & $\begin{array}{l}\text { Fuel Supply shutdown - shutdown } \\
\text { Activities }\end{array}$ & 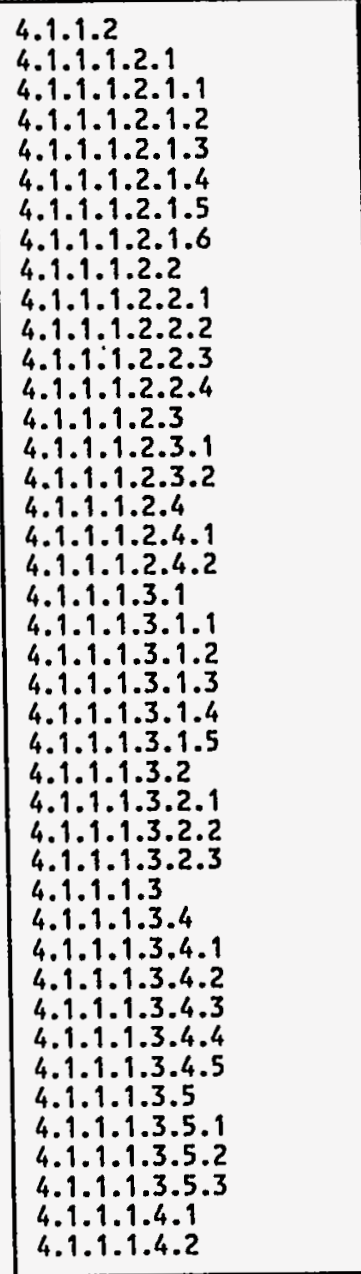 & $\begin{array}{l}\text { Manage and execute the actions necessary to } \\
\text { meet shutdown criteria including the } \\
\text { establ ishment of this criteria. Includes } \\
\text { updates to shutdown plan and other shutdown } \\
\text { documents. Provides packaging, handl ing, } \\
\text { inspecting and shipping of waste drums, and } \\
\text { burial boxes non RCRA radioactive } \\
\text { hazardous, non-regulated and radioactive } \\
\text { mixed waste and their corresponding waste } \\
\text { disposal assessments. Provides for the } \\
\text { packaging and transportation of SNM for } \\
\text { relocation. }\end{array}$ \\
\hline
\end{tabular}


TRANSITION PROJECTS

FY 1996 MYPP

1.3.1/7.1/6.12

2.A.1 Technicaf Documents Tree $\&$ Products Structure

\begin{tabular}{|c|c|c|c|c|c|}
\hline $\begin{array}{l}\text { PROGRAM } \\
\text { ELEMENT }\end{array}$ & ACTIVITY & COST ACCOUNT & TITLE & $\begin{array}{l}\text { SYSTEM ENGINEERIHG } \\
\text { FUNCTION } \\
\end{array}$ & PRODUCT DESCRIPTION \\
\hline 7.1 .2 & & & 300 Area fuel supply shutdoun & י४४ & ४ै। \\
\hline & & $\underset{\mathrm{K} 3 \mathrm{AO} 04}{7.1 .2 .1 .04}$ & $\begin{array}{l}\text { Fuel Supply Shutdown } 313 \text { South } \\
\text { Building Isolation }\end{array}$ & 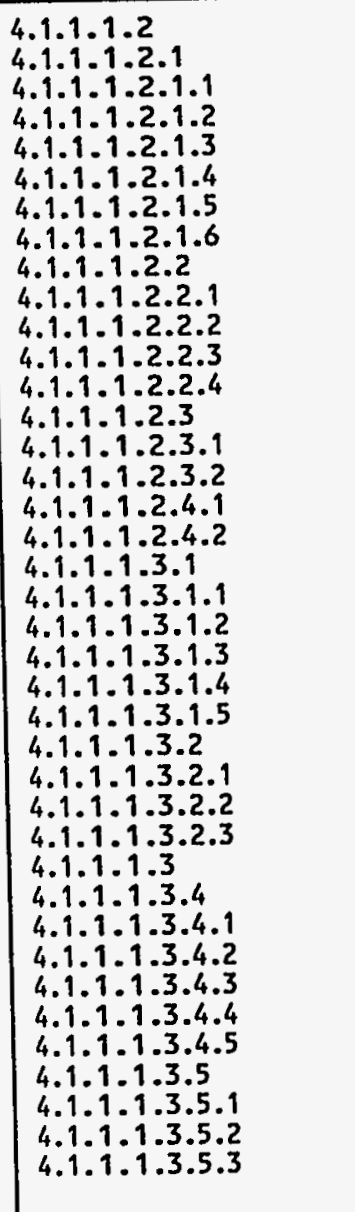 & $\begin{array}{l}\text { Includes deactivation and stabilization and } \\
\text { reconfiguration, including sampling and } \\
\text { analysis, data validation, project } \\
\text { management and engineering support, } \\
\text { modification to the structure, waste } \\
\text { characterization, packaging, } \\
\text { transportation, and assessments, and } \\
\text { turnover criteria elements. } \\
\qquad\end{array}$ \\
\hline
\end{tabular}


TRANSITION PROJECTS

FY 1996 MYPP

1.3.1/7.1/6.12

2.A.1 Technical Documents Tree \& Products Structure

\begin{tabular}{|c|c|c|c|c|c|}
\hline $\begin{array}{l}\text { PROGRAM } \\
\text { ELEMENT }\end{array}$ & ACTIVITY & COST ACCOUNT & TITLE & $\begin{array}{l}\text { SYSTEM ENGINEERING } \\
\text { FUNCTION }\end{array}$ & PRODUCT DESCRIPTIION \\
\hline 7.1 .2 & 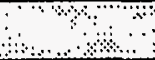 & & 300 Area fuelsupply shutdown & - & 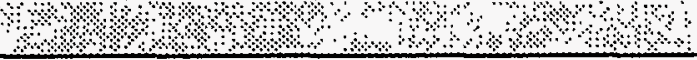 \\
\hline & - & ${ }_{\text {K3A05 }}^{7.1 .2 .1 .05}$ & $\begin{array}{l}\text { Fuel supply shutdown SNM Safe } \\
\text { Storage }\end{array}$ & 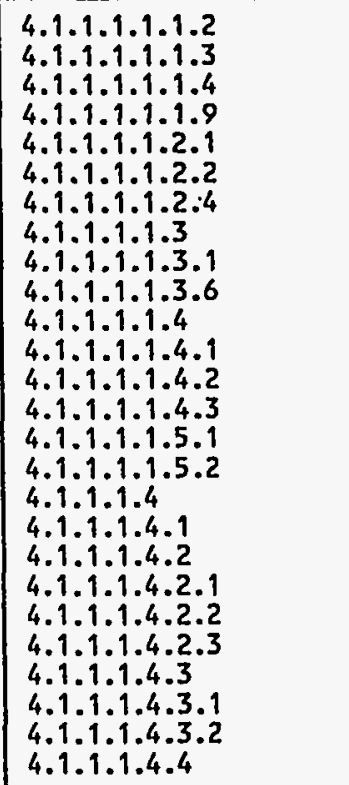 & $\begin{array}{l}\text { Includes the safe and compl iant storage of } \\
\text { Special Nuclear Materials, including } \\
\text { stabilization, work control, surveillances, } \\
\text { preventative and corrective maintenance. } \\
\text { Includes sNM management, Safeguards and } \\
\text { Security, inventory control/reports, } \\
\text { storage reports and other Documentation. } \\
\text { Also includes training, qualifications, and } \\
\text { certifications. Also facillity assessments } \\
\text { including fire maintenance that pertain to } \\
\text { these buildings. Provides the management } \\
\text { and administrative/program integration } \\
\text { support required for project direction and } \\
\text { control associated to this sNM activity. } \\
\text {. }\end{array}$ \\
\hline
\end{tabular}


TRANSITION PROJECTS

FY 1996 MYPP

1.3.1/7.1/6.12

\section{A.1 Technical Documents Tree \& Products Structure}

\begin{tabular}{|c|c|c|c|c|c|}
\hline $\begin{array}{l}\text { PROGRAM } \\
\text { ELEMENT }\end{array}$ & ACTIVITY & COST ACCOUNT & TITLE & $\begin{array}{l}\text { SYSTEN ENGINEERING } \\
\text { FUNCTION }\end{array}$ & PRODUCT DESCRIPTION \\
\hline \multirow[t]{7}{*}{7.1 .3} & & $\nabla$, & Plutonium Finishing Plant & 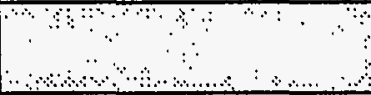 & - \\
\hline & $7.1 .3,1 \%$ & $\because \because$ & PFp surue i lance und Maintenance & as & ( \\
\hline & & $\underset{K 6 A 01}{7.1 .3 .1 .01}$ & Corrective Maintenance & 4.1 .1 .2 .1 .2 .1 & $\begin{array}{l}\text { OSR, Plant Essential, \& Balance of Plant } \\
\text { equipment repaired/replaced and operational } \\
\text { status verified. Excludes equipment } \\
\text { located in vaults and vault-type rooms. }\end{array}$ \\
\hline & . & ${ }_{K 6 A 02}^{7.1 .3 .1 .02}$ & Preventative Maintenance & 4.1 .1 .2 .1 .2 .2 & $\begin{array}{l}\text { OSR, Plant Essential, \& Balance of Plant } \\
\text { equipment calibrated and maintained with } \\
\text { operational condition/status verified. } \\
\text { Excludes equipment located in vaul ts and } \\
\text { vault-type rooms. }\end{array}$ \\
\hline & & $\underset{K 6 \mathrm{~A} 03}{7.1 .3 .1 .03}$ & $\begin{array}{l}\text { Nuclear Process/Radiation } \\
\text { Surveillances }\end{array}$ & 4.1 .1 .2 .1 .1 .1 .4 & $\begin{array}{l}\text { Surveillance for proper operation performed } \\
\text { on all OSR and essential equipment, } \\
\text { CAM/room air filter systems and radiation } \\
\text { surveys performed with associated data } \\
\text { sheets filled out and verified. Excludes } \\
\text { equipment located in vaults and vault-type } \\
\text { rooms. }\end{array}$ \\
\hline & & $\frac{7.1 .3 .1 .04}{K 6 A 04}$ & Ventilation/Power Surveillances & 4.1 .1 .2 .1 .1 .1 .4 & $\begin{array}{l}\text { Surveillance for proper operation performed } \\
\text { on all ventilation/power equipment systems } \\
\text { with associated data sheets filled out and } \\
\text { verified. Excludes equipment located in } \\
\text { vaults and vault-type rooms. }\end{array}$ \\
\hline & & $\underset{K 6 A 05}{7.1 .3 .1 .05}$ & Environmental Compliance & $\begin{array}{l}4.1 \cdot 1 \cdot 2 \cdot 1 \cdot 1 \cdot 1.4 \\
4.1 .1 .2 .1 .3 .5\end{array}$ & $\begin{array}{l}\text { Surveillance for proper operation performed } \\
\text { on all Environmental monitoring systems and } \\
\text { associated equipment; modifications for } \\
\text { environmental compl iance completed with } \\
\text { operational condition/status verified; all } \\
\text { required environmental compl iance } \\
\text { documentation (Permits, etc.) generated and } \\
\text { submitted to state and government agencies. }\end{array}$ \\
\hline
\end{tabular}


TRANSITION PROJECTS

FY 1996 MYPP

1.3.1/7.1/6.12

\section{A.1 Technicaf Documents Tree \& Products Structure}

\begin{tabular}{|c|c|c|c|c|c|}
\hline $\begin{array}{l}\text { PROGRAM } \\
\text { ELEMENT }\end{array}$ & ACTIVITY & COST ACCOUNT & TITLE & $\begin{array}{l}\text { SYSTEM ENGINEERING } \\
\text { FUNCTION }\end{array}$ & PRODUCT DESCRIPTION \\
\hline \multirow[t]{6}{*}{7.1 .3} & & is & Plutonium Finishing ptant & ४ै। & १ै \\
\hline & & $\underset{K 6 \mathrm{AOS}}{7.1 .3 .1 .06}$ & $\begin{array}{l}\text { Safety Boundary \& Emergency } \\
\text { Planning }\end{array}$ & 4.1 .1 .2 .1 .3 .1 & $\begin{array}{l}\text { FSARs and CSERs updated and maintained; } \\
\text { Unreviewed Safety Question (USA) screening } \\
\text { performed and documented (including } \\
\text { resolution of any identified USQS); } \\
\text { emergency plans and documentation } \\
\text { maintained and emergency drills performed. }\end{array}$ \\
\hline & & 7.1.3.1.07 & Procedure Maintenance & 4.1 .1 .2 .1 .3 & $\begin{array}{l}\text { All Operational, Laboratory, Maintenance, } \\
\text { and Administrative procedures maintained } \\
\text { with all reviews and revisions documented. }\end{array}$ \\
\hline & & $\underset{\mathrm{K} 6 \mathrm{A08}}{7.1 .3 .1 .08}$ & Plant Specific Support & $\begin{array}{l}4.1 .1 .2 .1 .2 \\
4.1 .1 .2 .1 .1\end{array}$ & $\begin{array}{l}\text { Protective clothing inventoried and } \\
\text { distributed; consumable equipment and } \\
\text { supplies inventoried, ordered, received, } \\
\text { stocked and distributed; waste drum } \\
\text { handling (includes NDA assays, packaging, } \\
\text { shipping, accountabil ity and required } \\
\text { documentat ion); fire systems maintenance } \\
\text { activities supported; general operational } \\
\text { condition of Labs maintained and } \\
\text { control led. }\end{array}$ \\
\hline & . & $\underset{K 6 A 09}{7.1 .3 .1 .09}$ & Project Management & $\begin{array}{l}4.1 \cdot 1 \cdot 2 \cdot 1.1 .1 \\
4.1 .1 .2 .1 .5 \\
4.1 .1 .2 .1 .4\end{array}$ & $\begin{array}{l}\text { Projects, work tasks, etc. managed; status } \\
\text { reports issued, administer personnel } \\
\text { training, plant coordination of all } \\
\text { maintenance \& operations activities, plan \& } \\
\text { schedule plant tours per DOE request, } \\
\text { corrective action management, administer } \\
\text { occurrence reporting and perform management } \\
\text { self assessments. }\end{array}$ \\
\hline & $\begin{array}{l}7.1 .3 .2 \\
\mathrm{~K} 6 \mathrm{~B}\end{array}$ & & $\begin{array}{l}\text { Stabi i izè/Reconfigure for Minimum } \\
\text { Surve il lance. }\end{array}$ & $4.1 .1 .2 .3 \quad$ an & 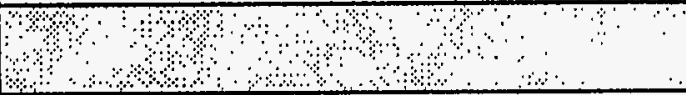 \\
\hline
\end{tabular}




\section{A.1 Technical Documents Tree \& Products Structure}

\begin{tabular}{|c|c|c|c|c|c|}
\hline $\begin{array}{l}\text { PROGRAM } \\
\text { ELEMENT }\end{array}$ & ACTIVITY & COST ACCOUNT & TITLE & $\begin{array}{l}\text { SYSTEM ENGINEERING } \\
\text { FUNCTION }\end{array}$ & PRODUCT DESCRIPTION \\
\hline \multirow[t]{5}{*}{$\begin{array}{c}7.1 .3 \\
K 6\end{array}$} & $\because \because 3$ & : & plutonium finishing plant & क्या & \% \\
\hline & & $\underset{K 6 B 0 A}{7.1 .3 .2 .01}$ & Thermal Treatment & $\begin{array}{l}4.1 \cdot 1 \cdot 2.4 .3 \\
4.7 .1 .4 \cdot 1.2\end{array}$ & $\begin{array}{l}\text { Treatment of Pu-bearing materials to } \\
\text { transform them into a stable Pu oxide form } \\
\text { suitable for } 50-y e a r \text { storage. It includes } \\
\text { treatment, when necessary, for organic } \\
\text { destruction by hydrolysis prior to furnace } \\
\text { treatment. Thermal treatment includes } \\
\text { stabilization in two existing and/or nine } \\
\text { new muffle furnaces. Also provided will be } \\
\text { a feasibility determination for thermal } \\
\text { treatment via a continuous calciner, to be } \\
\text { used primarily for the stabilization of Pu } \\
\text { oxides. }\end{array}$ \\
\hline & & $\underset{K G B O B}{7.1 .3 .2 .02}$ & Solution Treatment & $\begin{array}{l}4 \cdot 1 \cdot 1 \cdot 2.4 .3 \\
4.7 .1 .4 \cdot 1.2\end{array}$ & $\begin{array}{l}\text { Provides the design, procurement, } \\
\text { installation, testing and operation of a } \\
\text { continuously operating vertical calciner } \\
\text { with batch-operated precipitation and } \\
\text { filtration system. This process will be } \\
\text { designed to attain a throughput of } \\
\text { approximately } 275 \text { liters of feed per month. }\end{array}$ \\
\hline & & $\begin{array}{l}7.1 .3 .2 .03 \\
\text { K6BOC }\end{array}$ & Pyrolysis furnace & $\begin{array}{l}4 \cdot 1 \cdot 1 \cdot 2 \cdot 4 \cdot 3 \\
4.7 \cdot 1 \cdot 4 \cdot 1.2\end{array}$ & $\begin{array}{l}\text { Pyrolysis Furnaces ( } 2 \text { stage) designed, } \\
\text { constructed, tested and verified } \\
\text { operational. Provides activities directly } \\
\text { associated Hith transforming the current } \\
\text { inventory of Pu-bearing polycubes and } \\
\text { miscellaneous combustible materials to a } \\
\text { stable oxide form suitable for 50-year } \\
\text { storage. }\end{array}$ \\
\hline & & $\begin{array}{l}7.1 .3 .2 .04 \\
\mathrm{~K} 6 \mathrm{BOD}\end{array}$ & Miscellaneous Treatment Facility & $\begin{array}{l}4 \cdot 1 \cdot 1 \cdot 2 \cdot 4.3 \\
4.7 .1 .4 .1 .2\end{array}$ & $\begin{array}{l}\text { Development, testing and implementation of } \\
\text { any applicable pretreatment systems } \\
\text { necessary to support stabilization of } \\
\text { unusual or unique plutonium-bearing } \\
\text { materials in inventory at PFP. } \\
\text { Pretreatment may involve characterization, } \\
\text { the development of a pretreatment process, } \\
\text { appropriate testing and the pretreatment } \\
\text { itself. This activity does Nor include the } \\
\text { final stabilization of the material. }\end{array}$ \\
\hline
\end{tabular}




\section{TRANSITION PROJECTS}

\section{FY 1996 MYPP}

\subsection{1/7.1/6.12}

\section{A. 1 Technical Documents Tree \& Products Structure}

\begin{tabular}{|c|c|c|c|c|c|}
\hline $\begin{array}{l}\text { PROGRAM } \\
\text { ELEMENT }\end{array}$ & ACTIVITY & COST ACCOUNT & TITLE & $\begin{array}{l}\text { SYSTEM ENGINEERING } \\
\text { FUNCTION }\end{array}$ & PRODUCT DESCRIPTION \\
\hline \multirow[t]{5}{*}{$\begin{array}{r}713 \\
\times 6 \\
\end{array}$} & $\because$ & \begin{tabular}{r}
4 \\
\hdashline \\
\end{tabular} & pífoniun F inishing ṕlant & \% & Pै। \\
\hline & & $\begin{array}{l}7.1 .3 .2 .05 \\
\text { X6BOE }\end{array}$ & Cleanup \& Terminal Cleanout (TCO) & $\begin{array}{l}4 \cdot 1 \cdot 1 \cdot 2 \cdot 3 \cdot 2 \cdot 2 \\
4.1 \cdot 1 \cdot 2 \cdot 3 \cdot 2 \cdot 3 \\
4 \cdot 1 \cdot 1 \cdot 5 \cdot 3 \cdot 3 \cdot 1\end{array}$ & $\begin{array}{l}\text { The TCO of residue plutonium-bearing } \\
\text { materials from identified locations } \\
\text { throughout the PFP Complex and cleanup of } \\
\text { the identified location from which the } \\
\text { residue plutonium-bearing material has been } \\
\text { removed. Involves removal of equipment, } \\
\text { systems, lines, gloveboxes and/or } \\
\text { structures as necessary to properly remove } \\
\text { the residual plutonium. Gloveboxes will be } \\
\text { cleaned up, placed in stored condition and } \\
\text { verified to meet EM- } 40 \text { acceptance criteria. }\end{array}$ \\
\hline & & $\begin{array}{l}7.1 .3 .2 .06 \\
\text { K6BOF }\end{array}$ & $\begin{array}{l}\text { DNFSB 94-1 Facility Modifications } \\
\text { (ADS 6625) }\end{array}$ & $\begin{array}{l}4 \cdot 1 \cdot 1 \cdot 2 \cdot 2 \\
4 \cdot 1 \cdot 1 \cdot 2 \cdot 3 \\
4 \cdot 1 \cdot 1 \cdot 2.4 \\
4 \cdot 7 \cdot 1 \cdot 4 \cdot 1 \\
4.7 \cdot 1 \cdot 4 \cdot 2 \\
4.7 \cdot 1 \cdot 5 \cdot 1\end{array}$ & $\begin{array}{l}\text { Facility modifications (Line Items) funded } \\
\text { through ADS } 6625 \text { which are necessary to } \\
\text { support implementation of DNFSB } \\
\text { Recomendation } 94-1 \text { activities within the } \\
\text { PFP Complex. Specifically, modifications } \\
\text { to vaults, packaging system } \\
\text { modifications, and material container } \\
\text { changes. }\end{array}$ \\
\hline & · & $\begin{array}{l}7.1 .3 .2 .07 \\
K 680 G\end{array}$ & Laboratory Support \& Upgrades & $\begin{array}{l}4 \cdot 1 \cdot 1 \cdot 2.1 \\
4.1 \cdot 1 \cdot 2.2 \\
4 \cdot 7 \cdot 1 \cdot 4 \cdot 1 \\
4.7 .1 .5 .1\end{array}$ & $\begin{array}{l}\text { Analytical laboratory support, including } \\
\text { material analyses and maintaining } \\
\text { standards, and necessary laboratory } \\
\text { upgrades, including both Analytical Lab and } \\
\text { Plutonium Processing Support Lab, to } \\
\text { support DNFSB } 94-1 \text { Project. }\end{array}$ \\
\hline & & $\begin{array}{l}7.1 .3 .2 .08 \\
K 6 B 02\end{array}$ & $\begin{array}{l}\text { Facility Modification for Minimum } \\
\text { Surveillance }\end{array}$ & $4.1 \cdot 1.2 .3 .1$ & $\begin{array}{l}\text { Facility modifications necessary to } \\
\text { stabilize and reconfigure the facility for } \\
\text { minimum surveillance while continuing with } \\
\text { the safe deactivation of nonessential } \\
\text { systems, components and physical } \\
\text { structures. }\end{array}$ \\
\hline & & $\begin{array}{l}7.1 .3 .2 .09 \\
\mathrm{~K} 6 \mathrm{~B} 03\end{array}$ & Engineering Studies/Evaluations & 4.1 .1 .2 .2 .4 & $\begin{array}{l}\text { Engineering studies and/or evaluations } \\
\text { necessary to stabilize and reconfigure the } \\
\text { facility while continuing with the safe } \\
\text { deactivation of nonessential systems, } \\
\text { components and physical structures. }\end{array}$ \\
\hline
\end{tabular}


TRANSITION PROJECTS

FY 1996 MYPP

1.3.1/7.1/6.12

2.A.1 Technjoal Documents Tree \& Products structure

\begin{tabular}{|c|c|c|c|c|c|}
\hline $\begin{array}{l}\text { PROGRAM } \\
\text { ELEMENT }\end{array}$ & ACTIVITY & COST ACCOUNT' & TITLE & $\begin{array}{l}\text { SYSTEM ENGINEERING } \\
\text { FUNCTION } \\
\end{array}$ & PRODUCT DESCRIPTION \\
\hline 7.91 .3 & sis & @ & Plutonium finishing plant & मे० & 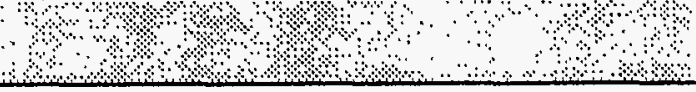 \\
\hline & & $\underset{K 6 B 04}{7.1 .3 .2 .010}$ & $\begin{array}{l}\text { Environmental Documentation and } \\
\text { Regulatory Negotiations }\end{array}$ & $4.1 \cdot 1 \cdot 2.2 .4$ & $\begin{array}{l}\text { Environmental compl iance documentation, } \\
\text { including any additional negotiations for } \\
\text { related environmental compl iance } \\
\text { activities, necessary to stabilize and } \\
\text { reconf igure the facility while cont inuing } \\
\text { with safe deactivation of nonessential } \\
\text { systems, components and physical } \\
\text { structures. Includes all environmental } \\
\text { documentation, except the PFP Cleanup EIs, } \\
\text { necessary to support ONFSB Recommendation } \\
94-1 \text { at PFP. }\end{array}$ \\
\hline & & $\underset{K 6805}{7.1 .3 .2 .11}$ & EIS Project Support & 4.1 .1 .2 .2 .4 & $\begin{array}{l}\text { Provides all pFP Transition Project support } \\
\text { to the preparation, negotiation, rel ease } \\
\text { and final approval of the PFP Environmental } \\
\text { Impact Statement. }\end{array}$ \\
\hline & & $\underset{K 6806}{7.1 .3 .2 .12}$ & Project Management & $\begin{array}{l}4.1 \cdot 1 \cdot 2 \cdot 1 \cdot 1.1 \\
4.1 \cdot 1 \cdot 2 \cdot 1.5 \\
4.1 .1 .2 \cdot 1.4\end{array}$ & $\begin{array}{l}\text { Project management, administration support } \\
\text { (such as secretaries and clerks) and } \\
\text { training necessary for the implementation } \\
\text { of DNFSB Recommendation } 94-1 \text { at PFP. } \\
\text { Project management support necessary to } \\
\text { safely deactivate nonessential systems, } \\
\text { system components, and physical structures } \\
\text { in coordination with implementation of } \\
\text { oNFSB } 94-1 \text { activities. Includes formal } \\
\text { training, oJT raining, and all job } \\
\text { specific training needed to perform the } \\
\text { identified activities. }\end{array}$ \\
\hline & $7: 1.3 .3$ & & SNM Safe Storage and Treatment & $\because \because$ & $\begin{array}{l}0 \\
\cdots\end{array}$ \\
\hline
\end{tabular}




\section{TRANSITION PROJECTS}

FY 1996 MYPP

1.3.1/7.1/6.12

\section{A.1 Technicat Documentes Tree $\&$ products structure}

\begin{tabular}{|c|c|c|c|c|c|}
\hline $\begin{array}{l}\text { PROGRAM } \\
\text { ELEMENT }\end{array}$ & ACTIVITY & COST ACCOUNT & TITLE & $\begin{array}{l}\text { SYSTEH ENGINEERING } \\
\text { FUNCTION }\end{array}$ & PRODUCT DESCRIPTION \\
\hline \multirow[t]{4}{*}{$\begin{array}{l}7.1 .3 \\
K 6\end{array}$} & $\because: \because$ & $\because$ & Plutonium finishing plant & a & Pै? \\
\hline & & $\begin{array}{l}7.1 .3 .3 .02 \\
\mathrm{~K} 6 \mathrm{CO2}\end{array}$ & SNM Shipments/Receipts & 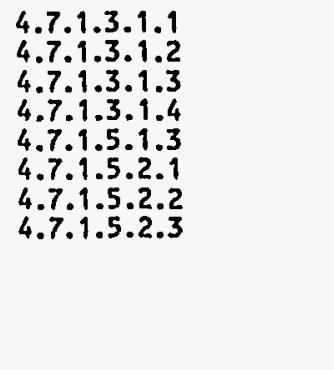 & $\begin{array}{l}\text { Provides the documentation and technical } \\
\text { verification for the receipt and shipment } \\
\text { of SNM to and from the PFP Complex. } \\
\text { Technical verification of received material } \\
\text { is to include NDA and/or other confirmatory } \\
\text { measurement activities as required. } \\
\text { Ensures proper transfer of custody, } \\
\text { verifies manifest and material } \\
\text { docurientation and performs necessary } \\
\text { conf irmatory measurements to place the } \\
\text { received material into a safe and secure } \\
\text { storage configuration. }\end{array}$ \\
\hline & & $\begin{array}{l}7.1 .3 .3 .03 \\
\mathrm{~K} 6 \mathrm{CO} 3\end{array}$ & SNM Inventories & $\begin{array}{l}4 \cdot 1 \cdot 1 \cdot 2 \cdot 1.3 \\
4 \cdot 7 \cdot 1 \cdot 1 \cdot 1.2 \\
4 \cdot 7 \cdot 1 \cdot 4 \cdot 2 \cdot 1\end{array}$ & $\begin{array}{l}\text { Performs required routine SNM inventories, } \\
\text { and special SNM inventories, necessary to } \\
\text { satisfy requirements and regulations. } \\
\text { Inventories are substantiated through } \\
\text { nondestructive assays or other measurement } \\
\text { methodologies and any inventory differences } \\
\text { reconciled to standards. }\end{array}$ \\
\hline & & $\begin{array}{l}7.1 .3 .3 .04 \\
\text { K6C04 }\end{array}$ & Vault Modifications & 4.7 .1 .1 .2 .4 & $\begin{array}{l}\text { Provides review of vault, and vault-type } \\
\text { room, conditions and viability to meet } \\
\text { plant mission, comply with } \\
\text { regulations/requirements and to minimize } \\
\text { costs. Performs studies to determine and } \\
\text { evaluate al ternatives for vault } \\
\text { modifications and acts on the evaluations } \\
\text { as needed. }\end{array}$ \\
\hline & & $\begin{array}{l}7.1 .3 .3 .05 \\
\mathrm{~K} 6 \mathrm{CO} 5\end{array}$ & $\begin{array}{l}\text { Environmental Compliance } \\
\text {. }\end{array}$ & $\begin{array}{l}4.7 .1 .1 .2 .4 \\
4.7 .1 .1 .3 .5\end{array}$ & $\begin{array}{l}\text { Support to assure compliance to new and } \\
\text { existing environmental regulations } \\
\text { pertaining to vault storage facilities and } \\
\text { long term storage of SNM/NM/NF materials. } \\
\text { Addresses any identified environnental } \\
\text { issues, such as required NEPA } \\
\text { documentation, Permitting, et cetera, } \\
\text { required to support vault operations. }\end{array}$ \\
\hline
\end{tabular}




\section{TRANSITION PROJECTS}

\section{A.1 Techinical Documents Tree \& Products structure}

\begin{tabular}{|c|c|c|c|c|c|}
\hline $\begin{array}{l}\text { PROGRAM } \\
\text { ELEMENT }\end{array}$ & ACTIVITY & COST ACCOUNT & TITLE & $\begin{array}{l}\text { SYSTEN ENGINEERING } \\
\text { FUNCTION }\end{array}$ & PRODUCT DESCRIPTIOH \\
\hline \multirow[t]{4}{*}{$\begin{array}{c}7.1 .3 \\
\times 6 . \\
\end{array}$} & & $\begin{array}{ccc}\ddots & \ddots \\
\ddots & \ddots \\
1\end{array}$ & P.lutonium Finishing plant & & 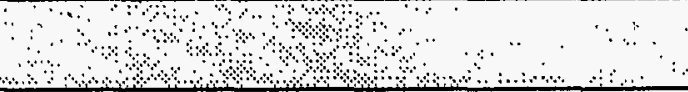 \\
\hline & & ${ }_{\mathrm{K} 6 \mathrm{C} 06}^{7.1 .3 .3 .06}$ & IAEA Support Activities & $\begin{array}{l}4 \cdot 7 \cdot 1 \cdot 1 \cdot 1 \\
4 \cdot 7 \cdot 1 \cdot 1.2 \\
4.7 \cdot 1 \cdot 1.3 \\
4.7 \cdot 1 \cdot 1.5\end{array}$ & $\begin{array}{l}\text { Supports the intent of Implementation of } \\
\text { International Safeguards at PFP and places } \\
\text { fissile materials no longer needed for } \\
\text { deterrent under the U.S. - International } \\
\text { Atomic Energy Agency (IAEA) Voluntary } \\
\text { Safeguards Agreement. Provides support for } \\
\text { IAEA-related inspections (monthly, annual, } \\
\text { etc.), tours, and Ad Hoc visits. Also } \\
\text { covered is all support for operation and } \\
\text { maintenance of Vault \#3 consistent with } \\
\text { IAEA Safeguards requirements. }\end{array}$ \\
\hline & & $\underset{\mathrm{K} 6 \mathrm{CO} 7}{7.1 .3 .3 .07}$ & Engineering Studies/Assessments & 4.1 .1 .2 .4 & $\begin{array}{l}\text { Provides engineering studies and } \\
\text { assessments as required to determine vault } \\
\text { storage alternatives and options necessary } \\
\text { to support deactivation of the PFP Complex. }\end{array}$ \\
\hline & & $\underset{K 6 C 08}{7.1 .3 .3 .08}$ & Corrective Maintenance & $\begin{array}{l}4.7 .1 .1 .2 .1 \\
4.7 .1 .1 .2 .2\end{array}$ & $\begin{array}{l}\text { Corrective maintenance for the } 2736-2 / 2736- \\
2 B \text { facilities, all } 2 B / 2-v a u l t \text { support } \\
\text { facilities, such as the } 2721-z \text { facility, } \\
\text { and all } 234-52 \text { vaults and/or vault-type } \\
\text { rooms, that brings systems and equipment } \\
\text { back to their operational states after } \\
\text { failure. }\end{array}$ \\
\hline & · & $\underset{K 6 C 09}{7.1 .3 .3 .09}$ & $\begin{array}{l}\text { Preventative Maintenance } \\
\text {. }\end{array}$ & $\begin{array}{l}4.7 .1 .1 .2 .1 \\
4.7 .1 .1 .2 .2\end{array}$ & $\begin{array}{l}\text { Preventative maintenance activities to } \\
2736-2 / 2736-2 B \text { facilities, all } 2 B / Z-v a u l t \\
\text { support facilities, such as the } 2721-2 \\
\text { facility, and all } 234-52 \text { vaults and/or } \\
\text { vault-type rooms necessary to minimize all } \\
\text { unplanned events and premature equipment } \\
\text { failures. }\end{array}$ \\
\hline
\end{tabular}




\section{TRANSITION PROJECTS}

FY 1996 MYPP

1.3.1/7.1/6.12

2.A.1 Technical Documents Tree \& Products Structure

\begin{tabular}{|c|c|c|c|c|c|}
\hline $\begin{array}{l}\text { PROGRAM } \\
\text { ELEMENT }\end{array}$ & ACTIVITY & COST ACCOUNT & TITLE & $\begin{array}{l}\text { SYSTEM ENGINEERING } \\
\text { FUNCTION }\end{array}$ & PRODUCT DESCRIPTION \\
\hline \multirow[t]{5}{*}{7.1 .3} & $\begin{array}{lll}\because & \cdots \\
\because & \because & \cdots \\
\end{array}$ & & Putonium finishing plant & : & Pै। \\
\hline & & $\begin{array}{l}7.1 .3 .3 .10 \\
K 6 C 10\end{array}$ & $\begin{array}{l}\text { Nucl ear Process/Radiation } \\
\text { Surveillances }\end{array}$ & 4.7 .1 .1 .1 .1 & $\begin{array}{l}\text { Provides nuclear process and radiation } \\
\text { surveillances of facility operations and } \\
\text { operating systems in PFP complex vault- } \\
\text { related facilities and develops acutely } \\
\text { unsafe condition action plans for nuclear } \\
\text { processes and radiation activities. Also } \\
\text { included is the implementation of oSR } \\
\text { surveillance procedures and the performance } \\
\text { of surveillances for safety analysis } \\
\text { compliance for nuclear processes and } \\
\text { radiation activities. }\end{array}$ \\
\hline & . & $\underset{\mathrm{K} 6 \mathrm{C} 11}{7.1 .3 .3 .11}$ & Ventilation/Power Surveillances & 4.7 .1 .1 .1 .1 & $\begin{array}{l}\text { Provides ventilation- and power-related } \\
\text { surveillances of facility operations and } \\
\text { operating systems in PFP complex vault- } \\
\text { related facilities. Develops acutely } \\
\text { unsafe condition action plans, implements } \\
\text { vault related oSR surveillance procedures } \\
\text { and provides surveillances for safety } \\
\text { analysis compliance of vault-related power } \\
\text { and ventilation systems. }\end{array}$ \\
\hline & & $\underset{K 6 C 13}{7.1 .3 .3 .13}$ & Project Management & $\begin{array}{l}4.7 .1 .1 .4 \\
4.7 .1 .2\end{array}$ & $\begin{array}{l}\text { Provides the management and administrative } \\
\text { support required for project direction and } \\
\text { control. Provides training associated with } \\
\text { the project activities pertaining to this } \\
\text { SNM Vault Activity. }\end{array}$ \\
\hline & $\begin{array}{l}7.1: 3.4 \\
K 60\end{array}$ & & $\begin{array}{l}\text { Deactivation Plahning \& Turnover } \\
\text { Endpoint. }\end{array}$ & & 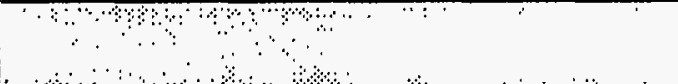 \\
\hline
\end{tabular}




\section{TRANSITION PROJECTS}

FY 1996 MYPP

1.3.1/7.1/6.12

\section{A.1 Technical Documents Tree \& Products structure}

\begin{tabular}{|c|c|c|c|c|c|}
\hline $\begin{array}{l}\text { PROGRAM } \\
\text { ELEMENT }\end{array}$ & ACTIVITY & COST ACCOUNT & TITLE & $\begin{array}{l}\text { SYSTEM ENGINEERING } \\
\text { FUNCTION }\end{array}$ & PRODUCT DESCRIPTION \\
\hline \multirow[t]{6}{*}{$\begin{array}{l}7.1 .3 \\
K 6\end{array}$} & 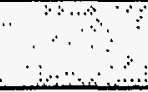 & \begin{tabular}{lll}
$\ddots$ \\
\hdashline \\
\hdashline
\end{tabular} & Plutoniun finishing Plant & $\therefore \quad \cdots$ & ४ै। \\
\hline & & $\begin{array}{l}7.1 .3 .4 .01 \\
K 6001\end{array}$ & $\begin{array}{l}\text { Characterization/Remediation } \\
\text { Planning }\end{array}$ & 4.1 .1 .2 .2 & $\begin{array}{l}\text { Characterization and remediation planning } \\
\text { for the identified facility infrastructure, } \\
\text { process systems and other facility systems, } \\
\text { facility contents, equipment, } \\
\text { instrumentation and utilities to support } \\
\text { deactivation. Includes the initial } \\
\text { characterization of the turnover end point } \\
\text { state for the identified facility } \\
\text { infrastructure, process systems and other } \\
\text { facility systems, facility contents, } \\
\text { equipment, instrumentation and utilities. }\end{array}$ \\
\hline & & $\begin{array}{l}7.1 .3 .4 .02 \\
\mathrm{k} 6002\end{array}$ & Facility Deactivation Planning & 4.1 .1 .2 .2 & $\begin{array}{l}\text { Provides development of facility } \\
\text { deactivation plans. Specifically, } \\
\text { determines how to best implement } \\
\text { deactivation requirements for the PFP } \\
\text { Complex, plans the facility deactivation } \\
\text { and identifies/negotiates, the facility } \\
\text { equipment disposition requi rements. }\end{array}$ \\
\hline & & $\begin{array}{l}7.1 .3 .4 .03 \\
\times 6003\end{array}$ & Environmental Compliance & 4.1 .1 .2 .2 & $\begin{array}{l}\text { Provides applicable deactivation } \\
\text { environmental compliance documentation and } \\
\text { negotiates any related enviromental } \\
\text { compliance issues, and/or activities, } \\
\text { necessary for continued deactivation while } \\
\text { continuing with the safe-deactivation of } \\
\text { nonessential systems, components and } \\
\text { physical structures. }\end{array}$ \\
\hline & & $\begin{array}{l}7.1 .3 .4 .04 \\
K 6004\end{array}$ & Project Management & 4.1 .1 .2 .2 & $\begin{array}{l}\text { Project management support to assess the } \\
\text { current state of the facility, identify and } \\
\text { negotiate material and equipment } \\
\text { disposition requirements, develop plans to } \\
\text { deactivate facilities and to negotiate and } \\
\text { maintain the desired facility turnover } \\
\text { endpoint specifications and long-term } \\
\text { archiving of facility information. }\end{array}$ \\
\hline & 7.1 .3 .5 & 1. & General Plant Support & & 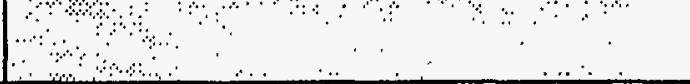 \\
\hline
\end{tabular}




\section{TRANSITION PROJECTS}

FY 1996 MYPP

1.3.1/7.1/6.12

\section{A.1 Technical Documents Tree \& Products Structure}

\begin{tabular}{|c|c|c|c|c|c|}
\hline $\begin{array}{l}\text { PROGRAM } \\
\text { ELEMENT }\end{array}$ & ACTIVITY & COST ACCOUNT & TITLE & $\begin{array}{l}\text { SYSTEM ENGINEERING } \\
\text { FUNCTION }\end{array}$ & PRODUCT DESCRIPTION \\
\hline \multirow[t]{7}{*}{$\begin{array}{c}7.1 .3 \\
K 6\end{array}$} & & & Plutoniun Finishing plant : & $\begin{array}{c}\because \cdots \\
\because \cdots \\
\cdots\end{array} \because \cdots$ & Pै. \\
\hline & & $\begin{array}{l}7.1 .3 .5 .01 \\
\text { KGE01 }\end{array}$ & General Assessments & 4.1 .1 .2 .1 .1 .4 & $\begin{array}{l}\text { Provides the basic services and outside } \\
\text { facility support, such as electricity, } \\
\text { steam, water, etc. to the PFP Complex. } \\
\text { Resource usage cannot be tied directly to } \\
\text { an individual product. }\end{array}$ \\
\hline & & $\begin{array}{l}7.1 .3 .5 .02 \\
\text { KGEO2 }\end{array}$ & Environmental Assessments & 4.1 .1 .2 .1 .1 .4 & $\begin{array}{l}\text { Provides interim disposition of facility } \\
\text { hazardous, radiological and mixed Haste. } \\
\text { Service resources are distributed on a rate } \\
\text { basis per unit of measure, i.e., dollars } \\
\text { per cubic foot. }\end{array}$ \\
\hline & · & $\begin{array}{l}7.1 .3 .5 .03 \\
\text { K } 6 \mathrm{E} 03\end{array}$ & $\begin{array}{l}\text { Facility Baseline Control } \\
\text { Management }\end{array}$ & $\begin{array}{l}4.1 \cdot 1.2 .1 \\
4.1 .1 .2 .2 \\
4.7 .1 .1 \\
4.7 .1 .2\end{array}$ & $\begin{array}{l}\text { Provides the general support to the PFP } \\
\text { Complex that is not directly attributable } \\
\text { to a specific portion of the PFP Transition } \\
\text { Project. This cost account includes } \\
\text { management administrative activities, } \\
\text { general janitorial and housekeeping, and } \\
\text { the generic training required to obtain } \\
\text { facility entrance. }\end{array}$ \\
\hline & & $\begin{array}{l}7.1 .3 .5 .04 \\
\text { KGEO4 }\end{array}$ & Spares Inventory Change & $\begin{array}{l}4.1 .1 .2 .1 .2 \\
4.7 .1 .1 .2\end{array}$ & $\begin{array}{l}\text { Provides an accounting tool for tracking } \\
\text { the net change in spare parts inventory. }\end{array}$ \\
\hline & $7.1: 3: 6$ & : & Safeguards and Security & $\begin{array}{c}\cdots \\
\cdots\end{array}:$ & की \\
\hline & & $\begin{array}{l}7.1 .3 .6 .01 \\
\text { K6F01 }\end{array}$ & Basic Security & $\begin{array}{l}4.1 \cdot 1 \cdot 2 \cdot 1 \cdot 1.3 \\
4.7 \cdot 1 \cdot 1.1 .2\end{array}$ & $\begin{array}{l}\text { Provides patrol services specific to the } \\
\text { PFP complex. Prevents the theft, sabotage, } \\
\text { diversion or unauthorized control of } \\
\text { special nuclear material and provides } \\
\text { access control to the PFP protected area } \\
\text { and all vault related PFP facilities. Also } \\
\text { enforces county, state and federal laws and } \\
\text { statutes. }\end{array}$ \\
\hline
\end{tabular}


TRANSITION PROJECTS

1.3.1/7.1/6.12

FY 1996 MYPP

structure

\section{A.1 Technical Documents Tree \& Products Structure}

\begin{tabular}{|c|c|c|c|c|c|}
\hline $\begin{array}{l}\text { PROGRAM } \\
\text { ELEMENT }\end{array}$ & ACTIVITY & COST ACCOUNT & TITLE & $\begin{array}{l}\text { SYSTEM ENGINEERING } \\
\text { FUNCTION }\end{array}$ & PRODUCT DESCRIPTION \\
\hline \multirow[t]{6}{*}{7.9 .3} & & \begin{tabular}{llll}
$\because$ & $\ddots$ & $\ddots$ & $\ddots$ \\
\hdashline & $\ddots$ & $\ddots$
\end{tabular} & plutoniun prishing plant & 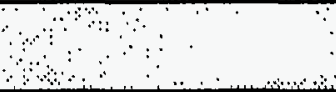 & अ \\
\hline & . & $\begin{array}{l}7.1 .3 .6 .02 \\
\text { K6F02 }\end{array}$ & SNM Custodial support & $\begin{array}{l}4.1 \cdot 1 \cdot 2 \cdot 1 \cdot 1.3 \\
4.7 .1 .1 .1 .2\end{array}$ & $\begin{array}{l}\text { Provides SNM custodial services to all PFP } \\
\text { complex facilities, including ZB/Z-Vault } \\
\text { facilities and } 234-52 \text { vaul ts and/or vault- } \\
\text { type rooms. Provides the necessary support } \\
\text { to operate systems for material accounting } \\
\text { and reporting, oversight requirements, } \\
\text { internal audits of Material Balance Area } \\
\text { (MBA) operations and maintains the Tamper } \\
\text { Indicating Device (TID) program. Maintains } \\
\text { accountability of special nuclear material } \\
\text { and includes all computer systems required } \\
\text { for the accountability. }\end{array}$ \\
\hline & & ${ }_{\mathrm{K} 6 \mathrm{FO3}}^{7.1 .3 .63}$ & $\begin{array}{l}\text { Security Control Documentation and } \\
\text { Studies }\end{array}$ & $\begin{array}{l}4 \cdot 1 \cdot 1 \cdot 2 \cdot 1 \cdot 3 \cdot 7 \\
4 \cdot 7 \cdot 1 \cdot 1.3\end{array}$ & $\begin{array}{l}\text { Maintains accounting and material control } \\
\text { records and appropriate manuals and } \\
\text { procedures to show compl iance with } \\
\text { regulations and procedures for the PFP } \\
\text { Complex. Provides for security control } \\
\text { studies and evaluations (VA, MSSA, etc.). }\end{array}$ \\
\hline & & $\underset{\text { K6F04 }}{7.1 .3 .6 .04}$ & SAS Life Extension Modifications & $\begin{array}{l}4 \cdot 7 \cdot 1 \cdot 1 \cdot 1.2 \\
4 \cdot 7 \cdot 1 \cdot 1 \cdot 2.4 \\
4 \cdot 7 \cdot 1 \cdot 1.3\end{array}$ & $\begin{array}{l}\text { Provides review of safeguards and security } \\
\text { system conditions and viability to meet } \\
\text { plant mission and regulations/requirements. } \\
\text { Performs studies necessary to determine and } \\
\text { evaluate al ternatives for sAS modifications } \\
\text { and then acts upon approved evaluations. }\end{array}$ \\
\hline & & $\begin{array}{l}7.1 .3 .6 .05 \\
\text { K6F05 }\end{array}$ & SAS Maintenance & $\begin{array}{l}4.1 .1 .2 .1 .2 \\
4.7 .1 .1 .2\end{array}$ & $\begin{array}{l}\text { Safeguards and security maintenance } \\
\text { activities necessary to bring S\&s systems } \\
\text { and equipment back to operational status } \\
\text { after failure and to minimize all unplanned } \\
\text { events and premature s\&s equipment failures } \\
\text { in PFP complex facilities. }\end{array}$ \\
\hline & & $\begin{array}{l}7.1 .3 .6 .06 \\
\text { K6F06 }\end{array}$ & Project Management & $\begin{array}{l}4 \cdot 1 \cdot 1 \cdot 2 \cdot 1 \\
4 \cdot 1 \cdot 1 \cdot 2.2 \\
4 \cdot 7 \cdot 1 \cdot 1 \cdot 1.3 \\
4 \cdot 7 \cdot 1 \cdot 1.4 \\
4.7 \cdot 1.2\end{array}$ & $\begin{array}{l}\text { Provides management and administrative } \\
\text { support for project direction and control } \\
\text { for tasks identified in this Activity. } \\
\text { Also provides the training associated with } \\
\text { this activity. }\end{array}$ \\
\hline
\end{tabular}




\section{TRANSITION PROJECTS}

FY 1996 MYPP

1.3.1/7.1/6.12

\section{A.1 Technical Documents Tree \& Products Structure}

\begin{tabular}{|c|c|c|c|c|c|}
\hline $\begin{array}{l}\text { PROGRAM } \\
\text { ELEMENT }\end{array}$ & ACTIVITY & COST ACCOUNT & TITLE & $\begin{array}{l}\text { SYSTEM ENGINEERING } \\
\text { FUNCTION. }\end{array}$ & PRODUCT DESCRIPTION \\
\hline \multirow[t]{12}{*}{7.1 .6} & 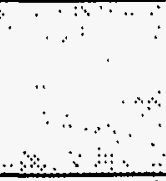 & $\because$ & 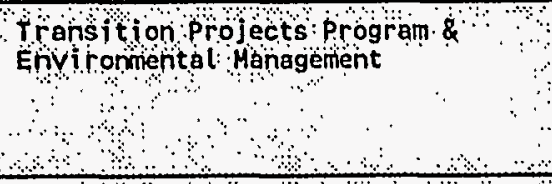 & 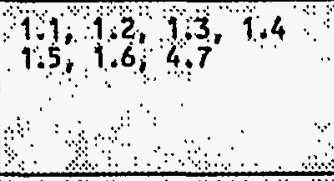 & 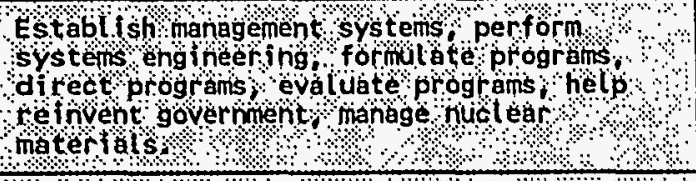 \\
\hline & $\begin{array}{r}71.6 .1 \\
K 1 A \\
1\end{array}$ & : & 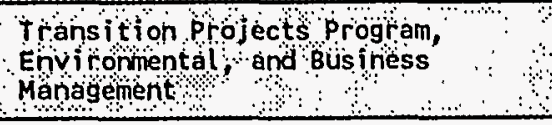 & 11913140 & 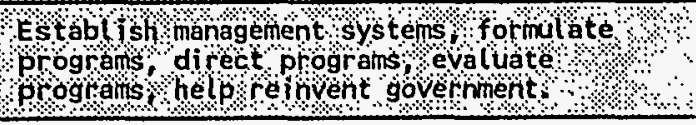 \\
\hline & & $\underset{\text { X1A01 }}{7.1 .6 .1 .01}$ & $\begin{array}{l}\text { Transition Projects Program } \\
\text { Management }\end{array}$ & & \\
\hline & & $\begin{array}{l}7.1 .6 .1 .02 \\
K 1 A 02\end{array}$ & $\begin{array}{l}\text { Transition Projects Business } \\
\text { Management }\end{array}$ & & \\
\hline & & $\begin{array}{l}7.1 .6 .1 .03 \\
\text { K1A03 }\end{array}$ & Conduct of Operations & & \\
\hline & & $\underset{\mathrm{K} 1 \mathrm{AO4}}{7.1 .6 .1 .04}$ & Environmental Management & & \\
\hline & $\begin{array}{c}7.16 .2 \\
k 1 B\end{array}$ & & $\begin{array}{l}\text { Special Nuclear Materials } \\
\text { Management }\end{array}$ & 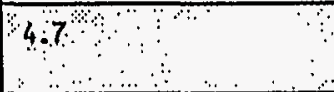 & hanage Nuclear Materials \\
\hline & & $\underset{K 1801}{7.1 .6 .2 .01}$ & $\begin{array}{l}\text { Defense Programs Off-site } \\
\text { Representative }\end{array}$ & & \\
\hline & & $\underset{K 1 B 02}{7.1 .6 .2 .02}$ & Safeguards and Security & & \\
\hline & & $\underset{\text { K1803 }}{7.1 .6 .2 .03}$ & Special Nuclear Materials & & \\
\hline & & $\begin{array}{c}7.1 .6 .2 .04 \\
\mathrm{~K} 1 \mathrm{~B} 04\end{array}$ & Plutonium Strategic Plan & & \\
\hline & $\begin{array}{l}7.1 .6 .3 \\
\mathrm{KIC}\end{array}$ & & Systems Engineering & 1.2 & Perform systems Englneer tng \\
\hline & & $\begin{array}{l}\text { K1C01 } \\
\text { K1.6.3.01 }\end{array}$ & Site and Program Support & & \\
\hline
\end{tabular}




\section{TRANSITION PROJECTS}

FY 1996 MYPP

1.3.1/7.1/6.12

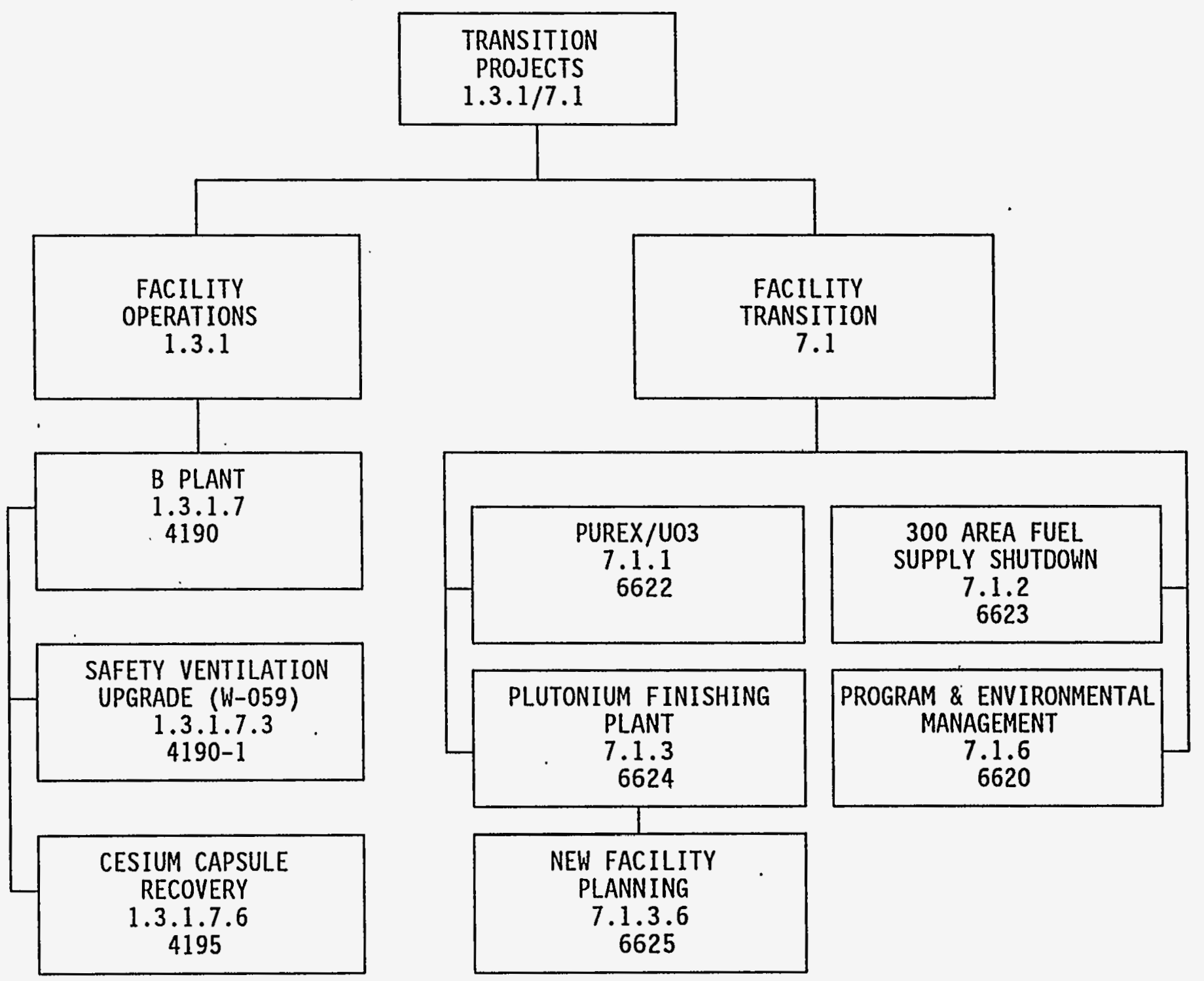




\section{TRANSITION PROJECTS}

FY 1996 MYPP

1.3.1/7.1/6.12

\section{A.2 WBS Structure}

The Work Breakdown Structure for the B Plant / WESF Transition Project has been restructured to separate costs for $B$ Plant and its auxiliary facilities from those for WESF and is aligned to clearly differentiate requirements for minimum safe operations from facility deactivation and the incremental work scope, such as WESF upgrades and Cesium and Strontium capsule recovery. Project-wide program management, administration and regulatory compliance functions have been centralized for visibility and efficiency in cost accounting. The Work Breakdown Structure is as follows:

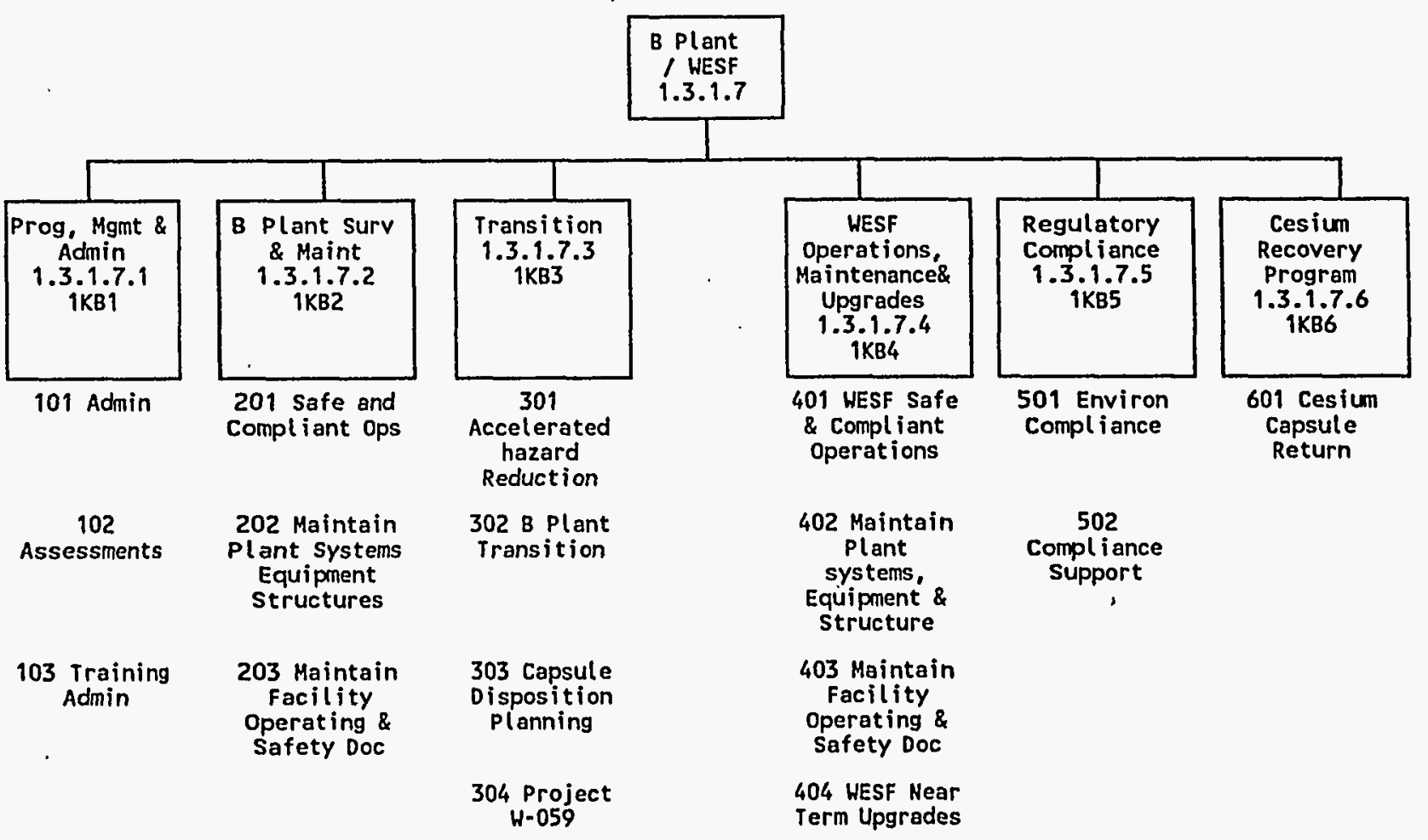




\section{TRANSITION PROJECTS}

FY 1996 MYPP

1.3.1/7.1/6.12

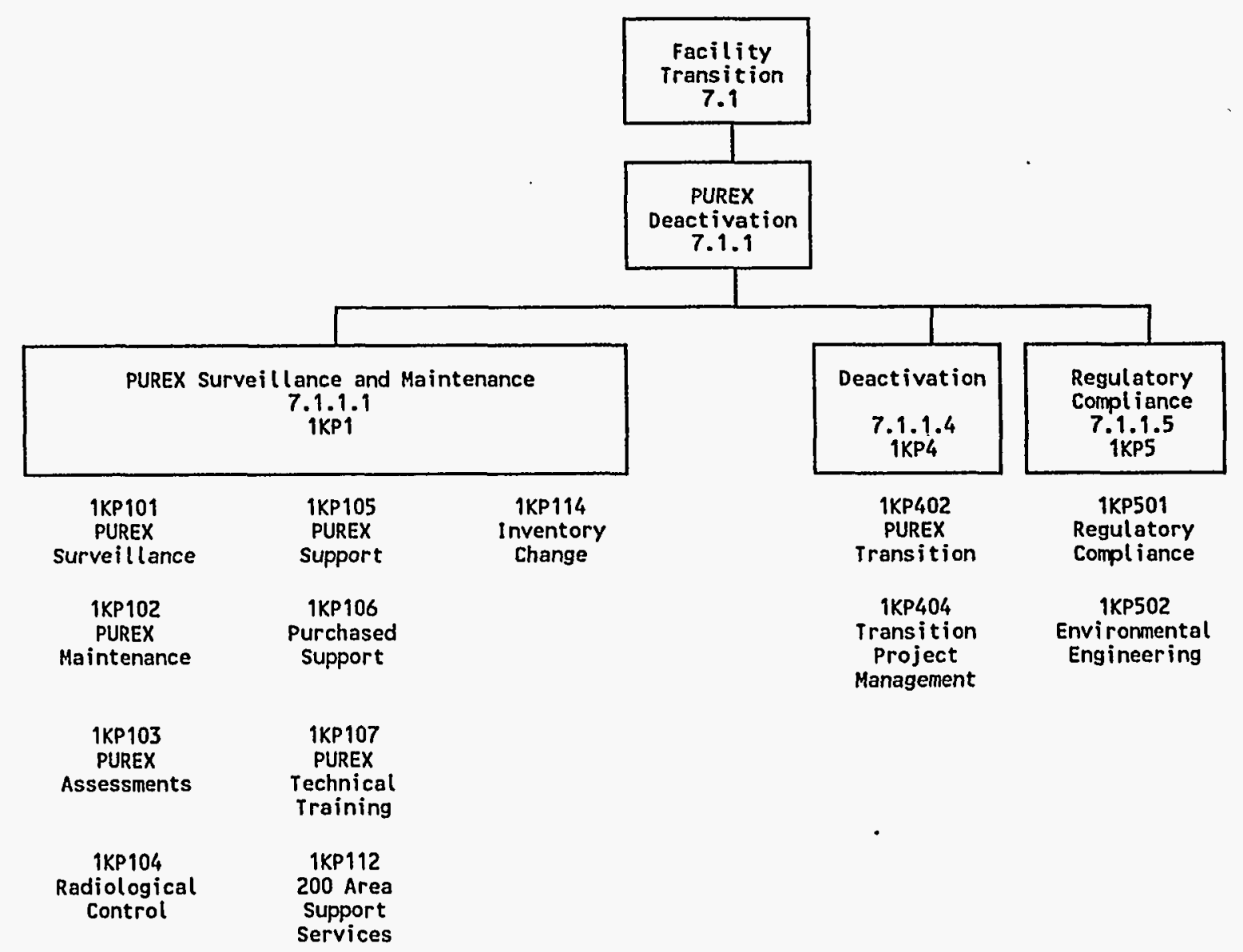




\section{TRANSITION PROJECTS}

FY 1996 MYPP

1.3.1/7.1/6.12

Facility 7.1

300 Area Fuel Supply Shutdown

Program

300 Area Fuel Supply Shutdown 7.1.2.1

Surveillance and

Maintenance
7.1 .2 .1 .1

RCRA Activities

7.1.2.1.2

Shutdown

Activities

7.1.2.1.3

313s Building
Isolation

7.1.2.1.4

SNM Safe storage

7.1.2.1.5 


\section{TRANSITION PROJECTS}

\begin{tabular}{|c|c|c|c|c|c|c|}
\hline & & & $\begin{array}{c}\text { Facility } \\
\text { Transition } \\
7.1\end{array}$ & & & \\
\hline & & & $\begin{array}{l}\text { PEP } \\
7.1 .3\end{array}$ & & & \\
\hline $\begin{array}{l}\text { PFP Surv } \\
\& \text { Maint } \\
7.1 .3 .1 \\
1 \mathrm{~K} 6 \mathrm{~A}\end{array}$ & $\begin{array}{l}\text { Stab/Recon- } \\
\text { figure for } \\
\text { Min Surv } \\
7.1 .3 .2 \\
1 \times 68\end{array}$ & $\begin{array}{c}\text { SNM Safe } \\
\text { Storage } \\
\text { and } \\
\text { Treatment } \\
7.1 .3 .3 \\
\text { IKGC }\end{array}$ & & $\begin{array}{c}\text { Deactivation } \\
\text { Planning \& } \\
\text { Turnover } \\
\text { Endpoint } \\
7.1 .3 .4 \\
1 \mathrm{~K} 60\end{array}$ & $\begin{array}{c}\text { General } \\
\text { Plant } \\
\text { Support } \\
7.11 .3 .5 \\
\text { 1KGE }\end{array}$ & $\begin{array}{c}\text { Safeguards } \\
\text { and } \\
\text { security } \\
7.1 .3 .6 \\
1 \mathrm{~K} 6 \mathrm{~F}\end{array}$ \\
\hline $\begin{array}{c}1.01 \\
\text { Corrective } \\
\text { Maint }\end{array}$ & $\begin{array}{l}2.01 \\
\text { Thermal } \\
\text { Treatment }\end{array}$ & $\begin{array}{c}3.02 \\
\text { SNM Ship/ } \\
\text { Receipts }\end{array}$ & & $\begin{array}{c}4.01 \\
\text { Characteriza } \\
\text { tion/Remedia } \\
\text { tion } \\
\text { Planning }\end{array}$ & $\begin{array}{c}5.01 \\
\text { General } \\
\text { Assessments }\end{array}$ & $\begin{array}{c}6.01 \\
\text { Basic } \\
\text { Physical } \\
\text { Security }\end{array}$ \\
\hline $\begin{array}{l}1.02 \\
\text { Preventive } \\
\text { Maint }\end{array}$ & $\begin{array}{l}2.02 \\
\text { Solution } \\
\text { Treatment }\end{array}$ & $\begin{array}{c}3.03 \\
\text { SNM } \\
\text { Inventories }\end{array}$ & & $\begin{array}{c}4.02 \\
\text { Develop } \\
\text { Deactivation } \\
\text { Plans }\end{array}$ & $\begin{array}{c}5.02 \\
\text { Environment } \\
\text { Assessments }\end{array}$ & $\begin{array}{c}6.02 \\
\text { SNM } \\
\text { Custodial } \\
\text { Support }\end{array}$ \\
\hline $\begin{array}{l}1.03 \\
\text { Nuclear } \\
\text { Process/ } \\
\text { Radiation } \\
\text { Surveill }\end{array}$ & $\begin{array}{l}2.03 \\
\text { Pyrolysis } \\
\text { Furnace }\end{array}$ & $\begin{array}{l}3.04 \\
\text { Vault } \\
\text { Mods }\end{array}$ & & $\begin{array}{l}4.03 \\
\text { Environment } \\
\text { Compl iance }\end{array}$ & $\begin{array}{c}5.03 \\
\text { Facility } \\
\text { Baseline } \\
\text { Control } \\
\text { Management }\end{array}$ & $\begin{array}{l}6.03 \\
\text { Security } \\
\text { Control } \\
\text { Doc \& } \\
\text { Studies }\end{array}$ \\
\hline $\begin{array}{c}1.04 \\
\text { Vent/Power } \\
\text { Surveill }\end{array}$ & $\begin{array}{c}2.04 \\
\text { Misc } \\
\text { Treatment } \\
\text { Facility }\end{array}$ & $\begin{array}{l}.3 .05 \\
\text { Environment } \\
\text { al } \\
\text { Compl iance }\end{array}$ & & $\begin{array}{c}4.04 \\
\text { Project } \\
\text { Management }\end{array}$ & $\begin{array}{c}5.04 \\
\text { Spares } \\
\text { Inventory } \\
\text { Change }\end{array}$ & $\begin{array}{l}6.04 \\
\text { SAS Life } \\
\text { Extension } \\
\text { Mods }\end{array}$ \\
\hline $\begin{array}{l}1.05 \\
\text { Environmen } \\
\text { tal } \\
\text { Compl i ance }\end{array}$ & $\begin{array}{c}2.05 \\
\text { Cleanup \& } \\
\text { TCO }\end{array}$ & $\begin{array}{l}3.06 \\
\text { IAEA } \\
\text { SUpport }\end{array}$ & & & & $\begin{array}{l}6.05 \\
\text { SAS Maint }\end{array}$ \\
\hline
\end{tabular}




\title{
TRANSITION PROJECTS
}

FY 1996 MYPP

\subsection{1/7.1/6.12}

PFP (Continued)
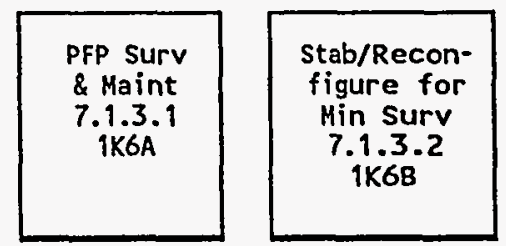

2.06

1.06

Soundary/

Emergency

Planning

DNFSB $94-1$

Facility

Mods (ADS

6625)

1.07

Procedure

2.07

Maịnt

Lab Support

\& Upgrades

1.08

Specific

2.08

Facility

Mods for

Surveill

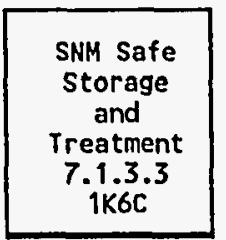

\begin{tabular}{|c|c|}
\hline $\begin{array}{c}\text { Deactivation } \\
\text { Planning \& } \\
\text { Turnover } \\
\text { Endpoint } \\
7.1 .3 .4 \\
1 \mathrm{~K} 60\end{array}$ & $\begin{array}{c}\text { General } \\
\text { Plant } \\
\text { Support } \\
7.1 .3 .5 \\
\text { IKGE }\end{array}$ \\
\hline
\end{tabular}

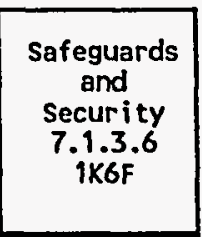

3.07

Engr

Studies/

Assessments

6.06

Project

Management

3.08

Corrective

Maint

Support

3.09

Preventive

Maintenance

1.09

Project
Management

\begin{abstract}
2.09
Engr studies

Evaluations
\end{abstract}

2.10

Environment

Doc and

Doc and
Regulatory

Negotiation

$$
2.11
$$

EIS Project support

2.12

Project

Management

3.10

Nuclear
Proc/Rad

Surveill

3.11

Ventilation

/Power

Surveill

3.13

Project

Management 
TRANSITION PROJECTS

FY 1996 MYPP

$\stackrel{N}{N}$
1.3.1/7.1/6.12

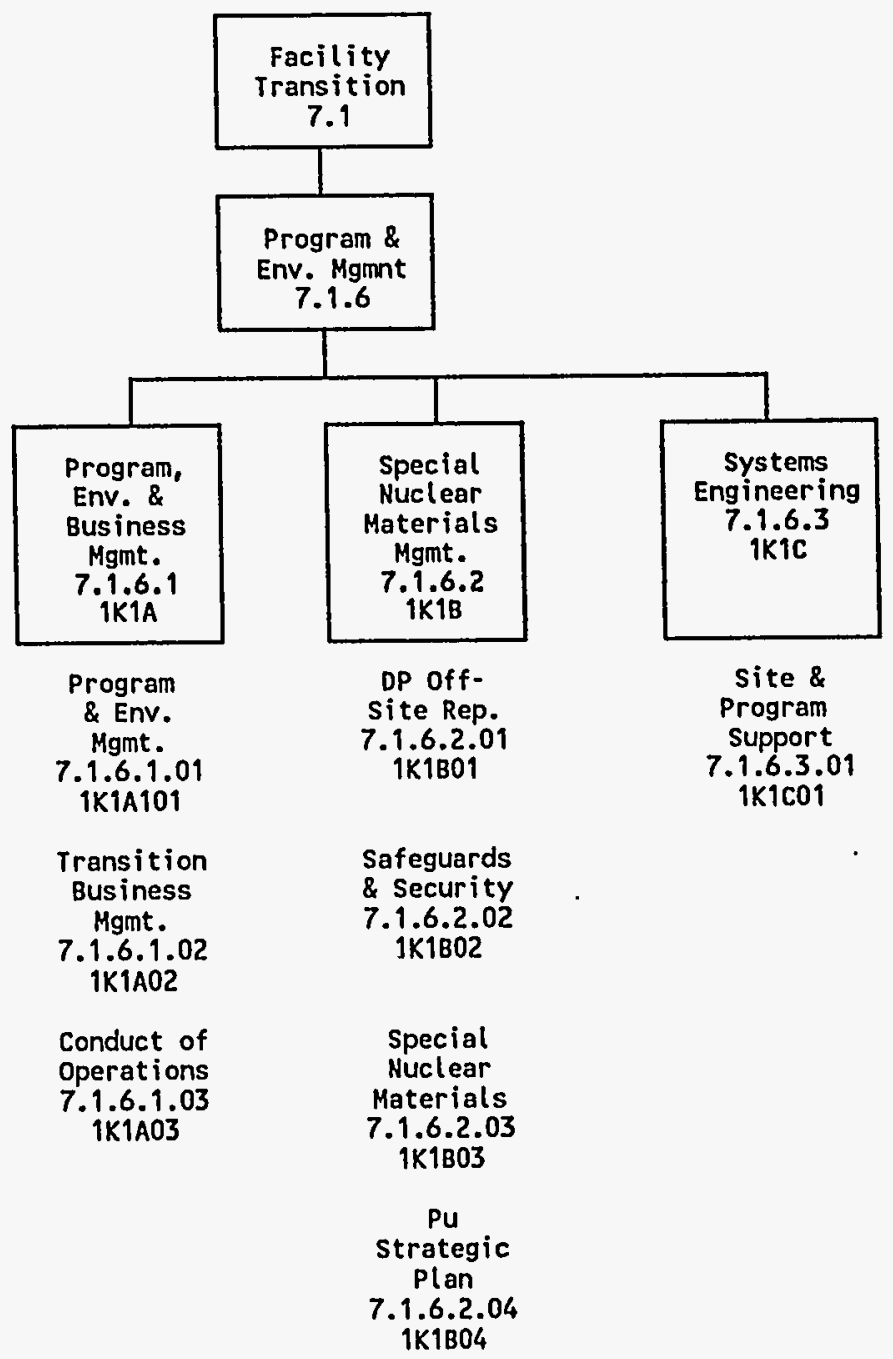


WHC-SP-1126, Rev. 1

\section{TRANSITION PROJECTS}

\section{A.3 WBS Dictionary}

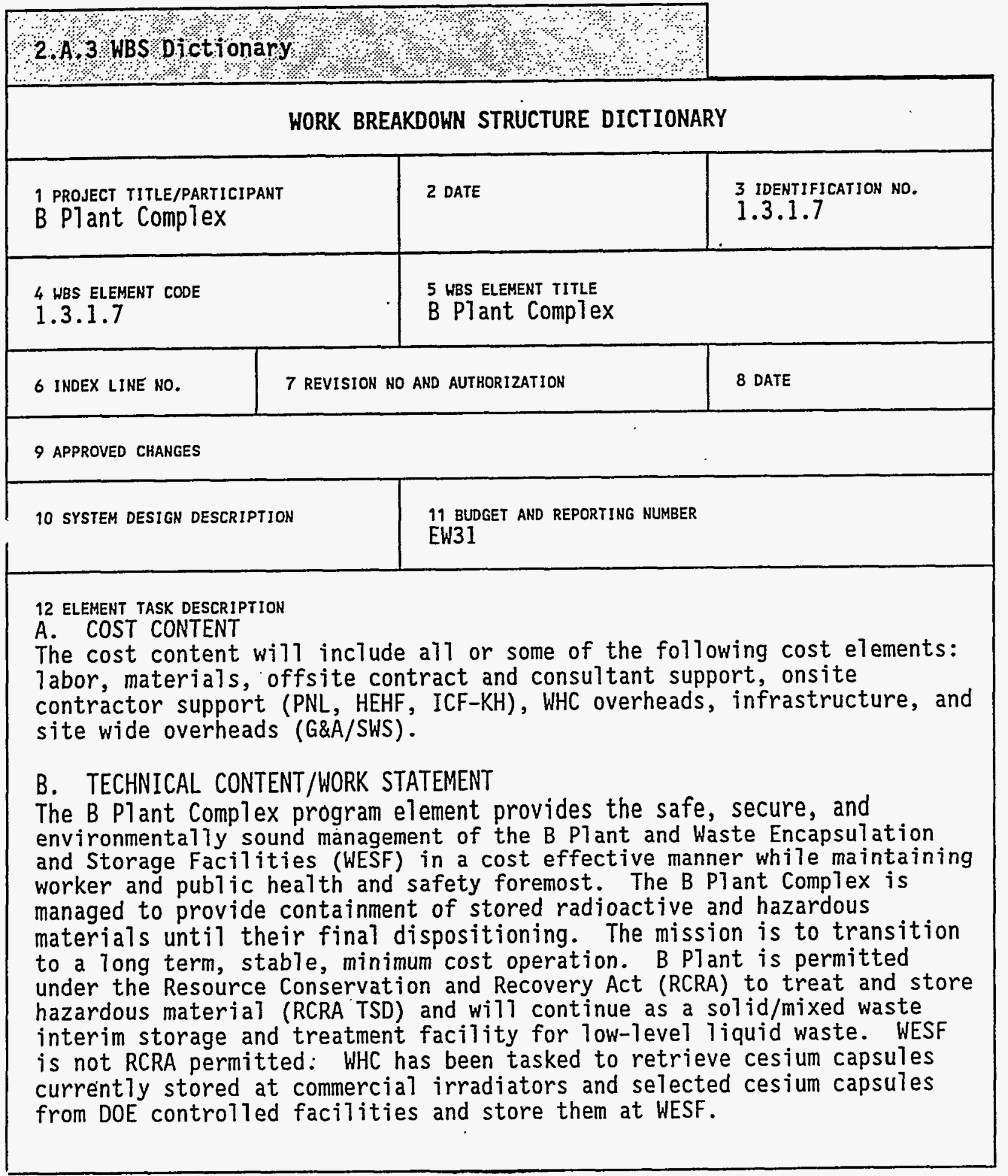


WHC-SP-1126, Rev. 1

\section{TRANSITION PROJECTS}

FY 1996 MYPP

1.3.1/7.1/6.12

\begin{tabular}{|c|c|c|}
\hline \multicolumn{3}{|c|}{ WORK BREAKDOWN STRUCTURE DICTIONARY } \\
\hline $\begin{array}{l}1 \text { PROJECT TITLE/PARTICIPANT } \\
\text { B Plant Complex }\end{array}$ & 2 DATE & $\begin{array}{l}3 \text { IDENTIFICATION NO. } \\
1.3 .1 .7\end{array}$ \\
\hline $\begin{array}{l}4 \text { WBS ELEMENT CODE } \\
1.3 .1 .7 .1\end{array}$ & \multicolumn{2}{|c|}{$\begin{array}{l}5 \text { HBS ELEMENT TITLE } \\
\text { Program Management and Administration }\end{array}$} \\
\hline 6 INDEX LINE NO. & 7 REVISION NO AND AUTHORIZATION & 8 DATE \\
\hline \multicolumn{3}{|l|}{9 APPROVED CHANGES } \\
\hline 10 SYSTEM DESIGN DESCRIPTION & \multicolumn{2}{|c|}{$\begin{array}{l}11 \text { BUDGET AND REPORTING NUMBER } \\
\text { EW31 }\end{array}$} \\
\hline \multicolumn{3}{|c|}{$\begin{array}{l}\text { 12 ELEMENT TASK DESCRIPTION } \\
\text { A. COST CONTENT } \\
\text { The cost content wi } 11 \text { include all or some of the following cost elements: } \\
\text { labor, materials, offsite contract and consultant support, onsite } \\
\text { contractor support (PNL, HEHF, ICF-KH), WHC overheads, infrastructure, and } \\
\text { site-wide overheads (G\&A/SWS). } \\
\text { B. TECHNICAL CONTENT/WORK STATEMENT } \\
\text { The Program Management and Administration activity includes program } \\
\text { management and administrative support; site assessments; and training } \\
\text { administration and in-plant training services. Program management and } \\
\text { administrative support includes schedule development and maintenance, } \\
\text { budget/cost development and tracking, change control support, and } \\
\text { corrective action management support. Site assessments provides funds for } \\
\text { utilities, laundry, rail service, waste disposal, and computer network } \\
\text { services. Training Services develops, presents, and evaluates training } \\
\text { materials and services necessary to maintain qualified plant personnel per } \\
\text { DOE Orders and state and federal regulations. }\end{array}$} \\
\hline
\end{tabular}


WHC-SP-1126, Rev. 1

TRANSITION PROJECTS.

FY 1996 MYPP

1.3.1/7.1/6.12

2.A.3. WBS Dictionary

WORK BREAKDOWN STRUCTURE DICTIONARY

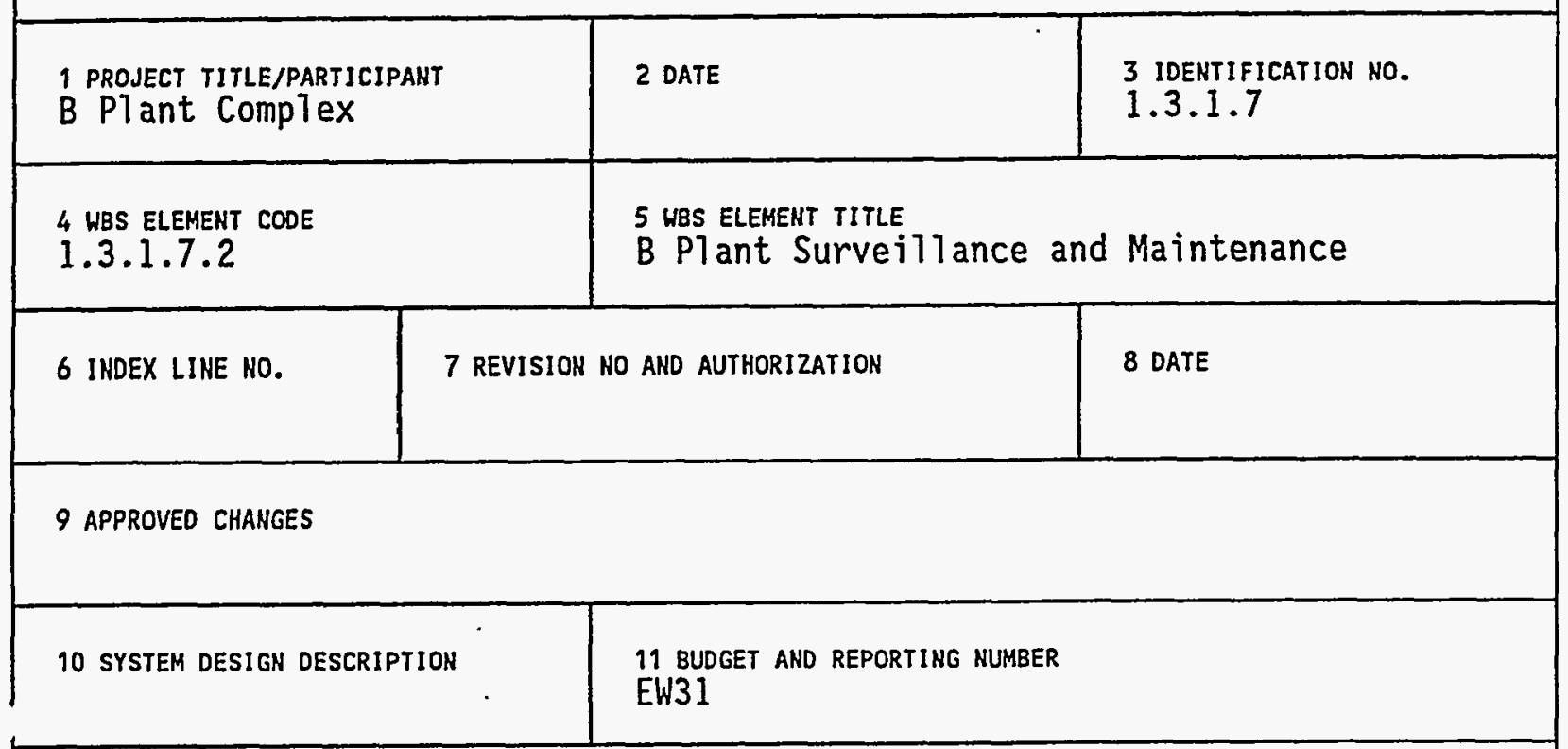

12 ELEMENT TASK DESCRIPTION

A. COST CONTENT

The cost content will include all or some of the following cost elements: labor, materials, offsite contract and consultant support, onsite contractor support (PNL, HEHF, ICF-KH), WHC overheads, infrastructure, and site-wide overheads (G\&A/SWS).

B. TECHNICAL CONTENT/WORK STATEMENT

Provide for the safe and compliant maintenance operation of the B PIant canyon facility and its associated ancillary buildings pending deactivation. Complete surveillance and maintenance activities on those systems necessary to ensure operations are conducted with in the facilities' safety and compliance envelopes which results in proper control of the facility radiological and hazardous inventories. Ensure operational and safety documentation is current and staff is trained in its implementation. 
WHC-SP-1126, Rev. 1

\section{TRANSITION PROJECTS}

FY 1996 MYPP

1.3.1/7.1/6.12

2.A.3. WBS Dictionary

\section{WORK BREAKDOWN STRUCTURE DICTIONARY}

\begin{tabular}{|c|c|c|c|}
\hline \multicolumn{2}{|c|}{$\begin{array}{l}1 \text { PROJECT TITLE/PARTICIPANT } \\
\text { B Plant Complex }\end{array}$} & 2 DATE & $\begin{array}{l}3 \text { IDENTIFICATION NO. } \\
1.3 .1 .7\end{array}$ \\
\hline \multicolumn{2}{|l|}{$\begin{array}{l}4 \text { HBS ELEMENT COOE } \\
1.3 .1 .7 .3\end{array}$} & \multicolumn{2}{|l|}{$\begin{array}{l}5 \text { WBS ELEMENT TITLE } \\
\text { Transition }\end{array}$} \\
\hline 6 INDEX LINE NO. & \multicolumn{2}{|c|}{7 REVISION NO AND AUTHORIZATION } & 8 DATE \\
\hline \multicolumn{4}{|l|}{9 APPROVED CHANGES } \\
\hline \multicolumn{2}{|c|}{10 SYSTEM DESIGN DESCRIPTION } & \multicolumn{2}{|c|}{$\begin{array}{l}11 \text { BUDGET AND REPORTING NUMBER } \\
\text { EW3I }\end{array}$} \\
\hline \multicolumn{4}{|c|}{$\begin{array}{l}12 \text { ELEMENT TASK DESCRIPTION } \\
\text { A. COST CONTENT } \\
\text { The cost content will include all or some of the following cost elements: } \\
\text { labor, materials, offsite contract and consultant support, onsite } \\
\text { contractor support (PNL, HEHF, ICF-KH), WHC overheads, infrastructure, and } \\
\text { site-wide overheads (G\&A/SWS). }\end{array}$} \\
\hline \multicolumn{4}{|c|}{$\begin{array}{l}\text { B. TECHNICAL CONTENT/WORK STATEMENT } \\
\text { Transition B Plant and its auxiliary facilities to a safe and cost- } \\
\text { effective surveillance and maintenance configuration and effect an orderly } \\
\text { turnover to the Hanford Surplus Facilities Program by } 2002 \text {. Plan for } \\
\text { disposition of the cesium and strontium capsules. Transition WESF and its } \\
\text { auxiliary facilities to a safe and cost-effective surveillance and } \\
\text { maintenance configuration and effect an orderly turnover to the Hanford } \\
\text { Surplus Facilities by 2013. }\end{array}$} \\
\hline
\end{tabular}


WHC-SP-1126, Rev. 1

\section{TRANSITION PROJECTS}

FY 1996 MYPP

1.3.1/7.1/6.12

2. A. 3 WBSDictoonary

WORK BREAKDOWN STRUCTURE DICTIONARY

\begin{tabular}{|c|c|c|c|}
\hline \multicolumn{2}{|c|}{$\begin{array}{l}1 \text { PROJECT TITLE/PARTICIPANT } \\
\text { B Plant CompleX }\end{array}$} & 2 DATE & $\begin{array}{l}3 \text { IDENTIFICATION NO. } \\
1.3 .1 .7\end{array}$ \\
\hline \multicolumn{2}{|l|}{$\begin{array}{l}4 \text { WBS ELEMENT COOE } \\
1.3 .1 .7 .4\end{array}$} & \multicolumn{2}{|c|}{$\begin{array}{l}5 \text { HBS ELEMENT TITLE } \\
\text { WESF Operation, Maintenance, and Upgrades }\end{array}$} \\
\hline 6 INDEX LINE NO. & 7 REVISION & AND AUTHORIZATION & 8 DATE \\
\hline \multicolumn{4}{|l|}{9 APPROVED CHANGES } \\
\hline \multicolumn{2}{|c|}{10 SYSTEM DESIGN DESCRIPTION } & \multicolumn{2}{|l|}{$\begin{array}{l}11 \text { BUDGET AND REPORTING NUMBER } \\
\text { EW31 }\end{array}$} \\
\hline
\end{tabular}

12 ELEMENT TASK DESCRIPTION

A. COST CONTENT

The cost content will include all or some of the following cost elements: labor, materials, offsite contract and consultant support, onsite contractor support (PNL, HEHF, ICF-KH), WHC overheads, infrastructure, and site-wide overheads (G\&A/SWS).

\section{B. TECHNICAL CONTENT/WORK STATEMENT}

Provide safe and compliant management of the cesium and strontium inventory within the WESF pool cells, hot cells, and ventilation systems pending their removal. Complete surveillance and maintenance activities on those systems necessary to ensure operations are conducted within the facilities' safety and compliance envelopes. Update and implement operational and safety documentation to reflect current facility operations and conditions. Complete facility upgrades necessary for safe, cost effective operation of the pool cells. 
WHC-SP-1126, Rev. 1

\section{TRANSITION PROJECTS}

\begin{tabular}{|c|c|c|}
\hline \multicolumn{3}{|c|}{ 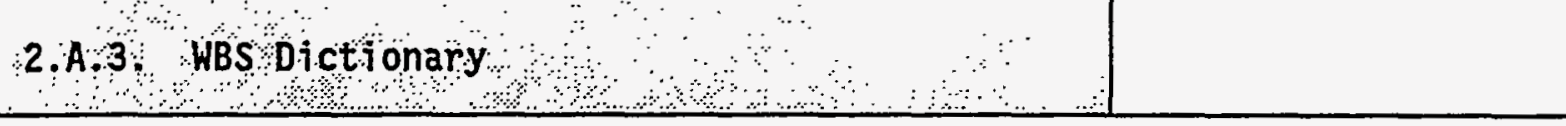 } \\
\hline \multicolumn{3}{|c|}{ WORK BREAKDOWN STRUCTURE DICTIONARY } \\
\hline $\begin{array}{l}1 \text { PROJECT TITLE/PARTICIPANT } \\
\text { B Plant Complex }\end{array}$ & 2 DATE & $\begin{array}{l}3 \text { IDENTIFICATION NO. } \\
1.3 .1 .7\end{array}$ \\
\hline $\begin{array}{l}4 \text { HBS ELEMENT COOE } \\
1.3 .1 .7 .5\end{array}$ & \multicolumn{2}{|c|}{$\begin{array}{l}5 \text { UBS ELEMENT TITLE } \\
\text { Regulatory Compliance }\end{array}$} \\
\hline 6 INDEX LIHE NO. & YO AND AUTHORIZATION & 8 DATE \\
\hline \multicolumn{3}{|l|}{9 APPROVED CHANGES } \\
\hline 10 SYSTEM DESIGN DESCRIPTION & \multicolumn{2}{|c|}{$\begin{array}{l}11 \text { BUDGET AND REPORTING NUMBER } \\
\text { EW31 }\end{array}$} \\
\hline \multicolumn{3}{|c|}{$\begin{array}{l}\text { B. TECHNICAL CONTENT/WORK STATEMENT } \\
\text { Ensure operations are conducted in accordance with environmental, safety, } \\
\text { quality assurance, and security requirements including Resource, } \\
\text { Conservation and Recovery Act, Clean Air Act, Clean Water Act, Safe } \\
\text { Drinking Water Act, National Environmental Policy Act, National Historic } \\
\text { Preservation Act, safety, rad con, and QA CFRs, and DOE Orders; perform } \\
\text { regulatory self-assessments; provide safety and QA support; maintain } \\
\text { qualified compliance staff; and administer regulatory compliance officer } \\
\text { responsibilities. }\end{array}$} \\
\hline
\end{tabular}


WHC-SP-1126, Rev. 1

TRANSITION PROJECTS

FY 1996 MYPP

1.3.1/7.1/6.12

2.A. 3 WBS Dictionary

WORK BREAKDOWN STRUCTURE DICTIONARY

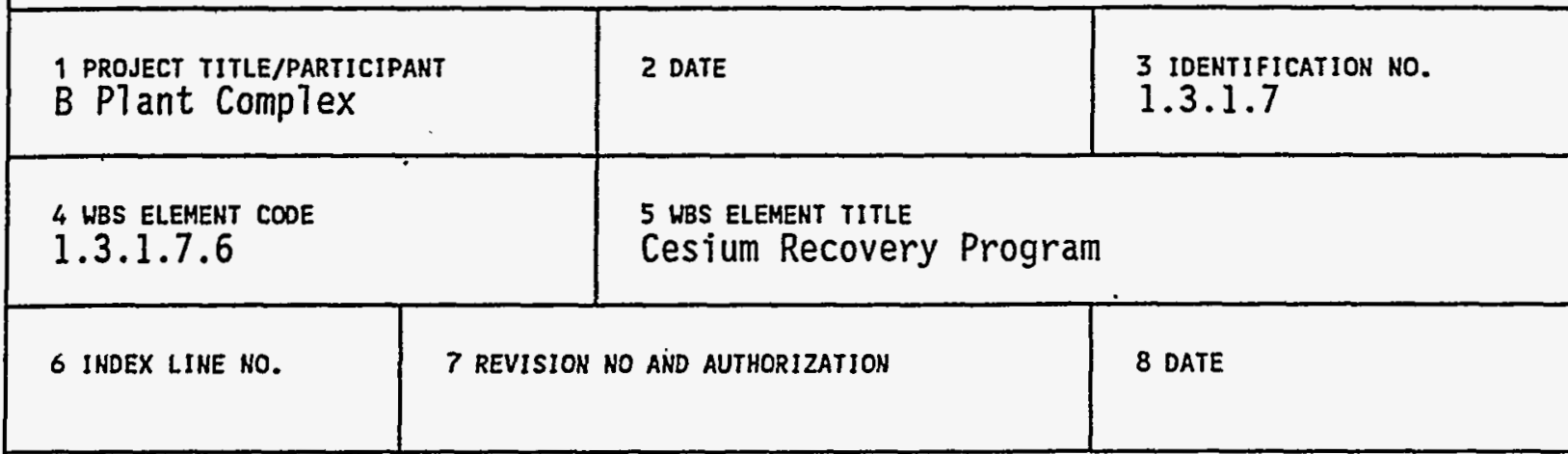

9 APPROVED CHANGES

10 SYSTEM DESIGN DESCRIPTION

11 BUDGET AND REPORTING NUMBER EW31

12 ELEMENT TASK DESCRIPTION

A. COST CONTENT

The cost content will include all or some of the following cost elements: labor, materials, offsite contract and consultant support, onsite contractor support (PNL, HEHF, ICF-KH), WHC overheads, infrastructure, and site-wide overheads (G\&A/SWS).

B. TECHNICAL CONTENT/WORK STATEMENT

Return a 71 capsules leased to private companies to WESF for long-term storage. Provide for the reencapsulation of failed and suspect capsules currentiy located at WESF and the Pacific Northwest Laboratories to achieve a configuration suitable for long-term storage at WESF. Reencapsulation will be performed by PNL under the direction of Westinghouse utilizing equipment and procedures developed during fiscal year 1996 under Activity Data Sheet 8400. Maintain the capability to ship capsules from offsite and onsite locations as necessary to support the previously noted missions. Provide for the surveillance of capsules at PNL until removal to WESF. 
WHC-SP-1126, Rev. I

TRANSITION PROJECTS

FY 1996 MYPP

1.3.1/7.1/6.12

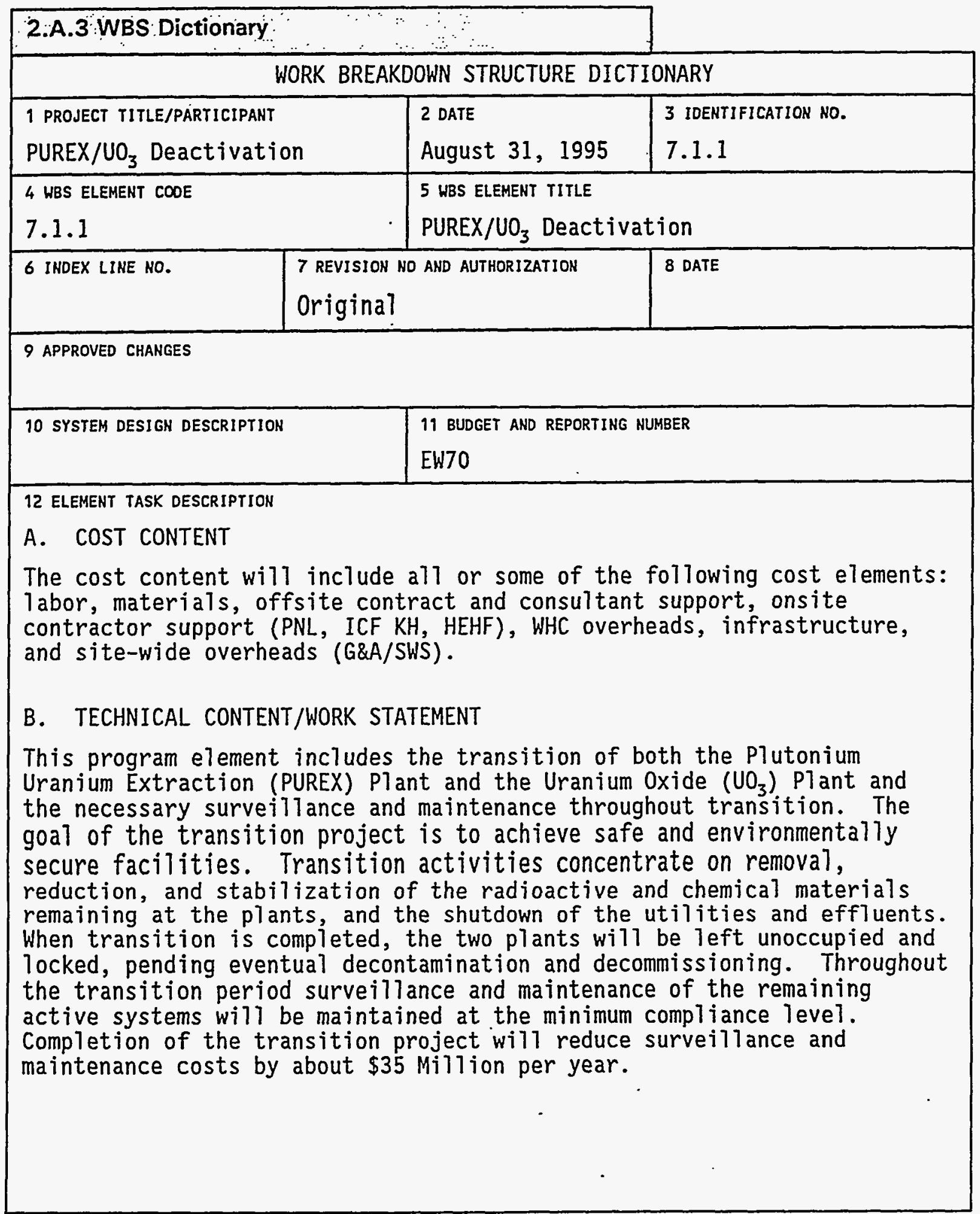


WHC-SP-1126, Rev. I

TRANSITION PROJECTS

FY 1996 MYPP

1.3.1/7.1/6.12

\begin{tabular}{|c|c|c|}
\hline \multicolumn{3}{|c|}{$\frac{2 \text { A } 3 \text { WBS Dictionary }}{\text { WORK BREAKDOWN STRUCTURE DICTIONARY }}$} \\
\hline $\begin{array}{l}1 \text { PROJECT TITLE/PARTICIPANT } \\
\text { PUREX } / \mathrm{UO}_{3} \text { Deactivation }\end{array}$ & $\begin{array}{l}2 \text { DATE } \\
\text { August } 31,1995\end{array}$ & $\begin{array}{l}3 \text { IDENTIFICATION NO. } \\
7.1 .1\end{array}$ \\
\hline $\begin{array}{l}4 \text { WBS ELEMENT CODE } \\
7.1 .1 .1\end{array}$ & \multicolumn{2}{|c|}{$\begin{array}{l}5 \text { KBS ELEMENT TITLE } \\
\text { PUREX SUrveill ance and Maintenance }\end{array}$} \\
\hline \begin{tabular}{l|l}
6 INDEX LINE NO. & (i)
\end{tabular} & $\begin{array}{l}7 \text { REVISION NO AND AUTHORIZATION } \\
\text { Original }\end{array}$ & 8 DATE \\
\hline \multicolumn{3}{|l|}{9 APPROVED CHANGES } \\
\hline 10 SYSTEM DESIGN DESCRIPTION & \multicolumn{2}{|c|}{$\begin{array}{l}11 \text { BUDGET AND REPORTING NUMBER } \\
\text { EW70 }\end{array}$} \\
\hline \multicolumn{3}{|c|}{$\begin{array}{l}\text { 12 ELEMENT TASK DESCRIPTION } \\
\text { A. COST CONTENT } \\
\text { The cost content will include all or some of the following cost elements: } \\
\text { labor, materials, offsite contract and consultant support, onsite } \\
\text { contractor support (PNL, ICF KH, HEHF), WHC overheads, infrastructure, } \\
\text { and site-wide overheads (G\&A/SWS). } \\
\text { B. TECHNICAL CONTENT/WORK STATEMENT } \\
\text { Direct and manage all tasks required to support minimum PUREX PIant } \\
\text { surveillance and maintenance of critical utility and safety systems while } \\
\text { the Plant is undergoing transition. Operate the HVAC, utility and } \\
\text { effluent monitoring systems. Maintain minimum compliance with applicable } \\
\text { State and Federal regulations and DOE Orders. Maintain all equipment and } \\
\text { complete all preventative maintenance, corrective maintenance and } \\
\text { calibrations to maintain the plant within the OSD limits. Maintain a } \\
\text { comprehensive scheduled survey program for both dose rate and } \\
\text { contamination levels throughout PUREX. }\end{array}$} \\
\hline
\end{tabular}


WHC-SP-1126, Rev. I

TRANSITION PROJECTS

FY 1996 MYPP

1.3.1/7.1/6.12

\begin{tabular}{|c|c|c|}
\hline \multicolumn{3}{|c|}{ 2.A3WBS Dictionary a } \\
\hline \multicolumn{3}{|c|}{ WORK BREAKDOWN STRUCTURE DICTIONARY } \\
\hline \multirow{2}{*}{$\begin{array}{l}1 \text { PROJECT TITLE/PARTICIPANT } \\
\text { PUREX } / \mathrm{UO}_{3} \text { Deactivation } \\
4 \text { WBS ELEMENT COOE } \\
7.1 .1 .4\end{array}$} & $\begin{array}{l}2 \text { DATE } \\
\text { August } 31,1995\end{array}$ & $\begin{array}{l}3 \text { IDENTIFICATION NO. } \\
7.1 .1\end{array}$ \\
\hline & $\begin{array}{l}5 \text { WBS ELEMENT TITLE } \\
\text { Transition }\end{array}$ & \\
\hline 6 INDEX LINE NO. & $\begin{array}{l}7 \text { REVISION NO AND AUTHORIZATION } \\
\text { Original }\end{array}$ & 8 DATE \\
\hline \multicolumn{2}{|l|}{9 APPROVED CHANGES } & \\
\hline 10 SYSTEM DESIGN DESCRIPTION & \multicolumn{2}{|c|}{$\begin{array}{l}\text { "I BUDGET AND REPORTING NUMBER } \\
\text { EW70 }\end{array}$} \\
\hline \multicolumn{3}{|c|}{$\begin{array}{l}\text { The cost content will include all or some of the following cost elements: } \\
\text { labor, materials, offsite contract and consultant support, onsite } \\
\text { contractor support (PNL, ICF KH, HEHF), WHC overheads, infrastructure, } \\
\text { and site-wide overheads (G\&A/SWS). }\end{array}$} \\
\hline \multicolumn{3}{|c|}{ B. TECHNICAL CONTENT/WORK STATEMENT } \\
\hline \multicolumn{3}{|c|}{$\begin{array}{l}\text { Deactivate the PUREX and } \mathrm{UO}_{3} \text { Plants. Transition execution activities } \\
\text { include: organic disposal recovered acid removal, cold chemical } \\
\text { disposal, radionuclide removal from waste concentrator systems, disposal } \\
\text { of plutonium nitrate/uranium nitrate solution. Activities also include: } \\
\text { decontamination of } \mathrm{N}-\mathrm{Ce} 1 \mathrm{l} \text {, Q-Cell, and product recovery room, flushing of } \\
\text { radionuclides and chemicals, disposition/disposal of silver reactors, } \\
\text { disposition of fuel elements in } \mathrm{A}, \mathrm{B} \text {, and C-Cells, disposition of } \\
\text { aluminum clad fuel in the slug storage basin, and transfer of solutions } \\
\text { within the plant to a permitted waste storage/treatment facility. } \\
\text { Facility utility systems will be deactivated and consolidated as } \\
\text { appropriate to support long-term low cost surveillance and maintenance.. }\end{array}$} \\
\hline
\end{tabular}


WHC-SP-1126, Rev. I

- TRANSITION PROJECTS

FY 1996 MYPP 1.3.1/7.1/6.12

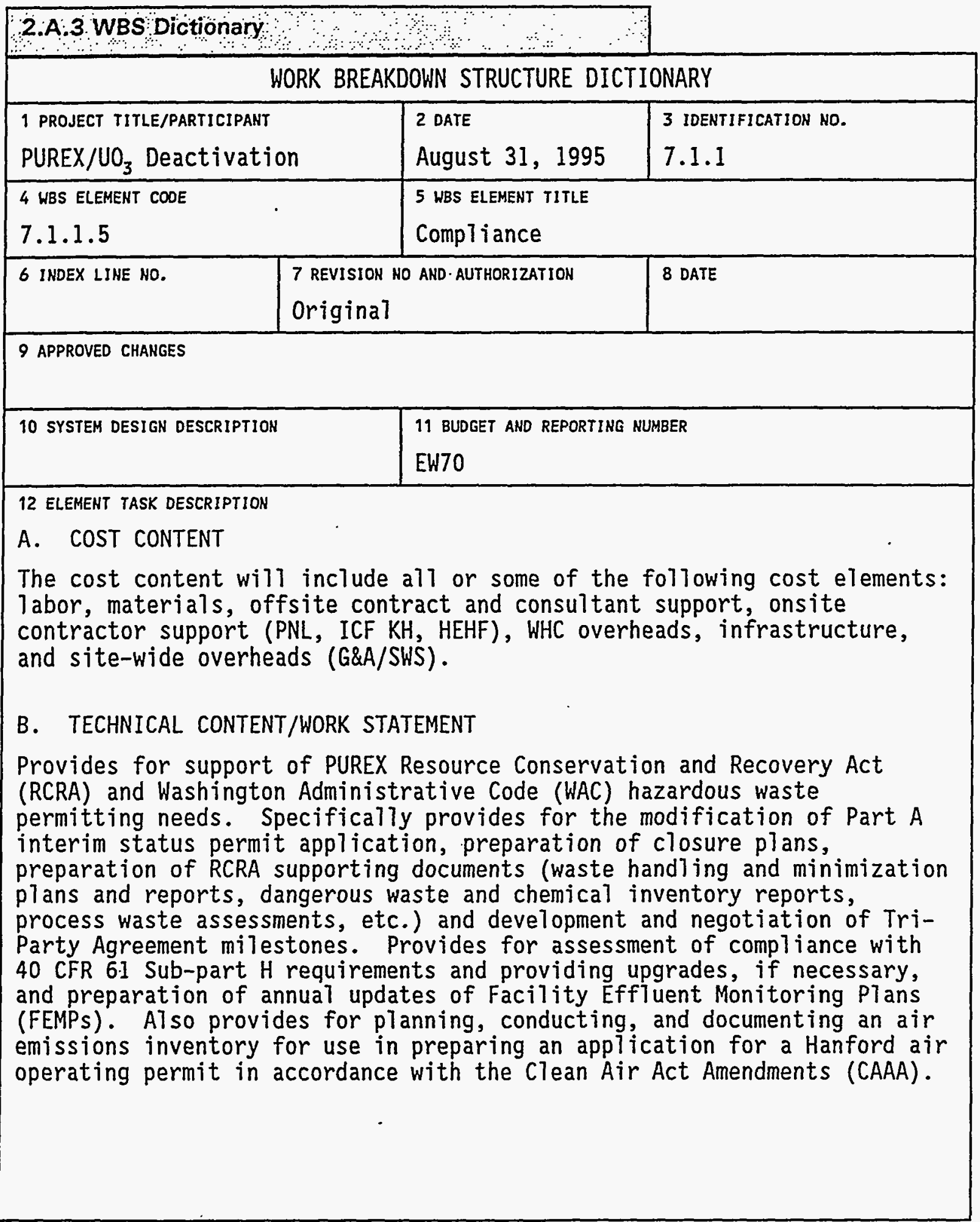


WHC-SP-1126, Rev.'1

\section{TRANSITION PROJECTS}

FY 1996 MYPP

1.3.1/7.1/6.12

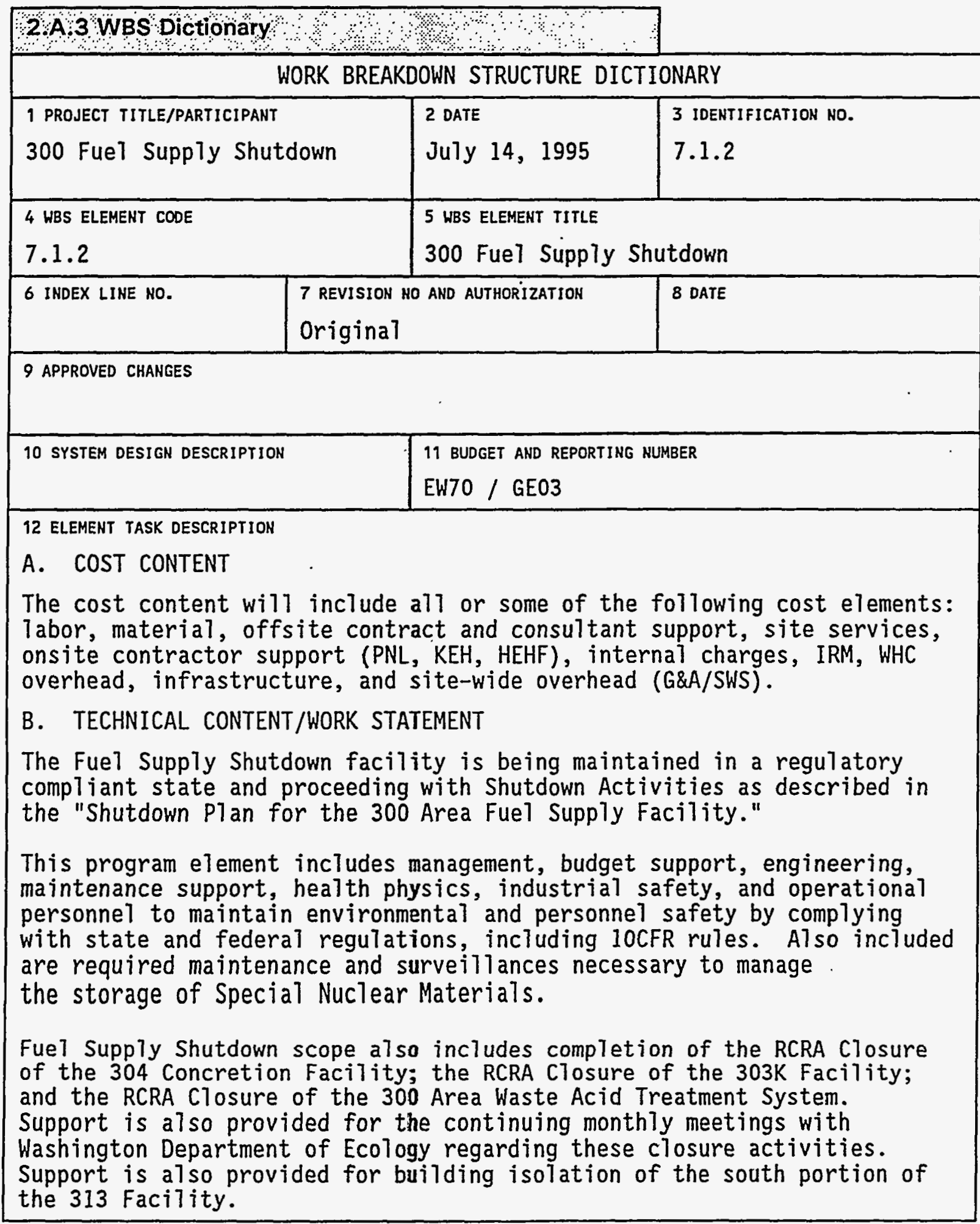


WHC-SP-1126, Rev. I

TRANSITION PROJECTS

FY 1996 MYPP

1.3.1/7.1/6.12

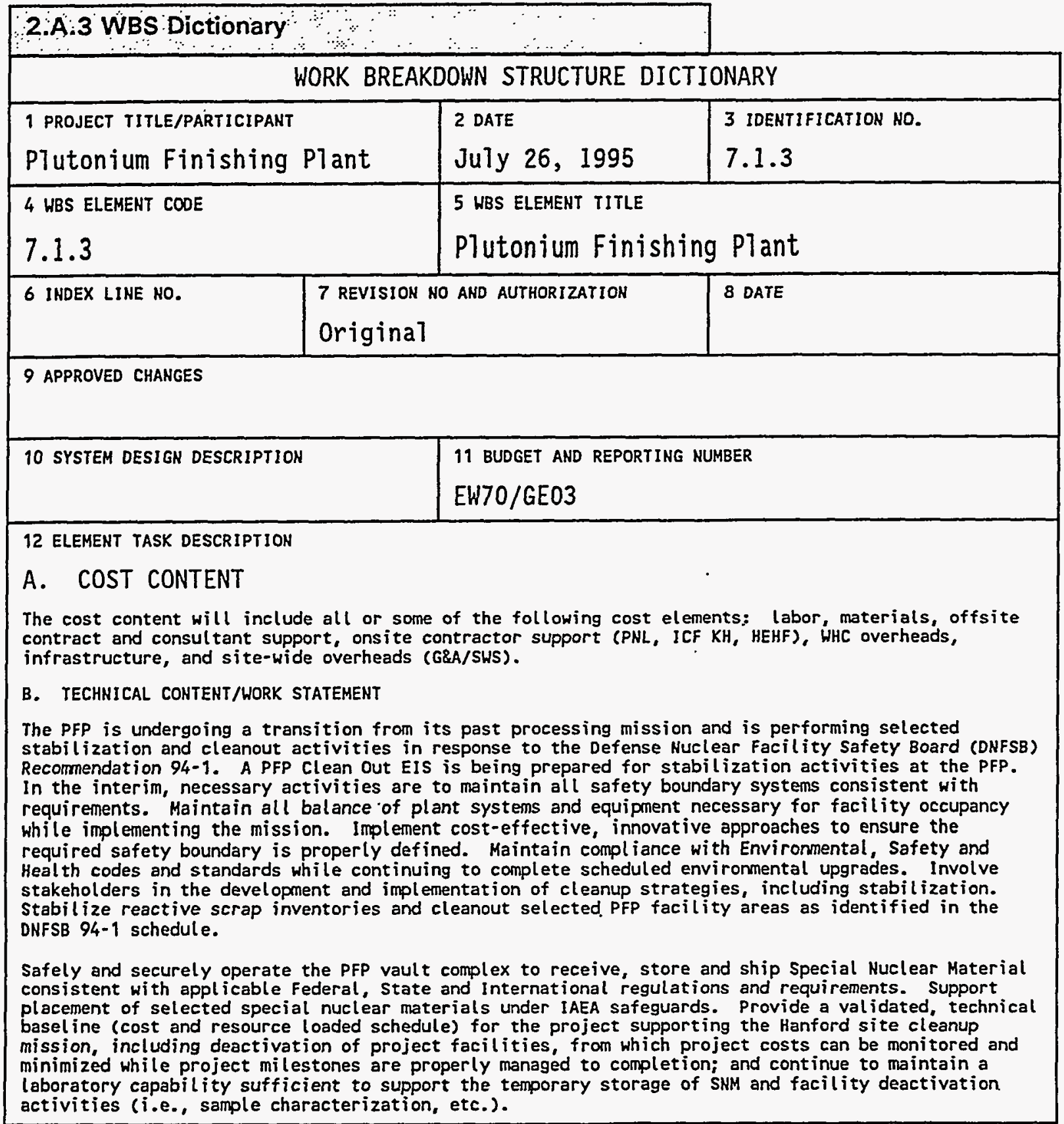


WHC-SP-1126, Rev. 1

\section{TRANSITION PROJECTS}

FY 1996 MYPP

1.3.1/7.1/6.12

\begin{tabular}{|c|c|c|}
\hline \multicolumn{3}{|c|}{ WORK BREAKDOWN STRUCTURE DICTIONARY } \\
\hline $\begin{array}{l}1 \text { PRDJECT TITLE/PARTICIPANT } \\
\text { Plutonium Finishing Plant }\end{array}$ & $\begin{array}{l}2 \text { DATE } \\
\text { JUTY } 26,1995\end{array}$ & $\begin{array}{l}3 \text { IDENTIFICATION NO. } \\
7.1 .3\end{array}$ \\
\hline $\begin{array}{l}4 \text { WBS ELEMENT CODE } \\
7.1 .3 .1\end{array}$ & \multicolumn{2}{|c|}{$\begin{array}{l}5 \text { HBS ELEMENT TITLE } \\
\text { PFP Surveillance and Maintenance }\end{array}$} \\
\hline 6 INDEX LINE NO. & $\begin{array}{l}7 \text { REVISION NO AND AUTHORIZATION } \\
\text { Original }\end{array}$ & 8 DATE \\
\hline \multicolumn{3}{|l|}{9 APPROVED CHANGES } \\
\hline 10 SYSTEM DESIGN DESCRIPTION & \multicolumn{2}{|c|}{$\begin{array}{l}11 \text { BUDGET AND REPORTING NUMBER } \\
\text { EW70/GE03 }\end{array}$} \\
\hline \multicolumn{3}{|c|}{$\begin{array}{l}\text { The cost content will include all or some of the following cost elements: } \\
\text { labor, materials, offsite contract and consultant support, onsite } \\
\text { contractor support (PNL, ICF KH, HEHF), WHC overheads, infrastructure, } \\
\text { and site-wide overheads (G\&A/SWS). }\end{array}$} \\
\hline \multicolumn{3}{|c|}{ B. TECHNICAL CONTENT/WORK STATEMENT } \\
\hline \multicolumn{3}{|c|}{$\begin{array}{l}\text { This activity entails all planned tasks to maintain the safety boundary } \\
\text { for the PFP Complex, EXCEPT FOR THE } 2736-Z / 2736-Z B \text { FACILITIES, ALL ZB/Z- } \\
\text { VAULT SUPPORT FACILITIES, SUCH AS THE 2721-Z FACILITY, AND ALL } 234-5 Z \\
\text { VAULTS AND/OR VAULT-TYPE ROOMS AS IDENTIFIED, and includes a } 11 \text { safety } \\
\text { boundary maintenance, operation surveillances and environmental } \\
\text { compliance tasks. This activity maintains the facility structure, } \\
\text { qual ified staff, safe and compliant equipment, documentation and provides } \\
\text { assessment of safety and compliance states. Provides all necessary } \\
\text { resources for safe and compliant operation in accordance with governing } \\
\text { safety codes and regulations. This activity includes the following: }\end{array}$} \\
\hline \multicolumn{3}{|c|}{$\begin{array}{ll}\text { *Corrective Maintenance } & \text { *Procedure Maintenance } \\
\text { *Preventative Maintenance } & * \text { Plant Specific Support } \\
\text { * Nuclear Process/Radiation Surveillance } & * \text { Project Management } \\
\text { *Ventilation/Power Surveillances } & \\
\text { *Environmental Compliance } & \text { *Safety Boundary \& Emergency Planning Activities }\end{array}$} \\
\hline
\end{tabular}


WHC-SP-1126, Rev. 1

\section{TRANSITION PROJECTS}

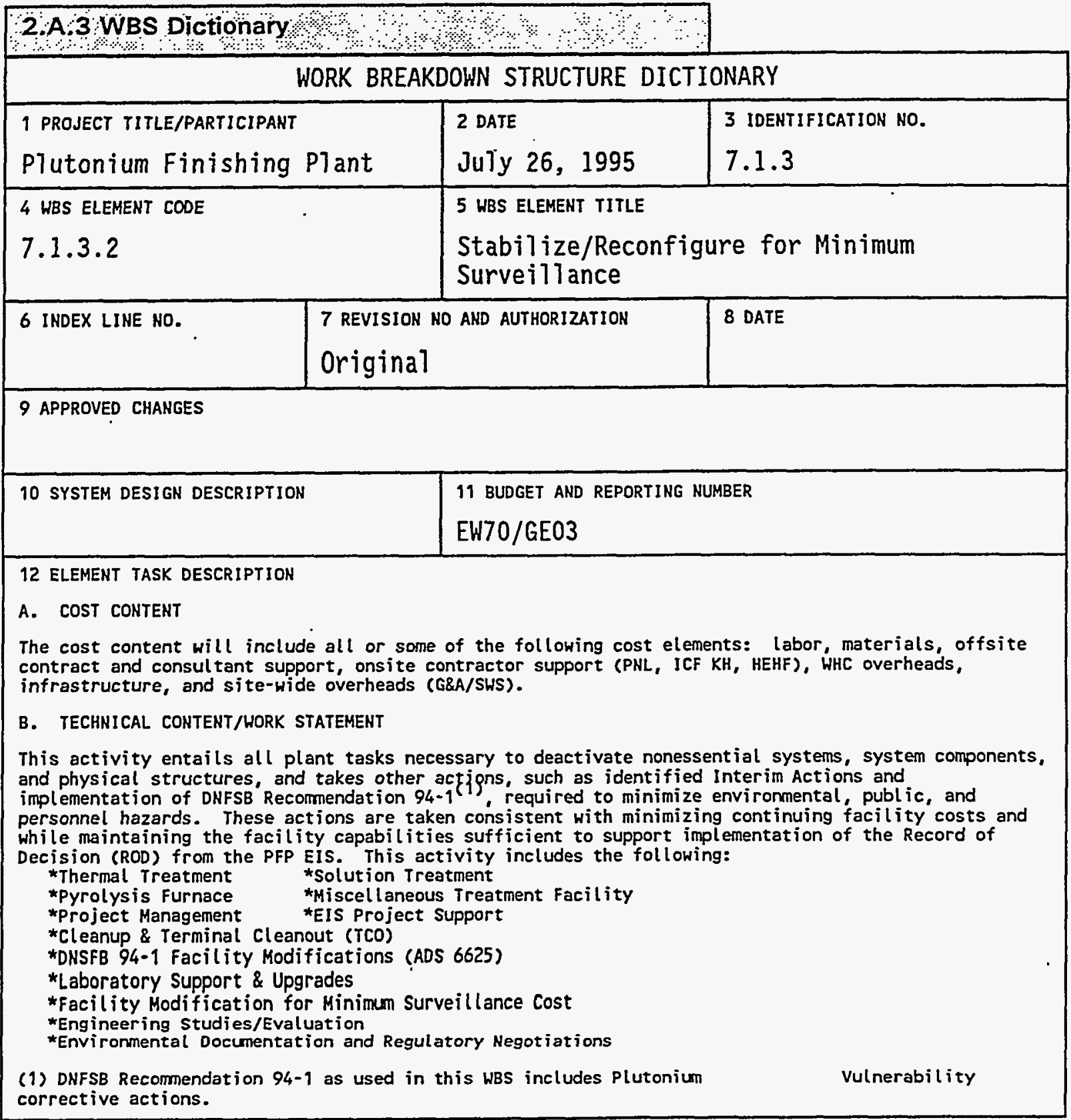


WHC-SP-1126, Rev. 1

TRANSITION PROJECTS

FY 1996 MYPP

1.3.1/7.1/6.12

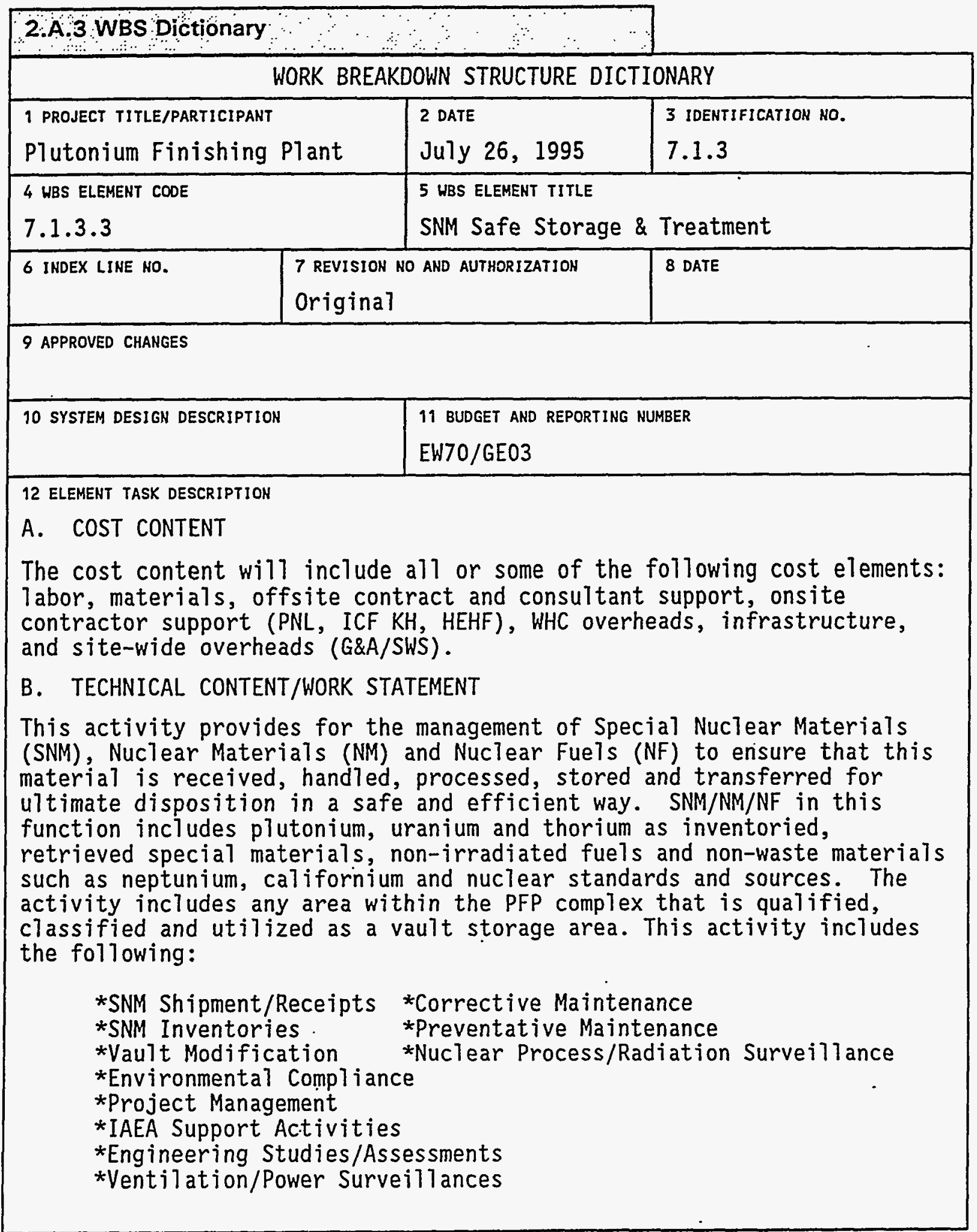


WHC-SP-1126, Rev. 1

\section{TRANSITION PROJECTS}

FY 1996 MYPP

\subsection{1/7.1/6.12}

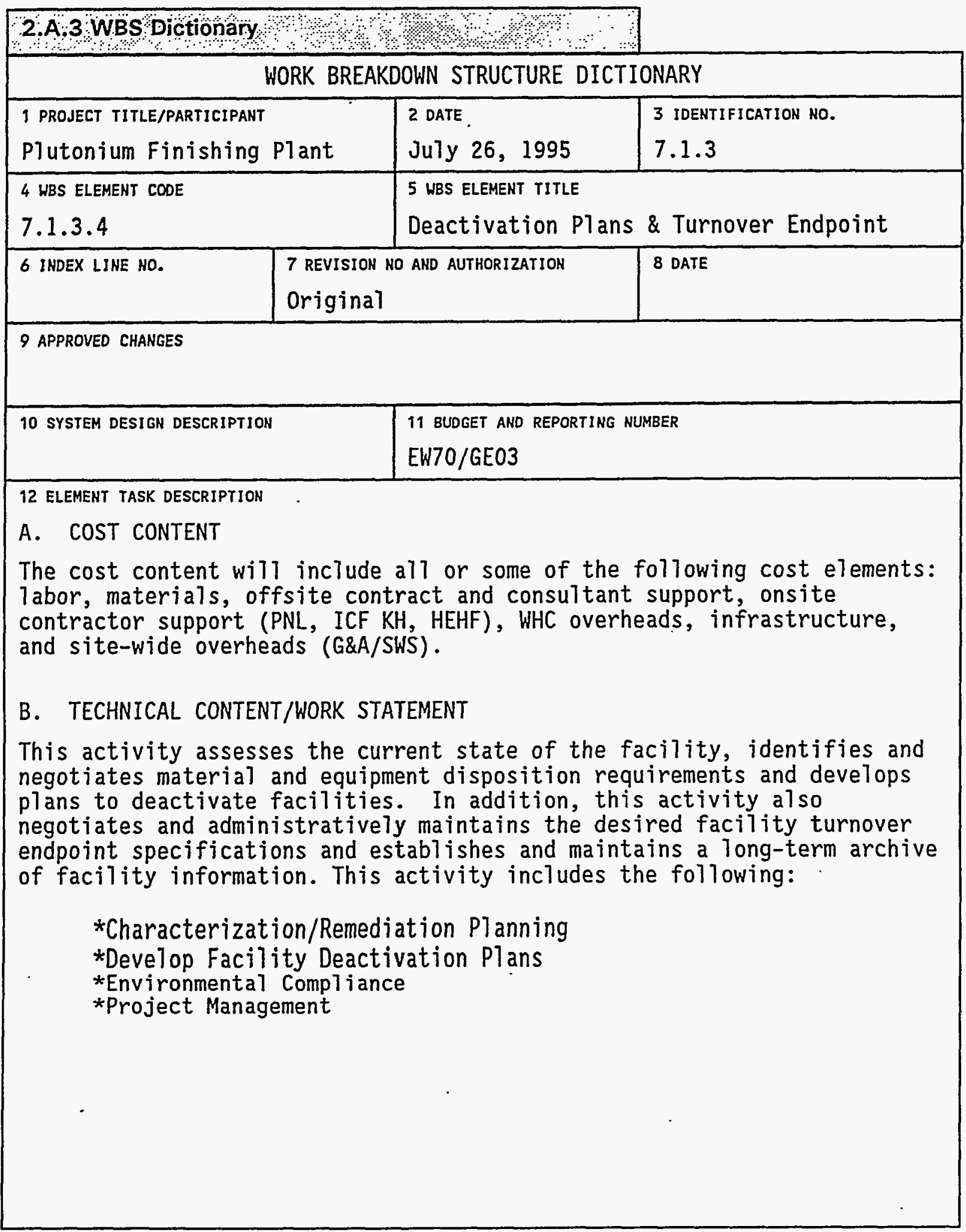


WHC-SP-1126, Rev. 1

TRANSITION PROJECTS

FY 1996 MYPP

1.3.1/7.1/6.12

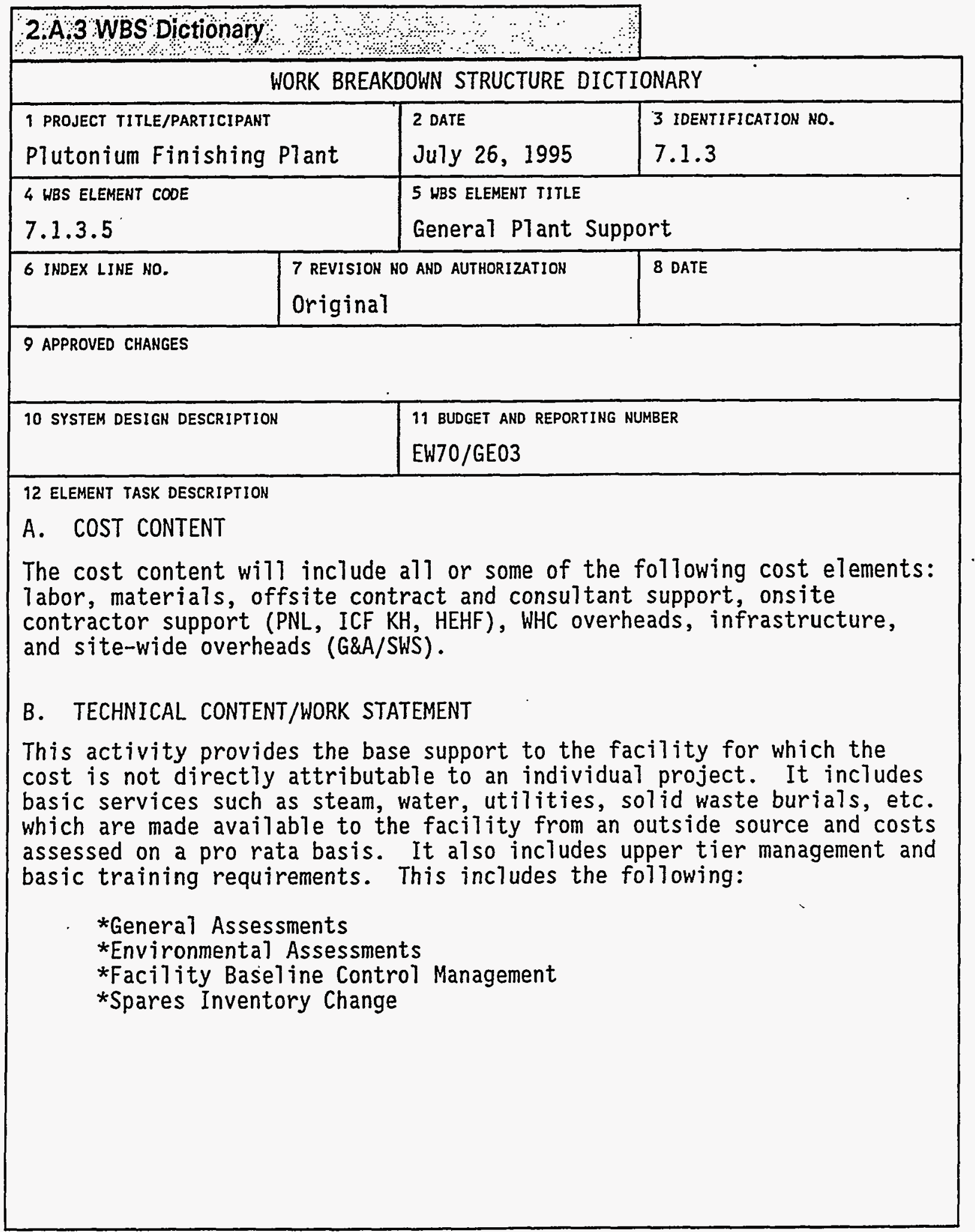


WHC-SP-1126, Rev. 1

TRANSITION PROJECTS

FY 1996 MYPP

1.3.1/7.1/6.12

\begin{tabular}{|c|c|c|}
\hline \multicolumn{3}{|c|}{ 2A 3 WBS Dictionary } \\
\hline \multicolumn{3}{|c|}{ WORK BREAKDOWN STRUCTURE DICTIONARY } \\
\hline $\begin{array}{l}1 \text { PROJECT IITLE/PARTICIPANT } \\
\text { Plutonium Finishing Plant }\end{array}$ & $\begin{array}{l}2 \text { DATE } \\
\text { JUTy } 26,1995\end{array}$ & $\begin{array}{l}3 \text { IDENTIFICATION NO. } \\
7.1 .3\end{array}$ \\
\hline $\begin{array}{l}4 \text { WBS ELEMENT CODE } \\
7.1 .3 .6\end{array}$ & \multicolumn{2}{|c|}{$\begin{array}{l}5 \text { WBS ELEMENT TITLE } \\
\text { Safeguards and Security }\end{array}$} \\
\hline 6 INDEX LINE NO. & $\begin{array}{l}7 \text { REVISION NO AND AUTHORIZATION } \\
\text { Original }\end{array}$ & 8 DATE \\
\hline \multicolumn{3}{|l|}{9 APPROVED CHANGES } \\
\hline 10 SYSTEM DESIGN DESCRIPTION & \multicolumn{2}{|c|}{$\begin{array}{l}11 \text { BUDGET AND REPORTING NUMBER } \\
\text { EW70/GEO3 }\end{array}$} \\
\hline \multicolumn{3}{|c|}{$\begin{array}{l}12 \text { ELEMENT TASK DESCRIPTION } \\
\text { A. COST CONTENT } \\
\text { The cost content will include all or some of the following cost elements: } \\
\text { labor, materials, offsite contract and consultant support, onsite } \\
\text { contractor support (PNL, ICF KH, HEHF), WHC overheads, infrastructure, } \\
\text { and site-wide overheads (G\&A/SWS). } \\
\text { B. TECHNICAL CONTENT/WORK STATEMENT } \\
\text { This activity provides the safeguards and security resources, direction } \\
\text { and oversight to provide safe, secure and compliant storage of SNM/NM/NF } \\
\text { until final disposition of the material is accomplished. Contains } \\
\text { physical security, safeguards accounting and material control, record } \\
\text { keeping, studies, evaluations and assessments. Also included is any } \\
\text { facility modifications required to maintain compliance/safety and to meet } \\
\text { Site mission goals. This includes the following: }\end{array}$} \\
\hline \multicolumn{3}{|c|}{$\begin{array}{l}\text { *Basic Physical Security } \\
\text { *SNM Custodial Support } \\
\text { *Security Control Documentation and Studies } \\
\text { *SAS Life Extension Modification } \\
\text { *SAS Maintenance } \\
\text { *Project Management }\end{array}$} \\
\hline
\end{tabular}


WHC-SP-1126, Rev. 1

\section{TRANSITION PROJECTS}

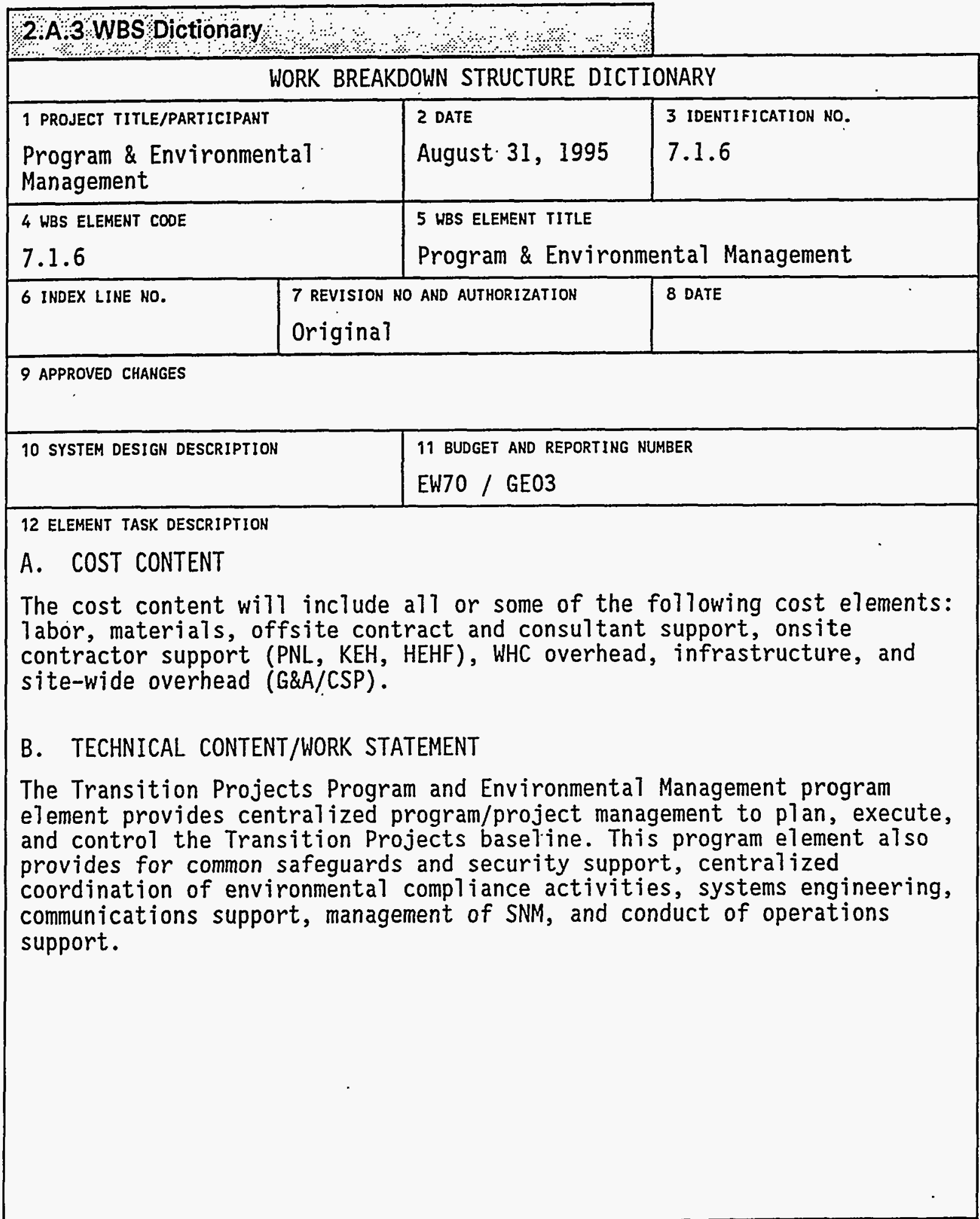


WHC-SP-1126, Rev. I

TRANSITION PROJECTS

FY 1996 MYPP

1.3.1/7.1/6.12

\begin{tabular}{|c|c|c|c|}
\hline \multicolumn{4}{|c|}{ 2A 3 WBS Dictionany } \\
\hline \multicolumn{4}{|c|}{ WORK BREAKDOWN STRUCTURE DICTIONARY } \\
\hline \multirow{2}{*}{\multicolumn{2}{|c|}{$\begin{array}{l}1 \text { PROJECT TITLE/PARTICIPANT } \\
\text { Program \& Environmental } \\
\text { Management }\end{array}$}} & 2 DATE & 3 IDENTIFICATION NO. \\
\hline & & August 31,1995 & 7.1 .6 \\
\hline \multicolumn{2}{|l|}{4 HBS ELEMENT CODE } & \multicolumn{2}{|l|}{5 HBS ELEMENT TITLE } \\
\hline \multicolumn{2}{|l|}{7.1 .6 .1} & \multicolumn{2}{|c|}{$\begin{array}{l}\text { Transition Projects Program, Environmental } \\
\text { and Business Management }\end{array}$} \\
\hline 6 INDEX LINE NO. & \multicolumn{2}{|c|}{$\begin{array}{l}7 \text { REVISION NO AND AUTHORIZATION } \\
\text { Original }\end{array}$} & 8 DATE \\
\hline \multicolumn{4}{|l|}{9 APPROVED CHANGES } \\
\hline \multicolumn{2}{|l|}{10 SYSTEM DESIGK DESCRIPTION } & \multicolumn{2}{|c|}{$\begin{array}{l}11 \text { BUDGET AND REPORTING NUMBER } \\
\text { EW70 }\end{array}$} \\
\hline \multicolumn{4}{|l|}{12 ELEMENT TASK DESCRIPTION } \\
\hline \multicolumn{4}{|c|}{$\begin{array}{l}\text { The cost content will include all or some of the following cost elements: } \\
\text { labor, materials, offsite contract and consultant support, onsite } \\
\text { contractor support (PNL, KEH, HEHF), WHC overhead, infrastructure, and } \\
\text { site-wide overhead (G\&A/CSP). }\end{array}$} \\
\hline \multicolumn{4}{|c|}{ B. TECHNICAL CONTENT/WORK STATEMENT } \\
\hline \multicolumn{4}{|c|}{$\begin{array}{l}\text { The Transition Projects Program Administration element provides } \\
\text { management of common resources such as program and environmental } \\
\text { management, program control, conduct of operations, DOE-HQ support, } \\
\text { financial management and commitment planning, and communications support. } \\
\text { Specific tasks include the standardized preparation of activity data } \\
\text { sheets (ADS), Fiscal Year Work Plans and Multi-Year Program Plans, } \\
\text { regular performance reporting to the U.S. Department of Energy-Richland } \\
\text { Field Office (RL), administration of the SMS and Progress Tracking System } \\
\text { (PTS). The Transition Projects Environmental Management provides for } \\
\text { the centralized integrating, coordinating, negotiating and planning of } \\
\text { Transition Projects environmental compliance activities. }\end{array}$} \\
\hline
\end{tabular}


WHC-SP-1126, Rev. 1

TRANSITION PROJECTS

FY 1996 MYPP

1.3.1/7.1/6.12

\section{A 3 WBS Dictionary}

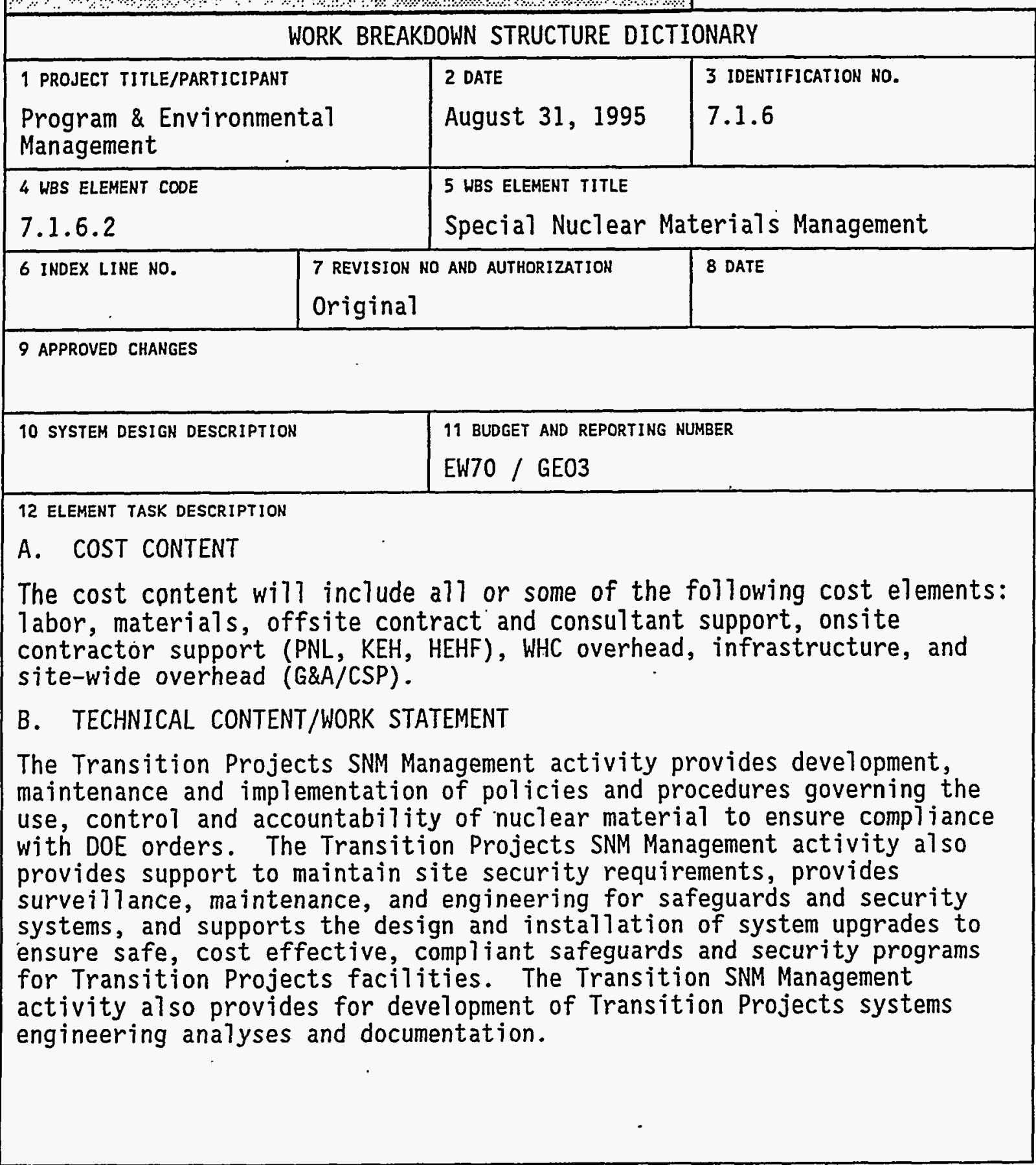


WHC-SP-1126, Rev. 1

\section{TRANSITION PROJECTS}

2.A.3 WBS Dictionary

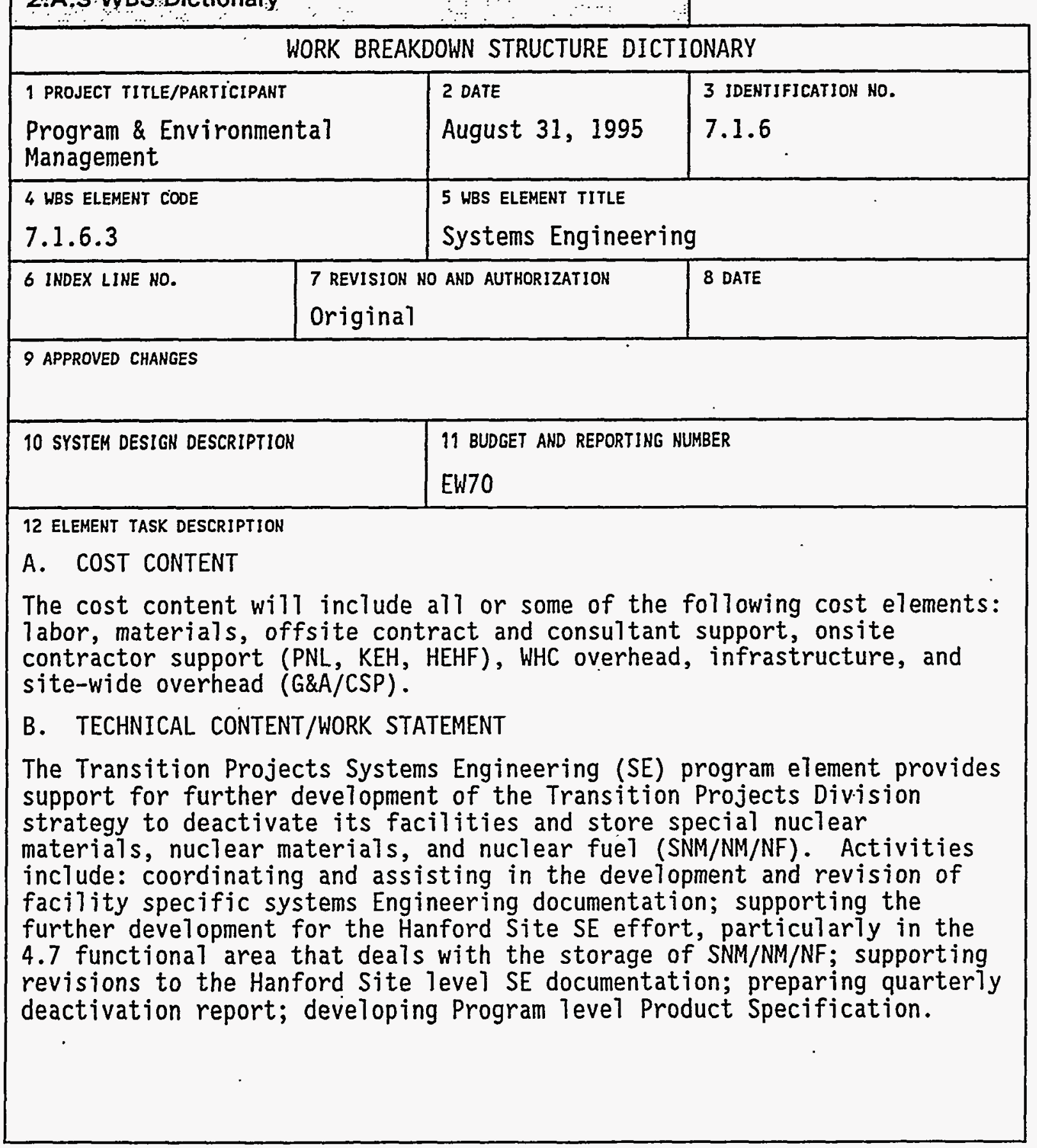




\section{TRANSITION PROJECTS}

FY 1996 MYPP

1.3.1/7.1/6.12

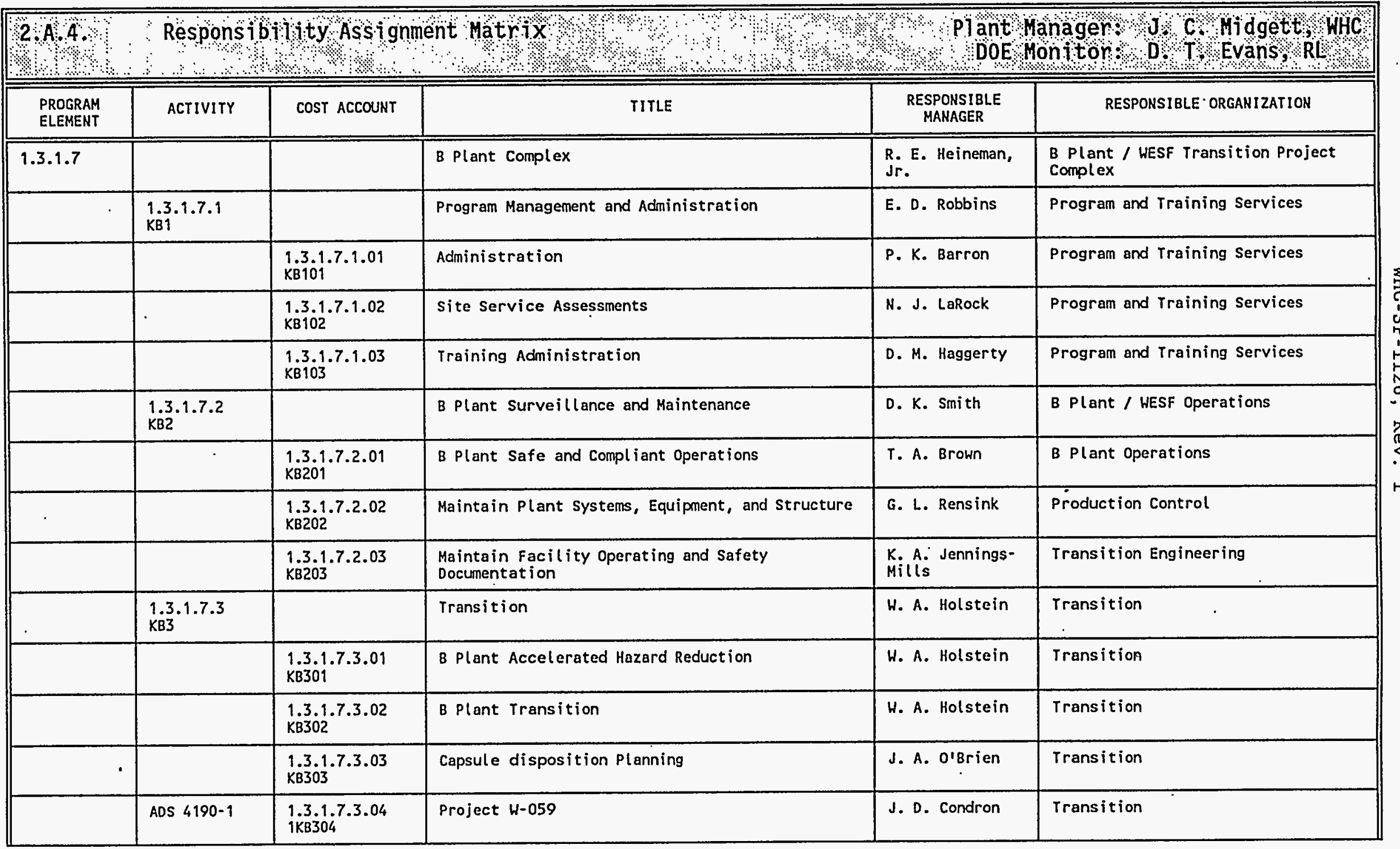




\section{TRANSITION PROJECTS}

FY 1996 MYPP

1.3.1/7.1/6.12

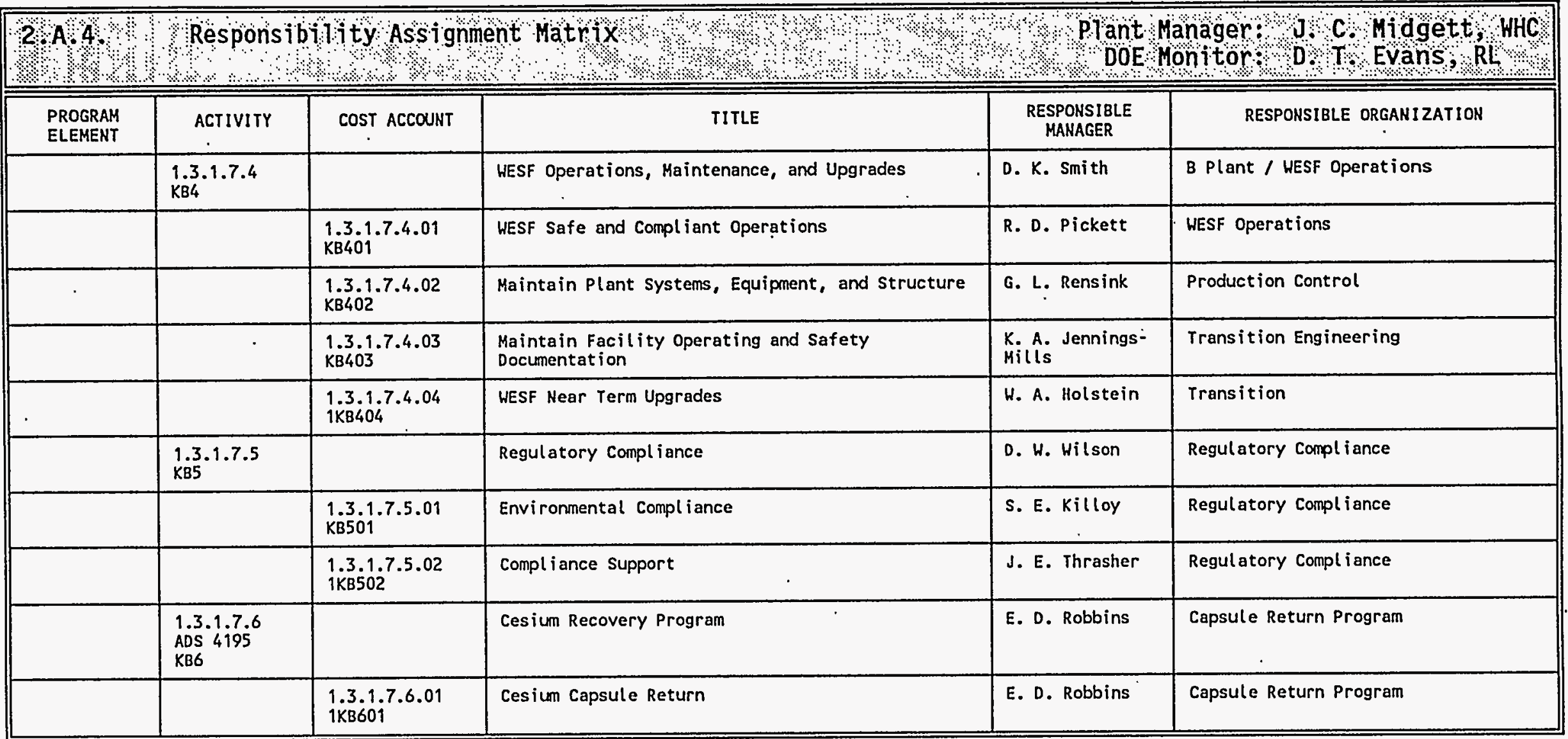


TRANSITION PROJECTS

1.3.1/7.1/6.12

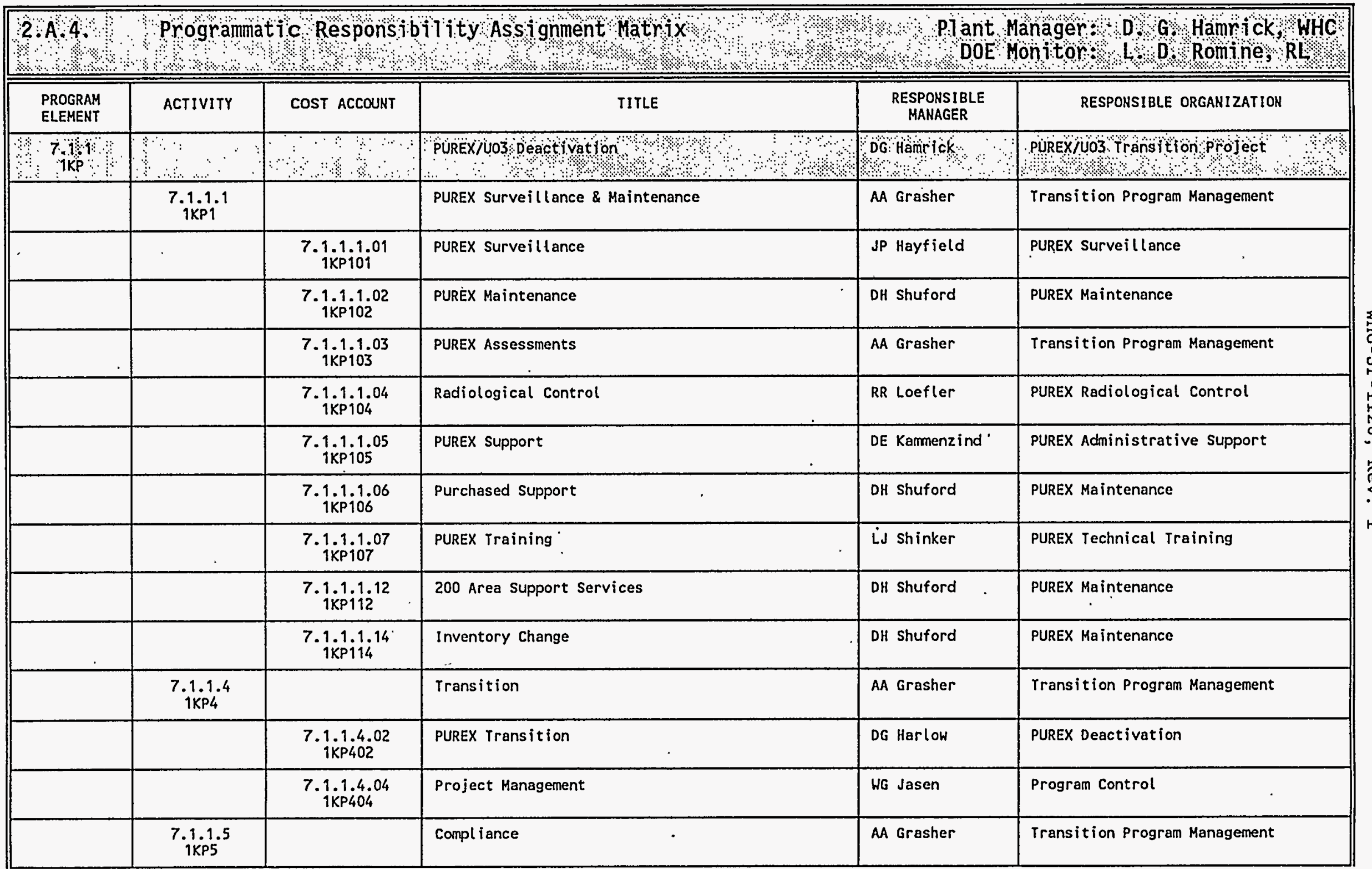




\section{TRANSITION PROJECTS}

FY 1996 MYPP

1.3.1/7.1/6.12

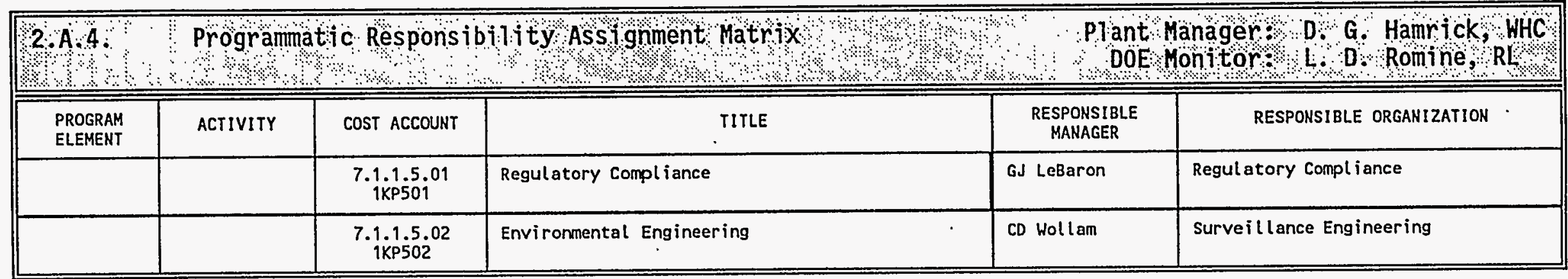




\section{TRANSITION PROJECTS}

\section{FY 1996 MYPP}

1.3.1/7.1/6.12

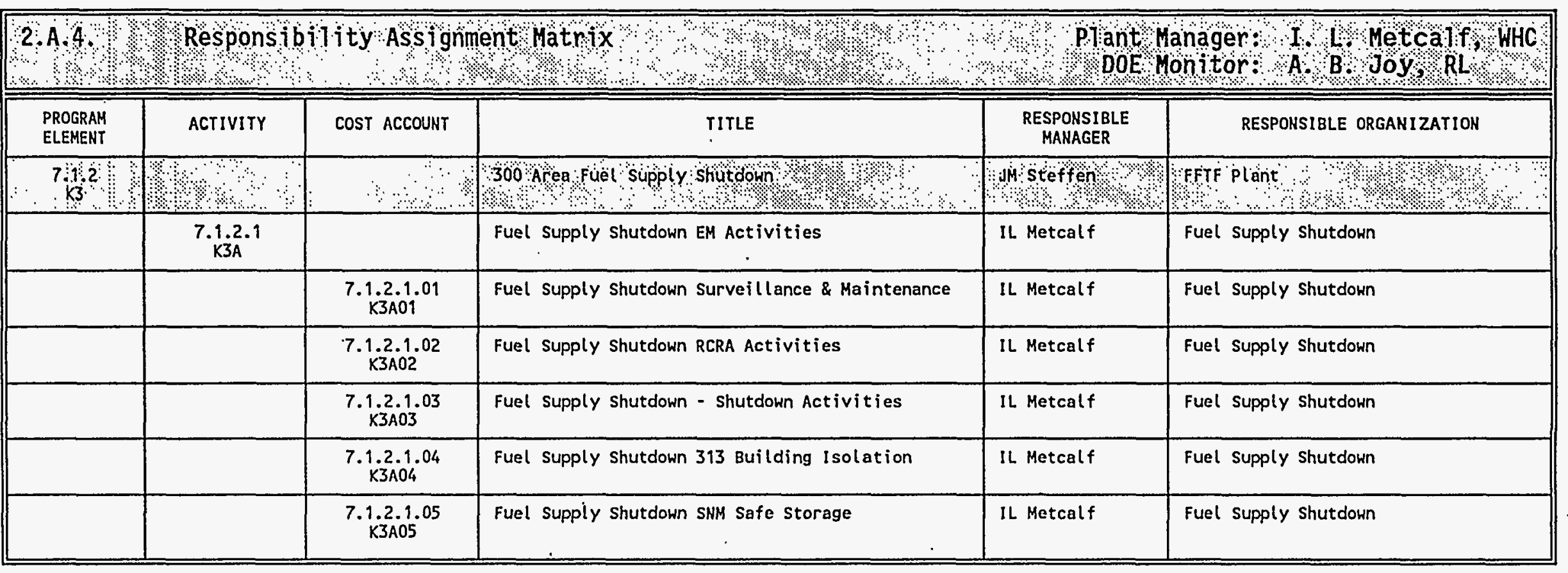




\section{TRANSITION PROJECTS}

FY 1996 MYPP

1.3.1/7.1/6.12

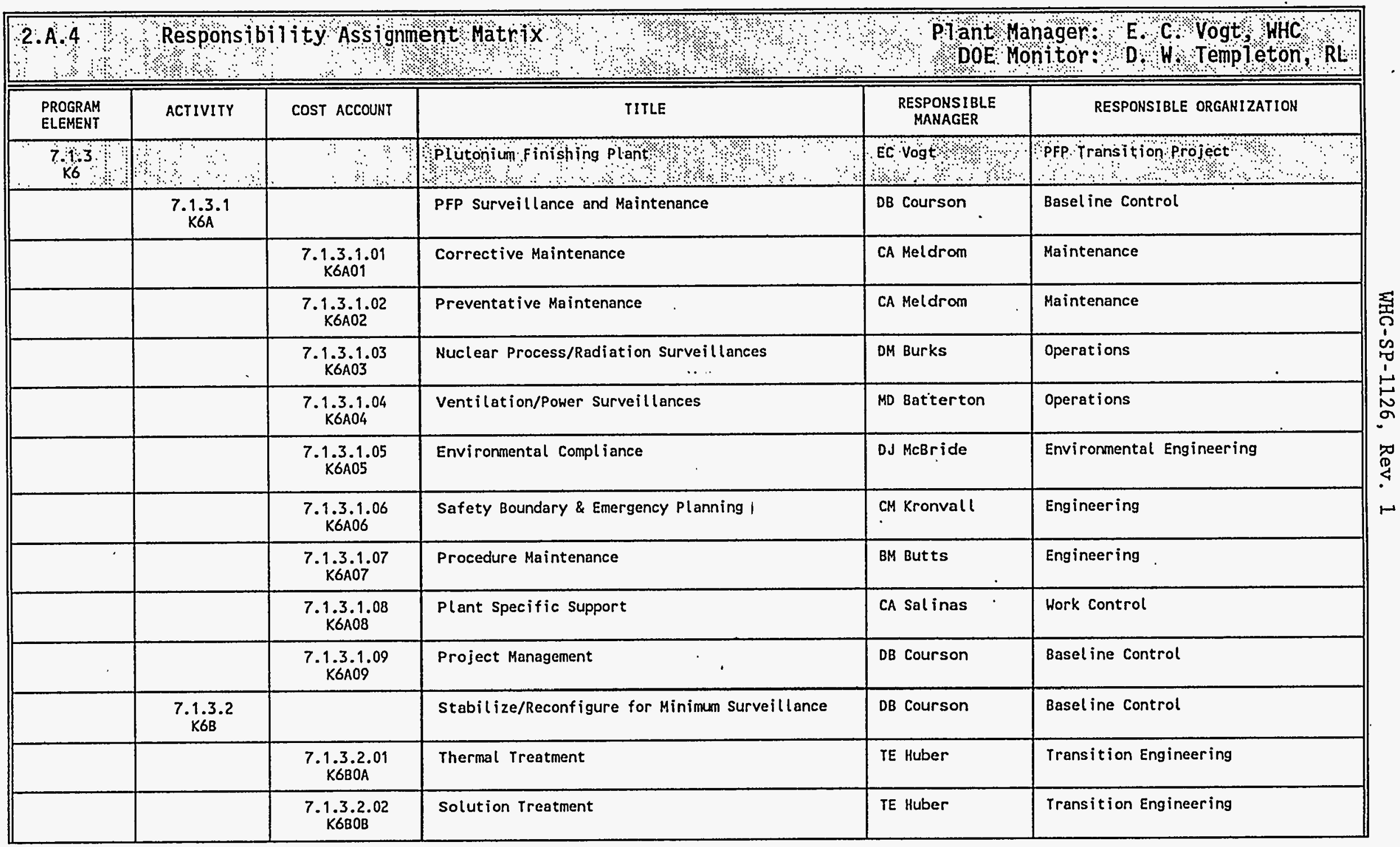


TRANSITION PROJECTS

1.3.1/7.1/6.12

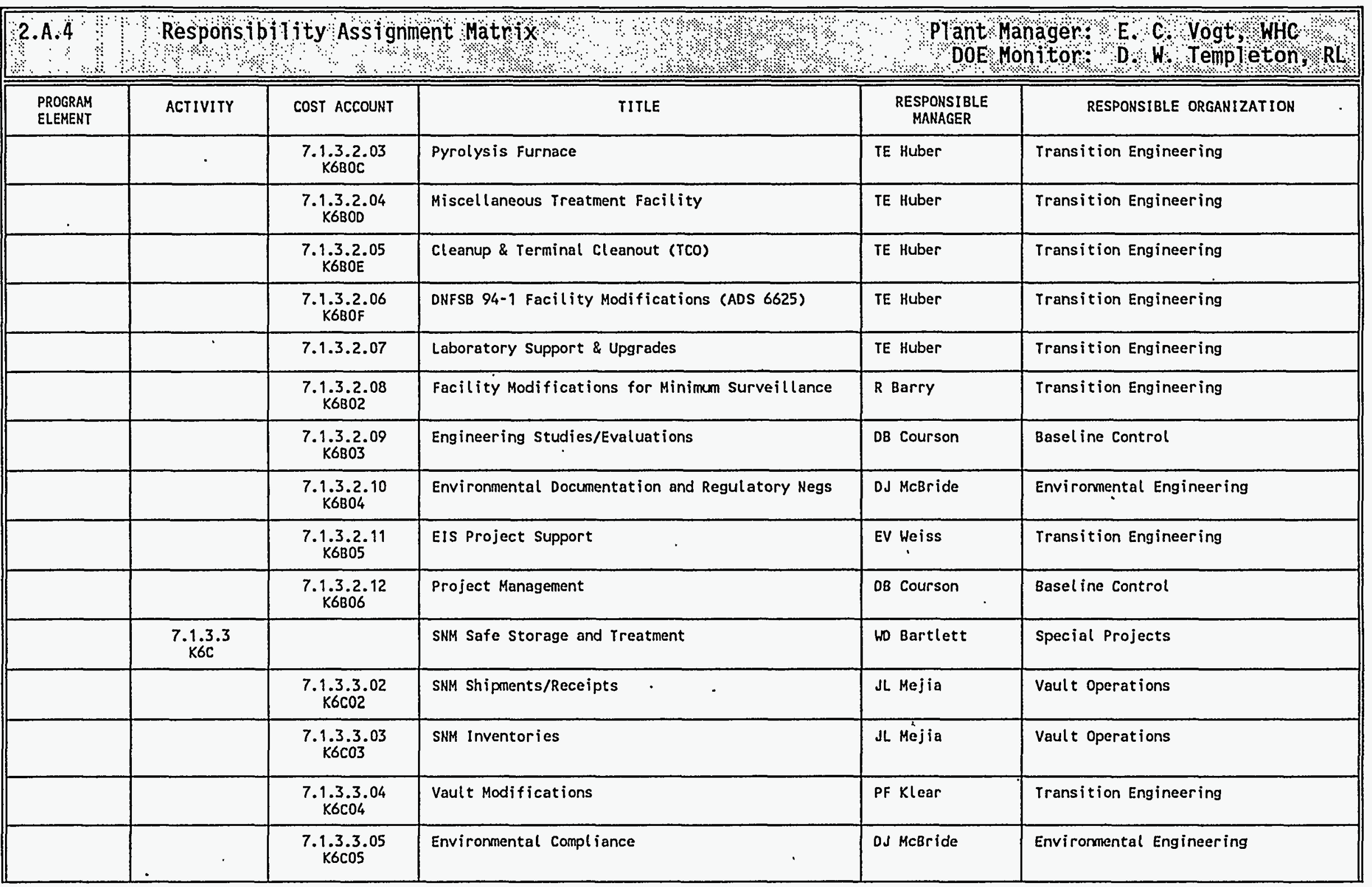




\section{FY 1996 MYPP}

TRANSITION PROJECTS

1.3.1/7.1/6.12

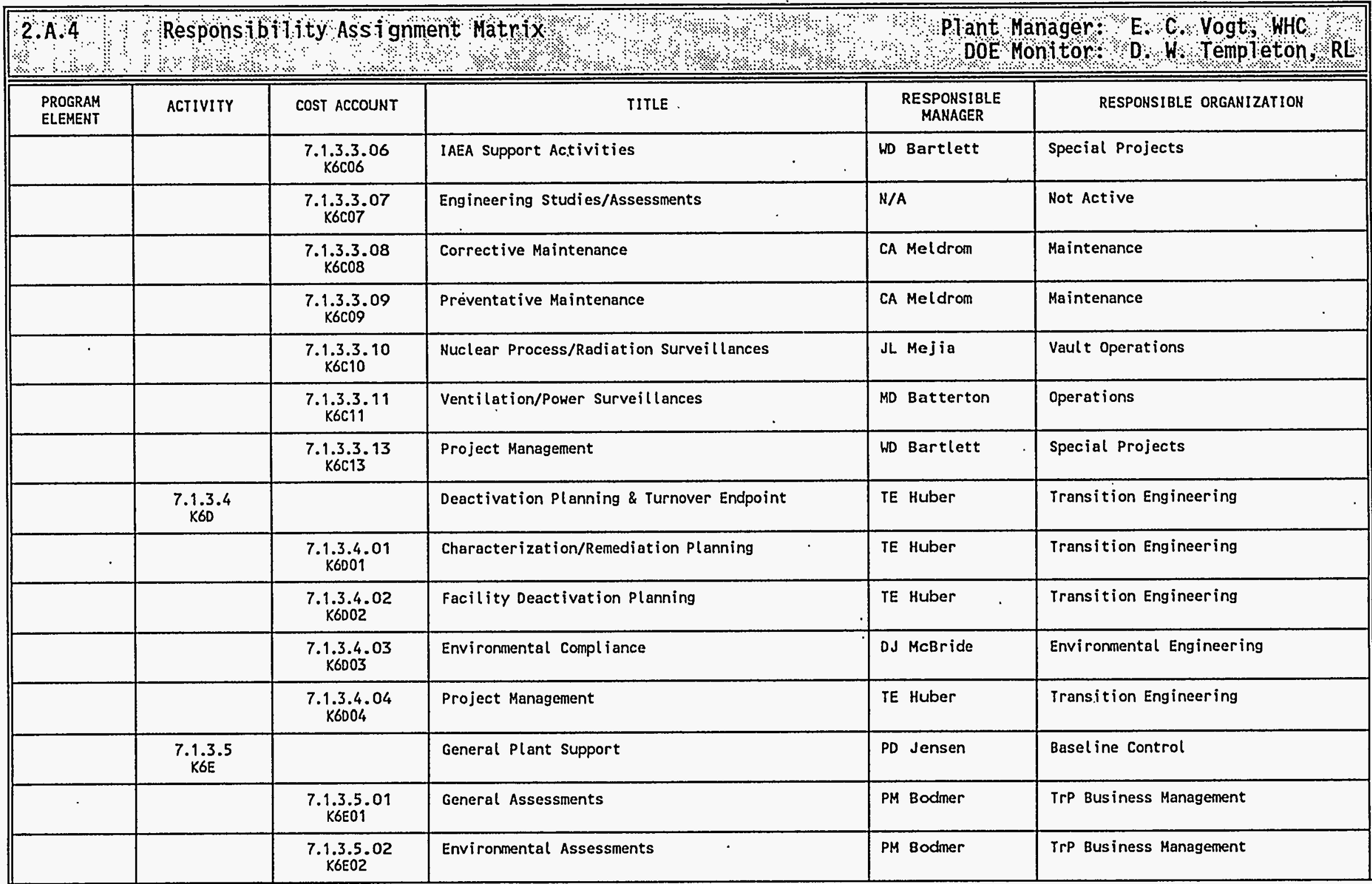


TRANSITION PROJECTS

1.3.1/7.1/6.12

FY 1996 MYP.P

Plant Nanager $\mathrm{E}$. C Yogt, WHC

DoE Honitor $\mathrm{D}$. W Templeton, RL

\begin{tabular}{|c|c|c|c|c|c|}
\hline $\begin{array}{l}\text { PROGGRAM } \\
\text { ELEMENT }\end{array}$ & ACTIVITY & COST ACCOUNT & TITLE & $\begin{array}{l}\text { RESPONSIBLE } \\
\text { MANAGER }\end{array}$ & RESPONSIBLE ORGANIZATION \\
\hline & & $\begin{array}{l}7.1 .3 .5 .03 \\
\text { K } 6 E 03\end{array}$ & Facility Baseline Control Management & PD Jensen & Basel ine Control \\
\hline & & $\underset{\mathrm{K} 6 \mathrm{EO4}}{7.1 .3 .5 .04}$ & Spares Inventory Change & PD Jensen & Basel ine Control \\
\hline & $\begin{array}{c}7.1 .3 .6 \\
\mathrm{~K} 6 \mathrm{~F}\end{array}$ & . & Safeguards and Security & WF Russell & Baseline Control \\
\hline & & $\begin{array}{c}7.1 .3 .6 .01 \\
\text { K6FO1 }\end{array}$ & Basic Security & WF Russell & Baseline Control \\
\hline & & $7.1_{K 6 F 02} .3 .6 .02$ & SNM Custodial Support & BR Fitzpatrick & Safeguards Material Contral \\
\hline & & $\begin{array}{c}7.1 .3 .6 .03 \\
\text { X6FO3 }\end{array}$ & Security Control Documentation and Studies & WF Russell & Baseline Control \\
\hline & & $\underset{\text { K6FO4 }}{7.1 .3 .6 .04}$ & SAS Life Extension Modifications. & PF Klear & Transition Engineering \\
\hline & & $\begin{array}{c}7.1 .3 .6 .05 \\
\text { K6F05 }\end{array}$ & SAS Maintenance & JW Gideon & Northern Security Operations \\
\hline & & $\begin{array}{l}7.1 .3 .6 .06 \\
\text { K6F06 }\end{array}$ & Project Management & WF Russell & Basel ine Control \\
\hline
\end{tabular}




\section{TRANSITION PROJECTS}

FY 1996 MYPP

1.3.1/7.1/6.12

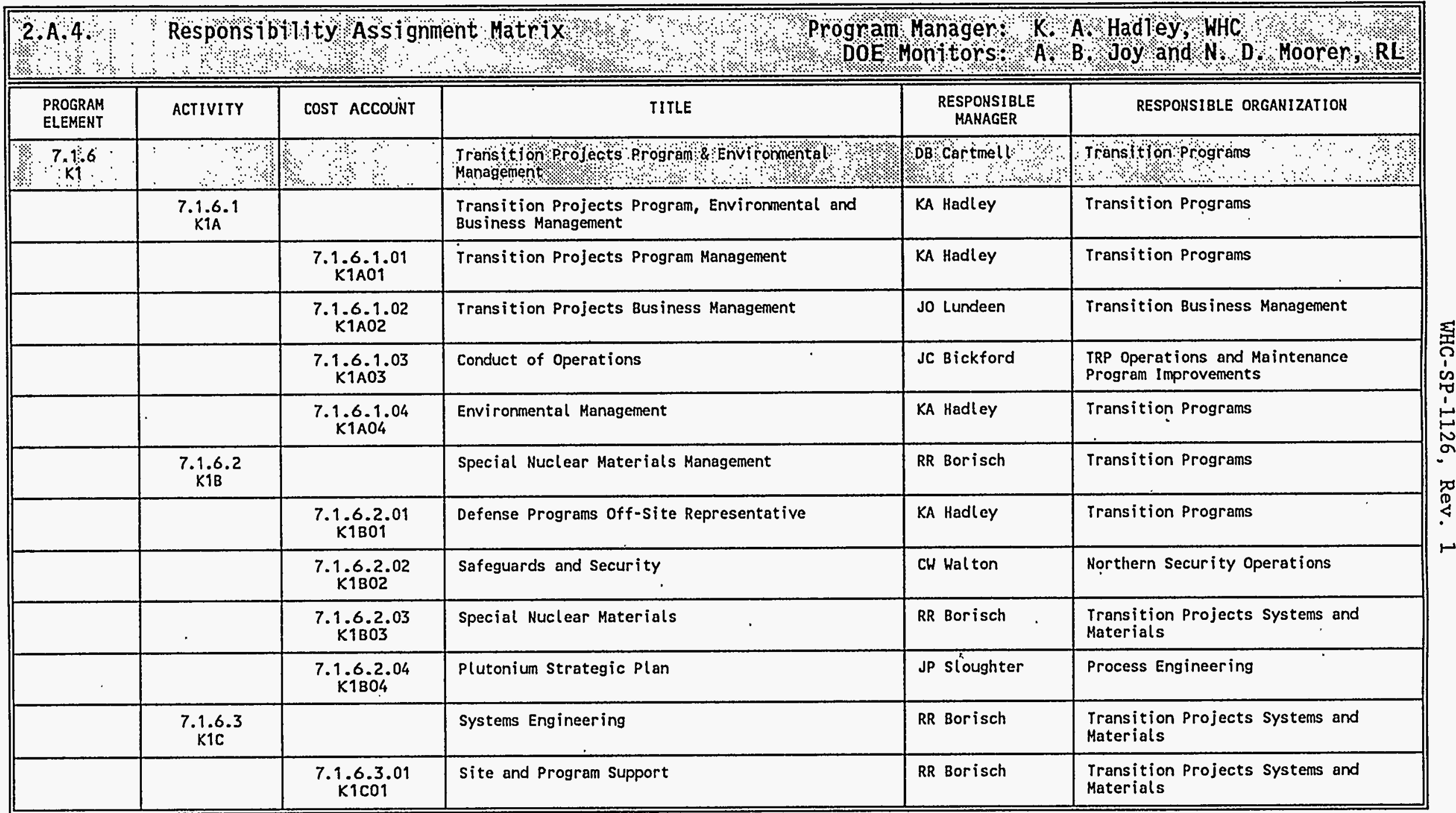


2.A.5 Techinology Requirentents

This section intentionally left blank.

$n$
$\vdots$
$i$
1 
WHC-SP-1126, Rev. 1

TRANSITION PROJECTS

FY 1996 MYPP

1.3.1/7.1/6.12

2. A 6 Waste Type Data

FY 1996 Waste Type/Spent Fuel Volume Projections for WBS: 1.3.1.7.

Program Name: B Plant/WESF Transition Project

\begin{tabular}{|c|c|c|c|c|c|c|}
\hline \multicolumn{4}{|c|}{ Period Start Date:10/1/95 } & \multicolumn{3}{|c|}{ Period End Date: 9/30/96 } \\
\hline $\begin{array}{l}\text { Waste } \\
\text { Type }\end{array}$ & $\begin{array}{l}\text { Beginning } \\
\text { Inventory }\end{array}$ & $\begin{array}{l}\text { Waste } \\
\text { Generated }\end{array}$ & $\begin{array}{l}\text { Waste } \\
\text { Received }\end{array}$ & $\begin{array}{l}\text { Haste } \\
\text { Reduction }\end{array}$ & $\begin{array}{l}\text { Haste } \\
\text { Transferred }\end{array}$ & $\begin{array}{l}\text { Waste } \\
\text { Stored }\end{array}$ \\
\hline HLW & 0 & 0 & 0 & 0 & 0 & 0 \\
\hline$L L W(s)$ & 0 & 237 & 0 & 0 & 237 & 0 \\
\hline LLW (1) & 0 & 0 & 0 & 0 & 0 & 0 \\
\hline LLMH (s) & 5.2 & 2.1 & 0 & 0 & 2.1 & 0 \\
\hline LLMH (1) & 0 & 114 & 0 & 0 & 114 & 0 \\
\hline $\begin{array}{l}\text { LLW } \\
\text { (GTC3) }\end{array}$ & 0 & 0 & 0 & 0 & 0 & 0 \\
\hline TRU & 0 & 0 & 0 & .0 & 0 & 0 \\
\hline TRUM & 0 & 0 & 0 & 0 & 0 & 0 \\
\hline$H A Z$ & 0 & 4.2 & 0 & 0 & 4.2 & 0 \\
\hline Landfil1 & 0 & 950 & 0 & 0 & 950 & 0 \\
\hline Asbestos & 0 & 4.25 & 0 & 0 & 4.25 & 0 \\
\hline $\begin{array}{l}\text { San. } \\
\text { (Liq) }\end{array}$ & 0 & 265,000 & 0 & 0 & 265,000 & 0 \\
\hline
\end{tabular}

Note: Volumetric units for values entered in the table are cubic meters, except for spent fuel and spent fuel by-products where the value are reported in metric tons of heavy metal. 
WHC-SP-1126, Rev. 1

\section{TRANSITION PROJECTS}

FY 1996 MYPP

1.3.1/7.1/6.12

2. A.6. Waste Type Data

FY 1997 Waste Type/Spent Fuel Volume Projections for WBS: 1.3.1.7

Program Name: B Plant/WESF Transition Projects

\begin{tabular}{|c|c|c|c|c|c|c|}
\hline \multicolumn{4}{|c|}{ Perjod Start Date: 10/1/96 } & \multicolumn{3}{|c|}{ Period End Date: $9 / 30 / 97$} \\
\hline $\begin{array}{l}\text { Waste } \\
\text { Type }\end{array}$ & $\begin{array}{l}\text { Beginning } \\
\text { Inventory }\end{array}$ & $\begin{array}{l}\text { Waste } \\
\text { Generated }\end{array}$ & $\begin{array}{l}\text { Haste } \\
\text { Received }\end{array}$ & $\begin{array}{l}\text { Haste } \\
\text { Reduction }\end{array}$ & $\begin{array}{l}\text { Waste } \\
\text { Transferred }\end{array}$ & $\begin{array}{l}\text { Waste } \\
\text { Stored }\end{array}$ \\
\hline HLH & 0 & 0 & 0 & 0 & 0 & 0 \\
\hline LLH .(s) & 0 & 187 & 0 & 0 & 187 & 0 \\
\hline $\operatorname{LLW}(1)$ & 0 & 0 & 0 & 0 & 0 & 0 \\
\hline LLMW (s) & 5.2 & 2.1 & 0 & 0 & 2.1 & 0 \\
\hline LLMH (1) & 0 & 114 & 0 & 0 & 114 & 0 \\
\hline $\begin{array}{l}\text { LLW } \\
\text { (GTC3) }\end{array}$ & 0 & 0 & 0 & 0 & 0 & 0 \\
\hline TRU & 0 & 0 & 0 & 0 & 0 & 0 \\
\hline TRUM & 0 & 0 & 0 & 0 & 0 & 0 \\
\hline HAZ & 0 & 4.2 & 0 & 0 & 4.2 & 0 \\
\hline Landfill & 0 & 950 & 0 & 0 & 950 & 0 \\
\hline Asbestos & 0 & 4.25 & 0 & 0 & 4.25 & 0 \\
\hline $\begin{array}{l}\text { San. } \\
\text { (Liq) }\end{array}$ & 0 & 265,000 & 0 & 0 & 265,000 & 0 \\
\hline
\end{tabular}

Note: Volumetric units for values entered in the table are cubic meters, except for spent fuel and spent fuel by-products where the value are reported in metric tons of heavy metal. 
WHC-SP-1126, Rev. 1

\section{TRANSITION PROJECTS}

FY 1996 MYPP

2. A.6 Waste Type Data

FY 1998 Waste Type/Spent Fuel Volume Projections for WBS: 1.3.1.7

Program Name: B Plant/WESF Transition Projects

\begin{tabular}{|c|c|c|c|c|c|c|}
\hline \multicolumn{4}{|c|}{ Period Start Date:10/1/97 } & \multicolumn{3}{|c|}{ Period End Date: 9/30/98 } \\
\hline $\begin{array}{l}\text { Haste } \\
\text { Type }\end{array}$ & $\begin{array}{l}\text { Beginning } \\
\text { Inventory }\end{array}$ & $\begin{array}{l}\text { Waste } \\
\text { Generated }\end{array}$ & $\begin{array}{l}\text { Waste } \\
\text { Received }\end{array}$ & $\begin{array}{l}\text { Haste } \\
\text { Reduction }\end{array}$ & $\begin{array}{l}\text { Waste } \\
\text { Transferred }\end{array}$ & $\begin{array}{l}\text { Haste } \\
\text { Stored }\end{array}$ \\
\hline HLH & 0 & 0 & 0 & 0 & 0 & 0 \\
\hline $\operatorname{LLW}(s)$ & 0 & 187 & 0 & 0 & 187 & 0 \\
\hline LLW (1) & 0 & 0 & 0 & 0 & 0 & 0 \\
\hline LLMW (s) & 5.2 & 2.1 & 0 & 0 & 2.1 & 0 \\
\hline LLMW (1) & 0 & 356 & 0 & 0 & 356 & 0 \\
\hline $\begin{array}{l}\text { LLW } \\
\text { (GTC3) }\end{array}$ & 0 & 0 & 0 & 0 & 0 & 0 \\
\hline TRU & 0 & 0 & 0 & 0 & 0 & 0 \\
\hline TRUM & 0 & 0 & 0 & 0 & 0 & 0 \\
\hline HAZ & 0 & 4.2 & 0 & 0 & 4.2 & 0 \\
\hline Landfill & 0 & 950 & 0 & 0 & 950 & 0 \\
\hline Asbestos & 0 & 4.25 & 0 & 0 & 4.25 & 0 \\
\hline $\begin{array}{l}\text { San. } \\
\text { (Liq) }\end{array}$ & 0 & 265,000 & 0 & 0 & 265,000 & 0 \\
\hline
\end{tabular}

Note: Volumetric units for values entered in the table are cubic meters, except for spent fuel and spent fuel by-products where the value are reported in metric tons of heavy metal. 
WHC-SP-1126, Rev. 1

\section{TRANSITION PROJECTS}

FY 1996 MYPP

2. A. 6 Waste Type Data

FY 1999 Waste Type/Spent Fuel Volume Projections for WBS: 1.3.1.7

Program Name: B Plant/WESF Transition Projects

Period Start Date: 10/1/98

\begin{tabular}{|c|c|c|c|c|c|c|}
\hline $\begin{array}{l}\text { Waste } \\
\text { Type }\end{array}$ & $\begin{array}{l}\text { Beginning } \\
\text { Inventory }\end{array}$ & $\begin{array}{l}\text { Waste } \\
\text { Generated }\end{array}$ & $\begin{array}{l}\text { Haste } \\
\text { Received }\end{array}$ & $\begin{array}{l}\text { Haste } \\
\text { Reduction }\end{array}$ & $\begin{array}{l}\text { Waste } \\
\text { Transferred }\end{array}$ & $\begin{array}{l}\text { Waste } \\
\text { Stored }\end{array}$ \\
\hline HLW & 0 & 0 & 0 & 0 & 0 & 0 \\
\hline LLW (s) & 0 & 187 & 0 & 0 & 187 & 0 \\
\hline LLW (1) & 0 & 0 & 0 & 0 & 0 & 0 \\
\hline LLMH (s) & 5.2 & 2.1 & 0 & 0 & 2.1 & 0 \\
\hline LLMH (1) & 0 & 436 & 0 & 0 & 436 & 0 \\
\hline $\begin{array}{l}\text { LLW } \\
\text { (GTC3) }\end{array}$ & 0 & 0 & 0 & 0 & 0 & 0 \\
\hline TRU & 0 & 0 & 0 & 0 & 0 & 0 \\
\hline TRUM & 0 & 0 & 0 & 0 & 0 & 0 \\
\hline HAZ & 0 & 4.2 & 0 & 0 & 4.2 & 0 \\
\hline Landfil1 & 0 & 950 & 0 & 0 & 950 & 0 \\
\hline Asbestos & 0 & 4.25 & 0 & 0 & 4.25 & 0 \\
\hline $\begin{array}{l}\text { San. } \\
\text { (Liq) }\end{array}$ & 0 & 26,500 & 0 & 0 & 27,000 & 0 \\
\hline
\end{tabular}

Note: Volumetric units for values entered in the table are cubic meters, except for spent fuel and spent fuel by-products where the value are reported in metric tons of heavy metal. 
WHC-SP-1126, Rev. I

\section{TRANSITION PROJECTS}

FY 1996 MYPP

1.3.1/7.1/6.12

2A. 6 Waste Type Data

FY 2000 Waste Type/Spent Fuel Volume Projections for WBS: 1.3 .1 .7

Program Name: B PTant/WESF Transition Projects

\begin{tabular}{|c|c|c|c|c|c|c|}
\hline \multicolumn{4}{|c|}{ Period Start Date:10/1/99 } & \multicolumn{3}{|c|}{ Period End Date: $9 / 30 / 2000$} \\
\hline $\begin{array}{l}\text { Haste } \\
\text { Type }\end{array}$ & $\begin{array}{l}\text { Beginning } \\
\text { Inventory }\end{array}$ & $\begin{array}{l}\text { Waste } \\
\text { Generated }\end{array}$ & $\begin{array}{l}\text { Waste } \\
\text { Received }\end{array}$ & $\begin{array}{l}\text { Haste } \\
\text { Reduction }\end{array}$ & $\begin{array}{l}\text { Waste } \\
\text { Transferred }\end{array}$ & $\begin{array}{l}\text { Waste } \\
\text { Stored }\end{array}$ \\
\hline HLW & 0 & 0 & 0 & 0 & 0 & 0 \\
\hline$L L W(s)$ & 0 & 62.3 & 0 & 0 & 62.3 & 0 \\
\hline $\operatorname{LLW}(1)$ & 0 & 0 & 0 & 0 & 0 & 0 \\
\hline LLMW (s) & 5.2 & 0.7 & 0 & 0 & 0.7 & 0 \\
\hline LLMW (1) & 0 & 150 & 0 & 0 & 150 & 0 \\
\hline $\begin{array}{l}\text { LLW } \\
\text { (GTC3) }\end{array}$ & 0 & 0 & 0 & 0 & 0 & 0 \\
\hline TRU & 0 & 0 & 0 & 0 & 0 & 0 \\
\hline TRUM & 0 & 0 & 0 & 0 & 0 & 0 \\
\hline$H A Z$ & 0 & 1.4 & 0 & 0 & 1.4 & 0 \\
\hline Landfill & 0 & 950 & 0 & 0 & 950 & 0 \\
\hline Asbestos & 0 & 4.25 & 0 & 0 & 4.25 & 0 \\
\hline $\begin{array}{l}\text { San. } \\
\text { (Liq) }\end{array}$ & 0 & 26,500 & 0 & 0 & 27,000 & 0 \\
\hline
\end{tabular}

Note: Volumetric units for values entered in the table are cubic meters, except for spent fuel and spent fuel by-products where the value are reported in metric tons of heavy metal. 
WHC-SP-1126, Rev. 1

\section{TRANSITION PROJECTS}

FY 1996 MYPP

1.3.1/7.1/6.12

2.A.6. Waste, Type Data

To End of Life Cycle - Waste Type/Spent Fuel Volume Projections for WBS:1.3.1.7

Program Name: B Plant/WESF Transition Projects

\begin{tabular}{|c|c|c|c|c|c|c|}
\hline \multicolumn{4}{|c|}{ Period Start Date:10/1/2000 } & \multicolumn{3}{|c|}{ Period End Date: 2025} \\
\hline $\begin{array}{l}\text { Waste } \\
\text { Type }\end{array}$ & $\begin{array}{l}\text { Beginning } \\
\text { Inventory }\end{array}$ & $\begin{array}{l}\text { Haste } \\
\text { Generated }\end{array}$ & $\begin{array}{l}\text { Waste } \\
\text { Received }\end{array}$ & $\begin{array}{l}\text { Waste } \\
\text { Reduction }\end{array}$ & $\begin{array}{l}\text { Haste } \\
\text { Transferred }\end{array}$ & $\begin{array}{l}\text { Waste } \\
\text { Stored }\end{array}$ \\
\hline HLH & 0 & 0 & 0 & 0 & 0 & 0 \\
\hline LLW (s) & 0 & 1560 & 0 & 0 & 1560 & 0 \\
\hline LLW (1) & 0 & 0 & 0 & 0 & 0 & 0 \\
\hline LLMW (s) & 5.2 & 17.5 & 0 & 0 & 22.7 & 0 \\
\hline LLMH (1) & 0 & 3750 & 0 & 0 & 3750 & 0 \\
\hline $\begin{array}{l}\text { LLK } \\
\text { (GTC3) }\end{array}$ & 0 & 0 & 0 & 0 & 0 & 0 \\
\hline TRU & 0 & 0 & 0 & 0 & 0 & 0 \\
\hline TRUM & 0 & 0 & 0 & 0 & 0 & 0 \\
\hline HAZ & 0 & 35 & 0 & 0 & 35 & 0 \\
\hline Landfill & 0 & 2850 & 0 & 0 & 2850 & 0 \\
\hline Asbestos & 0 & 51 & 0 & 0 & 51 & 0 \\
\hline $\begin{array}{l}\text { San. } \\
\text { (Liq) }\end{array}$ & 0 & 636,000 & 0 & 0 & 636,000 & 0 \\
\hline
\end{tabular}

Note: Volumetric units for values entered in the table are cubic meters, except for spent fuel and spent fuel by-products where the value are reported in metric tons of heavy metal. 
WHC-SP-1126, Rev. 1

\section{TRANSITION PROJECTS}

FY 1996 MYPP

1.3.1/7.1/6.12

2.A.6 Waste Type Data

FY 1996 Waste/SNM Type Volume Projections for WBS: 7.1.1

Program Name: PUREX

\begin{tabular}{|c|c|c|c|c|c|c|}
\hline \multicolumn{4}{|c|}{ Period Start Date: $10 / 1 / 95$} & \multicolumn{3}{|c|}{ Period End Date: $9 / 30 / 96$} \\
\hline $\begin{array}{l}\text { Waste/ } \\
\text { SNM } \\
\text { Type }\end{array}$ & $\begin{array}{l}\text { Beginning } \\
\text { Inventory }\end{array}$ & $\begin{array}{l}\text { Waste/ } \\
\text { SNM } \\
\text { Generated }\end{array}$ & $\begin{array}{l}\text { Haste/ SNH } \\
\text { Received }\end{array}$ & $\begin{array}{l}\text { Haste/ SNM } \\
\text { Reduction }\end{array}$ & $\begin{array}{l}\text { Waste/ SNM } \\
\text { Transferred }\end{array}$ & $\begin{array}{l}\text { Waste/ SNM } \\
\text { Stored/ } \\
\text { Dispositioned }\end{array}$ \\
\hline HLW & 500 & 500 & & & 1,000 & \\
\hline $\operatorname{LLH}(\mathrm{s})$ & & 195 & & & 195 & \\
\hline LLW (1) & & & & & & \\
\hline LLMH (s) & & 12.2 & & & 12.2 & \\
\hline LLMW (1) & & & & & & \\
\hline LLW (GTC3) & & & & & & \\
\hline SNM & & & & & & \\
\hline TRU & & 9.62 & & & 9.62 & \\
\hline TRUM $\cdot$ & & 2.09 & & & 2.09 & \\
\hline$H A Z$ & & 4.81 & & & 4.81 & \\
\hline Landfill & & 953 & & & 953 & \\
\hline Asbestos & & 8 & & & 8 & \\
\hline San. (Liq) & & 500,000 & & & 500,000 & \\
\hline
\end{tabular}

Note: Volumetric units for values entered in the table shall be cubic meters, except for SNM where the value shall be reported in metric tons of heavy metal. If the units are not specified, the units for the value shall be assumed to be cubic meters. 
WHC-SP-1126, Rev. 1

\section{TRANSITION PROJECTS}

FY 1996 MYPP

1.3.1/7.1/6.12

2.A.6 Waste Type Data

FY 1997 Waste/SNM Type Volume Projections for WBS: 7.1.1

Program Name: PUREX

\begin{tabular}{|c|c|c|c|c|c|c|}
\hline \multicolumn{4}{|c|}{ Period Start Date:10/1/96 } & \multicolumn{3}{|c|}{ Period End Date: $9 / 30 / 97$} \\
\hline $\begin{array}{l}\text { Waste/ } \\
\text { SNM } \\
\text { Type }\end{array}$ & $\begin{array}{l}\text { Beginning } \\
\text { Inventory }\end{array}$ & $\begin{array}{l}\text { Waste/ } \\
\text { SNM } \\
\text { Generated }\end{array}$ & $\begin{array}{l}\text { Haste/ SHM } \\
\text { Received }\end{array}$ & $\begin{array}{l}\text { Haste/ SNM } \\
\text { Reduction }\end{array}$ & $\begin{array}{l}\text { Waste/ SNM } \\
\text { Transferred }\end{array}$ & $\begin{array}{l}\text { Waste/ SNM } \\
\text { Stored/ } \\
\text { Dispositioned }\end{array}$ \\
\hline HLW & & & & & & \\
\hline LLH (s) & & 393 & & & 393 & \\
\hline LLW (1) & & & & & & \\
\hline LLMW (s) & & 12 & & & 12 & \\
\hline LLMW (1) & & & & & & \\
\hline LLH (GTC3) & & . & & & & \\
\hline SNM & & & & & & \\
\hline TRU & & & & & & \\
\hline TRUM & & & & & & \\
\hline $\mathrm{HAZ}$ & & 4.25 & & & 4.25 & \\
\hline Landfill & & 953 & & & 953 & \\
\hline Asbestos & & 8 & & & 8 & \\
\hline San. (Liq) & & 500,000 & & & 500,000 & \\
\hline
\end{tabular}

Note: Volumetric units for values entered in the table shall be cubic meters, except for SNM where the value shall be reported in metric tons of heavy metal. If the units are not specified, the units for the value shall be assumed to be cubic meters. 
WHC-SP-1126, Rev. I

\section{TRANSITION PROJECTS}

\section{FY 1996 MYPP \\ 1.3.1/7.1/6.12}

2. A. Waste Type Data

FY 1998 Waste/SNH Type Volume Projections for WBS: 7.1.1

Program Name: PUREX

\begin{tabular}{|c|c|c|c|c|c|c|}
\hline \multicolumn{4}{|c|}{ Period Start Date:10/1/97 } & \multicolumn{3}{|c|}{ Period End Date: 9/30/98 } \\
\hline $\begin{array}{l}\text { Waste/ } \\
\text { SHM } \\
\text { Type }\end{array}$ & $\begin{array}{l}\text { Beginning } \\
\text { Inventory }\end{array}$ & $\begin{array}{l}\text { Haste/ } \\
\text { SNH } \\
\text { Generated }\end{array}$ & $\begin{array}{l}\text { Waste/ SNM } \\
\text { Received }\end{array}$ & $\begin{array}{l}\text { Haste/ SNM } \\
\text { Reduction }\end{array}$ & $\begin{array}{l}\text { Haste/ SNM } \\
\text { Transferred }\end{array}$ & $\begin{array}{l}\text { Waste/ SNM } \\
\text { Stored/ } \\
\text { Dispositioned }\end{array}$ \\
\hline$H L W$ & & & & & & \\
\hline $\operatorname{LLH}(\mathrm{s})$ & & & & & & \\
\hline LLH (1) & & & & & & \\
\hline LLMW (s) & & & & & & \\
\hline -LMW (1) & & & & & & \\
\hline LLW (GTC3) & & & & & & \\
\hline SNM & & & & & & \\
\hline TRU & & & & & & · \\
\hline TRUM & & & & & & \\
\hline HAZ & & & & & & \\
\hline Landfil & & & & & & \\
\hline Asbestos & & & & & & \\
\hline San. (Liq) & & & & & . & \\
\hline
\end{tabular}

Note: Volumetric units for values entered in the table shall be cubic meters, except for SNM where the value shall

be reported in metric tons of heavy metal. If the units are not specified, the units for the value shall be assumed

to be cubic meters. 
WHC-SP-1126, Rev. 1

TRANSITION PROJECTS

FY 1996 MYPP 1.3.1/7.1/6.12

2.A.6. Waste Type Data

\section{SUPPORTING DATA \& ASSUMPTIONS \\ PROGRAM: PUREX Transition \\ WBS: 7.1 .1}

\section{Assumptions: Waste Type, Quantities and Schedule}

HLW forecast is based on the current canyon liquid inventory plus project flush volumes.

Solid waste projections are based on the Interface Control Document (ICD) between PUREX $/ \mathrm{UO}_{3}$ Plant Transition and Solid Waste Disposal Division (WHC-SD-WM-PICD-002) updated with internal memo 17730-95-022 dated June 28, 1995, from JR Hilliard to KL H ladek, "PUREX SOLLID WASTE FORECAST".

San. (Liq) volume is based on projected PUREX Chemical Sewer (CSL) flow rates. 
WHC-SP-1126, Rev. 1

TRANSITION PROJECTS

FY 1996 MYPP

1.3.1/7.1/6.12

2. A. Waste Type Data

FY 1996 Waste Type/Spent Fuel Volume Projections for WBS: IK3

Program Name: 300 Fuel Supply Shutdown

\begin{tabular}{|c|c|c|c|c|c|c|}
\hline \multicolumn{4}{|c|}{ Period Start Date:10/1/95 } & \multicolumn{3}{|c|}{ Period End Date: 9/30/96 } \\
\hline $\begin{array}{l}\text { Waste } \\
\text { Type }\end{array}$ & $\begin{array}{l}\text { Beginning } \\
\text { Inventory }\end{array}$ & $\begin{array}{l}\text { Waste } \\
\text { Generated }\end{array}$ & $\begin{array}{l}\text { Haste } \\
\text { Received }\end{array}$ & $\begin{array}{l}\text { Waste } \\
\text { Reduction }\end{array}$ & $\begin{array}{l}\text { Haste } \\
\text { Transferred }\end{array}$ & $\begin{array}{l}\text { Haste } \\
\text { Stored }\end{array}$ \\
\hline HLW & & & & & & \\
\hline $\operatorname{LLW}(s)$ & & $159.6 \mathrm{~m}^{3}$ & & & $159.6 \mathrm{~m}^{3}$ & \\
\hline LLW (1) & - & & & & & \\
\hline LLMW (s) & & $8.0 \mathrm{~m}^{3}$ & & & $8.0 \mathrm{~m}^{3}$ & \\
\hline LLMH (1) & & & & & & \\
\hline LLW (GTC3 & & & & & & \\
\hline TRU & & & & & & \\
\hline TRUM & & & & & & \\
\hline HAZ & & $1.0 \mathrm{~m}^{3}$ & & & $1.0 \mathrm{~m}^{3}$ & \\
\hline Landfi 11 & & & & & & \\
\hline Asbestos & & & & & & \\
\hline San. (Liq & & & & & & \\
\hline
\end{tabular}


WHC-SP-1126, Rev. 1

TRANSITION PROJECTS

FY 1996 MYPP

1.3.1/7.1/6.12

2. A.6 Waste Type Data

FY 1997 Waste Type/Spent Fuel Volume Projections for WBS: 1K3

Program Name: 300 Area Fuel Supply Shutdown

\begin{tabular}{|c|c|c|c|c|c|c|}
\hline \multicolumn{4}{|c|}{ Period Start Date:10/1/96 } & \multicolumn{3}{|c|}{ Period End Date: $9 / 30 / 97$} \\
\hline $\begin{array}{l}\text { Waste } \\
\text { Type }\end{array}$ & $\begin{array}{l}\text { Beginning } \\
\text { Inventory }\end{array}$ & $\begin{array}{l}\text { Haste } \\
\text { Generated }\end{array}$ & $\begin{array}{l}\text { Waste } \\
\text { Received }\end{array}$ & $\begin{array}{l}\text { Haste } \\
\text { Reduction }\end{array}$ & $\begin{array}{l}\text { Haste } \\
\text { Transferred }\end{array}$ & $\begin{array}{l}\text { Waste } \\
\text { Stored }\end{array}$ \\
\hline \multicolumn{7}{|l|}{ HLW } \\
\hline LLH (s) & & $56.0 \mathrm{~m}^{3}$ & & & $56.0 \mathrm{~m}^{3}$ & \\
\hline \multicolumn{7}{|l|}{ LLW (1) } \\
\hline LLMW (s) & & $.4 \mathrm{~m}^{3}$ & & & $.4 \mathrm{~m}^{3}$ & \\
\hline \multicolumn{7}{|l|}{ LLMW (1) } \\
\hline \multicolumn{7}{|l|}{ LLW (GTC3) } \\
\hline \multicolumn{7}{|l|}{ TRU } \\
\hline \multicolumn{7}{|l|}{ TRUM } \\
\hline HAZ & & $.6 \mathrm{~m}^{3}$ & & & $.6 \mathrm{~m}^{3}$ & \\
\hline \multicolumn{7}{|l|}{ Landfil1 } \\
\hline \multicolumn{7}{|l|}{ Asbestos } \\
\hline San. (Liq) & & & & & & \\
\hline
\end{tabular}


WHC-SP-1126, Rev. 1

\section{TRANSITION PROJECTS}

FY 1996 MYPP 1.3.1/7.1/6.12 .

2.A.6 Waste Type Data

FY 1998 Waste Type/Spent Fuel Volume Projections for WBS: IK3

Program Name: 300 Area Fuel Supply Shutdown

\begin{tabular}{|c|c|c|c|c|c|c|}
\hline \multicolumn{4}{|c|}{ Period Start Date:10/1/97 } & \multicolumn{3}{|c|}{ Period End Date: 9/30/98 } \\
\hline $\begin{array}{l}\text { Waste } \\
\text { Type }\end{array}$ & $\begin{array}{l}\text { Beginning } \\
\text { Inventory }\end{array}$ & $\begin{array}{l}\text { Waste } \\
\text { Generated }\end{array}$ & $\begin{array}{l}\text { Haste } \\
\text { Received }\end{array}$ & $\begin{array}{l}\text { Waste } \\
\text { Reduction }\end{array}$ & $\begin{array}{l}\text { Waste } \\
\text { Transferred }\end{array}$ & $\begin{array}{l}\text { Waste } \\
\text { Stored }\end{array}$ \\
\hline \multicolumn{7}{|l|}{ HLW } \\
\hline $\operatorname{LLW}(s)$ & & $33.2 \mathrm{~m}^{3}$ & & & $33.2 \mathrm{~m}^{3}$ & \\
\hline \multicolumn{7}{|l|}{ LLH (1) } \\
\hline LLMW" (s) & & $10.0 \mathrm{~m}^{3}$ & & & $10.0 \mathrm{~m}^{3}$ & \\
\hline \multicolumn{7}{|l|}{ LLMH (1) } \\
\hline \multicolumn{7}{|l|}{ LLH (GTC3) } \\
\hline \multicolumn{7}{|l|}{ TRU } \\
\hline \multicolumn{7}{|l|}{ TRUH } \\
\hline \multicolumn{7}{|l|}{ HAZ } \\
\hline Landfil1 & & $22.39 \mathrm{~m}^{3}$ & & & $22.39 \mathrm{~m}^{3}$ & \\
\hline \multicolumn{7}{|l|}{ Asbestos } \\
\hline San. (Liq) & & & & & & \\
\hline
\end{tabular}


WHC-SP-1126, Rev. 1

\section{TRANSITION PROJECTS}

FY 1996 MYPP

1.3.1/7.1/6.12

2. A.6. Waste Type Data

SUPPORTING DATA \& ASSUMPTIONS

PROGRAM: 300 FUEL SUPPLY SHUTDOWN

WBS: IK3

Date Prepared: August 18, 1995

Preparer:J. M. Bishop

1. Assumptions: Waste Type, Quantities and Schedule

The Waste Type Data Projections assume deactivation scheduled for 3-31-99.

Low Level Waste:

FY 1996: 38 (each) $4^{\prime} \times 4^{\prime} \times 8^{\prime}$ plywood burial boxes -38 (ea.) 55 gallon drums generated from the clean-up of the $313,303 \mathrm{M}$ and the 333 building.

Fy 1997: 14 (each) $4^{\prime} \times 4^{\prime} \times 8^{\prime}$ plywood burial boxes generated from the clean-up of the piping int the $303 \mathrm{~F}$ building and misc contaminated items in the 3135 building.

Fy 1998: 6 (each) 55 gallon drums and 8 (ea.) 4'x4'x8' plywood burial boxes generated from the clean-up of decontaminated piping and miscellaneous waste from the 333 building.

MIXED WASTE:

FY 1996: 20 (each) 55 gallon drums and 1 (ea.) 4'x4'x8' plywood burial box from clean-up $303 \mathrm{M}$ and 313 sludge recovery area (RCRA).

FY 1997: 2 (each) 55 gallon drums generated from the decontamination of the $303 F$, 333 , and the $334 \mathrm{~A}$ buildings.

FY 1998: 30 (each) 55 gallon drums and 1 (ea.) $4^{\prime} \times 4^{\prime} \times 8^{\prime}$ plywood burial box. generated from the clean-up of piping from the 333 building and tank 40 and 50 decontamination.

HAZARDOUS WASTE:

FY 1996: 5 (each) 55 gallon drums from the 333 building.

FY 1997: 3 (each) 55 gallon drums generated from the clean-up of the 334 building.

LANDFILL:

FY 1998: $22.39 \mathrm{~m}^{3}$ of trench cover concrete blocks.

A11 waste/spent fuel assumptions generated from WHC-SD-FL-SSP-002, "Shutdown P1an for the 300 Area Fuel Supply Facilities." 
WHC-SP-1126, Rev. 1

TRANSITION PROJECTS

FY 1996 MYPP

1.3.1/7.1/6.12

2.A.6 Haste Type Data

FY 1996 Waste/Material Type Volume Projections for WBS: 7.1 .3

Program Name: Plutonium Finishing Plant

Period Start Date: 10/1/95

\begin{tabular}{|c|c|c|c|c|c|c|}
\hline $\begin{array}{l}\text { Waste/ } \\
\text { Material } \\
\text { Type }\end{array}$ & $\begin{array}{l}\text { Beginning } \\
\text { Inventory }\end{array}$ & $\begin{array}{l}\text { Haste/ } \\
\text { Material } \\
\text { Generated }\end{array}$ & $\begin{array}{l}\text { Hastef } \\
\text { Material } \\
\text { Received }\end{array}$ & $\begin{array}{l}\text { Waste/ } \\
\text { Material } \\
\text { Reduction }\end{array}$ & $\begin{array}{l}\text { Waste/ Material } \\
\text { Transferred }\end{array}$ & $\begin{array}{l}\text { Waste/ Material } \\
\text { Stored/ } \\
\text { Dispositioned }\end{array}$ \\
\hline HLW & 0 & 0 & 0 & 0 & 0 & 0 \\
\hline LLH (s) & 0 & 962 & 0 & 586 & 376 & 0 \\
\hline LLW (T) & 0 & 19,800 & 0 & 19,800 & 0 & 0 \\
\hline LLMW (s) & 0 & 129 & 0 & 0 & 129 & 0 \\
\hline .LMW (1) & 0 & 0 & 0 & 0 & 0 & 0 \\
\hline $\begin{array}{l}\text { LLW } \\
\text { (GTC3) }\end{array}$ & 0 & 0 & 0 & 0 & 0 & 0 \\
\hline Material & NA & NA & NA & NA & NA & NA \\
\hline TRU & 0 & 126 & 0 & 0 & 126 & 0 \\
\hline TRUM & 8.44 & 43.2 & 0 & 0 & 43.2 & 8.44 \\
\hline HAZ & 0 & 44.9 & 0 & 0 & 44.9 & 0 \\
\hline Landfill & 0 & 1826 & 0 & 0 & 1826 & 0 \\
\hline Asbestos & 0 & 16.6 & 0 & 0 & 16.6 & 0 \\
\hline $\begin{array}{l}\text { San. } \\
\text { (Liq) }\end{array}$ & 0 & 24,400 & 19,800 & 0 & 44,200 & 0 \\
\hline
\end{tabular}

Note: Volumetric units for values entered in the table shall be cubic meters, except for Material where the value shall be reported in metric tons of heavy metal. If the units are not specified, the units for the value shall be assumed to be cubic meters. 
WHC-SP-1126, Rev. 1

\section{TRANSITION PROJECTS}

FY 1996 MYPP

\subsection{1/7.1/6.12}

2. A.6 Waste.Type Data

FY 1997 Waste/Material Type Volume Projections for WBS: 7.1.3

Program Name: Plutonium Finishing Plant

\begin{tabular}{|c|c|c|c|c|c|c|}
\hline \multicolumn{4}{|c|}{ Period Start Date:10/1/96 } & \multicolumn{3}{|c|}{ Period End Date: 9/30/97 } \\
\hline $\begin{array}{l}\text { Waste/ } \\
\text { Material } \\
\text { Type }\end{array}$ & $\begin{array}{l}\text { Beginning } \\
\text { Inventory }\end{array}$ & $\begin{array}{l}\text { Haste/ } \\
\text { Material } \\
\text { Generated }\end{array}$ & $\begin{array}{l}\text { Haste/ } \\
\text { Material } \\
\text { Received }\end{array}$ & $\begin{array}{l}\text { Haste/ } \\
\text { Material } \\
\text { Reduction }\end{array}$ & $\begin{array}{l}\text { Waste/ Material } \\
\text { Transferred } \\
\end{array}$ & $\begin{array}{l}\text { Waste/ Material } \\
\text { Stored/ } \\
\text { Dispositioned }\end{array}$ \\
\hline HLW & 0 & 0 & 0 & 0 & 0 & 0 \\
\hline LLH (s) & 0 & 1030 & 0 & 612 & 418 & 0 \\
\hline LLW (1) & 0 & 19,800 & 0 & 19,800 & 0 & 0 \\
\hline LLMH (s) & 0 & 131 & 0 & 0 & 131 & 0 \\
\hline LLMH (1) & 0 & 0 & 0 & 0 & 0 & 0 \\
\hline LLW (GTC3) & 0 & 0 & 0 & 0 & 0 & 0 \\
\hline Material & NA & NA & NA & NA & NA & NA \\
\hline TRU & 0 & 272 & 0 & 0 & 272 & 0 \\
\hline TRUM & 8.44 & 63.5 & 0 & 0 & 63.5 & 8.44 \\
\hline HAZ & 0 & 44.9 & 0 & 0 & 44.9 & 0 \\
\hline Landfill & 0 & 1826 & 0 & 0 & 1826 & 0 \\
\hline Asbestos & 0 & 16.6 & 0 & 0 & 16.6 & 0 \\
\hline San. (Liq) & 0 & 24,400 & 19,800 & 0 & 44,200 & 0 \\
\hline
\end{tabular}

Note: Volumetric units for values entered in the table shall be cubic meters, except for Material where the value shall be reported in metric tons of heavy metal. If the units are not specified, the units for the value shall be assumed to be cubic meters. 
WHC-SP-1126, Rev. 1

\section{TRANSITION PROJECTS}

FY 1996 MYPP

1.3.1/7.1/6.12

2. A 6 . Waste Type Data

FY 1998 Waste/Material Type Volume Projections for WBS: 7.1.3

\section{Program Name: Plutonium Finishing Plant}

\begin{tabular}{|c|c|c|c|c|c|c|}
\hline \multicolumn{4}{|c|}{ Period Start Date:10/1/97 } & \multicolumn{3}{|c|}{ Period End Date: 9/30/98 } \\
\hline $\begin{array}{l}\text { Waste/ } \\
\text { Material } \\
\text { Type }\end{array}$ & $\begin{array}{l}\text { Beginning } \\
\text { Inventory }\end{array}$ & $\begin{array}{l}\text { Waste/ } \\
\text { Material } \\
\text { Generated }\end{array}$ & $\begin{array}{l}\text { Haste/ } \\
\text { Material } \\
\text { Received }\end{array}$ & $\begin{array}{l}\text { Waste/ } \\
\text { Material } \\
\text { Reduction }\end{array}$ & $\begin{array}{l}\text { Haste/ Material } \\
\text { Transferred }\end{array}$ & $\begin{array}{l}\text { Haste/ Material } \\
\text { Stored/ } \\
\text { Dispositioned }\end{array}$ \\
\hline HLW & 0 & 0 & 0 & 0 & 0 & 0 \\
\hline$L L W(s)$ & 0 & 1138 & 0 & 670 & 468 & 0 \\
\hline LLW (1) & 0 & 19,800 & 0 & 19,800 & 0 & 0 \\
\hline LLMW (s) & 0 & 129 & 0 & 0 & 129 & 0 \\
\hline LLMH (1) & 0 & 0 & 0 & 0 & 0 & 0 \\
\hline $\begin{array}{l}\text { LLW } \\
\text { (GTC3) }\end{array}$ & 0 & 0 & 0 & 0 & 0 & 0 \\
\hline Material & NA & $N A$ & NA & NA & NA & NA \\
\hline TRU & 0 & 383 & 0 & 0 & 383 & 0 \\
\hline TRUM & 8.44 & 63.5 & 0 & 0 & 63.5 & 8.44 \\
\hline HAZ & 0 & 44.9 & 0 & 0 & 44.9 & 0 \\
\hline Landfill & 0 & 1826 & 0 & 0 & 1826 & 0 \\
\hline Asbestos & 0 & 16.6 & 0 & 0 & 16.6 & 0 \\
\hline $\begin{array}{l}\text { San. } \\
\text { (Liq) }\end{array}$ & 0 & 24,400 & 19,800 & 0 & 44,200 & 0 \\
\hline
\end{tabular}

Note: Volumetric units for values entered in the table shall be cubic meters, except for Material where the value shall be reported in metric tons of heavy metal. If the units are not specified, the units for the value shall be assumed to be cubic meters. 
WHC-SP-1126, Rev. I

TRANSITION PROJECTS

FY 1996 MYPP

1.3.1/7.1/6.12

2. A.6. Waste Type Data

FY 1999 Waste/Material Type Volume Projections for WBS: 7.1 .3

Program Name: Plutonium Finishing Plant

\begin{tabular}{|c|c|c|c|c|c|c|}
\hline \multicolumn{4}{|c|}{ Period Start Date:10/1/98 } & \multicolumn{3}{|c|}{ Period End Date: 9/30/99 } \\
\hline $\begin{array}{l}\text { Haste/ } \\
\text { Material } \\
\text { Type }\end{array}$ & $\begin{array}{l}\text { Beginning } \\
\text { Inventory }\end{array}$ & $\begin{array}{l}\text { Waste/ } \\
\text { Material } \\
\text { Generated }\end{array}$ & $\begin{array}{l}\text { Haste/ } \\
\text { Material } \\
\text { Received }\end{array}$ & $\begin{array}{l}\text { Waste/ } \\
\text { Material } \\
\text { Reduction }\end{array}$ & $\begin{array}{l}\text { Waste/ Material } \\
\text { Transferred }\end{array}$ & $\begin{array}{l}\text { Waste/ Material } \\
\text { Stored/ } \\
\text { Dispositioned }\end{array}$ \\
\hline$H L W$ & 0 & 0 & 0 & 0 & 0 & 0 \\
\hline LLW (s) & 0 & 1163 & 0 & 692 & 471 & 0 \\
\hline $\operatorname{LLW}(1)$ & 0 & 19,800 & 0 & 19,800 & 0 & 0 \\
\hline LLMW (s) & 0 & 141 & 0 & 0 & 141 & 0 \\
\hline LLMW (1) & 0 & 0 & 0 & 0 & 0 & 0 \\
\hline $\begin{array}{l}\text { LLH } \\
\text { (GTC3) }\end{array}$ & 0 & 0 & 0 & 0 & 0 & 0 \\
\hline Material & NA & $N A$ & NA & $N A$ & NA & $N A$ \\
\hline TRU & 0 & 334 & 0 & 0 & 334 & 0 \\
\hline TRUM & 8.44 & 51.0 & 0 & 0 & 51.0 & 8.44 \\
\hline HAZ & 0 & 44.9 & 0 & 0 & 44.9 & 0 \\
\hline Landfill. & 0 & 1826 & 0 & 0 & 1826 & 0 \\
\hline Asbestos & 0 & 16.6 & 0 & 0 & 16.6 & 0 \\
\hline San. & 0 & 24,400 & 19,800 & 0 & 44,200 & 0 \\
\hline
\end{tabular}

Note: Volumetric units for values entered in the table shall be cubic meters, except for Material where the value shall be reported in metric tons of heavy metal. If the units are not specified, the units for the value shall be assumed to be cubic meters. 
WHC-SP-1126, Rev. 1

TRANSITION PROJECTS

FY 1996 MYPP

1.3.1/7.1/6.12

2. A.6. Waste Type Data

FY 2000 Waste/Material Type Volume Projections for WBS: 7.1.3

Program Name: Plutonium Finishing Plant

\begin{tabular}{|c|c|c|c|c|c|c|}
\hline \multicolumn{4}{|c|}{ Period Start Date:10/1/99 } & \multicolumn{3}{|c|}{ Period-End Date: $9 / 30 / 2000$} \\
\hline $\begin{array}{l}\text { Waste/ } \\
\text { Material } \\
\text { Type }\end{array}$ & $\begin{array}{l}\text { Beginning } \\
\text { Inventory }\end{array}$ & $\begin{array}{l}\text { Waste/ } \\
\text { Material } \\
\text { Generated }\end{array}$ & $\begin{array}{l}\text { Waste/ } \\
\text { Material } \\
\text { Received }\end{array}$ & $\begin{array}{l}\text { Waste/ } \\
\text { Material } \\
\text { Reduction }\end{array}$ & $\begin{array}{l}\text { Waste/ Material } \\
\text { Transferred }\end{array}$ & $\begin{array}{l}\text { Waste/ Material } \\
\text { Stored/ } \\
\text { Dispositioned }\end{array}$ \\
\hline HLW & 0 & 0 & 0 & 0 & 0 & 0 \\
\hline LLH (s) & 0 & 1184 & 0 & 744 & 440 & 0 \\
\hline $\operatorname{LLW}(1)$ & 0 & 19,800 & 0 & 19,800 & 0 & 0 \\
\hline LLMW (s) & 0 & 129 & 0 & 0 & 129 & 0 \\
\hline LMW (1) & 0 & 0 & 0 & 0 & 0 & 0 \\
\hline $\begin{array}{l}\text { LLW } \\
\text { (GTC3) }\end{array}$ & 0 & 0 & 0 & 0 & 0 & 0 \\
\hline Material & NA & NA & NA & $N A$ & NA & NA \\
\hline TRU & 0 & 294 & 0 & 0 & 294 & 0 \\
\hline TRUM & 8.44 & 48.4 & 0 & 0 & 48.4 & 8.44 \\
\hline$H A Z$ & 0 & 44.9 & 0 & 0 & 44.9 & 0 \\
\hline Landfill & 0 & 1826 & 0 & 0 & 1826 & 0 \\
\hline Asbestos & 0 & 16.6 & 0 & 0 & 16.6 & 0 \\
\hline $\begin{array}{l}\text { San. } \\
\text { (Liq) }\end{array}$ & 0 & 24,400 & 19,800 & 0 & 44,200 & 0 \\
\hline
\end{tabular}

Note: Volumetric units for values entered in the table shall be cubic meters, except for Material where the value shall be reported in metric tons of heavy metal. If the units are not specified, the units for the value shall be assumed to be cubic meters. 
WHC-SP-1126, Rev. 1

\section{TRANSITION PROJECTS}
FY 1996 MYPP
1.3.1/7.1/6.12

2.6. Haste Type Data

FY 2000 To End Of Facility/Activity Life Cycle Waste/Material Type Volume Projections for WBS: 7.1 .3

Program Name: Plutonium Finishing Plant

Period Start Date: $10 / 1 / 2000$

Period End Date: End of Facility Activ Life Cycle

\begin{tabular}{|c|c|c|c|c|c|c|}
\hline $\begin{array}{l}\text { Hastel } \\
\text { Material } \\
\text { Type }\end{array}$ & $\begin{array}{l}\text { Beginning } \\
\text { Inventory }\end{array}$ & $\begin{array}{l}\text { Waste/ } \\
\text { Material } \\
\text { Generated }\end{array}$ & $\begin{array}{l}\text { Haste/ } \\
\text { Material } \\
\text { Received }\end{array}$ & $\begin{array}{l}\text { Waste/ } \\
\text { Material } \\
\text { Reduction }\end{array}$ & $\begin{array}{l}\text { Waste/ Material } \\
\text { Transferred }\end{array}$ & $\begin{array}{l}\text { Waste/ Material } \\
\text { Stored/ } \\
\text { Dispositioned }\end{array}$ \\
\hline HLW & 0 & 0 & 0 & 0 & 0 & 0 \\
\hline LLW (s) & 0 & & 0 & & & 0 \\
\hline LLW (1) & 0 & & 0 & & & 0 \\
\hline LLMW (s) & 0 & & 0 & & & 0 \\
\hline LLMW (1) & 0 & & 0 & & & 0 \\
\hline $\begin{array}{l}\text { LLH } \\
\text { (GTC3) }\end{array}$ & 0 & & 0 & & & 0 \\
\hline Material & $N A$ & NA & NA & $N A$ & $N A$ & NA \\
\hline TRU & 0 & & 0 & & & 0 \\
\hline TRUM & 8.44 & & 0 & & & \\
\hline $\mathrm{HAZ}$ & 0 & & 0 & & & 0 \\
\hline Landfill & 0 & . & 0 & & & 0 \\
\hline Asbestos & 0 & & 0 & & & 0 \\
\hline $\begin{array}{l}\text { San. } \\
\text { (Liq) }\end{array}$ & 0 & & 0 & & & 0 \\
\hline
\end{tabular}

Note: Volumetric units for values entered in the table shall be cubic meters, except for Material where the value shall be reported in metric tons of heavy metal. If the units are not specified, the units for the value shall be assumed to be cubic meters. 
WHC-SP-1126, Rev. 1

\section{TRANSITION PROJECTS}

FY 1996 MYPP

1.3.1/7.1/6.12

2.A.6. Wastettype Data

SUPPORTING DATA \& ASSUMPTIONS

PROGRAM: PIutonium Finishing Plant

WBS: 7.1 .3

1.Assumptions: Waste Type, Quantities and ScheduTe

Date Prepared: August 11, 1995

Preparer:E. G. Backlund

All types, FY 2000 to End Of Life Cycle- End of life cycle is undefined and therefore an endpoint does not exist to use for estimates. WHC-EP-0853 Volume 1 Draft, DNFSB Recommendation 94-1, Hanford Site Integrated Stabi7ization Management Plan, July 1995 states vault storage will be for 30 to 50 years. This range of numbers is used to indicate indefinitely. An average cubic meter/year value estimated on the stable value for the year 2025 is given except for TRUM which is projected to zero at 2008.

HLW, all years- HLW does not exist at PFP as Beta emitters were removed prior to feed being sent to Plutonium Finishing Plant.

LLW (s) - Waste Transferred is sum of LLW 1 and LLW III from Plutonium Finishing Plant 199530 Year Solid Waste Forecast. $1996 \quad 293+83=376$

$1997306+112=418$

$1998335+133=468$

$1999346+125=471$

LLW (s) - Waste Reduction is assumed volume reduced at compactor, assumption 3

generated/I shipped ratio of LLW I. Generated is; $19963(293)+83=962$

$\begin{array}{ll}1997 & 3(306)+112=1030 \\ 1998 & 3(335)+133=1138 \\ 1999 & 3(346)+125=1163\end{array}$

LLW (s)- Reduced is the volume compacted, the difference between generation and shipped.

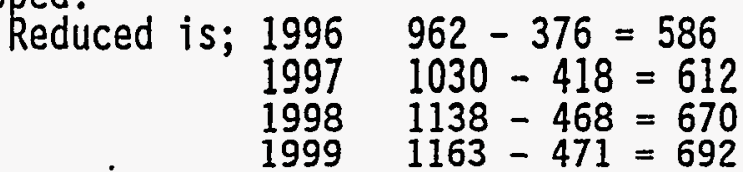

LLW (1)-Assumption is the volume for a year, referenced in letter 15530-95-GGB-044, from $G$. Bergquist to $M$. Gibson, for the $243-Z$ LLWTF wi7l be constant for the years of .this projection.

$5,229,453$ gailons $\times 1 \mathrm{~m}^{3} / 264.17=19,800 \mathrm{~m}^{3}$. The $L L W(1)$ is made nonradioactive (reduced) at $243-Z$ and then becomes part of the San. (Liq) stream.

San. (Liq)- Assumption is the volume transferred for the month of July 1995 will be constant for al7 months and years of this projection. Volume is from DSI of $8 / 4 / 95$, To K. Iwatate from D. R. Hirzel.

972250 gallons/month $\times 12$ months $\times 1 \mathrm{~m}^{3} / 264.17$ gallons $=44,200 \mathrm{~m}^{3}$.

The waste generated is the waste transferred less the waste received; $44,200-19,800=24,400 \mathrm{~m}^{3}$

LLMW (s) volumes are from the Plutonium Finishing Plant 199530 Year Solid Waste Forecast.

LLMW (?) is not generated, received, transferred, etc. at Plutonium Finishing Plant. 
WHC-SP-1126, Rev. 1

\section{TRANSITION PROJECTS}

FY 1996 MYPP

1.3.1/7.1/6.12

2. A. 6 Wast te Type Data

LLW (GTC3) does not exist for a TRU only facility. GTC3 is TRU.

Material has been deemed an inappropriate category by the programs office, and is therefore labeled NA.

TRU volumes are from the Plutonium Finishing Plant 199530 Year Solid Waste Forecast.

TRUM is a sum of solid waste volume from the Plutonium Finishing Plant 199530 Year Sol id Waste Forecast and liquid waste to Double Shell Tanks. The liquid volume is from Tetter 15530-95-GGB-003, G. Bergquist to J. Strode. Beginning inventory is an estimate of the volume that is in the $241-Z$ facility at this time. Assumption is that waste will be transferred as it accumulates, so that the same amount is in the tank on each Oct. 1st.

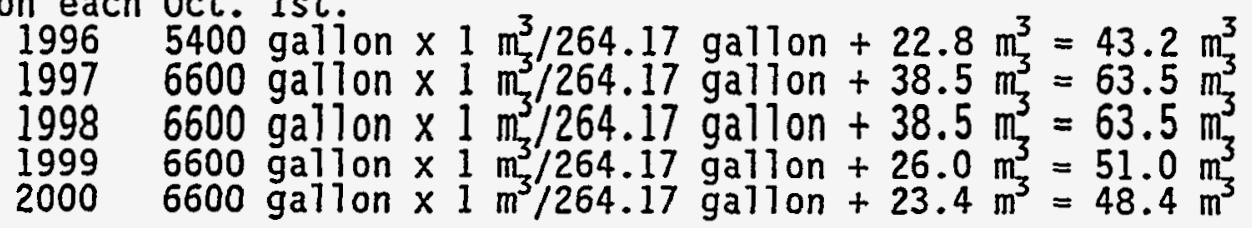

Beginning inventory;

42 weight factor $\times 201$ i iters/weight factor $\times 1 \mathrm{~m}^{3} / 1000$ i iters $=8.44 \mathrm{~m}^{3}$

HAZ volumes are from the Plutonium Finishing Plant 199530 Year Solid Waste Forecast.

Landfill is an estimate based on 7-8 $y d^{3}$ dumpsters and 1-4 yd $\mathrm{d}^{3}$ emptied once per week. Assumption is that each dumpster is $3 / 4$ ful7. Added to this is a small volume of waste shipped to Landfill by PFP Solid Waste Shippers. The volume by shippers was an average of 1991 through 1994 and a projected volume for 1995 .

Assumed is that these volumes will be the same through this projection.

$\begin{array}{cr}1991 & 99.5 \\ 1992 & 119 \\ 1993 & 0 \\ 1994 & 0 \\ 1995 \text { (proj) } & 26.2\end{array}$

$244.7 / 5=48,9 \mathrm{yd}^{3}$

$48.9 \mathrm{yd}^{3} \times 0.7646 \mathrm{~m}^{3} / \mathrm{yd}^{3}=37.4 \mathrm{~m}^{3}$

52 weeks $\left[\left[7(6)+1(3) \mathrm{yd}^{3}\right] \times 0.7646 \mathrm{~m}^{3} / \mathrm{yd}^{3}+37.4=1826 \mathrm{~m}^{3}\right.$.

Asbestos volume is assumed to be constant in the years of this projection and is an average of volumes shipped from 1988 through the present with the volume for 1995

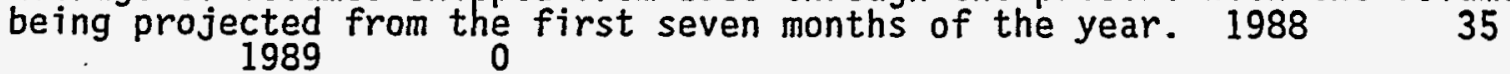

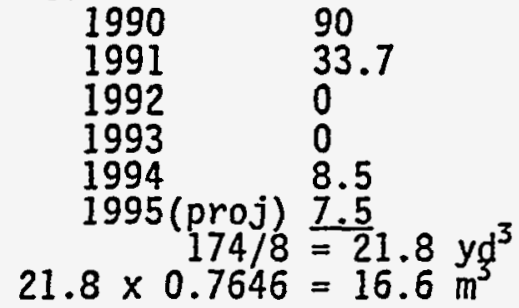


WHC-SP-1126, Rev. 1

\section{TRANSITION PROJECTS}

FY 1996 MYPP

\subsection{1/7.1/6.12}

2. 6 Waster Type Data

FY 1996 Haste/SNM Type Volume Projections for WBS: 7.1.6

Program Name: PROGRAM AND ENVIRONMENTAL MANAGEMENT

\begin{tabular}{|c|c|c|c|c|c|c|}
\hline \multicolumn{4}{|c|}{ Period Start Date:10/1/95 } & \multicolumn{3}{|c|}{ Period End Date: 9/30/96 } \\
\hline $\begin{array}{l}\text { Waste/ } \\
\text { SNM } \\
\text { Type }\end{array}$ & $\begin{array}{l}\text { Beginning } \\
\text { Inventory }\end{array}$ & $\begin{array}{l}\text { Waste/ } \\
\text { SNM } \\
\text { Generated }\end{array}$ & $\begin{array}{l}\text { Waste/ } \\
\text { SNM } \\
\text { Received }\end{array}$ & $\begin{array}{l}\text { Haste/ } \\
\text { SNM } \\
\text { Reduction }\end{array}$ & $\begin{array}{l}\text { Waste/ SNM } \\
\text { Transferred }\end{array}$ & $\begin{array}{l}\text { Waste/ SNM } \\
\text { Stored/ } \\
\text { Dispositione }\end{array}$ \\
\hline
\end{tabular}

Program and Environmental Management has no Waste Type Data to compile. 


\section{TRANSITION PROJECTS}

\section{FY 1996 MYPP}

\subsection{1/7.1/6.12}

\section{B.1 Program Master Baseline Schedule}

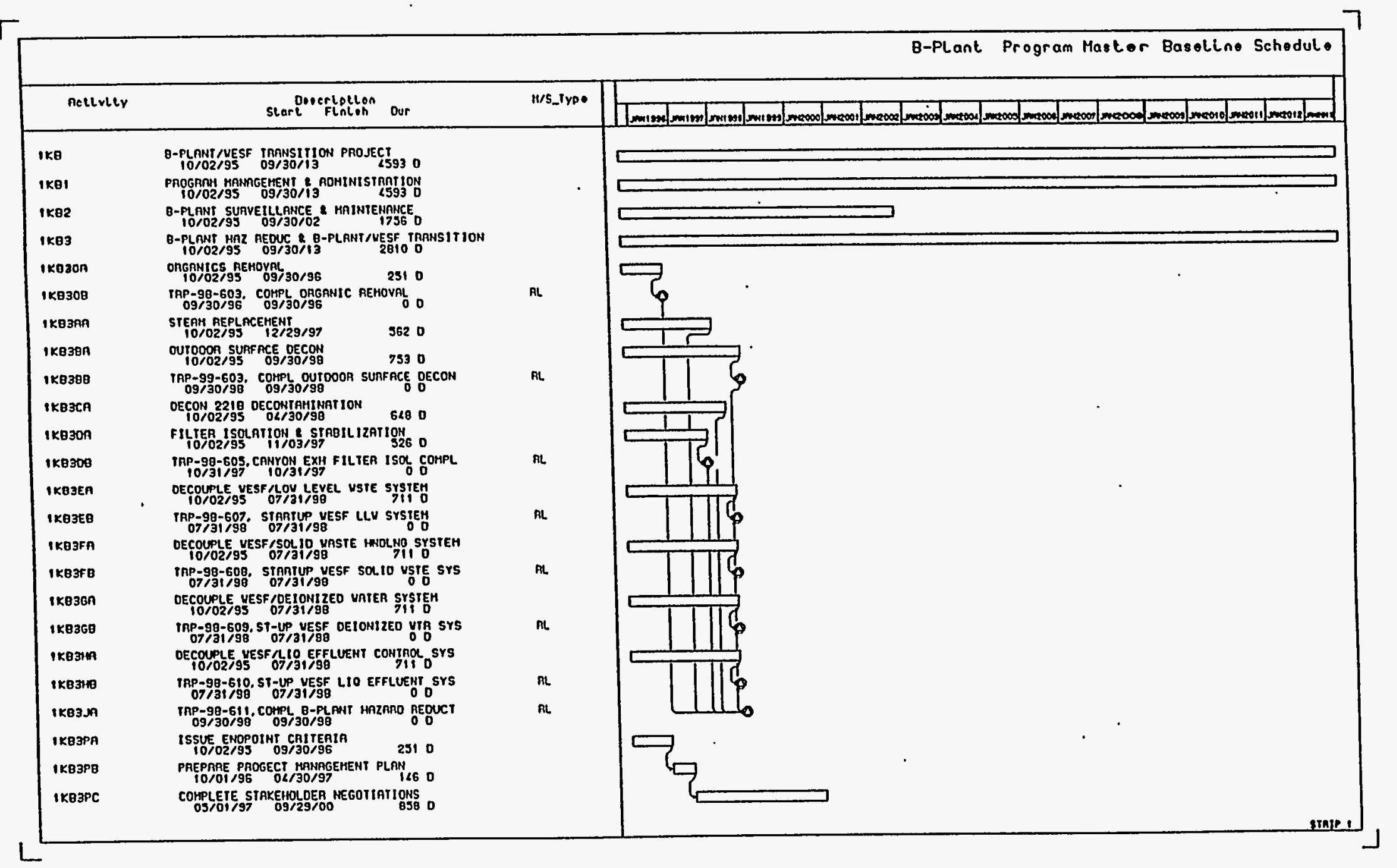




\section{TRANSITIUN PROJECTS}

\section{FY 1996 MYPP}

\subsection{1/7.1/6.12}

\section{B.1 Program Master Basel ine Schedule}

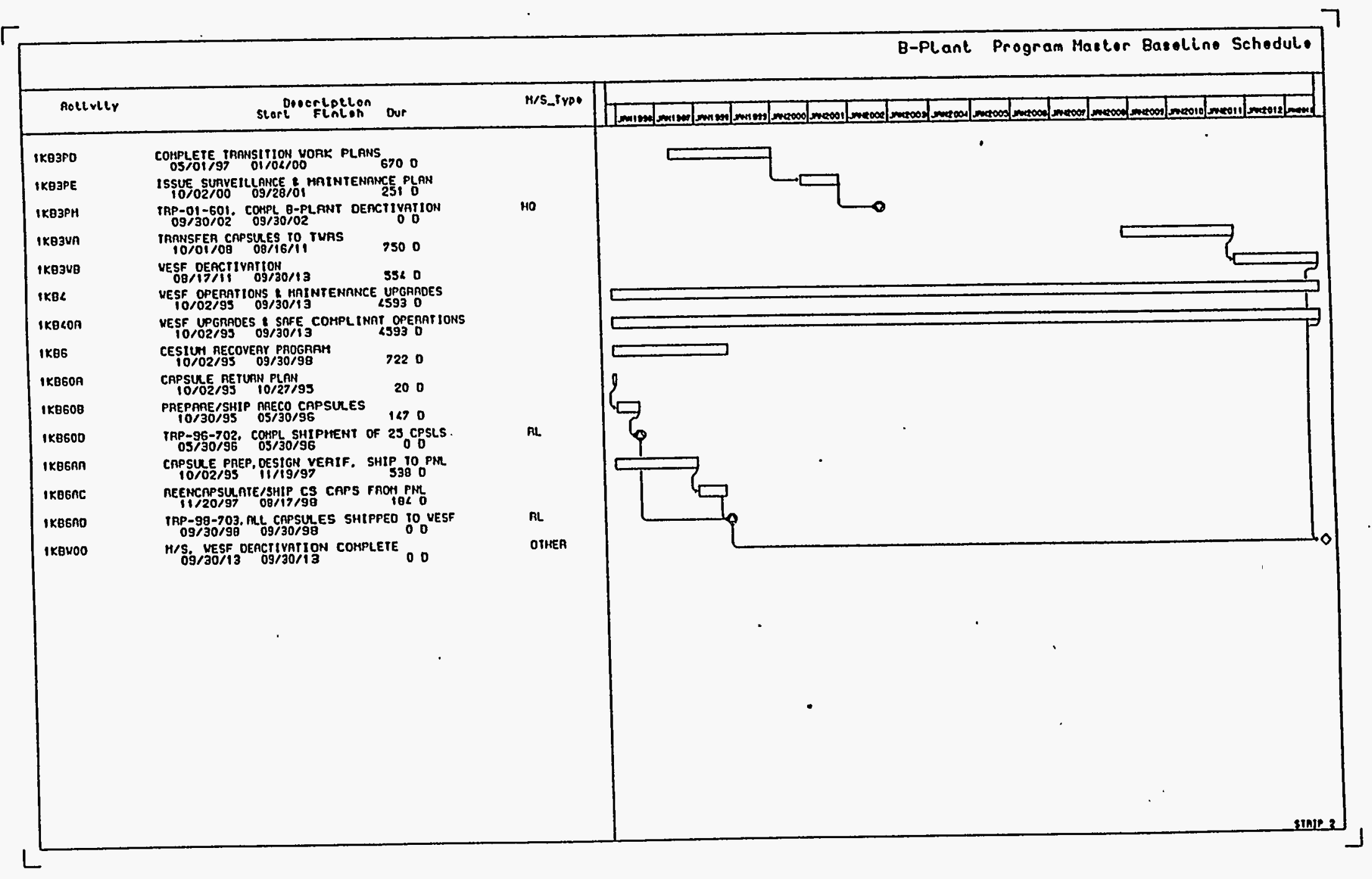




\section{TRANSITION PROJECTS}

\section{FY 1996 MYPP}

1.3.1/7.1/6.12

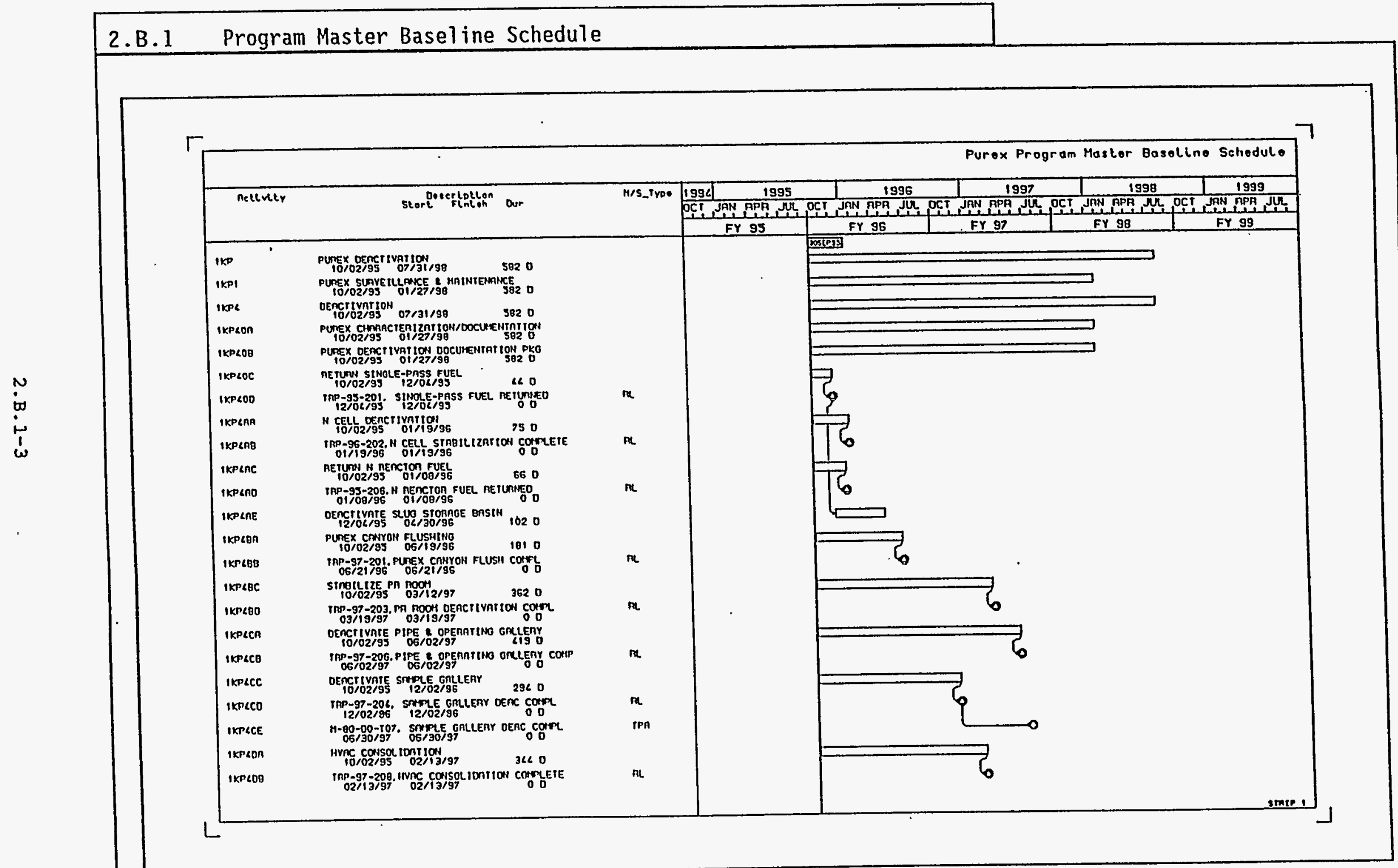


TRANSITION PROJECTS

FY 1996 MYPP

1.3.1/7.1/6.12

2.B.1 Program Master Baseline Schedule

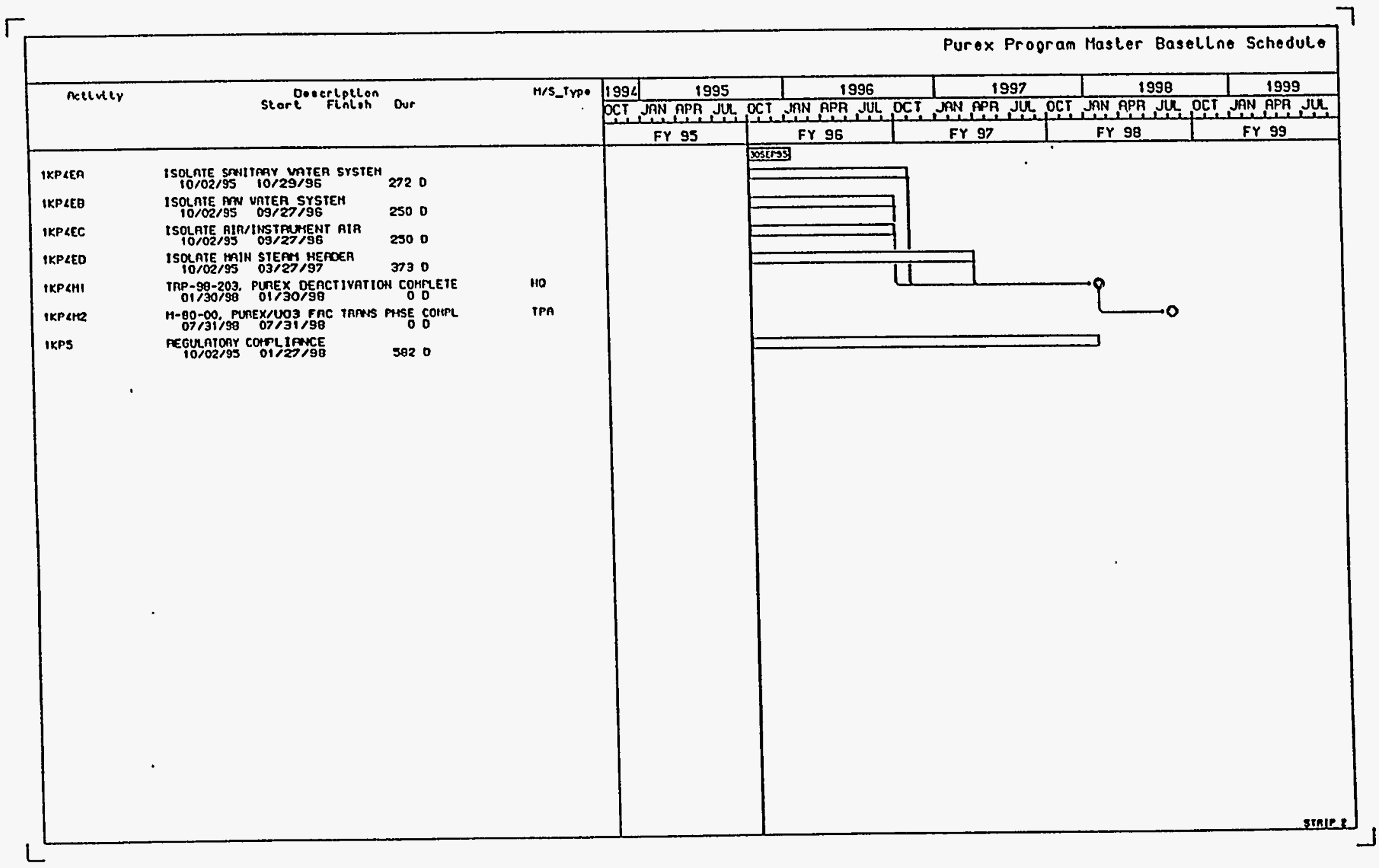




\section{TRANSITION PROJECTS}

FY 1996 MYPP

\subsection{1/7.1/6.12}

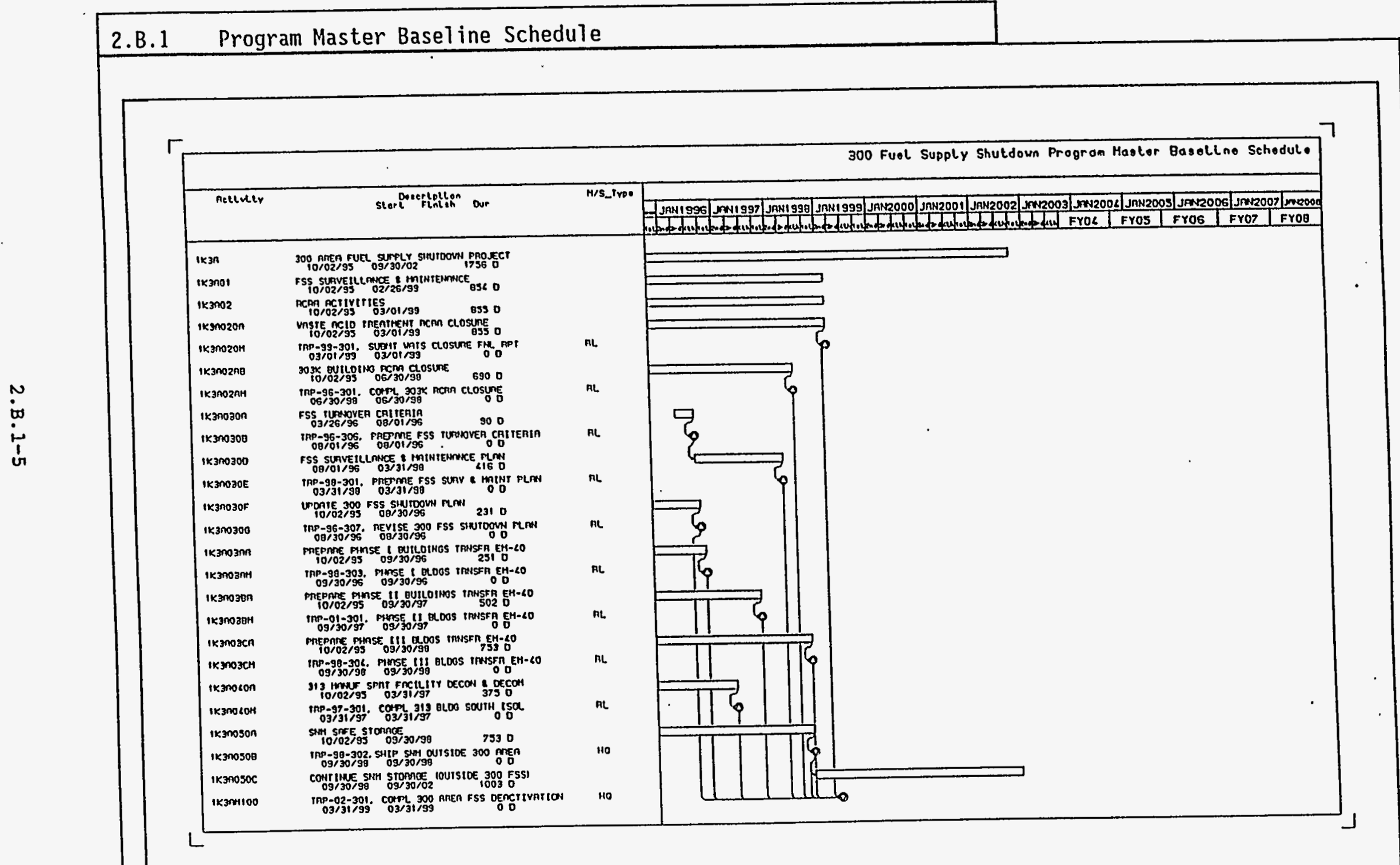

2: 771000\FY95 MYYFYHP TPP-MYPP.MST 


\section{TRANSITION PROJECTS}

\section{FY 1996 MYPP}

\subsection{1/7.1/6.12}

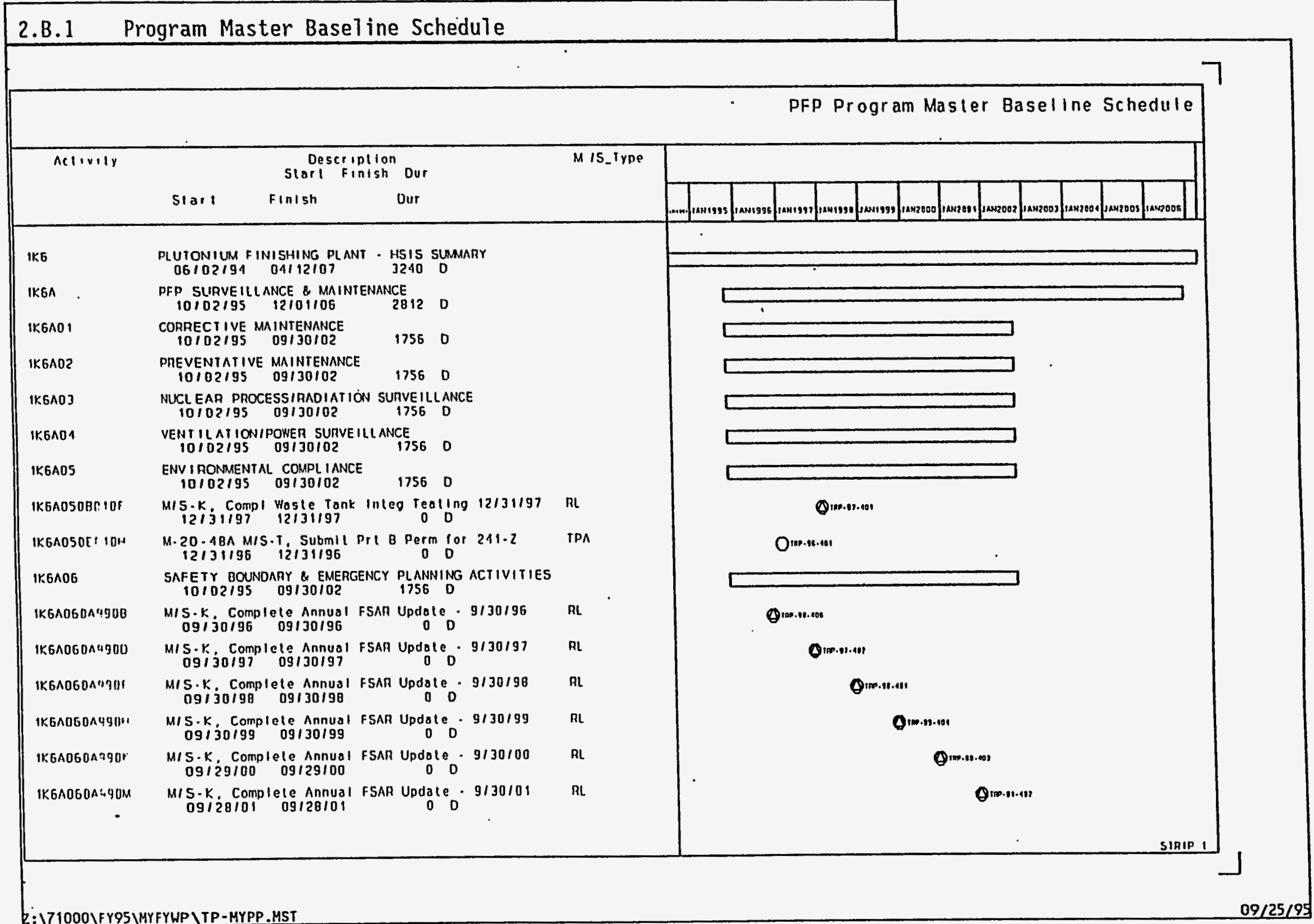


FY 1996 MYPP

\section{TRANSITION PROJECTS \\ 1.3.1/7.1/6.12}

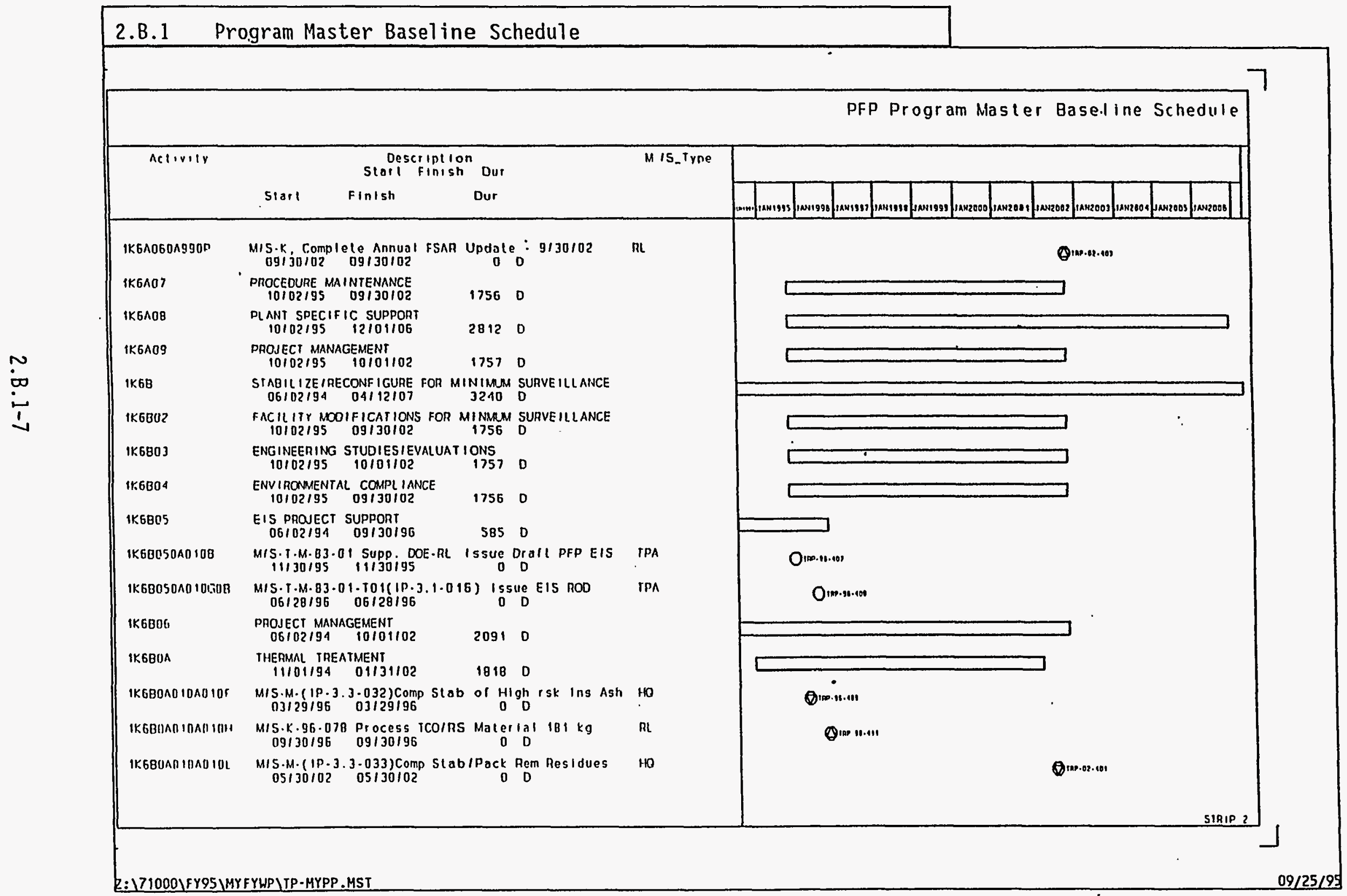




\section{TRANSITION PROJECTS}

FY 1996 MYPP

1.3.1/7.1/6.12

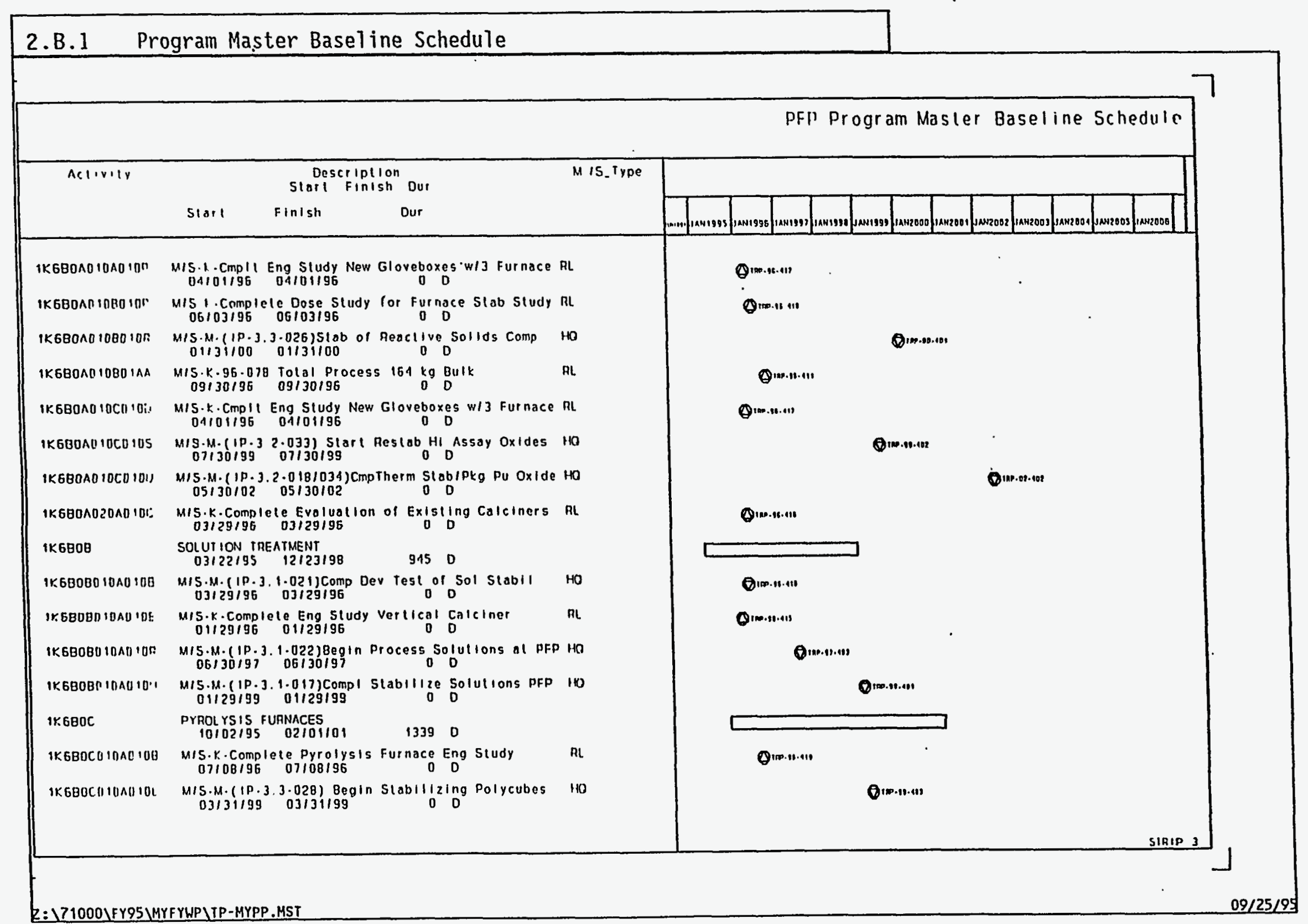


TRANSITION PROJECTS

FY 1996 MYPP

\subsection{1/7.1/6.12}

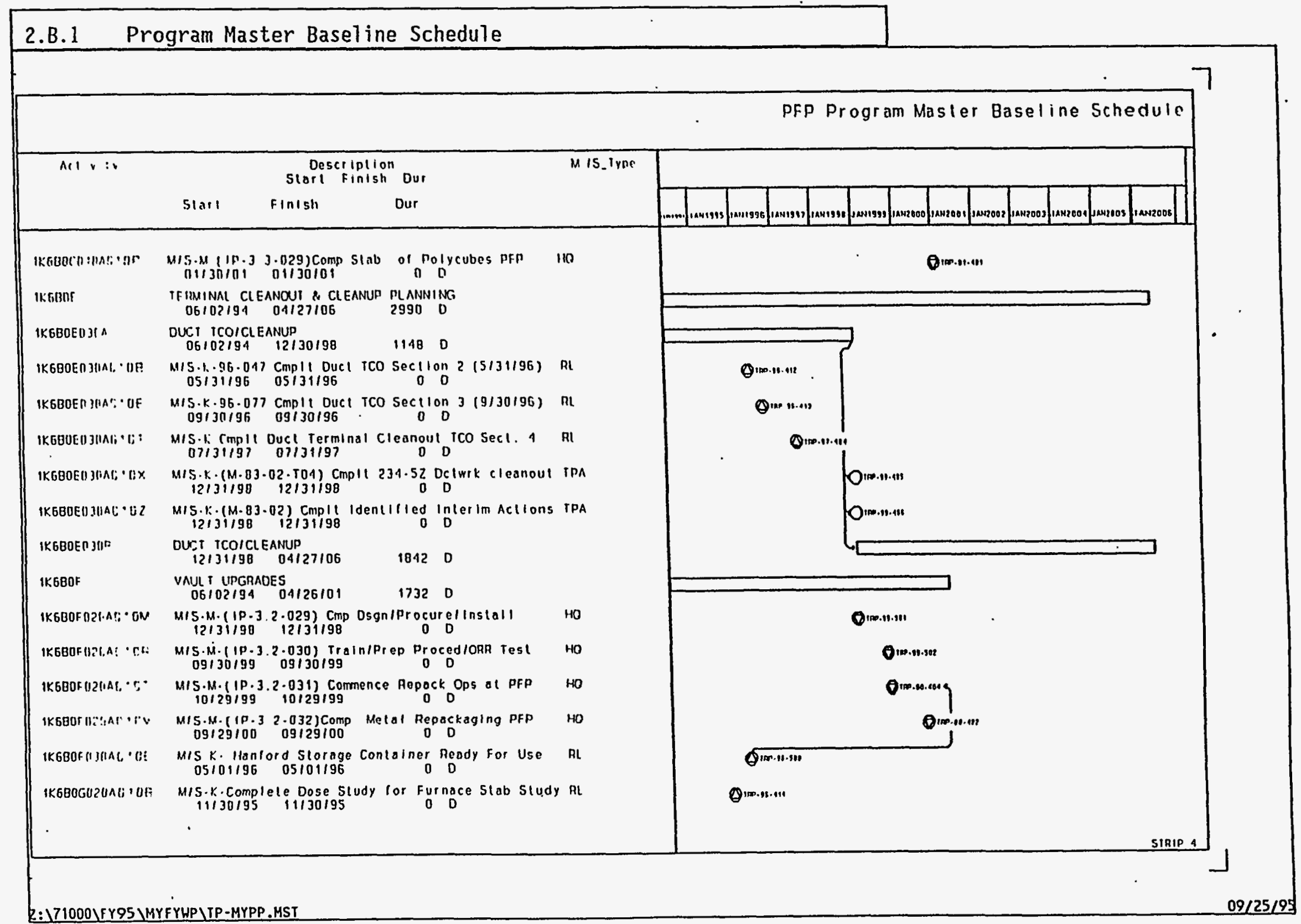




\section{FY 1996 MYPP}

TRANSITION PROJECTS

1.3.1/7.1/6.12

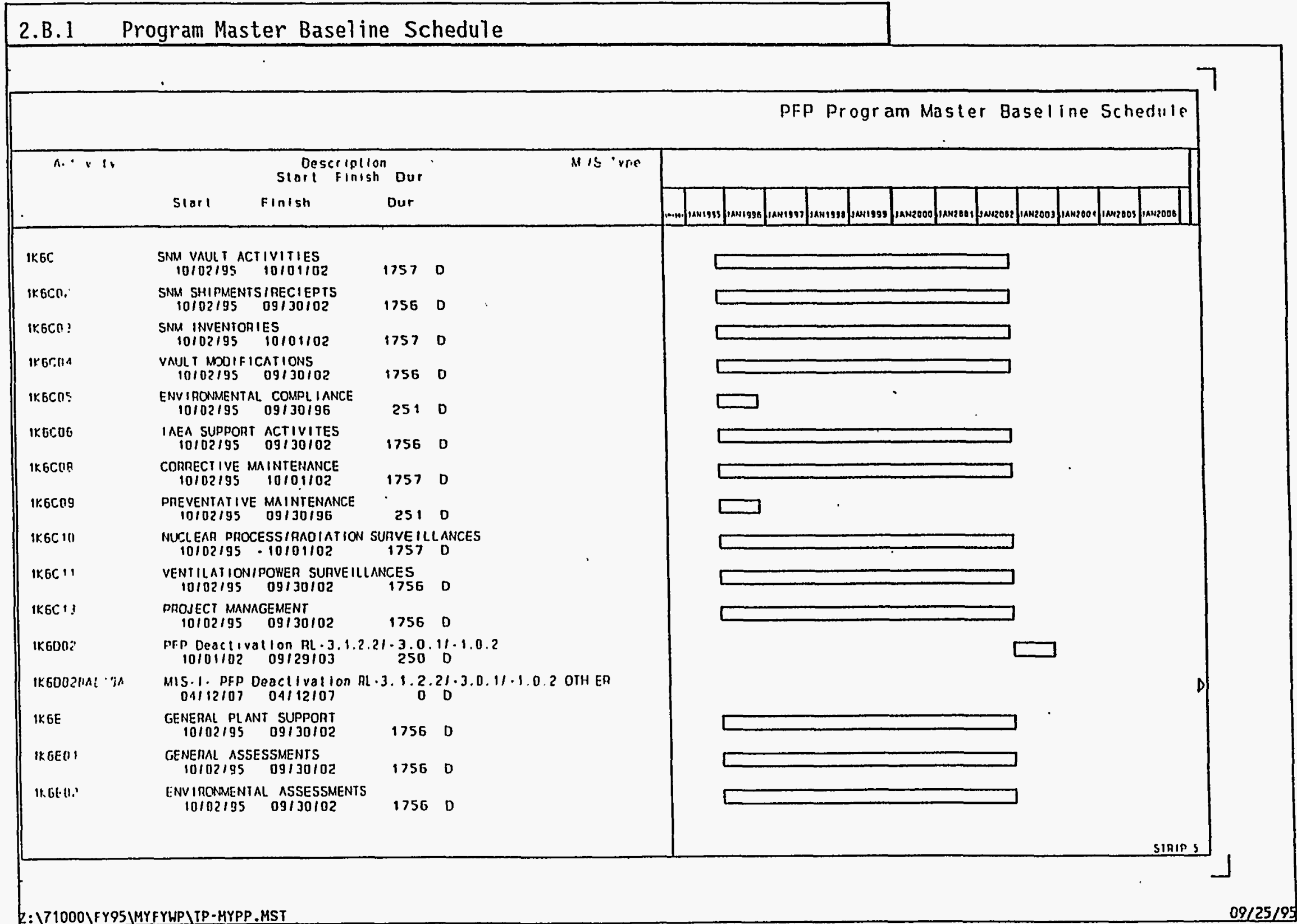




\section{TRANSITION PROJECTS}

\section{FY 1996 MYPP}

\subsection{1/7.1/6.12}

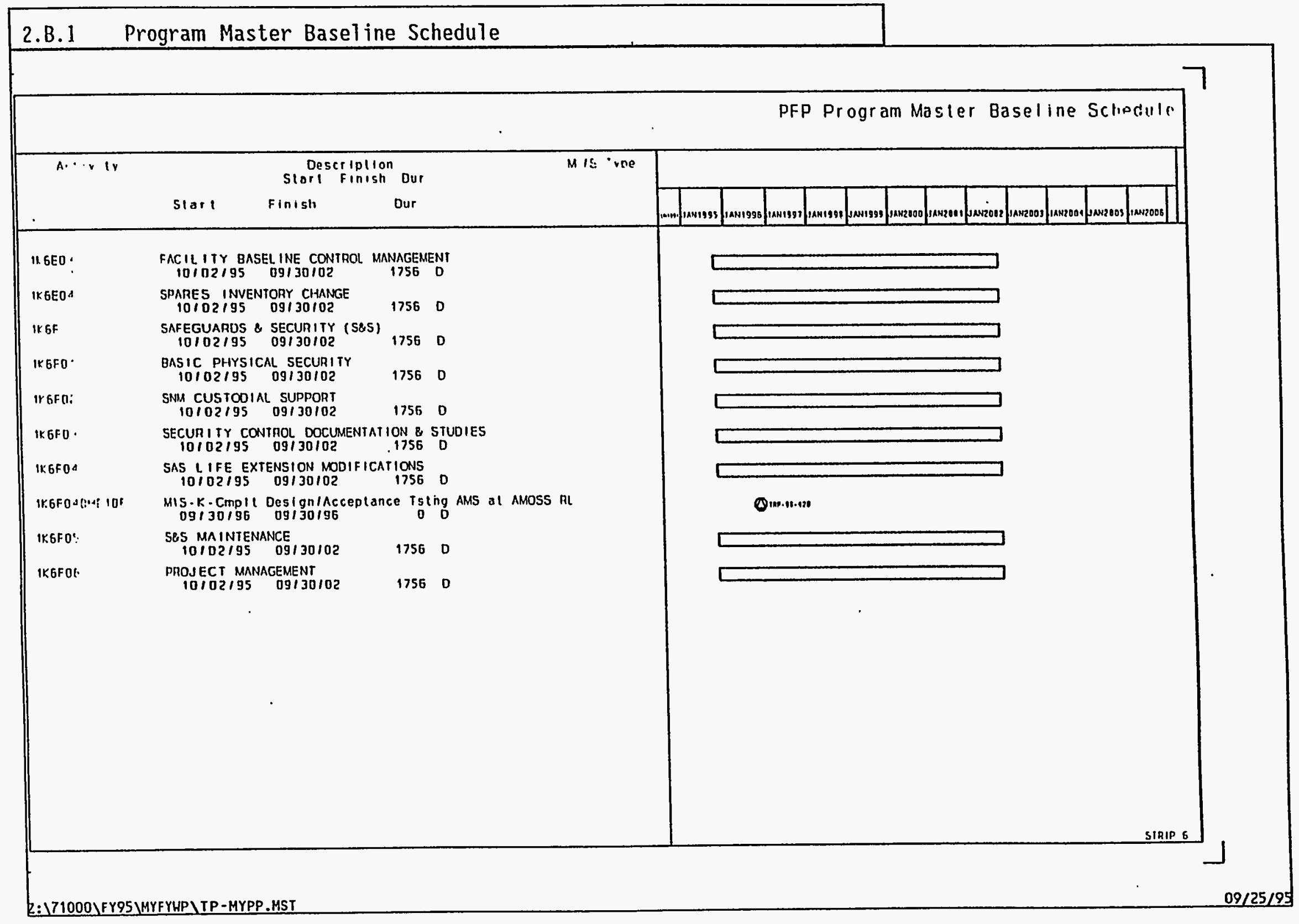




\section{TRANSITION PROJECTS}

\section{FY 1996 MYPP \\ 1.3.1/7.1/6.12}

2.B.1 Program Master Baseline Schedule

Program and Environmental Management (7.1.6)

The Program and Environmental Management program element provides management support for resources and activities that are common to all Transition Projects plants. These activities consists primarily of level-of-effort tasks which are repetitive from year to year and, thus, no Program Master Baseline Schedule is provided here. 


\section{B.1 Program Master Basel ine Schedule}

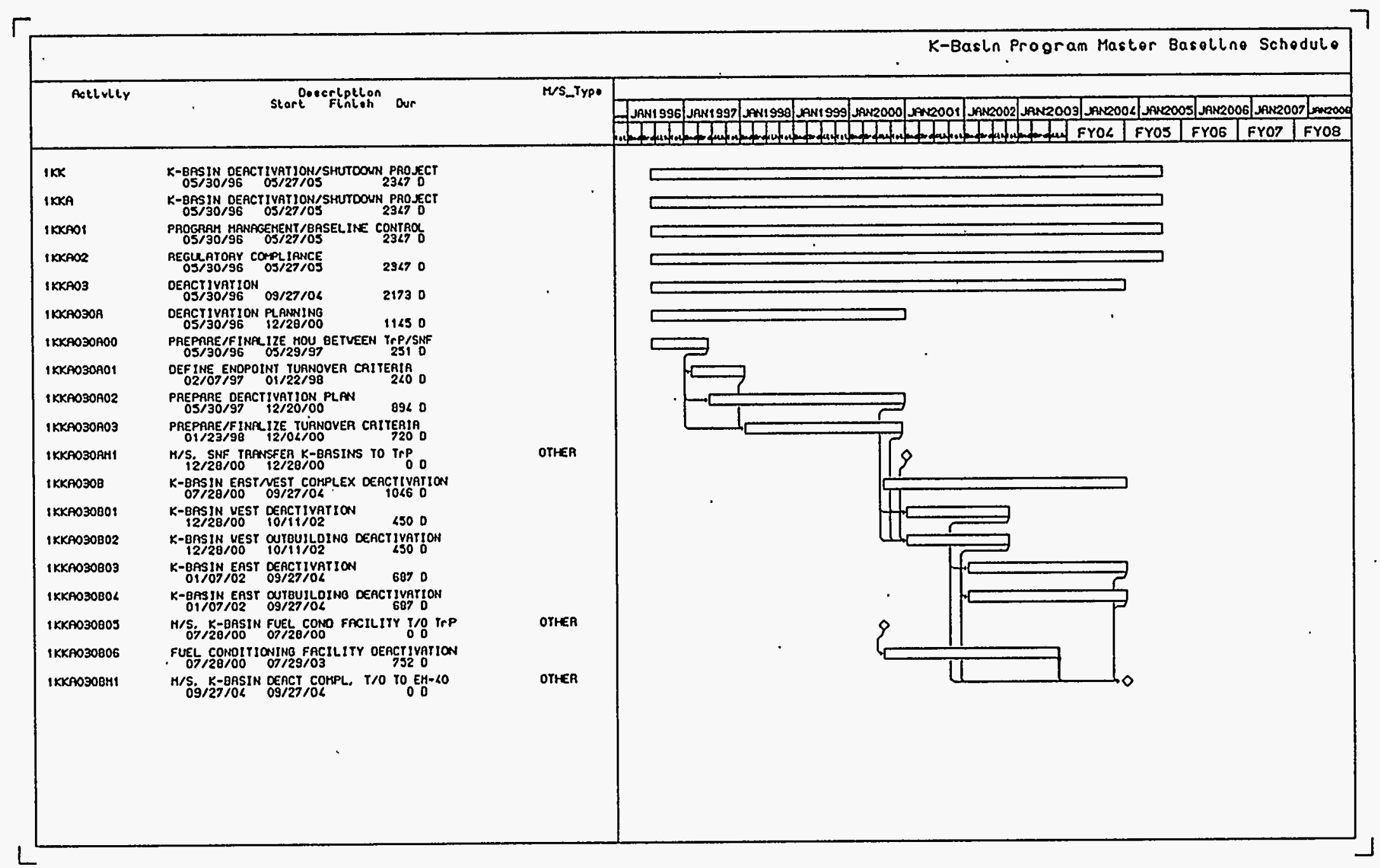




\section{TRANSITI N PROJECTS}

\begin{tabular}{|c|c|c|c|c|c|c|c|}
\hline \multicolumn{8}{|c|}{ 2.B.2 Milestone $\mathrm{Ljst}$} \\
\hline $\begin{array}{l}\text { Control } \\
\text { Number }\end{array}$ & $\begin{array}{l}\text { Milestone } \\
\text { Type }\end{array}$ & RL WBS & $\begin{array}{l}\text { ADS } \\
\text { NUMBER }\end{array}$ & Milestone Description & $\begin{array}{l}\text { Original } \\
\text { Basel ine } \\
\text { Date }\end{array}$ & $\begin{array}{l}\text { Revised } \\
\text { Basel ine } \\
\text { Date }\end{array}$ & $\begin{array}{l}\text { CiN } \\
\text { Change } \\
\text { Authority }\end{array}$ \\
\hline \multicolumn{8}{|c|}{ 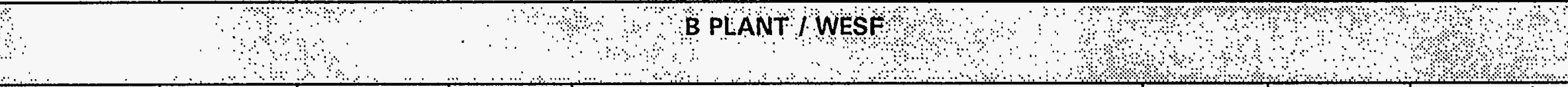 } \\
\hline$T R P-95-622$ & RL & 1.3.1.7 & 4190-0 & Clean and remove inventory on $\mathrm{K}-3$ Ductwork & $09 / 30 / 95$ & TSD & $T P-95-057$ \\
\hline$T R P-96-601$ & TPA & 1.3.1.7 & $4190-0$ & Complete B Plant Tank Integrity Assessment Report & $12 / 31 / 95$ & & \\
\hline TRP-96-606 & IPA & 1.3.1.7 & $4190-0$ & $\begin{array}{l}\text { Complete chemical separation of radionuclides from } \\
\text { legacy organics in B Plant }\end{array}$ & $12 / 31 / 95$ & & \\
\hline TRP-96-602 & RL & 1.3.1.7 & $4190-0$ & Award WESF Ion Exchange Contract & $01 / 31 / 96$ & & \\
\hline TRP-95-616 & RL. & 1.3.1.7 & $4190-0$ & Submit B Plant / WESF S/RID to RL for approval & $09 / 29 / 95$ & $03 / 29 / 96$ & $T P-95-057$ \\
\hline TRP-96-603 & RL & 1.3.1.7 & $4190-0$ & Implement B Plant ISB & $07 / 31 / 96$ & & \\
\hline TRP-98-603 & RL & 1.3.1.7 & $4190-0$ & Complete Removal of B Plant Organic inventory & $09 / 15 / 98$ & $09 / 30 / 96$ & $T P-95-057$ \\
\hline TRP-95-618 & RL & 1.3.1.7 & $4190-0$ & $\begin{array}{l}\text { Submit B Plant / WESF S/RID Phase I Assessment } \\
\text { Report to RL for approval }\end{array}$ & $09 / 30 / 96$ & & \\
\hline TRP-96-604 & RL & 1.3.1.7 & $4190-0$ & B Plant / WESF Procedures Improvement & $09 / 30 / 96$ & & \\
\hline TRP-97-601 & RL & 1.3.1.7 & $4190-0$ & $\begin{array}{l}\text { Projected FY97 \& Plant Surveillance and Maintenance } \\
\text { Costs Reduced by } 20 \% \text { from FY } 66\end{array}$ & $10 / 01 / 96$ & & \\
\hline
\end{tabular}


TRANSII N PROJECTS

FY 1996 MYPP

1.3.1/7.1/6.12

\begin{tabular}{|c|c|c|c|c|c|c|c|}
\hline $\begin{array}{l}\text { Control } \\
\text { Number }\end{array}$ & $\begin{array}{l}\text { Milestone } \\
\text { Type }\end{array}$ & RL WBS & $\begin{array}{l}\text { ADS } \\
\text { NUMBER }\end{array}$ & Milestone Description & $\begin{array}{l}\text { Original } \\
\text { Basel ine } \\
\text { Date }\end{array}$ & $\begin{array}{c}\text { Revised } \\
\text { Basel ine } \\
\text { Date }\end{array}$ & $\begin{array}{l}\text { CIN } \\
\text { Change } \\
\text { Authority }\end{array}$ \\
\hline TRP-98-604. & RL & 1.3.1.7 & $4190-0$ & $\begin{array}{l}\text { Projected FY98 \& Plant Surveillance and Maintenance } \\
\text { Costs Reduced by } 50 \% \text { from FY96 }\end{array}$ & $10 / 01 / 97$ & & \\
\hline TRP-98-605 & RL & 1.3.1.7 & 4190-0 & Canyon Exhaust filter isolation and Stabilization & $11 / 01 / 97$ & & \\
\hline TRP-98-602 & RL & 1.3.1.7 & $4190-1$ & $\begin{array}{l}\text { Complete Definitive Design B Plant Safety Class Vent } \\
\text { Upgrade }(H-059)\end{array}$ & $01 / 31 / 98$ & & \\
\hline TRP-98-606 & RL & 1.3.1.7 & $4190-0$ & Deactivate Steam Supply to the B Plant Complex & $01 / 31 / 98$ & & \\
\hline TRP-95-606 & RL & 1.3.1.7 & $4190-0$ & $\begin{array}{l}\text { Submit Project Management Plan (PMP) Outline to RL } \\
\text { for Approval }\end{array}$ & $04 / 29 / 95$ & Deleted & $T P-95-057$ \\
\hline TRP-95-612 & RL & 1.3.1.7 & $4190-0$ & $\begin{array}{l}\text { Submit Project Management Plan (PMP) to RL for } \\
\text { Approval }\end{array}$ & $04 / 30 / 98$ & & \\
\hline TRP-98-607 & RL & 1.3.1.7 & $4190-0$ & Startup the WESF Low Level Liquid Haste System & $07 / 31 / 98$ & & \\
\hline TRP-98-608 & RL & 1.3.1.7 & 4190-0 & Startup the WESF Solid Waste Systems & $07 / 31 / 98$ & & \\
\hline TRP-98-609 & RL & 1.3.1.7 & $4190-0$ & Startup the WESF Deionized Water Systems & $07 / 31 / 98$ & & \\
\hline TRP-98-610 & RL & 1.3.1.7 & $4190-0$ & Startup the WESF Liquid Effluent Systems & $07 / 31 / 98$ & & \\
\hline TRP-99-601 & RL & 1.3.1.7 & $4190-1$ & $\begin{array}{l}\text { Complete Construction of B Plant Canyon Ventilation } \\
\text { Upgrade }(H-059)\end{array}$ & $09 / 30 / 99$ & $09 / 30 / 98$ & TP-95-057 \\
\hline
\end{tabular}


TRANSIT: \PROJECTS

1.3.1/7.1/6.12

\begin{tabular}{|c|c|c|c|c|c|c|c|}
\hline \multicolumn{8}{|c|}{ 2.B:2 Milestone List } \\
\hline $\begin{array}{l}\text { Control } \\
\text { Number }\end{array}$ & $\begin{array}{l}\text { Milestone } \\
\text { Type }\end{array}$ & RL WBS & $\begin{array}{l}\text { ADS } \\
\text { NUMBER }\end{array}$ & Milestone Description & $\begin{array}{l}\text { Original } \\
\text { Basel ine } \\
\text { Date }\end{array}$ & $\begin{array}{l}\text { Revised } \\
\text { Baseline } \\
\text { Date }\end{array}$ & $\begin{array}{l}\text { CIN } \\
\text { Change } \\
\text { Authority }\end{array}$ \\
\hline TRP-98-611 & $\mathrm{RL}$ & 1.3.1.7 & $4190-0$ & Complete Accelerated Hazard Reduction Activities & $09 / 30 / 98$ & & \\
\hline TRP-99-602 & RL & 1.3.1.7 & $4190-0$ & $\begin{array}{l}\text { Projected FY99 B Plant Surveillance and Maintenance } \\
\text { Costs Reduced by } 65 \% \text { from FY96 }\end{array}$ & $10 / 01 / 98$ & & \\
\hline TRP-99-603 & RL & 1.3.1.7 & 4190-0 & Outdoor Surface Decontamination Complete & $02 / 28 / 99$ & & \\
\hline TRP-00-601 & RL & 1.3.1.7 & $4190-0$ & $\begin{array}{l}\text { Projected FYOO B Plant Surveillance and Maintenance } \\
\text { Costs Reduced by } 75 \% \text { from FY96 }\end{array}$ & $10 / 01 / 99$ & & \\
\hline$T R P-0 i-603$ & RL & 1.3.1.7 & $4190-0$ & $\begin{array}{l}\text { Projected FY01 B Plant Surveil lance and Maintenance } \\
\text { Costs Reduced by } 80 \% \text { from FY96 }\end{array}$ & $10 / 01 / 00$ & & \\
\hline TRP-01-601 & HQ & 1.3.1.7 & $4190-0$ & Complete B Plant Deactivation & 09/30/02 & & \\
\hline & & & & CESIUM CAPSULERECOUERY & & & \\
\hline TRP-96-702 & RL & 1.3.1.7.5 & 4195-0 & Complete ARECO Capsule Shipments to WESF & $09 / 30 / 96$ & $05 / 30 / 96$ & $T P-95-057$ \\
\hline TRP-98-701 & HO & 1.3.1.7.5 & 4195-0 & Complete ORNL Shipment of HESF Residue & $09 / 30 / 98$ & Deleted & TP-95-057 \\
\hline TRP- $98-702$ & RL & 1.3.1.7.5 & $4195-0$ & $\begin{array}{l}\text { Initiate Re-encapsulation of Residue and SHollen } \\
\text { Capsules at WESF }\end{array}$ & $09 / 30 / 98$ & Deleted & $T P-95-057$ \\
\hline TRP-98-703 & RL & 1.3.1.7.5 & $4195 \cdot 0$ & Complete Return of all Cesium Capsules to WESF & $09 / 30 / 98$ & & \\
\hline
\end{tabular}


TRANSIT N PROJECTS

FY 1996 MYPP

1.3.1/7.1/6.12

\begin{tabular}{|c|c|c|c|c|c|c|c|}
\hline $\begin{array}{l}\text { Control } \\
\text { Number }\end{array}$ & $\begin{array}{l}\text { Milestone } \\
\text { Type }\end{array}$ & RL WBS & $\begin{array}{l}\text { ADS } \\
\text { NUMBER }\end{array}$ & Milestone Description & $\begin{array}{l}\text { Original } \\
\text { Basel ine } \\
\text { Date }\end{array}$ & $\begin{array}{c}\text { Revised } \\
\text { Basel ine } \\
\text { Date }\end{array}$ & $\begin{array}{l}\text { CIN } \\
\text { Change } \\
\text { Authority }\end{array}$ \\
\hline$\therefore$ & & & $\because \because$ & PUREXI UOBDEACTIVATION & & ४ै। & $\because$ \\
\hline TRP-95-201 & RL & 7.1 .1 & $6622-0$ & Return Single-Pass fuel & 10/17/94 & 12/04/95 & TP-95-001 \\
\hline TRP-95-206 & RL. & 7.1 .1 & $6622-0$ & Return N Reactor Fuel & $5 / 03 / 95$ & $1 / 08 / 96$ & TP-95-001 \\
\hline TRP-96-201 & RL & 7.1 .1 & $6622-0$ & Complete Nitric Acid Disposal & $2 / 24 / 96$ & $4 / 01 / 96$ & $T P-95-009$ \\
\hline TRP-96-202 & RL & 7.1 .1 & $6622-0$ & Complete N Cell Stabilization & $6 / 17 / 96$ & 1/19/96 & F0-94-019 \\
\hline TRP-96-203 & RL & 7.1 .1 & $6622-0$ & Complete Pu-U Solution Disposal & $7 / 22 / 96$ & 10/05/95 & F0-94-019 \\
\hline TRP-96-204 & TPA-RL & 7.1 .1 & $6622-0$ & $\begin{array}{l}\text { Submit a PUREX Preclosure Hork Plan to EPA and Ecology (M-20- } \\
24 A)\end{array}$ & $7 / 31 / 96$ & & $M-20-94-07$ \\
\hline TRP-96-205 & TPA-RL & 7.1 .1 & $6622-0$ & $\begin{array}{l}\text { Complete removal of concentrated (recovered) 203-A Nitric Acid } \\
\text { at PUREX }(M-80-00-T 04)\end{array}$ & $6 / 30 / 96$ & & $M-80-94-1$ \\
\hline TRP-96-206 & TPA-RL & 7.1 .1 & $6622-0$ & $\begin{array}{l}\text { Submit the end point criteria and surveillance and maintenance } \\
\text { plan in support of the PUREX Preclosure Work Plan }(M-80-02)\end{array}$ & $7 / 31 / 96$ & & $M-80-94-1$ \\
\hline TRP-96-207 & TPA-RL & 7.1 .1 & $6622-0$ & Submit PUREX Surveillance and Maintenance Plan $(\mathrm{M}-80-02-\mathrm{TOZ})$ & $5 / 31 / 96$ & & $M-80-94-1$ \\
\hline TRP-97-201 & RL & 7.1 .1 & $6622-0$ & Complete PUREX Canyon Flushing & 12/04/96 & $6 / 21 / 96$ & $T P-95-009$ \\
\hline TRP-97-202 & RL & 7.1.1 & $6622-0$ & Complete Isolation of Tank Farm Haste Lines & $12 / 04 / 96$ & & \\
\hline TRP-97-203 & RL & 7.1 .1 & $6622-0$ & Complete PR Room Deactivation & $3 / 19 / 97$ & & \\
\hline
\end{tabular}


TRANSIT. JN PROJECTS

1.3.1/7.1/6.12

\begin{tabular}{|c|c|c|c|c|c|c|c|}
\hline $\begin{array}{l}\text { Control } \\
\text { Number }\end{array}$ & $\begin{array}{l}\text { Milestone } \\
\text { Type }\end{array}$ & RL. WBS & $\begin{array}{c}\text { ADS } \\
\text { NUMBER }\end{array}$ & Milestone Description & $\begin{array}{c}\text { Original } \\
\text { Basel ine } \\
\text { Date }\end{array}$ & $\begin{array}{c}\text { Revised } \\
\text { Basel ine } \\
\text { Date }\end{array}$ & $\begin{array}{c}\text { CIN } \\
\text { Change } \\
\text { Authority }\end{array}$ \\
\hline TRP-97-204 & RL & 7.1 .1 & $6622-0$ & Complete Sample Gallery Deactivation & $4 / 22 / 97$ & 12/02/96 & TP-95-009 \\
\hline TRP-97-205 & RL & 7.1 .1 & $6622-0$ & Reconcile PUREX SNM Final Accountability & $5 / 16 / 97$ & & \\
\hline TRP-97-206 & RL & 7.1 .1 & $6622-0$ & Complete Deactivation of Pipe and Operating Gallery & 6/02/97 & & \\
\hline TRP-97-207 & RL & 7.1 .1 & $6622-0$ & Complete PUREX/UO 3 Plant Surveillance and Maintenance Plan & $6 / 24 / 97$ & $5 / 30 / 96$ & $T P-95-009$ \\
\hline TRP-97-208 & RL & 7.1 .1 & $6622-0$ & Complete HVAC System Consolidation & $6 / 25 / 97$ & $2 / 13 / 97$ & TP-95-009 \\
\hline$T R P=97-209$ & TPA-RL & 7.1 .1 & $6622-0$ & $\begin{array}{l}\text { Complete implementation of selected alternative for management } \\
\text { of Spent Fuel from PUREX (M-80-00-T05) }\end{array}$ & $12 / 31 / 96$ & & $M-80-94-1$ \\
\hline TRP-97-210 & TPA-RL & 7.1.1 & 6622-0 & $\begin{array}{l}\text { Complete Deactivation of the PUREX Plant 211-A Area (M-80-00- } \\
\text { T06) }\end{array}$ & $4 / 30 / 97$ & & $M-80-94-1$ \\
\hline TRP-97-211 & TPA-RL & 7.1 .1 & $6622-0$ & $\begin{array}{l}\text { Complete Deactivation of the PUREX Plant Sample Gallery } \\
(M-80-00-T 07)\end{array}$ & $6 / 30 / 97$ & & $M-80-94-1$ \\
\hline TRP-97-212 & TPA-RL & 7.1 .1 & $6622-0$ & Remove Process Waste Solutions from Tanks $D 5$ and $E 6(H-80-03)$ & $1 / 31 / 97$ & & $M-80-94-1$ \\
\hline TRP-97-213 & TPA-RL & 7.1 .1 & $6622-0$ & $\begin{array}{l}\text { Complete Deactivation of the PUREX Plant U-Cell/Fractionator } \\
(M-80-04)\end{array}$ & $4 / 30 / 97$ & & $H-80-94-1$ \\
\hline TRP-97-214 & TPA-RL & 7.1 .1 & $6622-0$ & $\begin{array}{l}\text { Complete Deactivation of the PUREX Plant Aqueous Makeup Area } \\
(M-80-05)\end{array}$ & $6 / 30 / 97$ & & $M-80-94-1$ \\
\hline TRP-97-215 & TPA-RL & 7.1 .1 & $6622-0$ & Complete Deactivation of the PUREX Plant Canyon (M-80-06) & $7 / 31 / 97$ & & $M-80-94-1$ \\
\hline TRP-98-201 & RL & 7.1 .1 & $6622-0$ & Complete Deactivation of Ancillary Buildings & $6 / 01 / 98$ & $8 / 22 / 97$ & TP-95-009 \\
\hline TRP-98-202 & RL & 7.1 .1 & $6622-0$ & Discontinue PUREX Liquid Effluent Discharge & $6 / 17 / 98$ & $1 / 23 / 98$ & $T P-95-009$ \\
\hline
\end{tabular}


TRANSITI I PROJECTS

FY 1996 MYPP

1.3.1/7.1/6.12

\begin{tabular}{|c|c|c|c|c|c|c|c|}
\hline $\begin{array}{l}\text { Control } \\
\text { Number }\end{array}$ & $\begin{array}{l}\text { Milestone } \\
\text { Type }\end{array}$ & RL WBS & $\begin{array}{l}\text { ADS } \\
\text { NUMBER }\end{array}$ & Milestone Description & $\begin{array}{c}\text { Original } \\
\text { Basel ine } \\
\text { Date }\end{array}$ & $\begin{array}{c}\text { Revised } \\
\text { Basel ine } \\
\text { Date }\end{array}$ & $\begin{array}{l}\text { CIN } \\
\text { Change } \\
\text { Authority }\end{array}$ \\
\hline$T R P=98-203$ & HO & 7.1 .1 & $6622-0$ & Complete PUREX Deactivation & $7 / 31 / 98$ & $1 / 30 / 98$ & $T P-95-009$ \\
\hline TRP-98-204 & TPA-RL & 7.1 .1 & $6622-0$ & Complete Deactivation of the PUREX Plant 203-A Area (M-80-07) & $4 / 30 / 98$ & & $H-80-94-1$ \\
\hline$T R P-98-205$ & TPA-RL & 7.1 .1 & $6622-0$ & $\begin{array}{l}\text { Document Hazardous Substances/Dangerous Hastes Remaining Within } \\
\text { the PUREX Plant }(M-80-08)\end{array}$ & $7 / 31 / 98$ & & $M-80-94-1$ \\
\hline TRP-98-206 & TPA-HQ & 7.1 .1 & $6622-0$ & $\begin{array}{l}\text { Complete PUREX and } \mathrm{UO}_{3} \text { Plant Facility Transition Phase and } \\
\text { initiate the Surveillance and Maintenance Phase }(\mathrm{M}-80-00)\end{array}$ & $7 / 31 / 98$ & & $M-80-94-1$ \\
\hline
\end{tabular}

$N$
$\vdots$
$\dot{\sigma}$ 


\begin{tabular}{|c|c|c|c|c|c|c|c|}
\hline $\begin{array}{l}\text { Control } \\
\text { Number }\end{array}$ & $\begin{array}{l}\text { Milestone } \\
\text { Type }\end{array}$ & RL HBS & ADS NUMBER & Milestone Description & $\begin{array}{c}\text { Original } \\
\text { Basel ine } \\
\text { Date }\end{array}$ & $\begin{array}{c}\text { Revised } \\
\text { Basel ine } \\
\text { Date }\end{array}$ & $\begin{array}{c}\text { CIN } \\
\text { Change } \\
\text { Authority }\end{array}$ \\
\hline & & & & 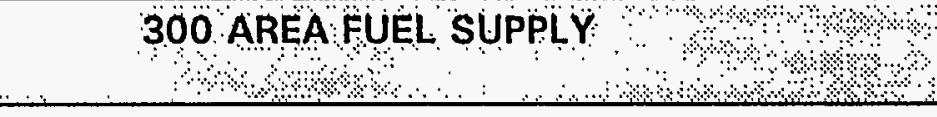 & \$? & & \\
\hline TRP-96-303 & RL & 7.1 .2 & $6623-0$ & $\begin{array}{l}\text { Resolve Ecology comments on } 304 \text { Concretion Final Report and } \\
\text { Submit Closed Part A Permit to Ecology. }\end{array}$ & $3-29-96$ & & TP-95-057 \\
\hline TRP-96-306 & RL & 7.1 .2 & $6623-0$ & Prepare Fuel Supply Shutdown Turnover criteria. & $8 / 1 / 96$ & & TP-95-057 \\
\hline TRP-96-3.07 & RL & 7.1 .2 & $6623-0$ & Revise Fuel Supply Shutdown Plan with FY 1996 activities. & $8 / 30 / 96$ & & $T P-95-057$ \\
\hline TRP-98-301 & RL & 7.1 .2 & $6623-0$ & $\begin{array}{l}\text { Prepare Fuel Supply Shutdown Surveillance and Maintenance } \\
\text { Plan for activities after deactivation. }\end{array}$ & $3 / 31 / 98$ & & $T P-95-057$ \\
\hline TRP-96-304 & RL & 7.1 .2 & $6623-0$ & Submit NOD Resolved HATS Closure Plan to RL. & $8 / 15 / 96$ & & \\
\hline TRP-97-303 & RL & 7.1.2 & $6623-0$ & $\begin{array}{l}\text { Complete HATS closure Field Activities in } \\
303 F, 334 \text {, and } 333 \text { Building. }\end{array}$ & 9/30/97 & & \\
\hline TRP-96-301 & RL. & 7.1 .2 & $6623-0$ & Complete 303K Facility RCRA Closure Activities. & 9/30/96 & $6 / 30 / 98$ & $T P-95-057$ \\
\hline TRP-96-302 & RL & 7.1 .2 & $6623-0$ & Complete 3135 Portion of WATS RCRA Closure Field Activities. & 9/30/96 & , & \\
\hline TRP-97-301 & RL & 7.1 .2 & $6623-0$ & Complete Isolation of 313 Building South Side. & $3 / 31 / 97$ & & TP-95-057 \\
\hline TRP-99-301 & RL & 7.1 .2 & $6623-0$ & Submit Final Report on WATS Closure Activities to RL. & 3/1/99 & & \\
\hline TRP-98-302 & Ha & 7.1 .2 & $6623-0$ & Ship balance of SNM to Harehouse Outside 300 Area. & 9/30/98 & & TP-95-057 \\
\hline TRP-98-303 & RL & 7.1 .2 & $6623-0$ & Prepare Phase 1 Buildings for Transfer to EM 40. & 9/30/98 & 9/30/96 & TP-95-057 \\
\hline TRP-01-301 & RL & 7.1 .2 & $6623-0$ & Prepare Phase 11 Buildings for Transfer to EM 40 . & 9/30/01 & 9/30/97 & TP-95-057 \\
\hline TRP-98-304 & RL & 7.1 .2 & $6623-0$ & Prepare Phase III Buildings for Transfer to EM 40 . & 9/30/98 & & $T P-95-057$ \\
\hline
\end{tabular}


TRANSIT. . PROJECTS

FY 1996 MYPP

1.3.1/7.1/6.12

\begin{tabular}{|c|c|c|c|c|c|c|c|}
\hline $\begin{array}{l}\text { Control } \\
\text { Number }\end{array}$ & $\begin{array}{l}\text { Milestone } \\
\text { Type }\end{array}$ & RL WBS & ADS NUMBER & Milestone Description & $\begin{array}{c}\text { Original } \\
\text { Basel ine } \\
\text { Date }\end{array}$ & $\begin{array}{c}\text { Revised } \\
\text { Basel ine } \\
\text { Date }\end{array}$ & $\begin{array}{c}\text { CIN } \\
\text { Change } \\
\text { Authority }\end{array}$ \\
\hline TRP-01-302 & RL & 7.1 .2 & $6623-0$ & Complete Haste Acid Treatment RCRA Closure Field Activities. & 9/30/2001 & 9/30/98 & TP-95-057 \\
\hline TRP-02-301 & HQ & 7.1 .2 & $6623-0$ & Complete 300 Area Fuel Supply Deactivation. & 9/30/2002 & $3 / 31 / 99$ & TP-95-057 \\
\hline
\end{tabular}


TRANSI ${ }^{-}$IN PROJECTS

FY 1996 MYPP

1.3.1/7.1/6.12

\begin{tabular}{|c|c|c|c|c|c|c|c|}
\hline $\begin{array}{l}\text { Control } \\
\text { Number }\end{array}$ & $\begin{array}{l}\text { Milestone } \\
\text { Type }\end{array}$ & RL HBS & ADS NUMBER & Milestone Description & $\begin{array}{l}\text { Original } \\
\text { Basel ine } \\
\text { Date }\end{array}$ & $\begin{array}{l}\text { Revised } \\
\text { Basel ine } \\
\text { Date }\end{array}$ & $\begin{array}{l}\text { CIN } \\
\text { Change } \\
\text { Authority }\end{array}$ \\
\hline \multicolumn{8}{|c|}{ 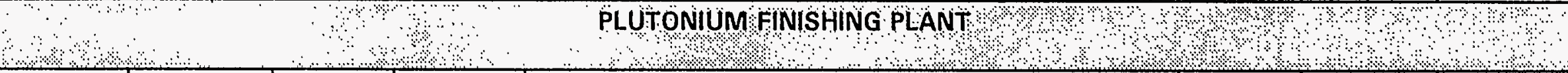 } \\
\hline$T R P-96-401$ & TPA - RL & 7.1 .3 & $6624-0$ & Submit Part B Permit or Closure Plan for $241-\mathrm{Z}(\mathrm{M}-20-48 \mathrm{~A})$ & $5 / 31 / 96$ & 12/31/96 & $M-83-94-1$ \\
\hline TRP-96-405 & $T P A-R L$ & 7.1 .3 & $6624-0$ & Complete $10-\mathrm{L}$. Solution Downloading (M-83-02-T03) & $6 / 30 / 96$ & & $M-83-94-1$ \\
\hline TRP-96-406 & RL & 7.1 .3 & $6624-0$ & Complete Annual FSAR Update & 9/30/96 & & \\
\hline TRP-96-407 & $\begin{array}{c}\mathrm{TPA}-\mathrm{RL} \\
\cdot\end{array}$ & 7.1 .3 & $6624-0$ & $\begin{array}{l}\text { SUpport DOE to issue DRAFT PFP Envirormental impact Statement } \\
(M-83-01)\end{array}$ & $11 / 30 / 95$ & & $M-83-94-1$ \\
\hline TRP-96-408 & $T P A-R L$ & 7.1 .3 & $6624-0$ & SUpport DOE to Issue EIS Record of Decision (M-83-01-T01) & $6 / 30 / 96$ & & $M-83-94-1$ \\
\hline TRP-96-409 & HQ & 7.1 .3 & $6624-0$ & Complete Stabilization of High-Risk Incinerator Ash & $3 / 31 / 96$ & & \\
\hline $\operatorname{TRP}-96-410$ & HQ & 7.1 .3 & $6624-0$ & $\begin{array}{l}\text { Complete Developmental Testing of Solution Stabilization } \\
\text { Technologies }\end{array}$ & $3 / 31 / 96$ & & \\
\hline TRP-96-411 & RL & 7.1 .3 & $6624-0$ & Complete Stabilization of $345 \mathrm{Kg}$ of TCO/RS Material & 9/30/96 & & \\
\hline TRP-96-412 & RL & 7.1 .3 & $6624-0$ & Complete Duct Terminal Cleanout (TCO), Section $\# 2$ & $5 / 31 / 96$ & & \\
\hline TRP-96-413 & RL & 7.1 .3 & $6624-0$ & Complete Duct Terminal Cleanout (TCO), Section $\# 3$ & $9 / 30 / 96$ & & \\
\hline TRP-96-414 & RL & 7.1 .3 & $6624-0$ & Complete PFP Laboratory Support Assessment & $11 / 30 / 95$ & & \\
\hline TRP-96-415 & RL & 7.1 .3 & $6624-0$ & Complete Engineering Study for Vertical Calciner & $1 / 29 / 96$ & & \\
\hline TRP-96-416 & RL & 7.1 .3 & $6624-0$ & Complete Evaluation of Existing Calciners & $3 / 29 / 96$ & & \\
\hline TRP-96-417 & RL & 7.1 .3 & $6624-0$ & $\begin{array}{l}\text { Complete Engineering Study for New Gloveboxes with Three } \\
\text { Muffle Furnaces }\end{array}$ & 4/01/96 & & \\
\hline TRP-96-418 & RL & 7.1 .3 & $6624-0$ & Complete Dose Study for Furnace Stabilization Operation & 6/03/96 & & \\
\hline TRP-96-419 & RL & 7.1 .3 & $6624-0$ & Complete Pyrolysis Furnace Engineering Study & $7 / 08 / 96$ & & \\
\hline
\end{tabular}




\section{FY 1996 MYPP}

TRANSI: ,N PROJECTS

1.3.1/7.1/6.12

\begin{tabular}{|c|c|c|c|c|c|c|c|}
\hline $\begin{array}{l}\text { Control } \\
\text { Number }\end{array}$ & $\begin{array}{l}\text { Milestone } \\
\text { Type }\end{array}$ & RL WBS & ADS NUMBER & Milestone Description & $\begin{array}{l}\text { Original } \\
\text { Baseline } \\
\text { Date }\end{array}$ & $\begin{array}{c}\text { Revised } \\
\text { Basel ine } \\
\text { Date }\end{array}$ & $\begin{array}{c}\text { CIN } \\
\text { Change } \\
\text { Authority }\end{array}$ \\
\hline TRP-96-420 & RL & 7.1 .3 & $6624-0$ & $\begin{array}{l}\text { Complete Design/Acceptance Test of New PC-Based Alarm } \\
\text { Monitoring System (AMS) at the AMS Mock-up (AMOSS) }\end{array}$ & 9/30/96 & & \\
\hline TRP-97-401 & RL & 7.1 .3 & $6624-0$ & Complete Waste Tank Integrity Assessment & 9/30/97 & 12/31/97 & TP-95-035 \\
\hline$T R P=97-402$ & RL. & 7.1 .3 & $.6624-0$ & Complete Annual FSAR Update & 9/30/97 & & \\
\hline$T R P-97-403$ & HO & 7.1 .3 & $6624-0$ & Begin Processing Solutions at PFP & $6 / 30 / 97$ & & \\
\hline TRP-97-404 & RL & 7.1 .3 & $6624-0$ & Complete Duct Terminal cleanout (TCO), Section $\# 4$ & $7 / 31 / 97$ & & \\
\hline$T R P=98=401$ & RL. & 7.1 .3 & $6624 \cdot 0$ & Complete Annual FSAR Update & $9 / 30 / 98$ & & \\
\hline TRP-99-401 & HQ & 7.1 .3 & $6624-0$ & Complete Stabilization of Remaining Solutions $(4,800 \mathrm{~L})$ at PFP & $1 / 31 / 99$ & & \\
\hline TRP-99-402 & HQ & 7.1 .3 & $6624-0$ & Start Restabilizing High Assay Oxides at PFP & $7 / 31 / 99$ & & \\
\hline TRP-99-403 & HQ & 7.1 .3 & $6624-0$ & Start Stabilization of Polycubes & $3 / 31 / 99$ & & \\
\hline$T R P-99 \cdot-404$ & RL & 7.1 .3 & $6624-0$ & Complete Annual FSAR Update & 9/30/99 & & \\
\hline TRP-99-405 & TPA - RL & 7.1 .3 & $6624-0$ & Complete 234-52 Ductwork Cleanout (M-83-02-T04) & 12/31/98 & & $M-83-94-1$ \\
\hline TRP-99-406 & TPA - RL & 7.1 .3 & $6624-0$ & Complete Identified Interim Actions (M-83-02) & $12 / 31 / 98$ & & $M-83-94-1$ \\
\hline$T R P-00-401$ & Ha & 7.1 .3 & $6624-0$ & $\begin{array}{l}\text { Complete Stabilization of Reactive Solid Residues (Sand, slag } \\
\& \text { crucible, poorly characterized items) at PFP }\end{array}$ & $1 / 31 / 00$ & & \\
\hline$T R P-00-402$ & Ha & 7.1 .3 & $6624-0$ & Complete Metal Repackaging at PFP & $9 / 30 / 00$ & & \\
\hline$T R P-00-403$ & RL & 7.1 .3 & $6624-0$ & Complete Annual FSAR Update & $9 / 30 / 00$ & & \\
\hline TRP $-00-404$ & HO & 7.1 .3 & $6624-0$ & Commence Repackaging Operations at PFP & $10 / 31 / 99$ & & . \\
\hline TRP $-01-401$ & HQ & 7.1 .3 & $6624-0$ & Complete Stabilization of Polycubes & $1 / 31 / 01$ & & \\
\hline TRP-01-402 & RL & 7.1 .3 & $6624-0$ & Complete Annual FSAR Update & $9 / 30 / 01$ & & \\
\hline TRP $-02-401$ & Ha & 7.1 .3 & $6624-0$ & $\begin{array}{l}\text { Complete Stabilization of and Packaging of all Remaining } \\
\text { Residues at PFP }\end{array}$ & $5 / 31 / 02$ & & \\
\hline
\end{tabular}


t

FY 1996 MYPP
TRANSI $\quad$ N PROJECTS

1.3.1/7.1/6.12

\begin{tabular}{|c|c|c|c|c|c|c|c|}
\hline $\begin{array}{l}\text { Control } \\
\text { Number }\end{array}$ & $\begin{array}{l}\text { Milestone } \\
\text { Type }\end{array}$ & RL WBS & ADS NUMBER & Milestone Description & $\begin{array}{l}\text { Original } \\
\text { Baseline } \\
\text { Date }\end{array}$ & $\begin{array}{l}\text { Revised } \\
\text { Basel ine } \\
\text { Date }\end{array}$ & $\begin{array}{l}\text { CIN } \\
\text { Change } \\
\text { Authority }\end{array}$ \\
\hline TRP-02-402 & HQ & 7.1 .3 & $6624-0$ & $\begin{array}{l}\text { Complete Thermal Stabilization/Repackaging of all } \mathrm{PuO}_{2} \text { to Meet } \\
\text { Metal/Oxide Storage Standard at PFP. }\end{array}$ & $5 / 31 / 02$ & & \\
\hline TRP $-02-403$ & RL & 7.1 .3 & $6624-0$ & Complete Annual FSAR Update & $9 / 30 / 02$ & & \\
\hline \multicolumn{8}{|c|}{ NEW FACILITY PLANNING } \\
\hline$T R P-96-500$ & RL & 7.1 .3 .6 & $6625-0$ & Hanford Material Container Ready for Use & $5 / 01 / 96$ & & \\
\hline TRP-99-501 & Ha & 7.1 .3 .6 & $6625-0$ & $\begin{array}{l}\text { Complete Design, Procurement \& Installation of new Repackaging } \\
\text { system at PFP }\end{array}$ & $12 / 31 / 98$ & & \\
\hline
\end{tabular}


TRANSI] N PROJECTS

FY 1996 MYPP

1.3.1/7.1/6.12

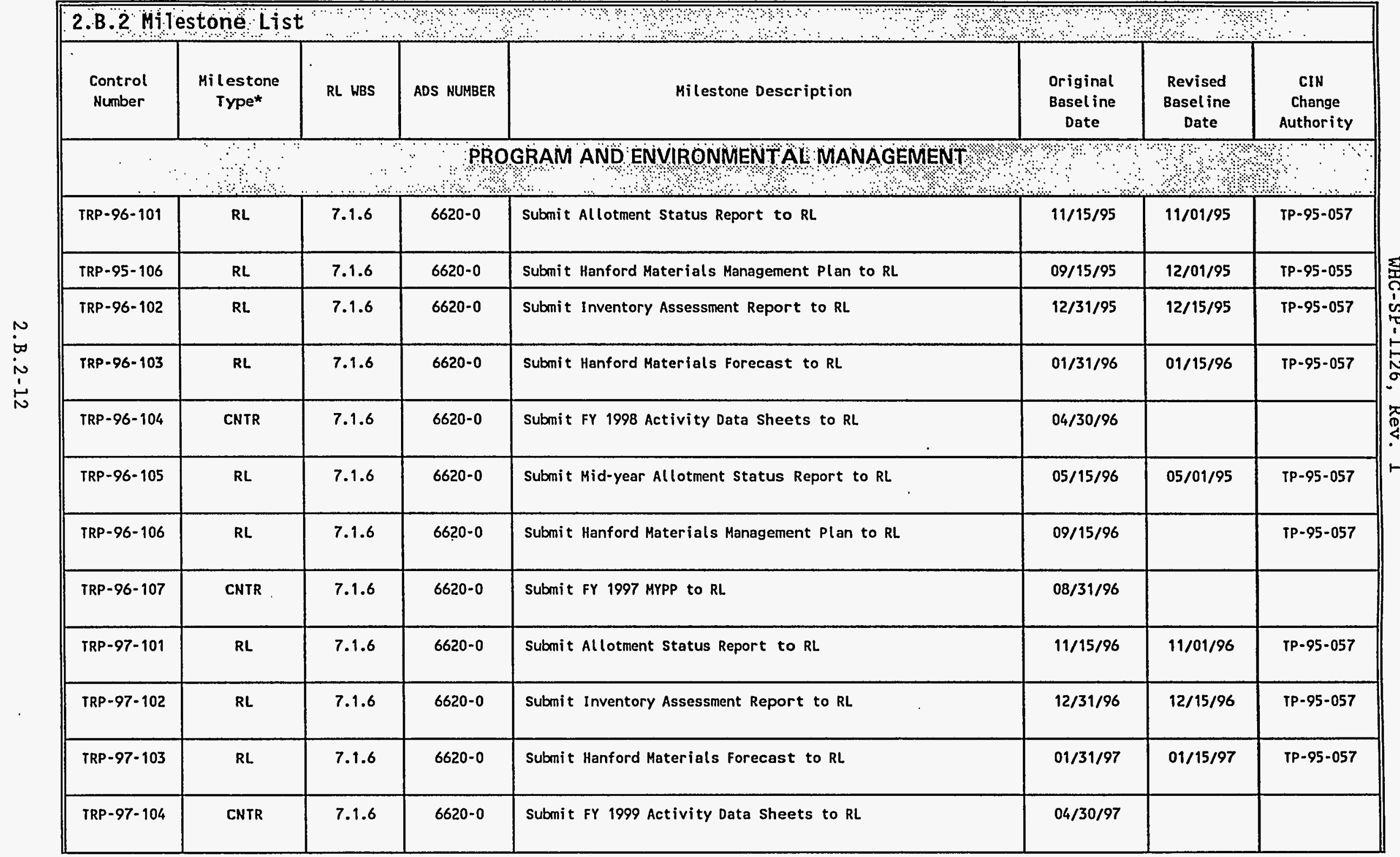


TRANSI: , N PROJECTS

FY 1996 MYPP

1.3.1/7.1/6.12

\begin{tabular}{|c|c|c|c|c|c|c|c|}
\hline $\begin{array}{l}\text { Control } \\
\text { Number }\end{array}$ & $\begin{array}{c}\text { Milestone } \\
\text { Type* }\end{array}$ & RL WBS & ADS NUMBER & Milestone Description & $\begin{array}{l}\text { Original } \\
\text { Basel ine } \\
\text { Date }\end{array}$ & $\begin{array}{c}\text { Revised } \\
\text { Basel ine } \\
\text { Date }\end{array}$ & $\begin{array}{l}\text { CIN } \\
\text { Chenge } \\
\text { Authority }\end{array}$ \\
\hline TRP-97-105 & RL & 7.1 .6 & $6620-0$ & Submit Mid-year Allotment Status Report to RL & $05 / 15 / 97$ & 05/01/97 & TP-95-057 \\
\hline TRP-97-106 & RL & 7.1 .6 & $6620-0$ & Submit Hanford Materials Management Plan to RL & $09 / 15 / 97$ & & TP-95-057 \\
\hline TRP-97-107 & CNTR & 7.1 .6 & $6620-0$ & Submit FY 1998 MYPP to RL & $08 / 31 / 97$ & & \\
\hline TRP-98-101 & RL & 7.1 .6 & $6620-0$ & Submit Allotment Status Report to RL & $11 / 15 / 97$ & 11/01/97 & TP-95-057 \\
\hline TRP-98-102 & RL & 7.1 .6 & $6620-0$ & Submit Inventory Assessment Report to RL & $12 / 31 / 97$ & 12/15/97 & $T P-95-057$ \\
\hline TRP-98-103 & RL & 7.1 .6 & $6620-0$ & Submit Hanford Materials Forecast to RL & $01 / 31 / 98$ & $01 / 15 / 98$ & $T P-95-057$ \\
\hline TRP-98-104 & CNTR & 7.1 .6 & $6620-0$ & Submit FY 2000 Activity Data Sheets to RL & $04 / 30 / 98$ & & \\
\hline TRP-98-105 & RL & 7.1 .6 & $6620-0$ & Submit Mid-year Allotment Status Report to RL & $05 / 15 / 98$ & 05/01/98 & $T P-95-057$ \\
\hline TRP-98-106 & RL & 7.1 .6 & $6620-0$ & Submit Hanford Materials Management Plan to RL & $09 / 15 / 98$ & & TP-95-057 \\
\hline TRP-98-107 & CNTR & 7.1 .6 & $6620-0$ & Submit FY 1999 MYPP to RL & $08 / 31 / 98$ & & \\
\hline TRP-99-101 & RL & 7.1 .6 & $6620-0$ & Submit Allotment Status Report to RL & $11 / 15 / 98$ & $11 / 01 / 98$ & TP-95-057 \\
\hline TRP-99-102 & RL & 7.1 .6 & $6620-0$ & Submit Inventory Assessment Report to RL & $12 / 31 / 98$ & $12 / 15 / 98$ & TP-95-057 \\
\hline TRP-99-103 & RL & 7.1 .6 & $6620-0$ & Submit Hanford Materials Forecast to RL. & 01/31/99 & $01 / 15 / 99$ & TP-95-057 \\
\hline
\end{tabular}


TRANSI: N PROJECTS

FY 1996 MYPP

1.3.1/7.1/6.12

\begin{tabular}{|c|c|c|c|c|c|c|c|}
\hline \multicolumn{8}{|c|}{ 2.B.2 Milestone List } \\
\hline $\begin{array}{l}\text { Control } \\
\text { Number }\end{array}$ & $\begin{array}{l}\text { Milestone } \\
\text { Type* }\end{array}$ & RL WBS & ADS NUMBER & Milestone Description & $\begin{array}{c}\text { Original } \\
\text { Basel ine } \\
\text { Date }\end{array}$ & $\begin{array}{c}\text { Revised } \\
\text { Baseline } \\
\text { Date }\end{array}$ & $\begin{array}{c}\text { CIN } \\
\text { Change } \\
\text { Authority }\end{array}$ \\
\hline TRP-99-104 & CNTR & 7.1 .6 & $6620-0$ & Submit FY 2001 Activity Data Sheets to RL & $04 / 30 / 99$ & & \\
\hline TRP-99-105 & RL & 7.1 .6 & $6620-0$ & Submit Mid-year Allotment Status Report to RL & $05 / 1.15 / 99$ & 05/01/99 & $T P-95-057$ \\
\hline TRP-90-106 & RL & 7.1 .6 & $6620-0$ & Submit Hanford Materials Management Plan to RL & $09 / 15 / 99$ & & $T P-95-057$ \\
\hline TRP-99-107 & CNTR & 7.1 .6 & $6620-0$ & Submit FY $2000 \mathrm{MYPP}$ to RL. & $08 / 31 / 99$ & & \\
\hline$T R P-00-101$ & RL & 7.1 .6 & $6620-0$ & Subrmit Allotment Status Report to RL & $11 / 15 / 99$ & $11 / 01 / 99$ & $T P-95-057$ \\
\hline$T R P-00-102$ & RL & 7.1 .6 & $6620-0$ & Submit Inventory Assessment Report to RL & $12 / 31 / 99$ & $12 / 15 / 99$ & $T P-95-057$ \\
\hline$T R P-00-103$ & RL & 7.1 .6 & $6620-0$ & Submit Hanford Materials Forecast to RL & $01 / 31 / 2000$ & $01 / 15 / 00$ & TP-95-057 \\
\hline TRP $-00-104$ & CNTR & 7.1 .6 & $6620-0$ & Submit FY 2002 Activity Data Sheets to RL & $04 / 30 / 2000$ & & \\
\hline$T R P-00-105$ & RL & 7.1 .6 & $6620-0$ & Submit Mid-year Allotment Status Report to RL & $05 / 15 / 2000$ & $05 / 01 / 00$ & $T P-95-057$ \\
\hline$T R P-00-106$ & RL & 7.1 .6 & $6620-0$ & Submit Manford Materials Management Plan to RL & $09 / 15 / 2000$ & & $T P-95-057$ \\
\hline TRP-00-107 & CNTR & 7.1 .6 & $6620-0$ & Submit FY 2001 MYPP to RL & $08 / 31 / 2000$ & & \\
\hline TRP-01-101 & RL & 7.1 .6 & $6620-0$ & Submit Allotment Status Report to RL & $11 / 15 / 2000$ & $11 / 01 / 00$ & TP-95-057 \\
\hline TRP-01-102 & RL & 7.1 .6 & $6620-0$ & Submit Inventory Assessment Report to RL & $12 / 31 / 2000$ & $12 / 15 / 00$ & TP-95-057 \\
\hline
\end{tabular}


FY 1996 MYPP

TRANSII $N$ PROJECTS

1.3.1/7.1/6.12

\begin{tabular}{|c|c|c|c|c|c|c|c|}
\hline \multicolumn{8}{|c|}{$2 \cdot B_{2}$ Milestone lost } \\
\hline $\begin{array}{l}\text { Control } \\
\text { Number }\end{array}$ & $\begin{array}{c}\text { Milestone } \\
\text { Type* }\end{array}$ & RL WBS & ADS NUMBER & Milestone Description & $\begin{array}{c}\text { Original } \\
\text { Basel ine } \\
\text { Date }\end{array}$ & $\begin{array}{c}\text { Revised } \\
\text { Basel ine } \\
\text { Date }\end{array}$ & $\begin{array}{l}\text { CIN } \\
\text { Change } \\
\text { Authority }\end{array}$ \\
\hline TRP-01-103 & RL & 7.1 .6 & $6620-0$ & Submit Hanford Materials Forecast to RL & $01 / 31 / 2001$ & $01 / 15 / 01$ & $T P-95-057$ \\
\hline TRP-01-104 & CNTR & 7.1 .6 & $6620-0$ & Submit FY 2003 Activity Data Sheets to RL & $04 / 30 / 2001$ & & \\
\hline TRP-01-105 & RL & 7.1 .6 & $6620-0$ & Submit Mid-year Allotment Status Report to RL & $05 / 15 / 2001$ & 05/01/01 & $T P-95-057$ \\
\hline TRP-01-106 & RL & 7.1 .6 & $6620-0$ & $\begin{array}{l}\text { Submit Hanford Materials Management Plan to RL } \\
\text { - }\end{array}$ & $09 / 15 / 2001$ & & $T P-95-057$ \\
\hline TRP-01-107 & CNTR & 7.1 .6 & $6620-0$ & Submit FY 2002 MYPP to RL & $08 / 31 / 2001$ & & \\
\hline TRP-02-101 & RL & 7.1 .6 & $6620-0$ & Submit Allotment Status Report to RL & $11 / 15 / 2001$ & $11 / 01 / 01$ & $T P-95-057$ \\
\hline TRP-02-102 & RL & 7.1 .6 & $6620-0$ & Submit Inventory Assessment Report to RL & $12 / 31 / 2001$ & $12 / 15 / 01$ & TP-95-057 \\
\hline TRP-02-103 & RL & 7.1 .6 & $6620-0$ & Submit Hanford Materials Forecast to RL & $01 / 31 / 2002$ & $01 / 15 / 02$ & TP-95-057 \\
\hline TRP-02-104 & CNTR & 7.1 .6 & $6620-0$ & Submit FY 2004 Activity Data Sheets to RL. & $04 / 30 / 2002$ & & \\
\hline TRP-02-105 & $\mathrm{RL}$ & 7.1 .6 & $6620-0$ & Submit Mid-year Alloṭent Status Report to RL & 05/15/2002 & $05 / 01 / 02$ & $T P-95-057$ \\
\hline TRP-02-106 & RL. & 7.1 .6 & $6620-0$ & Submit Hanford Materials Management Plan to RL. & $09 / 15 / 2002$ & & $T P-95-057$ \\
\hline TRP-02-107 & CNTR & 7.1 .6 & $6620-0$ & Submit FY 2003 MYPP to RL: & $08 / 31 / 2002$ & & \\
\hline
\end{tabular}


FY 1996 MYPP

WHC-SP-1126, Rev. 1

TRANSITION PROJECTS

1.3.1/7.1/6.12

\begin{tabular}{||l|l||}
\hline \multicolumn{3}{|c|}{ Westinghouse Hanford Company } \\
MILESTONE DESCRIPTION SHEET
\end{tabular}


WHC-SP-1126, Rev. I

FY 1996 MYPP

TRANSITION PROJECTS

1.3.1/7.1/6.12

\begin{tabular}{|c|c|c|c|}
\hline \multicolumn{4}{|c|}{$\begin{array}{l}\text { Westinghouse Hanford Company } \\
\text { MILESTONE DESCRIPTION SHEET }\end{array}$} \\
\hline \multicolumn{3}{|c|}{ Title: Complete B Plant Tank Integrity Assessment } & Date: $08 / 31 / 94$ \\
\hline \multicolumn{3}{|c|}{ Assigned To: Transition Projects / B Plant Complex } & CIN: \\
\hline \multicolumn{3}{|c|}{ Program WBS Designator: $1.3 .1 .7(1 \mathrm{~KB})$} & Due Date: $12 / 31 / 95$ \\
\hline \multicolumn{3}{|c|}{ Control Number: TRP-96-601 } & Rev: Original \\
\hline MILESTONE TYPE: & DIVISION: & DELIVERABLE: & ADDRESS TO: \\
\hline $\begin{array}{l}\square \text { DOE-HQ } \\
\text { X DOE-RL } \\
\square \text { CONTRACTOR }\end{array}$ & $\begin{array}{l}\square \text { State } \\
\square \text { Federal } \\
\square \text { DOE } \\
\square \text { RCRA } \\
\text { X TPA Number } \\
\text { M-32-07-T04 }\end{array}$ & $\begin{array}{l}\square \text { Report } \\
\text { X Letter } \\
\square \text { Drawings } \\
\square \text { Other (specify) }\end{array}$ & $\begin{array}{l}\square \text { DOE-HQ } \\
\text { X DOE-RL } \\
\square \text { Other (specify) }\end{array}$ \\
\hline \multicolumn{4}{|l|}{ Milestone Description: } \\
\hline \multicolumn{4}{|c|}{$\begin{array}{l}\text { Perform external visual inspection and structural integrity test of tanks } 24-1 \text { and } 24-2 \text { and } \\
\text { associated tank supports and prepare and submit a report of findings. }\end{array}$} \\
\hline \multirow{2}{*}{\multicolumn{4}{|c|}{$\begin{array}{l}\text { Description of what constitutes completion of this milestone: } \\
\text { Transmittal of the report to RL } 10 \text { working days }(12 / 14 / 95) \text { prior to the above date will } \\
\text { constitute completion of this milestone. The submission to RL on } 12 / 14 / 95 \text { allows for } \\
\text { concurrence and submittal of the report to the Department of Ecology by the Tri-Party } \\
\text { Agreement milestone date of } 12 / 31 / 95 \text {. }\end{array}$}} \\
\hline & & & \\
\hline \multirow{2}{*}{\multicolumn{2}{|c|}{$\begin{array}{l}\text { Cost Account Manager } \\
\text { S. E. Killoy }\end{array}$}} & \multirow{2}{*}{\multicolumn{2}{|c|}{$\begin{array}{l}\text { Program/Project Manager } \quad \text { Date } \\
\text { R. E. Heineman, Jr. }\end{array}$}} \\
\hline & & & \\
\hline \multicolumn{2}{|c|}{$\begin{array}{l}\text { Program Element Manager } \\
\text { D. W. Wilson }\end{array}$} & \multicolumn{2}{|c|}{$\begin{array}{ll}\text { DOE Monitor } & \text { Date } \\
\text { D. T. Evans } & \\
\end{array}$} \\
\hline
\end{tabular}


WHC-SP-1126, Rev. I

FY 1996 MYPP

TRANSITION PROJECTS

1.3.1/7.1/6.12

\begin{tabular}{|c|c|c|c|}
\hline \multicolumn{4}{|c|}{$\begin{array}{l}\text { Westinghouse Hanford Company } \\
\text { MICESTONE DESCRIPTION SHEET }\end{array}$} \\
\hline \multicolumn{3}{|c|}{$\begin{array}{l}\text { Title: Complete Chemical Separation of Radionuclides from Legacy } \\
\text { Organics in B Plant }\end{array}$} & Date: $9 / 8 / 95$ \\
\hline \multicolumn{3}{|c|}{ Assigned To: Transition Projects / B Plant Complex } & CIN: \\
\hline \multicolumn{3}{|c|}{ Program WBS Designator: $1 \cdot 3.1 .7(1 \mathrm{~KB})$} & Due Date:12/31/95 \\
\hline \multicolumn{3}{|c|}{ Control Number: TRP-96-606 } & Rev: Original \\
\hline $\begin{array}{l}\text { MILESTONE TYPE: } \\
\square \text { DOE-HQ } \\
\text { X DOE-RL } \\
\square \text { CONTRACTOR }\end{array}$ & 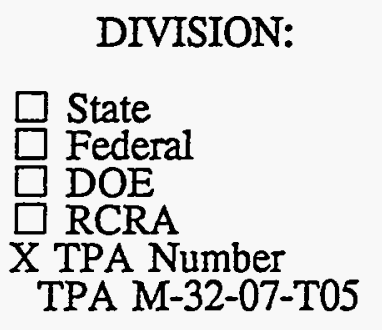 & $\begin{array}{l}\text { DELIVERÁBLE: } \\
\square \text { Report } \\
\text { X Letter } \\
\square \text { Drawings } \\
\square \text { Other (specify) }\end{array}$ & $\begin{array}{l}\text { ADDRESS TO: } \\
\square \text { DOE-HQ } \\
\text { X DOE-RL } \\
\square \text { Other (specify) }\end{array}$ \\
\hline \multicolumn{4}{|c|}{$\begin{array}{l}\text { Milestone Description: } \\
\text { Perform chemical separation of radionuclides from the organic solvent waste to support a future } \\
\text { engineering study of alternatives regarding disposition of the organic solvent waste. }\end{array}$} \\
\hline \multicolumn{4}{|c|}{$\begin{array}{l}\text { Description of what constitutes completion of this milestone: } \\
\text { Radionuclide contamination has been removed from the legacy organics in sufficient quantities } \\
\text { as to render the organics suitable for shipping as Low Specific Activity material as defined by } \\
\text { Department of Transportation shipping regulations. }\end{array}$} \\
\hline \multicolumn{2}{|l|}{$\begin{array}{l}\text { Cost Account Manager } \\
\text { W. A. Holstein }\end{array}$} & \multicolumn{2}{|c|}{$\begin{array}{l}\text { Program/Project Manager } \\
\text { R. E. Heineman, Jr. } \\
\end{array}$} \\
\hline \multicolumn{2}{|c|}{$\begin{array}{l}\text { Program Element Manager } \\
\text { W. A. Holstein }\end{array}$} & \multicolumn{2}{|c|}{$\begin{array}{l}\text { DOE Monitor Date } \\
\text { D. T. Evans }\end{array}$} \\
\hline
\end{tabular}


WHC-SP-1126, Rev. I

FY 1996 MYPP

TRANSITION PROJECTS

1.3.1/7.1/6.12

\begin{tabular}{|c|c|c|c|}
\hline \multicolumn{4}{|c|}{$\begin{array}{l}\text { Westinghouse Hanford Company } \\
\text { MILESTONE DESCRIPTION SHEET }\end{array}$} \\
\hline \multicolumn{3}{|c|}{ Title: Award a Contract for WESF Ion Exchange Services } & Date: $08 / 14 / 95$ \\
\hline \multicolumn{3}{|c|}{ Assigned To: Transition Projects / B Plant Complex } & CIN: \\
\hline \multicolumn{3}{|c|}{ Program WBS Designator: 1.3.1.7 (1KB) } & Due Date:01/31/96 \\
\hline \multicolumn{3}{|c|}{ Control Number: TRP-96-602 } & Rev: Original \\
\hline $\begin{array}{l}\text { MILESTONE TYPE: } \\
\text { D DOE-HQ } \\
\text { X DOE-RL } \\
\text { a DOE-FO } \\
\text { C CONTRACTOR }\end{array}$ & $\begin{array}{l}\quad \text { DIVISION: } \\
\text { 口 State } \\
\text { G Federal } \\
\text { x DOE } \\
\text { a RCRA } \\
\square \text { TPA Number }\end{array}$ & $\begin{array}{l}\text { DELIVERABLE: } \\
\square \text { Report } \\
\text { x Letter } \\
\text { a Drawings } \\
\square \text { Other (specify) }\end{array}$ & $\begin{array}{l}\text { ADDRESS TO: } \\
\text { D DOE-HQ } \\
\text { x DOE-RL } \\
\text { Q Other (specify) }\end{array}$ \\
\hline \multicolumn{4}{|c|}{$\begin{array}{l}\text { Milestone Description: } \\
\text { Obtain a service contract for Ion Exchange Services for the WESF pool cell. }\end{array}$} \\
\hline \multicolumn{4}{|c|}{$\begin{array}{l}\text { Description of what constitutes completion of this milestone: } \\
\text { Issuance of a contract to provide Ion exchange services for the WESF pool cells in the event of } \\
\text { a capsule leak. }\end{array}$} \\
\hline \multicolumn{2}{|l|}{$\begin{array}{l}\text { Cost Account Manager } \\
\text { M. M. Pereira }\end{array}$} & \multicolumn{2}{|c|}{$\begin{array}{l}\text { Program/Project Manager } \quad \text { Date } \\
\text { R. E. Heineman, Jr. }\end{array}$} \\
\hline \multicolumn{2}{|c|}{$\begin{array}{l}\text { Program Element Manager } \\
\text { W. A. Holstein }\end{array}$} & \multicolumn{2}{|c|}{$\begin{array}{ll}\text { DOE Monitor } & \text { Date } \\
\text { D. T. Evans } & \\
\end{array}$} \\
\hline
\end{tabular}


WHC-SP-1126, Rev. 1

FY 1996 MYPP

TRANSITION PROJECTS

1.3.1/7.1/6.12

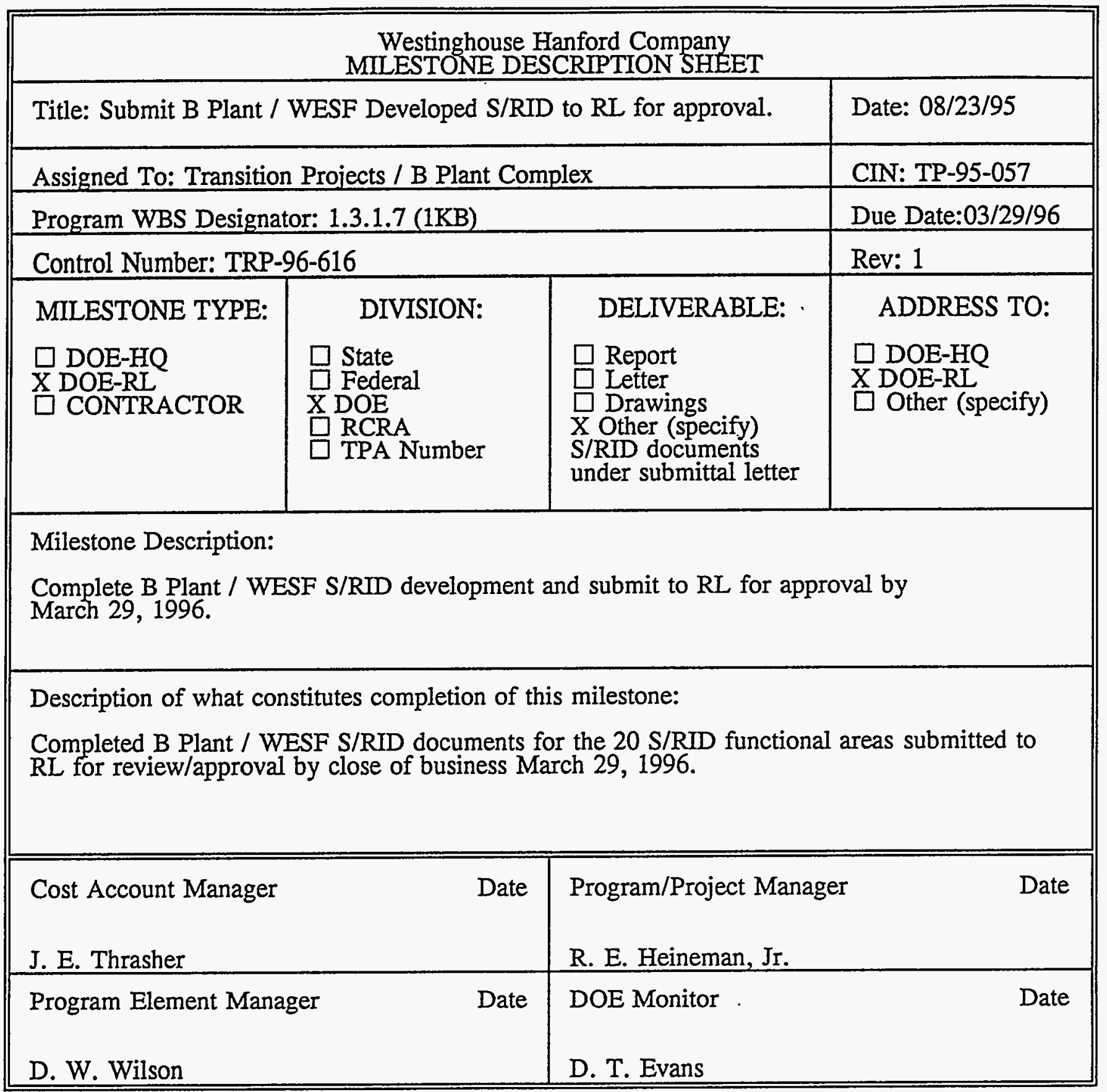


FY 1996 MYPP

\section{TRANSITION PROJECTS}

\subsection{1/7.1/6.12}

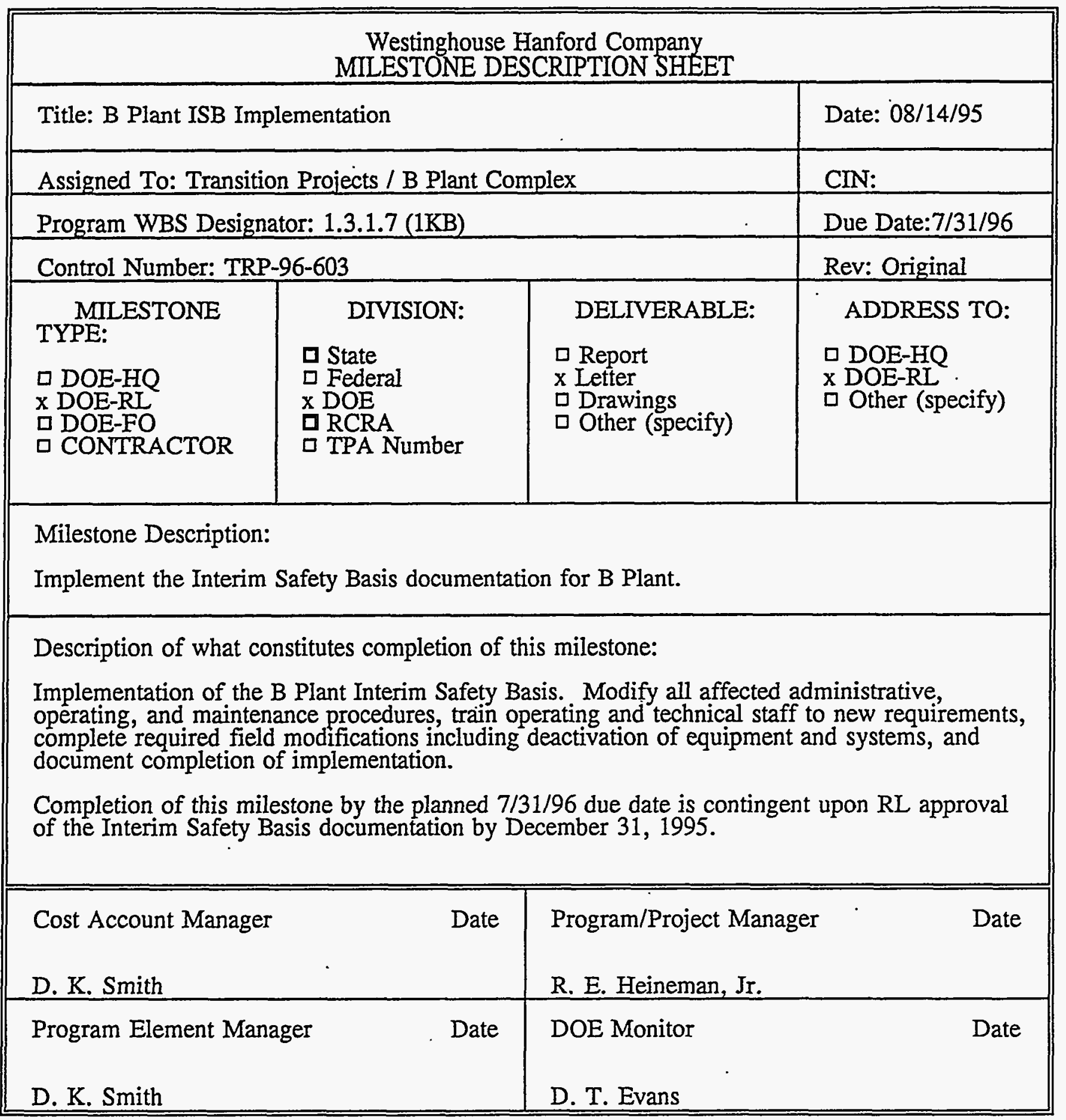


FY 1996 MYPP

\section{TRANSITION PROJECTS}

\subsection{1/7.1/6.12}

\begin{tabular}{|c|c|c|c|}
\hline \multicolumn{4}{|c|}{$\begin{array}{l}\text { Westinghouse Hanford Company } \\
\text { MILESTONE DESCRIPTION SHEET }\end{array}$} \\
\hline \multicolumn{3}{|c|}{ Title: Complete Organic Removal } & Date: $08 / 14 / 95$ \\
\hline \multicolumn{3}{|c|}{ Assigned To: Transition Projects / B Plant Complex } & CIN:TP-95-057 \\
\hline \multicolumn{3}{|c|}{ Program WBS Designator: 1.3.1.7 (1KB) } & $\begin{array}{l}\text { Due Date: } \\
09 / 30 / 96\end{array}$ \\
\hline \multicolumn{3}{|c|}{ Control Number: TRP-98-603 } & Rev: 1 \\
\hline $\begin{array}{l}\text { MILESTONE } \\
\text { TYPE: } \\
\text { प DOE-HQ } \\
\text { X DOE-RL } \\
\text { Q DOE-FO } \\
\square \text { CONTRACTOR }\end{array}$ & $\begin{array}{l}\text { DIVISION: } \\
\text { a State } \\
\text { Federal } \\
\text { x DOE } \\
\text { RCRA } \\
\text { RPA Number }\end{array}$ & $\begin{array}{l}\text { DELIVERABLE: } \\
\text { a Report } \\
\text { x Letter } \\
\text { a Drawings } \\
\square \text { Other (specify) }\end{array}$ & $\begin{array}{l}\text { ADDRESS TO: } \\
\text { a DOE-HQ } \\
\text { x DOE-RI } \\
\text { ם Other (specify) }\end{array}$ \\
\hline \multicolumn{4}{|c|}{$\begin{array}{l}\text { Milestone Description: } \\
\text { Transfer organics from the B Plant canyon to the } 211-\mathrm{B} \text { or off plant location. }\end{array}$} \\
\hline \multicolumn{4}{|c|}{$\begin{array}{l}\text { Description of what constitutes completion of this milestone: } \\
\text { Transfer the radioactive flammable legacy organics from two tanks inside B Plant Canyon to } \\
\text { compliant storage outside the Canyon. Completion of the milestone will occur when the } \\
\text { organic liquid is removed from tanks TK-27-3 and TK-28-3, and placed into compliant storage } \\
\text { outside of the Canyon. This milestone does not include the removal of the existing storage } \\
\text { tanks, transfer systems, or deactivation of systems currently supporting the storage of organics } \\
\text { in the Canyon. }\end{array}$} \\
\hline \multicolumn{2}{|c|}{ Cost Account Manager Date } & \multicolumn{2}{|c|}{$\begin{array}{l}\text { Program/Project Manager } \\
\text { R. E. Heineman, Jr. }\end{array}$} \\
\hline \multicolumn{2}{|c|}{$\begin{array}{l}\text { Program Element Manager } \\
\text { W. A. Holstein }\end{array}$} & \multicolumn{2}{|l|}{$\begin{array}{l}\text { DOE Monitor } \\
\text { D. T. Evans }\end{array}$} \\
\hline
\end{tabular}


WHC-SP-1126, Rev. I

\section{TRANSITION PROJECTS}

FY 1996 MYPP

1.3.1/7.1/6.12

\section{Westinghouse Hanford Company} MILESTONE DESCRIPTION SHEET

Title: Submit B Plant / WESF S/RID Phase I (Administrative) Assessment Report to DOE/RL.

Assigned To: Transition Projects / B Plant Complex

Program WBS Designator: 1.3.1.7 (1KB)

Control Number: TRP-96-618

MILESTONE TYPE:

$\square$ DOE-HQ

X DOE-RL

$\square$ CONTRACTOR

\begin{tabular}{|l|}
\hline DIVISION: \\
$\square$ State \\
$\square$ Federal \\
X DOE \\
$\square$ RCRA \\
$\square$ TPA Number \\
\end{tabular}

DELIVERABLE:

$\square$ Report

$\square$ Letter

Drawings

$X$ Other (specify)

S/RID Phase I

Assessment Report

under cover letter.

Milestone Description:

Submit completed B Plant / WESF S/RID Phase I Assessment to RL by close of business September 30, 1996.

Description of what constitutes completion of this milestone:

Submittal of completed B Plant / WESF S/RID Phase I Assessment Report with transmittal letter by close of business on September 30, 1996.

\begin{tabular}{||ll|lr||}
\hline \hline Cost Account Manager & Date & Program/Project Manager & Date \\
J. E. Thrasher & & R. E. Heineman, Jr. & \\
\hline Program Element Manager & Date & DOE Monitor & Date \\
D. W. Wilson & & D. T. Evans & \\
\hline
\end{tabular}


WHC-SP-1126, Rev. 1

FY 1996 MYPP

TRANSITION PROJECTS

1.3.1/7.1/6.12

\begin{tabular}{|c|c|c|c|}
\hline \multicolumn{4}{|c|}{$\begin{array}{l}\text { Westinghouse Hanford Company } \\
\text { MILESTONE DESCRIPTION SHEET }\end{array}$} \\
\hline \multicolumn{3}{|c|}{ Title: B Plant / WESF Procedures Improvement } & Date: 08/14/94 \\
\hline \multicolumn{3}{|c|}{ Assigned To: Transition Projects / B Plant Complex } & CIN: \\
\hline \multicolumn{3}{|c|}{ Program WBS Designator: 1.3.1.7 (1KB) } & $\begin{array}{l}\text { Due Date: } \\
\text { 09/30/96 }\end{array}$ \\
\hline \multicolumn{3}{|c|}{ Control Number: TRP-96-604 } & Rev: Original \\
\hline $\begin{array}{l}\text { MILESTONE } \\
\text { TYPE: } \\
\text { Q DOE-HQ } \\
\text { X DOE-RI } \\
\text { D DOE-FO } \\
\square \text { CONTRACTOR }\end{array}$ & $\begin{array}{l}\quad \text { DIVISION: } \\
\text { a State } \\
\text { G Federal } \\
\text { X DOE } \\
\text { वCRA } \\
\square \text { TPA Number }\end{array}$ & $\begin{array}{l}\text { DELIVERABLE: } \\
\text { a Report } \\
\text { a Letter } \\
\text { Drawings } \\
\text { X Other (specify) } \\
\text { Closed out HATS } \\
\text { items }\end{array}$ & $\begin{array}{l}\text { ADDRESS TO: } \\
\text { a DOE-HQ } \\
\text { a DOE-RL } \\
\text { X Other (specify) } \\
\text { RE Heineman, Jr. }\end{array}$ \\
\hline \multicolumn{4}{|c|}{$\begin{array}{l}\text { Revise procedures for B Plant / WESF to comply with WHC-CM-3-5, Section } 12.5 \text { and } \\
\text { subsequently closeout all Hanford Action Tracking System items per the Procedure Action Plan } \\
\text { issued as an attachment to letter 1690-KAH-95-014, KA Hedquist to RE Heineman, Jr. }\end{array}$} \\
\hline \multicolumn{4}{|c|}{$\begin{array}{l}\text { Description of what constitutes completion of this milestone: } \\
\text { Closeout of HATS items as identified in the above letter. }\end{array}$} \\
\hline \multicolumn{2}{|c|}{ Cost Account Manager Date } & \multicolumn{2}{|c|}{ Program/Project Manager Date } \\
\hline \multicolumn{2}{|c|}{$\begin{array}{l}\text { Program Element Manager } \\
\text { D. K. Smith }\end{array}$} & \multicolumn{2}{|l|}{$\begin{array}{l}\text { DOE Monitor } \\
\text { D. T. Evans }\end{array}$} \\
\hline
\end{tabular}




\section{TRANSITION PROJECTS}

FY 1996 MYPP

1.3.1/7.1/6.12

\begin{tabular}{|c|c|c|c|}
\hline \multicolumn{4}{|c|}{$\begin{array}{l}\text { Westinghouse Hanford Company } \\
\text { MILESTONE DESCRIPTION SHEET }\end{array}$} \\
\hline \multicolumn{3}{|c|}{$\begin{array}{l}\text { Title: Projected FY } 97 \text { B Plant S\&M Costs Reduced by } 20 \% \text { Compared } \\
\text { with FY } 96\end{array}$} & Date: $08 / 14 / 95$ \\
\hline \multicolumn{3}{|c|}{ Assigned To: Transition Projects / B Plant Complex } & CIN: \\
\hline \multicolumn{3}{|c|}{ Program WBS Designator: 1.3.1.7 (1KB) } & Due Date: $10 / 1 / 96$ \\
\hline \multicolumn{3}{|c|}{ Control Number: TRP-97-601 } & Rev: Original \\
\hline $\begin{array}{l}\text { MILESTONE } \\
\text { TYPE: } \\
\text { a DOE-HQ } \\
\text { प DOE-RL } \cdot \\
\text { X DOE-FO } \\
\square \text { CONTRACTOR }\end{array}$ & $\begin{array}{l}\quad \text { DIVISION: } \\
\text { a State } \\
\text { G Federal } \\
\text { x DOE } \\
\text {. RCRA } \\
\square \text { TPA Number }\end{array}$ & $\begin{array}{l}\text { DELIVERABLE: } \\
\text { Report } \\
\text { X Letter } \\
\text { व Drawings } \\
\square \text { Other (specify) }\end{array}$ & $\begin{array}{l}\text { ADDRESS TO: } \\
\text { Q DOE-HQ } \\
\text { x DOE-RI } \\
\text { 口 Other (specify) }\end{array}$ \\
\hline \multicolumn{4}{|c|}{$\begin{array}{l}\text { Milestone Description: } \\
\text { The projected overall Fiscal Year } 1997 \text { costs for Surveillance and Maintenance of B Plant will } \\
\text { be reduced by } 20 \% \text { when compared to the Fiscal Year } 1996 \text { costs. }\end{array}$} \\
\hline \multicolumn{4}{|c|}{$\begin{array}{l}\text { Description of what constitutes completion of this milestone: } \\
\text { The projected annual Surveillance and Maintenance costs for B Plant have been reduced as a } \\
\text { result of completion of Accelerated Hazard Reduction activities and other initiatives. }\end{array}$} \\
\hline \multicolumn{2}{|c|}{$\begin{array}{l}\text { Cost Account Manager Date } \\
\text { D. K. Smith }\end{array}$} & \multicolumn{2}{|c|}{$\begin{array}{l}\text { Program/Project Manager } \\
\text { R. E. Heineman, Jr. }\end{array}$} \\
\hline \multicolumn{2}{|c|}{$\begin{array}{l}\text { Program Element Manager } \\
\text { D. K. Smith }\end{array}$} & \multicolumn{2}{|l|}{$\begin{array}{l}\text { DOE Monitor } \\
\text { D. T. Evans }\end{array}$} \\
\hline
\end{tabular}


WHC-SP-1126, Rev. 1

FY 1996 MYPP

TRANSITION PROJECTS

1.3.1/7.1/6.12

\begin{tabular}{|c|c|c|c|}
\hline \multicolumn{4}{|c|}{$\begin{array}{l}\text { Westinghouse Hanford Company } \\
\text { MILESTONE DESCRIPTION SHEET }\end{array}$} \\
\hline \multicolumn{3}{|c|}{$\begin{array}{l}\text { Title: Projected FY98 B Plant S\&M Costs Reduced by } 50 \% \text { Compared } \\
\text { with FY } 96\end{array}$} & Date: $08 / 14 / 95$ \\
\hline \multicolumn{3}{|c|}{ Assigned To: Transition Projects / B Plant Complex } & CIN: \\
\hline \multicolumn{3}{|c|}{ Program WBS Designator: 1.3.1.7 (1KB) } & Due Date: $10 / 1 / 97$ \\
\hline \multicolumn{3}{|c|}{ Control Number: TRP-98-604 } & Rev: Original \\
\hline $\begin{array}{l}\text { MILESTONE } \\
\text { TYPE: } \\
\text { DOE-HQ } \\
\text { D DOE-RI } \\
\text { X DOE-FO } \\
\square \text { CONTRACTOR }\end{array}$ & $\begin{array}{l}\quad \text { DIVISION: } \\
\text { a State } \\
\text { Gederal } \\
\times \text { DOE } \\
\text { Q RCRA } \\
\square \text { TPA Number }\end{array}$ & $\begin{array}{l}\text { DELIVERABLE: } \\
\text { Q Report } \\
\text { x Letter } \\
\text { a Drawings } \\
\square \text { Other (specify) }\end{array}$ & $\begin{array}{l}\text { ADDRESS TO: } \\
\text { व DOE-HQ } \\
\text { x DOE-RI } \\
\text { 口 Other (specify) }\end{array}$ \\
\hline \multicolumn{4}{|c|}{$\begin{array}{l}\text { Milestone Description: } \\
\text { The projected overall Fiscal Year } 1998 \text { costs for Surveillance and Maintenance of B Plant will } \\
\text { be reduced by } 50 \% \text { when compared to the Fiscal Year } 1995 \text { costs. }\end{array}$} \\
\hline \multicolumn{4}{|c|}{$\begin{array}{l}\text { Description of what constitutes completion of this milestone: } \\
\text { The projected annual Surveillance and Maintenance costs for B Plant have been reduced as a } \\
\text { result of completion of Accelerated Hazard Reduction activities and other initiatives. }\end{array}$} \\
\hline \multicolumn{2}{|c|}{ Cost Account Manager Date } & \multicolumn{2}{|c|}{$\begin{array}{l}\text { Program/Project Manager } \\
\text { R. E. Heineman, Jr. }\end{array}$} \\
\hline \multicolumn{2}{|c|}{$\begin{array}{l}\text { Program Element Manager } \\
\text { D. K. Smith }\end{array}$} & \multicolumn{2}{|l|}{$\begin{array}{l}\text { DOE Monitor } \\
\text { D. T. Evans }\end{array}$} \\
\hline
\end{tabular}


WHC-SP-1126, Rev. I

FY 1996 MYPP

\section{TRANSITION PROJECTS}

1.3.1/7.1/6.12

Westinghouse Hanford Company

MILESTONE DESCRIPTION SHEET

Title: Canyon Exhaust Filter Isolation and Stabilization

Assigned To: Transition Projects / B Plant Complex

Program WBS Designator: 1.3.1.7 (1KB)

Control Number: TRP-98-605

MILESTONE TYPE:

$\square$ DOE-HQ

$X$ DOE-RL

CONTRACTOR

\begin{tabular}{|l|} 
DIVISION: \\
$\square$ State \\
Federal \\
XOE \\
$\square$ RCRA \\
$\square$ TPA Number \\
\hline
\end{tabular}

Date: $8 / 29 / 95$

CIN:

Due Date:11/01/97

Rev: Original

DELIVERABLE:

$X$ Report

$\square$ Letter

Drawings

Other (specify)

ADDRESS TO:

口DOE-HQ

X DOE-RI

$\checkmark$ Other (specify)

Milestone Description:

The sand filter system for the B Plant canyon exhaust filtration system have been deactivated and stabilized awaiting final decommissioning.

Description of what constitutes completion of this milestone:

All work packages for the deactivation of the sand filter for the B Plant exhaust system have been completed and closed out.

\begin{tabular}{||ll|ll||}
\hline \hline Cost Account Manager & Date & Program/Project Manager & Date \\
W. A. Holstein & & R. E. Heineman, Jr. & \\
\hline Program Element Manager & Date & DOE Monitor & Date \\
W. A. Holstein & & D. T. Evans & \\
\hline
\end{tabular}


WHC-SP-1126, Rev. 1

\section{TRANSITION PROJECTS}

FY 1996 MYPP

1.3.1/7.1/6.12

\begin{tabular}{|c|c|c|c|}
\hline \multicolumn{4}{|c|}{$\begin{array}{l}\text { Westinghouse Hanford Company } \\
\text { MILESTONE DESCRIPTION SHEET }\end{array}$} \\
\hline \multicolumn{3}{|c|}{$\begin{array}{l}\text { Title: Complete Definitive Design B Plant Safety Class Vent Upgrade } \\
(\text { W-059) }\end{array}$} & Date: $8 / 29 / 95$ \\
\hline \multicolumn{3}{|c|}{ Assigned To: Transition Projects / B Plant Complex } & CIN: \\
\hline \multicolumn{3}{|c|}{ Program WBS Designator: $1.3 .1 .7(1 \mathrm{~KB})$} & Due Date:01/31/98 \\
\hline \multicolumn{3}{|c|}{ Control Number: TRP-98-602 } & Rev: Original \\
\hline $\begin{array}{l}\text { MILESTONE TYPE: } \\
\square \text { DOE-HQ } \\
\text { X DOE-RL } \\
\square \text { CONTRACTOR }\end{array}$ & 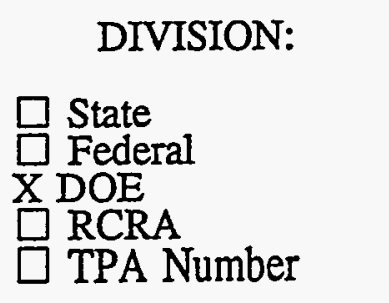 & $\begin{array}{l}\text { DELIVERABLE: } \\
\text { R Report } \\
\text { L Letter } \\
\text { X Drawings } \\
\square \text { Other (specify) }\end{array}$ & $\begin{array}{l}\text { ADDRESS TO: } \\
\square \text { DOE-HQ } \\
\text { X DOE-RI } \\
\square \text { Other (specify) }\end{array}$ \\
\hline \multirow{2}{*}{\multicolumn{4}{|c|}{$\begin{array}{l}\text { Milestone Description: } \\
\text { Complete the definitive design for the upgrading of the B Plant Safety Class Ventilation system. }\end{array}$}} \\
\hline & & & \\
\hline \multirow{2}{*}{\multicolumn{4}{|c|}{$\begin{array}{l}\text { Description of what constitutes completion of this milestone: } \\
\text { Definitive design documents and drawings have been completed and approved. }\end{array}$}} \\
\hline & & & \\
\hline \multicolumn{2}{|c|}{$\begin{array}{ll}\text { Cost Account Manager } \quad \text { Date }\end{array}$} & \multicolumn{2}{|c|}{ Program/Project Manager Date } \\
\hline \multicolumn{2}{|l|}{ W. A. Holstein } & \multicolumn{2}{|l|}{ R. E. Heineman, Jr. } \\
\hline \multicolumn{2}{|c|}{ Program Element Manager } & \multicolumn{2}{|l|}{ DOE Monitor } \\
\hline \multicolumn{2}{|l|}{ W. A. Holstein } & \multicolumn{2}{|l|}{ D. T. Evans } \\
\hline
\end{tabular}


WHC-SP-1126, Rev. I

FY 1996 MYPP

TRANSITION PROJECTS

1.3.1/7.1/6.12

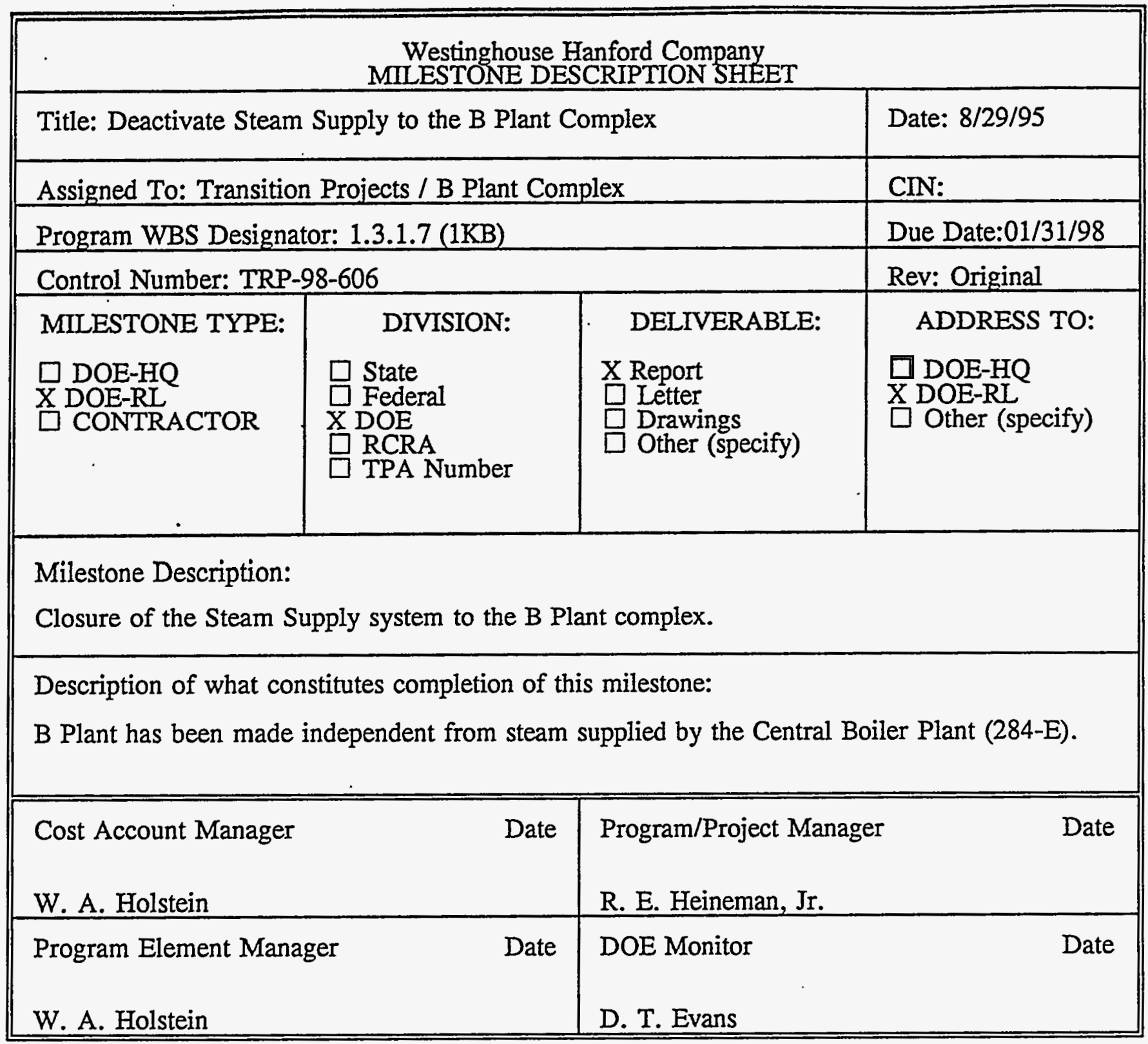


WHC-SP-1126, Rev. 1

FY 1996 MYPP

TRANSITION PROJECTS

1.3.1/7.1/6.12

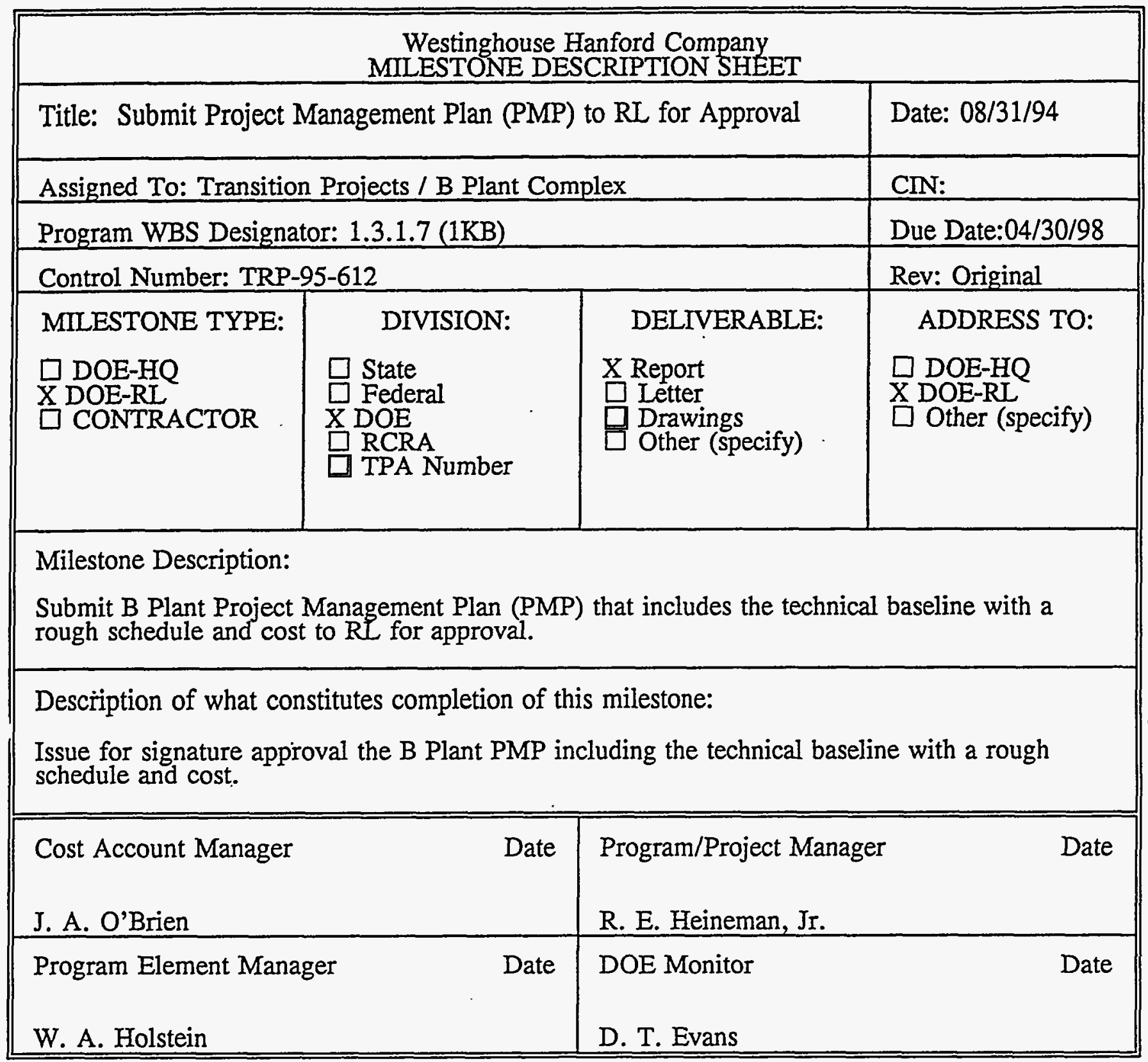


WHC-SP-1126, Rev. I

TRANSITION PROJECTS

FY 1996 MYPP

1.3.1/7:1/6.12

Westinghouse Hanford Company MILESTONE DESCRIPTION SHEET

Title: Startup the WESF Low Level Liquid Waste System.

Date: $8 / 29 / 95$

Assigned To: Transition Projects / B Plant Complex

CIN:

Program WBS Designator: 1.3.1.7 (1KB) Due Date:07/31/98

Control Number: TRP-98-607

MILESTONE TYPE:

$\square$ DOE-HQ

$X$ DOE-RL

CONTRACTOR

\begin{tabular}{l} 
DIVISION: \\
$\square$ State \\
$\square$ Federal \\
DOE \\
$\square$ RCRA \\
$\square$ TPA Number \\
\hline
\end{tabular}
Rev: Original

DELIVERABLE:

ADDRESS TO:

$\square$ Report

$X$ Letter

Drawings

Other (specify)

DOE-HQ

X DOE-RL Other (specify)

Milestone Description:

The new system has been installed for the storage, treatment, and monitoring of WESF Low Level Liquid Waste .

Description of what constitutes completion of this milestone:

The system has been declared operational by WESF Plant Operations.

\begin{tabular}{||ll|ll||}
\hline \hline Cost Account Manager & Date & Program/Project Manager & Date \\
W. A. Holstein & & R. E. Heineman, Jr. & \\
\hline Program Element Manager & Date & DOE Monitor & Date \\
W. A. Holstein & & D. T. Evans & \\
\hline
\end{tabular}


WHC-SP-1126, Rev. 1

FY 1996 MYPP

\section{TRANSITION PROJECTS}

1.3.1/7.1/6.12

\begin{tabular}{|c|c|c|c|}
\hline \multicolumn{4}{|c|}{$\begin{array}{l}\text { Westinghouse Hanford Company } \\
\text { MLESTONE DESCRIPTION SHEET }\end{array}$} \\
\hline \multicolumn{3}{|c|}{ Title: Startup of the WESF Solid Waste Systems } & Date: $8 / 29 / 95$ \\
\hline \multicolumn{3}{|c|}{ Assigned To: Transition Projects / B Plant Complex } & CIN: \\
\hline \multicolumn{3}{|c|}{ Program WBS Designator: 1.3.1.7 (1KB) } & Due Date:07/31/98 \\
\hline \multicolumn{3}{|c|}{ Control Number: TRP-98-608 } & Rev: Original \\
\hline $\begin{array}{l}\text { MILESTONE TYPE: } \\
\text { D DOE-HQ } \\
\text { X DOE-RL } \\
\square \text { CONTRACTOR }\end{array}$ & $\begin{array}{l}\text { DIVISION: } \\
\square \text { State } \\
\square \text { Federal } \\
\text { X DOE } \\
\square \text { RCRA } \\
\square \text { TPA Number }\end{array}$ & $\begin{array}{l}\text { DELIVERABLE: } \\
\text { R Report } \\
\text { X Letter } \\
\square \text { Drawings } \\
\square \text { Other (specify) }\end{array}$ & $\begin{array}{l}\text { ADDRESS TO: } \\
\square \text { DOE-HQ } \\
\text { X DOE-RI } \\
\square \text { Other (specify) }\end{array}$ \\
\hline \multicolumn{4}{|c|}{$\begin{array}{l}\text { Milestone Description: } \\
\text { The new system for handling solid waste from WESF has been designed, constructed, and is } \\
\text { ready to place in service. }\end{array}$} \\
\hline \multicolumn{4}{|c|}{$\begin{array}{l}\text { Description of what constitutes completion of this milestone: } \\
\text { The new system is declared operational by WESF Plant Operations Management. }\end{array}$} \\
\hline \multicolumn{2}{|c|}{$\begin{array}{l}\text { Cost Account Manager Date } \\
\text { W. A. Holstein }\end{array}$} & \multicolumn{2}{|c|}{$\begin{array}{l}\text { Program/Project Manager Date } \\
\text { R. E. Heineman, Jr. }\end{array}$} \\
\hline \multicolumn{2}{|c|}{$\begin{array}{l}\text { Program Element Manager } \\
\text { W. A. Holstein }\end{array}$} & \multicolumn{2}{|c|}{$\begin{array}{l}\text { DOE Monitor } \\
\text { D. T. Evans }\end{array}$} \\
\hline
\end{tabular}


WHC-SP-1126, Rev. 1

FY 1996 MYPP

\section{TRANSITION PROJECTS}

\subsection{1/7.1/6.12}

Westinghouse Hanford Company MILESTONE DESCRIPTION SHEET

\begin{tabular}{|c|c|c|c|}
\hline \multicolumn{3}{|c|}{ Title: Startup of the WESF Deionized Water Systems } & Date: $8 / 29 / 95$ \\
\hline \multicolumn{3}{|c|}{ Assigned To: Transition Projects / B Plant Complex } & CIN: \\
\hline \multicolumn{3}{|c|}{ Program WBS Designator: $1.3 .1 .7(1 \mathrm{~KB})$} & Due Date:07/31/98 \\
\hline \multicolumn{3}{|c|}{ Control Number: TRP-98-609 } & Rev: Original \\
\hline MILESTONE TYPE: & DIVISION: & DELIVERABLE: & ADDRESS TO: \\
\hline $\begin{array}{l}\square \text { DOE-HQ } \\
\text { X DOE-RL } \\
\square \text { CONTRACTOR }\end{array}$ & $\begin{array}{l}\square \text { State } \\
\square \text { Federal } \\
\text { X DOE } \\
\square \text { RCRA } \\
\square \text { TPA Number }\end{array}$ & $\begin{array}{l}\square \text { Report } \\
\text { X Letter } \\
\square \text { Drawings } \\
\square \text { Other (specify) }\end{array}$ & $\begin{array}{l}\square \text { DOE-HQ } \\
\text { X DOE-RL } \\
\square \text { Other (specify) }\end{array}$ \\
\hline
\end{tabular}

Milestone Description:

A new system for providing deionized water to WESF has been designed, constructed and is ready to place into service.

Description of what constitutes completion of this milestone:

The new system has been declared ready for service by WESF Operations Management.

\begin{tabular}{|c|c|c|c|}
\hline $\begin{array}{l}\text { Cost Account Manager } \\
\text { W. A. Holstein }\end{array}$ & Date & $\begin{array}{l}\text { Program/Project Manager } \\
\text { R. E. Heineman, Jr. }\end{array}$ & Date \\
\hline $\begin{array}{l}\text { Program Element Manager } \\
\text { W. A. Holstein }\end{array}$ & Date & $\begin{array}{l}\text { DOE Monitor } \\
\text { D. T. Evans }\end{array}$ & Date \\
\hline
\end{tabular}


WHC-SP-1126, Rev. 1

FY 1996 MYPP

\section{TRANSITION PROJECTS}

1.3.1/7.1/6.12

Westinghouse Hanford Company

MILESTONE DESCRIPTION SHEET

Title: Startup the WESF Liquid Effluent Systems

Assigned To: Transition Projects / B Plant Complex

Program WBS Designator: 1.3.1.7 (1KB)

Control Number: TRP-98-610

MILESTONE TYPE:

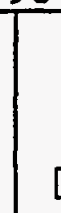

DIVISION:

DOE-HQ

$X$ DOE-RL

State

Federal

Report

$\mathrm{X}$ Letter

CONTRACTOR

$X D O E$

RCRA

$\square$ Drawings

$\square$ Other (specify)

Date: $8 / 29 / 95$

TPA Number

CIN:

Due Date:07/31/98

Rev: Original

ADDRESS TO:

$\square$ DOE-HQ

$X$ DOE-RL

Other (specify)

A

A new system for the monitoring and treatment of liquid effluents from WESF has been designed, and constructed.

Description of what constitutes completion of this milestone:

The new system has been declared ready for use by WESF Operations Management.

\begin{tabular}{||ll|lc||}
\hline \hline Cost Account Manager & Date & Program/Project Manager & Date \\
W. A. Holstein & & R. E. Heineman, Jr. & \\
\hline Program Element Manager & Date & $\begin{array}{l}\text { DOE Monitor } \\
\text { W. A. Holstein }\end{array}$ & D. T. Evans \\
\hline
\end{tabular}


WHC-SP-1126, Rev. I

FY 1996 MYPP

TRANSITION PROJECTS

1.3.1/7.1/6.12

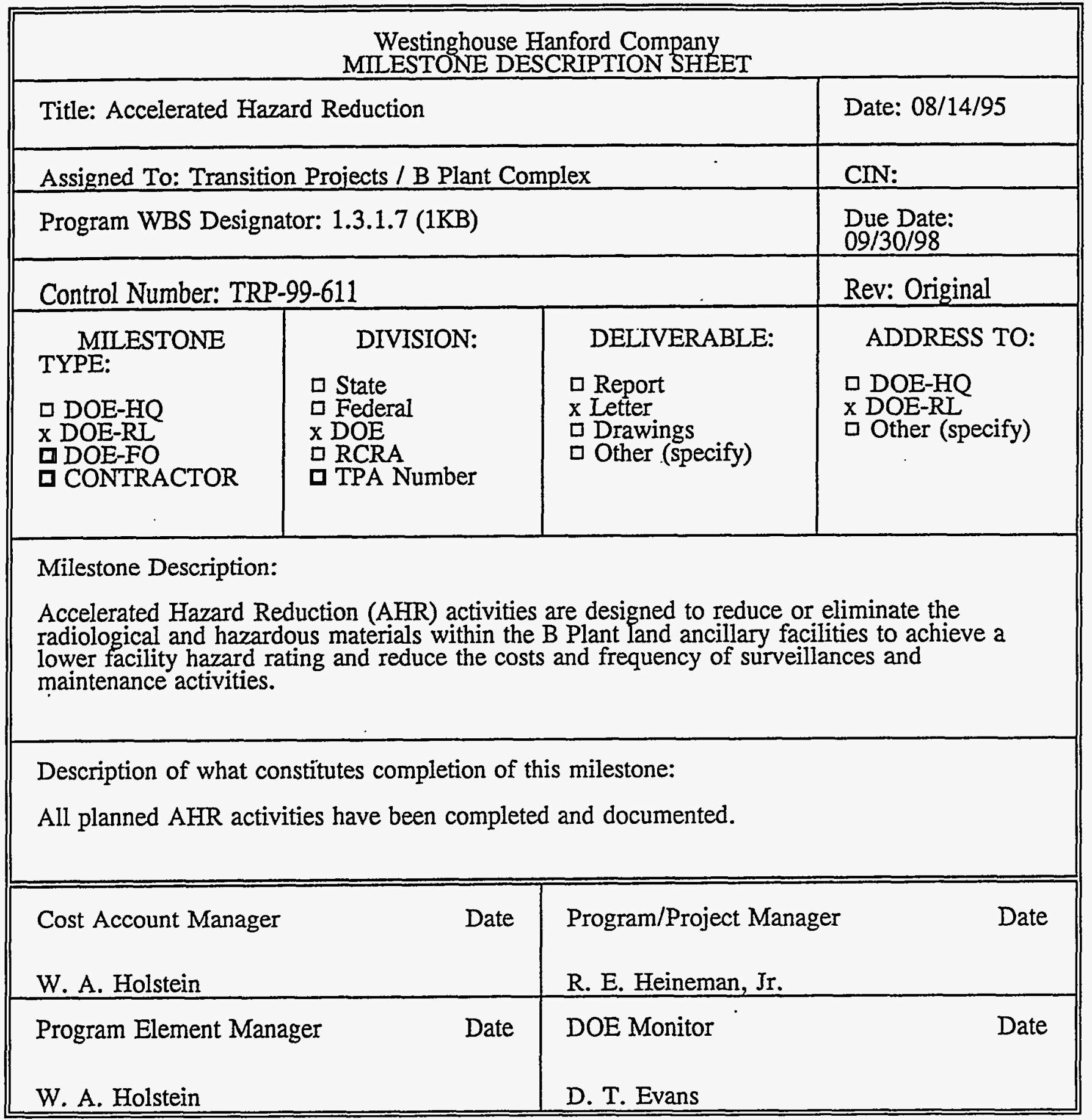


WHC-SP-1126, Rev. 1

FY 1996 MYPP

TRANSITION PROJECTS

1.3.1/7.1/6.12

\begin{tabular}{|c|c|c|c|}
\hline \multicolumn{4}{|c|}{$\begin{array}{l}\text { Westinghouse Hanford Company } \\
\text { MILESTONE DESCRIPTION SHEET }\end{array}$} \\
\hline \multicolumn{3}{|c|}{$\begin{array}{l}\text { Title: Complete Construction of B Plant Canyon Ventilation Upgrade } \\
\text { (W-059) }\end{array}$} & Date: $08 / 14 / 95$ \\
\hline \multicolumn{3}{|c|}{ Assigned To: Transition Projects / B Plant Complex } & CIN:TP-95-057 \\
\hline \multicolumn{3}{|c|}{ Program WBS Designator: 1.3.1.7 (1KB) } & $\begin{array}{l}\text { Due Date: } \\
09 / 30 / 98\end{array}$ \\
\hline \multicolumn{3}{|c|}{ Control Number: TRP-99-601 } & Rev: 1 \\
\hline $\begin{array}{l}\text { MILESTONE } \\
\text { TYPE: } \\
\text { प DOE-HQ } \\
\text { X DOE-RL } \\
\text { व DOE-FO } \\
\text { D CONTRACTOR }\end{array}$ & $\begin{array}{l}\quad \text { DIVISION: } \\
\text { State } \\
\text { Gederal } \\
\text { x DOE } \\
\text { RCRA } \\
\square \text { TPA Number }\end{array}$ & $\begin{array}{l}\text { DELIVERABLE: } \\
\text { Report } \\
x \text { Letter } \\
\text { a Drawings } \\
\square \text { Other (specify) }\end{array}$ & $\begin{array}{l}\text { ADDRESS TO: } \\
\text { a DOE-HQ } \\
\text { x DOE-RL } \\
\text { 口 Other (specify) }\end{array}$ \\
\hline \multicolumn{4}{|c|}{$\begin{array}{l}\text { Milestone Description: } \\
\text { Complete construction of Project W-059 to upgrade the B Plant canyon ventilation system. }\end{array}$} \\
\hline \multicolumn{4}{|c|}{$\begin{array}{l}\text { Description of what constitutes completion of this milestone: } \\
\text { The Project Official Acceptance of Construction (OAC), Part II has been approved. }\end{array}$} \\
\hline \multicolumn{2}{|c|}{ Cost Account Manager Date } & \multicolumn{2}{|c|}{$\begin{array}{l}\text { Program/Project Manager } \\
\text { R. E. Heineman, Jr. }\end{array}$} \\
\hline \multicolumn{2}{|c|}{$\begin{array}{l}\text { Program Element Manager } \\
\text { W. A. Holstein }\end{array}$} & \multicolumn{2}{|l|}{$\begin{array}{l}\text { DOE Monitor } \\
\text { D. T. Evans }\end{array}$} \\
\hline
\end{tabular}


WHC-SP-1126, Rev. 1

FY 1996 MYPP

TRANSITION PROJECTS

1.3.1/7.1/6.12

Westinghouse Hanford Company

MILESTONE DESCRIPTION SHEET

Title: Projected FY99 B Plant S\&M Costs Reduced by $65 \%$ Compared with FY96

Assigned To: Transition Projects / B Plant Complex

Program WBS Designator: 1.3.1.7 (1KB)

Control Number: TRP-99-602 TYPE:

MILESTONE

$\square$ DOE-HQ

$\square$ DOE-RL

$X$ DOE-FO

¿ CONTRACTOR

\section{DIVISION:}

$\square$ State

$\square$ Federal

$x$ DOE

口 RCRA

$\square$ TPA Number

Date: $08 / 14 / 95$

CIN:

Due Date: 10/1/98

Rev: Original

DELIVERABLE:

ADDRESS TO:

$\square$ Report

$x$ Letter

$\square$ Drawings

$\square$ Other (specify)

DOE-HQ

$x$ DOE-RL

$\square$ Other (specify)

Milestone Description:

The projected overall Fiscal Year 1999 costs for Surveillance and Maintenance of B Plant will be reduced by $65 \%$ when compared to the Fiscal Year 1996 costs.

Description of what constitutes completion of this milestone:

The projected annual Surveillance and Maintenance costs for B Plant have been reduced as a result of completion of Accelerated Hazard Reduction activities, B Plant Transition Activities, and other initiatives.

\begin{tabular}{||ll|ll|}
\hline \hline Cost Account Manager & Date & Program/Project Manager & Date \\
D. K. Smith & & R. E. Heineman, Jr. & \\
\hline Program Element Manager & Date & DOE Monitor & Date \\
D. K. Smith & & D. T. Evans & \\
\hline
\end{tabular}


WHC-SP-1126, Rev. 1

FY 1996 MYPP

\section{TRANSITION PROJECTS}

\subsection{1/7.1/6.12}

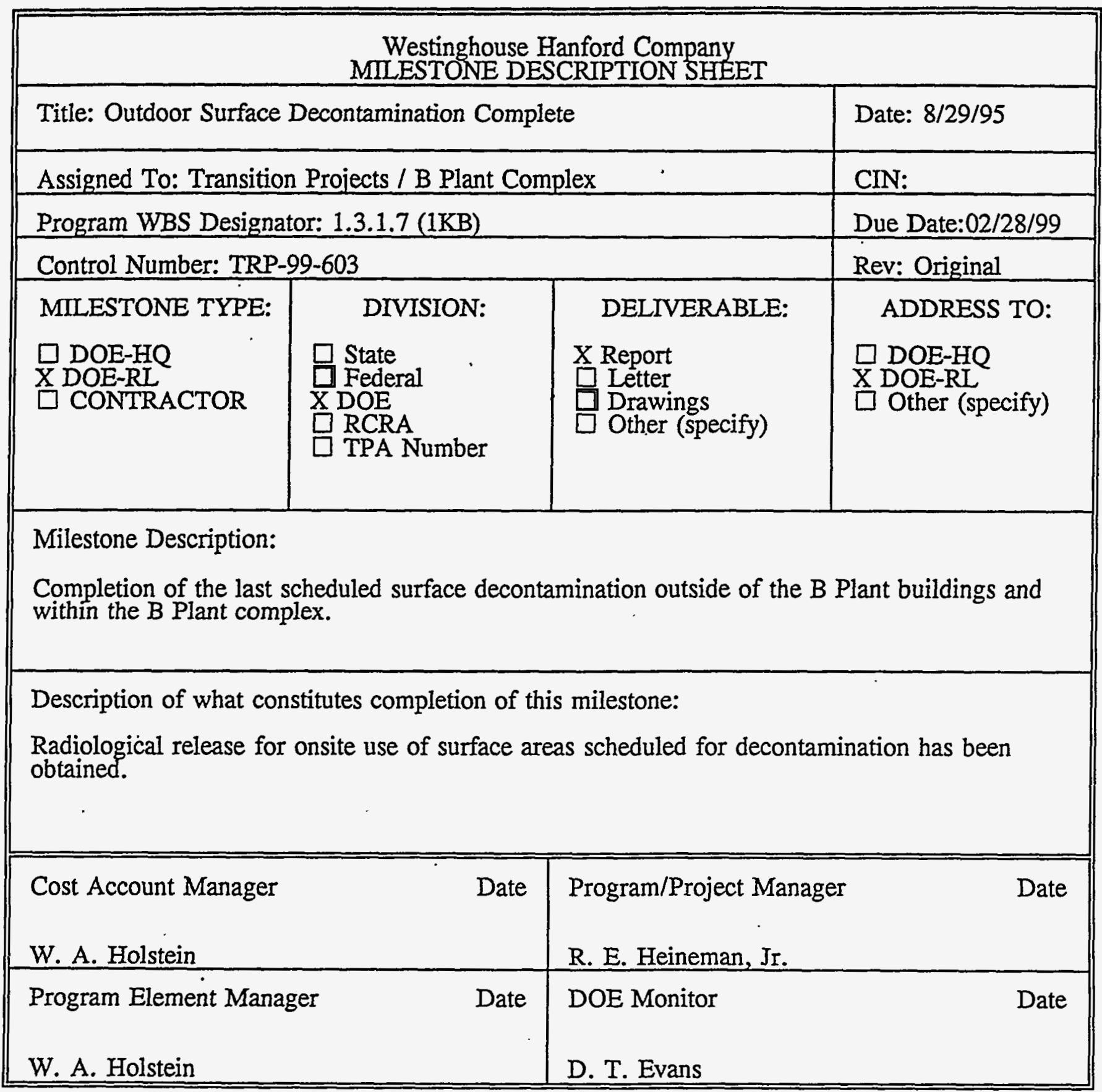


WHC-SP-1126, Rev. 1

FY 1996 MYPP

TRANSITION PROJECTS

1.3.1/7.1/6.12

\begin{tabular}{|c|c|c|c|}
\hline \multicolumn{4}{|c|}{$\begin{array}{l}\text { Westinghouse Hanford Company } \\
\text { MILESTONE DESCRIPTION SHEET }\end{array}$} \\
\hline \multicolumn{3}{|c|}{$\begin{array}{l}\text { Title: Projected FY00 B Plant S\&M Costs Reduced by } 75 \% \text { Compared } \\
\text { with FY } 96\end{array}$} & Date: $08 / 14 / 95$ \\
\hline \multicolumn{3}{|c|}{ Assigned To: Transition Projects / B Plant Complex } & CIN: \\
\hline \multicolumn{3}{|c|}{ Progiam WBS Designator: 1.3.1.7 (1KB) } & Due Date: $10 / 1 / 99$ \\
\hline \multicolumn{3}{|c|}{ Control Number: TRP-00-601 } & Rev: Original \\
\hline $\begin{array}{l}\text { MILESTONE } \\
\text { TYPE: } \\
\text { D DOE-HQ } \\
\text { D DOE-RL } \\
\text { X DOE-FO } \\
\square \text { CONTRACTOR }\end{array}$ & $\begin{array}{l}\quad \text { DIVISION: } \\
\text { Q State } \\
\text { a Federal } \\
\text { x DOE } \\
\square \text { RCRA } \\
\square \text { TPA Number }\end{array}$ & $\begin{array}{l}\text { DELIVERABLE: } \\
\text { a Report } \\
\text { x Letter } \\
\text { a Drawings } \\
\square \text { Other (specify) }\end{array}$ & $\begin{array}{l}\text { ADDRESS TO: } \\
\text { D DOE-HQ } \\
\text { x DOE-RI } \\
\text { 口 Other (specify) }\end{array}$ \\
\hline \multicolumn{4}{|c|}{$\begin{array}{l}\text { Milestone Description: } \\
\text { The projected overall Fiscal Year } 2000 \text { costs for Surveillance and Maintenance of B Plant will } \\
\text { be reduced by } 75 \% \text { when compared to the Fiscal Year } 1996 \text { costs. }\end{array}$} \\
\hline \multicolumn{4}{|c|}{$\begin{array}{l}\text { Description of what constitutes completion of this milestone: } \\
\text { The projected annual Surveillance and Maintenance costs for B Plant have been reduced as a } \\
\text { result of completion of Accelerated Hazard Reduction activities, B Plant Transition Activities, } \\
\text { and other initiatives. }\end{array}$} \\
\hline \multicolumn{2}{|c|}{$\begin{array}{l}\text { Cost Account Manager Date } \\
\text { D. K. Smith }\end{array}$} & \multicolumn{2}{|c|}{$\begin{array}{l}\text { Program/Project Manager } \\
\text { R. E. Heineman, Jr. }\end{array}$} \\
\hline \multicolumn{2}{|c|}{$\begin{array}{l}\text { Program Element Manager } \\
\text { D. K. Smith }\end{array}$} & \multicolumn{2}{|l|}{$\begin{array}{l}\text { DOE Monitor } \\
\text { D. T. Evans } \\
\end{array}$} \\
\hline
\end{tabular}


WHC-SP-1126, Rev. I

FY 1996 MYPP

TRANSITION PROJECTS

1.3.1/7.1/6.12

\begin{tabular}{|c|c|c|c|c|}
\hline \multicolumn{5}{|c|}{$\begin{array}{l}\text { Westinghouse Hanford Company } \\
\text { MILESTONE DESCRIPTION SHEET }\end{array}$} \\
\hline \multicolumn{4}{|c|}{$\begin{array}{l}\text { Title: Projected FY01 B Plant S\&M Costs Reduced by } 80 \% \text { Compared } \\
\text { with FY } 96\end{array}$} & Date: $08 / 14 / 95$ \\
\hline \multicolumn{4}{|c|}{ Assigned To: Transition Projects / B Plant Complex } & CIN: \\
\hline \multicolumn{4}{|c|}{ Program WBS Designator: 1.3.1.7 (1KB) } & Due Date: $10 / 1 / 00$ \\
\hline \multicolumn{4}{|c|}{ Control Number: TRP-01-603 } & Rev: Original \\
\hline $\begin{array}{l}\text { MILESTONE } \\
\text { TYPE: } \\
\text { D DOE-HO } \\
\text { 口 DOE-RI } \\
\text { X DOE-FO } \\
\text { 口 CONTRACTOR } \\
\end{array}$ & & & $\begin{array}{l}\text { DELIVERABLE: } \\
\text { a Report } \\
\text { x Letter } \\
\text { व Drawings } \\
\square \text { Other (specify) }\end{array}$ & $\begin{array}{l}\text { ADDRESS TO: } \\
\text { } \text { DOE-HQ } \\
\text { x DOE-RL } \\
\square \text { Other (specify) }\end{array}$ \\
\hline \multicolumn{5}{|c|}{$\begin{array}{l}\text { Milestone Description: } \\
\text { The projected overall Fiscal Year } 2001 \text { costs for Surveillance and Maintenance of B Plant will } \\
\text { be reduced by } 80 \% \text { when compared to the Fiscal Year } 1996 \text { costs. }\end{array}$} \\
\hline \multicolumn{5}{|c|}{$\begin{array}{l}\text { Description of what constitutes completion of this milestone: } \\
\text { The projected annual Surveillance and Maintenance costs for B Plant have been reduced as a } \\
\text { result of completion of Accelerated Hazard Reduction activities, B Plant Transition Activities, } \\
\text { and other initiatives. }\end{array}$} \\
\hline \multicolumn{3}{|c|}{ Cost Account Manager Date } & \multicolumn{2}{|c|}{$\begin{array}{l}\text { Program/Project Manage } \\
\text { R. E. Heineman, Jr. }\end{array}$} \\
\hline \multicolumn{3}{|c|}{$\begin{array}{l}\text { Program Element Manager } \\
\text { D. K. Smith }\end{array}$} & \multicolumn{2}{|l|}{$\begin{array}{l}\text { DOE Monitor } \\
\text { D. T. Evans }\end{array}$} \\
\hline
\end{tabular}


WHC-SP-1126, Rev. 1.

FY 1996 MYPP

TRANSITION PROJECTS

1.3.1/7.1/6.12

Westinghouse Hanford Company MILESTONE DESCRIPTION SHEET

Title: Complete B Plant Deactivation

Date: $08 / 14 / 95$

Assigned To: Transition Projects / B Plant Complex

CIN:

Program WBS Designator: 1.3.1.7 (1KB)

Due Date:09/30/02

Control Number: TRP-01-601

MILESTONE TYPE:

$x$ DOE-HQ

DOE-RL

$\square$ DOE-FO

$\square$ CONTRACTOR

DIVISION:

$\square$ State

$\square$ Federal

$x$ DOE

$\square$ RCRA

$\square$ TPA Number Rev: Original

DELIVERABLE:

Report

$x$ Letter

$\square$ Drawings

Other (specify)
ADDRESS TO:

$X$ DOE-HQ

DOE-RI

$\square$ Other (specify)

Milestone Description:

Transition B Plant and ancillary facilities to a safe shutdown condition and prepare for transfer to the site decommissioning contractor.

Description of what constitutes completion of this milestone:

B Plant and ancillary facilities have been deactivated and placed in a condition suitable for long term storage pending final decommissioning.

\begin{tabular}{||ll|lc||}
\hline \hline Cost Account Manager & Date & Program/Project Manager & Date \\
W. A. Holstein & & R. E. Heineman, Jr. & \\
\hline Program Element Manager & Date & DOE Monitor & Date \\
W. A. Holstein & & D. T. Evans & \\
\hline
\end{tabular}


WHC-SP-1126, Rev. 1

FY 1996 MYPP

\section{TRANSITION PROJECTS}

1.3.1/7.1/6.12

Westinghouse Hanford Company

MILESTONE DESCRIPTION SHEET

\begin{tabular}{|c|c|c|c|}
\hline \multicolumn{3}{|c|}{ Title: Complete ARECO Capsule Shipments to WESF } & Date: $08 / 14 / 95$ \\
\hline \multicolumn{3}{|c|}{ Assigned To: Transition Projects / B Plant Complex } & CIN: \\
\hline \multicolumn{3}{|c|}{ Program WBS Designator: 1.3.1.7 (1KB) } & Due Date:05/30/96 \\
\hline \multicolumn{3}{|c|}{ Control Number: TRP-96-702 } & Rev: 1 \\
\hline MILESTONE TYPE: & DIVISION: & DELIVERABLE: & ADDRESS TO: \\
\hline $\begin{array}{l}\text { X DOE-HQ } \\
\square \text { DOE-RI } \\
\square \text { CONTRACTOR }\end{array}$ & $\begin{array}{l}\square \text { State } \\
\square \text { Federal } \\
\text { X DOE } \\
\square \text { RCRA } \\
\square \text { TPA Number }\end{array}$ & $\begin{array}{l}\square \text { Report } \\
\text { X Letter } \\
\square \text { Drawings } \\
\square \text { Other (specify) }\end{array}$ & $\begin{array}{l}\square \text { DOE-HQ } \\
\text { X DOE-RL } \\
\square \text { Other (specify) }\end{array}$ \\
\hline
\end{tabular}

Milestone Description:

Complete the return of all 25 cesium capsules from the ARECO. Two total shipments will be made.

Description of what constitutes completion of this milestone:

Complete the transportation of all 25 cesium capsules from ARECO to WESF, assuming BUSS Cask operational with a valid Certificate of Compliance from DOE/NRC and all capsules are in special form.

\begin{tabular}{|ll|lr|}
\hline Cost Account Manager & Date & Program/Project Manager & Date \\
E. D. Robbins & & R. E. Heineman, Jr. & \\
\hline Program Element Manager & Date & DOE Monitor & Date \\
E. D. Robbins & & D. T. Evans & \\
\hline
\end{tabular}


WHC-SP-1126, Rev. 1

FY 1996 MYPP

TRANSITION PROJECTS

1.3.1/7.1/6.12

Westinghouse Hanford Company MILESTONE DESCRIPTION SHEET

\begin{tabular}{|c|c|c|c|}
\hline \multicolumn{3}{|c|}{ Title:Complete the return of all cesium capsules to WESF } & Date: $8 / 14 / 95$ \\
\hline \multicolumn{3}{|c|}{ Assigned To: Transition Projects / B Plant Complex } & CIN: \\
\hline \multicolumn{3}{|c|}{ Program WBS Designator: $1.3 .1 .7(1 \mathrm{~KB})$} & Due Date: $9 / 30 / 98$ \\
\hline \multicolumn{3}{|c|}{ Control Number: TRP-98-703 } & Rev: Original \\
\hline MILESTONE TYPE: & DIVISION: & DELIVERABLE: & ADDRESS TO: \\
\hline $\begin{array}{l}\square \text { DOE-HQ } \\
\text { X DOE-RL } \\
\square \text { CONTRACTOR }\end{array}$ & $\begin{array}{l}\square \text { State } \\
\square \text { Federal } \\
X \text { DOE } \\
\square \text { RCRA } \\
\square \text { TPA Number }\end{array}$ & $\begin{array}{l}\text { X Report } \\
\square \text { Letter } \\
\square \text { Drawings } \\
\square \text { Other (specify) }\end{array}$ & $\begin{array}{l}\square \text { DOE-HQ } \\
X \text { DOE-RL } \\
\square \text { Other (specify) }\end{array}$ \\
\hline
\end{tabular}

Milestone Description:

All formerly leased cesium capsules have been returned to WESF for long term storage.

Description of what constitutes completion of this milestone:

All capsules have been returned and placed into the WESF pool cells and a final report prepared and issued.

\begin{tabular}{|c|c|c|c|}
\hline $\begin{array}{l}\text { Cost Account Manager } \\
\text { E. D. Robbins }\end{array}$ & Date & $\begin{array}{l}\text { Program/Project Manager } \\
\text { R. E. Heineman, Jr. }\end{array}$ & Date \\
\hline Program Element Manager & Date & DOE Monitor & Date \\
\hline E. D. Robbins & & D. T. Evans & \\
\hline
\end{tabular}


WHC-SP-1126, Rev. 1

\section{TRANSITION PROJECTS}

FY 1996 MYPP

1.3.1/7.1/6.12

\begin{tabular}{|c|c|c|c|}
\hline \multicolumn{4}{|c|}{$\begin{array}{l}\text { Westinghouse Hanford Company } \\
\text { MILESTONE DESCRIPTION SHEET }\end{array}$} \\
\hline \multicolumn{3}{|c|}{ Title: Return Single-Pass Fuel } & Date: 09/20/94 \\
\hline \multicolumn{3}{|c|}{ Assigned To: Transition Projects / PUREX/UO ${ }_{3}$ Deactivation } & CIN: TP-95-001 \\
\hline \multicolumn{3}{|c|}{ Program WBS Designator: 7.1 .1 (1KP) } & Due Date: $12 / 04 / 95$ \\
\hline \multicolumn{3}{|c|}{ Control Number: TRP-95-201 } & Rev: 1 \\
\hline $\begin{array}{l}\text { MILESTONE TYPE: } \\
\square \text { DOE-HQ } \\
\text { X DOE-RL } \\
\square \text { CONTRACTOR }\end{array}$ & 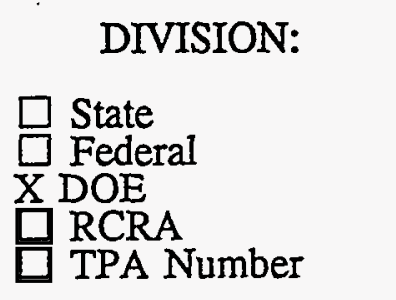 & $\begin{array}{l}\text { DELIVERABLE: } \\
\square \text { Report } \\
\text { X Letter } \\
\square \text { Drawings } \\
\square \text { Other (specify) }\end{array}$ & $\begin{array}{l}\text { ADDRESS TO: } \\
\square \text { DOE-HQ } \\
\text { X DOE-RL } \\
\square \text { Other (specify) }\end{array}$ \\
\hline \multicolumn{4}{|c|}{$\begin{array}{l}\text { Milestone Description: } \\
\text { Spent aluminum clad fuel is stored in the PUREX slug storage basin. The fuel will be removed } \\
\text { from PUREX and transferred to the } 100 \mathrm{KE} \text { Basin in cask cars for storage. }\end{array}$} \\
\hline \multicolumn{4}{|c|}{$\begin{array}{l}\text { Description of what constitutes completion of this milestone: } \\
\text { The milestone will be completed when all of the aluminum clad fuel is transferred to the } \mathrm{K} \\
\text { basins. }\end{array}$} \\
\hline \multicolumn{2}{|c|}{$\begin{array}{ll}\text { Cost Account Manager } & \text { Date } \\
\text { D. G. Harlow } & \\
\end{array}$} & \multicolumn{2}{|c|}{$\begin{array}{l}\text { Program/Project Manager Date } \\
\text { A. A. Grasher }\end{array}$} \\
\hline \multicolumn{2}{|c|}{$\begin{array}{l}\text { Program Element Manager } \\
\text { A. A. Grasher }\end{array}$} & $\begin{array}{l}\text { DOE Monitor } \\
\text { L. D. Romine }\end{array}$ & Date \\
\hline
\end{tabular}


WHC-SP-1126, Rev. 1

\title{
TRANSITION PROJECTS
}

FY 1996 MYPP

\author{
1.3.1/7.1/6.12
}

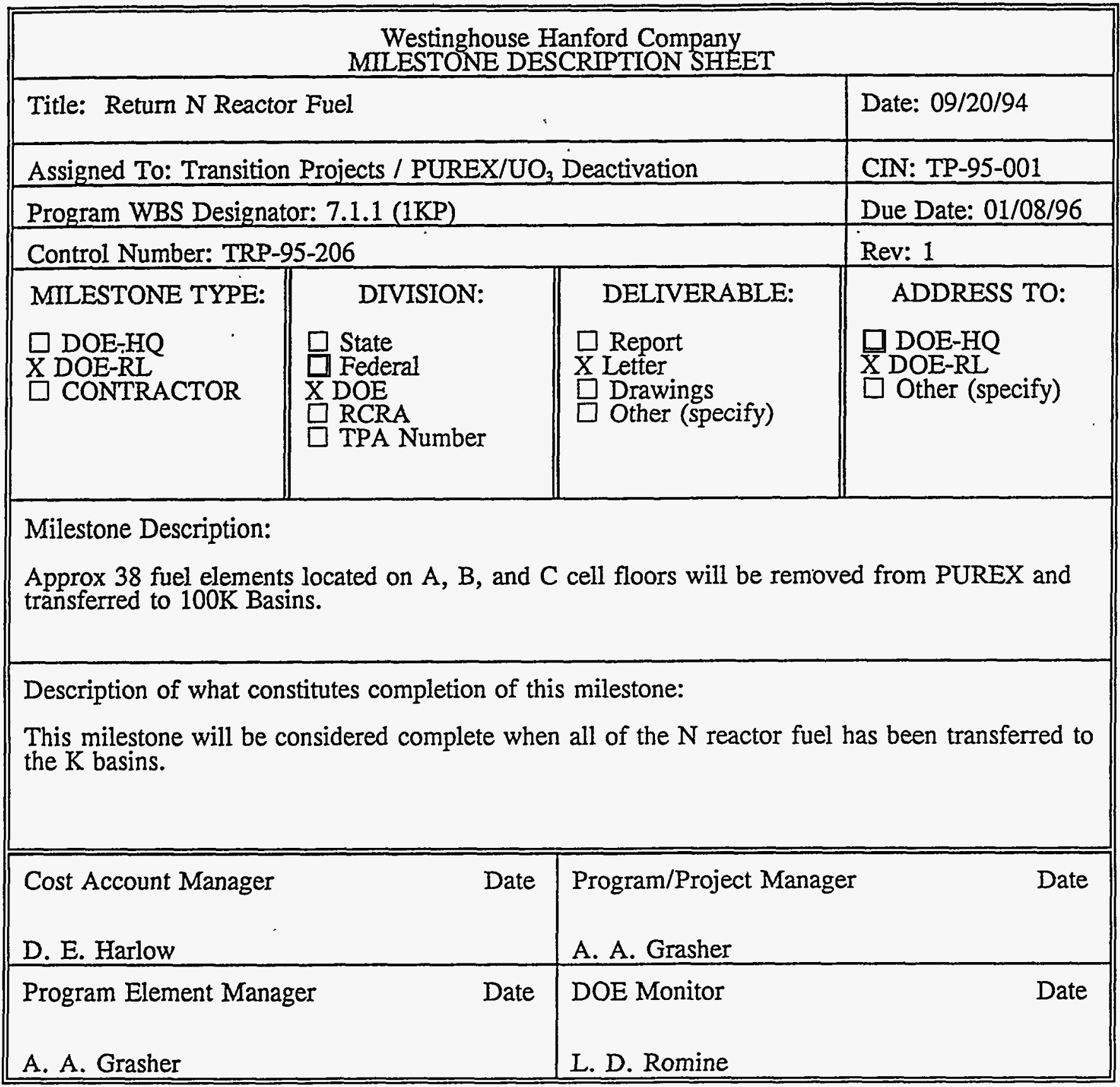


WHC-SP-1126, Rev. 1

FY 1996 MYPP

\section{TRANSITION PROJECTS}

\subsection{1/7.1/6.12}

\begin{tabular}{|c|c|c|c|}
\hline \multicolumn{4}{|c|}{$\begin{array}{l}\text { Westinghouse Hanford Company } \\
\text { MILESTONE DESCRIPTION SHET }\end{array}$} \\
\hline \multicolumn{3}{|c|}{ Title: Complete Nitric Acid Disposal } & Date: $10 / 18 / 95$ \\
\hline \multicolumn{3}{|c|}{ Assigned to: Transition Projects / PUREX/UO ${ }_{3}$ Deactivation } & CIN: TP-95-009 \\
\hline \multicolumn{3}{|c|}{ Program WBS Designator: 7.1 .1 (1KP) } & Due Date: $04 / 01 / 96$ \\
\hline \multicolumn{3}{|c|}{ Control Number: TRP-96-201 } & Rev: 2 \\
\hline MILESTONE TYPE: & DIVISION: & DELIVERABLE: & ADDRESS TO: \\
\hline $\begin{array}{l}\square \text { DOE-HQ } \\
\text { X DOE-RL } \\
\square \text { CONTRACTOR }\end{array}$ & $\begin{array}{l}\square \text { State } \\
\square \text { Federal } \\
\text { XOE } \\
\square \text { RCRA } \\
\square \text { TPA Number }\end{array}$ & $\begin{array}{l}\square \text { Report } \\
\text { X Letter } \\
\square \text { Drawings } \\
\square \text { Other (specify) }\end{array}$ & $\begin{array}{l}\square \text { DOE-HQ } \\
\text { X DOE-RL } \\
\square \text { Other (specify) }\end{array}$ \\
\hline \multirow{2}{*}{\multicolumn{4}{|c|}{$\begin{array}{l}\text { Milestone Description: } \\
\text { The recovered nitric acid at PUREX will be transferred to BNFL for }\end{array}$}} \\
\hline & & & \\
\hline \multirow{2}{*}{\multicolumn{4}{|c|}{$\begin{array}{l}\text { Description of what constitutes completion of this milestone: } \\
\text { This milestone will be completed when all of the recovered nitric acid has been dispositioned. }\end{array}$}} \\
\hline & & & \\
\hline \multicolumn{2}{|l|}{ Cost Account Manager } & \multicolumn{2}{|c|}{ Program/Project Manager } \\
\hline \multicolumn{2}{|l|}{ D. E. Harlow } & \multicolumn{2}{|l|}{ A. A. Grasher } \\
\hline \multicolumn{2}{|c|}{ Program Element Manager } & \multicolumn{2}{|l|}{ DOE Monitor } \\
\hline \multicolumn{2}{|l|}{ A. A. Grasher } & \multicolumn{2}{|l|}{ L. D. Romine } \\
\hline
\end{tabular}


WHC-SP-1126, Rev. I

FY 1996 MYPP

TRANSITION PROJECTS

1.3.1/7.1/6.12

\begin{tabular}{|c|c|c|c|}
\hline \multicolumn{4}{|c|}{$\begin{array}{l}\text { Westinghouse Hanford Company } \\
\text { MILESTONE DESCRIPTION SHEET }\end{array}$} \\
\hline \multicolumn{3}{|c|}{ Title: Complete N Cell Stabilization } & Date: $05 / 15 / 95$ \\
\hline \multicolumn{3}{|c|}{ Assigned to: Transition Projects / PUREX/UO ${ }_{3}$ Deactivation } & CIN: FO-94-019 \\
\hline \multicolumn{3}{|c|}{ Program WBS Designator: $7.1 .1(1 \mathrm{KP})$} & Due Date: $01 / 19 / 96$ \\
\hline \multicolumn{3}{|c|}{ Control Number: TRP-96-202 } & Rev: 1 \\
\hline $\begin{array}{l}\text { MILESTONE TYPE: } \\
\square \text { DOE-HQ } \\
\text { X DOE-RL } \\
\square \text { CONTRACTOR }\end{array}$ & $\begin{aligned} & \text { DIVISION: } \\
& \square \text { State } \\
& \square \text { Federal } \\
& \text { X DOE } \\
& \square \text { RCRA } \\
& \square \text { TPA Number }\end{aligned}$ & $\begin{array}{l}\text { DELIVERABLE: } \\
\square \text { Report } \\
\text { X Letter } \\
\square \text { Drawings } \\
\square \text { Other (specify) }\end{array}$ & $\begin{array}{l}\text { ADDRESS TO: } \\
\text { D DOE-HQ } \\
\text { X DOE-RL } \\
\square \text { Other (specify) }\end{array}$ \\
\hline \multicolumn{4}{|c|}{$\begin{array}{l}\text { Milestone Description: } \\
\text { Equipment in } N \text { cell will be removed from the glove boxes and the residual plutonium contained } \\
\text { within the glove boxes removed or stabilized. }\end{array}$} \\
\hline \multicolumn{4}{|c|}{$\begin{array}{l}\text { Description of what constitutes completion of this milestone: } \\
\text { This milestone will be complete when the necessary equipment has been removed, the glove } \\
\text { boxes have been stabilized and the end point criteria has been met. }\end{array}$} \\
\hline \multicolumn{2}{|c|}{$\begin{array}{l}\text { Cost Account Manager Date } \\
\text { D. G. Harlow }\end{array}$} & \multicolumn{2}{|c|}{$\begin{array}{l}\text { Program/Project Manager Date } \\
\text { A. A. Grasher }\end{array}$} \\
\hline \multicolumn{2}{|c|}{$\begin{array}{l}\text { Program Element Manager } \\
\text { A. A. Grasher }\end{array}$} & \multicolumn{2}{|c|}{$\begin{array}{l}\text { DOE Monitor } \\
\text { L. D. Romine } \\
\end{array}$} \\
\hline
\end{tabular}


WHC-SP-1126, Rev. 1

\section{TRANSITION PROJECTS}

FY 1996 MYPP

1.3.1/7.1/6.12

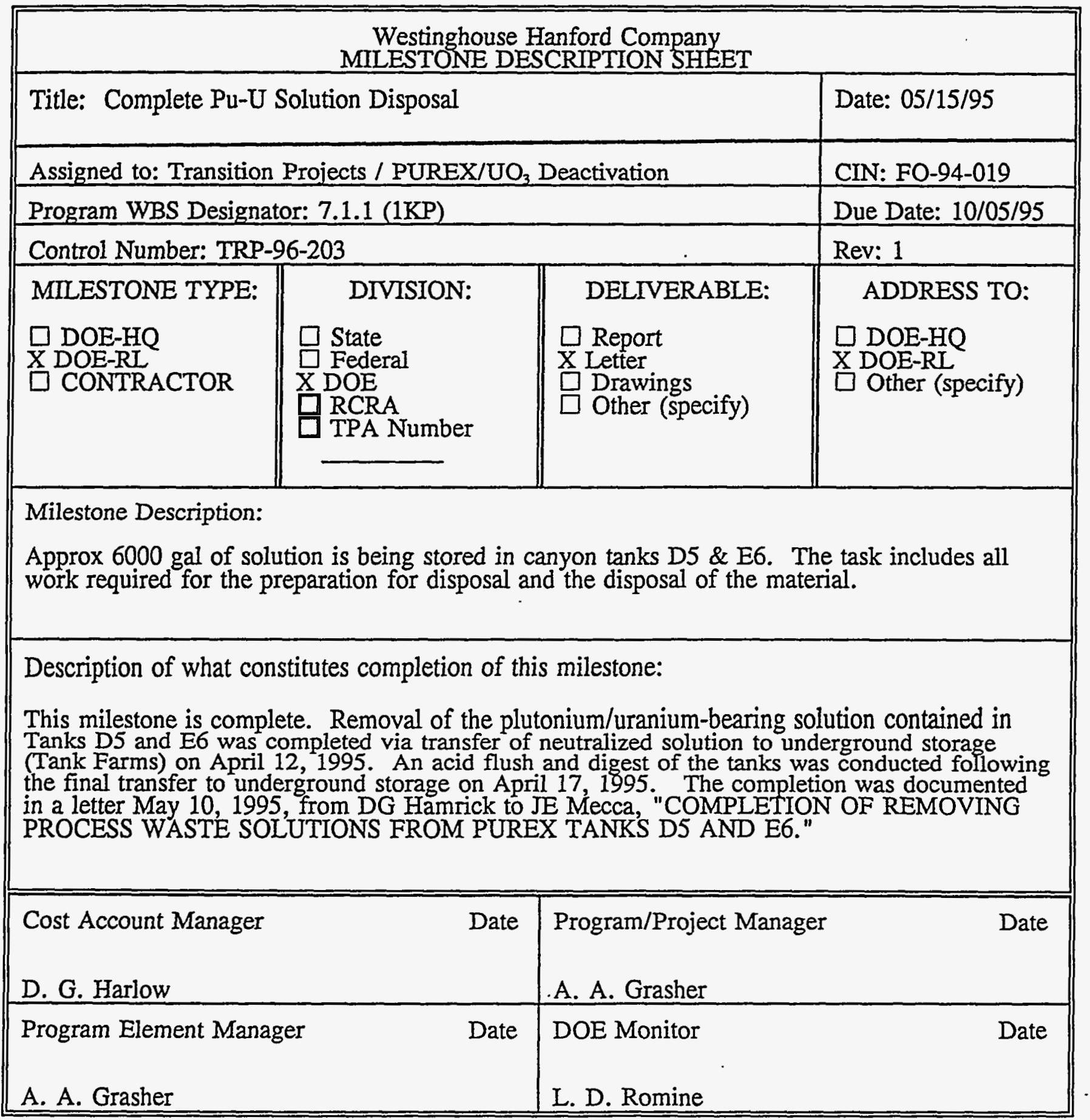


WHC-SP-1126, Rev. 1

\section{TRANSITION PROJECTS}

FY 1996 MYPP

1.3.1/7.1/6.12

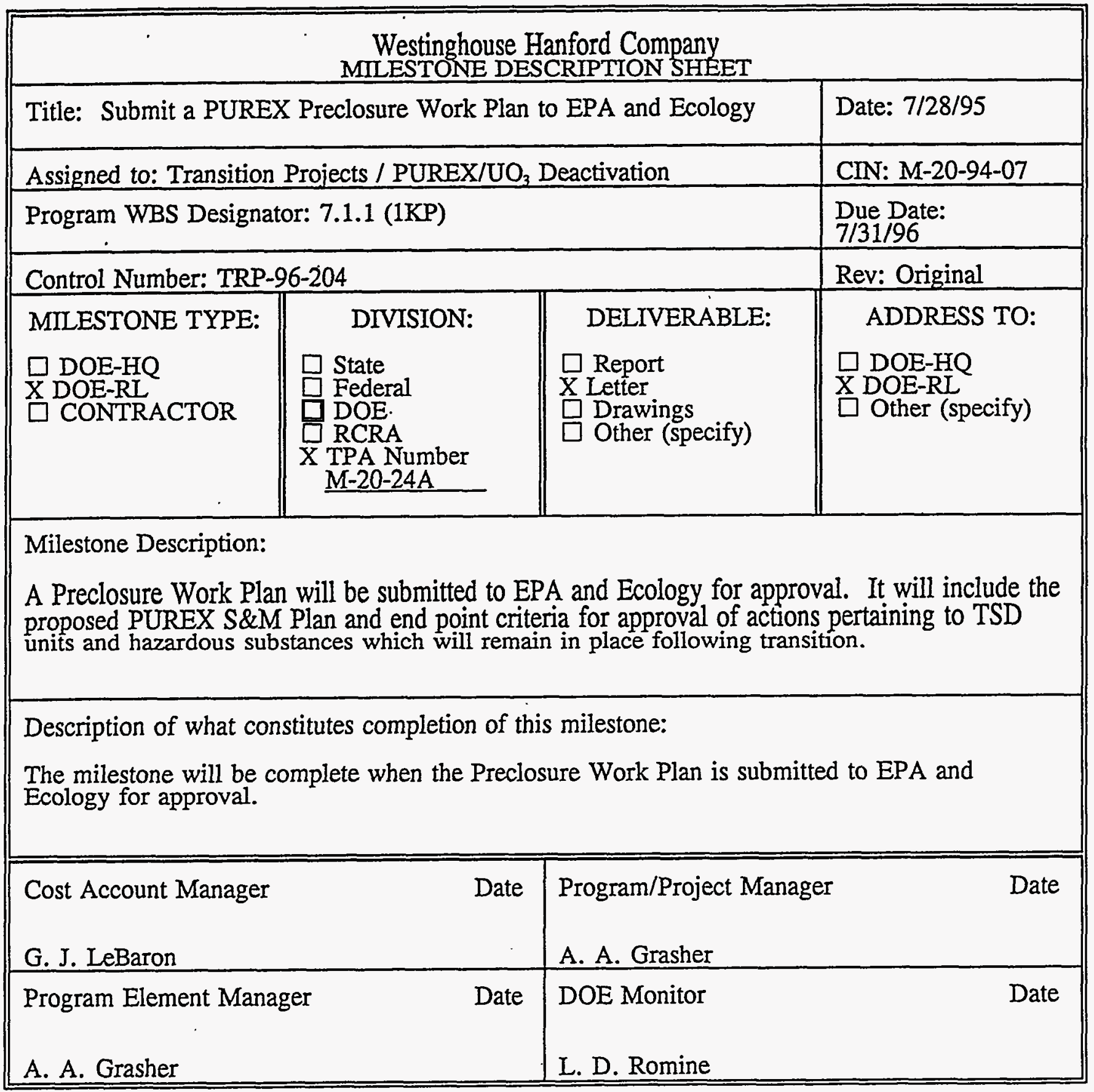


FY 1996 MYPP

WHC-SP-1126, Rev. I

TRANSITION PROJECTS

1.3.1/7.1/6.12

\begin{tabular}{|c|c|c|c|}
\hline \multicolumn{4}{|c|}{$\begin{array}{l}\text { Westinghouse Hanford Company } \\
\text { MILESTONE DESCRIPTION SHEET }\end{array}$} \\
\hline \multicolumn{3}{|c|}{$\begin{array}{l}\text { Title: Complete removal of concentrated (recovered) 203-A Nitric Acid } \\
\text { at PUREX }\end{array}$} & Date: $07 / 27 / 95$ \\
\hline \multicolumn{3}{|c|}{ Assigned to: Transition Projects / PUREX/UO ${ }_{3}$ Deactivation } & CIN: $M-80-94-0$ \\
\hline \multicolumn{3}{|c|}{ Program WBS Designator: 7.1.1 (1KP) } & $\begin{array}{l}\text { Due Date: } \\
6 / 30 / 96\end{array}$ \\
\hline \multicolumn{3}{|c|}{ Control Number: TRP-96-205 } & Rev: Original \\
\hline $\begin{array}{l}\text { MILESTONE TYPE: } \\
\square \text { DOE-HQ } \\
\text { X DOE-RL } \\
\square \text { CONTRACTOR }\end{array}$ & 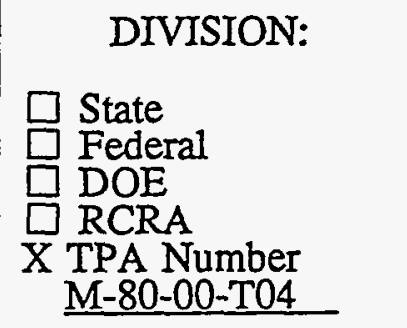 & $\begin{array}{l}\text { DELIVERABLE: } \\
\square \text { Report } \\
\text { X Letter } \\
\text { D Drawings } \\
\square \text { Other (specify) }\end{array}$ & $\begin{array}{l}\text { ADDRESS TO: } \\
\square \text { DOE-HQ } \\
\text { X DOE-RL } \\
\square \text { Other (specify) }\end{array}$ \\
\hline \multicolumn{4}{|c|}{$\begin{array}{l}\text { Milestone Description: } \\
\text { The recovered nitric acid at PUREX will be transferred to BNFL for beneficial use. This } \\
\text { includes completion of the National Environmental Policy Act (NEPA) process. }\end{array}$} \\
\hline \multicolumn{4}{|c|}{$\begin{array}{l}\text { Description of what constitutes completion of this milestone: } \\
\text { This milestone will be complete when all of the concentrated (recovered) acid has been removed } \\
\text { from the 203-A tanks. }\end{array}$} \\
\hline \multicolumn{2}{|c|}{$\begin{array}{l}\text { Cost Account Manager Date } \\
\text { D. G. Harlow }\end{array}$} & \multicolumn{2}{|c|}{$\begin{array}{l}\text { Program/Project Manager Date } \\
\text { A. A. Grasher }\end{array}$} \\
\hline \multicolumn{2}{|c|}{$\begin{array}{l}\text { Program Element Manager Date } \\
\text { A. A. Grasher }\end{array}$} & \multicolumn{2}{|c|}{$\begin{array}{l}\text { DOE Monitor } \\
\text { L. D. Romine }\end{array}$} \\
\hline
\end{tabular}


WHC-SP-1126, Rev. 1

TRANSITION PROJECTS

FY 1996 MYPP

\author{
1.3.1/7.1/6.12
}

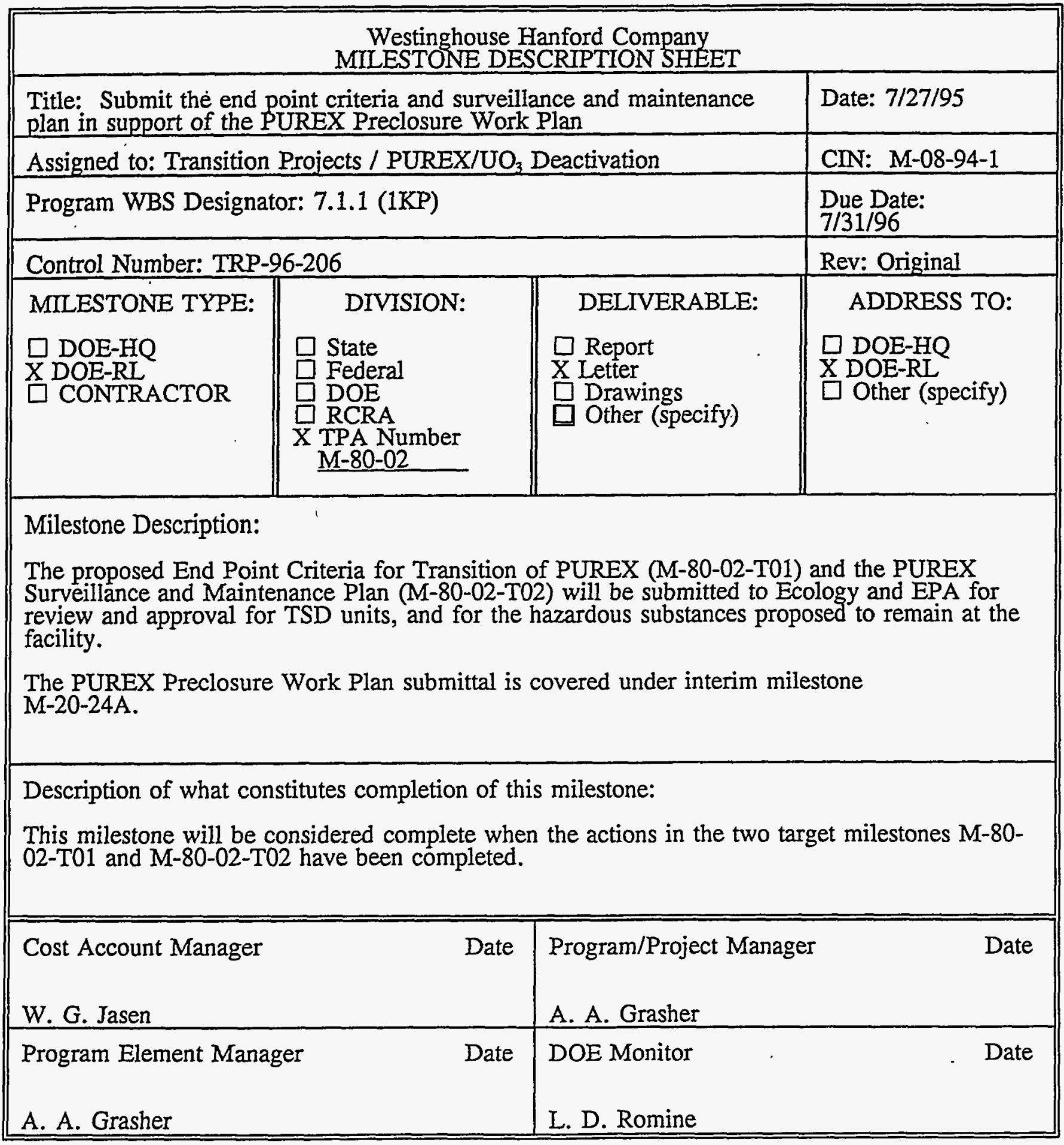


WHC-SP-1126, Rev. I

TRANSITION PROJECTS

FY 1996 MYPP

1.3.1/7.1/6.12

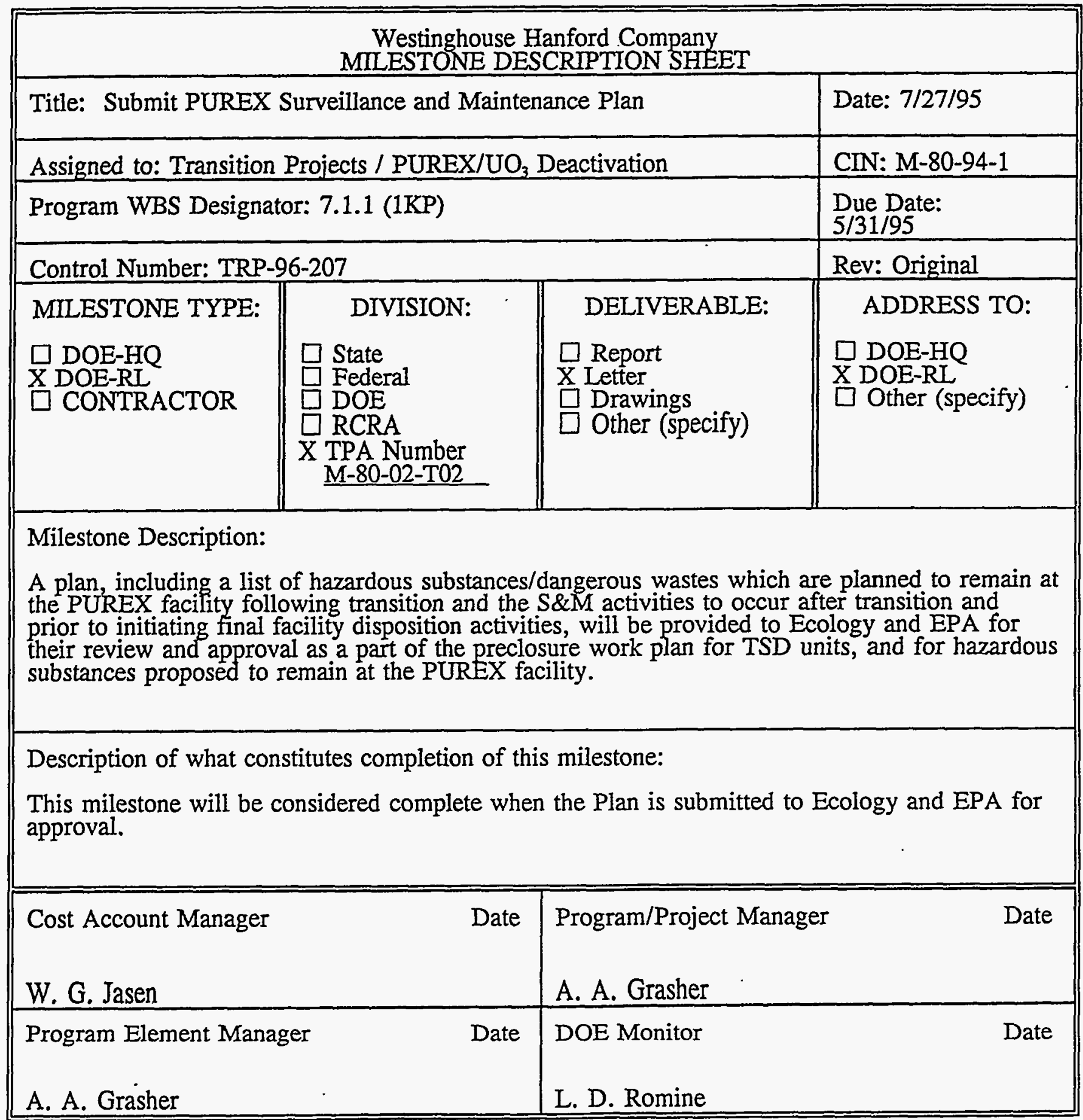


WHC-SP-1126, Rev. 1

\section{TRANSITION PROJECTS}

FY 1996 MYPP

1.3.1/7.1/6.12

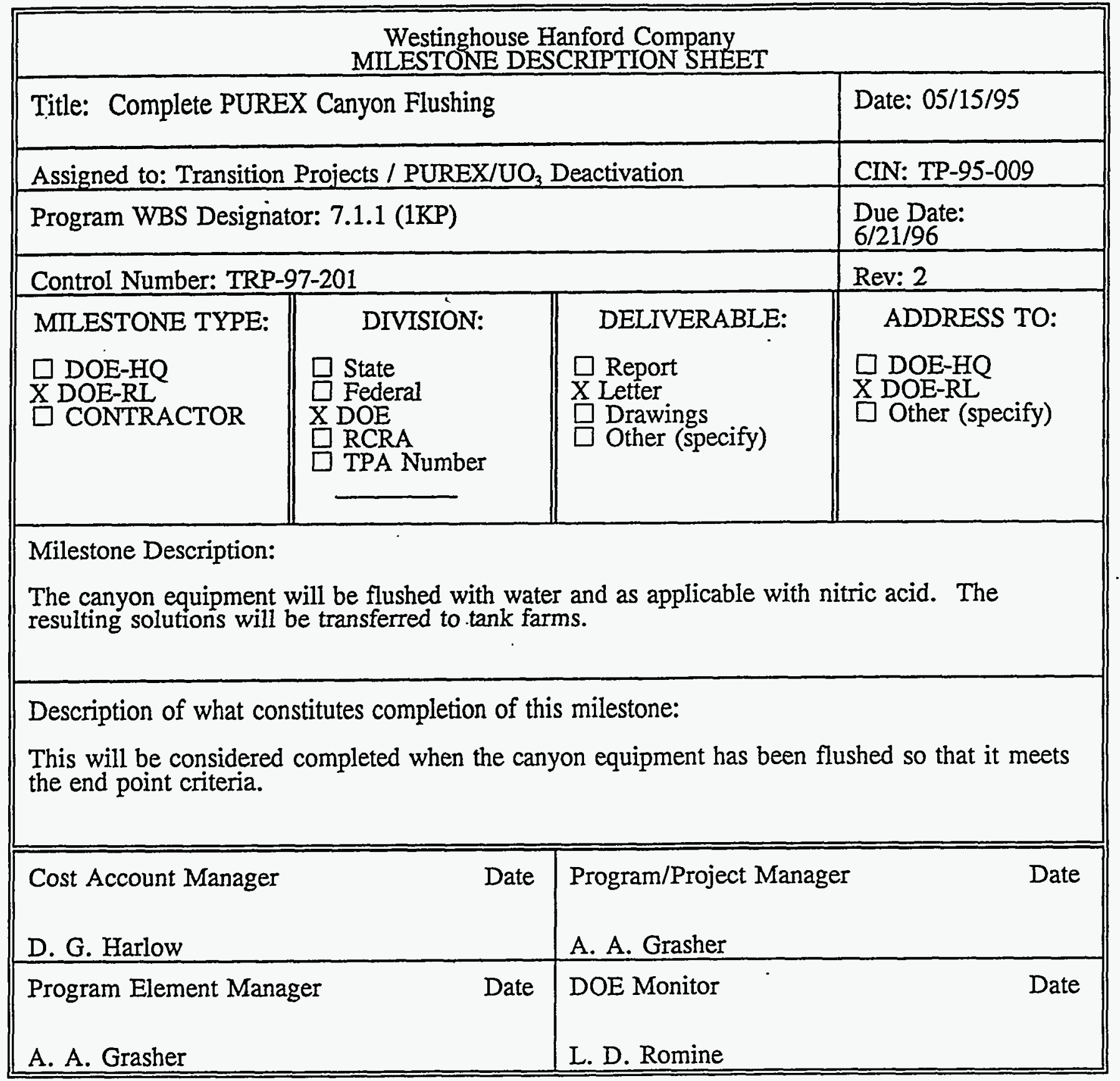


WHC-SP-1126, Rev. 1

\section{TRANSITION PROJECTS}

FY 1996 MYPP

1.3.1/7.1/6.12

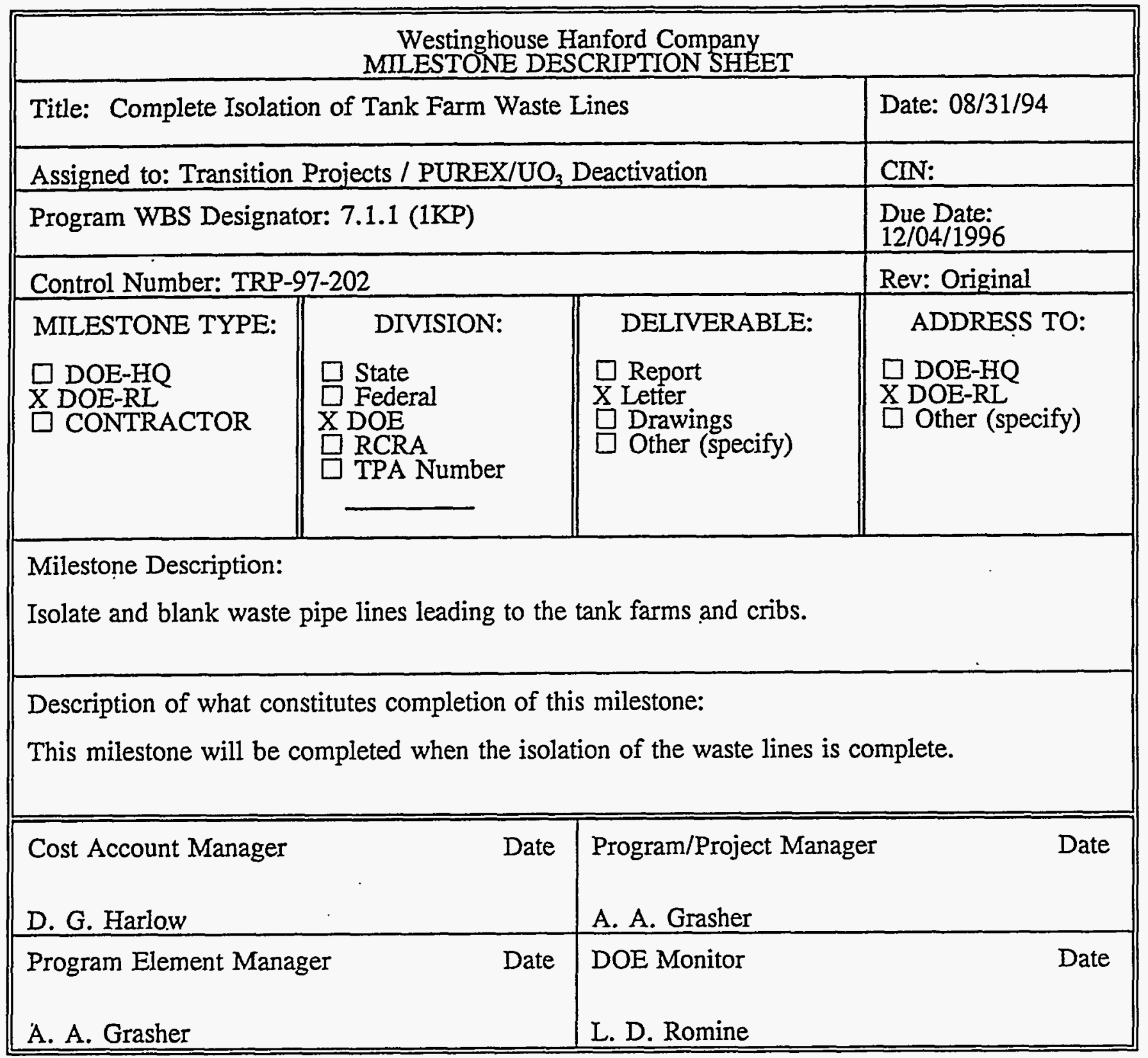


WHC-SP-1126, Rev. 1

FY 1996 MYPP

\section{TRANSITION PROJECTS}

\subsection{1/7.1/6.12}

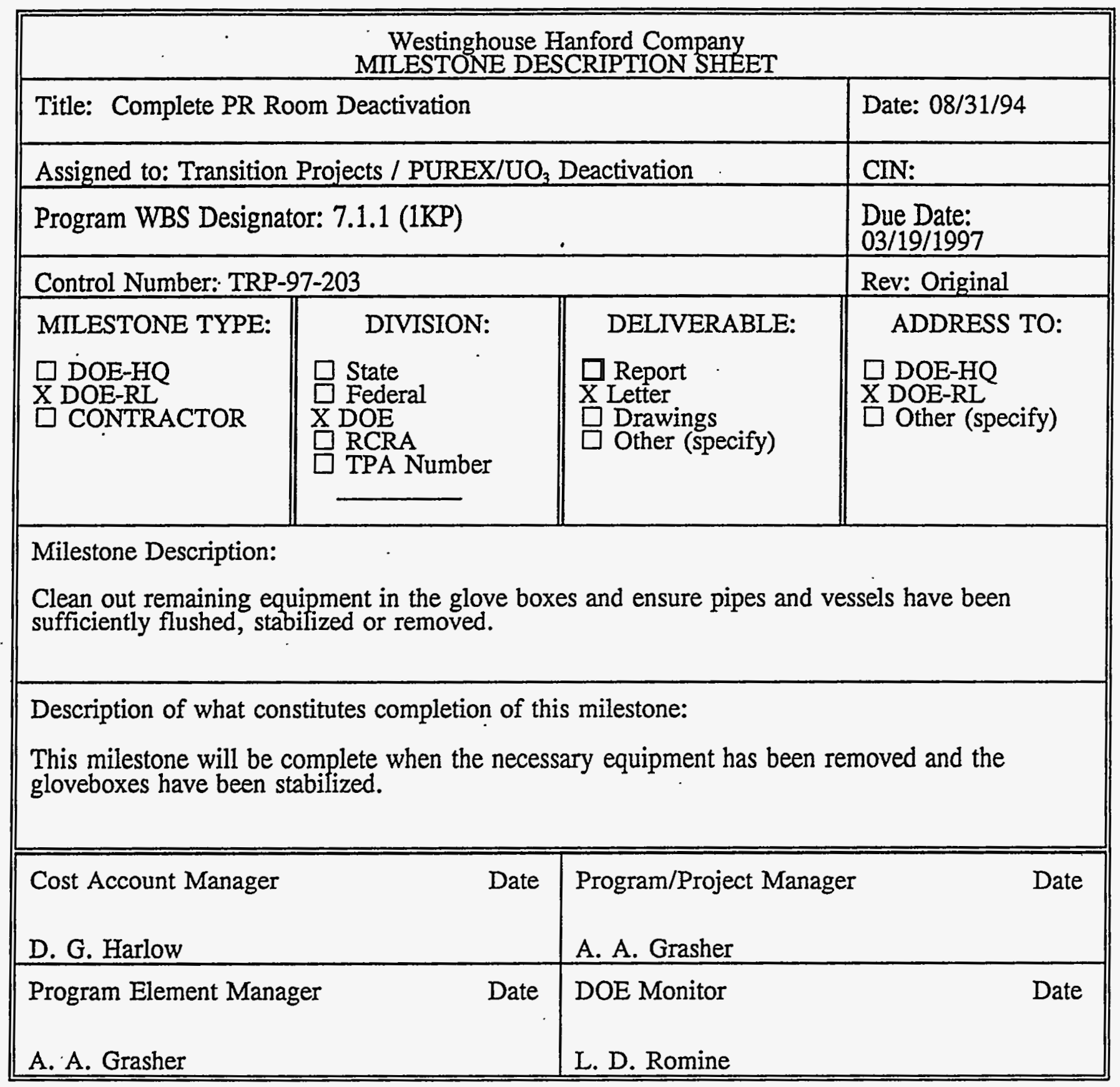


WHC-SP-1126, Rev. 1

\section{TRANSITION PROJECTS}

FY 1996 MYPP

1.3.1/7.1/6.12

\begin{tabular}{|c|c|c|c|}
\hline \multicolumn{4}{|c|}{$\begin{array}{l}\text { Westinghouse Hanford Company } \\
\text { MLESTONE DESCRIPTION SHEET }\end{array}$} \\
\hline \multicolumn{3}{|c|}{ Title: Complete Sample Gallery Deactivation } & Date: $05 / 15 / 95$ \\
\hline \multicolumn{3}{|c|}{ Assigned to: Transition Projects / PUREX/UO ${ }_{3}$ Deactivation } & CIN: TP-95-009 \\
\hline \multicolumn{3}{|c|}{ Program WBS Designator: 7.1.1 (1KP) } & Due Date: $12 / 02 / 96$ \\
\hline \multicolumn{3}{|c|}{ Control Number: TRP-97-204 } & Rev: 2 \\
\hline $\begin{array}{l}\text { MILESTONE TYPE: } \\
\square \text { DOE-HQ } \\
\text { X DOE-RI } \\
\square \text { CONTRACTOR }\end{array}$ & $\begin{array}{l}\text { DIVISION: } \\
\square \text { State } \\
\square \text { Federal } \\
\text { X DOE } \\
\square \text { RCRA } \\
\square \text { TPA Number }\end{array}$ & $\begin{array}{l}\text { DELIVERABLE: } \\
\square \text { Report } \\
\text { X Letter } \\
\square \text { Drawings } \\
\square \text { Other (specify) }\end{array}$ & $\begin{array}{l}\text { ADDRESS TO: } \\
\square \text { DOE-HQ } \\
\text { X DOE-RL } \\
\square \text { Other (specify) }\end{array}$ \\
\hline \multicolumn{4}{|c|}{$\begin{array}{l}\text { Milestone Description: } \\
\text { Clean out remaining solution and chemicals in the Sample Gallery tanks and pipes and stabilize } \\
\text { the radioactive contamination in the samplers. }\end{array}$} \\
\hline \multicolumn{4}{|c|}{$\begin{array}{l}\text { Description of what constitutes completion of this milestone: } \\
\text { This milestone will be completed when the sample gallery meets the end point criteria for } \\
\text { turnover to the EM-40. }\end{array}$} \\
\hline \multicolumn{2}{|l|}{$\begin{array}{l}\text { Cost Account Manager } \\
\text { D. G. Harlow }\end{array}$} & \multicolumn{2}{|c|}{ Program/Project Manager $\quad$ Date } \\
\hline \multicolumn{2}{|c|}{ Program Element Manager } & \multicolumn{2}{|l|}{$\begin{array}{l}\text { DOE Monitor } \\
\text { L. D. Romine }\end{array}$} \\
\hline
\end{tabular}


. WHC-SP-1126, Rev. 1

\section{TRANSITION PROJECTS}

FY 1996 MYPP
1.3.1/7.1/6.12

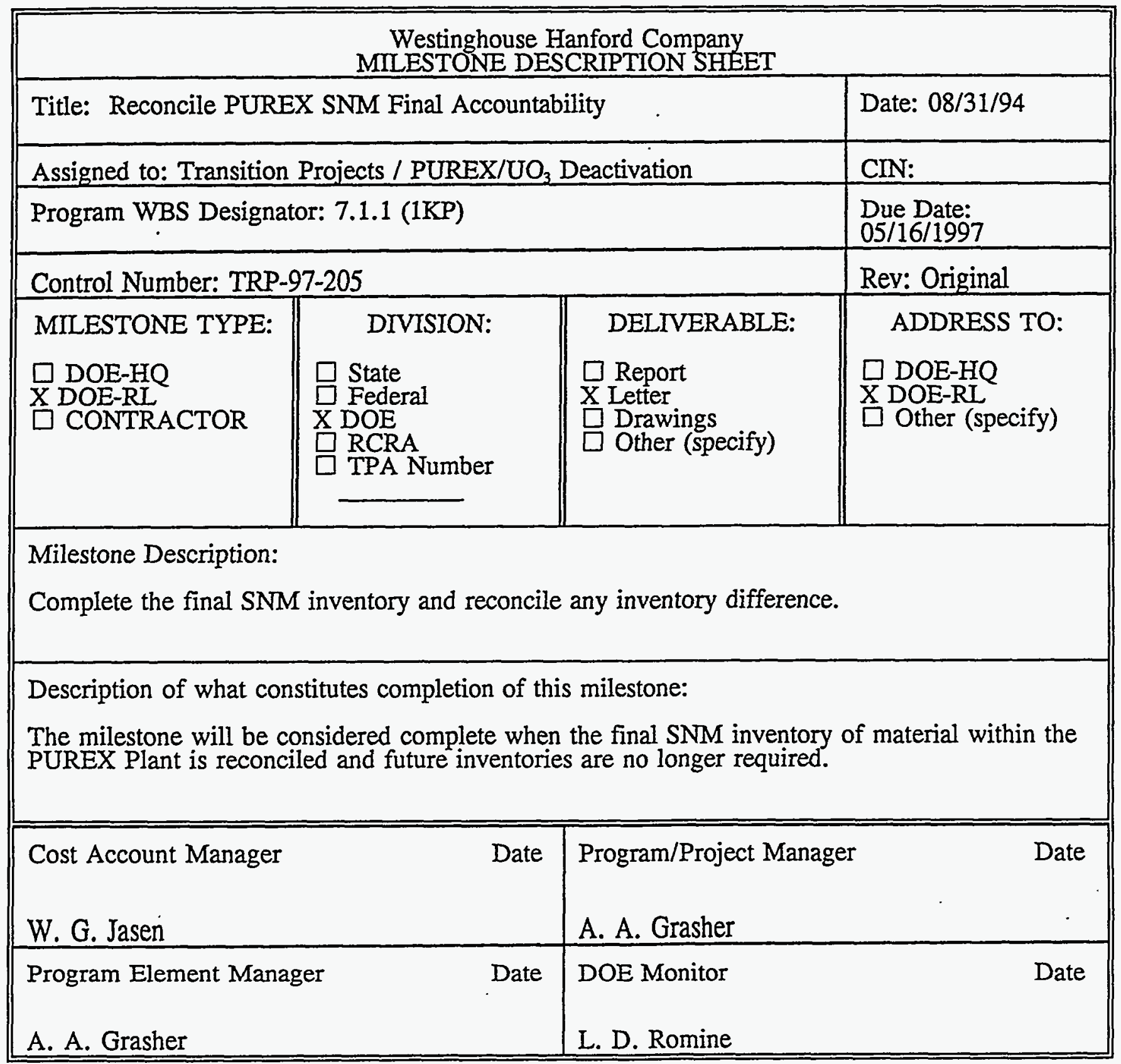


WHC-SP-1126, Rev. 1

FY 1996 MYPP

\title{
TRANSITION PROJECTS
}

\author{
1.3.1/7.1/6.12
}

Westinghouse Hanford Company MLESTONE DESCRIPTION SHEET

\begin{tabular}{|c|c|c|c|}
\hline \multicolumn{3}{|c|}{ Title: Complete Deactivation of Pipe \& Operating Gallery } & Date: $08 / 31 / 94$ \\
\hline \multicolumn{3}{|c|}{ Assigned to: Transition Projects / PUREX/UO 3 Deactivation } & CIN: \\
\hline \multicolumn{3}{|c|}{ Program WBS Designator: 7.1.1 (1KP) } & $\begin{array}{l}\text { Due Date: } \\
06 / 02 / 1997\end{array}$ \\
\hline \multicolumn{3}{|c|}{ Control Number: TRP-97-206 } & Rev: Original \\
\hline MILESTONE TYPE: & DIVISION: & DELIVERABLE: & ADDRESS TO: \\
\hline $\begin{array}{l}\square \text { DOE-HQ } \\
\text { X DOE-RL } \\
\square \text { CONTRACTOR }\end{array}$ & $\begin{array}{l}\square \text { State } \\
\square \text { Federal } \\
\text { XOE } \\
\square \text { RCRA } \\
\square \text { TPA Number }\end{array}$ & $\begin{array}{l}\text { Report } \\
\text { X Letter } \\
\square \text { Drawings } \\
\square \text { Other (specify) }\end{array}$ & $\begin{array}{l}\square \text { DOE-HQ } \\
\text { X DOE-RI } \\
\square \text { Other (specify) }\end{array}$ \\
\hline
\end{tabular}

Milestone Description:

The chemical supply lines and tanks in the gallery will be flushed with water and drained. Connections between the $\mathrm{P} \& \mathrm{O}$ gallery and other areas of the plant will be isolated to prevent material migration.

Description of what constitutes completion of this milestone:

This milestone will be completed when the end state criteria for the P\&O Gallery has been met.

\begin{tabular}{||ll|ll||}
\hline \hline Cost Account Manager & Date & Program/Project Manager & Date \\
D. G. Harlow & & A. A. Grasher & \\
\hline Program Element Manager & Date & DOE Monitor & Date \\
A. A. Grasher & & L. D. Romine & \\
\hline
\end{tabular}


WHC-SP-1126, Rev. 1

\section{TRANSITION PROJECTS}

FY 1996 MYPP

1.3.1/7.1/6.12

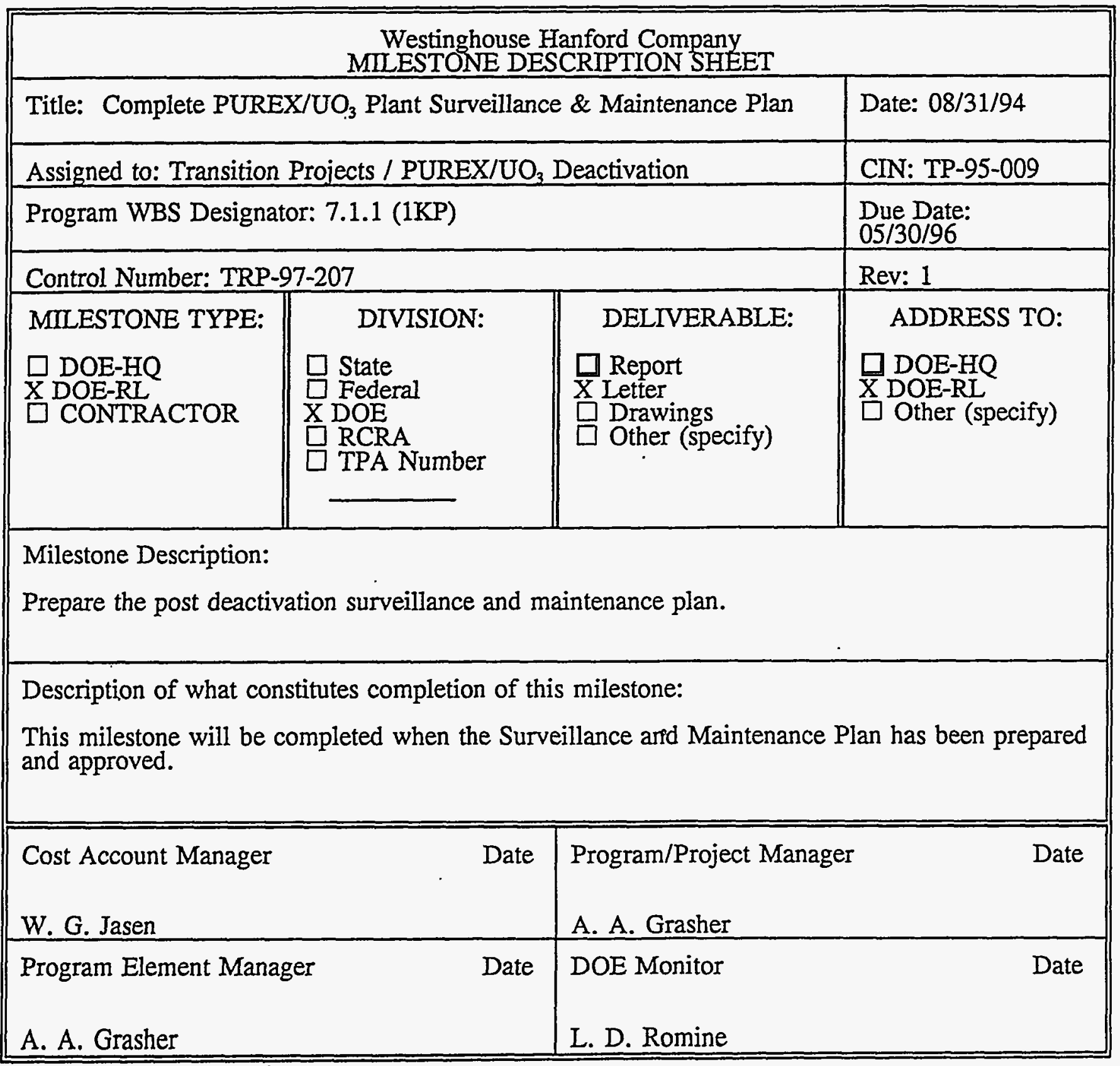


FY 1996 MYPP

WHC-SP-1126, Rev. 1

\section{TRANSITION PROJECTS}

\subsection{1/7.1/6.12}

\begin{tabular}{|c|c|c|c|}
\hline \multicolumn{4}{|c|}{$\begin{array}{l}\text { Westinghouse Hanford Company } \\
\text { MILESTONE DESCRIPTION SHEET }\end{array}$} \\
\hline \multicolumn{3}{|c|}{ Title: Complete HVAC System Consolidation } & Date: $05 / 15 / 95$ \\
\hline \multicolumn{3}{|c|}{ Assigned to: Transition Projects / PUREX/UO ${ }_{3}$ Deactivation } & CIN: TP-95-009 \\
\hline \multicolumn{3}{|c|}{ Program WBS Designator: 7.1.1 (1KP) } & $\begin{array}{l}\text { Due Date: } \\
\text { 2/13/97 }\end{array}$ \\
\hline \multicolumn{3}{|c|}{ Control Number: TRP-97-208 } & Rev: 2 \\
\hline $\begin{array}{l}\text { MILESTONE TYPE: } \\
\square \text { DOE-HQ } \\
\text { X DOE-RL } \\
\square \text { CONTRACTOR }\end{array}$ & 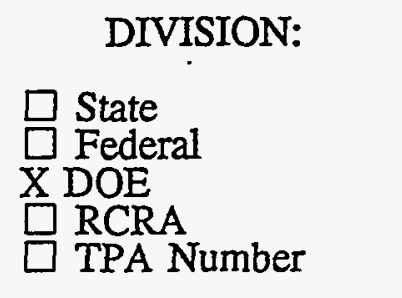 & $\begin{array}{l}\text { DELTVERABLE: } \\
\square \text { Report } \\
\text { X Letter } \\
\square \text { Drawings } \\
\square \text { Other (specify) }\end{array}$ & $\begin{array}{l}\text { ADDRESS TO: } \\
\square \text { DOE-HQ } \\
\text { X DOE-RI } \\
\square \text { Other (specify) }\end{array}$ \\
\hline \multicolumn{4}{|c|}{$\begin{array}{l}\text { Milestone Description: } \\
\text { HVAC and gaseous effluent flows will be terminated or redirected by engineered revisions } \\
\text { within the PUREX facility. }\end{array}$} \\
\hline \multicolumn{4}{|c|}{$\begin{array}{l}\text { Description of what constitutes completion of this milestone: } \\
\text { This milestone will be completed when the HVAC system modifications are completed to } \\
\text { eliminate or route all gaseous effluents through the PUREX main stack. }\end{array}$} \\
\hline \multicolumn{2}{|c|}{$\begin{array}{l}\text { Cost Account Manager Date } \\
\text { D. G. Harlow }\end{array}$} & \multicolumn{2}{|c|}{$\begin{array}{l}\text { Program/Project Manager Date } \\
\text { A. A. Grasher }\end{array}$} \\
\hline \multicolumn{2}{|c|}{$\begin{array}{l}\text { Program Element Manager } \\
\text { A. A. Grasher }\end{array}$} & \multicolumn{2}{|c|}{$\begin{array}{l}\text { DOE Monitor } \\
\text { L. D. Romine }\end{array}$} \\
\hline
\end{tabular}


WHC-SP-1126, Rev. 1

FY. 1996 MYPP

\section{TRANSITION PROJECTS}

1.3.1/7.1/6.12

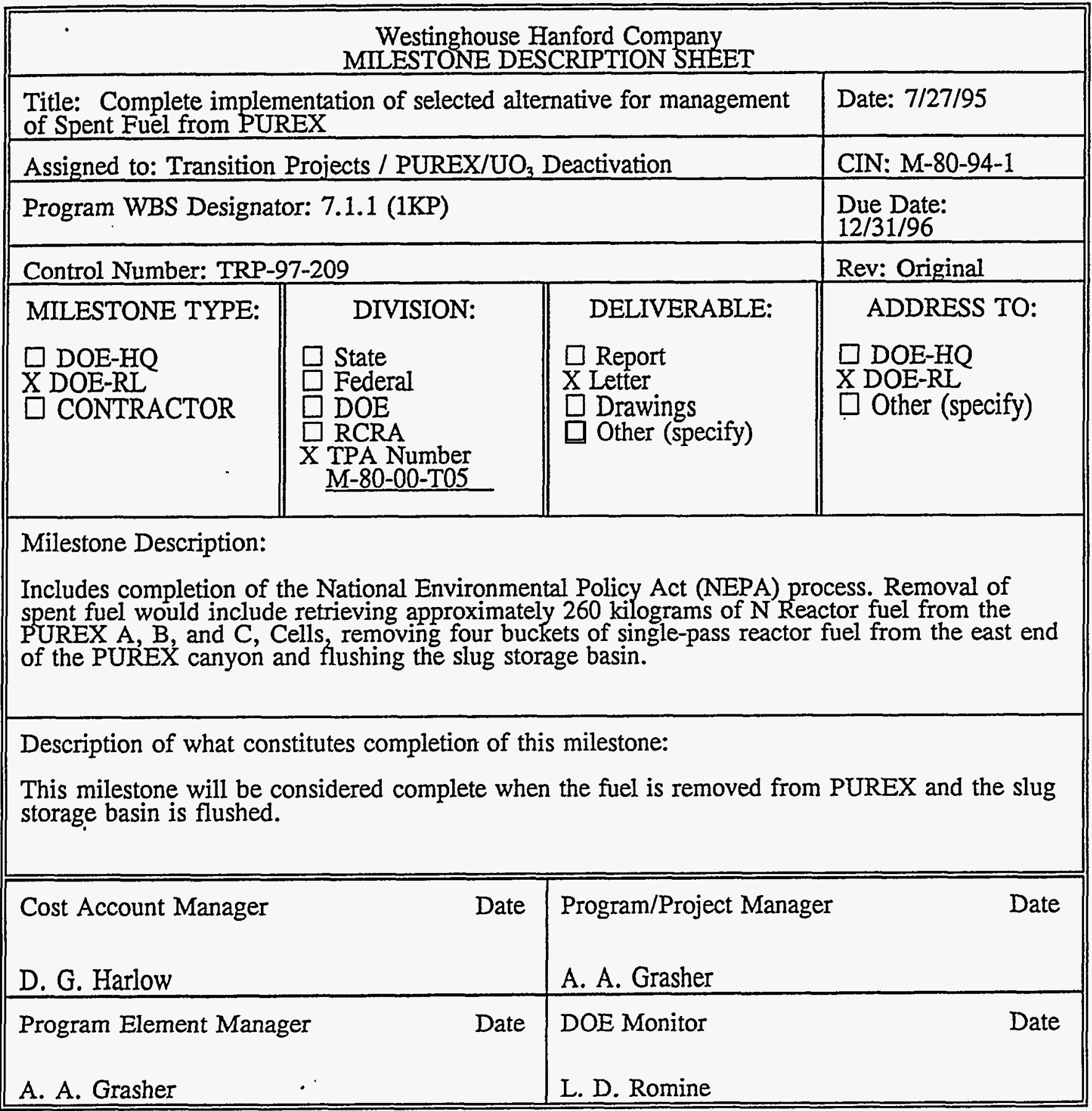


WHC-SP-1126, Rev. 1

FY 1996 MYPP

\section{TRANSITION PROJECTS}

1.3.1/7.1/6.12

\section{Westinghouse Hanford Company}

MILESTONE DESCRIPTION SHEET

Title: Complete Deactivation of the PUREX Plant 211-A Area

Assigned to: Transition Projects / PUREX/UO ${ }_{3}$ Deactivation

Program WBS Designator: 7.1.1 (1KP)

Control Number: TRP-97-210

MILESTONE TYPE:

$\square$ DOE-HQ

$\mathrm{X}$ DOE-RL

CONTRACTOR

\begin{tabular}{|l||} 
DIVISION: \\
State \\
Federal \\
\hline DOE \\
RCRA \\
X TPA Number \\
M-80-00-T06 \\
\hline
\end{tabular}

DELIVERABLE:

$\square$ Report

$\mathrm{X}$ Letter

Drawings

$\square$ Other (specify)

Date: $7 / 27 / 95$

CIN: M-80-94-1

Due Date:

4/30/97

Rev: Original

ADDRESS TO:

DOE-HQ

X DOE-RL

Other (specify)

Milestone Description:

Deactivation of the 211-A Area includes, but is not limited to, removing the chemical inventory, flushing tanks, removing tank heels, disposing of resins contained within isolated demineralizers, isolating utilities, and decontaminating/stabilizing surfaces contaminated with hazardous materials, as necessary. This target date does not include Tank-40 (see M-80-00-T03).

Description of what constitutes completion of this milestone:

This milestone will be considered complete when the necessary above actions have been taken and the 211-A Area meets the end point criteria.

\begin{tabular}{||ll|lr||}
\hline \hline Cost Áccount Manager & Date & Program/Project Manager & Date \\
D. G. Harlow & & A. A. Grasher & \\
\hline Program Element Manager & Date & DOE Monitor & Date \\
A. A. Grasher & & L. D. Romine & $\cdot$ \\
\hline
\end{tabular}




\section{WHC-SP-1126, Rev. I \\ TRANSITION PROJECTS}

FY 1996 MYPP

\subsection{1/7.1/6.12}

\begin{tabular}{|c|c|c|c|}
\hline \multicolumn{4}{|c|}{$\begin{array}{l}\text { Westinghouse Hanford Company } \\
\text { MMESTONE DESCRIPTION SHEET }\end{array}$} \\
\hline \multicolumn{3}{|c|}{ Title: Complete Deactivation of the PUREX Plant Sample Gallery } & Date: $7 / 27 / 95$ \\
\hline \multicolumn{3}{|c|}{ Assigned to: Transition Projects / PUREX/UO ${ }_{3}$ Deactivation } & CIN: $M-80-94-1$ \\
\hline \multicolumn{3}{|c|}{ Program WBS Designator: $7.1 .1(1 \mathrm{KP})$} & $\begin{array}{l}\text { Due Date: } \\
6 / 30 / 97\end{array}$ \\
\hline \multicolumn{3}{|c|}{ Control Nümber: TRP-97-211 } & Rev: Original \\
\hline $\begin{array}{l}\text { MILESTONE TYPE: } \\
\square \text { DOE-HQ } \\
\text { X DOE-RL } \\
\square \text { CONTRACTOR }\end{array}$ & $\begin{array}{l}\text { DIVISION: } \\
\square \text { State } \\
\text { Federal } \\
\text { DOE } \\
\text { DOE } \\
\text { XCRA } \\
\text { TPA Number } \\
\text { M-80-00-T07 }\end{array}$ & $\begin{array}{l}\text { DELIVERABLE: } \\
\square \text { Report } \\
\text { X Letter } \\
\square \text { Drawings } \\
\square \text { Other (specify) }\end{array}$ & $\begin{array}{l}\text { ADDRESS TO: } \\
\square \text { DOE-HQ } \\
\text { X DOE-RL } \\
\square \text { Other (specify) }\end{array}$ \\
\hline \multicolumn{4}{|c|}{$\begin{array}{l}\text { Milestone Description: } \\
\text { Deactivation of the Sample Gallery includes, but is not limited to, flushing headers and high } \\
\text { radiation samplers that may pose a contamination or dose problem, decontaminating and/or } \\
\text { stabilizing hoods containing significant quantities of special nuclear material, and } \\
\text { decontaminating/stabilizing and/or removing hood duct work. }\end{array}$} \\
\hline \multicolumn{4}{|c|}{$\begin{array}{l}\text { Description of what constitutes completion of this milestone: } \\
\text { This milestone will be considered complete when the Sample Gallery meets the end point } \\
\text { criteria. }\end{array}$} \\
\hline \multicolumn{2}{|c|}{$\begin{array}{l}\text { Cost Account Manager Date } \\
\text { D. G. Harlow }\end{array}$} & \multicolumn{2}{|c|}{$\begin{array}{l}\text { Program/Project Manager Date } \\
\text { A. A. Grasher }\end{array}$} \\
\hline \multicolumn{2}{|c|}{$\begin{array}{ll}\text { Program Element Manager } \quad \text { Date }\end{array}$} & \multicolumn{2}{|c|}{$\begin{array}{l}\text { DOE Monitor Date } \\
\text { L. D. Romine }\end{array}$} \\
\hline
\end{tabular}


WHC-SP-1126, Rev. 1

\section{TRANSITION PROJECTS}

FY 1996 MYPP

1.3.1/7.1/6.12

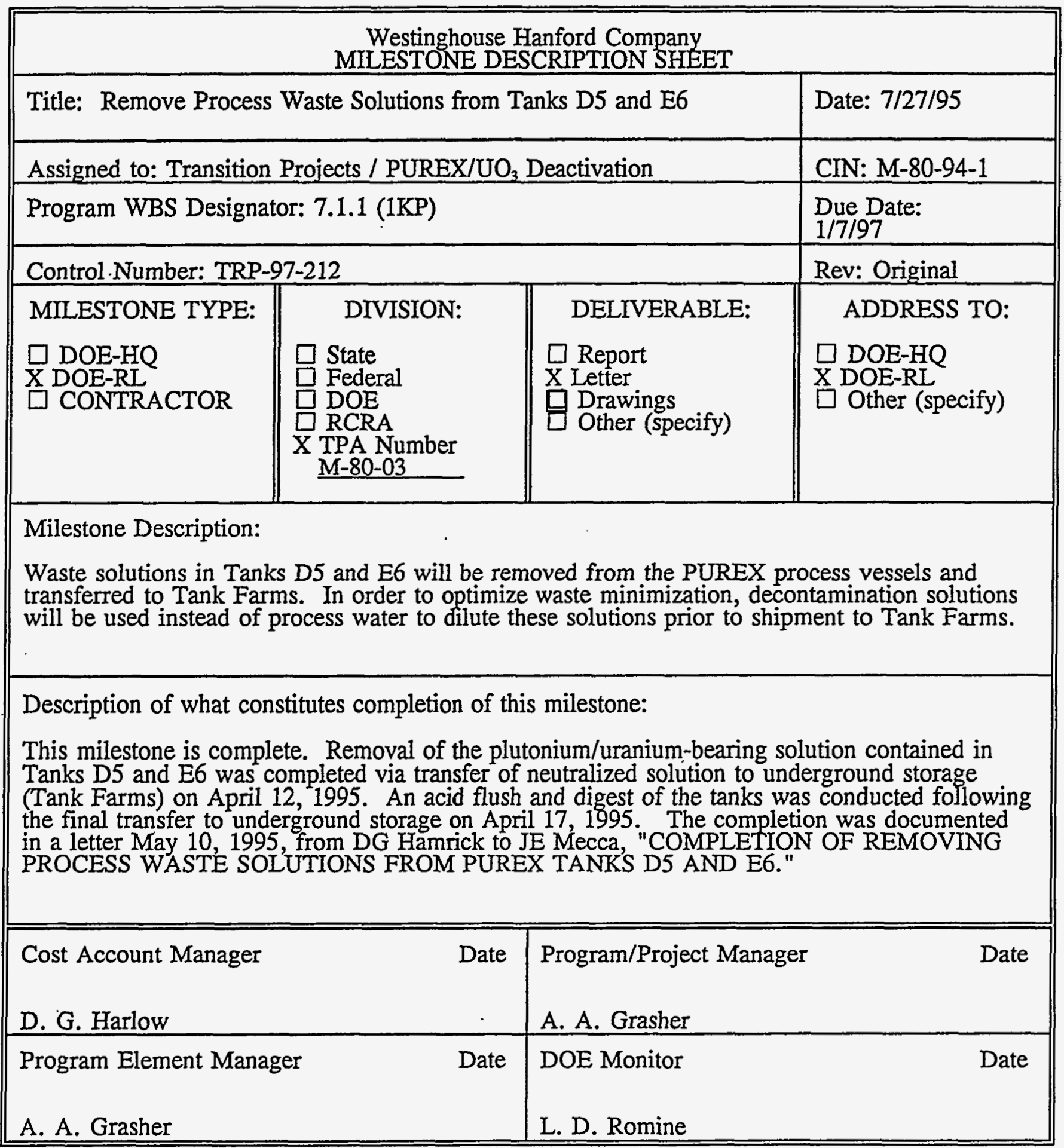


WHC-SP-1126, Rev. 1

\section{TRANSITION PROJECTS}

FY 1996 MYPP

1.3.1/7.1/6.12

Westinghouse Hanford Company MILESTONE DESCRIPTION SHEET

\begin{tabular}{|c|c|c|c|}
\hline \multicolumn{3}{|c|}{ Title: Complete Deactivation of the PUREX Plant U-Cell/Fractionator } & Date: $7 / 27 / 95$ \\
\hline \multicolumn{3}{|c|}{ Assigned to: Transition Projects / PUREX/UO ${ }_{3}$ Deactivation } & CIN: M-80-94-1 \\
\hline \multicolumn{3}{|c|}{ Program WBS Designator: 7.1 .1 (1KP) } & $\begin{array}{l}\text { Due Date: } \\
4 / 30 / 97\end{array}$ \\
\hline \multicolumn{3}{|c|}{ Control Number: TRP-97-213 } & Rev: Original \\
\hline MILESTONE TYPE: & DIVISION: & DELIVERABLE: & ADDRESS TO: \\
\hline $\begin{array}{l}\square \text { DOE-HQ } \\
\text { X DOE-RI } \\
\square \text { CONTRACTOR }\end{array}$ & $\begin{array}{l}\square \text { State } \\
\square \text { Federal } \\
\square \text { DOE } \\
\square \text { RCRA } \\
\text { X TPA Number } \\
\text { M-80-04 }\end{array}$ & $\begin{array}{l}\square \text { Report } \\
\text { X Letter } \\
\square \text { Drawings } \\
\square \text { Other (specify) }\end{array}$ & $\begin{array}{l}\square \text { DOE-HQ } \\
\text { X DOE-RL } \\
\square \text { Other (specify) }\end{array}$ \\
\hline
\end{tabular}

Milestone Description:

Deactivation of the U-Cell/Fractionator includes, but is not limited to removing recovered nitric acid, flushing vessels, and sealing $U$ cell cover blocks.

Description of what constitutes completion of this milestone:

The milestone will be considered complete when the U-Cell/fractionator systems meet the end point criteria.

\begin{tabular}{|ll|ll||}
\hline \hline Cost Account Manager & Date & Program/Project Manager & Date \\
D. G. Harlow & & A. A. Grasher & Date \\
\hline Program Element Manager & Date & DOE Monitor & \\
A. A. Grasher & & L. D. Romine & \\
\hline
\end{tabular}


WHC-SP-1126, Rev. 1

\section{TRANSITION PROJECTS}

FY 1996 MYPP

1.3.1/7.1/6.12

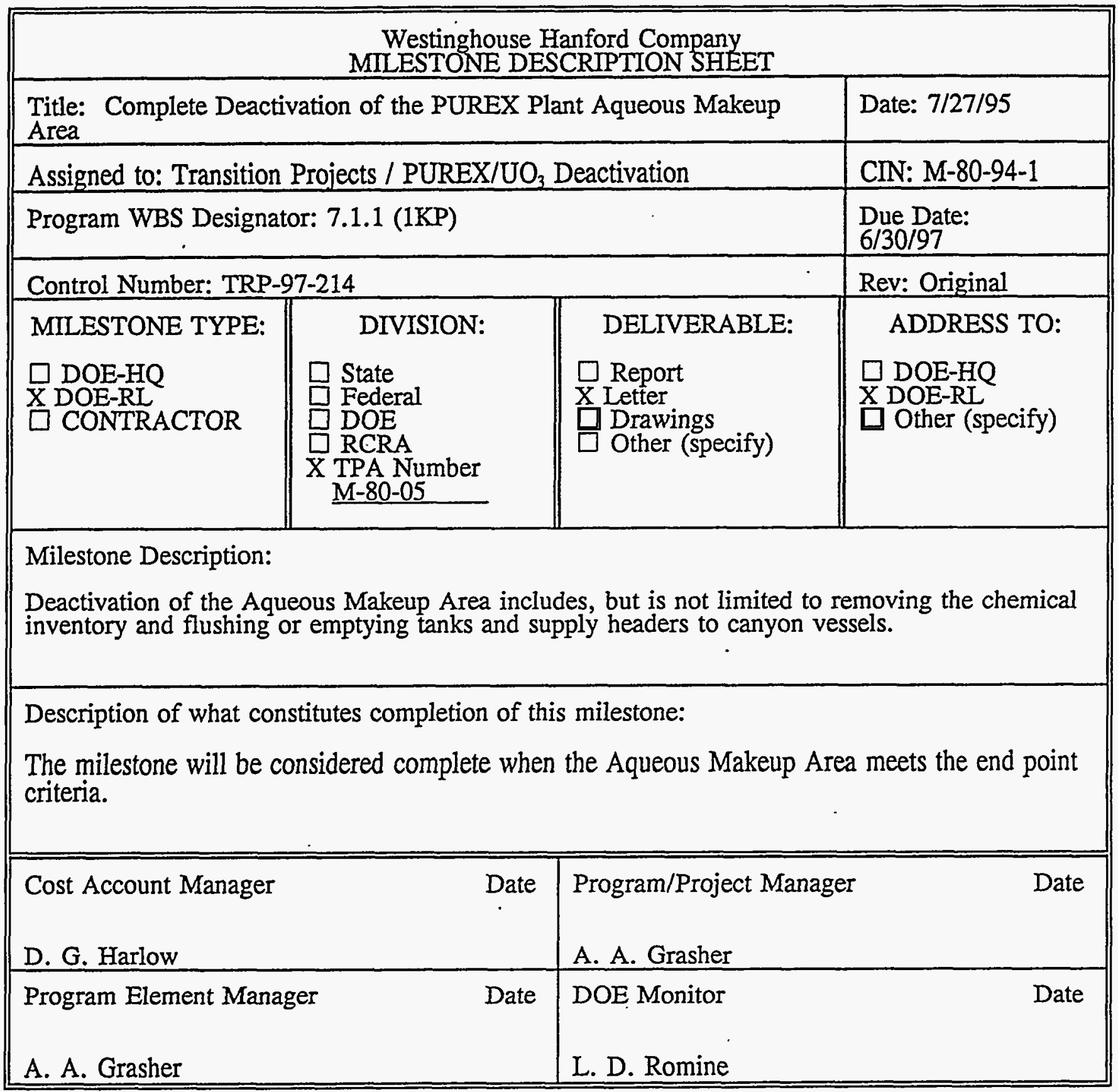


WHC-SP-1126, Rev. 1

\section{TRANSITION PROJECTS}

FY 1996 MYPP

\subsection{1/7.1/6.12}

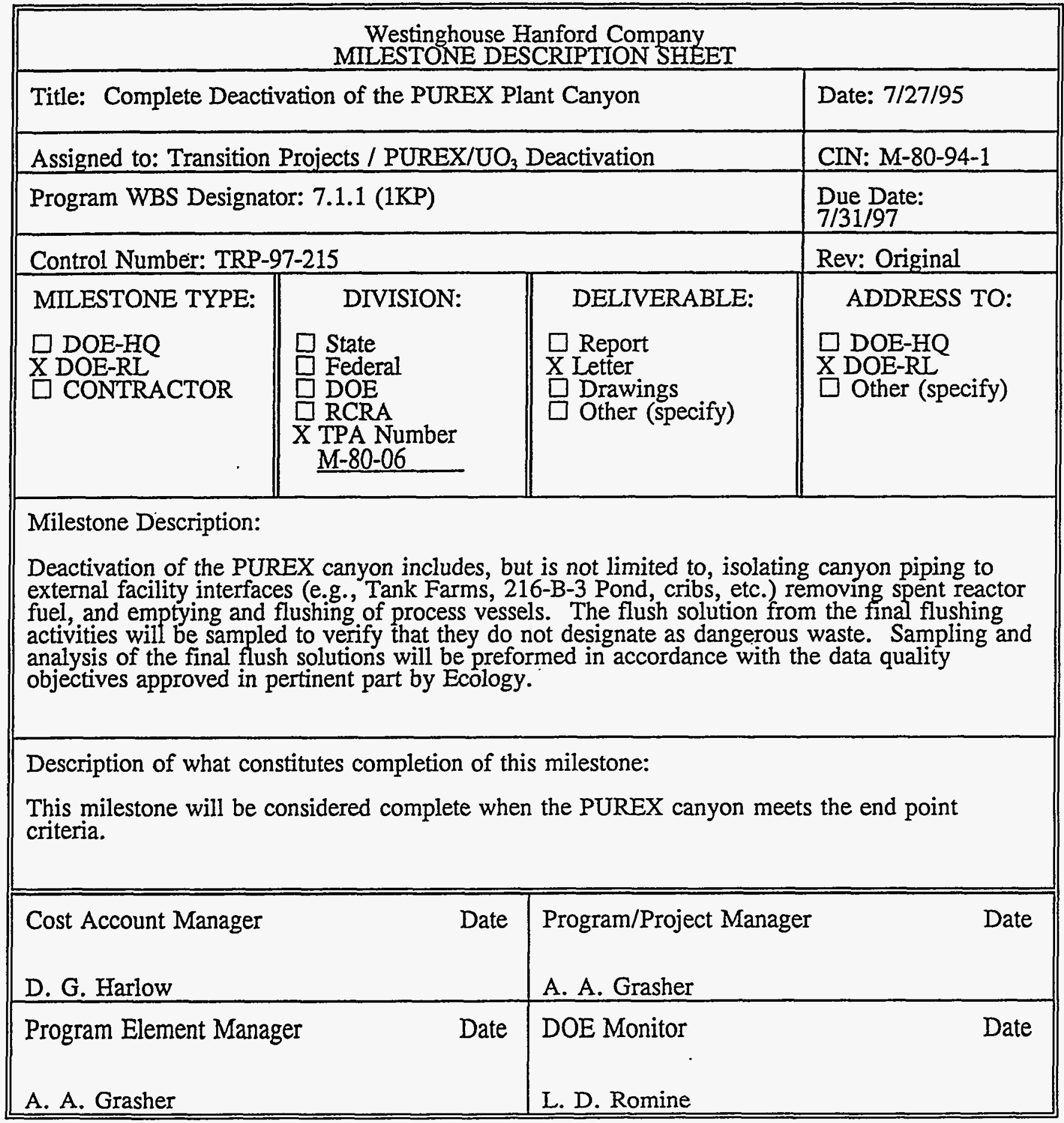


WHC-SP-1126, Rev. 1

\section{TRANSITION PROJECTS}

FY 1996 MYPP

1.3.1/7.1/6.12

Westinghouse Hanford Company MILESTONE DESCRIPTION SHEET

\begin{tabular}{|c|c|c|c|}
\hline \multicolumn{3}{|c|}{ Title: Complete Deactivation of Ancillary Buildings } & Date: $10 / 18 / 94$ \\
\hline \multicolumn{3}{|c|}{ Assigned to: Transition Projects / PUREX/UO 3 Deactivation } & CIN: TP-95-009 \\
\hline \multicolumn{3}{|c|}{ Program WBS Designator: 7.1 .1 (1KP) } & $\begin{array}{l}\text { Due Date: } \\
8 / 22 / 97\end{array}$ \\
\hline \multicolumn{3}{|c|}{ Control Number: TRP-98-201 } & Rev: 1 \\
\hline MILESTONE TYPE: & DIVISION: & DELIVERABLE: & ADDRESS TO: \\
\hline $\begin{array}{l}\square \text { DOE-HQ } \\
\text { X DOE-RL } \\
\square \text { CONTRACTOR }\end{array}$ & $\begin{array}{l}\square \text { State } \\
\square \text { Federal } \\
\text { X DOE } \\
\square \text { RCRA } \\
\square \text { TPA Number }\end{array}$ & $\begin{array}{l}\square \text { Report } \\
\text { X Letter } \\
\square \text { Drawings } \\
\square \text { Other (specify) }\end{array}$ & $\begin{array}{l}\square \text { DOE-HQ } \\
\text { X DOE-RI } \\
\square \text { Other (specify) }\end{array}$ \\
\hline
\end{tabular}

Milestone Description:

The ancillary buildings, structures and trailers at PUREX will be deactivated.

Description of what constitutes completion of this milestone:

This milestone will be completed when the ancillary buildings meet the end point criteria.

\begin{tabular}{|ll|ll|}
\hline \hline Cost Account Manager & Date & Program/Project Manager & Date \\
D. G. Harlow & & A. A. Grasher & \\
\hline Program Element Manager & Date & DOE Monitor & Date \\
A. A. Grasher & & L. D. Romine & \\
\hline
\end{tabular}


WHC-SP-1126, Rev. 1

\section{TRANSITION PROJECTS}

FY 1996 MYPP

1.3.1/7.1/6.12

\begin{tabular}{|c|c|c|c|}
\hline \multicolumn{4}{|c|}{$\begin{array}{l}\text { Westinghouse Hanford Company } \\
\text { MILESTONE DESCRIPTION SHEET }\end{array}$} \\
\hline \multicolumn{3}{|c|}{ Title: Discontinue PUREX Liquid Effluent Discharge } & Date: $10 / 18 / 94$ \\
\hline \multicolumn{3}{|c|}{ Assigned to: Transition Projects / PUREX/UO ${ }_{3}$ Deactivation } & CIN: TP-95-009 \\
\hline \multicolumn{3}{|c|}{ Program WBS Designator: $7.1 .1(1 \mathrm{KP})$} & $\begin{array}{l}\text { Due Date: } \\
1 / 23 / 98\end{array}$ \\
\hline \multicolumn{3}{|c|}{ Control Number: TRP-98-202 } & Rev: 1 \\
\hline $\begin{array}{l}\text { MILESTONE TYPE: } \\
\square \text { DOE-HQ } \\
\text { X DOE-RL } \\
\square \text { CONTRACTOR }\end{array}$ & 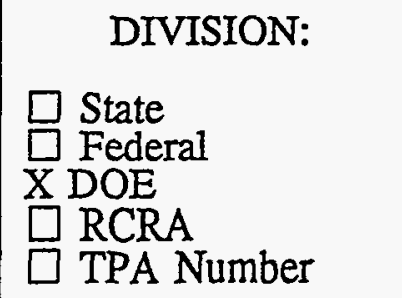 & $\begin{array}{l}\text { DELIVERABLE: } \\
\square \text { Report } \\
\text { X Letter } \\
\square \text { Drawings } \\
\square \text { Other (specify) }\end{array}$ & $\begin{array}{l}\text { ADDRESS TO: } \\
\square \text { DOE-HQ } \\
\text { X DOE-RI } \\
\square \text { Other (specify) }\end{array}$ \\
\hline \multicolumn{4}{|c|}{$\begin{array}{l}\text { Milestone Description: } \\
\text { All liquid effluents from the PUREX Plant will be discontinued. }\end{array}$} \\
\hline \multicolumn{4}{|c|}{$\begin{array}{l}\text { Description of what constitutes completion of this milestone: } \\
\text { This milestone will be completed when the liquid effluent system has been shut down and the } \\
\text { effluent line blanked. }\end{array}$} \\
\hline \multicolumn{2}{|c|}{$\begin{array}{l}\text { Cost Account Manager Date } \\
\text { D. G. Harlow }\end{array}$} & \multicolumn{2}{|c|}{$\begin{array}{l}\text { Program/Project Manager Date } \\
\text { A. A. Grasher }\end{array}$} \\
\hline \multicolumn{2}{|c|}{$\begin{array}{l}\text { Program Element Manager } \\
\text { A. A. Grasher }\end{array}$} & \multicolumn{2}{|l|}{$\begin{array}{l}\text { DOE Monitor } \\
\text { L. D. Romine }\end{array}$} \\
\hline
\end{tabular}




\section{TRANSITION PROJECTS}

WHC-SP-1126, Rev. 1

1.3.1/7.1/6.12

\begin{tabular}{|c|c|c|c|}
\hline \multicolumn{4}{|c|}{$\begin{array}{l}\text { Westinghouse Hanford Company } \\
\text { MMESTONE DESCRIPTION SHEET }\end{array}$} \\
\hline \multicolumn{3}{|c|}{ Title: Complete PUREX Deactivation } & Date: $10 / 18 / 94$ \\
\hline \multicolumn{3}{|c|}{ Assigned to: Transition Projects / PUREX/UO ${ }_{3}$ Deactivation } & CIN: TP-95-009 \\
\hline \multicolumn{3}{|c|}{ Program WBS Designator: $7.1 .1(1 \mathrm{KP})$} & $\begin{array}{l}\text { Due Date: } \\
1 / 30 / 98\end{array}$ \\
\hline \multicolumn{3}{|c|}{ Control Number: TRP-98-203 } & Rev: 1 \\
\hline $\begin{array}{l}\text { MILESTONE TYPE: } \\
\text { X DOE-HQ } \\
\square \text { DOE-RL } \\
\square \text { CONTRACTOR }\end{array}$ & $\begin{array}{l}\quad \text { DIVISION: } \\
\square \text { State } \\
\square \text { Federal } \\
\text { X DOE } \\
\square \text { RCRA } \\
\square \text { TPA Number }\end{array}$ & $\begin{array}{l}\text { DELIVERABLE: } \\
\square \text { Report } \\
\text { X Letter } \\
\square \text { Drawings } \\
\square \text { Other (specify) }\end{array}$ & $\begin{array}{l}\text { ADDRESS TO: } \\
\text { X DOE-HQ } \\
\square \text { DOE-RI } \\
\square \text { Other (specify) }\end{array}$ \\
\hline \multicolumn{4}{|c|}{$\begin{array}{l}\text { Milestone Description: } \\
\text { The PUREX Plant will be deactivated. }\end{array}$} \\
\hline \multicolumn{4}{|c|}{$\begin{array}{l}\text { Description of what constitutes completion of this milestone: } \\
\text { This milestone will be completed when the PUREX Plant meets the end point criteria. }\end{array}$} \\
\hline \multicolumn{2}{|c|}{$\begin{array}{l}\text { Cost Account Manager Date } \\
\text { D. G. Harlow }\end{array}$} & \multicolumn{2}{|c|}{$\begin{array}{l}\text { Program/Project Manager } \\
\text { A. A. Grasher }\end{array}$} \\
\hline \multicolumn{2}{|c|}{$\begin{array}{l}\text { Program Element Manager } \\
\text { A. A. Grasher }\end{array}$} & \multicolumn{2}{|l|}{$\begin{array}{l}\text { DOE Monitor } \\
\text { L. D. Romine } \\
\end{array}$} \\
\hline
\end{tabular}


WHC-SP-1126, Rev. 1

\title{
TRANSITION PROJECTS
}

FY 1996 MYPP

\author{
1.3.1/7.1/6.12
}

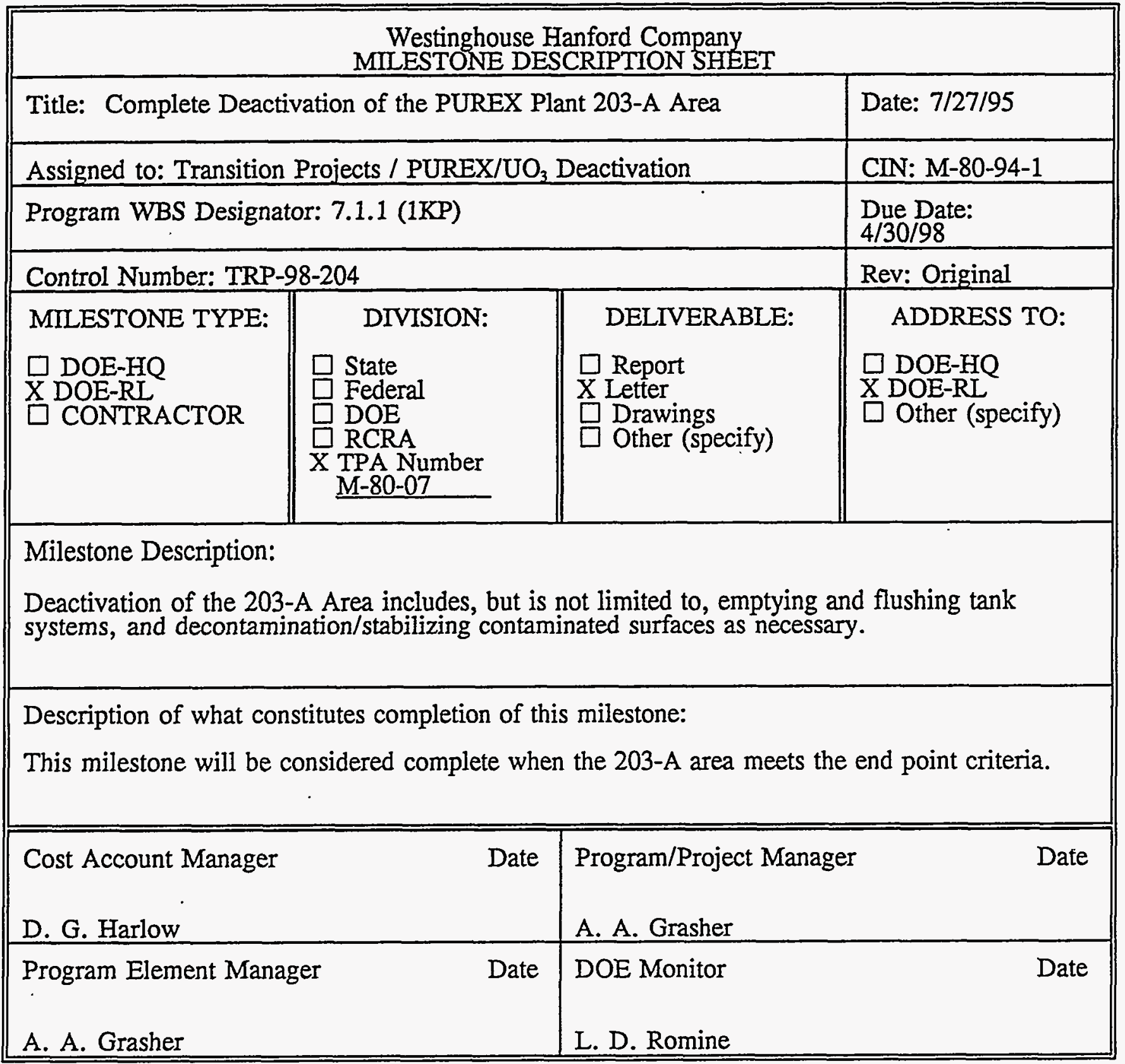


WHC-SP-1126, Rev. I

\title{
TRANSITION PROJECTS
}

FY 1996 MYPP

\author{
1.3.1/7.1/6.12
}

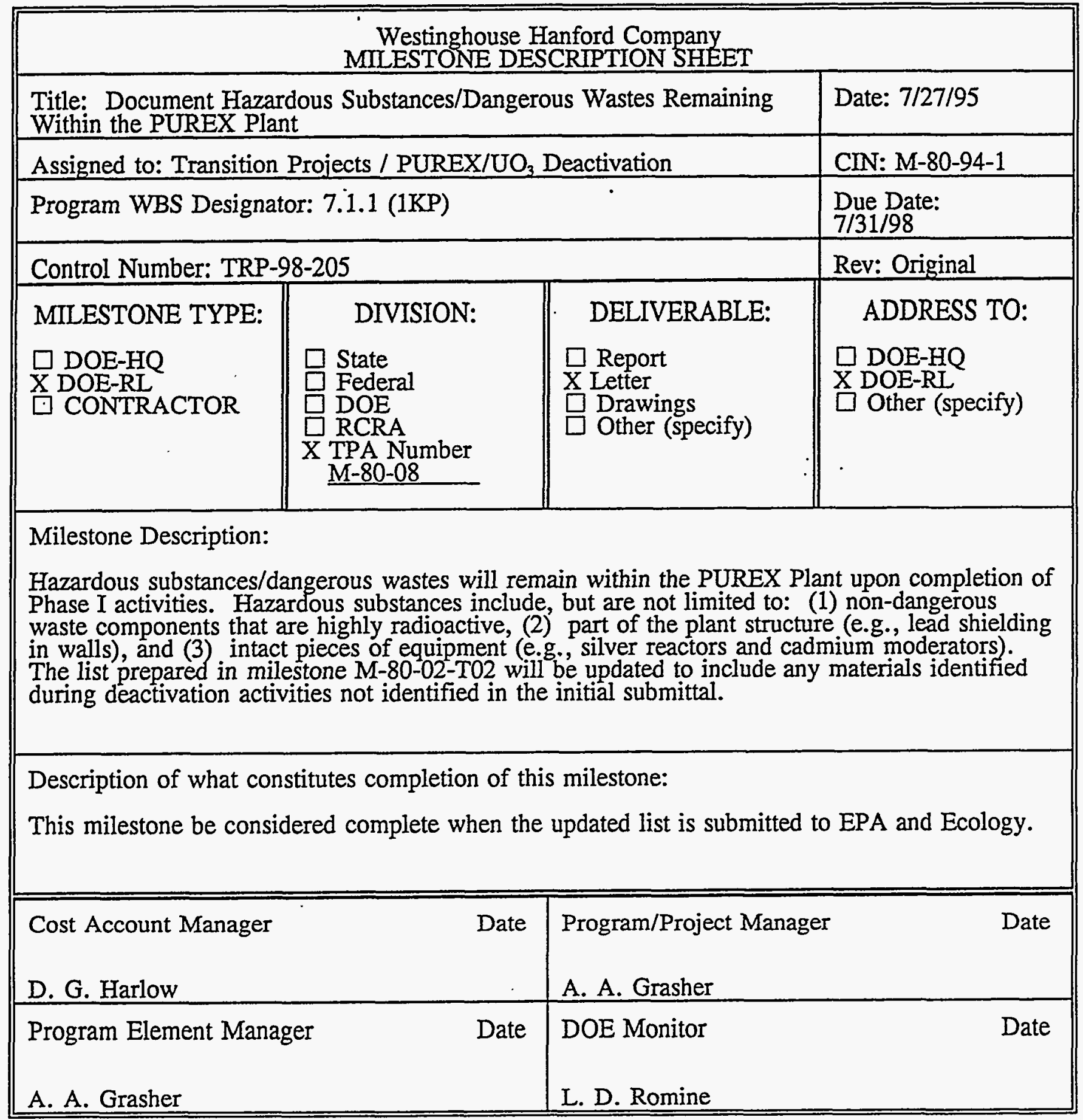


WHC-SP-1126, Rev. 1

\section{TRANSITION PROJECTS}

FY 1996 MYPP

\subsection{1/7.1/6.12}

\section{Westinghouse Hanford Company MILESTONE DESCRIPTION SHEET}

Title: Complete PUREX and $\mathrm{UO}_{3}$ Plant Facility Transition Phase and initiate the Surveillance and Maintenance Phase

Assigned to: Transition Projects / PUREX/UO ${ }_{3}$ Deactivation

Program WBS Designator: 7.1.1 (1KP)

Control Number: TRP-98-206

MILESTONE TYPE:

$\square$ DOE-HQ

X DOE-RL

CONTRACTOR

\begin{tabular}{||l||} 
DIVISION: \\
0 State \\
Federal \\
DOE \\
RCRA \\
X TPA Number \\
M-80-00 \\
\hline
\end{tabular}

DELIVERABLE:

$\square$ Report

X Letter

Drawings

Other (specify)
Date: $7 / 27 / 95$

CIN: M-80-94-1.

Due Date:

$7 / 31 / 98$

Rev: Original

ADDRESS TO:

$\square$ DOE-HQ

X DOE-RI

Other (specify)

\section{Milestone Description:}

Completion of this major milestone includes the following key elements: (1) completion of all activities necessary to achieve end point criteria for placing the PUREX/UO ${ }_{3}$ facilities in a safe and stable S\&M mode, and (2) completion of all activities described in the M-80 interim milestones and target actions.

Description of what constitutes completion of this milestone:

This milestone will be complete when all of the M-80 interim milestones and target actions are complete and the PUREX/UO 3 facilities meet the end point criteria.

\begin{tabular}{|ll|ll|}
\hline \hline Cost Account Manager & Date & Program/Project Manager & Date \\
D. G. Harlow & & A. A. Grasher & \\
\hline Program Element Manager & Date & DOE Monitor & Date \\
A. A. Grasher & & L. D. Romine & \\
\hline
\end{tabular}


WHC-SP-1126, Rev. 1

\section{TRANSITION PROJECTS}

FY 1996 MYPP

1.3.1/7.1/6.12

\begin{tabular}{|c|c|c|c|}
\hline \multicolumn{4}{|c|}{$\begin{array}{l}\text { Westinghouse Hanford Company } \\
\text { MLESTONE DESCRIPTION SHEET }\end{array}$} \\
\hline \multicolumn{3}{|c|}{$\begin{array}{l}\text { Title: Resolve Ecology Comments on } 304 \text { Final Report and Submit } 304 \\
\text { Part A Permit Stamped CLOSED to Ecology. }\end{array}$} & Date: $8 / 17 / 95$ \\
\hline \multicolumn{3}{|c|}{ Assigned To: Transition Projects / 300 Area Fuel Supply } & CIN: TP-95-057 \\
\hline \multicolumn{3}{|c|}{ Program WBS Designator: $7.1 .2(1 \mathrm{~K} 3)$} & Due Date: $3 / 29 / 96$ \\
\hline \multicolumn{3}{|c|}{ Control Number: TRP-96-303 } & \multirow{3}{*}{$\begin{array}{l}\text { Rev: } 1 \\
\text { ADDRESS TO: } \\
\square \text { DOE-HQ } \\
\text { X DOE-RL } \\
\square \text { Other }\end{array}$} \\
\hline MILESTONE TYPE: & DIVIȘION: & DELIVERABLE: & \\
\hline $\begin{array}{l}\square \text { DOE-HQ } \\
\text { X DOE-RL } \\
\square \text { CONTRACTOR }\end{array}$ & $\begin{array}{l}\text { X State } \\
\square \text { Federal } \\
\text { X DOE } \\
\text { X RCRA } \\
\square \text { TPA Number }\end{array}$ & $\begin{array}{l}\square \text { Report } \\
\text { X Letter } \\
\square \text { Drawings } \\
\square \text { Other }\end{array}$ & \\
\hline \multicolumn{4}{|c|}{$\begin{array}{l}\text { Milestone Description: } \\
\text { Resolve Ecology comments after their review of } 304 \text { final report and revise report, if needed. } \\
\text { Send Ecology copy of Part "A" Permit stamped "Closed" after Ecology agrees clean closure has } \\
\text { been achieved. }\end{array}$} \\
\hline \multicolumn{4}{|c|}{$\begin{array}{l}\text { Description of what constitutes completion of this milestone: } \\
\text { Transmittal letter to DOE-RL with Part "A" Permit stamped "Closed". }\end{array}$} \\
\hline \multicolumn{2}{|c|}{$\begin{array}{l}\text { Cost Account Manager Date } \\
\text { IL Metcalf }\end{array}$} & \multicolumn{2}{|c|}{$\begin{array}{l}\text { Program/Project Manager Date } \\
\text { IL Metcalf }\end{array}$} \\
\hline \multicolumn{2}{|c|}{$\begin{array}{l}\text { Program Element Manager } \\
\text { JM Steffen }\end{array}$} & \multicolumn{2}{|l|}{$\begin{array}{l}\text { DOE Monitor } \\
\text { MR Hahn }\end{array}$} \\
\hline
\end{tabular}


FY 1996 MYPP

WHC-SP-1126, Rev. 1

\section{TRANSITION PROJECTS}

\subsection{1/7.1/6.12}

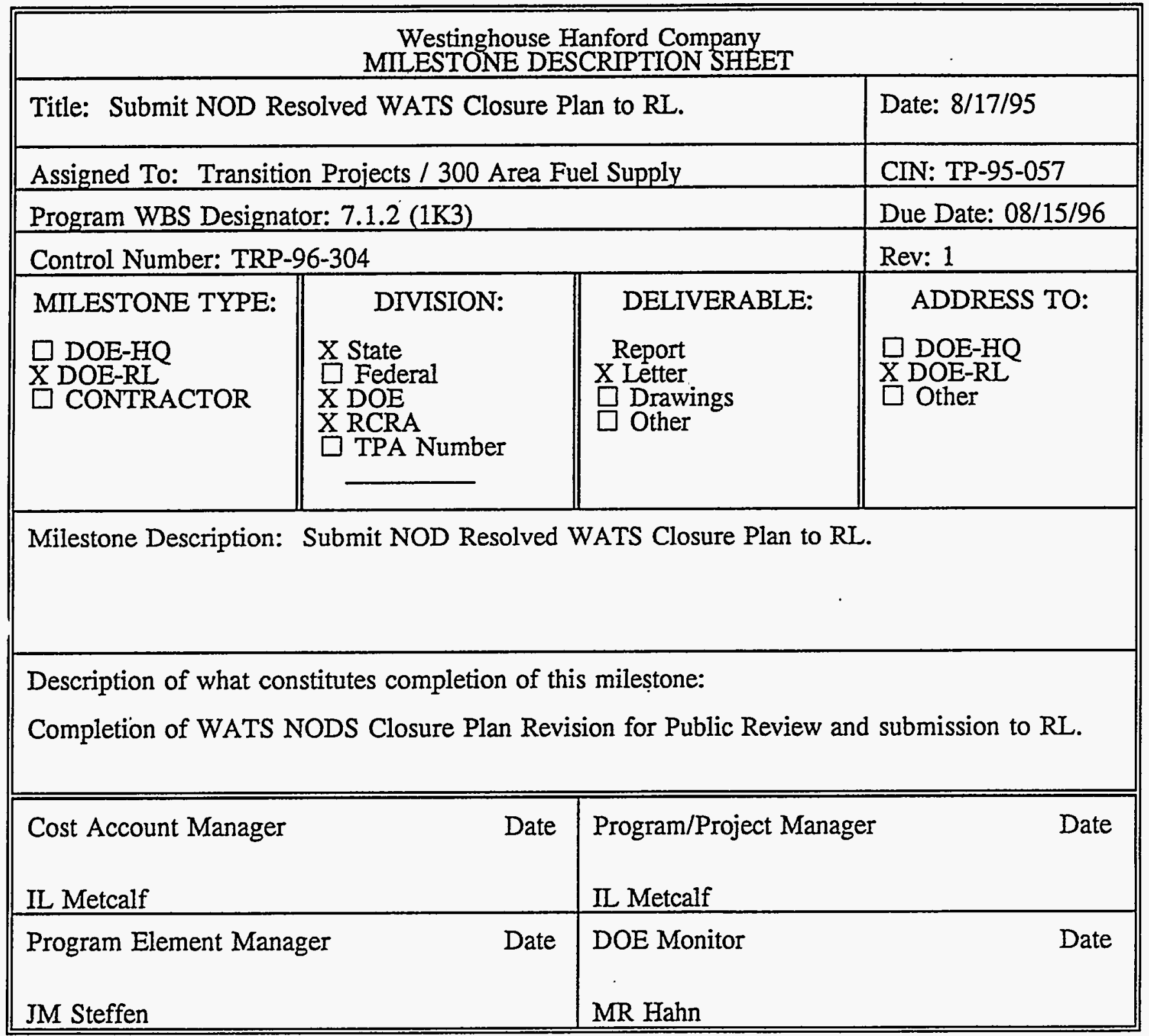


WHC-SP-1126, Rev. 1

TRANSITION PROJECTS

FY 1996 MYPP

1.3.1/7.1/6.12

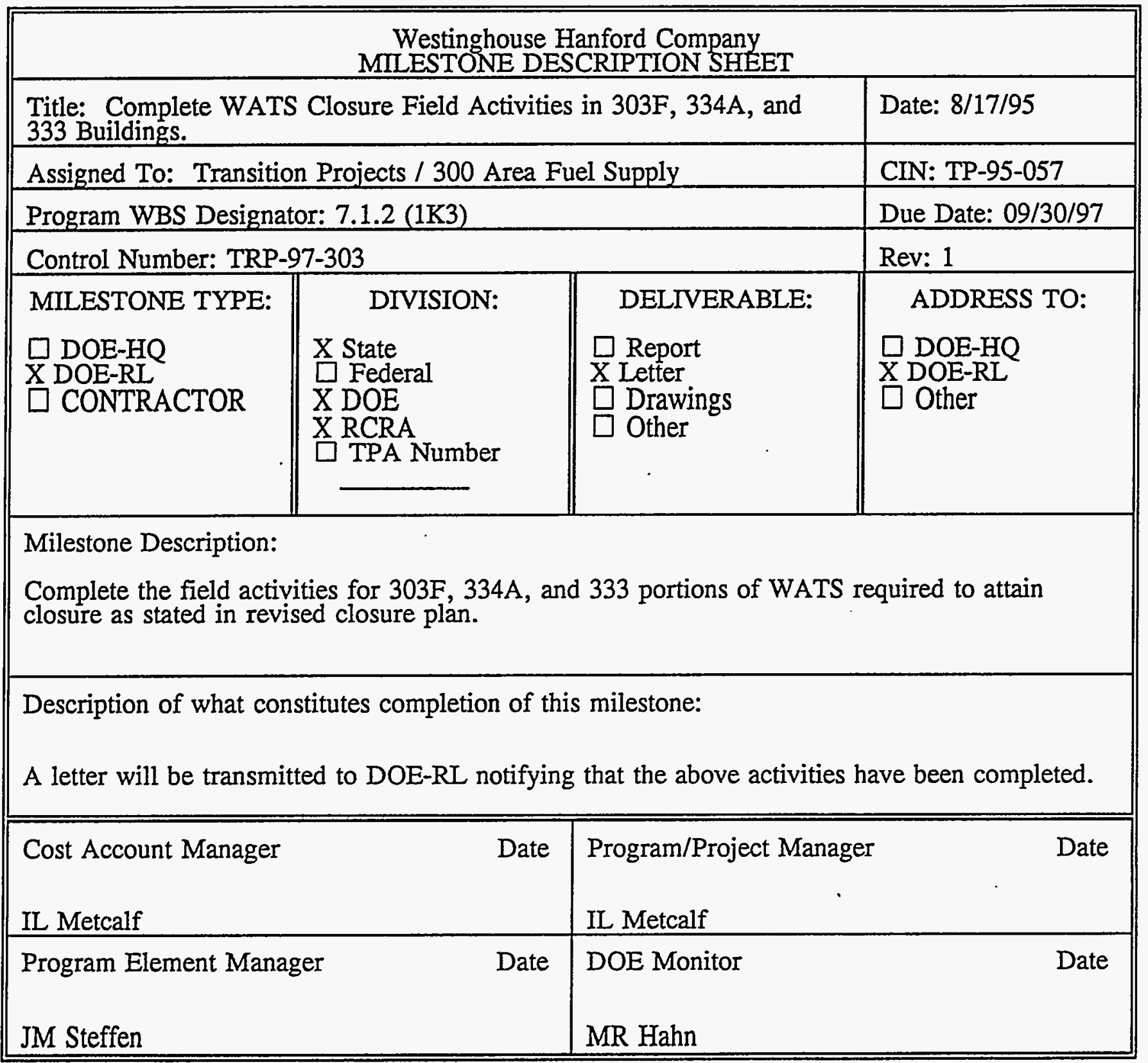


WHC-SP-1126, Rev. 1

\section{TRANSITION PROJECTS}

FY 1996 MYPP

1.3.1/7.1/6.12

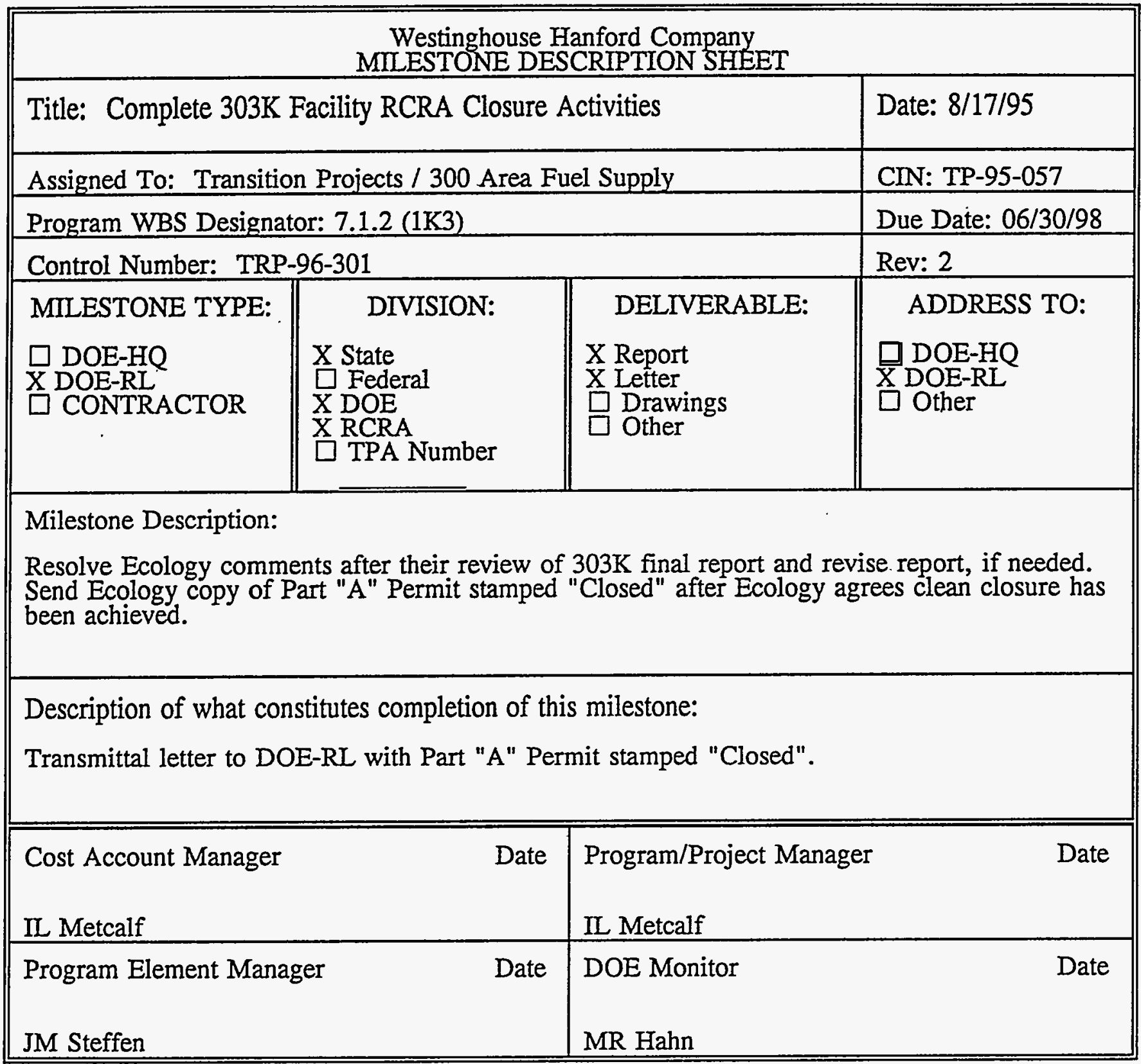


FY 1996 MYPP

WHC-SP-1126, Rev. 1

\section{TRANSITION PROJECTS}

\subsection{1/7.1/6.12}

\begin{tabular}{|c|c|c|c|}
\hline \multicolumn{4}{|c|}{$\begin{array}{l}\text { Westinghouse Hanford Company } \\
\text { MILESTONE DESCRIPTION SHEET }\end{array}$} \\
\hline \multicolumn{3}{|c|}{ Title: Complete 313 Portion of WATS RCRA Closure Field Activities. } & Date: $08 / 17 / 95$ \\
\hline \multicolumn{3}{|c|}{ Assigned To: Transition Projects / 300 Area Fuel Supply } & CIN: TP-95-057 \\
\hline \multicolumn{3}{|c|}{ Program WBS Designator: $7.1 .2(1 \mathrm{~K} 3)$} & Due Date: $09 / 30 / 96$ \\
\hline \multicolumn{3}{|c|}{ Control Number: TRP-96-302 } & Rev: 1 \\
\hline $\begin{array}{l}\text { MILESTONE TYPE: } \\
\square \text { DOE-HQ } \\
\text { X DOE-RL } \\
\square \text { CONTRACTOR }\end{array}$ & $\begin{array}{l}\text { DIVISION: } \\
\text { X State } \\
\square \text { Federal } \\
\text { X DOE } \\
\text { X RCRA } \\
\square \text { TPA Number }\end{array}$ & $\begin{array}{l}\text { DELIVERABLE: } \\
\square \text { Report } \\
\text { X Letter } \\
\square \text { Drawings } \\
\square \text { Other }\end{array}$ & $\begin{array}{l}\text { ADDRESS TO: } \\
\square \text { DOE-HQ } \\
\text { X DOE-RI } \\
\square \text { Other-Ecology }\end{array}$ \\
\hline \multicolumn{4}{|c|}{$\begin{array}{l}\text { Complete the } 313 \text { WATS field activities required to attain closure as stated in revised closure } \\
\text { plan. }\end{array}$} \\
\hline \multicolumn{4}{|c|}{$\begin{array}{l}\text { Description of what constitutes completion of this milestone: } \\
\text { Transmittal of letter to DOE-RL providing details of actions taken regarding the partial closure } \\
\text { of the WATS, will constitute completion of this milestone. This activity will cover the } 313 \\
\text { Building portion of WATS. }\end{array}$} \\
\hline \multicolumn{2}{|l|}{$\begin{array}{l}\text { Cost Account Manager } \\
\text { IL Metcalf }\end{array}$} & \multicolumn{2}{|l|}{ Program/Project Manager } \\
\hline \multicolumn{2}{|c|}{$\begin{array}{l}\text { Program Element Manager } \\
\text { JM Steffen }\end{array}$} & \multicolumn{2}{|l|}{$\begin{array}{l}\text { DOE Monitor } \\
\text { MR Hahn }\end{array}$} \\
\hline
\end{tabular}


WHC-SP-1126, Rev. I

FY 1996 MYPP

\section{TRANSITION PROJECTS}

1:3.1/7.1/6.12

\begin{tabular}{|c|c|c|c|}
\hline \multicolumn{4}{|c|}{$\begin{array}{l}\text { Westinghouse Hanford Company } \\
\text { MILESTONE DESCRIPTION SHEET }\end{array}$} \\
\hline \multicolumn{3}{|c|}{ Title: Complete Isolation of 313 Building South Side. } & Date: $8 / 17 / 95$ \\
\hline \multicolumn{3}{|c|}{ Assigned To: Transition Projects / 300 Area Fuel Supply } & CIN: TP-95-057 \\
\hline \multicolumn{3}{|c|}{ Program WBS Designator: $7.1 .2(1 \mathrm{~K} 3)$} & Due Date: $3 / 31 / 97$ \\
\hline \multicolumn{3}{|c|}{ Control Number: TRP-97-301 } & Rev: Original \\
\hline $\begin{array}{l}\text { MILESTONE TYPE: } \\
\square \text { DOE-HQ } \\
\text { X DOE-RL } \\
\square \text { CONTRACTOR }\end{array}$ & $\begin{array}{l}\text { DIVISION: } \\
\square \text { State } \\
\square \text { Federal } \\
\text { X DOE } \\
\square \text { RCRA } \\
\square \text { TPA Number }\end{array}$ & $\begin{array}{l}\text { DELIVERABLE: } \\
\text { } \square \text { Report } \\
\text { X Leter } \\
\text { Drawings } \\
\square \text { Other }\end{array}$ & $\begin{array}{l}\text { ADDRESS TO: } \\
\square \text { DOE-HQ } \\
\text { X DOE-RL } \\
\square \text { Other }\end{array}$ \\
\hline \multicolumn{4}{|c|}{$\begin{array}{l}\text { Milestone Description: } \\
\text { As an alternate plan to } 313 \text { Building demolition, isolation of the south portion will be completed } \\
\text { to decrease a serious safety hazard caused by an unsafe roof. }\end{array}$} \\
\hline \multicolumn{4}{|c|}{$\begin{array}{l}\text { Description of what constitutes completion of this milestone: } \\
\text { Completion of the following activities will constitute completion: isolation of } 313 \text { South Building } \\
\text { access, removal of } 313 \text { South Building utilities, removal of } 313 \text { South Building roof loadings } \\
\text { (i.e. HVAC units, etc), completion of NEPA, historical studies, and samplingcharacterization } \\
\text { activities, and removal of unattached radiologically contaminated materials. The completion date } \\
\text { is directly dependent upon the findings of the sampling/characterization results and follow on } \\
\text { project plan, cost, and schedule. A change control will be prepared to reflect changes necessary. }\end{array}$} \\
\hline \multicolumn{2}{|l|}{ Cost Account Manager } & \multicolumn{2}{|c|}{ Program/Project Manager } \\
\hline \multicolumn{2}{|c|}{$\begin{array}{l}\text { Program Element Manager } \\
\text { JM Steffen }\end{array}$} & \multicolumn{2}{|l|}{$\begin{array}{l}\text { DOE Monitor } \\
\text { MR Hahn }\end{array}$} \\
\hline
\end{tabular}


WHC-SP-1126, Rev. I

FY 1996 MYPP

TRANSITION PROJECTS

1.3.1/7.1/6.12

\begin{tabular}{|c|c|c|c|}
\hline \multicolumn{4}{|c|}{$\begin{array}{l}\text { Westinghouse Hanford Company } \\
\text { MILESTONE DESCRIPTION SHEET }\end{array}$} \\
\hline \multicolumn{3}{|c|}{ Title: Submit Final Report on WATS Closure Activities to RL. } & Date: $8 / 17 / 95$ \\
\hline \multicolumn{3}{|c|}{ Assigned To: Transition Projects / 300 Area Fuel Supply } & CIN: TP-95-057 \\
\hline \multicolumn{3}{|c|}{ Program WBS Designator: $7.1 .2(1 \mathrm{~K} 3)$} & Due Date: $03 / 01 / 99$ \\
\hline \multicolumn{3}{|c|}{ Control Number: TRP-99-301 } & Rev: 1 \\
\hline $\begin{array}{l}\text { MILESTONE TYPE: } \\
\square \text { DOE-HQ } \\
\text { X DOE-RL } \\
\square \text { CONTRACTOR }\end{array}$ & $\begin{array}{l}\text { DIVISION: } \\
\text { X State } \\
\text { ' Federal } \\
\text { X DOE } \\
\text { X RCRA } \\
\square \text { TPA Number }\end{array}$ & $\begin{array}{l}\text { DELIVERABLE: } \\
\text { X Report } \\
\text { Retter. } \\
\text { Drawings } \\
\text { Other }\end{array}$ & $\begin{array}{l}\text { ADDRESS TO: } \\
\square \text { DOE-HQ } \\
\text { X DOE-RL }\end{array}$ \\
\hline \multicolumn{4}{|c|}{$\begin{array}{l}\text { Milestone Description: } \\
\text { Resolve Ecology comments after their review of WATS final report and revise report, if needed. } \\
\text { Send Ecology copy of Part "A" Permit stamped "Closed" after Ecology agrees clean closure has } \\
\text { been achieved. }\end{array}$} \\
\hline \multicolumn{4}{|c|}{$\begin{array}{l}\text { Description of what constitutes completion of this milestone: } \\
\text { Transmittal letter to DOE-RL with Part "A" Permit stamped "Closed". }\end{array}$} \\
\hline \multicolumn{2}{|l|}{$\begin{array}{l}\text { Cost Account Manager } \\
\text { IL Metcalf }\end{array}$} & \multicolumn{2}{|c|}{$\begin{array}{l}\text { Program/Project Manager } \\
\text { IL Metcalf }\end{array}$} \\
\hline \multicolumn{2}{|c|}{$\begin{array}{l}\text { Program Ėlement Manager } \\
\text { JM Steffen }\end{array}$} & $\begin{array}{l}\text { DOE Monitor } \\
\text { MR Hahn }\end{array}$ & \\
\hline
\end{tabular}


WHC-SP-1126, Rev. 1

FY 1996 MYPP

TRANSITION PROJECTS

1.3.1/7.1/6.12

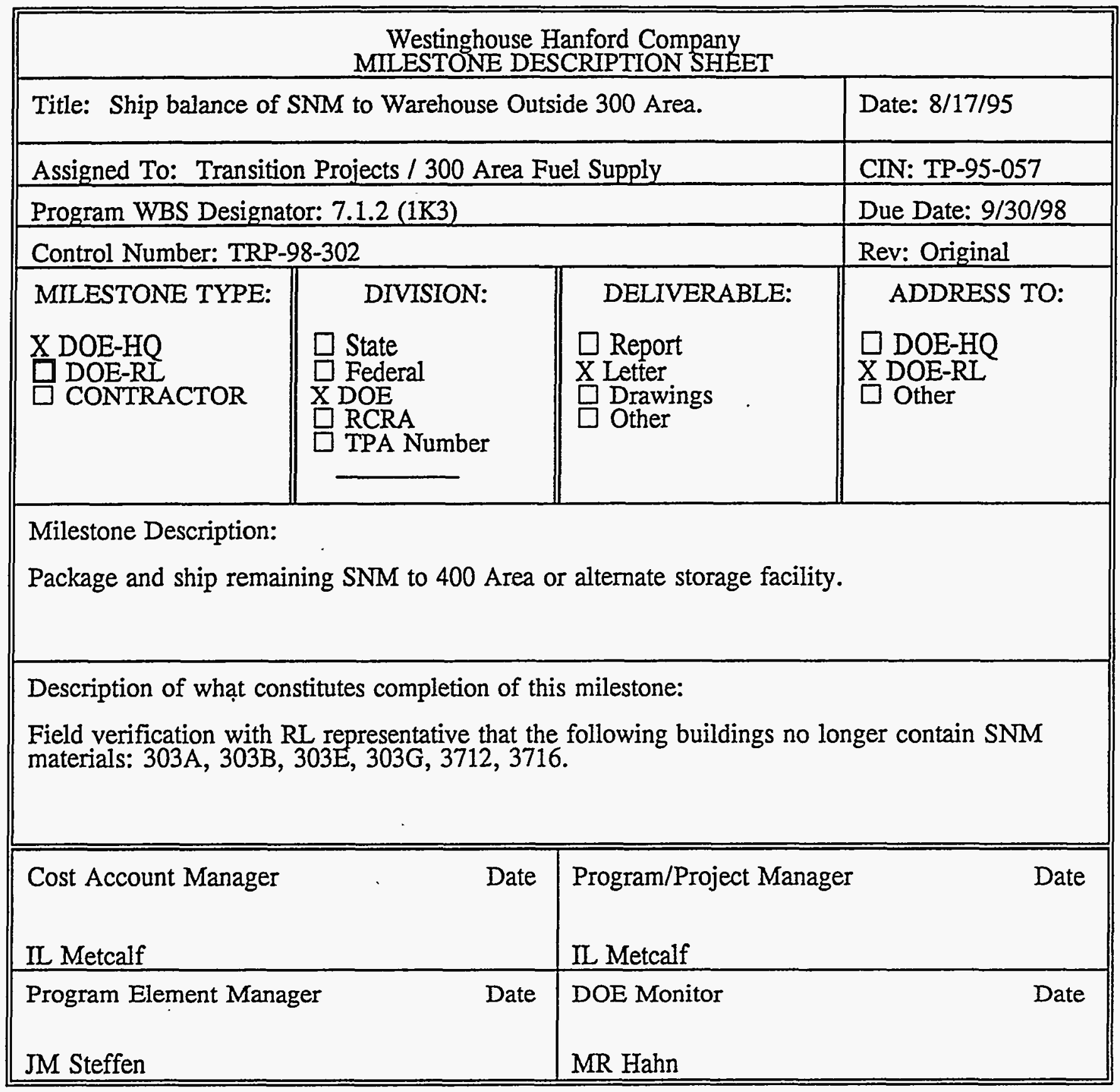


WHC-SP-1126, Rev. 1

FY 1996 MYPP

TRANSITION PROJECTS

1.3.1/7.1/6.12

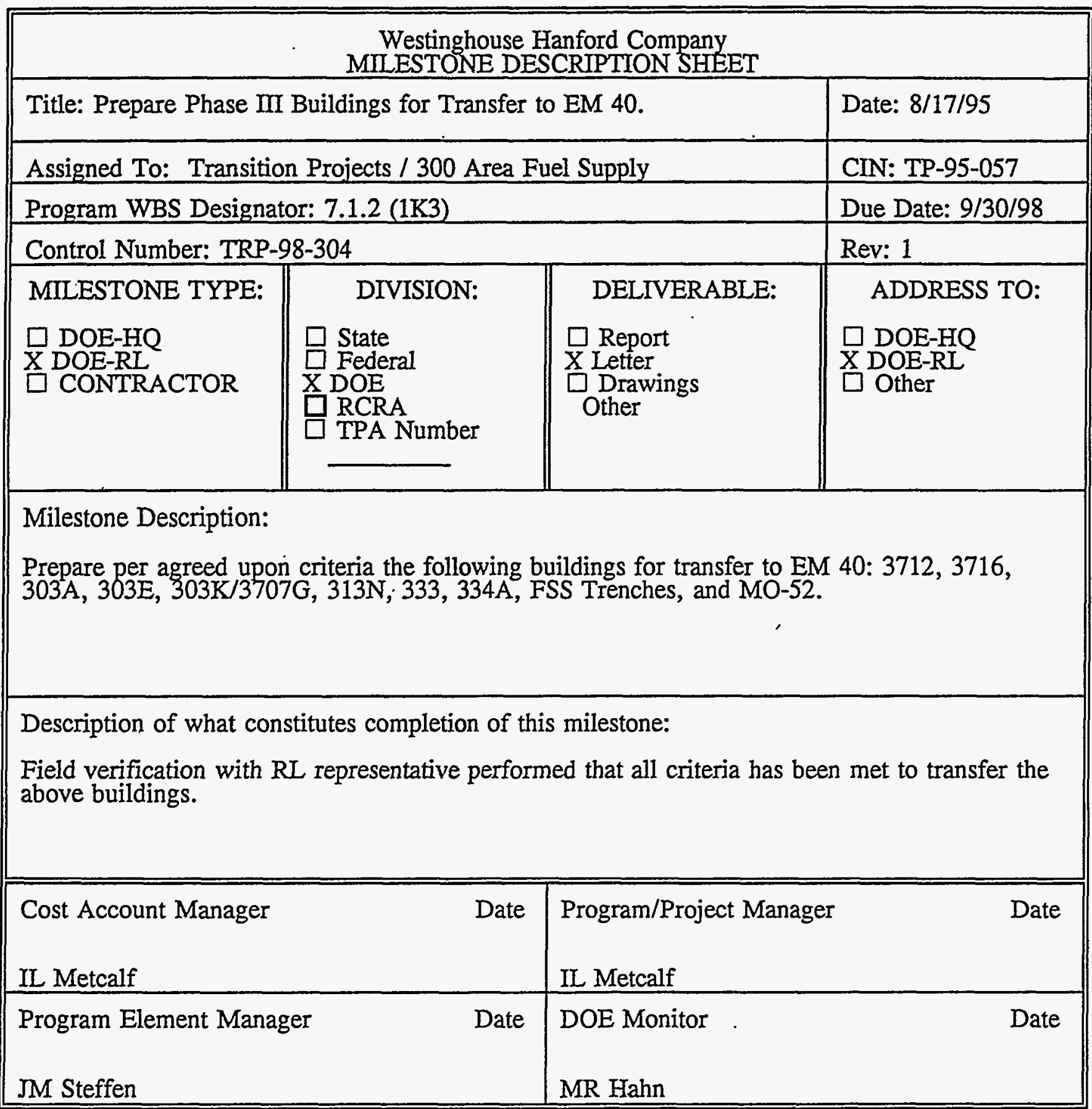


WHC-SP-1126, Rey. 1

FY 1996 MYPP

\section{TRANSITION PROJECTS}

1.3.1/7.1/6.12

\begin{tabular}{|c|c|c|c|}
\hline \multicolumn{4}{|c|}{$\begin{array}{l}\text { Westinghouse Hanford Company } \\
\text { MILESTONE DESCRIPTION SHEET }\end{array}$} \\
\hline \multicolumn{3}{|c|}{ Title: Prepare Phase 1 Buildings for Transfer to EM- 40 . } & Date: $08 / 17 / 95$ \\
\hline \multicolumn{3}{|c|}{ Assigned To: Transition Projects / 300 Area Fuel Supply } & CIN: TP-95-057 \\
\hline \multicolumn{3}{|c|}{ Program WBS Designator: $7.1 .2(1 \mathrm{~K} 3)$} & Due Date: $9 / 30 / 96$ \\
\hline \multicolumn{3}{|c|}{ Control Number: TRP-98-303 } & \multirow{2}{*}{$\begin{array}{l}\text { Rev: } 1 \\
\text { ADDRESS TO: } \\
\square \text { DOE-HQ } \\
\text { X DOE-RL } \\
\square \text { Other }\end{array}$} \\
\hline $\begin{array}{l}\text { MILESTONE TYPE: } \\
\square \text { DOE-HQ } \\
\text { X DOE-RL } \\
\square \text { CONTRACTOR }\end{array}$ & $\begin{aligned} & \text { DIVISION: } \\
& \text { State } \\
& \text { Federal } \\
& \text { X DOE } \\
& \text { DCRA } \\
& \square \text { TPA Number }\end{aligned}$ & $\begin{array}{l}\text { DELIVERABLE: } \\
\square \text { Report } \\
\text { X Letter } \\
\square \text { Drawings } \\
\text { Other }\end{array}$ & \\
\hline \multicolumn{4}{|c|}{$\begin{array}{l}\text { Milestone Description: } \\
\text { Prepare the following buildings in accordance with the shutdown criteria for transfer to EM-40: } \\
\text { 303M and East Storage Pad, 304/304A, and } 311 \text { Tank Farm. }\end{array}$} \\
\hline \multicolumn{4}{|c|}{ stitutes completion of this } \\
\hline \multicolumn{2}{|c|}{$\begin{array}{l}\text { Cost Account Manager Date } \\
\text { IL Metcalf }\end{array}$} & \multicolumn{2}{|c|}{$\begin{array}{l}\text { Program/Project Manager Date } \\
\text { II Metcalf }\end{array}$} \\
\hline \multicolumn{2}{|c|}{$\begin{array}{l}\text { Program Element Manager Date } \\
\text { JM Steffen }\end{array}$} & \multicolumn{2}{|c|}{$\begin{array}{l}\text { DOE Monitor } \\
\text { MR Hahn }\end{array}$} \\
\hline
\end{tabular}


WHC-SP-1126, Rev. 1

\section{TRANSITION PROJECTS}

FY 1996 MYPP

1.3.1/7.1/6.12

\begin{tabular}{|c|c|c|c|}
\hline \multicolumn{4}{|c|}{$\begin{array}{l}\text { Westinghouse Hanford Company } \\
\text { MILESTONE DESCRIPTION SHEET }\end{array}$} \\
\hline \multicolumn{3}{|c|}{ Title: Prepare Phase II Buildings for Transfer to EM-40. } & Date: $8 / 17 / 95$ \\
\hline \multicolumn{3}{|c|}{ Assigned To: Transition Projects / 300 Area Fuel Supply } & CIN: TP-95-057 \\
\hline \multicolumn{3}{|c|}{ Program WBS Designator: $7.1 .2(1 \mathrm{~K} 3)$} & Due Date: $9-30-97$ \\
\hline \multicolumn{3}{|c|}{ Control Number: TRP-01-301 } & Rev: 1 \\
\hline $\begin{array}{l}\text { MILESTONE TYPE: } \\
\square \text { DOE-HQ } \\
\text { X DOE-RL } \\
\square \text { CONTRACTOR }\end{array}$ & $\begin{array}{l}\text { DIVISION: } \\
\square \text { State } \\
\square \text { Federal } \\
\text { X DOE } \\
\square \text { RCRA } \\
\square \text { TPA Number }\end{array}$ & $\begin{array}{l}\text { DELIVERABLE: } \\
\square \text { Report } \\
\text { X Letter. } \\
\text { Drawings. } \\
\square \text { Other }\end{array}$ & $\begin{array}{l}\text { ADDRESS TO: } \\
\square \text { DOE-HQ } \\
\text { X DOE-RL } \\
\square \text { Other }\end{array}$ \\
\hline \multirow{2}{*}{\multicolumn{4}{|c|}{$\begin{array}{l}\text { Milestone Description: } \\
\text { Prepare the following buildings in accordance with the shutdown criteria for transfer to EM-40: } \\
313 \mathrm{~S}, 303 \mathrm{~B}, 303 \mathrm{G}, 303 \mathrm{~F}, 334 \text {, and } 334 \text { Tank Farm. } \\
\text { Note: Activities for the } 334 \text { Tank Farm do not include RCRA clean closure activities, which will } \\
\text { be completed at a later date. }\end{array}$}} \\
\hline & & & \\
\hline \multicolumn{4}{|c|}{$\begin{array}{l}\text { Description of what constitutes completion of this milestone: } \\
\text { Field verification with RL representative that criteria has been met for transfer of the above } \\
\text { buildings. }\end{array}$} \\
\hline \multicolumn{2}{|c|}{$\begin{array}{l}\text { Cost Account Manager Date } \\
\text { IL Metcalf }\end{array}$} & \multicolumn{2}{|c|}{$\begin{array}{l}\text { Program/Project Manager Date } \\
\text { IL Metcalf }\end{array}$} \\
\hline \multicolumn{2}{|c|}{$\begin{array}{l}\text { Program Element Manager } \\
\text { JM Steffen }\end{array}$} & \multicolumn{2}{|c|}{$\begin{array}{l}\text { DOE Monitor } \\
\text { MR Hahn }\end{array}$} \\
\hline
\end{tabular}




\section{WHC-SP-1126, Rev. 1 \\ TRANSITION PROJECTS \\ 1.3.1/7.1/6.12}

FY 1996 MYPP

\begin{tabular}{|c|c|c|c|}
\hline \multicolumn{4}{|c|}{$\begin{array}{l}\text { Westinghouse Hanford Company } \\
\text { MILESTONE DESCRIPTION SHEET }\end{array}$} \\
\hline \multicolumn{3}{|c|}{ Title: Completion Waste Acid Treatment RCRA Closure Field Activities } & Date: $8-17-95$ \\
\hline \multicolumn{3}{|c|}{ Assigned To: Transition Projects / 300 Area Fuel Supply } & CIN: TP-95-057 \\
\hline \multicolumn{3}{|c|}{ Program WBS Designator: $7.1 .2(1 \mathrm{~K} 3)$} & Due Date: $9 / 30 / 98$ \\
\hline \multicolumn{3}{|c|}{ Control Number: TRP-01-302 } & Rev: 1 \\
\hline $\begin{array}{l}\text { MILESTONE TYPE: } \\
\square \text { DOE-HQ } \\
\text { X DOE-RL } \\
\square \text { CONTRACTOR }\end{array}$ & $\begin{array}{l}\text { DIVISION: } \\
\text { X State } \\
\square \text { Federal } \\
\text { X DOE } \\
\text { X RCRA } \\
\square \text { TPA Number }\end{array}$ & $\begin{array}{l}\text { DELIVERABLE: } \\
\square \text { Report } \\
\text { X Letter } \\
\square \text { Drawings } \\
\square \text { Other }\end{array}$ & $\begin{array}{l}\text { ADDRESS TO: } \\
\square \text { DOE-HQ } \\
\text { X DOE-RL } \\
\square \text { Other }\end{array}$ \\
\hline \multicolumn{4}{|c|}{$\begin{array}{l}\text { Milestone Description: } \\
\text { Complete the activities for } 334 \text { and } 311 \text { Tank Farms and piping trench portions of WATS } \\
\text { required to attain closure, as stated in Revised Closure Plan. }\end{array}$} \\
\hline \multicolumn{4}{|c|}{$\begin{array}{l}\text { Description of what constitutes completion of this milestone: } \\
\text { A letter will be transmitted to DOE-RL notifying that the above activities have been completed. }\end{array}$} \\
\hline \multicolumn{2}{|c|}{ Cost Account Manager Date } & \multicolumn{2}{|c|}{$\begin{array}{l}\text { Program/Project Manager Date } \\
\text { IL Metcalf }\end{array}$} \\
\hline \multicolumn{2}{|c|}{$\begin{array}{l}\text { Program Element Manager } \\
\text { JM Steffen }\end{array}$} & \multicolumn{2}{|l|}{$\begin{array}{l}\text { DOE Monitor } \\
\text { MR Hahn } \\
\end{array}$} \\
\hline
\end{tabular}


WHC-SP-1126, Rev. 1

TRANSITION PROJECTS

FY 1996 MYPP

1.3.1/7.1/6.12

\begin{tabular}{|c|c|c|c|}
\hline \multicolumn{4}{|c|}{$\begin{array}{l}\text { Westinghouse Hanford Company } \\
\text { MILESTONE DESCRIPTION SHEET }\end{array}$} \\
\hline \multicolumn{3}{|c|}{ Title: Complete 300 Area Fuel Supply Deactivation } & Date: $8 / 17 / 95$ \\
\hline \multicolumn{3}{|c|}{ Assigned To: Transition Projects / 300 Area Fuel Supply } & CIN: TP-95-057 \\
\hline \multicolumn{3}{|c|}{ Program WBS Designator: $7.1 .2(1 \mathrm{~K} 3)$} & Due Date: $3 / 31 / 99$ \\
\hline \multicolumn{3}{|c|}{ Control Number: TRP-02-301 } & Rev: 1 \\
\hline $\begin{array}{l}\text { MILESTONE TYPE: } \\
\text { X DOE-HQ } \\
\text { DOE-RI } \\
\square \text { CONTRACTOR }\end{array}$ & 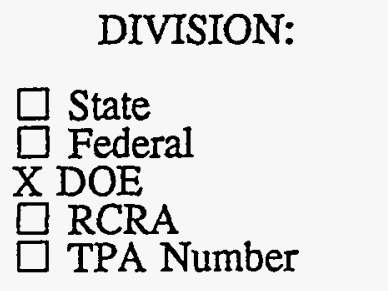 & $\begin{array}{l}\text { DELIVERABLE: } \\
\square \text { Report } \\
\text { X Letter } \\
\square \text { Drawings } \\
\square \text { Other }\end{array}$ & $\begin{array}{l}\text { ADDRESS TO: } \\
\text { X DOE-HQ } \\
\square \text { DOE-RI } \\
\square \text { Other }\end{array}$ \\
\hline \multicolumn{4}{|c|}{$\begin{array}{l}\text { Milestone Description: } \\
\text { Completion of closure activities for the } 300 \text { Area Fuel Supply Program. }\end{array}$} \\
\hline \multicolumn{4}{|c|}{$\begin{array}{l}\text { Description of what constitutes completion of this milestone: } \\
\text { Completion of activities described in the "Shutdown Plan for the } 300 \text { Area Fuel Supply } \\
\text { Facilities". }\end{array}$} \\
\hline \multicolumn{2}{|c|}{$\begin{array}{l}\text { Cost Account Manager } \quad \text { Date } \\
\text { IL Metcalf }\end{array}$} & \multicolumn{2}{|c|}{$\begin{array}{l}\text { Program/Project Manager } \quad \text { Date } \\
\text { IL Metcalf }\end{array}$} \\
\hline \multicolumn{2}{|c|}{$\begin{array}{l}\text { Program Element Manager } \\
\text { JM Steffen }\end{array}$} & \multicolumn{2}{|c|}{$\begin{array}{l}\text { DOE Monitor Date } \\
\text { MR Hahn }\end{array}$} \\
\hline
\end{tabular}


WHC-SP-1126, Rev. 1

TRANSITION PROJECTS

FY 1996 MYPP

1.3.1/7.1/6.12

Westinghouse Hanford Company MILESTONE DESCRIPTION SHEET

Title: Prepare Fuel Supply Shutdown Surveillance and Maintenance Plan $\quad$ Date: 08/17/95

Assigned To: Transition Projects / 300 Area Fuel Supply

Program WBS Designator: 7.1.2 (1K3)

CIN: TP-95-057

Control Number: TRP-98-301

MILESTONE TYPE:

DIVISION:

DELIVERABLE:

Due Date: 03/31/98

$\square$ DOE-HQ

$X$ DOE-RL

CONTRACTOR

\begin{tabular}{l} 
DIVISION: \\
$\square$ State \\
$\square$ Federal \\
DOE \\
RCRA \\
TPA Number \\
\hline
\end{tabular}

Rev: Original

X Report

$\square$ Letter

Drawings

Other

ADDRESS TO:

$\square$ DOE-HQ

$X$ DOE-RL

Other

Milestone Description:

Prepare a supporting document which establishes the Surveillance and Maintenance (S\&M) activities after deactivation of the FSS complex.

Description of what constitutes completion of this milestone:

Transmittal of S\&M plan to DOE-RL for approval.

\begin{tabular}{||lc|lc||}
\hline \hline Cost Account Manager & Date & Program/Project Manager & Date \\
IL Metcalf & & IL Metcalf & \\
\hline Program Element Manager & Date & DOE Monitor & Date \\
JM Steffen & & MR Hahn & \\
\hline
\end{tabular}


WHC-SP-1126, Rev. 1

TRANSITION PROJECTS

FY 1996 MYPP

1.3.1/7.1/6.12

\begin{tabular}{|c|c|c|c|}
\hline \multicolumn{4}{|c|}{$\begin{array}{l}\text { Westinghouse Hanford Company } \\
\text { MILESTONE DESCRIPTION SHEET }\end{array}$} \\
\hline \multicolumn{3}{|c|}{ Title: Revise Fuel Supply Shutdown Plan } & Date: $08 / 17 / 95$ \\
\hline \multicolumn{3}{|c|}{ Assigned To: Transition Projects / 300 Area Fuel Supply } & CIN: TP-95-057 \\
\hline \multicolumn{3}{|c|}{ Program WBS Designator: $7.1 .2(1 \mathrm{~K} 3)$} & Due Date: $08 / 30 / 96$ \\
\hline \multicolumn{3}{|c|}{ Control Number: TRP-96-307 } & Rev: Original \\
\hline $\begin{array}{l}\text { MILESTONE TYPE: } \\
\square \text { DOE-HQ } \\
\text { X DOE-RL } \\
\square \text { CONTRACTOR }\end{array}$ & $\begin{array}{l}\text { DIVISION: } \\
\text { State } \\
\text { Federal } \\
\text { X DOE } \\
\square \text { RCRA } \\
\square \text { TPA Number }\end{array}$ & $\begin{array}{l}\text { DELIVERABLE: } \\
\text { X Report } \\
\square \text { Letter } \\
\square \text { Drawings } \\
\square \text { Other }\end{array}$ & $\begin{array}{l}\text { ADDRESS TO: } \\
\square \text { DOE-HQ } \\
\text { X DOE-RL } \\
\square \text { Other }\end{array}$ \\
\hline \multicolumn{4}{|c|}{$\begin{array}{l}\text { Milestone Description: } \\
\text { Revise the Fuel Supply Shutdown Plan WHC-SD-FL-SSP-002 to reflect FY } 1996 \text { activities, post } \\
\text { work scope completion and current direction. }\end{array}$} \\
\hline \multicolumn{4}{|c|}{$\begin{array}{l}\text { Description of what constitutes completion of this milestone: } \\
\text { Transmittal of the supporting document to DOE-RL for approval. }\end{array}$} \\
\hline $\begin{array}{l}\text { Cost Account Manager } \\
\text { IL Metcalf }\end{array}$ & Date & $\begin{array}{l}\text { Program/Project Manager } \\
\text { IL Metcalf }\end{array}$ & Date \\
\hline $\begin{array}{l}\text { Program Element Manc } \\
\text { JM Steffen }\end{array}$ & Date & $\begin{array}{l}\text { DOE Monitor } \\
\text { MR Hahn }\end{array}$ & Date \\
\hline
\end{tabular}


WHC-SP-1126, Rev.'I

\section{TRANSITION PROJECTS}

FY 1996 MYPP

1.3.1/7.1/6.12

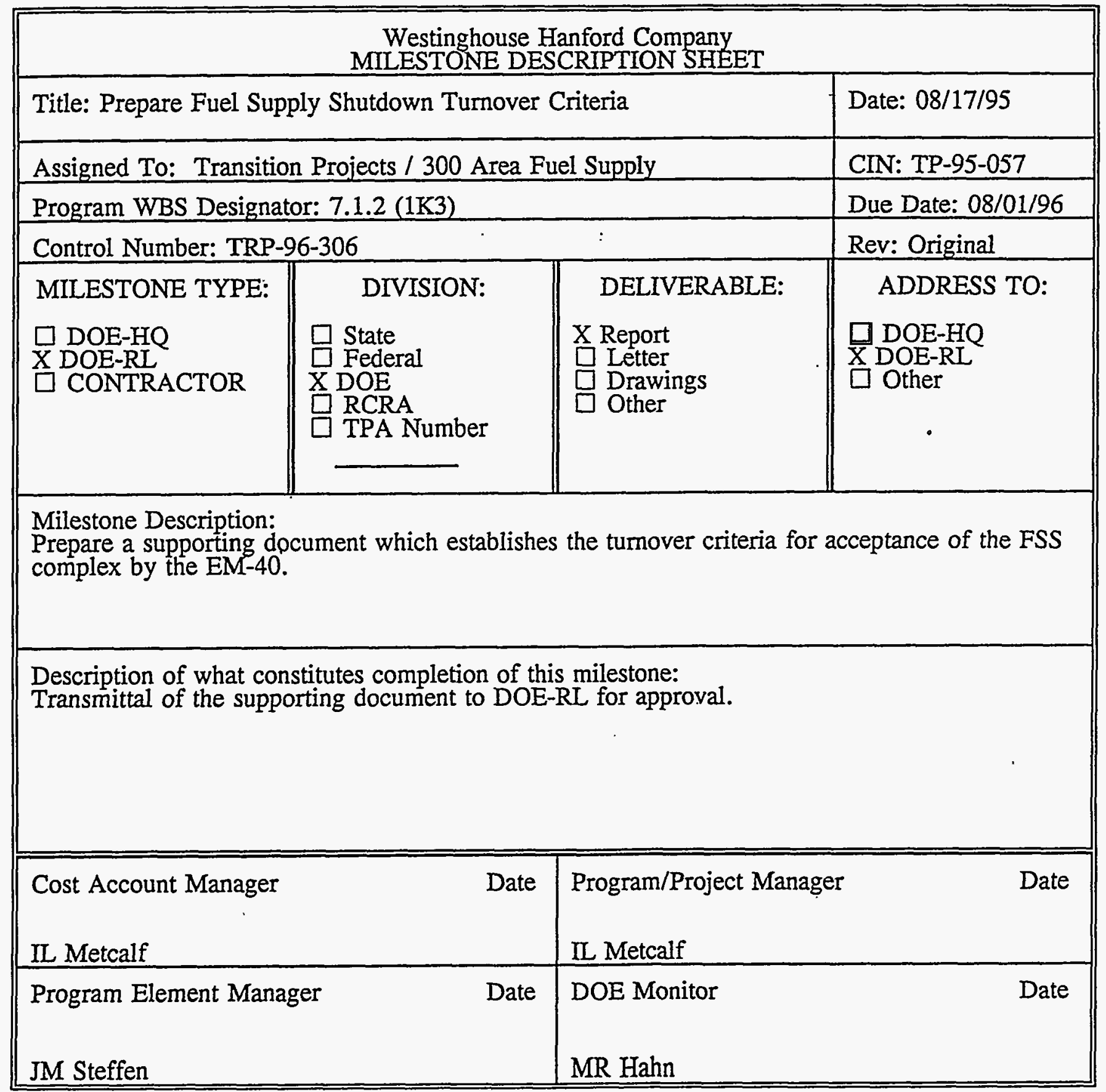


WHC-SP-1126, Rev. 1

FY 1996 MYPP

TRANSITION PROJECTS

1.3.1/7.1/6.12

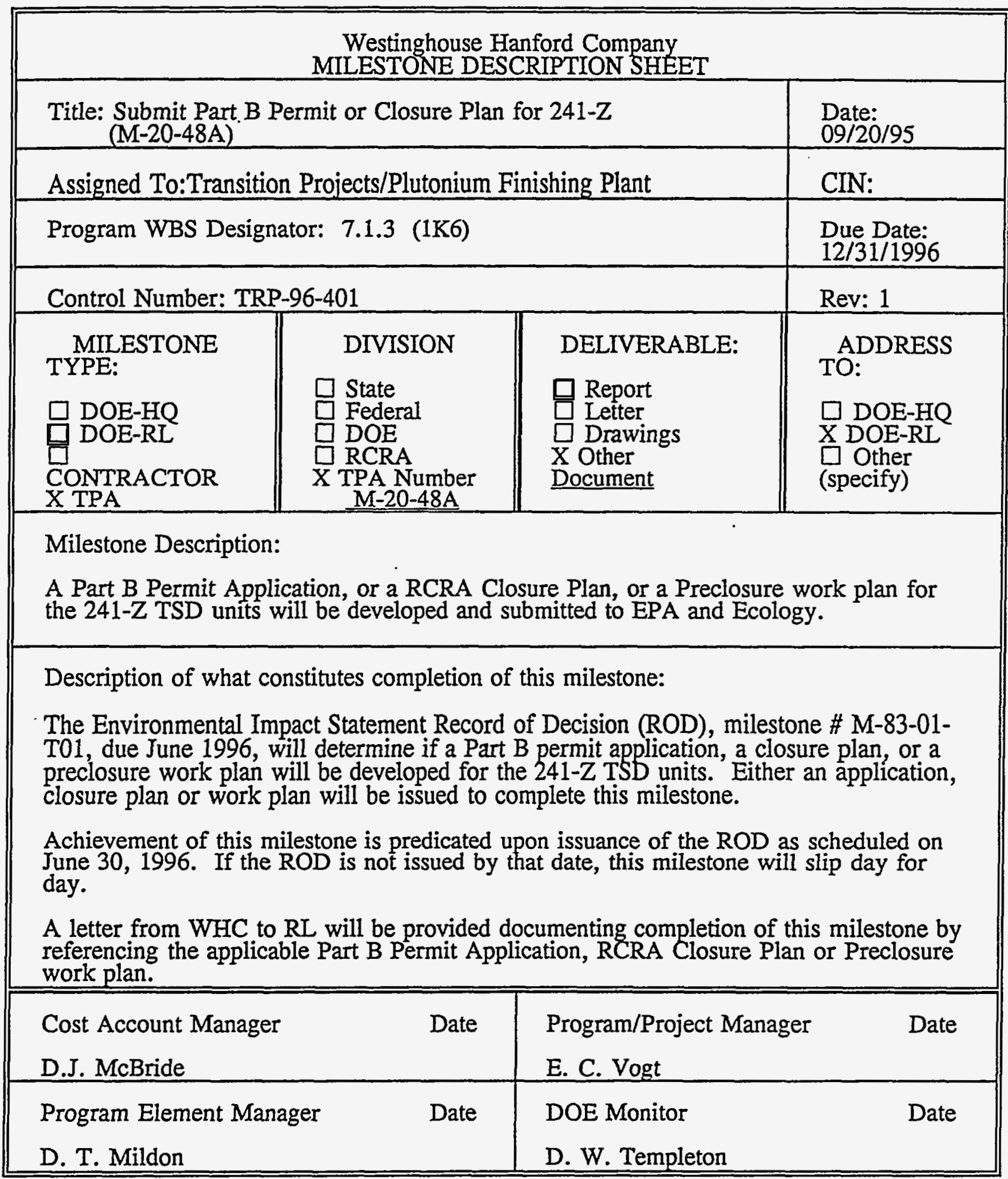


WHC-SP-1126, Rev. I

\section{TRANSITION PROJECTS}

FY 1996 MYPP

1.3.1/7.1/6.12

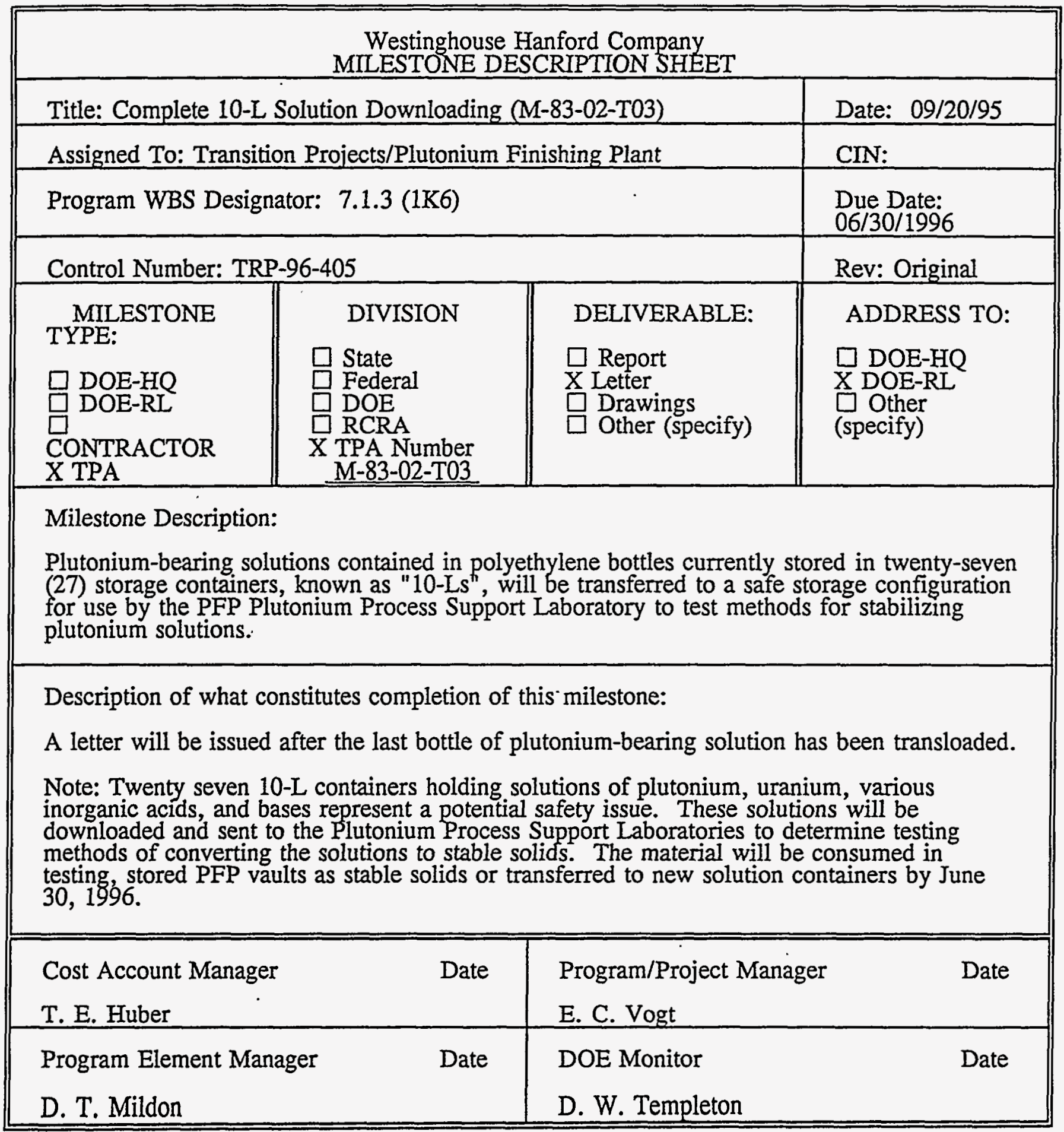


WHC-SP-1126, Rev. 1

\section{TRANSITION PROJECTS}

FY 1996 MYPP

1.3.1/7.1/6.12

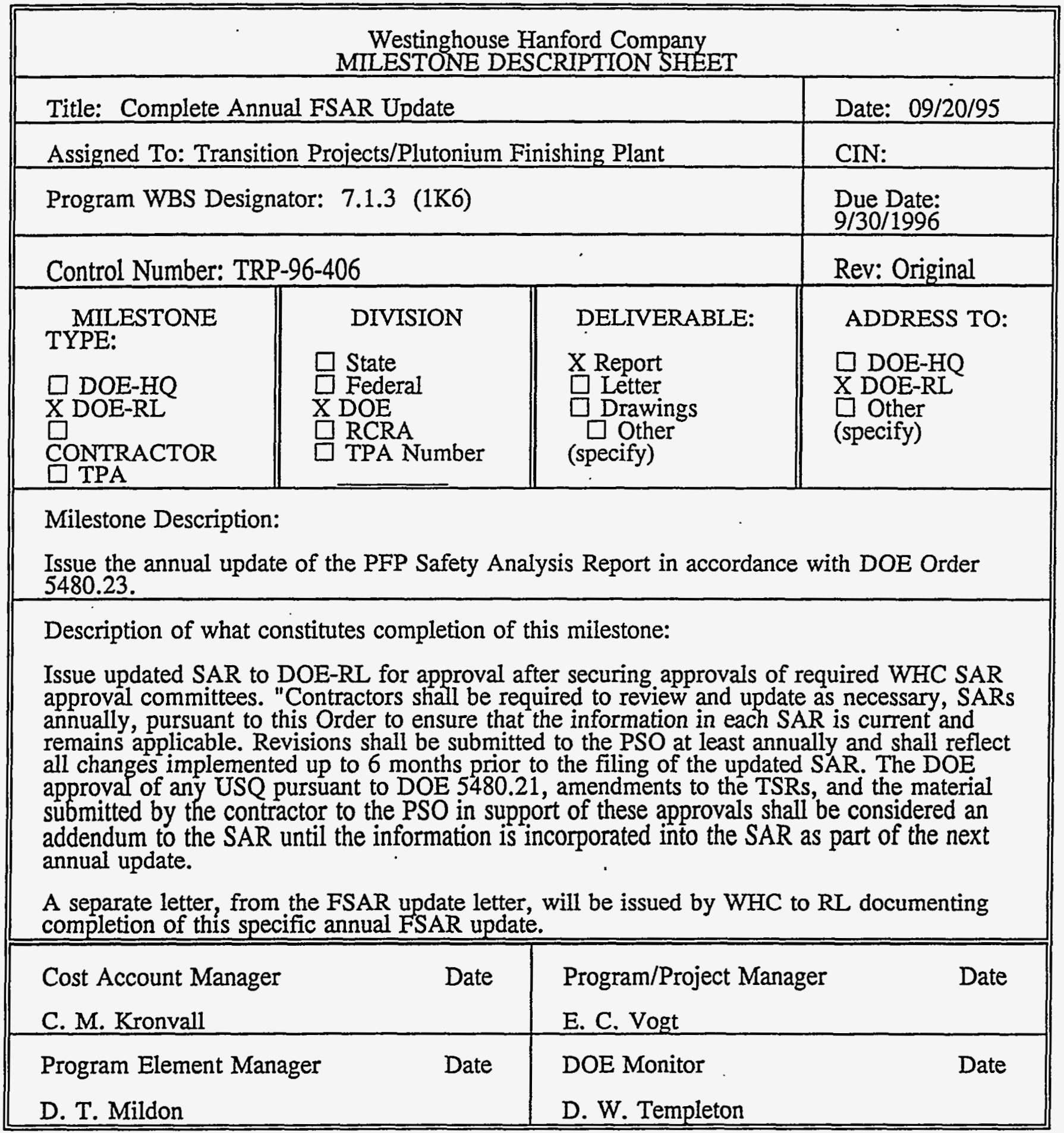


WHC-SP-1126, Rev. 1

TRANSITION PROJECTS

FY 1996 MYPP

\subsection{1/7.1/6.12}

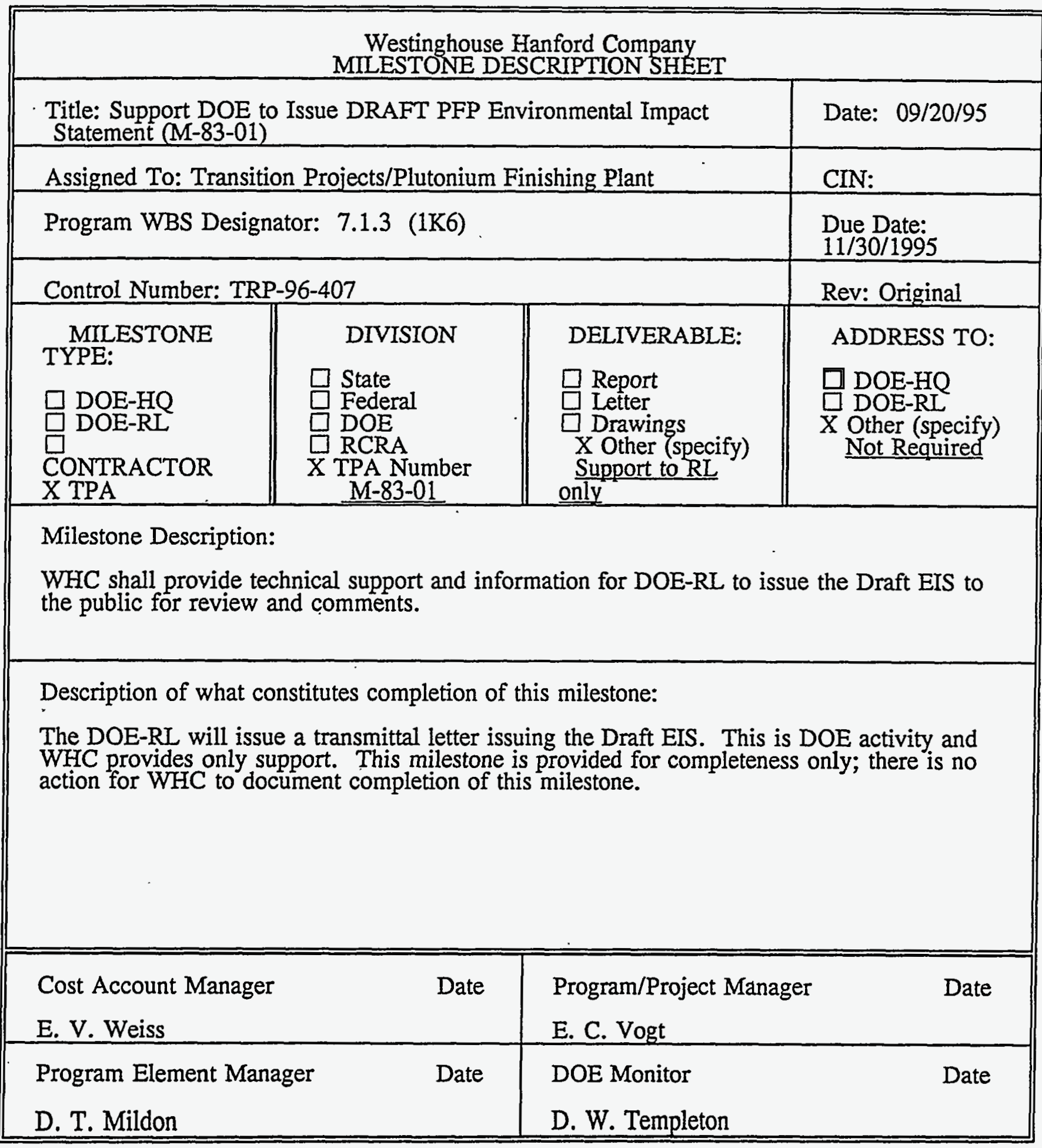


HHC-SP-1126, Rev. 1

TRANSITION PROJECTS

FY 1996 MYPP

1.3.1/7.1/6.12

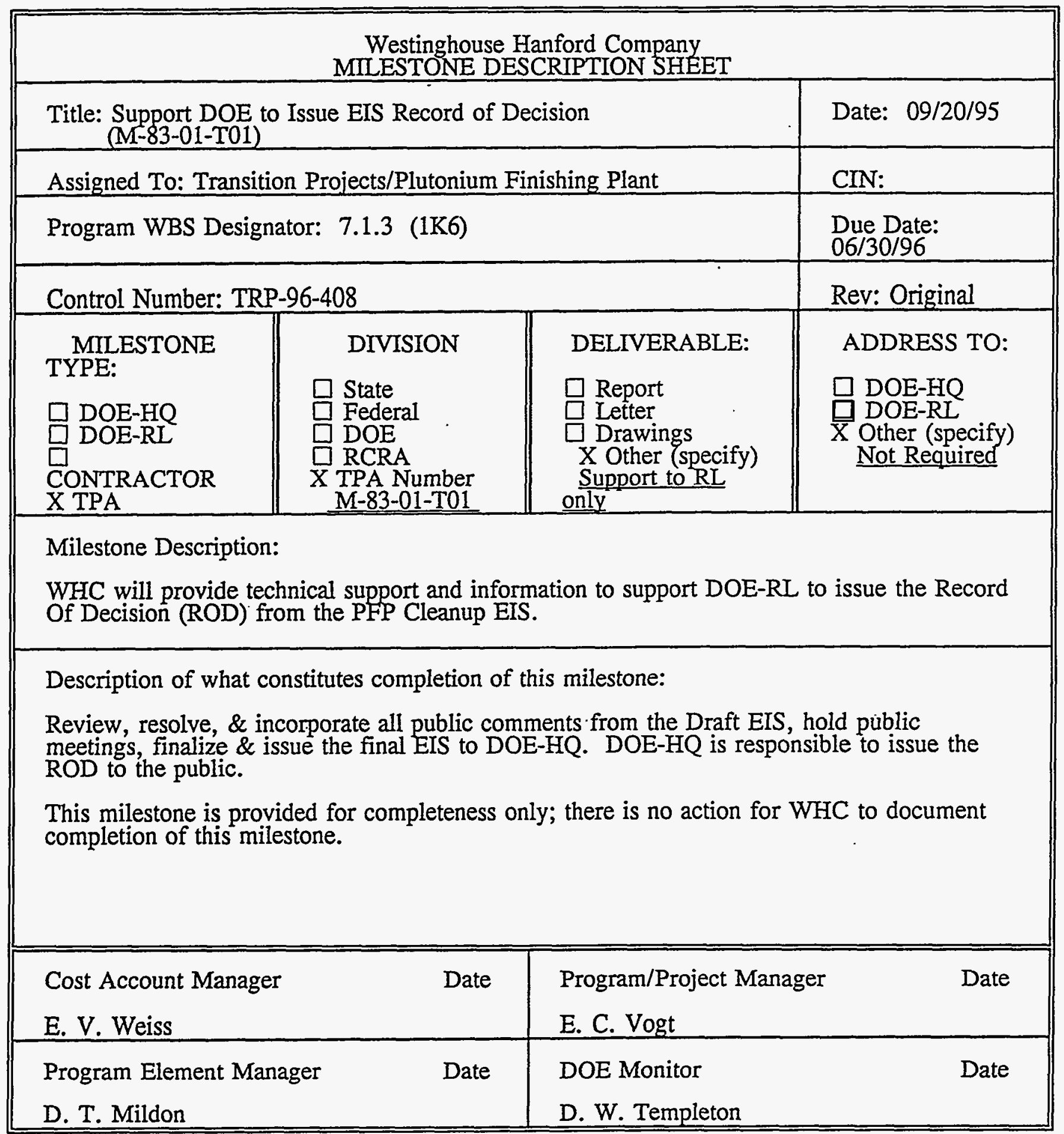


WHC-SP-1126, Rev. 1

\section{TRANSITION PROJECTS}

FY 1996 MYPP

1.3.1/7.1/6.12

\begin{tabular}{|c|c|c|c|}
\hline \multicolumn{4}{|c|}{$\begin{array}{l}\text { Westinghouse Hanford Company } \\
\text { MILESTONE DESCRIPTION SHEET }\end{array}$} \\
\hline \multicolumn{3}{|c|}{ Title: Complete Stabilization of High-Risk Incinerator $\quad$ Ash } & Date: $09 / 20 / 95$ \\
\hline \multicolumn{3}{|c|}{ Assigned To: Transition Projects/Plutonium Finishing Plant } & CIN: \\
\hline \multicolumn{3}{|c|}{ Program WBS Designator: 7.1 .3 (1K6) } & $\begin{array}{l}\text { Due Date: } \\
03 / 31 / 96\end{array}$ \\
\hline \multicolumn{3}{|c|}{ Control Number: TRP-96-409 } & Rev: Original \\
\hline $\begin{array}{l}\text { MILESTONE } \\
\text { TYPE: } \\
\text { X DOE-HQ } \\
\square \text { DOE-RL } \\
\text { CONTRACTOR } \\
\square \text { TPA } \\
\end{array}$ & $\begin{array}{l}\text { DIVISION } \\
\square \text { State } \\
\square \text { Federal } \\
\text { X DOE } \\
\text { DRCRA } \\
\square \text { TPA Number }\end{array}$ & $\begin{array}{l}\text { DELIVERABLE: } \\
\square \text { Report } \\
\text { X Letter } \\
\square \text { Drawings } \\
\square \text { Other } \\
\text { (specify) }\end{array}$ & $\begin{array}{l}\text { ADDRESS TO: } \\
\square \text { DOE-HQ } \\
\text { X DOE-RL } \\
\square \text { Other } \\
\text { (specify) }\end{array}$ \\
\hline \multicolumn{4}{|c|}{$\begin{array}{l}\text { Milestone Description: } \\
\text { Complete the stabilization, packaging and storage of } 46 \text { items (contained in } 52 \text { packages) of } \\
\text { High-Risk Incinerator Ash. }\end{array}$} \\
\hline \multicolumn{4}{|c|}{$\begin{array}{l}\text { Description of what constitutes completion of this milestone: } \\
\text { Stabilize Incinerator Ash to } 1,000 \text { degrees Centigrade and place into vault storage. The } \\
\text { stabilized material will be tested to ensure compliance with the PFP Vault Storage criteria, } \\
\text { placed into packaging that meets the vault OSD for interim storage, and placed into a PFP } \\
\text { vault. The total number of items stabilized is forty-six (contained in fifty-two packages). } \\
\text { A separate letter will be issued from WHC to RL documenting completion of this milestone } \\
\text { per the above description. }\end{array}$} \\
\hline \multicolumn{2}{|c|}{$\begin{array}{l}\text { Cost Account Manager } \\
\text { T. E. Huber }\end{array}$} & \multicolumn{2}{|c|}{$\begin{array}{l}\text { Program/Project Manager } \\
\text { E. C. Vogt }\end{array}$} \\
\hline \multicolumn{2}{|c|}{$\begin{array}{l}\text { Program Element Manager } \\
\text { D. T. Mildon }\end{array}$} & $\begin{array}{l}\text { DOE Monitor } \\
\text { D. W. Templeton }\end{array}$ & Date \\
\hline
\end{tabular}


WHC-SP-1126, Rev. 1

TRANSITION PROJECTS

FY 1996 MYPP

1.3.1/7.1/6.12

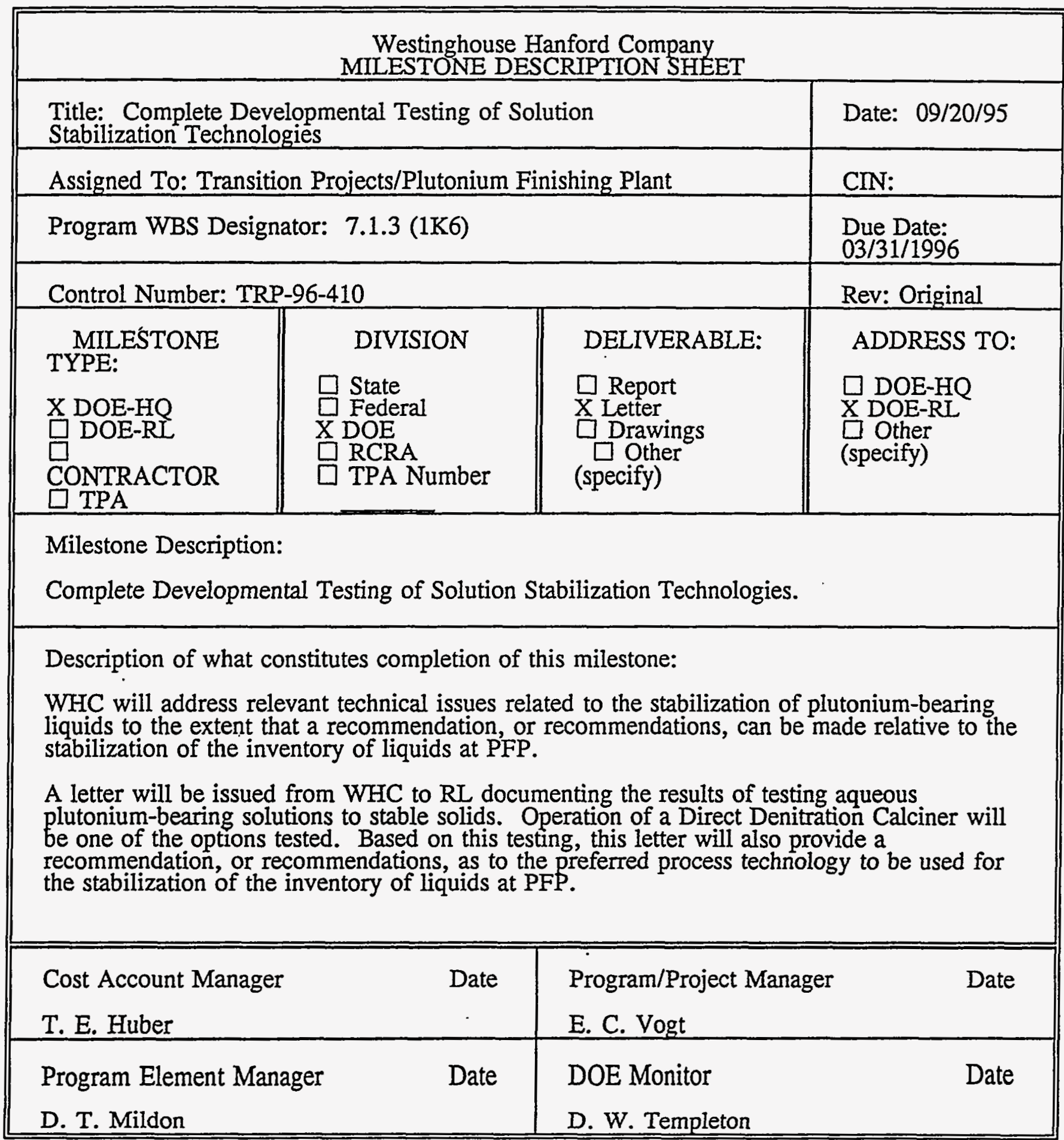


WHC-SP-1126, Rev. I

TRANSITION PROJECTS

FY 1996 MYPP

1.3.1/7.1/6.12

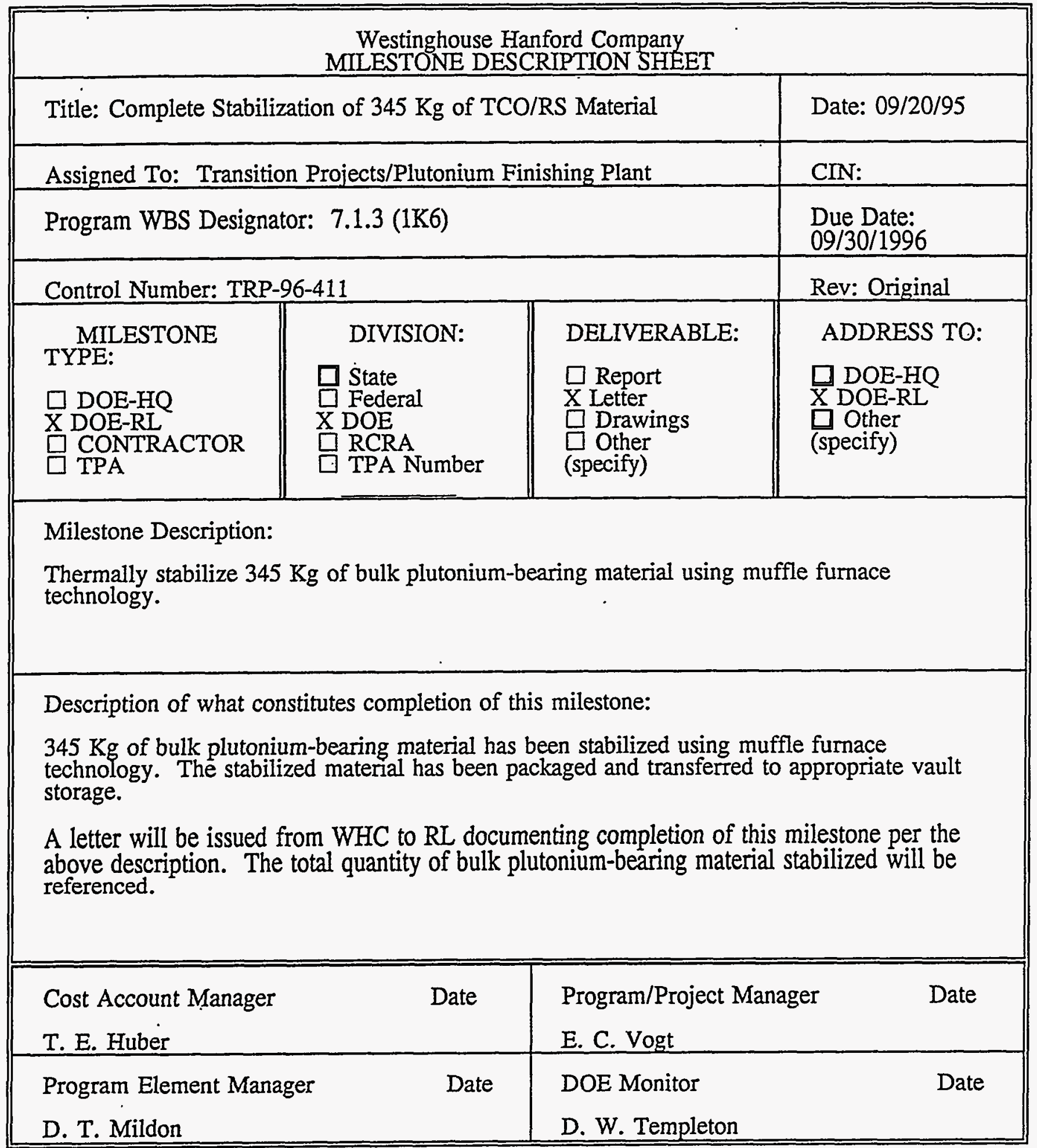


WHC-SP-1126, Rev. 1

\title{
TRANSITION PROJECTS
}

FY 1996 MYPP

\author{
1.3.1/7.1/6.12
}

Westinghouse Hanford Company

MILESTONE DESCRIPTION SHEET

Title: Complete Duct Terminal Cleanout (TCO), Section \#2

Date: $09 / 20 / 95$

Assigned To: Transition Projects/Plutonium Finishing Plant

Program WBS Designator: 7.1:3 (1K6)

CIN:

\begin{tabular}{|l||l||}
\hline \multicolumn{2}{||l}{ Control Number: TRP-96-412 } \\
\hline MILESTONE & DIVISION: \\
TYPE: & $\square$ State \\
$\square$ DOE-HQ & $\square$ Federal \\
X DOE-RL & X DOE \\
$\square$ CONTRACTOR & $\square$ RCRA \\
$\square$ TPA & $\square$ TPA Number \\
\hline
\end{tabular}

DELIVERABLE:

$\square$ Report

$\mathrm{X}$ Letter

$\square$ Drawings

$\square$ Other

(specify)
Due Date:

05/31/1996

Rev: Original

ADDRESS TO:

$\square$ DOE-HQ

X DOE-RL

$\square$ Other

(specify)

Milestone Description:

Complete the removal of Segment \#2, a -57 foot length of unused E-4 exhaust ventilation ducting in rooms 263 and 264 of the $234-5 Z$ building. Segment $\# 2$ is clearly defined in the Plutonium Finishing Plant (PFP) Remediation Project Management Plan, WHC-SD-CP-PMP003 , dated March 17, 1989, and is anticipated to contain approximately $1.8 \mathrm{Kg}$ of plutoniumbearing material.

Description of what constitutes completion of this milestone:

This milestone is considered complete when the $\sim 57$ feet of exhaust ventilation ducting, described above, has been removed, sectioned into pieces, packaged into appropriate containers and readied for disposal. Any plutonium material removed from the ducting will be stored in reactive scrap storage for stabilization at a later date, or discarded as TRU waste, as appropriate. A letter will be issued from WHC to RI documenting completion of this milestone per this description.

\begin{tabular}{|ll|lc|}
\hline \hline $\begin{array}{l}\text { Cost Account Manager } \\
\text { T. E. Huber }\end{array}$ & Date & $\begin{array}{l}\text { Program/Project Manager } \\
\text { E. C. Vogt }\end{array}$ & Date \\
\hline $\begin{array}{l}\text { Program Element Manager } \\
\text { D. T. Mildon }\end{array}$ & Date & $\begin{array}{l}\text { DOE Monitor } \\
\text { D. W. Templeton }\end{array}$ & Date \\
\hline
\end{tabular}


WHC-SP-1126, Rev. I

\section{TRANSITION PROJECTS}

FY 1996 MYPP

$$
\text { 1.3.1/7.1/6.12 }
$$

\begin{tabular}{|c|c|c|c|}
\hline \multicolumn{4}{|c|}{$\begin{array}{l}\text { Westinghouse Hanford Company } \\
\text { MILESTONE DESCRIPTION SHEET }\end{array}$} \\
\hline \multicolumn{3}{|c|}{ Title: Complete Duct Terminal Cleanout (TCO), Section \#3 } & Date: $09 / 20 / 95$ \\
\hline \multicolumn{3}{|c|}{ Assigned To: Transition Projects/Plutonium Finishing Plant } & CIN: \\
\hline \multicolumn{3}{|c|}{ Program WBS Designator: 7.1 .3 (1K6) } & $\begin{array}{l}\text { Due Date: } \\
09 / 30 / 1996 \\
\end{array}$ \\
\hline \multicolumn{3}{|c|}{ Control Number: TRP-96-413 } & Rev: Original \\
\hline $\begin{array}{l}\text { MILESTONE } \\
\text { TYPE: } \\
\square \text { DOE-HQ } \\
\text { X DOE-RL } \\
\square \text { CONTRACTOR } \\
\square \text { TPA }\end{array}$ & $\begin{array}{l}\text { DIVISION: } \\
\square \text { State } \\
\square \text { Federal } \\
\text { X DOE } \\
\square \text { RCRA } \\
\square \text { TPA Number }\end{array}$ & $\begin{array}{l}\text { DELIVERABLE: } \\
\square \text { Report } \\
\text { X Letter } \\
\square \text { Drawings } \\
\square \text { Other } \\
\text { (specify) }\end{array}$ & $\begin{array}{l}\text { ADDRESS TO: } \\
\square \text { DOE-HQ } \\
\text { X DOE-RL } \\
\square \text { Other } \\
\text { (specify) }\end{array}$ \\
\hline \multicolumn{4}{|c|}{$\begin{array}{l}\text { Milestone Description: } \\
\text { Complete the removal of Segment } \# 3 \text {, a }-140 \text { foot length of inactive E-4 exhaust ventilation } \\
\text { ducting in room } 262 \text { of the } 234-5 Z \text { building. Segment } \# 3 \text { is clearly defined in the Plutonium } \\
\text { Finishing Plant (PFP) Remediation Project Management Plan, WHC-SD-CP-PMP-003, dated } \\
\text { March } 17,1989 \text {, and is anticipated to contain approximately } 30 \text { grams of plutonium-bearing } \\
\text { material. }\end{array}$} \\
\hline \multicolumn{4}{|c|}{$\begin{array}{l}\text { Description of what constitutes completion of this milestone: } \\
\text { This milestone is considered complete when the } 140 \text { feet of exhaust ventilation ducting, } \\
\text { described above, has been removed, sectioned into pieces, packaged into appropriate } \\
\text { containers and readied for shipment. Any plutonium material removed is to be discarded as } \\
\text { TRU waste. A letter will be issued from WHC to RI documenting completion of this } \\
\text { milestone per this description. }\end{array}$} \\
\hline \multicolumn{2}{|c|}{$\begin{array}{l}\text { Cost Account Manager Date } \\
\text { T. E. Huber }\end{array}$} & \multicolumn{2}{|c|}{$\begin{array}{l}\text { Program/Project Manager } \\
\text { E. C. Vogt }\end{array}$} \\
\hline \multicolumn{2}{|c|}{$\begin{array}{l}\text { Program Element Manager } \\
\text { D. T. Mildon }\end{array}$} & \multicolumn{2}{|l|}{$\begin{array}{l}\text { DOE Monitor } \\
\text { D. W. Templeton }\end{array}$} \\
\hline
\end{tabular}


WHC-SP-1126, Rev: 1

TRANSITION PROJECTS

FY 1996 MYPP

1.3.1/7.1/6.12

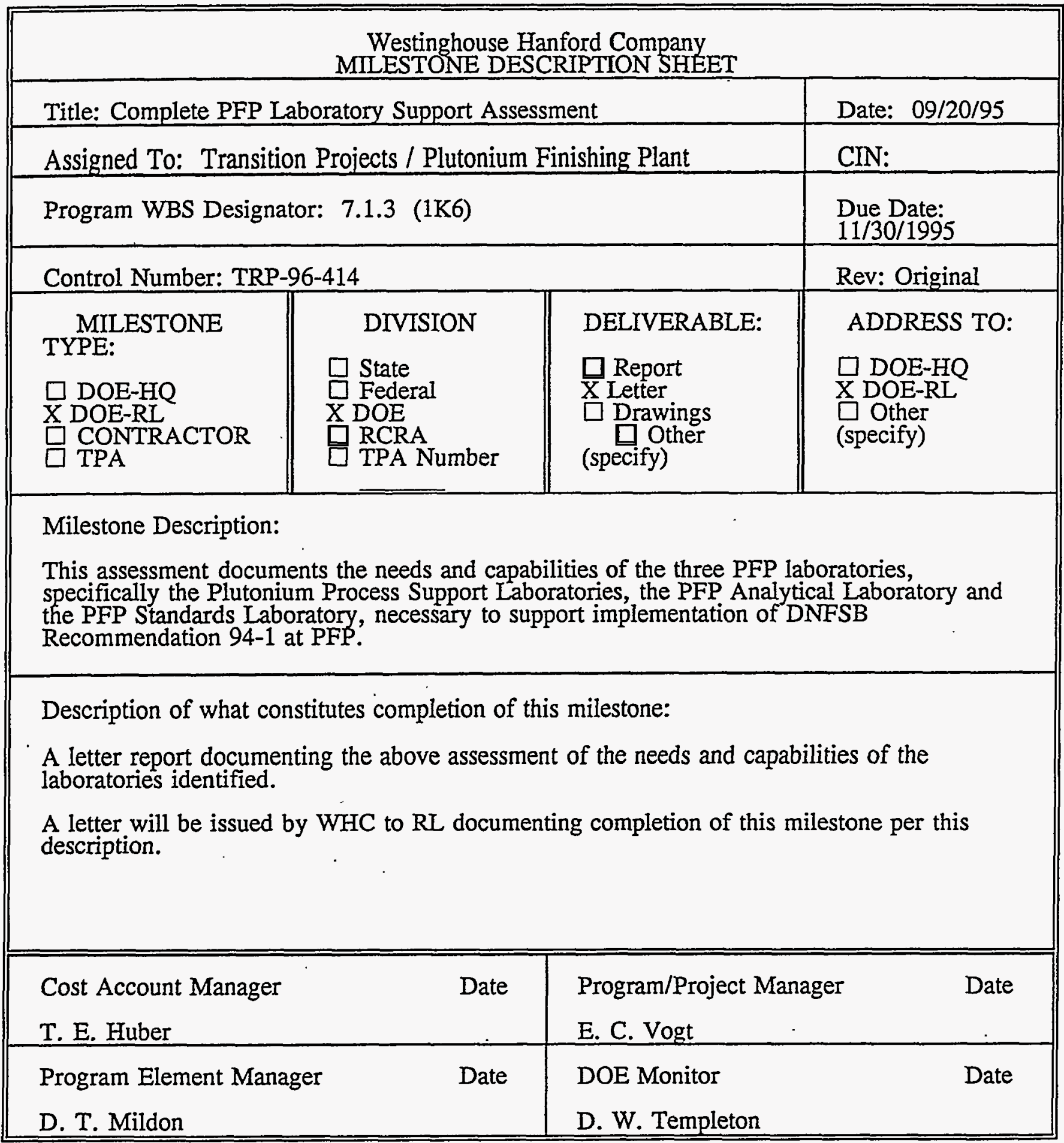


WHC-SP-1126, Rev. 1

TRANSITION PROJECTS

FY 1996 MYPP

1.3.1/7.1/6.12

Westinghouse Hanford Company

MILESTONE DESCRIPTION SHEET

Title: Complete Engineering Study for the Vertical Calciner

Assigned To: Transition Projects / Plutonium Finishing Plant

Program WBS Designator: 7.1.3 (1K6)

Control Number: TRP-96-415

MILESTONE TYPE:

$\square$ DOE-HQ

$\mathrm{X}$ DOE-RL

CONTRACTOR

TPA

\begin{tabular}{|l||} 
\\
$\square$ State \\
SFederal \\
X DOE \\
$\square$ RCRA \\
$\square$ TPA Number \\
\hline
\end{tabular}

DELIVERABLE:

$\square$ Report

X Letter

$\square$ Drawings

$\square$ Other

(specify)

Date: $09 / 20 / 95$

CIN:

Due Date:

$01 / 29 / 1996$

Rev: Original

Milestone Description:

This engineering study provides an assessment of the modifications required to the developmental vertical calciner in order to design and fabricate a product scale system. This study will also identity the location for the production scale system.

Description of what constitutes completion of this milestone:

A letter report documenting the required modifications to the developmental vertical calciner sufficient to design and fabricate a production scale system. The location for the production scale system within the PFP Complex will also be identified.

A letter will be issued by WHC to RL documenting completion of this milestone per this description.

\begin{tabular}{|ll|lc|}
\hline $\begin{array}{l}\text { Cost Account Manager } \\
\text { T. E. Huber }\end{array}$ & Date & $\begin{array}{l}\text { Program/Project Manager } \\
\text { E. C. Vogt }\end{array}$ & Date \\
\hline $\begin{array}{l}\text { Program Element Manager } \\
\text { D. T. Mildon }\end{array}$ & Date & $\begin{array}{l}\text { DOE Monitor } \\
\text { D. W. Templeton }\end{array}$ & Date \\
\hline
\end{tabular}


WHC-SP-1126, Rev. 1

\section{TRANSITION PROJECTS}

FY 1996 MYPP

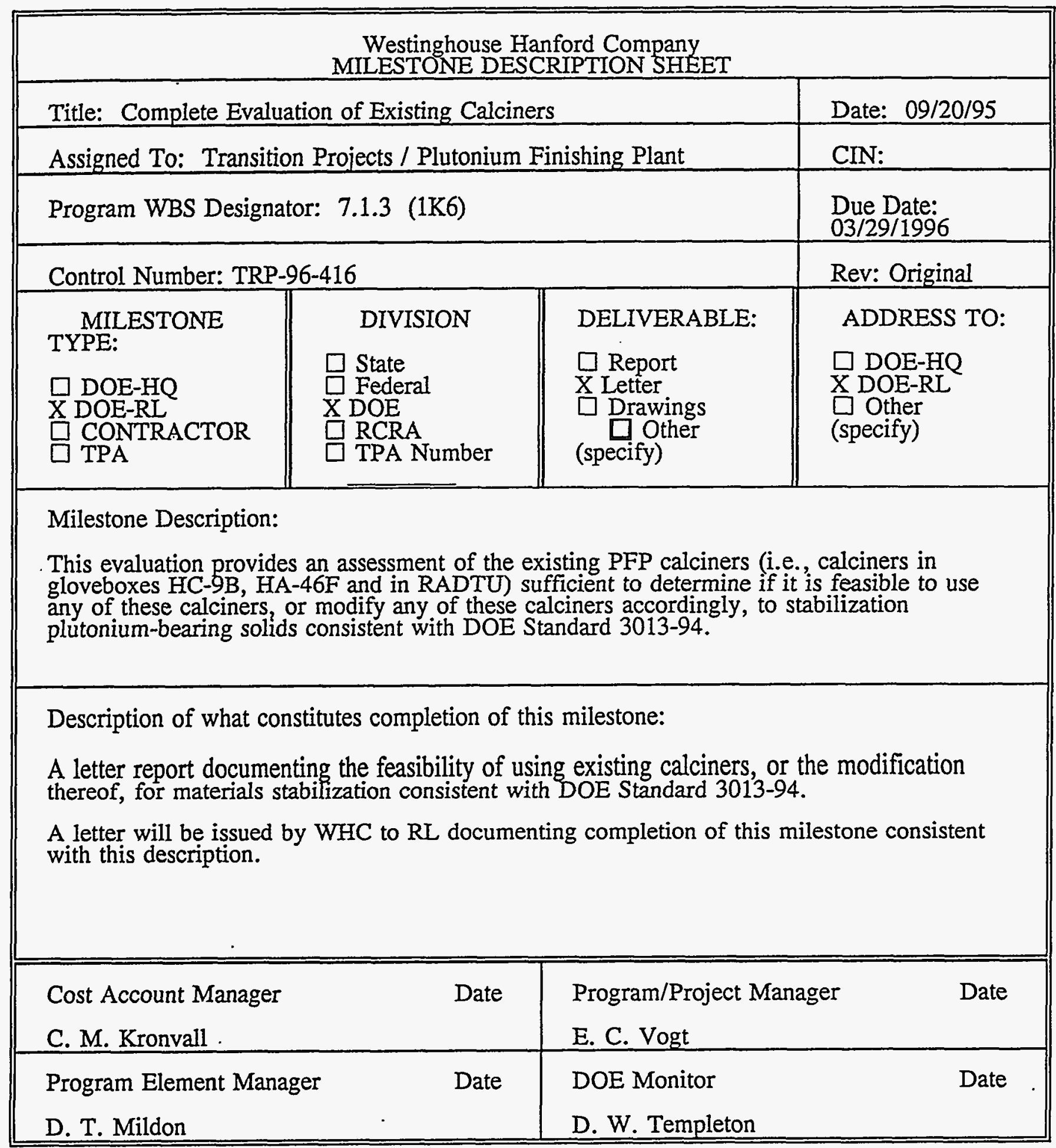


WHC-SP-1126, Rev. 1

\title{
TRANSITION PROJECTS
}

FY 1996 MYPP

\author{
1.3.1/7.1/6.12
}

Westinghouse Hanford Company MILESTONE DESCRIPTION SHEET

Title: Complete Engineering Study for New Glovebox with Three Muffle Furnaces

Assigned To: Transition Projects / Plutonium Finishing Plant

Program WBS Designator: 7.1.3 (1K6)

CIN:

\begin{tabular}{l}
\hline Control Number: TRP- \\
\hline MILESTONE \\
TYPE: \\
$\square$ DOE-HQ \\
X DOE-RL \\
$\square$ CONTRACTOR \\
$\square$ TPA
\end{tabular}

Milestone Description:

This engineering study provides an assessment of the need for an additional glovebox containing up to three muffle furnaces. The location of this new glovebox, if required, will also be identified.

Description of what constitutes completion of this milestone:

A letter report documenting the assessment for the need, and applicable location, for an addition glovebox and the number of muffle furnaces required.

A letter will be issued by WHC to RL documenting completion of this milestone consistent with this description.

\begin{tabular}{|ll|ll|}
\hline Cost Account Manager & Date & Program/Project Manager & Date \\
T. E. Huber & & E. C. Vogt & Date \\
\hline $\begin{array}{l}\text { Program Element Manager } \\
\text { D. T. Mildon }\end{array}$ & Date & $\begin{array}{l}\text { DOE Monitor } \\
\text { D. W. Templeton }\end{array}$ & \\
\hline
\end{tabular}


WHC-SP-1126, Rev. I

\section{TRANSITION PROJECTS}

FY 1996 MYPP

1.3.1/7.1/6.12

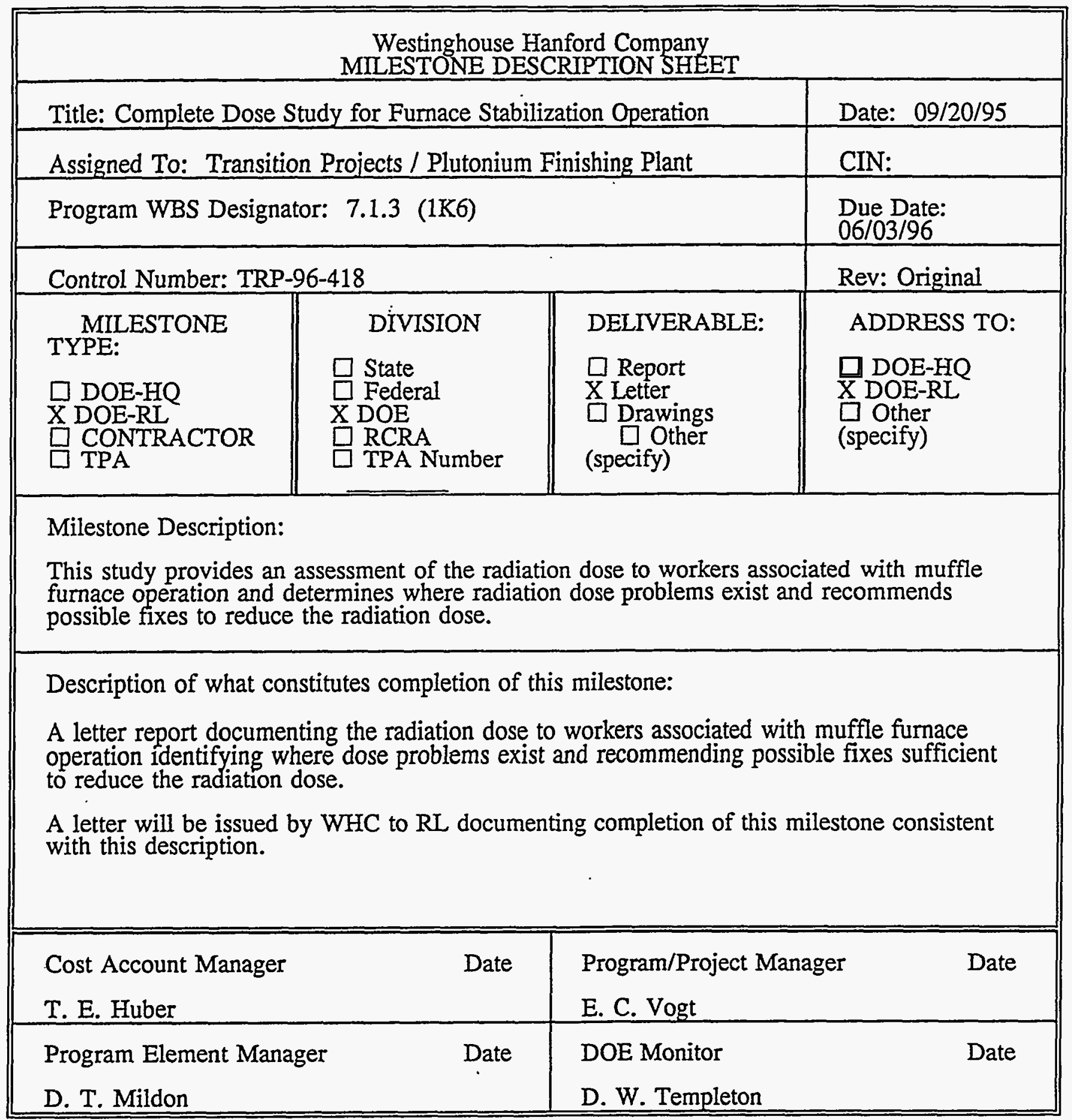


WHC-SP-1126, Rev. 1

\section{TRANSITION PROJECTS}

FY 1996 MYPP

1.3.1/7.1/6.12

Westinghouse Hanford Company

MILESTONE DESCRIPTION SHEET

Title: Complete Pyrolysis Furnace Engineering Study

Date: 09/20/95

Assigned To: Transition Projects / Plutonium Finishing Plant

CIN:

Program WBS Designator: 7.1 .3 (1K6)

Due Date: 07/08/96

Control Number: TRP-96-419

\begin{tabular}{l} 
MILESTONE \\
TYPE: \\
$\square$ DOE-HQ \\
X DOE-RL \\
$\square$ CONTRACTOR \\
$\square$ TPA \\
\hline
\end{tabular}

\begin{tabular}{l} 
DIVISION \\
$\square$ State \\
$\square$ Federal \\
X DOE \\
$\square$ RCRA \\
$\square$ TPA Number \\
\hline
\end{tabular}

DELIVERABLE:

$\square$ Report

$X$ Letter

$\square$ Drawings

$\square$ Other

(specify)
Rev: Original

ADDRESS TO:

$\square$ DOE-HQ

X DOE-RL

$\square$ Other

(specify)

\section{Milestone Description:}

This engineering study assesses the design modifications required to utilize a pyrolysis furnace system similar to Miscellaneous Treatment Hood \#4 (MT-4) for polycube

stabilization at PFP. This engineering study will also determine the location for this new system.

Description of what constitutes completion of this milestone:

A letter report assessing the design modifications, and the location, required to utilize a pyrolysis furnace system similar to MT-4 for polycube stabilization at PFP.

A letter will be issued from WHC to RL documenting completion of this milestone per the above description.

\begin{tabular}{||ll|ll||}
\hline Cost Account Manager & Date & $\begin{array}{l}\text { Program/Project Manager } \\
\text { E. C. Vogt }\end{array}$ & Date \\
T. E. Huber & & DOE Monitor & Date \\
Program Element Manager & Date & D. W. Templeton & \\
D. T. Mildon & & P.
\end{tabular}


WHC-SP-1126, Rev. 1

TRANSITION PROJECTS

FY 1996 MYPP

1.3.1/7.1/6.12

\begin{tabular}{|c|c|c|c|}
\hline \multicolumn{4}{|c|}{$\begin{array}{l}\text { Westinghouse Hanford Company } \\
\text { MILESTONE DESCRIPTION SHEET }\end{array}$} \\
\hline \multicolumn{3}{|c|}{$\begin{array}{l}\text { Title: Complete Design/Acceptance Testing of new PC Based Alarm } \\
\text { (AMOSS) }\end{array}$} & Date: $09 / 20 / 95$ \\
\hline \multicolumn{3}{|c|}{ Assigned To: Transition Projects/Plutonium Finishing Plant } & CIN: \\
\hline \multicolumn{3}{|c|}{ Program WBS Designator: 7.1 .3 (1K6) } & $\begin{array}{l}\text { Due Date: } \\
09 / 30 / 1996\end{array}$ \\
\hline \multicolumn{3}{|c|}{ Control Number: TRP-96-420 } & Rev: Original \\
\hline $\begin{array}{l}\text { MILESTONE } \\
\text { TYPE: } \\
\square \text { DOE-HQ } \\
\text { X DOE-RL } \\
\square \text { CONTRACTOR } \\
\square \text { TPA }\end{array}$ & $\begin{array}{l}\text { DIVISION: } \\
\text { State } \\
\square \text { Federal } \\
\text { X DOE } \\
\square \text { RCRA } \\
\square \text { TPA Number }\end{array}$ & $\begin{array}{l}\text { DELIVERABLE: } \\
\text { D Report } \\
\text { X Letter } \\
\square \text { Drawings } \\
\square \text { Other } \\
\text { (specify) }\end{array}$ & $\begin{array}{l}\text { ADDRESS TO: } \\
\text { D DOE-HQ } \\
\text { X DOE-RL } \\
\square \text { Other } \\
\text { (specify) }\end{array}$ \\
\hline \multicolumn{4}{|c|}{$\begin{array}{l}\text { Milestone Description: } \\
\text { Completed design, procurement, development and acceptance testing of the new PC-based } \\
\text { Alarm Monitoring System (AMS) at the AMS Mock-up, or Alarm Monitoring Operational } \\
\text { Support System (AMOSS). }\end{array}$} \\
\hline \multicolumn{4}{|c|}{$\begin{array}{l}\text { This milestone is complete upon the development of the acceptance test procedure, data } \\
\text { conversion, and successful acceptance testing of this new PC-based Alarm Monitoring System } \\
\text { at the AMOSS. Also, completion is based on the system being fully operational at the } \\
\text { completion of acceptance testing with only minor adjustments needed prior to installation at } \\
\text { PFP. }\end{array}$} \\
\hline \multicolumn{4}{|c|}{$\begin{array}{l}\text { A letter will be issued from WHC to RL documenting completion of this milestone } \\
\text { referencing the successful acceptance testing of this new PC-based AMS system. }\end{array}$} \\
\hline \multicolumn{2}{|c|}{$\begin{array}{l}\text { Cost Account Manager } \\
\text { T. E. Huber }\end{array}$} & \multicolumn{2}{|c|}{$\begin{array}{l}\text { Program/Project Manager } \\
\text { E. C. Vogt }\end{array}$} \\
\hline \multicolumn{2}{|c|}{$\begin{array}{l}\text { Program Element Manager } \\
\text { D. T. Mildon }\end{array}$} & \multicolumn{2}{|l|}{$\begin{array}{l}\text { DOE Monitor } \\
\text { D. W. Templeton }\end{array}$} \\
\hline
\end{tabular}


WHC-SP-1126, Rev. 1

\section{TRANSITION PROJECTS}

FY 1996 MYPP

1.3.1/7.1/6.12

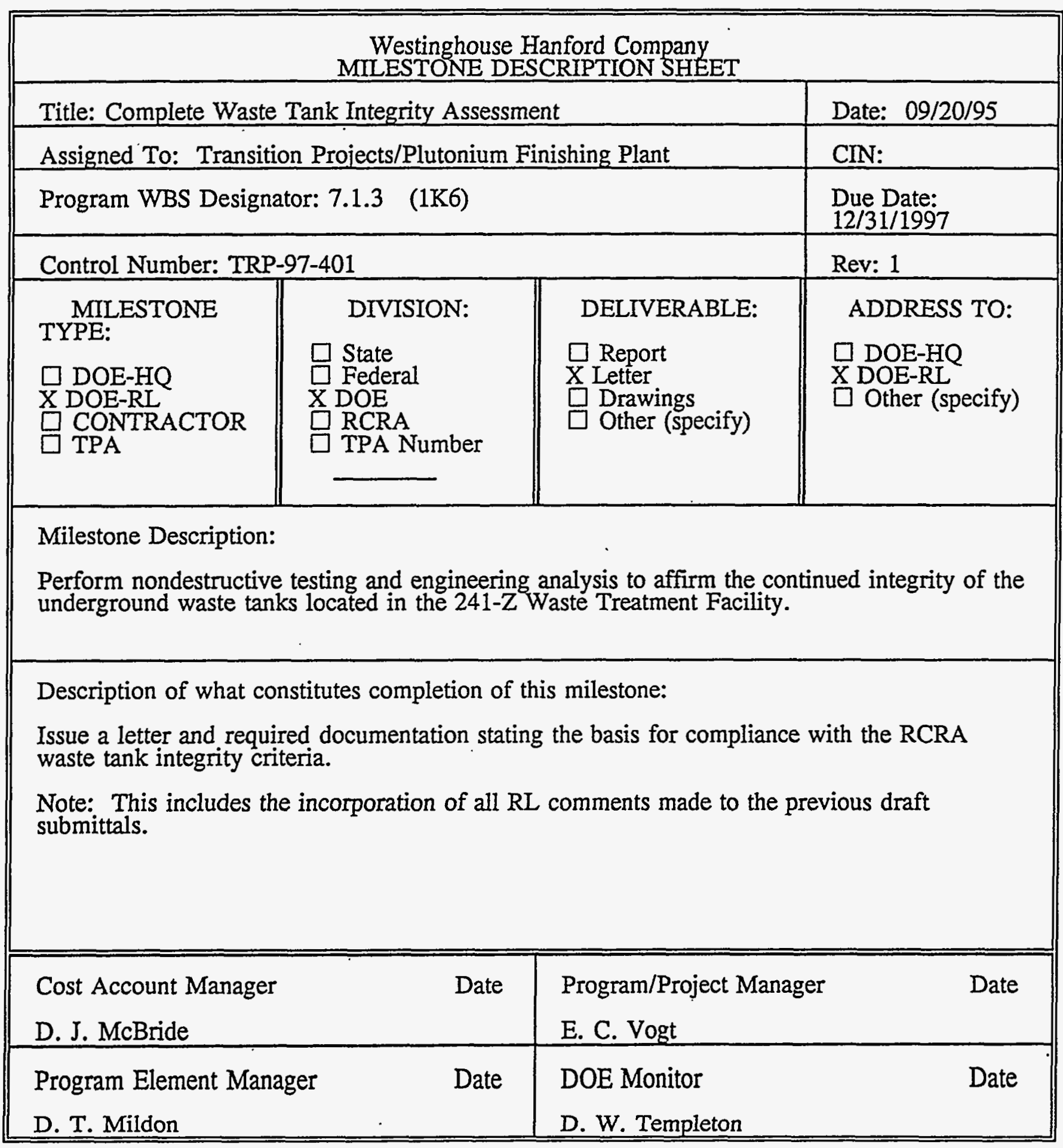


WHC-SP-1126, Rev. 1

TRANSITION PROJECTS

FY 1996 MYPP

1.3.1/7.1/6.12

Westinghouse Hanford Company MILESTONE DESCRIPTION SHEET

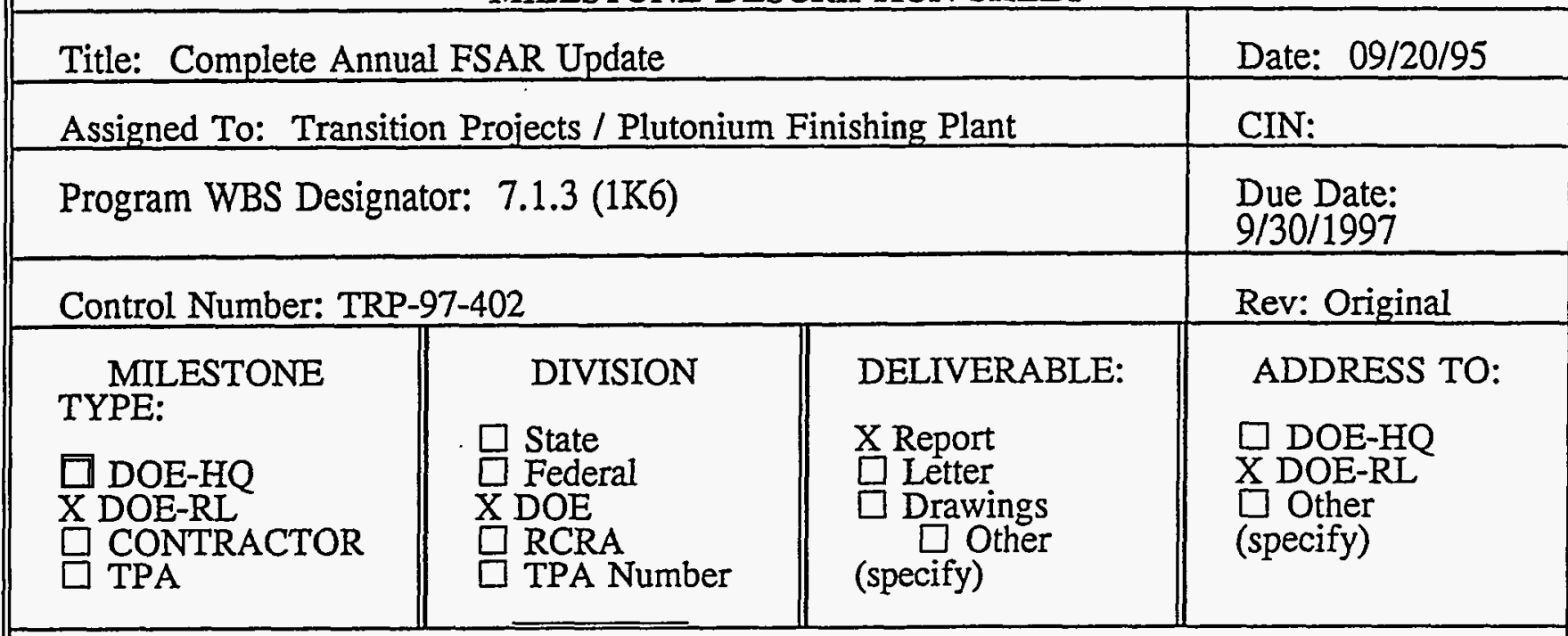

Milestone Description:

Issue the annual update of the PFP Safety Analysis Report in accordance with DOE Order 5480.23 .

Description of what constitutes completion of this milestone:

Issue updated SAR to DOE-RL for approval after securing approvals of required WHC SAR approval committees. "Contractors shall be required to review and update as necessary, SARs annually, pursuant to this Order to ensure that the information in each SAR is current and remains applicable. Revisions shall be submitted to the PSO at least annually and shall reflect all changes implemented up to 6 months prior to the filing of the updated SAR. The DOE approval of any USQ pursuant to DOE 5480.21, amendments to the TSRs, and the material submitted by the contractor to the PSO in support of these approvals shall be considered an addendum to the SAR until the information is incorporated into the SAR as part of the next annual update.

A separate letter, from the FSAR update letter, will be issued by WHC to RL documenting completion of this specific annual FSAR update.

\begin{tabular}{|ll|ll|}
\hline $\begin{array}{l}\text { Cost Account Manager } \\
\text { C.M. Kronvall }\end{array}$ & Date & $\begin{array}{l}\text { Program/Project Manager } \\
\text { E. C. Vogt }\end{array}$ & Date \\
\hline $\begin{array}{l}\text { Program Element Manager } \\
\begin{array}{l}\text { D. T. Mildon } \\
\hline\end{array}\end{array}$ & Date & $\begin{array}{l}\text { DOE Monitor } \\
\text { D. W. Templeton }\end{array}$ & Date \\
\hline
\end{tabular}


FY 1996 MYPP

WHC-SP-1126, Rev. I

TRANSITION PROJECTS

1.3.1/7.1/6.12

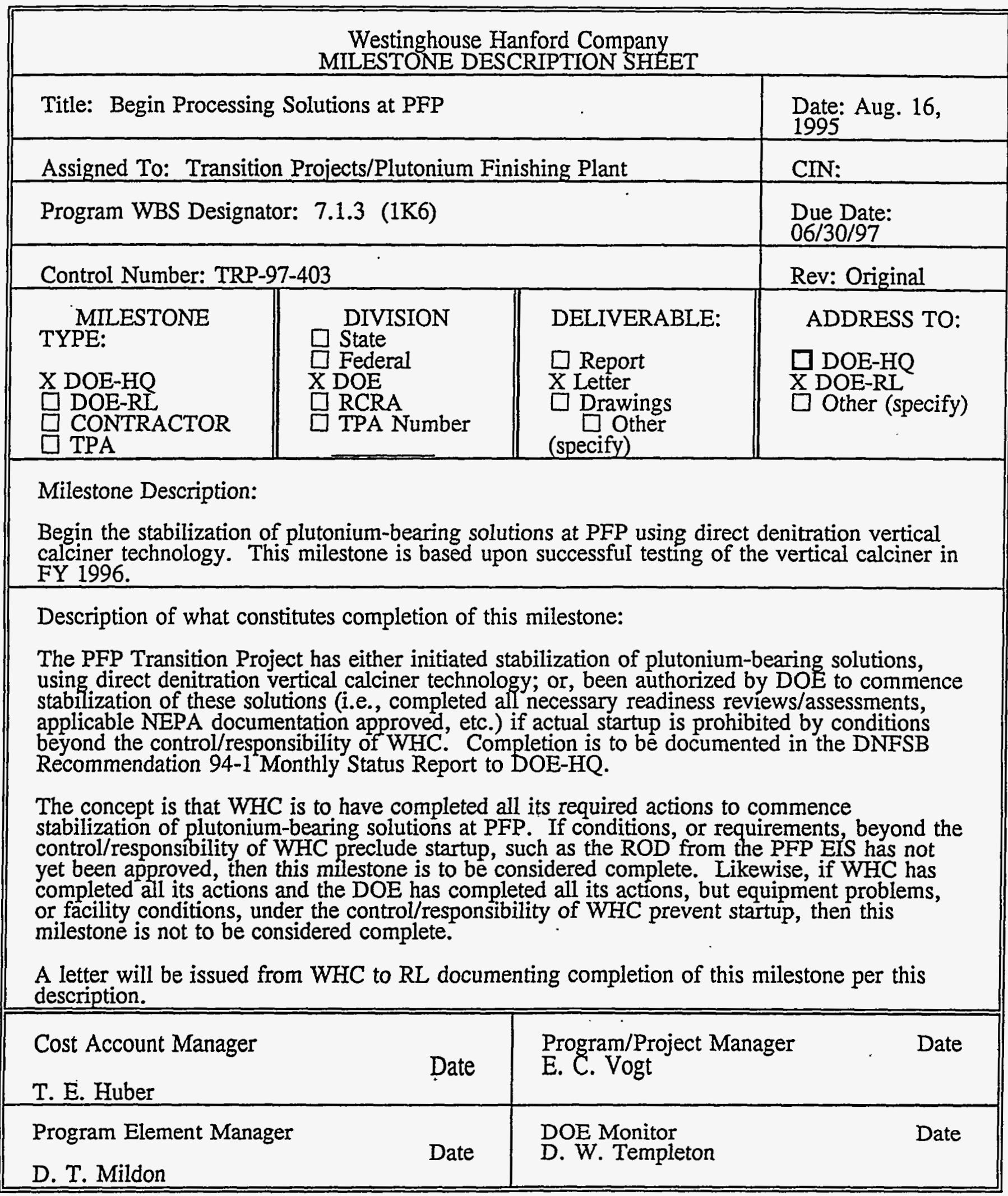


WHC-SP-1126, Rev. I

FY 1996 MYPP

\section{TRANSITION PROJECTS}

1.3.1/7.1/6.12

\section{Westinghouse Hanford Company MILESTONE DESCRIPTION SHEET}

Title: Complete Duct Terminal Cleanout (TCO), Section \#4 Date: $09 / 20 / 95$

Assigned To: Transition Projects/Plutonium Finishing Plant

Program WBS Designator: 7.1.3 (1K6) CIN:

Due Date: 07/31/1997

Control Number: TRP-97-404

\begin{tabular}{|l||l||}
\hline \multicolumn{1}{|c||}{ MILESTONE } & \multicolumn{1}{|c||}{ DIVISION: } \\
TYPE: & $\square$ State \\
D DOE-HQ & $\square$ Federal \\
X DOE-RL & X DOE \\
$\square$ CONTRACTOR & $\square$ RCRA \\
$\square$ TPA & $\square$ TPA Number \\
\hline
\end{tabular}

Rev: Original

Milestone Description:

Complete the removal of Segment \#4, a $\sim 150$ foot length of active process vacuum piping located primarily in room 262 of the 234-5Z building. Segment \#4 is clearly defined in the Plutonium Finishing Plant (PFP) Remediation Project Management Plan, WHC-SD-CP-PMP003 , dated March 17, 1989, and is anticipated to contain approximately $4.3 \mathrm{Kg}$ of plutoniumbearing material.

Description of what constitutes completion of this milestone:

This milestone is considered complete when the $\sim 150$ feet of active process vacuum piping, described above, has been removed, sectioned into pieces, packaged into appropriate containers and readied for shipment. Any plutonium material removed from this piping is to be either stored as reactive scrap for stabilization at a later date, or discarded as TRU waste, as appropriate. A letter will be issued from WHC to RL documenting completion of this milestone per this description.

\begin{tabular}{|ll|ll|}
\hline $\begin{array}{l}\text { Cost Account Manager } \\
\text { T. E. Huber }\end{array}$ & Date & $\begin{array}{l}\text { Program/Project Manager } \\
\text { E. C. Vogt }\end{array}$ & Date \\
\hline $\begin{array}{l}\text { Program Element Manager } \\
\text { D. T. Mildon }\end{array}$ & Date & $\begin{array}{l}\text { DOE Monitor } \\
\text { D. W. Templeton }\end{array}$ & Date \\
\hline
\end{tabular}


WHC-SP-1126, Rev. I

TRANSITION PROJECTS

FY 1996 MYPP

1.3.1/7.1/6.12

Westinghouse Hanford Company

MILESTONE DESCRIPTION SHEET

Title: Complete Annual FSAR Update

Date: $09 / 20 / 95$

Assigned To: Transition Projects/Plutonium Finishing Plant

Program WBS Designator: 7.1 .3 (1K6)

Control Number: TRP-98-401

MILESTONE TYPE:

$\square$ DOE-HQ

$\mathrm{X}$ DOE-RL

CONTRACTOR

TPA

\begin{tabular}{||l||} 
DIVISION \\
$\square$ State \\
$\square$ Federal \\
XOE \\
$\square$ RCRA \\
$\square$ TPA Number \\
\hline
\end{tabular}

DELIVERABLE:

CIN:

Due Date:

9/30/1998

Rev: Original

$X$ Report

$\square$ Letter

$\square$ Drawings

(specify)

ADDRESS TO:

$\square$ DOE-HQ

$X$ DOE-RL

$\square$ Other

(specify)

Milestone Description:

Issue the annual update of the PFP Safety Analysis Report in accordance with DOE Order 5480.23 .

Description of what constitutes completion of this milestone:

Issue updated SAR to DOE-RL for approval after securing approvals of required WHC SAR approval committees. "Contractors shall be required to review and update as necessary, SARs annually, pursuant to this Order to ensure that the information in each SAR is current and remains applicable. Revisions shall be submitted to the PSO at least annually and shall reflect all changes implemented up to 6 months prior to the filing of the updated SAR. The DOE approval of any USQ pursuant to DOE 5480.21, amendments to the TSRs, and the material submitted by the contractor to the PSO in support of these approvals shall be considered an addendum to the SAR until the information is incorporated into the SAR as part of the next annual update.

A separate letter, from the FSAR update letter, will be issued by WHC to RL documenting completion of this specific annual FSAR update.

\begin{tabular}{|ll|ll|}
\hline \hline $\begin{array}{l}\text { Cost Account Manager } \\
\text { C.M. Kronvall }\end{array}$ & Date & $\begin{array}{l}\text { Program/Project Manager } \\
\text { E. C. Vogt }\end{array}$ & Date \\
\hline $\begin{array}{l}\text { Program Element Manager } \\
\text { D. T. Mildon }\end{array}$ & Date & $\begin{array}{l}\text { DOE Monitor } \\
\text { D. W. Templeton }\end{array}$ & Date \\
\hline
\end{tabular}


WHC-SP-1126, Rev. 1

\section{TRANSITION PROJECTS}

FY 1996 MYPP

1.3.1/7.1/6.12

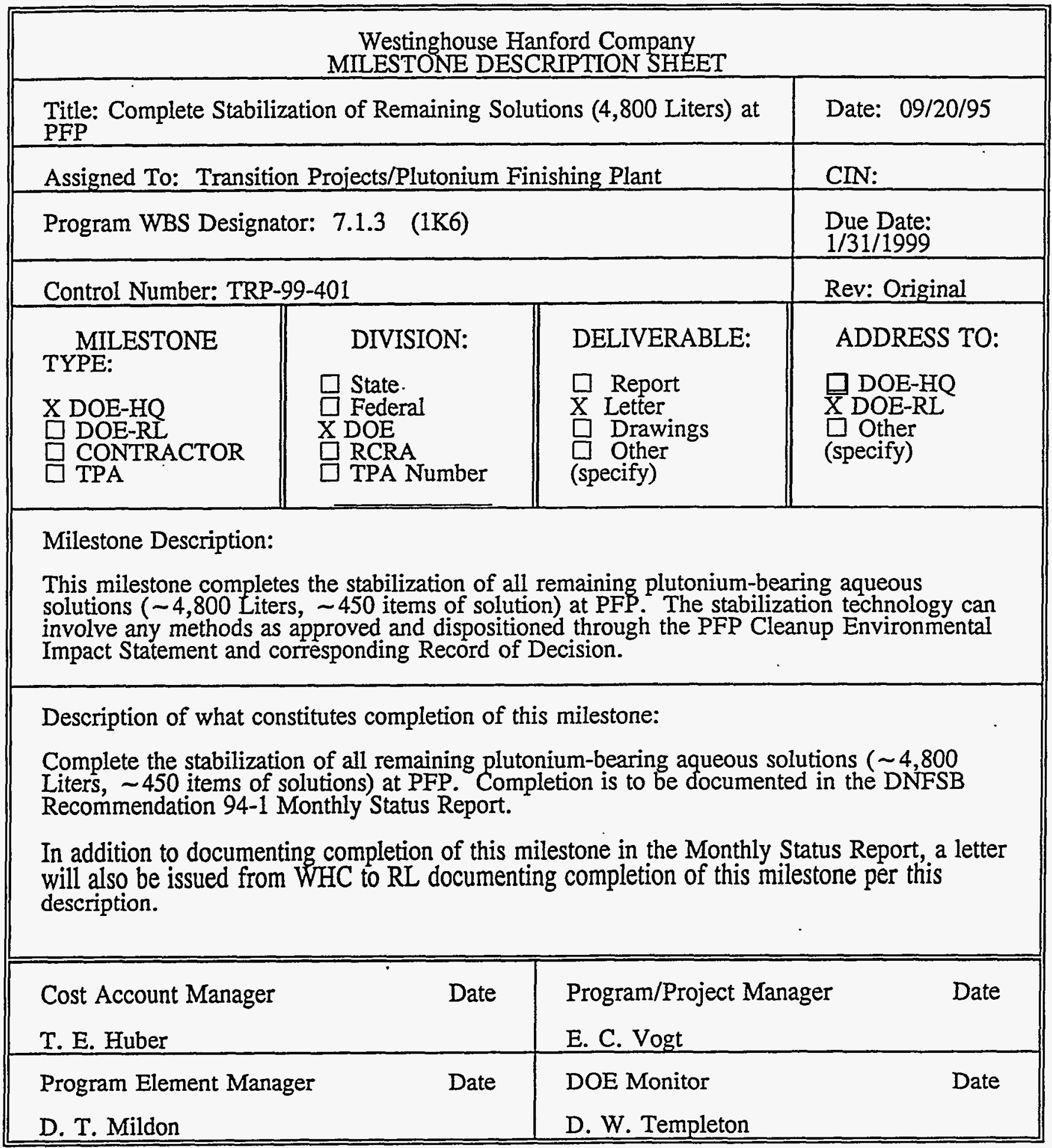


WHC-SP-1126, Rev. 1

FY 1996 MYPP

TRANSITION PROJECTS

1.3.1/7.1/6.12

\begin{tabular}{|c|c|c|c|}
\hline \multicolumn{4}{|c|}{$\begin{array}{l}\text { Westinghouse Hanford Company } \\
\text { MILESTONE DESCRIPTION SHEET }\end{array}$} \\
\hline \multicolumn{3}{|c|}{ Title: Start Restabilizing High Assay Oxides at PFP } & Date: $09 / 20 / 95$ \\
\hline \multicolumn{3}{|c|}{ Assigned To: Transition Projects/Plutonium Finishing Plant } & CIN: \\
\hline \multicolumn{3}{|c|}{ Program WBS Designator: 7.1.3 (1K6) } & $\begin{array}{l}\text { Due Date: } \\
\text { 7/31/1999 }\end{array}$ \\
\hline \multicolumn{3}{|c|}{ Control Number: TRP-99-402 } & Rev: Original \\
\hline $\begin{array}{l}\text { MILESTONE } \\
\text { TYPE: } \\
\text { X DOE-HQ } \\
\square \text { DOE-RI } \\
\square \text { CONTRACTOR } \\
\square \text { TPA }\end{array}$ & $\begin{array}{l}\text { DIVISION: } \\
\square \text { State } \\
\text { Federal } \\
\text { X DOE } \\
\square \text { RCRA } \\
\square \text { TPA Number }\end{array}$ & $\begin{array}{l}\text { DELIVERABLE: } \\
\text { Report } \\
\text { X Retter } \\
\square \text { Drawings } \\
\square \text { Other (specify) }\end{array}$ & $\begin{array}{l}\text { ADDRESS TO: } \\
\square \text { DOE-HQ } \\
\text { X DOE-RL } \\
\square \text { Other (specify) }\end{array}$ \\
\hline \multicolumn{4}{|c|}{$\begin{array}{l}\text { Milestone Description: } \\
\text { Commence restabilizing High Assay Oxides at the PFP utilizing muffle furnace and/or } \\
\text { continuous calciner technology with the new bagless transfer packaging system. }\end{array}$} \\
\hline \multicolumn{4}{|c|}{$\begin{array}{l}\text { Description of what constitutes completion of this milestone: } \\
\text { The PFP Transition Project has either initiated restabilizing High Assay Oxide using muffle } \\
\text { furnace and/or continuous calciner technology with the new bagless transfer packaging system; } \\
\text { or, been authorized by DOE to commence restabilizing operations (i.e., completed alf } \\
\text { necessary readiness reviews/assessments, applicable NEPA documentation approved, etc.) if } \\
\text { actual startup is prohibited by conditions beyond the control/responsibility of WHC. } \\
\text { Completion is to be documented in the DNFSB Recommendation 94-1 Monthly Status Report } \\
\text { to DOE-HQ. }\end{array}$} \\
\hline \multicolumn{4}{|c|}{$\begin{array}{l}\text { The concept is that WHC is to have completed all its required actions to commence } \\
\text { restabilizing operations at PFP. If conditions, or requirements, beyond the } \\
\text { control/responsibility of WHC preclude startup, such as required NEPA documentation } \\
\text { prepared by WHC as scheduled has not yet been approved, then this milestone is to be } \\
\text { considered complete. Likewise if WHC and DOE have completed anl their actions but } \\
\text { equipment problems, or facility conditions, under the control/responsibility of WHC prevent } \\
\text { startup, then this milestone is not to be considered complete. A letter will be issued from } \\
\text { WHC to RL documenting completion of this milestone per this description. }\end{array}$} \\
\hline \multicolumn{2}{|c|}{$\begin{array}{l}\text { Cost Account Manager } \\
\text { T. E. Huber }\end{array}$} & \multicolumn{2}{|c|}{$\begin{array}{l}\text { Program/Project Manager } \\
\text { E. C. Vogt }\end{array}$} \\
\hline \multicolumn{2}{|c|}{$\begin{array}{l}\text { Program Element Manager } \\
\text { D. T. Mildon }\end{array}$} & \multicolumn{2}{|l|}{$\begin{array}{l}\text { DOE Monitor. } \\
\text { D. W. Templeton }\end{array}$} \\
\hline
\end{tabular}


WHC-SP-1126, Rev. 1

FY 1996 MYPP

TRANSITION PROJECTS

1.3.1/7.1/6.12

\begin{tabular}{|c|c|c|c|}
\hline \multicolumn{4}{|c|}{$\begin{array}{l}\text { Westinghouse Hanford Company } \\
\text { MILESTONE DESCRIPTION SHEET }\end{array}$} \\
\hline \multicolumn{3}{|c|}{ Title: Start Stabilization of Polycubes } & Date: $09 / 20 / 95$ \\
\hline \multicolumn{3}{|c|}{ Assigned To: Transition Projects/Plutonium Finishing Plant } & CIN: \\
\hline \multicolumn{3}{|c|}{ Program WBS Designator: 7.1 .3 (1K6) } & $\begin{array}{l}\text { Due Date: } \\
07 / 31 / 1999\end{array}$ \\
\hline \multicolumn{3}{|c|}{ Control Number: TRP-99-403 } & Rev: Original \\
\hline $\begin{array}{l}\text { MILESTONE } \\
\text { TYPE: } \\
\text { X DOE-HQ } \\
\square \text { DOE-RI } \\
\square \text { CONTRACTOR } \\
\square \text { TPA }\end{array}$ & $\begin{aligned} & \text { DIVISION } \\
\square & \text { State } \\
\square & \text { Federal } \\
X & \text { DOE } \\
& \\
\square & \text { RCRA } \\
& \text { TPA Number }\end{aligned}$ & $\begin{array}{l}\text { DELIVERABLE: } \\
\square \text { Report } \\
\text { X Letter } \\
\square \text { Drawings } \\
\square \text { Other } \\
\text { (specify) }\end{array}$ & $\begin{array}{l}\text { ADDRESS TO: } \\
\square \text { DOE-HQ } \\
\text { X DOE-RL } \\
\square \text { Other } \\
\text { (specify) }\end{array}$ \\
\hline \multicolumn{4}{|c|}{$\begin{array}{l}\text { Milestone Description: } \\
\text { Begin the stabilization of polycubes, either at the PFP facility or at another authorized } \\
\text { facility, using appropriately approved technology. }\end{array}$} \\
\hline \multirow{2}{*}{\multicolumn{4}{|c|}{$\begin{array}{l}\text { The PFP Transition Project has either initiated stabilization of polycubes, using appropriate } \\
\text { approved technology; or, been authorized by DOE to commence stabilization of polycubes } \\
\text { (1.e., completed all necessary readines reviews/assessments, applicable NEPA documentation } \\
\text { approved, etc.) if actual startup is prohibited by conditions beyond the control/responsibility } \\
\text { of WHC. Or PFP/WHC has enabled the stabilization of PFP polycubes at another authorized } \\
\text { Facility to start by this date. } \\
\text { WHC is to have completed all its required actions to commence stabilization of polycubes at } \\
\text { PFP. If conditions, or requirements, beyond the control of WHC preclude startup, such as } \\
\text { lack of approval of the Record of Decision by its scheduled date (would prohibit from } \\
\text { commencing polycube stabilization), then this milestone is to be considered complete. } \\
\text { Likewise, if WHC and DOE have completed all their actions but equipment problems, or } \\
\text { facility conditions, under the control of WHC prevent startup, then this milestone is not to be } \\
\text { considered complete. A letter will be issued from WHC to RL documenting completion of } \\
\text { this milestone per this description. }\end{array}$}} \\
\hline & & & \\
\hline \multicolumn{2}{|c|}{$\begin{array}{l}\text { Cost Account Manager } \\
\text { T. E. Huber }\end{array}$} & \multicolumn{2}{|c|}{$\begin{array}{l}\text { Program/Project Manager } \\
\text { E. C. Vogt }\end{array}$} \\
\hline \multicolumn{2}{|c|}{$\begin{array}{l}\text { Program Element Manager } \\
\text { D. T. Mildon }\end{array}$} & \multicolumn{2}{|l|}{$\begin{array}{l}\text { DOE Monitor } \\
\text { D. W. Templeton }\end{array}$} \\
\hline
\end{tabular}


WHC-SP-1126, Rev. 1

\title{
TRANSITION PROJECTS
}

FY 1996 MYPP

\author{
1.3.1/7.1/6.12
}

Westinghouse Hanford Company MILESTONE DESCRIPTION SHEET

\begin{tabular}{|c|c|c|c|}
\hline \multicolumn{3}{|c|}{ Title: Complete Annual FSAR Update } & Date: $09 / 20 / 95$ \\
\hline \multicolumn{3}{|c|}{ Assigned To: Transition Projects / Plutonium Finishing Plant } & CIN: \\
\hline \multicolumn{3}{|c|}{ Program WBS Designator: $7.1 .3(1 \mathrm{~K} 6)$} & $\begin{array}{l}\text { Due Date: } \\
\text { 9/30/1999 }\end{array}$ \\
\hline \multicolumn{3}{|c|}{ Control Number: TRP-99-404 } & Rev: Original \\
\hline $\begin{array}{l}\text { MILESTONE } \\
\text { TYPE: } \\
\square \text { DOE-HQ } \\
\text { X DOE-RL } \\
\square \text { CONTRACTOR } \\
\square \text { TPA }\end{array}$ & $\begin{aligned} & \text { DIVISION } \\
& \text { Śtate } \\
& \square \text { Federal } \\
& \text { X DOE } \\
& \square \text { RCRA } \\
& \square \text { TPA Number }\end{aligned}$ & $\begin{array}{l}\text { DELTVERABLE: } \\
\text { X Report } \\
\square \text { Letter } \\
\square \text { Drawings } \\
\square \text { Other } \\
\text { (specify) }\end{array}$ & $\begin{array}{l}\text { ADDRESS TO: } \\
\square \text { DOE-HQ } \\
\square \text { DOE-RL } \\
\square \text { Other } \\
\text { (specify) }\end{array}$ \\
\hline
\end{tabular}

Milestone Description:

Issue the annual update of the PFP Safety Analysis Report in accordance with DOE Order 5480.23 .

Description of what constitutes completion of this milestone:

Issue updated SAR to DOE-RL for approval after securing approvals of required WHC SAR approval committees. "Contractors shall be required to review and update as necessary, SARs annually, pursuant to this Order to ensure that the information in each SAR is current and remains applicable. Revisions shall be submitted to the PSO at least annually and shall reflect all changes implemented up to 6 months prior to the filing of the updated SAR. The DOE approval of any USQ pursuant to DOE 5480.21, amendments to the TSRs, and the material submitted by the contractor to the PSO in support of these approvals shall be considered an addendum to the SAR until the information is incorporated into the SAR as part of the next annual update.

A separate letter, from the FSAR update letter, will be issued by WHC to RL documenting completion of this specific annual FSAR update.

\begin{tabular}{|ll|ll||}
\hline $\begin{array}{l}\text { Cost Account Manager } \\
\text { C. M. Kronvall }\end{array}$ & Date & $\begin{array}{l}\text { Program/Project Manager } \\
\text { E. C. Vogt }\end{array}$ & Date \\
\hline $\begin{array}{l}\text { Program Element Manager } \\
\text { D. T. Mildon }\end{array}$ & Date & $\begin{array}{l}\text { DOE Monitor } \\
\text { D. W. Templeton. }\end{array}$ & Date \\
\hline
\end{tabular}


WHC-SP-1126, Rev. 1

FY 1996 MYPP

TRANSITION PROJECTS

1.3.1/7.1/6.12

Westinghouse Hanford Company

MILESTONE DESCRIPTION SHEET

Title: Complete 234-5Z Ductwork Cleanout (M-83-02-T04)

Date: $09 / 20 / 95$

Assigned To: Transition Projects/Plutonium Finishing Plant

CIN:

Program WBS Designator: 7.1 .3 (1K6)

Due Date:

$12 / 31 / 1998$

Control Number: TRP-99-405

MILESTONE

TYPE:

DIVISION:

Rev: Original

$\square$ DOE-HQ

DOE-RL

CONTRACTOR

$\mathrm{X}$ TPA

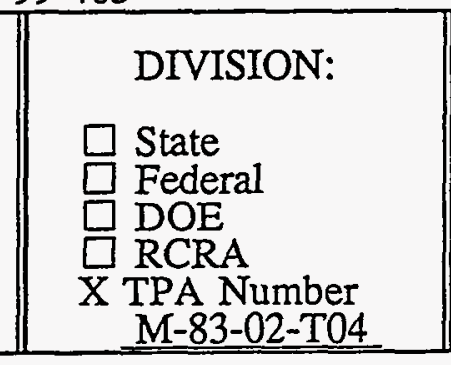

DELIVERABLE:

ADDRESS TO:

$\square$ Report

$X$ Letter

$\square$ Drawings

$\square$ Other

(specify)

$\square$ DOE-HQ

$X$ DOE-RL

$\square$ Other

(specify)

\section{Milestone Description:}

Residual Plutonium-bearing materials will be removed from identified exhaust ventilation ducting (two sections totalling approximately 60 meters [197 feet]) and selected process vacuum system piping (approximately 45 meters [150 feet]).

Description of what constitutes completion of this milestone:

This milestone is consider complete when the following DOE-RL milestones have been completed:
a. TRP-96-412, Complete Duct TCO, Section \#2
b. TRP-96-413, Complete Duct TCO, Section \#3
c. TRP-96-414, Complete Duct TCO, Section \#4

Completion of this milestone is to be documented in a letter prepared by WHC to DOE-RL upon completion of the last of the above three listed DOE-RL milestones. DOE-RL is to notify the appropriate Regulators in writing of the completion of this TPA milestone upon receipt of the WHC letter.

\begin{tabular}{|c|c|c|c|}
\hline $\begin{array}{l}\text { Cost Account Manager } \\
\text { T. E. Huber }\end{array}$ & Date & $\begin{array}{l}\text { Program/Project Manager } \\
\text { E. C. Vogt }\end{array}$ & Date \\
\hline $\begin{array}{l}\text { Program Element Manager } \\
\text { D. T. Mildon }\end{array}$ & Date & $\begin{array}{l}\text { DOE Monitor } \\
\text { D. W. Templeton }\end{array}$ & Date \\
\hline
\end{tabular}


WHC-SP-1126, Rev. 1

FY 1996 MYPP

TRANSITION PROJECTS

1.3.1/7.1/6.12

\begin{tabular}{|c|c|c|c|}
\hline \multicolumn{4}{|c|}{$\begin{array}{l}\text { Westinghouse Hanford Company } \\
\text { MILESTONE DESCRIPTION SHEET }\end{array}$} \\
\hline \multicolumn{3}{|c|}{ Title: Complete Identified Interim Actions (M-83-02) } & Date: $09 / 20 / 95$ \\
\hline \multicolumn{3}{|c|}{ Assigned To: Transition Projects/Plutonium Finishing Plant } & CIN: \\
\hline \multicolumn{3}{|c|}{ Program WBS Designator: 7.1 .3 (1K6). } & $\begin{array}{l}\text { Due Date: } \\
12 / 31 / 1998\end{array}$ \\
\hline \multicolumn{3}{|c|}{ Control Number: TRP-99-406 } & Rev: Original \\
\hline $\begin{array}{l}\text { MILESTONE } \\
\text { TYPE: } \\
\square \text { DOE-HQ } \\
\square \text { DOE-RI } \\
\text { X TPATRACTOR }\end{array}$ & 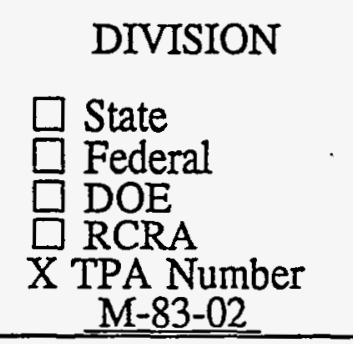 & $\begin{array}{l}\text { DELIVERABLE: } \\
\square \text { Report } \\
\text { X Letter } \\
\square \text { Drawings } \\
\square \text { Other } \\
\text { (specify) }\end{array}$ & $\begin{array}{l}\text { ADDRESS TO: } \\
\text { D DOE-HQ } \\
\text { X DOE-RI } \\
\square \text { Other } \\
\text { (specify) }\end{array}$ \\
\hline \multicolumn{4}{|c|}{$\begin{array}{l}\text { Milestone Description: } \\
\text { Completion of the following TPA target milestones: }\end{array}$} \\
\hline \multicolumn{4}{|c|}{$\begin{array}{l}\text { Description of what constitutes completion of this milestone: } \\
\text { A letter will be issued from WHC to RL verifying completion of the following TPA target } \\
\text { milestones: } \\
\text { 1) Submit Plan and Schedule for Additional Interim Actions (September 1995) } \\
\text { 2) Complete Sludge Stabilization (December 1995) } \\
\text { 3) Complete 10-L Solution Downloading (June 1996) } \\
\text { 4) Complete 234-5Z Ductwork Cleanout (December 1998) }\end{array}$} \\
\hline \multicolumn{2}{|c|}{$\begin{array}{l}\text { Cost Account Manager } \\
\text { D.J. McBride }\end{array}$} & \multicolumn{2}{|c|}{$\begin{array}{l}\text { Program/Project Manager } \\
\text { E. C. Vogt }\end{array}$} \\
\hline \multicolumn{2}{|c|}{$\begin{array}{l}\text { Program Element Manager } \\
\text { D. T. Mildon }\end{array}$} & \multicolumn{2}{|l|}{$\begin{array}{l}\text { DOE Monitor } \\
\text { D. W. Templeton }\end{array}$} \\
\hline
\end{tabular}


WHC-SP-1126, Rev. 1

TRANSITION PROJECTS

FY 1996 MYPP

\subsection{1/7.1/6.12}

Westinghouse Hanford Company

MILESTONE DESCRIPTION SHEET

\begin{tabular}{|c|c|c|c|}
\hline \multicolumn{3}{|c|}{$\begin{array}{l}\text { Title: Complete Stabilization of Reactive Solid Residues (Sand, slag } \\
\text { \& } \\
\text { crucible, poorly characterized items) at PFP }\end{array}$} & Date: $.09 / 20 / 95$ \\
\hline \multicolumn{3}{|c|}{ Assigned To: Transition Projects/Plutonium Finishing Plant } & CIN: \\
\hline \multicolumn{3}{|c|}{ Program WBS Designator: 7.1 .3 (1K6) } & $\begin{array}{l}\text { Due Date: } \\
1 / 31 / 2000\end{array}$ \\
\hline \multicolumn{3}{|c|}{ Control Number: TRP-00-401 } & Rev: Original \\
\hline $\begin{array}{l}\text { MILESTONE } \\
\text { TYPE: } \\
\text { X DOE-HQ } \\
\square \text { DOE-RI } \\
\text { CONTRACTOR } \\
\square \text { TPA }\end{array}$ & $\begin{array}{l}\text { DIVISION: } \\
\square \text { State } \\
\square \text { Federal } \\
\text { X DOE } \\
\square \text { RCRA } \\
\square \text { TPA Number }\end{array}$ & $\begin{array}{l}\text { DELIVERABLE: } \\
\square \text { Report } \\
\text { X Letter } \\
\text { Drawings } \\
\text { Other } \\
\text { (specify) }\end{array}$ & $\begin{array}{l}\text { ADDRESS TO: } \\
\text { D DOE-HQ } \\
\text { X DOE-RL } \\
\square \text { Other } \\
\text { (specify) }\end{array}$ \\
\hline
\end{tabular}

Milestone Description:

This milestone completes the stabilization of all remaining reactive solid residues (sand, slag \& crucible, poorly characterized items) at PFP. The stabilization technology can involve any methods as approved and dispositioned through the PFP Cleanup Environmental Impact Statement and corresponding Record of Decision.

Description of what constitutes completion of this milestone:

Complete the stabilization of all remaining reactive solid residues, as described above, at PFP. This material, once stabilized, is to packaged in accordance with the DOE Standard for $<50 \mathrm{wt}$.\% material (to be issued). Completion is to be documented in the DNFSB Recommendation 94-1 Monthly Status Report.

In addition to documenting completion of this milestone in the Monthly Status Report, a letter will also be issued from WHC to RL documenting completion of this milestone per this description.

\begin{tabular}{|c|c|c|c|}
\hline $\begin{array}{l}\text { Cost Account Manager } \\
\text { T. E. Huber }\end{array}$ & Date & $\begin{array}{l}\text { Program/Project Manager } \\
\text { E. C. Vogt }\end{array}$ & Date \\
\hline $\begin{array}{l}\text { Program Element Manager } \\
\text { D. T. Mildon }\end{array}$ & Date & $\begin{array}{l}\text { DOE Monitor } \\
\text { D. W. Templeton }\end{array}$ & Date \\
\hline
\end{tabular}


WHC-SP-1126, Rev. I

FY 1996 MYPP

TRANSITION PROJECTS

1.3.1/7.1/6.12

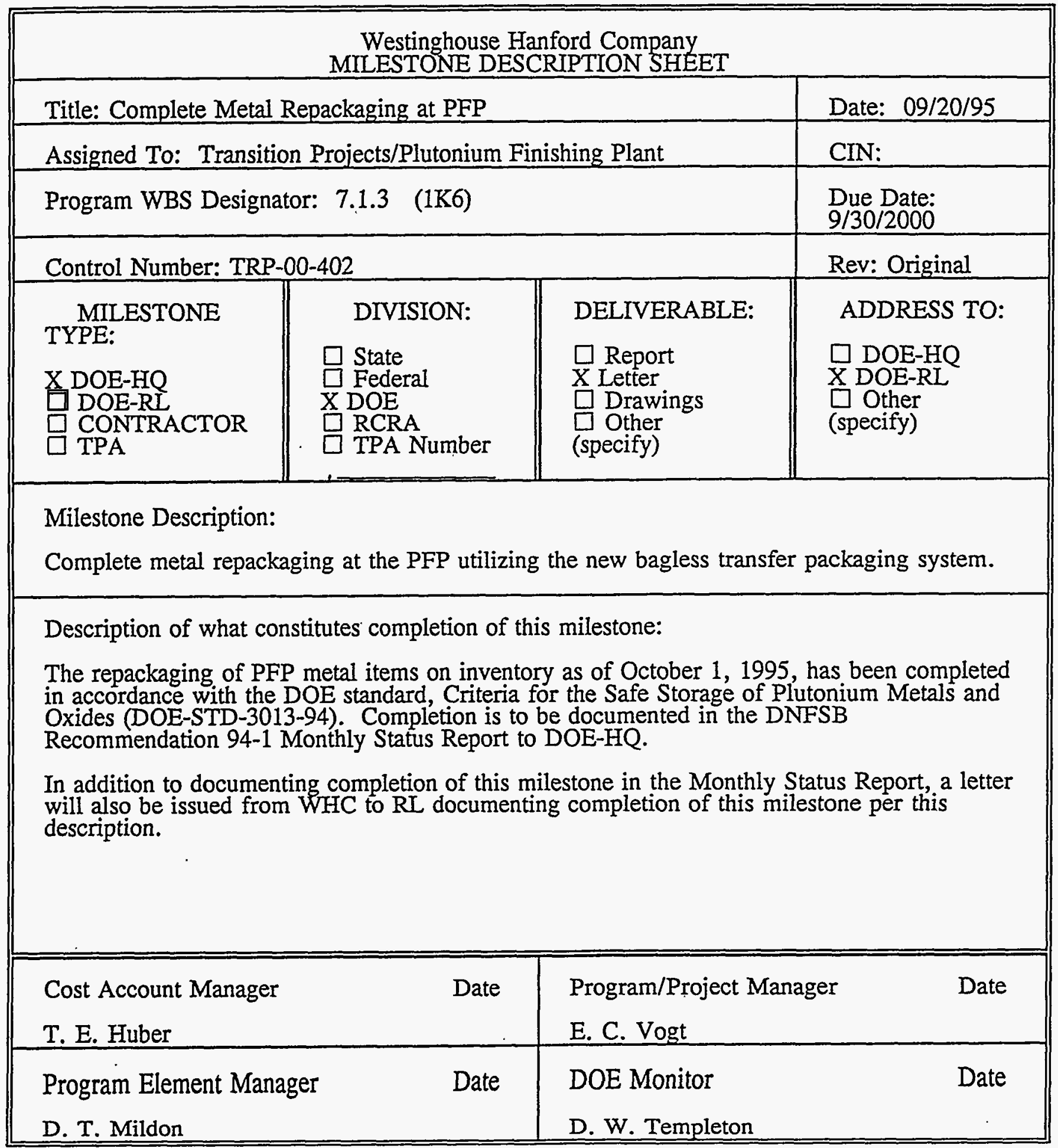


WHC-SP-1126, Rev. 1

\section{TRANSITION PROJECTS}

FY 1996 MYPP

1.3.1/7.1/6.12

Westinghouse Hanford Company MILESTONE DESCRIPTION SHEET

Title: Complete Annual FSAR Update

Assigned To: Transition Projects / Plutonium Finishing Plant

Program WBS Designator: 7.1 .3 (1K6)

Control Number: TRP-00-403

MILESTONE TYPE:

$\square$ DOE-HQ

X DOE-RL

CONTRACTOR TPA

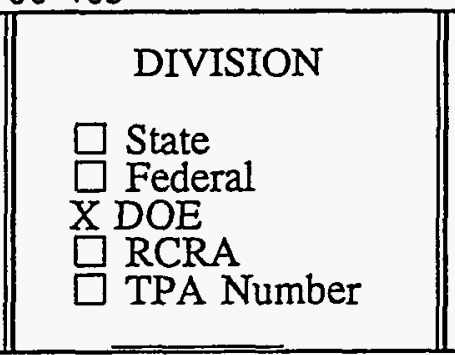

DELIVERABLE:

$X$ Report

$\square$ Letter

$\square$ Drawings

(specify)
Date: 09/20/95

CIN:

Due Date:

9/30/2000

Rev: Original

ADDRESS TO:

$\square$ DOE-HQ

X DOE-RL

$\square$ Other

(specify)

Milestone Description:

Issue the annual update of the PFP Safety Analysis Report in accordance with DOE Order 5480.23 .

Description of what constitutes completion of this milestone:

Issue updated SAR to DOE-RL for approval after securing approvals of required WHC SAR approval committees. "Contractors shall be required to review and update as necessary, SARs annually, pursuant to this Order to ensure that the information in each SAR is current and remains applicable. Revisions shall be submitted to the PSO at least annually and shall reflect all changes implemented up to 6 months prior to the filing of the updated SAR. The DOE approval of any USQ pursuant to DOE 5480.21, amendments to the TSRs, and the material submitted by the contractor to the PSO in support of these approvals shall be considered an addendum to the SAR until the information is incorporated into the SAR as part of the next annual update.

A separate letter, from the FSAR update letter, will be issued by WHC to RL documenting completion of this specific annual FSAR update.

Cost Account Manager.

Date

Program/Project Manager

Date

C. M. Kronvall

Program Element Manager

Date

E. C. Vogt

D. T. Mildon

DOE Monitor

Date

D. W. Templeton 
WHC-SP-1126, Rev. 1

TRANSITION PROJECTS

FY 1996 MYPP

1.3.1/7.1/6.12

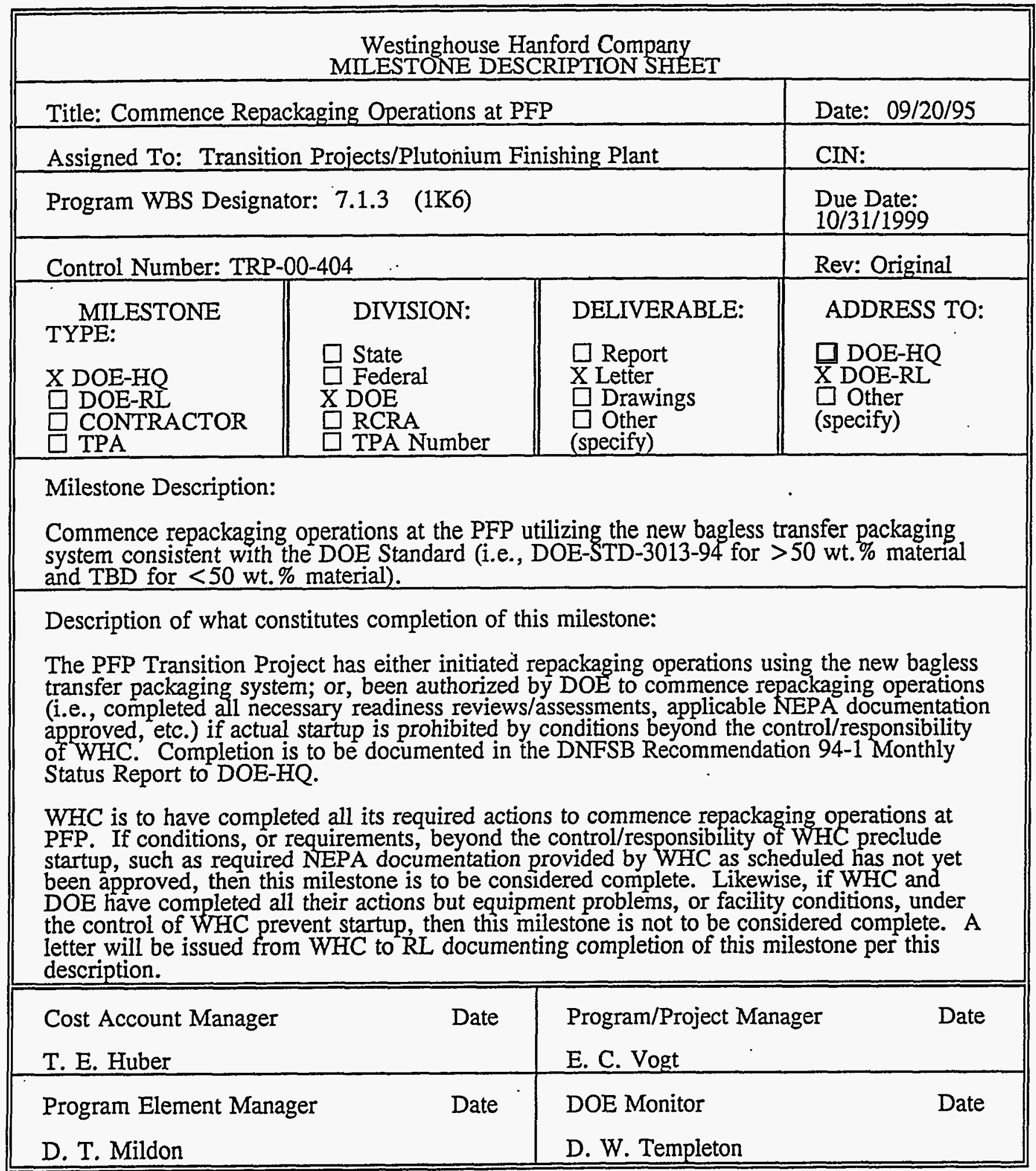


WHC-SP-1126, Rev. 1

FY 1996 MYPP

\section{TRANSITION PROJECTS}

\subsection{1/7.1/6.12}

Westinghouse Hanford Company MILESTONE DESCRIPTION SHEET

Title: Complete Stabilization of Polycubes

Assigned To: Transition Projects/Plutonium Finishing Plant

Program WBS Designator: 7.1 .3 (1K6)

Control Number: TRP-01-401

\begin{tabular}{|c||}
\hline MILESTONE \\
TYPE: \\
X DOE-HQ \\
$\square$ DOE-RL \\
$\square$ CONTRACTOR \\
$\square$ TPA \\
\hline
\end{tabular}

\begin{tabular}{l} 
DIVISION \\
$\square$ State \\
$\square$ Federal \\
X DOE \\
$\square$ RCRA \\
$\square$ TPA Number \\
\hline
\end{tabular}

DELIVERABLE:

$\square$ Report

$X$ Letter

$\square$ Drawings

$\square$ Other

(specify)

Date: 09/20/95

CIN:

Due Date:

$01 / 31 / 2001$

Rev: Original

Milestone Description:

Complete the stabilization of 2,700 pieces of polycubes, either at the PFP facility or at another authorized facility, using appropriately approved technology.

Description of what constitutes completion of this milestone:

The PFP Transition Project has completed the stabilization of 2,700 pieces of polycubes, using appropriate approved technology. Or PFP/WHC has shipped the 2,700 pieces of PFP polycubes to another authorized Facility by this date.

Completion is to be documented in the DNFSB Recommendation 94-1 Monthly Status Report to DOE-HQ.

In addition to documenting completion of this milestone in the Monthly Status Report, a letter will also be issued from WHC to RL documenting completion of this milestone per this description.

\begin{tabular}{|ll|ll|}
\hline $\begin{array}{l}\text { Cost Account Manager } \\
\text { T. E. Huber }\end{array}$ & Date & $\begin{array}{l}\text { Program/Project Manager } \\
\text { E. C. Vogt }\end{array}$ & Date \\
\hline $\begin{array}{ll}\text { Program Element Manager } \\
\text { D. T. Mildon }\end{array}$ & Date & DOE Monitor & Date \\
\hline
\end{tabular}


WHC-SP-1126, Rev. I

\section{TRANSITION PROJECTS}

FY 1996 MYPP

1.3.1/7.1/6.12

Westinghouse Hanford Company

MILESTONE DESCRIPTION SHEET

Title: Complete Annual FSAR Update

Date: $09 / 20 / 95$

Assigned To: Transition Projects/Plutonium Finishing Plant

CIN:

Program WBS Designator: 7.1.3 (1K6)

Due Date:

9/30/2001

Control Number: TRP-01-402 TYPE:

MILESTONE

DOE-HQ

X DOE-RL CONTRACTOR TPA

\section{DIVISION}

State

Federal

$\mathrm{X}$ DOE

$\square$ RCRA

TPA Number
DELIVERABLE:

$X$ Report

$\square$ Letter

$\square$ Drawings

$\square$ Other

(specify)

Rev: Original

ADDRESS TO:

$\square$ DOE-HQ

X DOE-RL

Other

(specify)

Milestone Description:

Issue the annual update of the PFP Safety Analysis Report in accordance with DOE Order 5480.23.

Description of what constitutes completion of this milestone:

Issue updated SAR to DOE-RL for approval after securing approvals of required WHC SAR approval committees. "Contractors shall be required to review and update as necessary, SARs annually, pursuant to this Order to ensure that the information in each SAR is current and remains applicable. Revisions shall be submitted to the PSO at least annually and shall reflect all changes implemented up to 6 months prior to the filing of the updated SAR. The DOE approval of any USQ pursuant to DOE 5480.21, amendments to the TSRs, and the material submitted by the contractor to the PSO in support of these approvals shall be considered an addendum to the SAR until the information is incorporated into the SAR as part of the next annual update.

A separate letter, from the FSAR update letter, will be issued by WHC to RL documenting completion of this specific annual FSAR update.

\begin{tabular}{|ll|ll||}
\hline \hline $\begin{array}{l}\text { Cost Account Manager } \\
\text { C. M. Kronvall }\end{array}$ & Date & $\begin{array}{l}\text { Program/Project Manager } \\
\text { E. C. Vogt }\end{array}$ & Date \\
\hline $\begin{array}{l}\text { Program Element Manager } \\
\text { D. T. Mildon }\end{array}$ & Date & $\begin{array}{l}\text { DOE Monitor } \\
\text { D. W. Templeton }\end{array}$ & Date \\
\hline
\end{tabular}


FY 1996 MYPP

\section{TRANSITION PROJECTS}

\subsection{1/7.1/6.12}

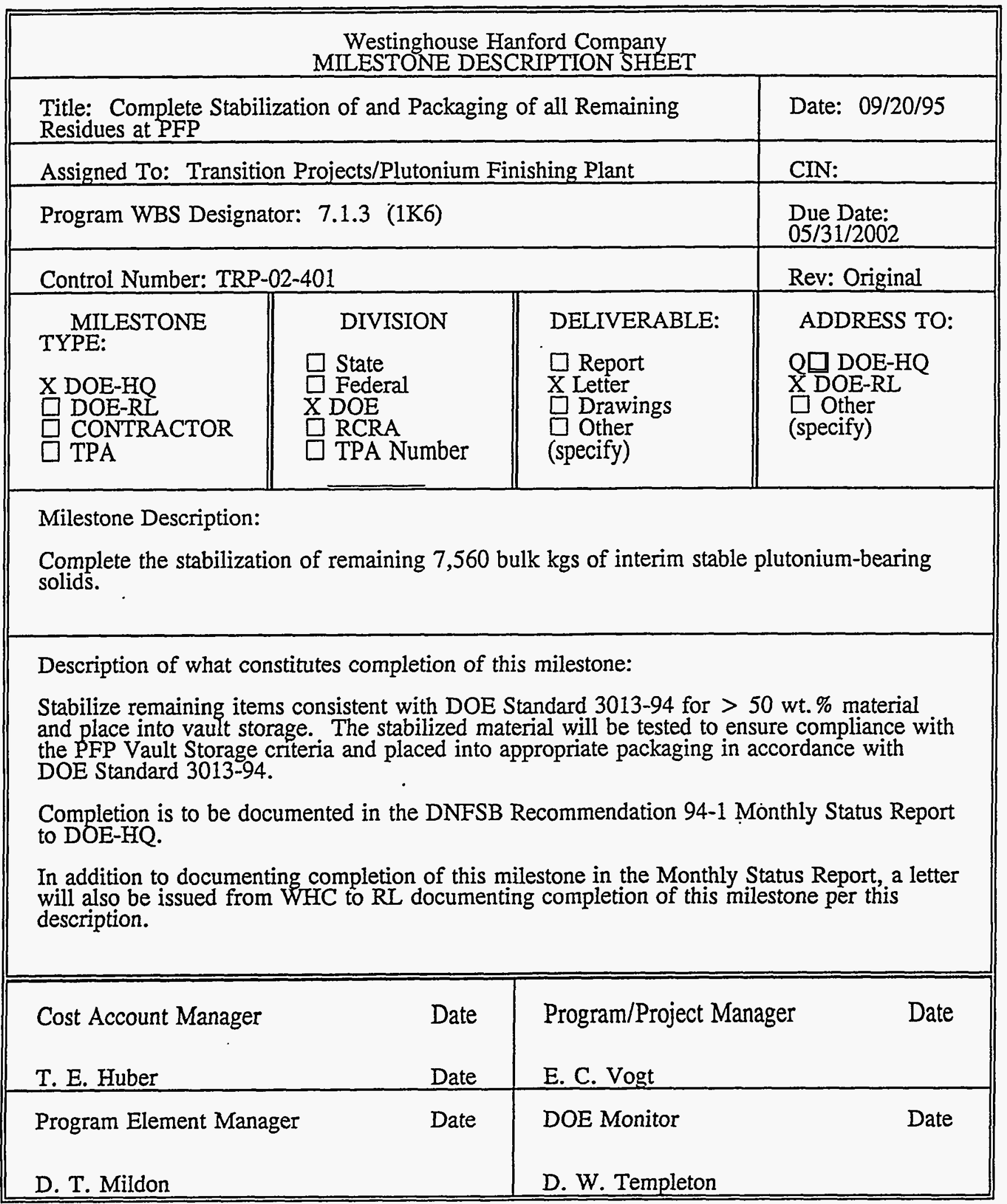


WHC-SP-1126, Rev. 1

\section{TRANSITION PROJECTS}

FY 1996 MYPP

1.3.1/7.1/6.12

Westinghouse Hanford Company MILESTONE DESCRIPTION SHEET

Title: Complete Thermal Stabilization/Repackaging of all $\mathrm{PuO}_{2}$ to meet Metal/Oxide Storage Standard at PFP

Assigned To: Transition Projects/Plutonium Finishing Plant

CIN:

Program WBS Designator: 7.1 .3 (1K6)

Due Date:

$05 / 31 / 2002$

Control Number: TRP-02-402

Rev: Original TYPE:

MILESTONE

$\mathrm{X}$ DOE-HQ

$\square$ DOE-RL

$\square$ CONTRACTOR TPA

\begin{tabular}{|c|}
\hline DIVISION \\
\hline $\begin{array}{l}\square \text { State } \\
\square \text { Federal } \\
\text { DOE } \\
\square \text { RCRA } \\
\square \text { TPA Number }\end{array}$ \\
\hline
\end{tabular}

DELIVERABLE:

$\square$ Report

$X$ Letter

$\square$ Drawings

$\square$ Other

(specify)

Date: $09 / 20 / 95$

Milestone Description:

Complete the stabilization of remaining High Assay plutonium metal/oxides.

Description of what constitutes completion of this milestone:

Stabilize remaining items consistent with DOE Standard 3013-94 for $>50 \mathrm{wt}$.\% material and place into vault storage. The stabilized material will be tested to ensure compliance with the PFP Vault Storage criteria and placed into appropriate packaging in accordance with DOE Standard 3013-94.

Completion is to be documented in the DNFSB Recommendation 94-1 Monthly Status Report to DOE-HQ.

In addition to documenting completion of this milestone in the Monthly Status Report, a letter will also be issued from WHC to RL documenting completion of this milestone per this description.

Cost Account Manager

T. E. Huber

Program Element Manager

D. T. Mildon

\section{Date}

Program/Project Manager

Date

E. C. Vogt

Date

DOE Monitor

Date

D. W. Templeton 
WHC-SP-1126, Rev. I

\section{TRANSITION PROJECTS}

FY 1996 MYPP

1.3.1/7.1/6.12

\begin{tabular}{|c|c|c|c|}
\hline \multicolumn{4}{|c|}{$\begin{array}{l}\text { Westinghouse Hanford Company } \\
\text { MILESTONE DESCRIPTION SHEET }\end{array}$} \\
\hline \multicolumn{3}{|c|}{ Title: Complete Annual FSAR Update } & Date: 09/20/95 \\
\hline \multicolumn{3}{|c|}{ Assigned To: Transition Projects/Plutonium Finishing Plant } & CIN: \\
\hline \multicolumn{3}{|c|}{ Program WBS Designator: 7.1 .3 (1K6) } & $\begin{array}{l}\text { Due Date: } \\
9 / 30 / 2002\end{array}$ \\
\hline \multicolumn{3}{|c|}{ Control Number: TRP-02-403 } & Rev: Original \\
\hline $\begin{array}{l}\text { MILESTONE } \\
\text { TYPE: } \\
\square \text { DOE-HQ } \\
\text { X DOE-RL } \\
\square \text { CONTRACTOR } \\
\square \text { TPA }\end{array}$ & $\begin{array}{l}\text { DIVISION } \\
\square \text { State } \\
\square \text { Federal } \\
\text { X DOE } \\
\square \text { RCRA } \\
\square \text { TPA Number }\end{array}$ & $\begin{array}{l}\text { DELIVERABLE: } \\
\text { X Report } \\
\square \text { Letter } \\
\square \text { Drawings } \\
\square \text { Other } \\
\text { (specify) }\end{array}$ & $\begin{array}{l}\text { ADDRESS TO: } \\
\square \text { DOE-HQ } \\
\text { X DOE-RL } \\
\square \text { Other } \\
\text { (specify) }\end{array}$ \\
\hline \multicolumn{4}{|c|}{$\begin{array}{l}\text { Milestone Description: } \\
\text { Issue the annual update of the PFP Safety Analysis Report in accordance with DOE Order } \\
5480.23 \text {. }\end{array}$} \\
\hline \multicolumn{4}{|c|}{ Description of what constitutes completion of this milestone: } \\
\hline \multicolumn{4}{|c|}{$\begin{array}{l}\text { Issue updated SAR to DOE-RL for approval after securing approvals of required WHC SAR } \\
\text { approval committees. "Contractors shall be required to review and update as necessary, SARs } \\
\text { annually, pursuant to this Order to ensure that the information in each SAR is current and } \\
\text { remains applicable. Revisions shall be submitted to the PSO at least annually and shall reflect } \\
\text { all changes implemented up to } 6 \text { months prior to the filing of the updated SAR. The DOE } \\
\text { approval of any USQ pursuant to DOE } 5480.21 \text {, amendments to the TSRs, and the material } \\
\text { submitted by the contractor to the PSO in support of these approvals shall be considered an } \\
\text { addendum to the SAR until the information is incorporated into the SAR as part of the next } \\
\text { annual update. }\end{array}$} \\
\hline \multicolumn{4}{|c|}{$\begin{array}{l}\text { A separate letter, from the FSAR update letter, will be issued by WHC to RL documenting } \\
\text { completion of this specific annual FSAR update. }\end{array}$} \\
\hline \multicolumn{2}{|c|}{$\begin{array}{l}\text { Cost Account Manager } \\
\text { C.M. Kronvall }\end{array}$} & \multicolumn{2}{|c|}{$\begin{array}{l}\text { Program/Project Manager } \\
\text { E. C. Vogt }\end{array}$} \\
\hline \multicolumn{2}{|c|}{$\begin{array}{l}\text { Program Element Manager } \\
\text { D. T. Mildon }\end{array}$} & $\begin{array}{l}\text { DOE Monitor } \\
\text { D. W. Templeton }\end{array}$ & Date \\
\hline
\end{tabular}


WHC-SP-1126, Rev. 1

FY 1996 MYPP

TRANSITION PROJECTS

1.3.1/7.1/6.12

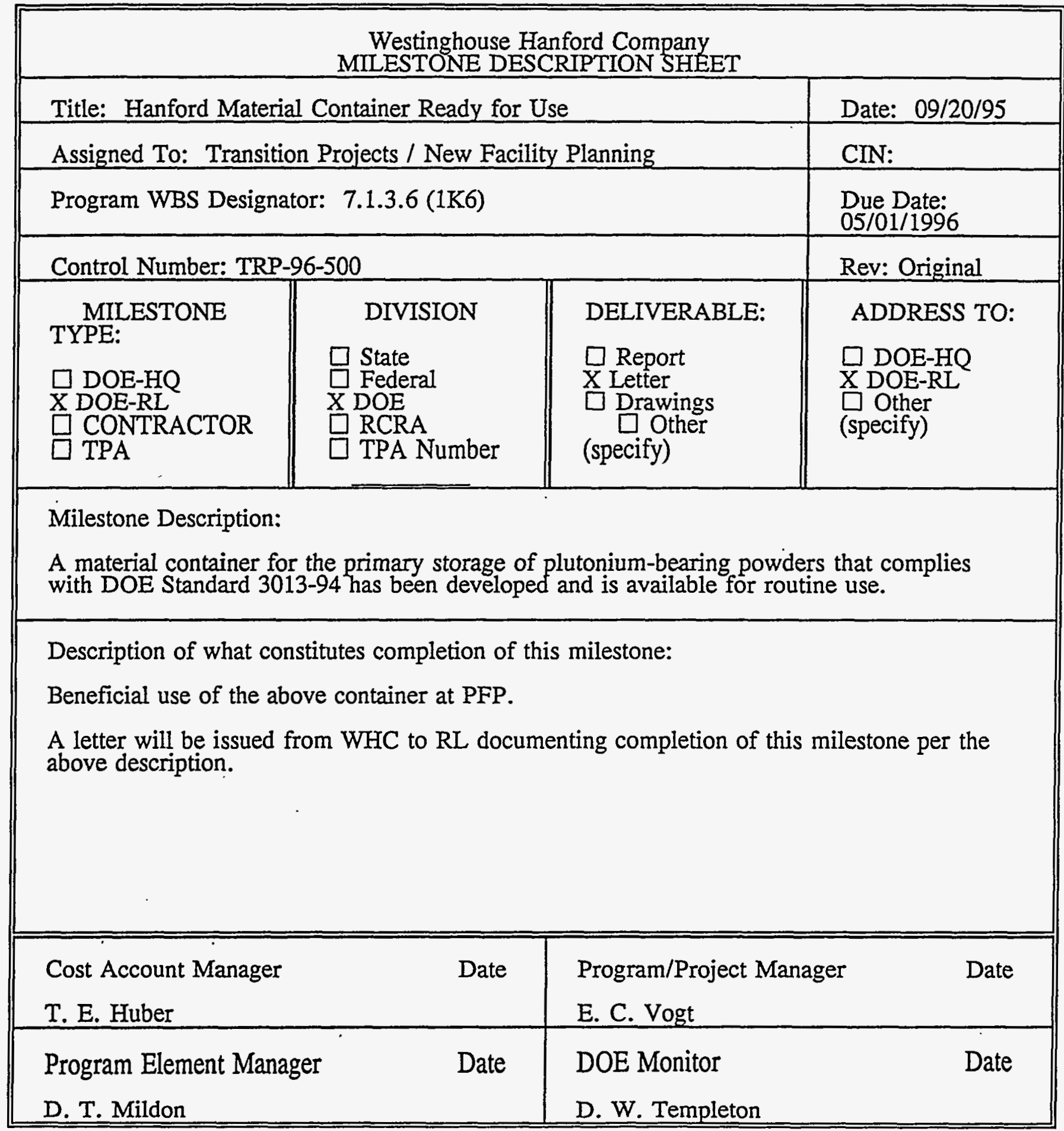


WHC-SP-1126, Rev. 1

FY 1996 MYPP

\section{TRANSITION PROJECTS}

1.3.1/7.1/6.12

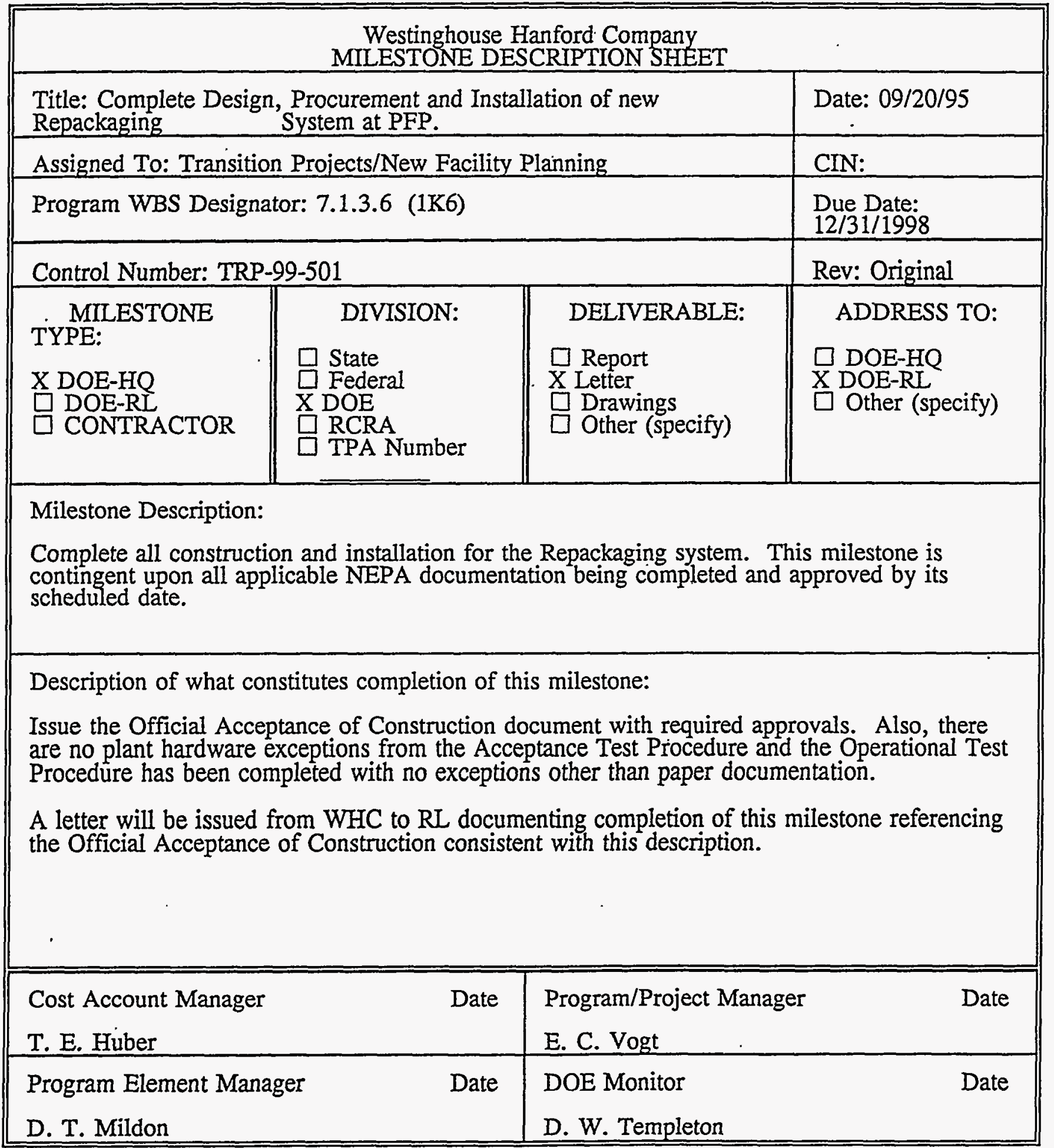


WHC-SP-1126, Rev. 1

FY 1996 MYPP

TRANSITION PROJECTS

1.3.1/7.1/6.12

\section{Westinghouse Hanford Company \\ MILESTONE DESCRIPTION SHEET}

\begin{tabular}{|c|c|c|c|}
\hline \multicolumn{3}{|c|}{$\begin{array}{l}\text { Title: Perform Operational Readiness Testing of PFP Repackaging } \\
\text { System }\end{array}$} & Date: 09/20/95 \\
\hline \multicolumn{3}{|c|}{ Assigned To: Transition Projects/New Facility Planning } & CIN: \\
\hline \multicolumn{3}{|c|}{ Program WBS Designator: 7.1.3.6 (1K6) } & $\begin{array}{l}\text { Due Date: } \\
\text { 9/30/1999 }\end{array}$ \\
\hline \multicolumn{3}{|c|}{ Control Number: TRP-99-502 } & Rev: Original \\
\hline $\begin{array}{l}\text { MILESTONE } \\
\text { TYPE: } \\
\text { X DOE-HQ } \\
\square \text { DOE-RL } \\
\square \text { CONTRACTOR }\end{array}$ & $\begin{array}{l}\text { DIVISION: } \\
\square \text { State } \\
\square \text { Federal } \\
\text { X DOE } \\
\square \text { RCRA } \\
\square \text { TPA Number }\end{array}$ & $\begin{array}{l}\text { DELIVERABLE: } \\
\square \text { Report } \\
\text { X Letter } \\
\square \text { Drawings } \\
\square \text { Other (specify) }\end{array}$ & $\begin{array}{l}\text { ADDRESS TO: } \\
\square \text { DOE-HQ } \\
\text { X DOE-RL } \\
\square \text { Other (specify) }\end{array}$ \\
\hline
\end{tabular}

Milestone Description:

Complete the operational readiness testing of the PFP Repackaging system.

Description of what constitutes completion of this milestone:

Perform all systems and operations checks on the PFP Repackaging system and fix, correct, or modify all deficiencies found. Issue document establishing the basis for certifying that all systems and operations function per the design requirements.

A letter will be issued from WHC to RL documenting completion of this milestone referencing the Official Acceptance of Construction consistent with this description.

\begin{tabular}{|ll|ll||}
\hline \hline Cost Account Manager & Date & Program/Project Manager & Date \\
T. E. Huber & & E. C. Vogt & Date \\
\hline Program Element Manager & Date & DOE Monitor & \\
D. T. Mildon & & D. W. Templeton & \\
\hline
\end{tabular}


WHC-SP-1126, Rev. 1

\section{TRANSITION PROJECTS}

FY 1996 MYPP

1.3.1/7.1/6.12

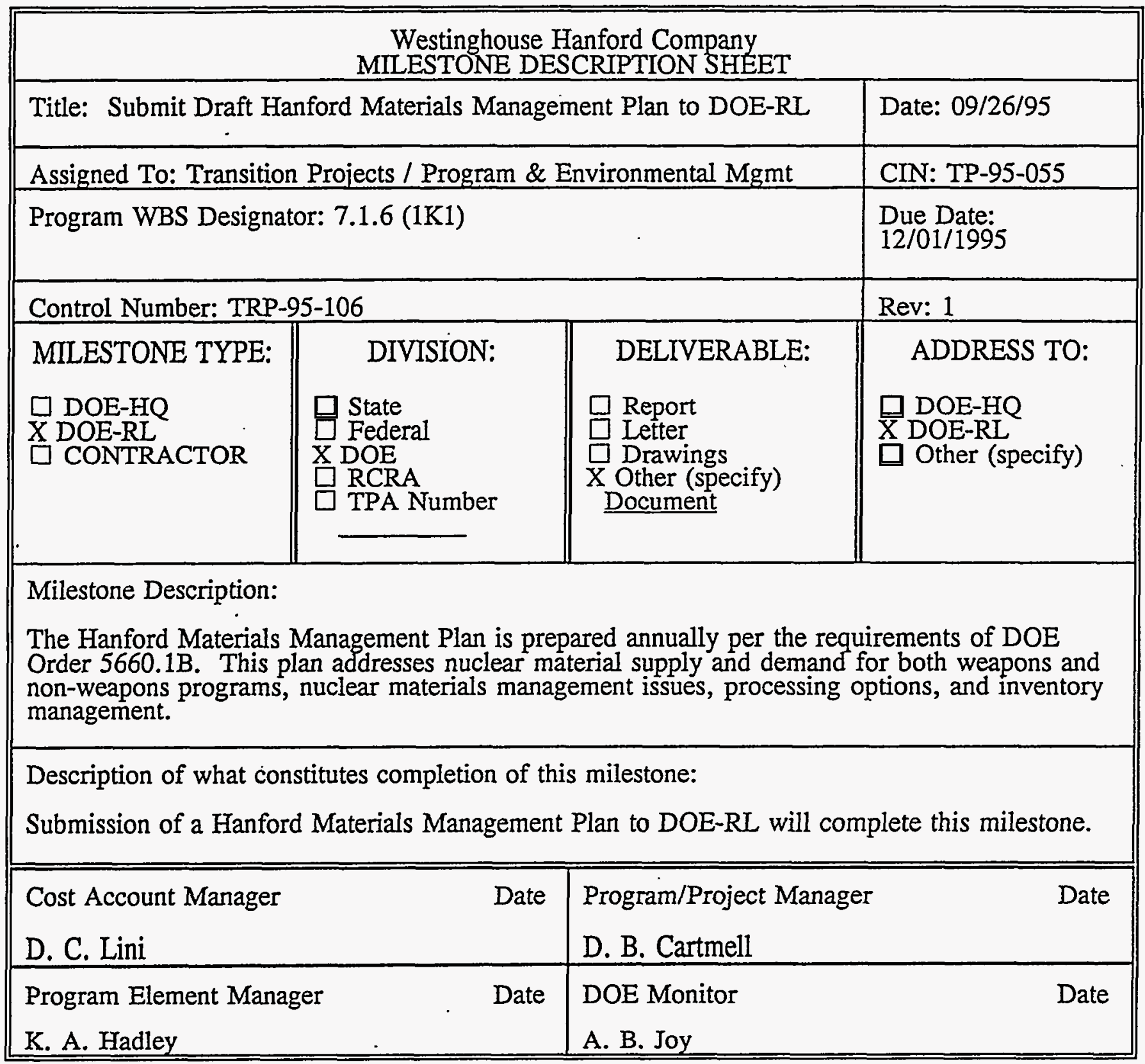


WHC-SP-1126, Rev. 1

\title{
TRANSITION PROJECTS
}

FY 1996 MYPP

\author{
1.3.1/7.1/6.12
}

\begin{tabular}{|c|c|c|c|}
\hline \multicolumn{4}{|c|}{$\begin{array}{l}\text { Westinghouse Hanford Company } \\
\text { MILESTONE DESCRIPTION SHEET }\end{array}$} \\
\hline \multicolumn{3}{|c|}{ Title: Submit Allotment Status Report to DOE-RL } & Date: $09 / 26 / 95$ \\
\hline \multicolumn{3}{|c|}{ Assigned To: Transition Projects / Program \& Environmental Mgmt } & CIN: TP-95-057 \\
\hline \multicolumn{3}{|c|}{ Program WBS Designator: 7.1.6 (1K1) } & $\begin{array}{l}\text { Due Date: } \\
\text { 11/01/1995 }\end{array}$ \\
\hline \multicolumn{3}{|c|}{ Control Number: TRP-96-101 } & Rev: 1 \\
\hline $\begin{array}{l}\text { MILESTONE TYPE: } \\
\square \text { DOE-HQ } \\
\text { X DOE-RL } \\
\square \text { CONTRACTOR }\end{array}$ & $\begin{aligned} & \text { DIVISION: } \\
& \text { State } \\
& \square \text { Federal } \\
& \text { X DOE } \\
& \square \text { RCRA } \\
& \square \text { TPA Number }\end{aligned}$ & $\begin{array}{l}\text { DELIVERABLE: } \\
\text { X Report } \\
\text { Letter } \\
\text { D Drawings } \\
\square \text { Other (specify) }\end{array}$ & $\begin{array}{l}\text { ADDRESS TO: } \\
\square \text { DOE-HQ } \\
\text { X DOE-RL } \\
\square \text { Other (specify) }\end{array}$ \\
\hline \multicolumn{4}{|c|}{$\begin{array}{l}\text { Milestone Description: } \\
\text { The Hanford Allotment Status Report is prepared annually per the requirements of DOE Order } \\
5660.1 \mathrm{~B} \text {. The delivery date of November } 01 \text { allows DOE-RL sufficient time for resubmission of } \\
\text { the Report by December } 1 \text {. }\end{array}$} \\
\hline \multicolumn{4}{|c|}{$\begin{array}{l}\text { Description of what constitutes completion of this milestone: } \\
\text { Submission of the Allotment Report to DOE-RL will complete this milestone. }\end{array}$} \\
\hline \multicolumn{2}{|l|}{$\begin{array}{l}\text { Cost Account Manager } \\
\text { D. C. Lini }\end{array}$} & \multicolumn{2}{|c|}{$\begin{array}{l}\text { Program/Project Manager . Date } \\
\text { D. B. Cartmell }\end{array}$} \\
\hline \multicolumn{2}{|c|}{$\begin{array}{l}\text { Program Element Manager } \\
\text { K. A. Hadley }\end{array}$} & \multicolumn{2}{|c|}{$\begin{array}{l}\text { DOE Monitor } \\
\text { A. B. Joy }\end{array}$} \\
\hline
\end{tabular}


WHC-SP-1126, Rev. I

\section{TRANSITION PROJECTS}

FY 1996 MYPP

\subsection{1/7.1/6.12}

Westinghouse Hanford Company MILESTONE DESCRIPTION SHEET

\begin{tabular}{|c|c|c|c|}
\hline \multicolumn{3}{|c|}{ Title: Submit Inventory Assessment Report to DOE-RL } & Date: $09 / 26 / 95$ \\
\hline \multicolumn{3}{|c|}{ Assigned To: Transition Projects / Program \& Environmental Mgmt } & CIN: TP-95-057 \\
\hline \multicolumn{3}{|c|}{ Program WBS Designator: 7.1.6 (1K1) } & $\begin{array}{l}\text { Due Date: } \\
12 / 15 / 1995\end{array}$ \\
\hline \multicolumn{3}{|c|}{ Control Number: TRP-96-102 } & Rev: 1 \\
\hline MILESTONE TYPE: & DIVISION: & DELIVERABLE: & ADDRESS TO: \\
\hline $\begin{array}{l}\square \text { DOE-HQ } \\
X \text { DOE-RL } \\
\square \text { CONTRACTOR }\end{array}$ & $\begin{array}{l}\square \text { State } \\
\square \text { Federal } \\
\text { X DOE } \\
\square \text { RCRA } \\
\square \text { TPA Number }\end{array}$ & $\begin{array}{l}\text { X Report } \\
\square \text { Letter } \\
\square \text { Drawings } \\
\square \text { Other (specify) }\end{array}$ & $\begin{array}{l}\square \text { DOE-HQ } \\
\text { X DOE-RL } \\
\square \text { Other (specify) }\end{array}$ \\
\hline
\end{tabular}

Milestone Description:

The Hanford Nuclear Materials Inventory Assessment Report is prepared annually per the requirements of DOE Order 5660.1B. The delivery date of December 15 allows DOE-RL sufficient time for submission of the report to DOE-HQ by January 15 .

Description of what constitutes completion of this milestone:

Submission of the Inventory Assessment Report to DOE-RL will complete this milestone.

\begin{tabular}{|ll|lr|}
\hline \hline Cost Account Manager & Date & $\begin{array}{l}\text { Program/Project Manager } \\
\text { D. B. Cartmell }\end{array}$ & Date \\
D. C. Lini & Date & $\begin{array}{l}\text { DOE Monitor } \\
\text { Program Element Manager }\end{array}$ & Date \\
K. A. Hadley & & \\
\hline
\end{tabular}


WHC-SP-1126, Rev. I

FY 1996 MYPP

TRANSITION PROJECTS

1.3.1/7.1/6.12

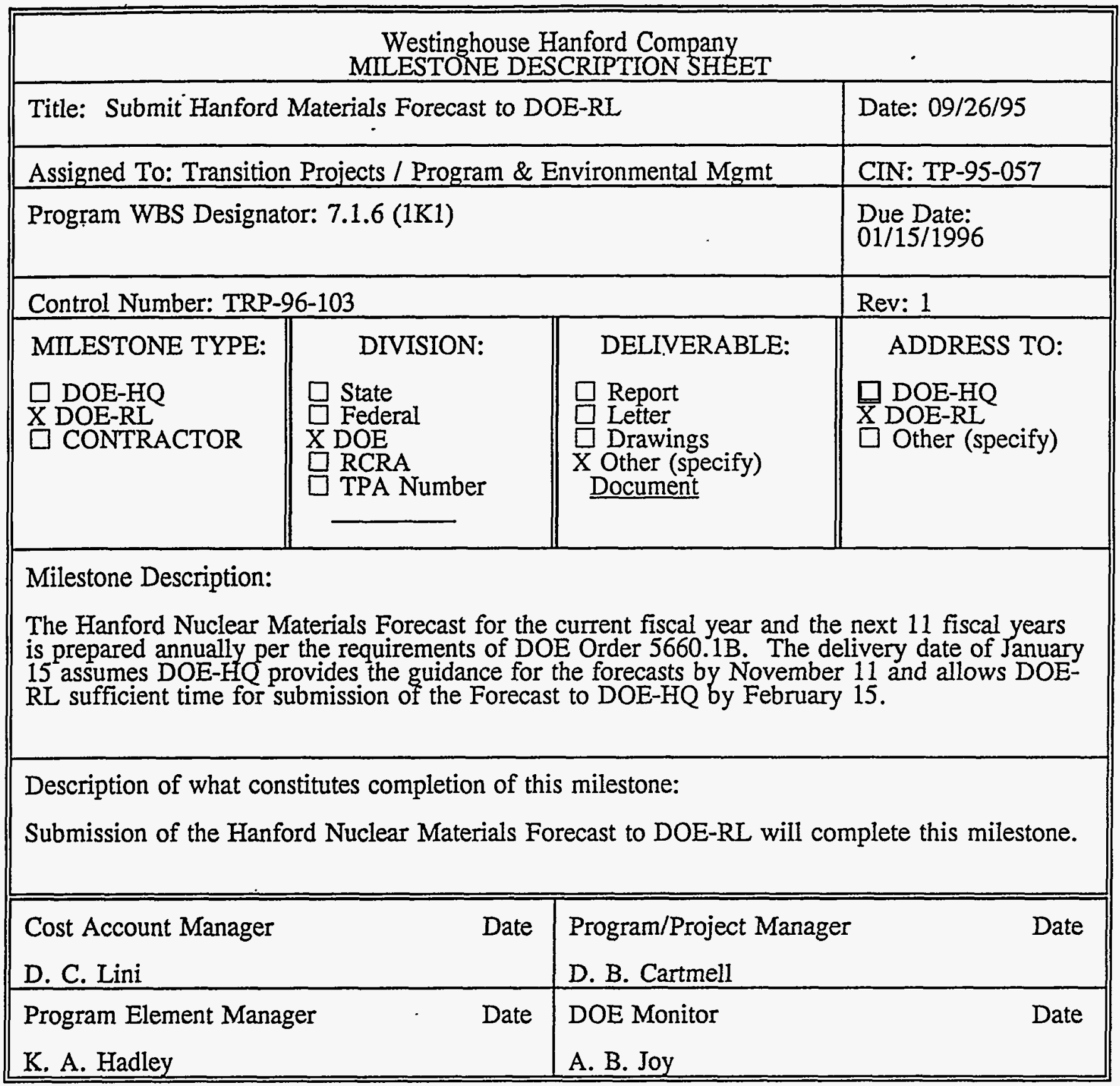


FY 1996 MYPP

\section{TRANSITION PROJECTS}

1.3.1/7.1/6.12

Westinghouse Hanford Company MILESTONE DESCRIPTION SHEET

Title: Submit FY 1998 Activity Data Sheets to DOE-RL

Date: $09 / 23 / 94$

Assigned To: Transition Projects / Program \& Environmental Mgmt

Program WBS Designator: 7.1.6 (1K1)

Control Number: TRP-96-104

MILESTONE TYPE:

DOE-HQ

DOE-RI

$X$ CONTRACTOR

\begin{tabular}{|c||} 
DIVISION: \\
$\square$ State \\
$\square$ Federal \\
X DOE \\
$\square$ RCRA \\
$\square$ TPA Number \\
\hline
\end{tabular}

DELIVERABLE:

Rev: Original

Due Date:

04/30/1996

$\square$ Report

$\square$ Letter

Drawings

$\mathrm{X}$ Other (specify)

Document

Milestone Description:

Submit FY 1998 Activity Data Sheets in support of the Five Year Plan to DOE-RL.

Description of what constitutes completion of this milestone:

Submission of the FY 1998 Activity Data Sheets in support of the Five Year Plan to DOE-RL will complete this milestone.

\begin{tabular}{|ll|lc|}
\hline \hline Cost Account Manager & Date & Program/Project Manager & Date \\
J. O. Lundeen & & D. B. Cartmell &. \\
\hline Program Element Manager & Date & DOE Monitor & Date \\
K. A. Hadley & & J. E. Mecca & \\
\hline
\end{tabular}


WHC-SP-1126, Rev. 1

FY 1996 MYPP

\section{TRANSITION PROJECTS}

1.3.1/7.1/6.12

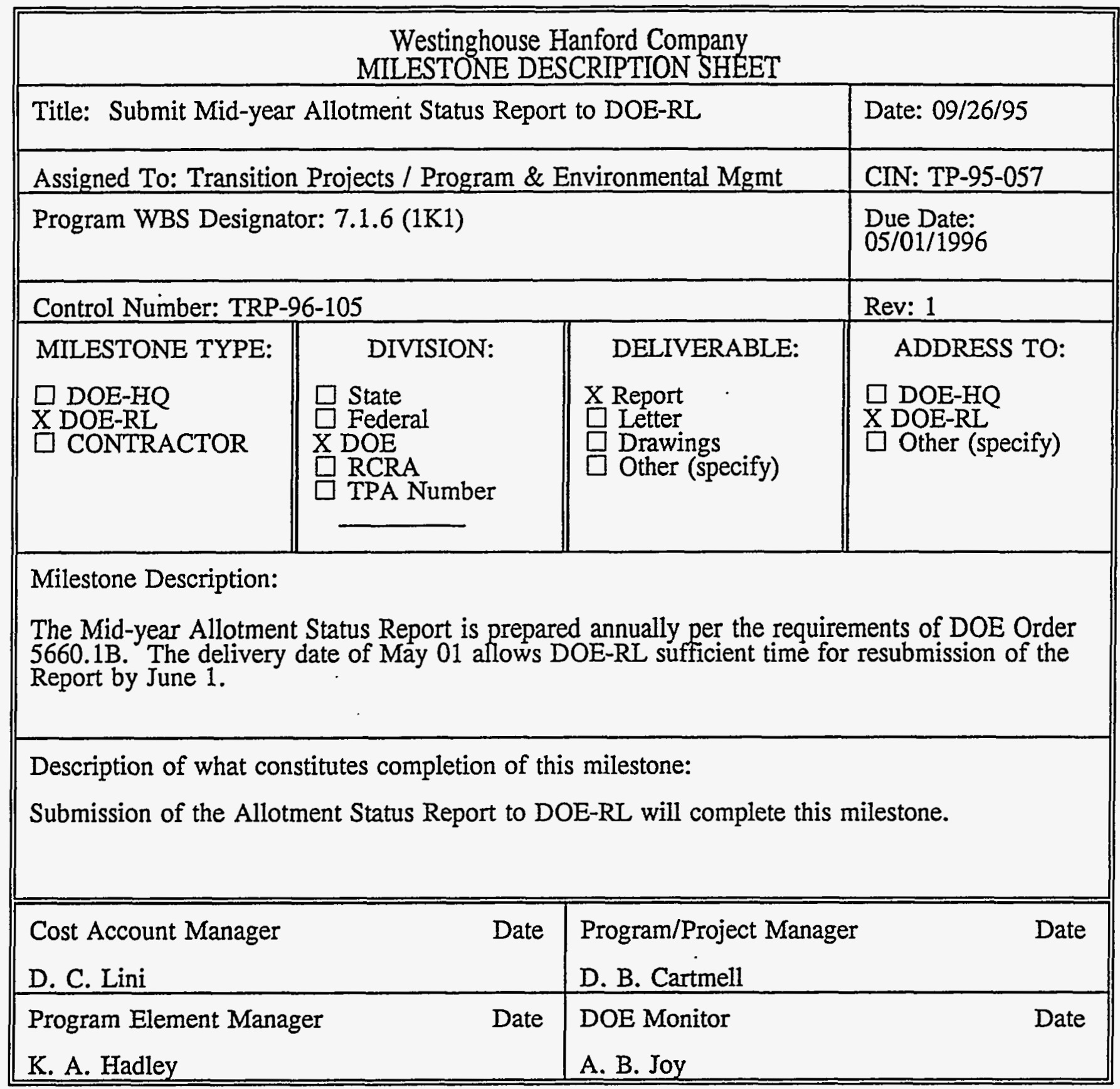


WHC-SP-1126, Rev. I

FY 1996 MYPP

\section{TRANSITION PROJECTS}

1.3.1/7.1/6.12

Westinghouse Hanford Company

MILESTONE DESCRIPTION SHEET

\begin{tabular}{|c|c|c|c|}
\hline \multicolumn{3}{|c|}{ Title: Submit Draft Hanford Materials Management Plan to DOE-RL } & Date: 09/26/95 \\
\hline \multicolumn{3}{|c|}{ Assigned To: Transition Projects / Program \& Environmental Mgmt } & CIN: TP-95-057 \\
\hline \multicolumn{3}{|c|}{ Program WBS Designator: 7.1.6 (1K1) } & $\begin{array}{l}\text { Due Date: } \\
09 / 15 / 1996\end{array}$ \\
\hline \multicolumn{3}{|c|}{ Control Number: TRP-96-106 } & Rev: 1 \\
\hline MILESTONE TYPE: & DIVISION: & DELIVERABLE: & ADDRESS TO: \\
\hline $\begin{array}{l}\square \text { DOE-HQ } \\
\text { X DOE-RL } \\
\square \text { CONTRACTOR }\end{array}$ & $\begin{array}{l}\square \text { State } \\
\square \text { Federal } \\
\text { X DOE } \\
\square \text { RCRA } \\
\square \text { TPA Number }\end{array}$ & $\begin{array}{l}\square \text { Report } \\
\text { Letter } \\
\text { Drawings } \\
\text { X Other (specify) } \\
\text { Document }\end{array}$ & $\begin{array}{l}\square \text { DOE-HQ } \\
\text { X DOE-RL } \\
\square \text { Other (specify) }\end{array}$ \\
\hline
\end{tabular}

Milestone Description:

The Hanford Materials Management Plan is prepared annually per the requirements of DOE Order 5660.1B. This plan addresses nuclear material supply and demand for both weapons and non-weapons programs, nuclear materials management issues, processing options, and inventory management. The delivery date will be approximately 8 weeks after DOE-HQ provides planning assumptions. For planning purposes it is assumed that DOE-HQ guidance will be provided no later than July 15, 1996. Changes to this assumption will be documented through change control.

Description of what constitutes completion of this milestone:

Submission of a Hanford Materials Management Plan to DOE-RL will complete this milestone.

\begin{tabular}{|c|c|c|c|}
\hline $\begin{array}{l}\text { Cost Account Manager } \\
\text { D. C. Lini }\end{array}$ & Date & $\begin{array}{l}\text { Program/Project Manager } \\
\text { D. B. Cartmell }\end{array}$ & Date \\
\hline $\begin{array}{l}\text { Program Element Manager } \\
\text { K. A. Hadley }\end{array}$ & Date & $\begin{array}{l}\text { DOE Monitor } \\
\text { A. B. Joy }\end{array}$ & Date \\
\hline
\end{tabular}


WHC-SP-1126, Rev. 1

FY 1996 MYPP

TRANSITION PROJECTS

1.3.1/7.1/6.12

\begin{tabular}{|c|c|c|c|}
\hline \multicolumn{4}{|c|}{$\begin{array}{l}\text { Westinghouse Hanford Company } \\
\text { MILESTONE DESCRIPTION SHEET }\end{array}$} \\
\hline \multicolumn{3}{|c|}{ Title: Submit FY 1997 MYPP to RL } & Date: 09/23/94 \\
\hline \multicolumn{3}{|c|}{ Assigned To: Transition Projects / Program \& Environmental Mgmt } & CIN: \\
\hline \multicolumn{3}{|c|}{ Program WBS Designator: 7.1.6 (1K1) } & $\begin{array}{l}\text { Due Date: } \\
08 / 31 / 1996\end{array}$ \\
\hline \multicolumn{3}{|c|}{ Control Number: TRP-96-107 } & Rev: Original \\
\hline $\begin{array}{l}\text { MILESTONE TYPE: } \\
\text { D DOE-HQ } \\
\text { X DOE-RL } \\
\text { CONTRACTOR }\end{array}$ & $\begin{aligned} & \text { DIVISION: } \\
& \square \text { State } \\
& \square \text { Federal } \\
& \text { X DOE } \\
& \square \text { RCRA } \\
& \square \text { TPA Number }\end{aligned}$ & $\begin{array}{l}\text { DELIVERABLE: } \\
\square \text { Report } \\
\square \text { Letter } \\
\square \text { Drawings } \\
\text { X Other (specify) } \\
\text { Document }\end{array}$ & $\begin{array}{l}\text { ADDRESS TO: } \\
\begin{array}{l}\text { DOE-HQ } \\
\text { D DOE-RL } \\
\square \text { Other (specify) }\end{array}\end{array}$ \\
\hline \multicolumn{4}{|c|}{$\begin{array}{l}\text { Milestone Description: } \\
\text { Submit combined FY } 1997 \text { Fiscal Year Work Plan/Multi-Year Program Plan to DOE-RL. }\end{array}$} \\
\hline \multicolumn{4}{|c|}{$\begin{array}{l}\text { Description of what constitutes completion of this milestone: } \\
\text { Submission of the FY } 1997 \text { Fiscal Year Work Plan/Multi-Year Program Plan to DOE-RL will } \\
\text { complete this milestone. }\end{array}$} \\
\hline \multicolumn{2}{|c|}{$\begin{array}{l}\text { Cost Account Manager } \\
\text { J. O. Lundeen }\end{array}$} & \multicolumn{2}{|c|}{$\begin{array}{l}\text { Program/Project Manager Date } \\
\text { D. B. Cartmell }\end{array}$} \\
\hline \multicolumn{2}{|c|}{$\begin{array}{l}\text { Program Element Manager } \quad \text { Date } \\
\text { K. A. Hadley }\end{array}$} & \multicolumn{2}{|c|}{$\begin{array}{l}\text { DOE Monitor } \\
\text { J. E. Mecca }\end{array}$} \\
\hline
\end{tabular}


WHC-SP-1126, Rev. 1

FY 1996 MYPP

\section{TRANSITION PROJECTS}

1.3.1/7.1/6.12

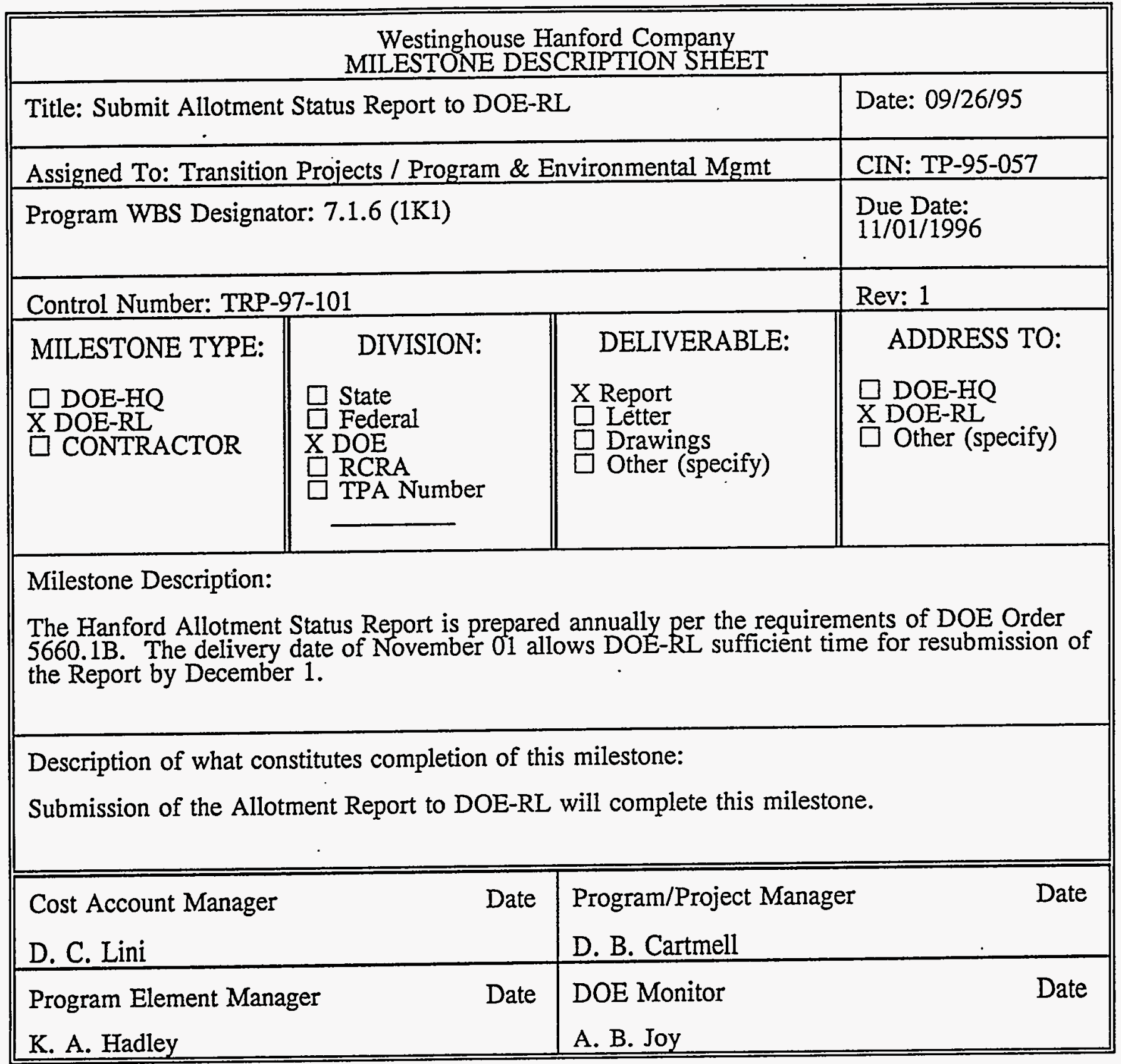


WHC-SP-1126, Rev. 1

FY 1996 MYPP

TRANSITION PROJECTS

1.3.1/7.1/6.12

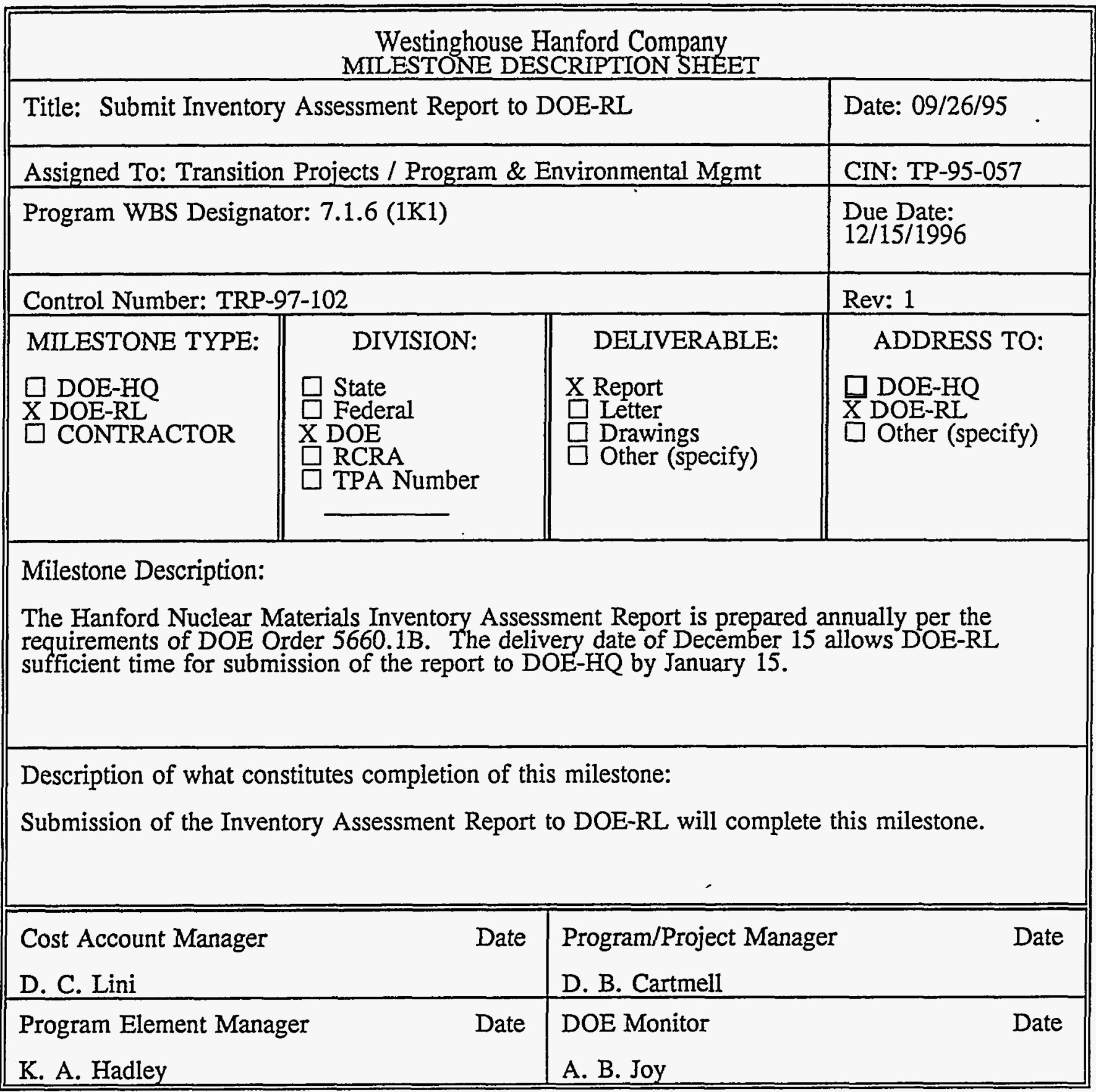


WHC-SP-1126, Rev. 1

TRANSITION PROJECTS

FY 1996 MYPP

1.3.1/7.1/6.12

\begin{tabular}{|c|c|c|c|}
\hline \multicolumn{4}{|c|}{$\begin{array}{l}\text { Westinghouse Hanford Company } \\
\text { MLESTONE DESCRIPTION SHET }\end{array}$} \\
\hline \multicolumn{3}{|c|}{ Title: Submit Hanford Materials Forecast to DOE-RL } & Date: $09 / 26 / 95$ \\
\hline \multicolumn{3}{|c|}{ Assigned To: Transition Projects / Program \& Environmental Mgmt } & CIN: TP-95-057 \\
\hline \multicolumn{3}{|c|}{ Program WBS Designator: $7.1 .6(1 \mathrm{~K} 1)$} & $\begin{array}{l}\text { Due Date: } \\
01 / 15 / 1997\end{array}$ \\
\hline \multicolumn{3}{|c|}{ Control Number: TRP-97-103 } & Rev: 1 \\
\hline MILESTONE TYPE: & DIVISION: & DELIVERABLE: & ADDRESS TO: \\
\hline $\begin{array}{l}\square \text { DOE-HQ } \\
\text { X DOE-RL } \\
\square \text { CONTRACTOR }\end{array}$ & $\begin{array}{l}\square \text { State } \\
\square \text { Federal } \\
\text { DOE } \\
\square \text { RCRA } \\
\square \text { TPA Number }\end{array}$ & $\begin{array}{l}\square \text { Report } \\
\square \text { Letter } \\
\square \text { Drawings } \\
\text { X Other (specify) } \\
\text { Document }\end{array}$ & $\begin{array}{l}\square \text { DOE-HQ } \\
\text { X DOE-RL } \\
\square \text { Other (specify) }\end{array}$ \\
\hline \multirow{2}{*}{\multicolumn{4}{|c|}{$\begin{array}{l}\text { Milestone Description: } \\
\text { The Hanford Nuclear Materials Forecast for the current fiscal year and the next } 11 \text { fiscal years } \\
\text { is prepared annually per the requirements of DOE Order } 5660 \text {. } 1 B \text {. The delivery date of January } \\
15 \text { assumes DOE-HQ provides the guidance for the forecasts by November } 11 \text { and allows DOE- } \\
\text { RL sufficient time for submission of the Forecast to DOE-HQ by February } 15 \text {. }\end{array}$}} \\
\hline & & & \\
\hline \multirow{2}{*}{\multicolumn{4}{|c|}{$\begin{array}{l}\text { Description of what constitutes completion of this milestone: } \\
\text { Submission of the Hanford Nuclear Materials Forecast to DOE-RL will complete this milestone. }\end{array}$}} \\
\hline & & & \\
\hline \multirow{2}{*}{\multicolumn{2}{|c|}{$\begin{array}{l}\text { Cost Account Manager Date } \\
\text { D. C. Lini }\end{array}$}} & \multirow{2}{*}{\multicolumn{2}{|c|}{$\begin{array}{l}\text { Program/Project Manager Date } \\
\text { D. B. Cartmell }\end{array}$}} \\
\hline & & & \\
\hline \multicolumn{2}{|c|}{$\begin{array}{l}\text { Program Element Manager } \\
\text { K. A. Hadley }\end{array}$} & \multicolumn{2}{|c|}{$\begin{array}{l}\text { DOE Monitor } \\
\text { A. B. Joy }\end{array}$} \\
\hline
\end{tabular}


WHC-SP-1126, Rev. 1

FY 1996 MYPP

TRANSITION PROJECTS

1.3.1/7.1/6.12

\begin{tabular}{|c|c|c|c|}
\hline \multicolumn{4}{|c|}{$\begin{array}{l}\text { Westinghouse Hanford Company } \\
\text { MILESTONE DESCRIPTION SHEET }\end{array}$} \\
\hline \multicolumn{3}{|c|}{ Title: Submit FY 1999 Activity Data Sheets to DOE-RL } & Date: $09 / 23 / 94$ \\
\hline \multicolumn{3}{|c|}{ Assigned To: Transition Projects / Program \& Environmental Mgmt } & CIN: \\
\hline \multicolumn{3}{|c|}{ Program WBS Designator: $7.1 .6(1 \mathrm{~K} 1)$} & $\begin{array}{l}\text { Due Date: } \\
04 / 30 / 1997\end{array}$ \\
\hline \multicolumn{3}{|c|}{ Control Number: TRP-97-104 } & Rev: Original \\
\hline $\begin{array}{l}\text { MILESTONE TYPE: } \\
\square \text { DOE-HQ } \\
\square \text { DOE-RL } \\
\text { X CONTRACTOR }\end{array}$ & $\begin{aligned} & \text { DIVISION: } \\
& \text { State } \\
& \text { Federal } \\
& \text { X DOE } \\
& \square \text { RCRA } \\
& \square \text { TPA Number }\end{aligned}$ & $\begin{array}{l}\text { DELIVERABLE: } \\
\square \text { Report } \\
\square \text { Letter } \\
\square \text { Drawings } \\
\text { X Other (specify) } \\
\text { Document }\end{array}$ & $\begin{array}{l}\text { ADDRESS TO: } \\
\square \text { DOE-HQ } \\
\text { X DOE-RL } \\
\square \text { Other (specify) }\end{array}$ \\
\hline \multicolumn{4}{|c|}{$\begin{array}{l}\text { Milestone Description: } \\
\text { Submit FY } 1999 \text { Activity Data Sheets in support of the Five Year Plan to DOE-RL. }\end{array}$} \\
\hline \multicolumn{4}{|c|}{$\begin{array}{l}\text { Description of what constitutes completion of this milestone: } \\
\text { Submission of the FY } 1999 \text { Activity Data Sheets in support of the Five Year Plan to DOE-RL } \\
\text { will complete this milestone. }\end{array}$} \\
\hline \multicolumn{2}{|l|}{$\begin{array}{l}\text { Cost Account Manager } \\
\text { J. O. Lundeen }\end{array}$} & \multicolumn{2}{|c|}{$\begin{array}{l}\text { Program/Project Manager } \\
\text { D. B. Cartmell }\end{array}$} \\
\hline \multicolumn{2}{|c|}{$\begin{array}{l}\text { Program Element Manager } \quad \text { Date } \\
\text { K. A. Hadley }\end{array}$} & \multicolumn{2}{|l|}{$\begin{array}{l}\text { DOE Monitor } \\
\text { J. E. Mecca } \\
\end{array}$} \\
\hline
\end{tabular}


WHC-SP-1126, Rev. I

FY 1996 MYPP

TRANSITION PROJECTS

1.3.1/7.1/6.12

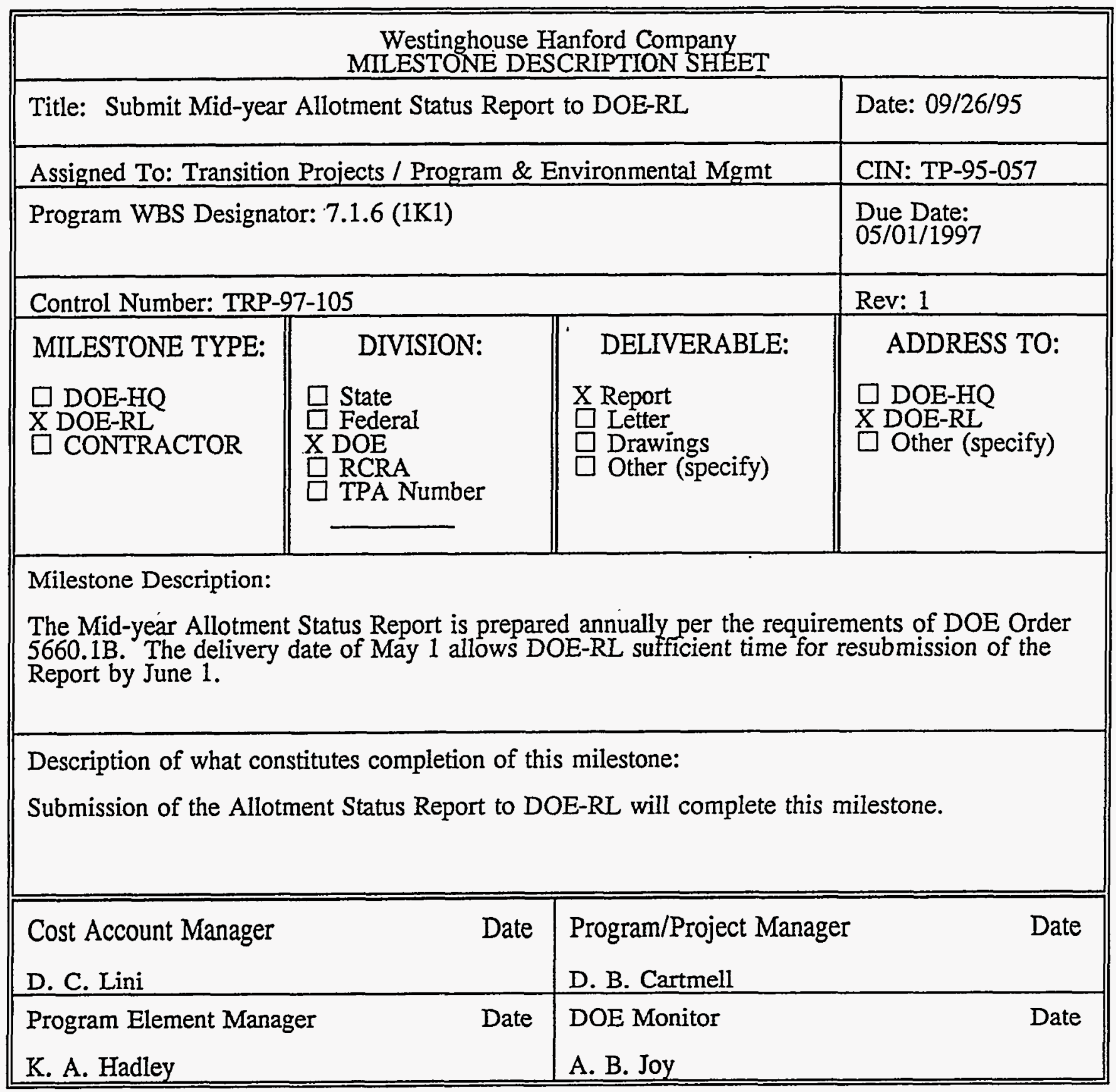


WHC-SP-1126, Rev. 1

FY 1996 MYPP

TRANSITION PROJECTS

1.3.1/7.1/6.12

\begin{tabular}{|c|c|c|c|}
\hline \multicolumn{4}{|c|}{$\begin{array}{l}\text { Westinghouse Hanford Company } \\
\text { MILESTONE DESCRIPTION SHEET }\end{array}$} \\
\hline \multicolumn{3}{|c|}{ Title: Submit Draft Hanford Materials Management Plan to DOE-RL } & Date: $09 / 26 / 95$ \\
\hline \multicolumn{3}{|c|}{ Assigned To: Transition Projects / Program \& Environmental Mgmt } & CIN: TP-95-057 \\
\hline \multicolumn{3}{|c|}{ Program WBS Designator: $7.1 .6(1 \mathrm{~K} 1)$} & $\begin{array}{l}\text { Due Date: } \\
\text { 09/15/1995 }\end{array}$ \\
\hline \multicolumn{3}{|c|}{ Control Number: TRP-97-106 } & Rev: 1 \\
\hline $\begin{array}{l}\text { MILESTONE TYPE: } \\
\square \text { DOE-HQ } \\
\text { X DOE-RL } \\
\square \text { CONTRACTOR }\end{array}$ & $\begin{aligned} & \text { DIVISION: } \\
& \\
& \text { State } \\
& \text { Federal } \\
& \text { X DOE } \\
& \square \text { RCRA } \\
& \square \text { TPA Number }\end{aligned}$ & $\begin{array}{l}\text { DELIVERABLE: } \\
\square \text { Report } \\
\square \text { Letter } \\
\square \text { Drawings } \\
\text { X Other (specify) } \\
\text { Document }\end{array}$ & $\begin{array}{l}\text { ADDRESS TO: } \\
\square \text { DOE-HQ } \\
\text { X DOE-RL } \\
\square \text { Other (specify) }\end{array}$ \\
\hline \multicolumn{4}{|c|}{$\begin{array}{l}\text { Milestone Description: } \\
\text { The Hanford Materials Management Plan is prepared annually per the requirements of DOE } \\
\text { Order } 5660.1 \mathrm{~B} \text {. This plan addresses nuclear material supply and demand for both weapons and } \\
\text { non-weapons programs, nuclear materials management issues, processing options, and inventory } \\
\text { management. The delivery date will be approxmmately } 8 \text { weeks after DOE-HQ provides planning } \\
\text { assumptions. For planning purposes it is assumed that DOE-HQ guidance will be provided no } \\
\text { later than July } 15,1995 \text {. Changes to this assumption will be documented through change } \\
\text { control. }\end{array}$} \\
\hline \multicolumn{4}{|c|}{$\begin{array}{l}\text { Description of what constitutes completion of this milestone: } \\
\text { Submission of a Hanford Materials Management Plan to DOE-RL will complete this milestone. }\end{array}$} \\
\hline \multicolumn{2}{|c|}{$\begin{array}{l}\text { Cost Account Manager } \quad \text { Date } \\
\text { D. C. Lini }\end{array}$} & \multicolumn{2}{|c|}{$\begin{array}{l}\text { Program/Project Manager Date } \\
\text { D. B. Cartmell }\end{array}$} \\
\hline \multicolumn{2}{|c|}{$\begin{array}{l}\text { Program Element Manager } \quad \text { Date } \\
\text { K. A. Hadley }\end{array}$} & \multicolumn{2}{|c|}{$\begin{array}{l}\text { DOE Monitor } \\
\text { A. B. Joy }\end{array}$} \\
\hline
\end{tabular}


WHC-SP-1126, Rev. 1

FY 1996 MYPP

TRANSITION PROJECTS

1.3.1/7.1/6.12

\begin{tabular}{|c|c|c|c|}
\hline \multicolumn{4}{|c|}{$\begin{array}{l}\text { Westinghouse Hanford Company } \\
\text { MILESTONE DESCRIPTION SHEET }\end{array}$} \\
\hline \multicolumn{3}{|c|}{ Title: Submit FY 1998 MYPP to RL } & Date: $09 / 23 / 94$ \\
\hline \multicolumn{3}{|c|}{ Assigned To: Transition Projects / Program \& Environmental Mgmt } & CIN: \\
\hline \multicolumn{3}{|c|}{ Program WBS Designator: 7.1.6 (1K1) } & $\begin{array}{l}\text { Due Date: } \\
\text { 08/31/1997 }\end{array}$ \\
\hline \multicolumn{3}{|c|}{ Control Number: TRP-97-107 } & Rev: Original \\
\hline $\begin{array}{l}\text { MILESTONE TYPE: } \\
\text { 口 DOE-HQ } \\
\square \text { DOE-RL } \\
\text { X CONTRACTOR }\end{array}$ & $\begin{array}{l}\text { DIVISION: } \\
\square \text { State } \\
\square \text { Federal } \\
\text { X DOE } \\
\square \text { RCRA } \\
\square \text { TPA Number. }\end{array}$ & $\begin{array}{l}\text { DELIVERABLE: } \\
\square \text { Report } \\
\square \text { Letter } \\
\square \text { Drawings } \\
\text { X Other (specify) } \\
\text { Document }\end{array}$ & $\begin{array}{l}\text { ADDRESS TO: } \\
\square \text { DOE-HQ } \\
\text { X DOE-RL } \\
\square \text { Other (specify) }\end{array}$ \\
\hline \multicolumn{4}{|c|}{$\begin{array}{l}\text { Milestone Description: } \\
\text { Submit combined FY } 1998 \text { Fiscal Year Work Plan/Multi-Year Program Plan to DOE-RL. }\end{array}$} \\
\hline \multicolumn{4}{|c|}{$\begin{array}{l}\text { Description of what constitutes completion of this milestone: } \\
\text { Submission of the FY } 1998 \text { Fiscal Year Work Plan/Multi-Year Program Plan to DOE-RL will } \\
\text { complete this milestone. }\end{array}$} \\
\hline \multicolumn{2}{|c|}{$\begin{array}{l}\text { Cost Account Manager } \quad \text { Date } \\
\text { J. O. Lundeen }\end{array}$} & \multicolumn{2}{|c|}{$\begin{array}{l}\text { Program/Project Manager Date } \\
\text { D. B. Cartmell }\end{array}$} \\
\hline \multicolumn{2}{|c|}{$\begin{array}{l}\text { Program Element Manager } \\
\text { K. A. Hadley }\end{array}$} & $\begin{array}{l}\text { DOE Monitor. } \\
\text { J. E. Mecca }\end{array}$ & Date \\
\hline
\end{tabular}




\begin{tabular}{|c|c|c|c|}
\hline \multicolumn{4}{|c|}{$\begin{array}{l}\text { Westinghouse Hanford Company } \\
\text { MIEESTONE DESCRIPTION SHEET }\end{array}$} \\
\hline \multicolumn{3}{|c|}{ Title: Submit Allotment Status Report to DOE-RL } & Date: 09/26/95 \\
\hline \multicolumn{3}{|c|}{ Assigned To: Transition Projects / Program \& Environmental Mgmt } & CIN: TP-95-057 \\
\hline \multicolumn{3}{|c|}{ Program WBS Designator: $7.1 .6(1 \mathrm{~K} 1)$} & $\begin{array}{l}\text { Due Date: } \\
\text { 11/01/1997 }\end{array}$ \\
\hline \multicolumn{3}{|c|}{ Control Number: TRP-98-101 } & Rev: 1 \\
\hline $\begin{array}{l}\text { MILESTONE TYPE: } \\
\text { D DOE-HQ } \\
\text { XOE-RL } \\
\square \text { CONTRACTOR }\end{array}$ & $\begin{array}{l}\text { DIVISION: } \\
\text { 몽 } \\
\text { State } \\
\text { Federal } \\
\text { DOE } \\
\text { DCRA } \\
\text { TPA Number } \\
\end{array}$ & $\begin{array}{l}\text { DELIVERABLE: } \\
\text { X Report } \\
\text { R Letter } \\
\text { Drawings } \\
\text { Other (specify) }\end{array}$ & $\begin{array}{l}\text { ADDRESS TO: } \\
\square \text { DOE-HQ } \\
\text { X DOE-RL } \\
\square \text { Other (specify) }\end{array}$ \\
\hline \multicolumn{4}{|c|}{$\begin{array}{l}\text { Milestone Description: } \\
\text { The Hanford Allotment Status Report is prepared annually per the requirements of DOE Order } \\
5660.1 \mathrm{~B} \text {. The delivery date of November } 01 \text { allows DOE-RL sufficient time for resubmission of } \\
\text { the Report by December } 1 \text {. }\end{array}$} \\
\hline \multicolumn{4}{|c|}{$\begin{array}{l}\text { Description of what constitutes completion of this milestone: } \\
\text { Submission of the Allotment Report to DOE-RL will complete this milestone. }\end{array}$} \\
\hline \multicolumn{2}{|c|}{$\begin{array}{ll}\text { Cost Account Manager } & \text { Date } \\
\text { D. C. Lini } & \\
\end{array}$} & \multicolumn{2}{|c|}{$\begin{array}{l}\text { Program/Project Manager } \quad \text { Date } \\
\text { D. B. Cartmell }\end{array}$} \\
\hline \multicolumn{2}{|c|}{$\begin{array}{ll}\text { Program Element Manager } & \text { Date } \\
\text { K. A. Hadley } & \\
\end{array}$} & \multicolumn{2}{|c|}{$\begin{array}{l}\text { DOE Monitor } \\
\text { A. B. Joy }\end{array}$} \\
\hline
\end{tabular}


WHC-SP-1126, Rev. 1

FY. 1996 MYPP

TRANSITION PROJECTS

1.3.1/7.1/6.12

\begin{tabular}{|c|c|c|c|}
\hline \multicolumn{4}{|c|}{$\begin{array}{l}\text { Westinghouse Hanford Company } \\
\text { MILESTONE DESCRIPTION SHEET }\end{array}$} \\
\hline \multicolumn{3}{|c|}{ Title: Submit Hanford Materials Forecast to DOE-RL } & Date: $09 / 26 / 95$ \\
\hline \multicolumn{3}{|c|}{ Assigned To: Transition Projects / Program \& Environmental Mgmt } & CIN: TP-95-057 \\
\hline \multicolumn{3}{|c|}{ Program WBS Designator: $7.1 .6(1 \mathrm{~K} 1)$} & $\begin{array}{l}\text { Due Date: } \\
01 / 15 / 1998\end{array}$ \\
\hline \multicolumn{3}{|c|}{ Control Number: TRP-98-103 } & Rev: 1 \\
\hline $\begin{array}{l}\text { MILESTONE TYPE: } \\
\square \text { DOE-HQ } \\
\text { X DOE-RL } \\
\square \text { CONTRACTOR }\end{array}$ & $\begin{aligned} & \text { DIVISION: } \\
& \text { State } \\
& \square \text { Federal } \\
& \text { X DOE } \\
& \square \text { RCRA } \\
& \square \text { TPA Number }\end{aligned}$ & $\begin{array}{l}\text { DELIVERABLE: } \\
\square \text { Report } \\
\square \text { Letter } \\
\square \text { Drawings } \\
\text { X Other (specify) } \\
\text { Document }\end{array}$ & 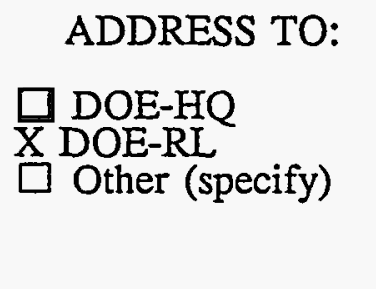 \\
\hline \multicolumn{4}{|l|}{ Milestone Description: } \\
\hline \multicolumn{4}{|c|}{$\begin{array}{l}\text { The Hanford Nuclear Materials Forecast for the current fiscal year and the next } 11 \text { fiscal years } \\
\text { is prepared annually per the requirements of DOE Order } 5660.1 \mathrm{~B} \text {. The delivery date of January } \\
31 \text { assumes DOE-HQ provides the guidance for the forecasts by November } 11 \text { and allows DOE- } \\
\text { RL sufficient time for submission of the Forecast to DOE-HQ by February } 15 \text {. }\end{array}$} \\
\hline \multirow{2}{*}{\multicolumn{4}{|c|}{$\begin{array}{l}\text { Description of what constitutes completion of this milestone: } \\
\text { Submission of the Hanford Nuclear Materials Forecast to DOE-RL will complete this milestone. }\end{array}$}} \\
\hline & & & \\
\hline \multicolumn{2}{|c|}{ Cost Account Manager $\quad$ Date } & \multicolumn{2}{|c|}{ Program/Project Manager Date } \\
\hline \multicolumn{2}{|c|}{$\begin{array}{l}\text { Program Element Manager } \\
\text { K. A. Hadley }\end{array}$} & \multicolumn{2}{|c|}{$\begin{array}{l}\text { DOE Monitor Date } \\
\text { A. B. Joy }\end{array}$} \\
\hline
\end{tabular}


WHC-SP-1126, Rev. 1

FY 1996 MYPP

TRANSITION PROJECTS

1.3.1/7.1/6.12

\begin{tabular}{|c|c|c|c|}
\hline \multicolumn{4}{|c|}{$\begin{array}{l}\text { Westinghouse Hanford Company } \\
\text { MILESTONE DESCRIPTION SHEET }\end{array}$} \\
\hline \multicolumn{3}{|c|}{ Title: Submit FY 1997 Activity Data Sheets to DOE-RL } & Date: $09 / 23 / 94$ \\
\hline \multicolumn{3}{|c|}{ Assigned To: Transition Projects / Program \& Environmental Mgmt } & CIN: \\
\hline \multicolumn{3}{|c|}{ Program WBS Designator: $7.1 .6(1 \mathrm{~K} 1)$} & $\begin{array}{l}\text { Due Date: } \\
04 / 30 / 1998\end{array}$ \\
\hline \multicolumn{3}{|c|}{ Control Number: TRP-98-104 } & Rev: Original \\
\hline $\begin{array}{l}\text { MILESTONE TYPE: } \\
\square \text { DOE-HQ } \\
\square \text { DOE-RL } \\
\text { X CONTRACTOR }\end{array}$ & $\begin{aligned} & \text { DIVISION: } \\
& \square \text { State } \\
& \square \text { Federal } \\
& \text { X DOE } \\
& \square \text { RCRA } \\
& \square \text { TPA Number }\end{aligned}$ & $\begin{array}{l}\text { DELIVERABLE: } \\
\square \text { Report } \\
\square \text { Letter } \\
\square \text { Drawings } \\
\text { X Other (specify) } \\
\text { Document }\end{array}$ & $\begin{array}{l}\text { ADDRESS TO: } \\
\square \text { DOE-HQ } \\
\text { X DOE-RL } \\
\square \text { Other (specify) }\end{array}$ \\
\hline \multicolumn{4}{|c|}{$\begin{array}{l}\text { Milestone Description: } \\
\text { Submit FY } 2000 \text { Activity Data Sheets in support of the Five Year Plan to DOE-RL. }\end{array}$} \\
\hline \multicolumn{4}{|c|}{$\begin{array}{l}\text { Description of what constitutes completion of this milestone: } \\
\text { Submission of the FY } 2000 \text { Activity Data Sheets in support of the Five Year Plan to DOE-RL } \\
\text { will complete this milestone. }\end{array}$} \\
\hline \multicolumn{2}{|c|}{$\begin{array}{l}\text { Cost Account Manager } \quad \text { Date } \\
\text { J. O. Lundeen }\end{array}$} & \multicolumn{2}{|c|}{$\begin{array}{l}\text { Program/Project Manager Date } \\
\text { D. B. Cartmell }\end{array}$} \\
\hline \multicolumn{2}{|c|}{$\begin{array}{l}\text { Program Element Manager } \\
\text { K. A. Hadley }\end{array}$} & \multicolumn{2}{|c|}{$\begin{array}{l}\text { DOE Monitor Date } \\
\text { J. E. Mecca }\end{array}$} \\
\hline
\end{tabular}


WHC-SP-1126, Rev. 1

FY 1996 MYPP

TRANSITION PROJECTS

1.3.1/7.1/6.12

\begin{tabular}{|c|c|c|c|}
\hline \multicolumn{4}{|c|}{$\begin{array}{l}\text { Westinghouse Hanford Company } \\
\text { MILESTONE DESCRIPTION SHEET }\end{array}$} \\
\hline \multicolumn{3}{|c|}{ Title: Submit Draft Hanford Materials Management Plan to DOE-RL } & Date: 09/26/95 \\
\hline \multicolumn{3}{|c|}{ Assigned To: Transition Projects / Program \& Environmental Mgmt } & CIN: TP-95-057 \\
\hline \multicolumn{3}{|c|}{ Program WBS Designator: 7.1.6 (1K1) } & $\begin{array}{l}\text { Due Date: } \\
09 / 15 / 1995\end{array}$ \\
\hline \multicolumn{3}{|c|}{ Control Number: TRP-98-106 } & Rev: 1 \\
\hline $\begin{array}{l}\text { MILESTONE TYPE: } \\
\square \text { DOE-HQ } \\
\text { X DOE-RL } \\
\square \text { CONTRACTOR }\end{array}$ & $\begin{aligned} & \text { DIVISION: } \\
& \square \text { State } \\
& \square \text { Federal } \\
& \text { X DOE } \\
& \square \text { RCRA } \\
& \square \text { TPA Number }\end{aligned}$ & $\begin{array}{l}\text { DELIVERABLE: } \\
\square . \text { Report } \\
\square \text { Letter } \\
\square \text { Drawings } \\
\text { X Other (specify) } \\
\text { Document }\end{array}$ & $\begin{array}{l}\text { ADDRESS TO: } \\
\square \text { DOE-HQ } \\
\text { X DOE-RL } \\
\square \text { Other (specify) }\end{array}$ \\
\hline \multicolumn{4}{|c|}{$\begin{array}{l}\text { Milestone Description: } \\
\text { The Hanford Materials Management Plan is prepared annually per the requirements of DOE } \\
\text { Order } 5660.1 \mathrm{~B} \text {. This plan addresses nuclear material supply and demand for both weapons and } \\
\text { non-weapons programs, nuclear materials management issues, processing options, and inventory } \\
\text { management. The delivery date will be approximately } 8 \text { weeks after DOE-HQ provides planning } \\
\text { assumptions. For planning purposes it is assumed that DOE-HQ guidance will be provided no } \\
\text { later than July 15, 1995. Changes to this assumption will be documented through change } \\
\text { control. }\end{array}$} \\
\hline \multicolumn{4}{|c|}{$\begin{array}{l}\text { Description of what constitutes completion of this milestone: } \\
\text { Submission of a Hanford Materials Management Plan to DOE-RL will complete this milestone. }\end{array}$} \\
\hline \multicolumn{2}{|c|}{$\begin{array}{l}\text { Cost Account Manager } \quad \text { Date } \\
\text { D. C. Lini }\end{array}$} & \multicolumn{2}{|c|}{$\begin{array}{l}\text { Program/Project Manager } \\
\text { D. B. Cartmell }\end{array}$} \\
\hline \multicolumn{2}{|c|}{$\begin{array}{l}\text { Program Element Manager } \quad \text { Date } \\
\text { K. A. Hadley }\end{array}$} & \multicolumn{2}{|c|}{$\begin{array}{l}\text { DOE Monitor } \quad \text { Date } \\
\text { A. B. Joy }\end{array}$} \\
\hline
\end{tabular}


WHC-SP-1126, Rev. 1

FY 1996 MYPP

\section{TRANSITION PROJECTS}

1.3.1/7.1/6.12

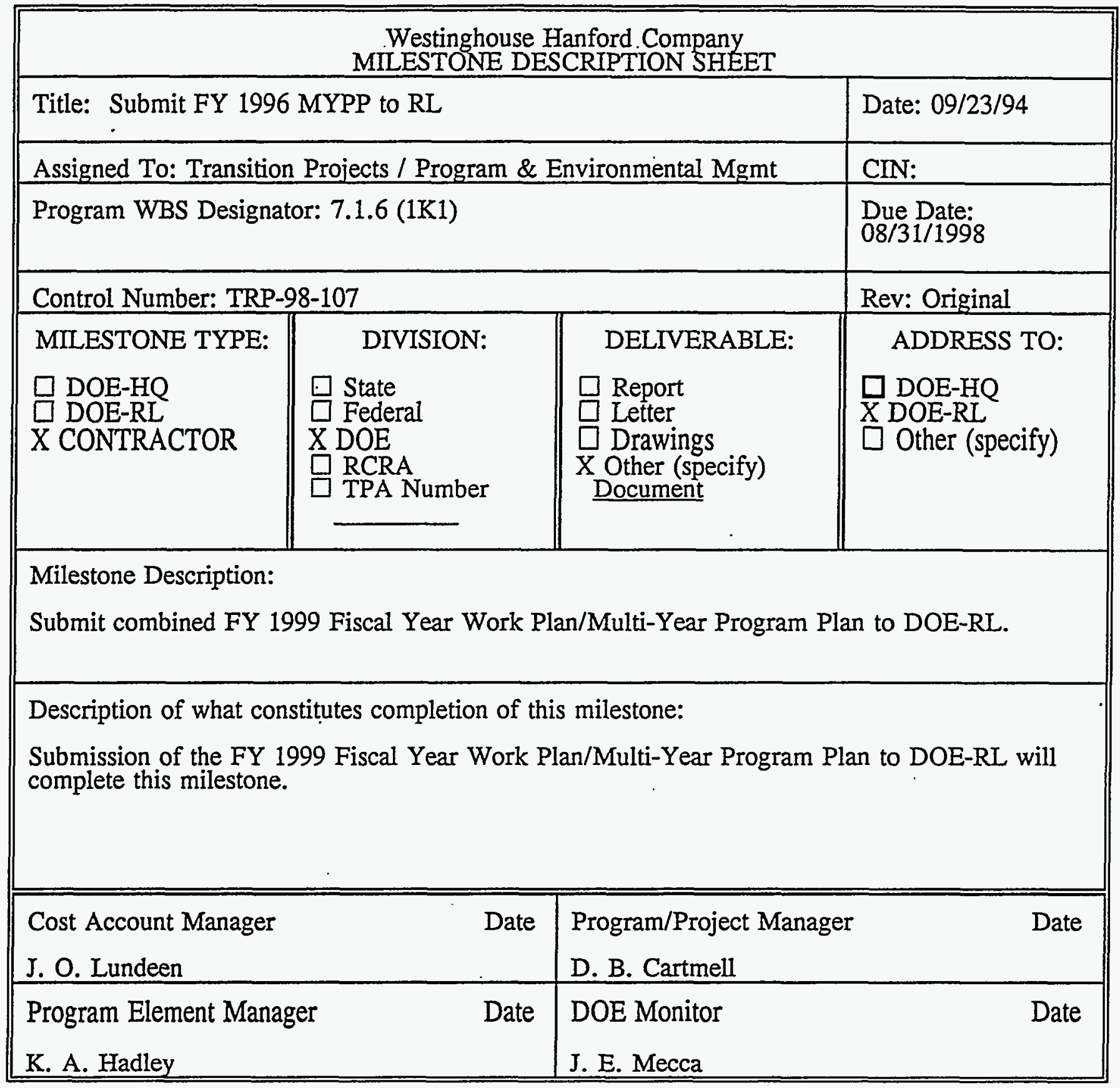




\section{C.1 Cost Baseline Summary By Year}

The cost baseline summary by year section defines the cost estimate baseline required to complete the technical work scope as scheduled. The baseline is derived from resource loaded schedules, historical data and developed cost account plans.

Each year, through life cycle, is included in this section. The last column, you will note, is titled "FY 2003 \& Beyond". This is the summation of all costs from FY 2003 to end point. A few things to note regarding this information:

B Plant/WESF (1.3.1.7): Deactivation completion for B Plant is scheduled for 9/30/02 (HQ milestone) and transferred to EM-40. Deactivation completion for WESF is scheduled for FY 2013 and transferred to EM-40. Minimum surveillance and maintenance costs beyond the deactivation completion dates will be included until they are incorporated into the EM-40 MYP.P.

PUREX (7.1.1): Deactivation completion for PUREX is scheduled for $1 / 30 / 98$ (HQ milestone), $7 / 31 / 98$ (TPA milestone), and transferred to EM-40.

300 Area Fuels (7.1.2): Supply

Deactivation completion for the 300 Area Fuels Supply Shutdown is scheduled for $3 / 31 / 99$ (HQ milestone) and transferred to EM-40. Minimum surve $i 17$ ance and maintenance costs beyond the deactivation. completion dates will be included until they are incorporated into the EM-40 MYPP.

Plutonium $(7.1 .3)$ :

Finishing Piant

PFP life cycle costs are reflected through FY 2025, consistent with the Baseline Environmental Management Report.

Program and $(7.1 .6)$ : Environmental Mgmt

K Basins $(7.1 . X)$ :
PFP life cycle costs are reflected through FY 2025, consistent with the Baseline Environmental Management Report.

Formal transition of the K Basins will begin in FY 2001 and 1 ife cycle costs are reflected through FY 2047. 
TRANSITII PROJECTS

FY 1996 MYPP

1.3.1/7.1/6.12

\section{C.1 Cost Basel ine Summary By Year}

( $\$$ in 000 's)

\begin{tabular}{c} 
WBS/ADS \\
TITLE \\
\hline
\end{tabular}

1.3.1.7/4190-0

B PLANT

1.3.1.7/4190-1

$\stackrel{N}{o}$ SAFETY VENTILATION UPGRADE

í

1.3.1.7 / 4195-0

CESIUM CAPSULE RECOVERY

$1.3 .1 / 41 \mathrm{xX}$

TRANSITION PROJJECTS

EM-30 subtotal
FY 2003

IYPE FY 1996 FY 1997 FY 1998 FY 1999 FY 2000 FY 2001 FY 2002 \& Beyond

\begin{tabular}{lrrrrrrrr} 
OE & 32736 & 35651 & 32569 & 31161 & 13705 & 13343 & 14058 & 332760 \\
CE & 250 & 256 & 500 & 500 & 0 & 0 & 0 & 0 \\
GPP & 0 & 0 & 0 & 0 & 0 & 0 & 0 & 0 \\
LI & 0 & 0 & 0 & 0 & 0 & 0 & 0 & 0 \\
\cline { 2 - 9 } & 32986 & 35907 & 33069 & 31661 & 13705 & 13343 & 14058 & 332760
\end{tabular}

\begin{tabular}{lrrrrrrrr} 
OE & 112 & 305 & 547 & 98 & 0 & 0 & 0 & 0 \\
CE & 0 & 0 & 0 & 0 & 0 & 0 & 0 & 0 \\
GPP & 0 & 0 & 0 & 0 & 0 & 0 & 0 & 0 \\
LI & 0 & 1500 & 2000 & 0 & 0 & 0 & 0 & 0 \\
\cline { 2 - 9 } & 112 & 1805 & 2547 & 98 & 0 & 0 & 0 & 0
\end{tabular}

\begin{tabular}{lrrrrrrrr} 
OE & 1987 & 638 & 4404 & 0 & 0 & 0 & 0 & 0 \\
$\mathrm{CE}$ & 0 & 0 & 0 & 0 & 0 & 0 & 0 & 0 \\
$\mathrm{GPP}$ & 0 & 0 & 0 & 0 & 0 & 0 & 0 & 0 \\
$\mathrm{LI}$ & 0 & 0 & 0 & 0 & 0 & 0 & 0 & 0 \\
\hline & 1987 & 638 & 4404 & 0 & 0 & 0 & 0 & 0
\end{tabular}

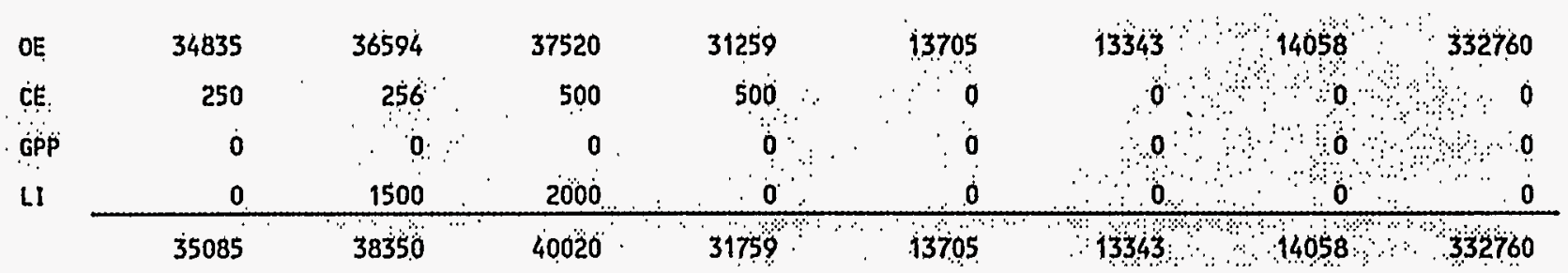


TRANSITIL.J PROJECTS

1.3.1/7.1/6.12

\section{C.1 Cost Basej ine Sümary By Year}

( $\$$ in 000 's)

WBS/ADS

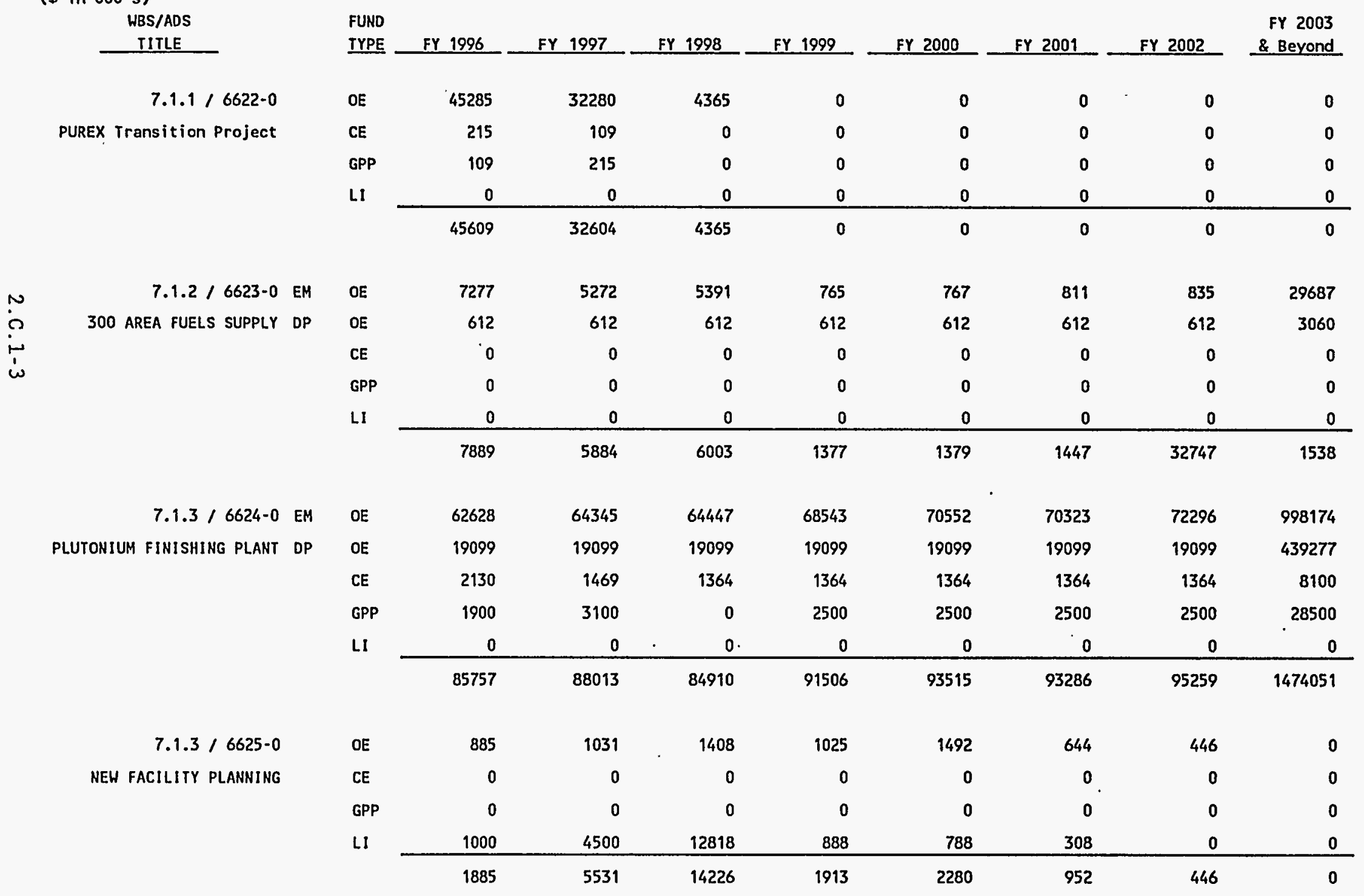

FUND

TYPE 
WHC-SP-1126, Rev. 1

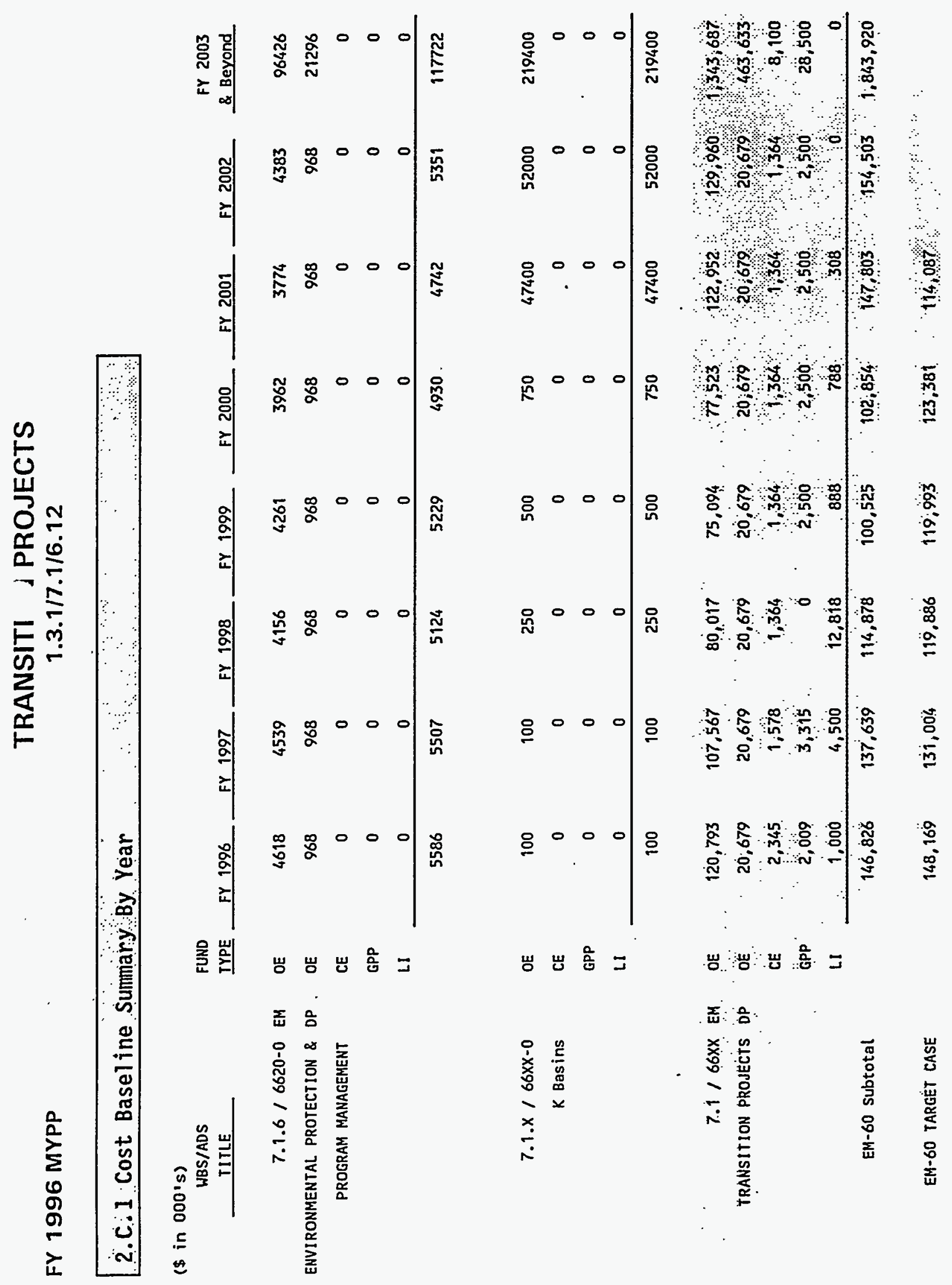


TRANSITI PROJECTS

FY 1996 MYPP

1.3.1/7.1/6.12

\section{C. 1 Cost Basel ine Summary By Year}

( $\$$ in $\left.000^{\prime} s\right)$

WBS/ADS

FUND

IYPE

1996 FY 1997 FY 1998 FY 1999

FY 2000

FY 2001

FY 2003

TITLE

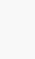

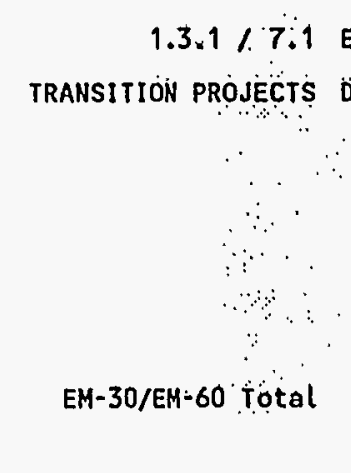

EM-30 \& EM-60 TARGET CASE

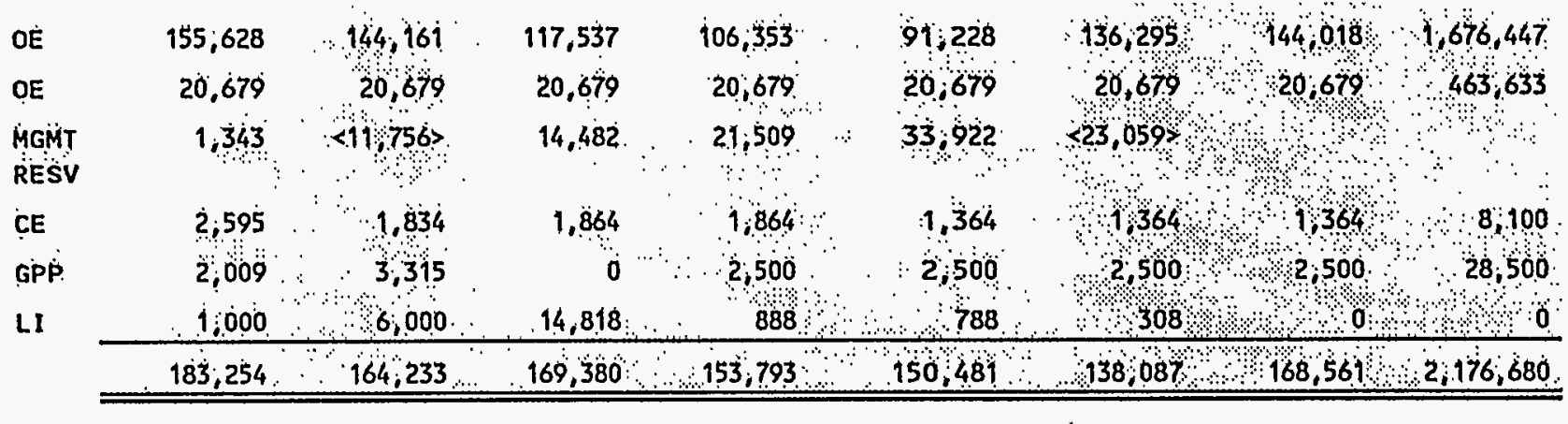

183,254

164,154

169,386

$15 \ddot{3}, 79 \ddot{3}$

$150 ; 481$

$13 \ddot{8}, 0 \ddot{87}$ 


\section{TRANSITION PROJECTS}

\subsection{Bàsis of Estimate}

General:

The basis of the Transition Projects cost estimate is the Activity Based Cost estimates. Upon completion of initial and ongoing critical analysis phases and finalization of the estimates, this plan will be revised with a change control package to reflect a final estimate.

Detailed Basis of Estimate information is available upon request from Transition Projects Business Management. PUREX:

The basis of the PUREX Surveillance \& Maintenance cost estimate is the Activity Based Cost estimate which is currently being prepared. The first draft of the estimate was used to prepare this plan. Upon completion of the critical analysis phase and finalization of the estimate, this plan will be revised with a change control package to reflect the final estimate.

The basis of the Transition cost estimate is the current project cost baseline and schedule baseline. This is based on the Activity Based Cost estimate for this project was completed in December 1993 and has been

1 modified with subsequent change control packages. 
TRANSITION PROJECTS

FY 1996 MYPP

1.3.1/7.1/6.12

2.C. 3 Planned Staffing Profiles

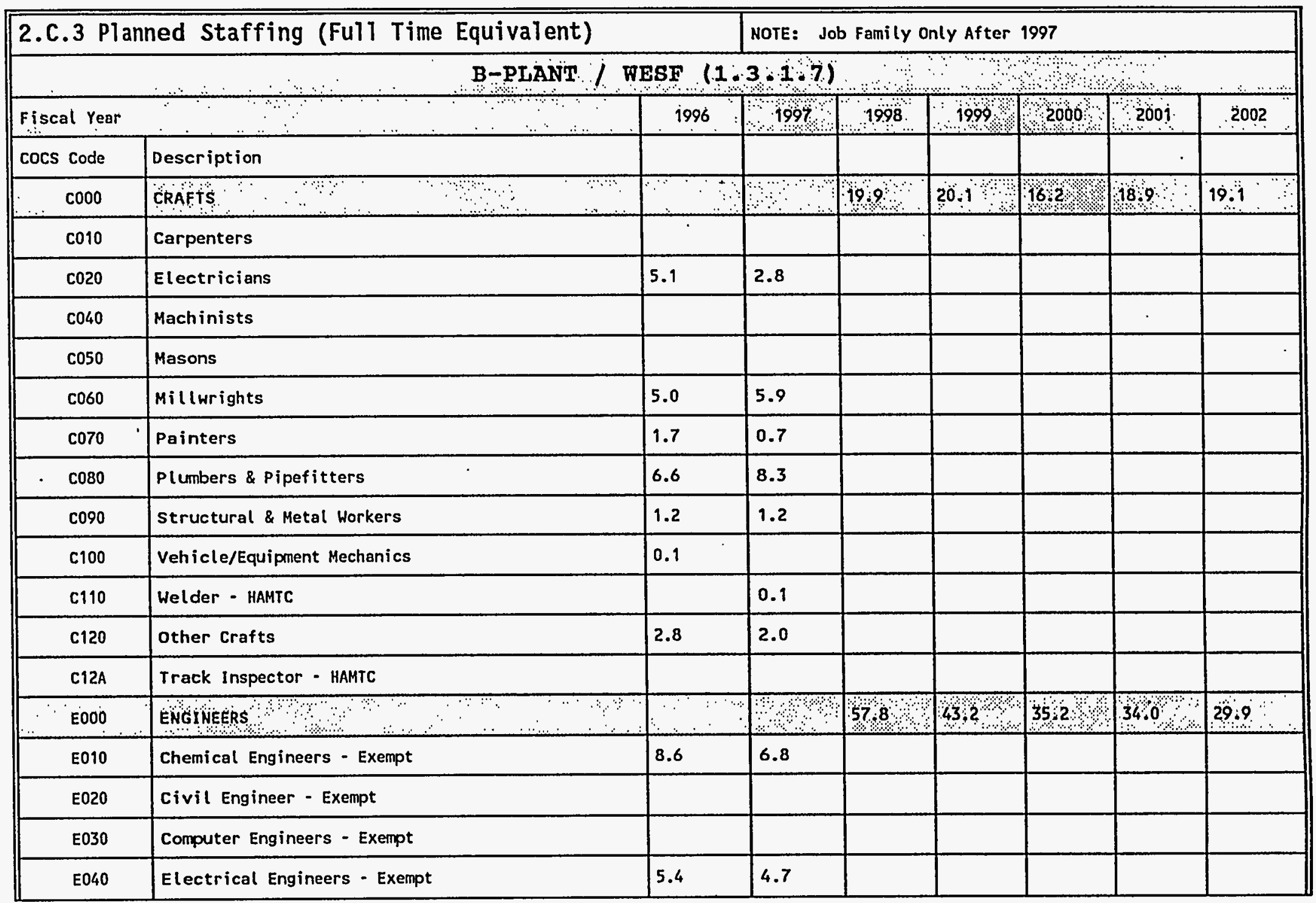


2.c.3 Planned staffing Proffles

\begin{tabular}{|c|c|c|c|c|c|c|c|c|}
\hline \multicolumn{3}{|c|}{ 2.C.3 Planned Staffing (Full Time Equivalent) } & \multicolumn{6}{|c|}{ NOTE: Job Family Only After 1997} \\
\hline \multicolumn{9}{|c|}{ 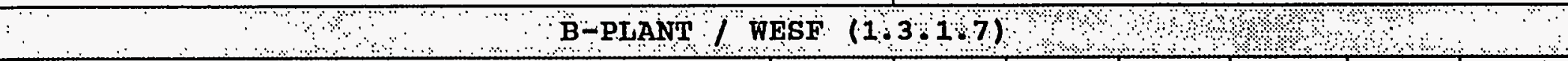 } \\
\hline \multicolumn{2}{|l|}{ Físcal Year. } & 1996 & $1997:$ & 1998: & 1999 & 2000 & zóoi & $2002:$ \\
\hline cocs code & Description & & & & & & & \\
\hline E050 & Environmental Engineers - Exempt & 7.6 & 8.1 & & & & & \\
\hline E060 & Industrial Engineers - Exempt & & & & & & & \\
\hline E070 & Mechanical Engineers - Exempt & 12.3 & 12.0 & & & & & \\
\hline E080 & Nuclear Engineers - Exempt & 5.4 & 4.4 & & & & & \\
\hline EO90 & Mining Engineers - Exempt & & & & & & & \\
\hline E100 & Plant Engineers & 19.7 & 15.9 & & & & & \\
\hline E110 & Quality Control Engineers & 1.8 & 1.3 & & & & & \\
\hline E120 & Safety Engineers & 4.5 & 3.7 & & & & & \\
\hline E130 & OTHER ENGINEERS & 9.8 & 14.8 & & & & & \\
\hline 6000 & GENERAL AOMINISTRATIVE & $\therefore$ & $\therefore$ & 1712 & 19.3 & 15.4 & 16.1 & 15.8 \\
\hline 6010 & Administrative Assistants & 0.9 & 0.9 & & & & & \\
\hline G020 & office Clerks (General) & 7.8 & 5.8 & & & & & \\
\hline 6030 & office Clerks (Specialists) & 2.7 & 2.5 & & & & & \\
\hline $6040^{\circ}$ & Secretaries & 11.5 & 9.5 & & & & & \\
\hline G050 & Clerk, Typist, \& Word Processing & & & & & & & \\
\hline LoÖ & LABORORS & "“ & $\because \because$ & is & अ & ?. & अ? & 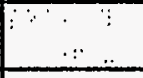 \\
\hline Lo10 & Firefighters & & & & & & & \\
\hline L020 & Janitors \& Cleaners & & & & & & & \\
\hline
\end{tabular}




\section{TRANSITION PROJECTS}

FY 1996 MYPP

1.3.1/7.1/6.12

2.C.3 Planned staffing profiles

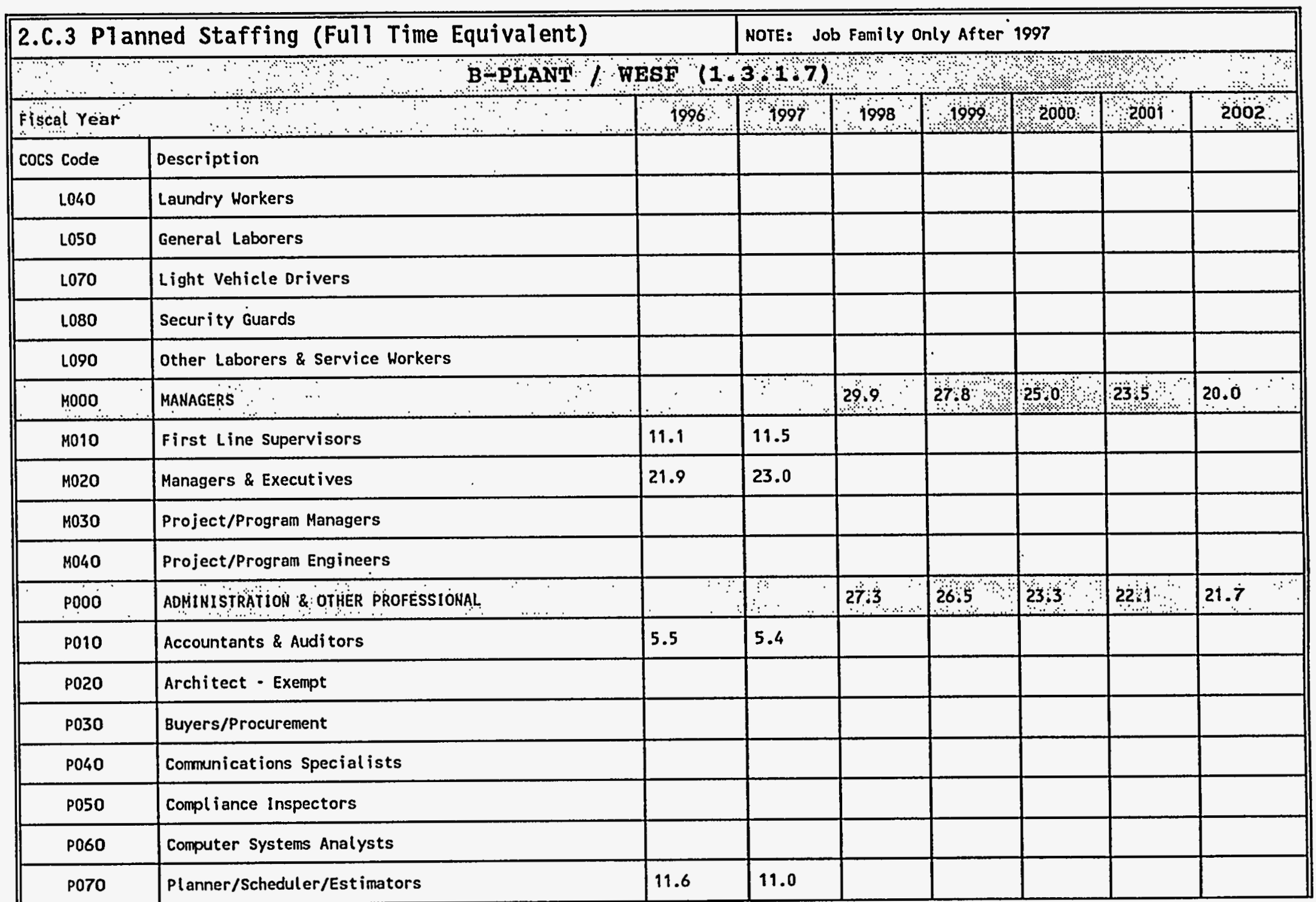


TRANSITION PROJECTS

1.3.1/7.1/6.12

2.C.3 PTanned Staffing profiles

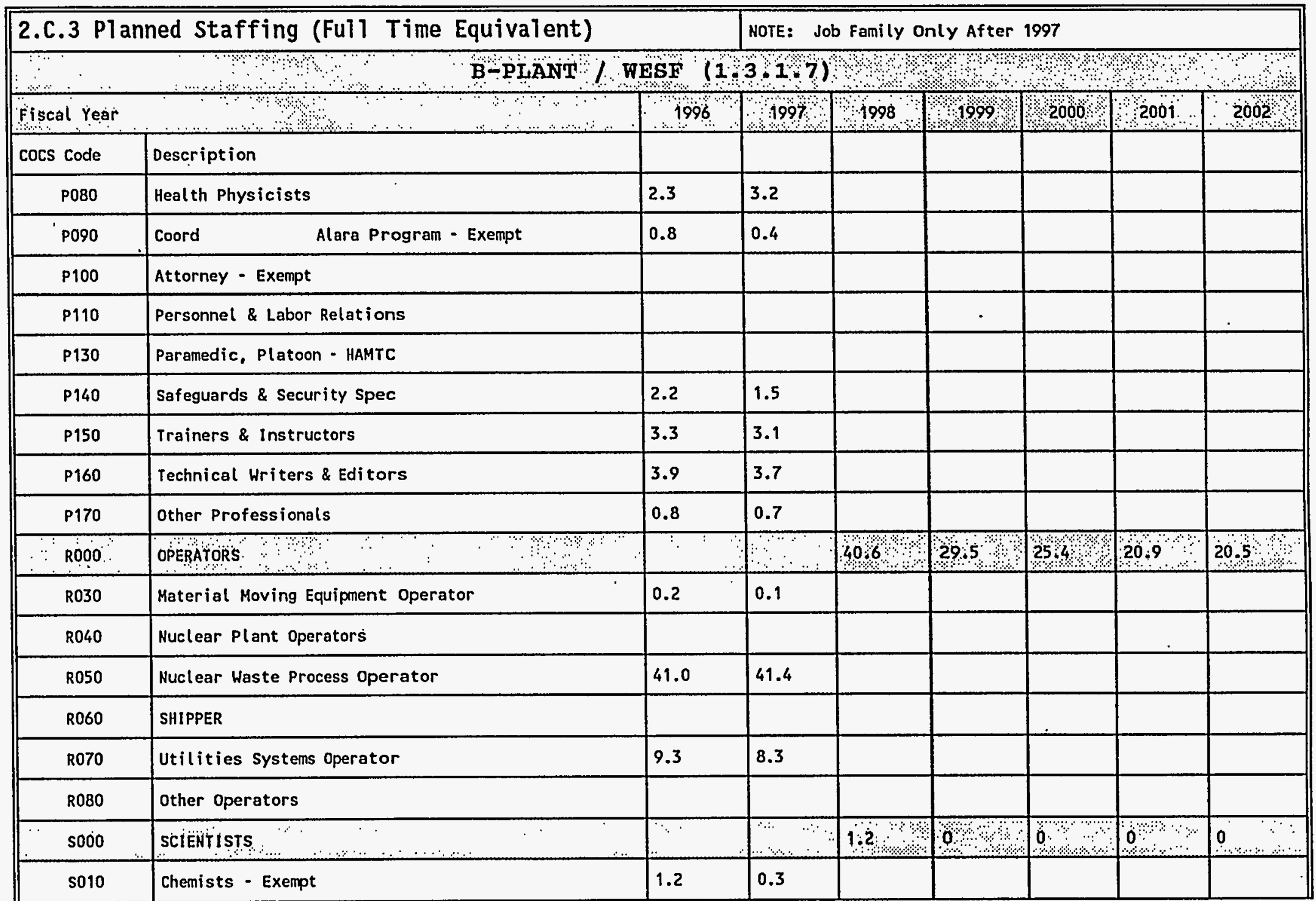


TRANSITION PROJECTS

1.3.1/7.1/6.12

2.C.3 planned staffing profiles

\begin{tabular}{|c|c|c|c|c|c|c|c|c|}
\hline \multicolumn{3}{|c|}{ 2.C.3 Planned Staffing (Full Time Equivalent) } & \multicolumn{6}{|c|}{ NOTE: Job Family Only After 1997} \\
\hline \multicolumn{9}{|c|}{ B-PLANT /WESF (1)31/7) } \\
\hline \multicolumn{2}{|c|}{ Fiscal Year $\quad \begin{array}{lllllll} & \\
\end{array}$} & 1996 & 1997 & 1998 & $1999:$ & 2000 & 20001 & 2002 \\
\hline cocs Code & Description & & & & & & & \\
\hline s020 & Environmental scientists - Exempt & 0.6 & 0.7 & & & & & \\
\hline $\mathbf{s 0 3 0}$ & Geologists - Exempt & & & & & & & \\
\hline 5040 & Life Scientists - Exempt & & & & & & & \\
\hline s050 & Materials Scientists - Exempt & & & & & & & \\
\hline so60 & Mathematicians - Exempt & & & & & & & \\
\hline so70 & Physicists - Exempt & & & & & & & \\
\hline s080 & Social - Exempt & & & & & & & \\
\hline s090 & Other Scient ists - Exempt & & & & & & & \\
\hline$\because$ T000 & TECHNICtANS & \begin{tabular}{ccc}
4 \\
\hdashline
\end{tabular} & 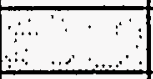 & $267 \div$ & $2+12$ & 150 & $15: 9$ & 15.0 \\
\hline T010 & Computer Operator/Coder & & & & & & & \\
\hline TO20 & Drafters & 0.4 & & & & & & \\
\hline T030 & Engineering Technician - NonExempt & 1.2 & 0.9 & & & & & \\
\hline T050 & Heal th Physics Technician - HAMTC & 14.7 & 15.1 & & & & & \\
\hline TO70 & Instruments \& Control Technician & 8.7 & 7.9 & & & & & \\
\hline T080 & Chemical Technologist - HAMTC & & & & & & & \\
\hline T090 & Media Technicians & & & & & & . & \\
\hline$T 110$ & other Technicians & 2.8 & 2.2 & & & & & \\
\hline
\end{tabular}




\section{C.3 Planined Staffing Profiles}

\begin{tabular}{|c|c|c|c|c|c|c|c|c|}
\hline \multicolumn{3}{|c|}{ 2.C.3 Planned Staffing (Full Time Equivalent) } & \multicolumn{6}{|c|}{ NOTE: Job Family Only After 1997} \\
\hline & . & $3(7 \cdot 1$ & 1) & अ? & 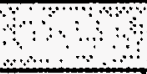 & (n) & no & \\
\hline Fiscal Year & $\therefore$ & 1996 & 1997 & 1998 & 1999 & 2000 & 2001 & 2002 \\
\hline cocs Code & Description & & & & & & & . \\
\hline $\operatorname{coso}$ & CRAFTS $\quad \because \quad \because \quad \quad \therefore \quad \therefore$ & 37 & 24 & $4+\cdots$ & ô & 003 & $0 .+3$ & 0 \\
\hline $\mathrm{CO10}$ & Carpenters & 2 & 1 & & & & & \\
\hline $\mathrm{CO20}$ & Electricians & 8 & 5 & & & & & \\
\hline $\mathrm{CO40}$ & Machinists & 2 & 2 & & & & & \\
\hline C050 & Masons & 1 & 1 & & & & & \\
\hline $\mathrm{C} 060$ & Millwrights & 5 & 3 & & & & & \\
\hline $\mathrm{CO70}$ & Painters & 3 & 1 & & & & & \\
\hline $\operatorname{cos0}$ & Plumbers \& Pipefitters & 7 & 4 & & & & & \\
\hline $\operatorname{coso}$ & Structural \& Metal Workers & 6 & 6 & & & & & \\
\hline C100 & Vehicle/Equipment Mechanics & 1 & 0 & & & & & \\
\hline c110 & Helder - HAMTC & & & & & & & \\
\hline$c 120$ & other Crafts - Insulators & 2 & 1 & & & & & \\
\hline C12A & Track Inspector - HAMTC & & & & & & & \\
\hline$E 000$ & ENGINEERS & 63 & 32 & 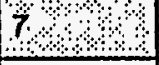 & 0 & 00 & $0+1$ & 0. \\
\hline E010 & Chemical Engineers - Exempt & 15 & 9 & & & & & \\
\hline E020 & Civil Engineer - Exempt & & & & & & & \\
\hline E030 & Computer Engineers - Exempt & & & & & & & \\
\hline EO40 & Electrical Engineers - Exempt & 3 & 2 & & & & & \\
\hline
\end{tabular}




\section{TRANSITION PROJECTS}

1.3.1/7.1/6.12

\section{C.3 Planned Staffing Profiles}

\begin{tabular}{|c|c|c|c|c|c|c|c|c|}
\hline \multicolumn{3}{|c|}{ 2.C.3 Planned Staffing (Full Time Equivalent) } & \multicolumn{6}{|c|}{ NOTE: Job Family Only After 1997} \\
\hline \multicolumn{9}{|c|}{ 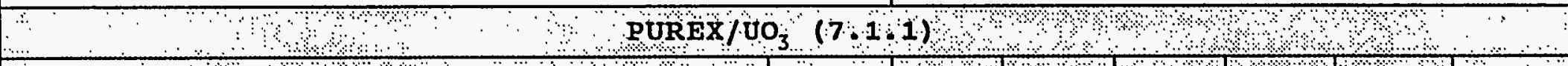 } \\
\hline Fiscal Year & 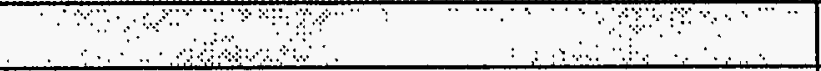 & 1996 & $1997 \%$ & 1998 & & & 2001 & 2002 \\
\hline cocs Code & Description & & & & & & & \\
\hline E050 & Environmental Engineers - Exempt & 3 & 2 & & & & & \\
\hline E060 & Industrial Engineers - Exempt & & & & & & & \\
\hline E070 & Mechanical Engineers - Exempt & 9 & 5 & & & & & \\
\hline E080 & Nuclear Engineers - Exempt & 4 & 2 & & & & & \\
\hline E090 & Mining Engineers - Exempt & & & & & & & \\
\hline$E 100$ & Plant Engineers & 20 & 8 & & & & & \\
\hline E110 & Quality Control Engineers & 3 & 2 & & & & & \\
\hline E120 & Safety Engineers & 4 & 2 & & & & & \\
\hline E130 & OTHER ENGINEERS & 2 & 0 & & & & & \\
\hline G000 & GENËRAL ADMINISSTRATIVE & 27 & $14: \vdots$ & i़। & 0 & 0 & +3: & 0 \\
\hline $\mathbf{G 0 1 0}$ & Administrative Assistants & 5 & 2 & & & & & \\
\hline $\mathbf{6 0 2 0}$ & Office Clerks (General) & 8 & 4 & & & & & \\
\hline 6030 & Office Clerks (Specialists) & 4 & 3 & & & & & \\
\hline G040 & Secretaries & 10 & 5 & & & & & \\
\hline 6050 & Clerk, Typist, \& Hord Processing & & & & & & & \\
\hline LOOO & 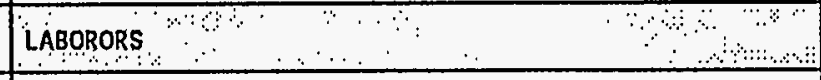 & $6 \quad \because \because$ & 3 & क्या & ০४७। & 0 & 0 & $0 \cdot 2:$ \\
\hline Lo10 & Firefighters & & & & & & & \\
\hline 1020 & Janitors \& Cleaners & 3 & 1 & & & & & \\
\hline
\end{tabular}


TRANSITION PROJECTS

1.3.1/7.1/6.12

\section{C.3 Planned Staffing Profiles}

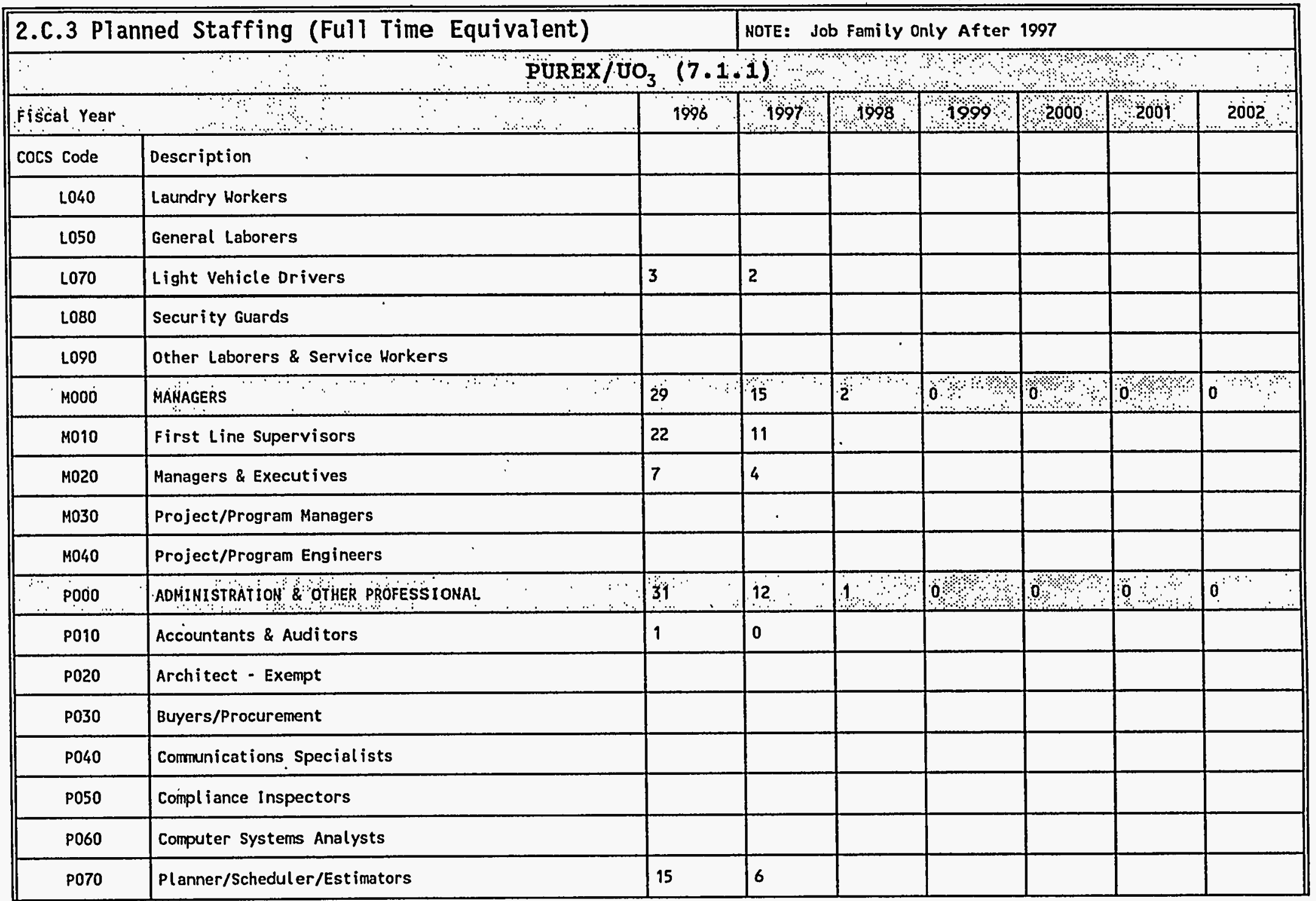


TRANSITION PROJECTS

FY 1996 MYPP

1.3.1/7.1/6.12

\section{C. 3 Planned Staffing Profiles}

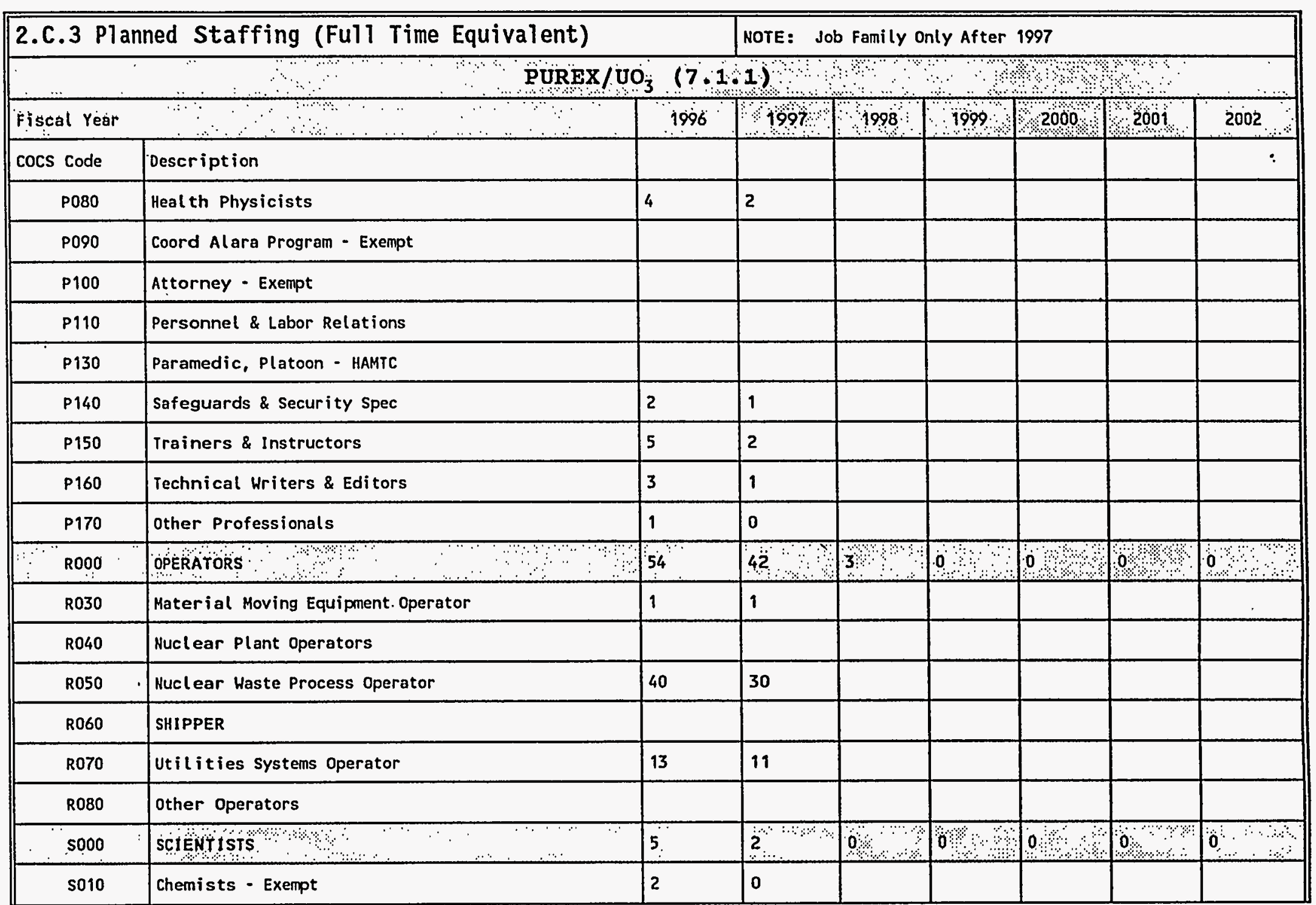




\section{C.3 Planied staffing profiles}

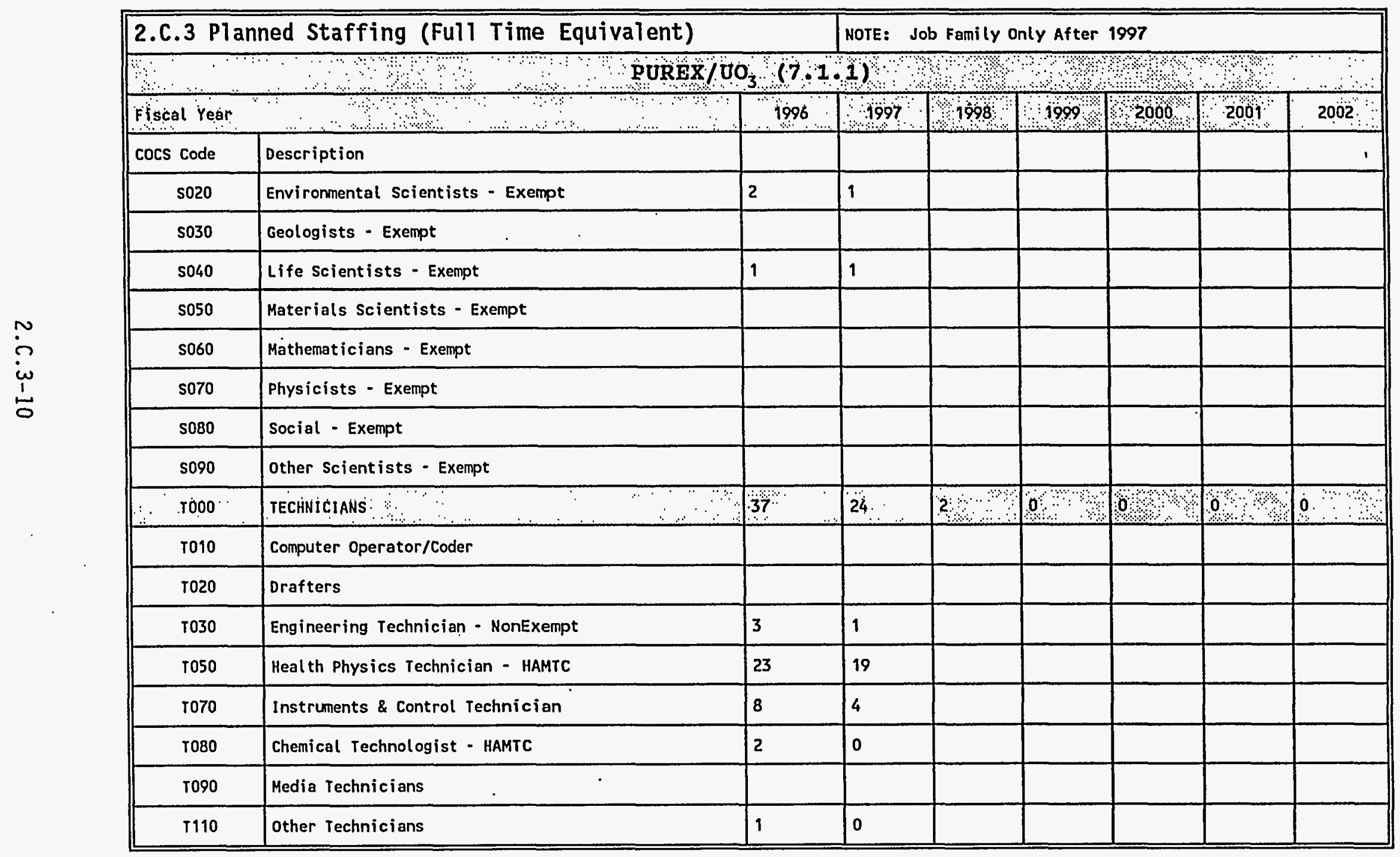


2.C. 3 Planned Staffing Profiles

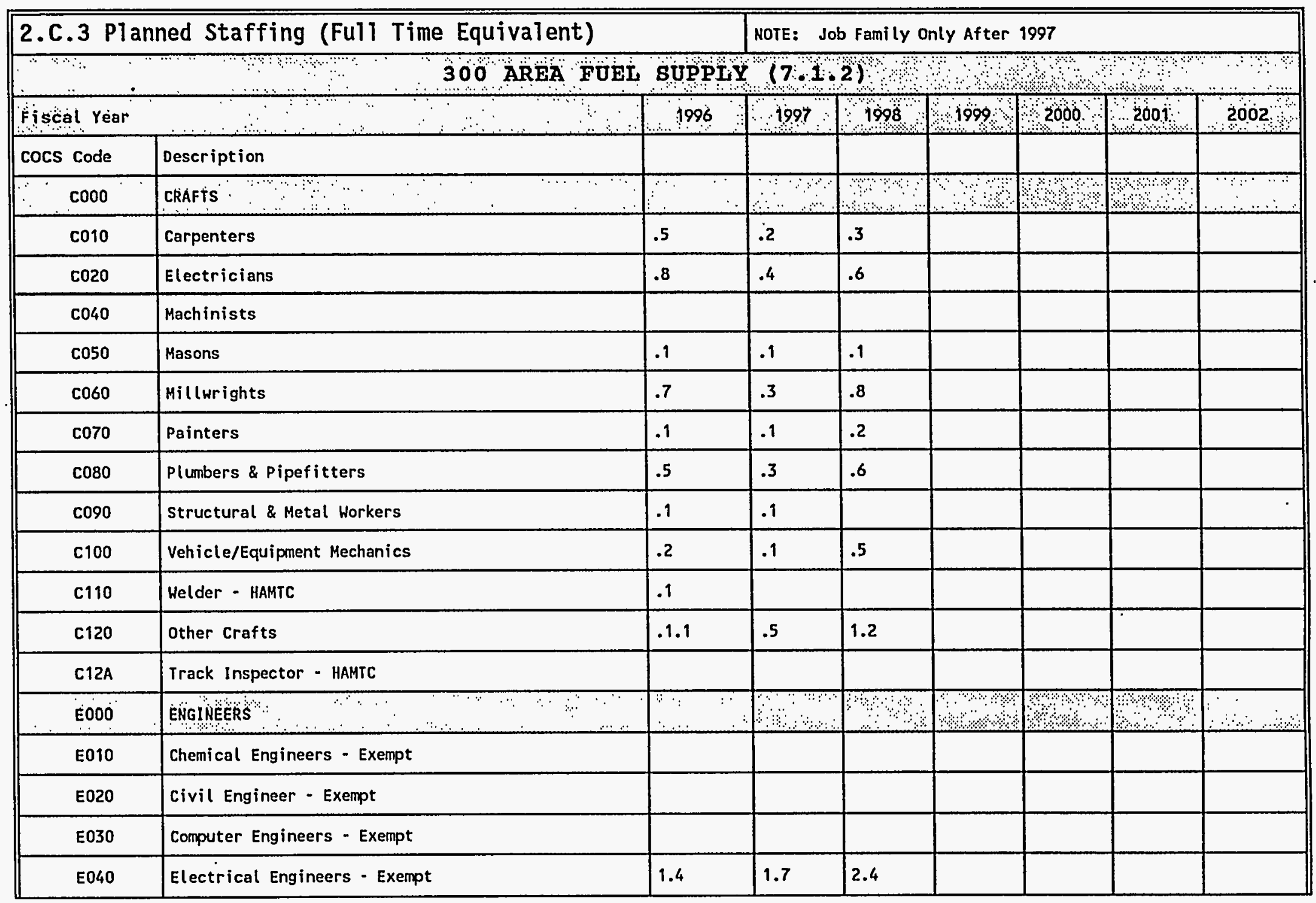


TRANSITION PROJECTS

FY 1996 MYPP

1.3.1/7.1/6.12

2.C. 3 Planned Staffing Profiles

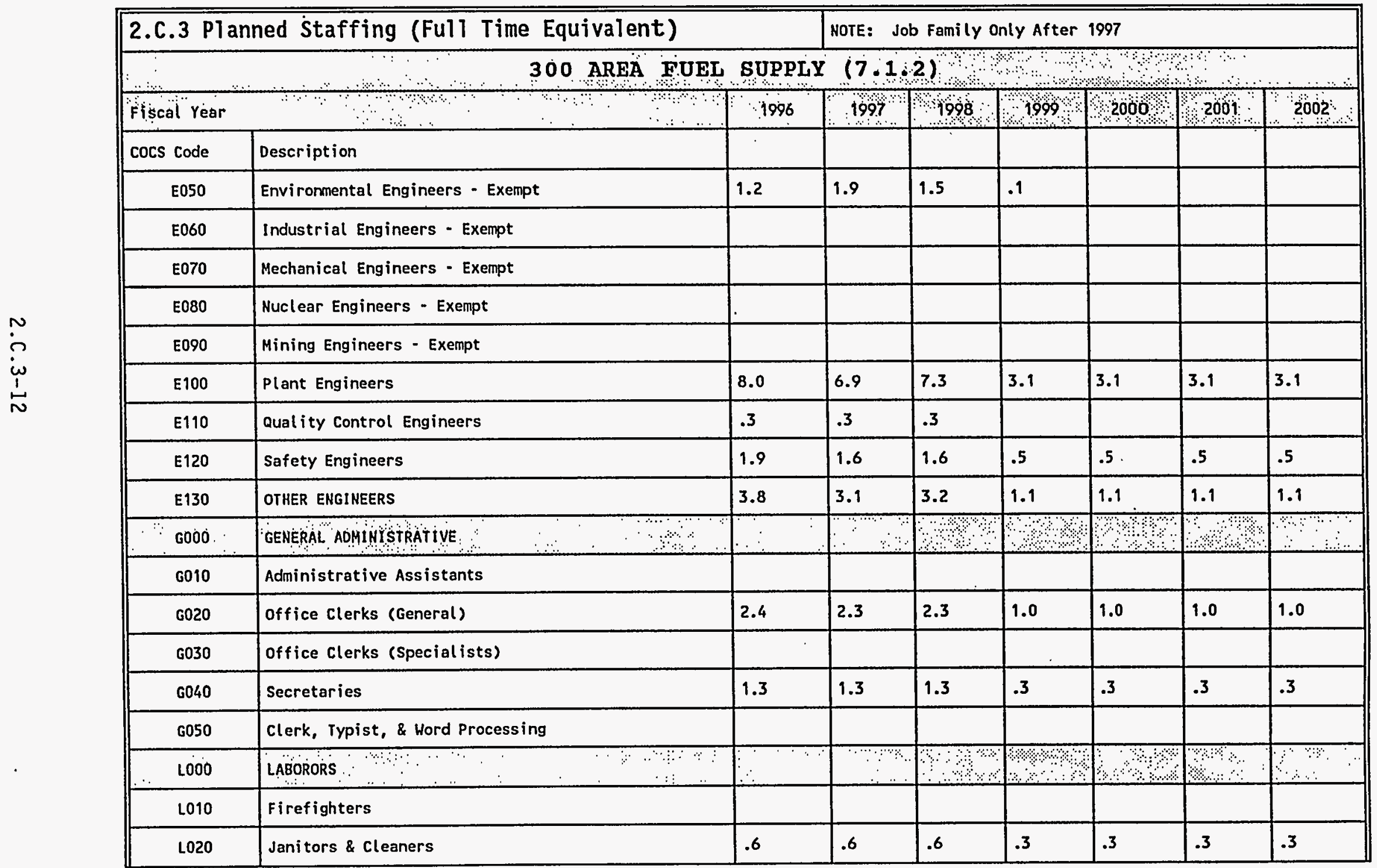


2. 3 Planned Staffing Profites

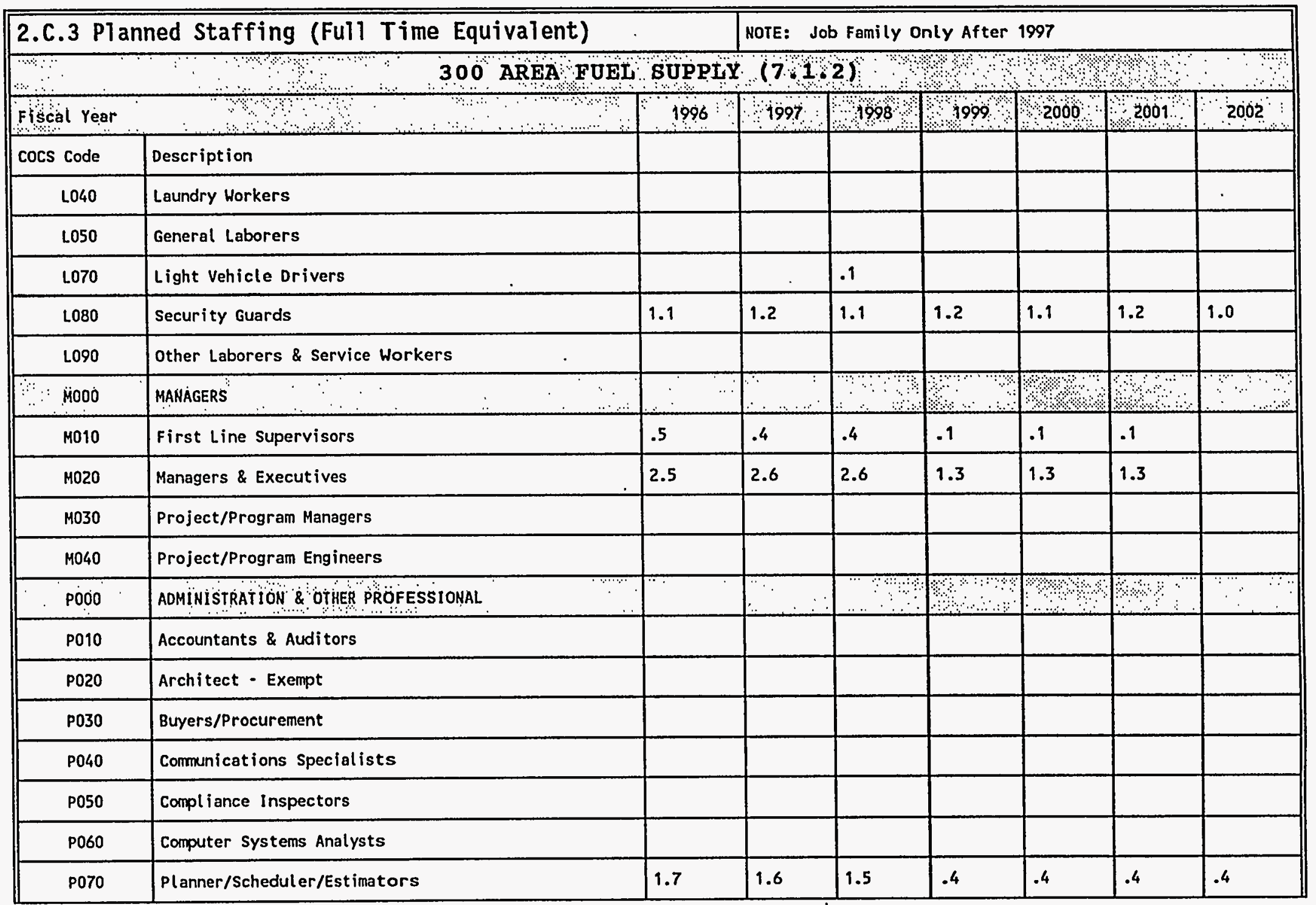




\section{TRANSITION PROJECTS}

\section{FY 1996 MYPP}

1.3.1/7.1/6.12

\section{C 3 Planned staffing Profiles}

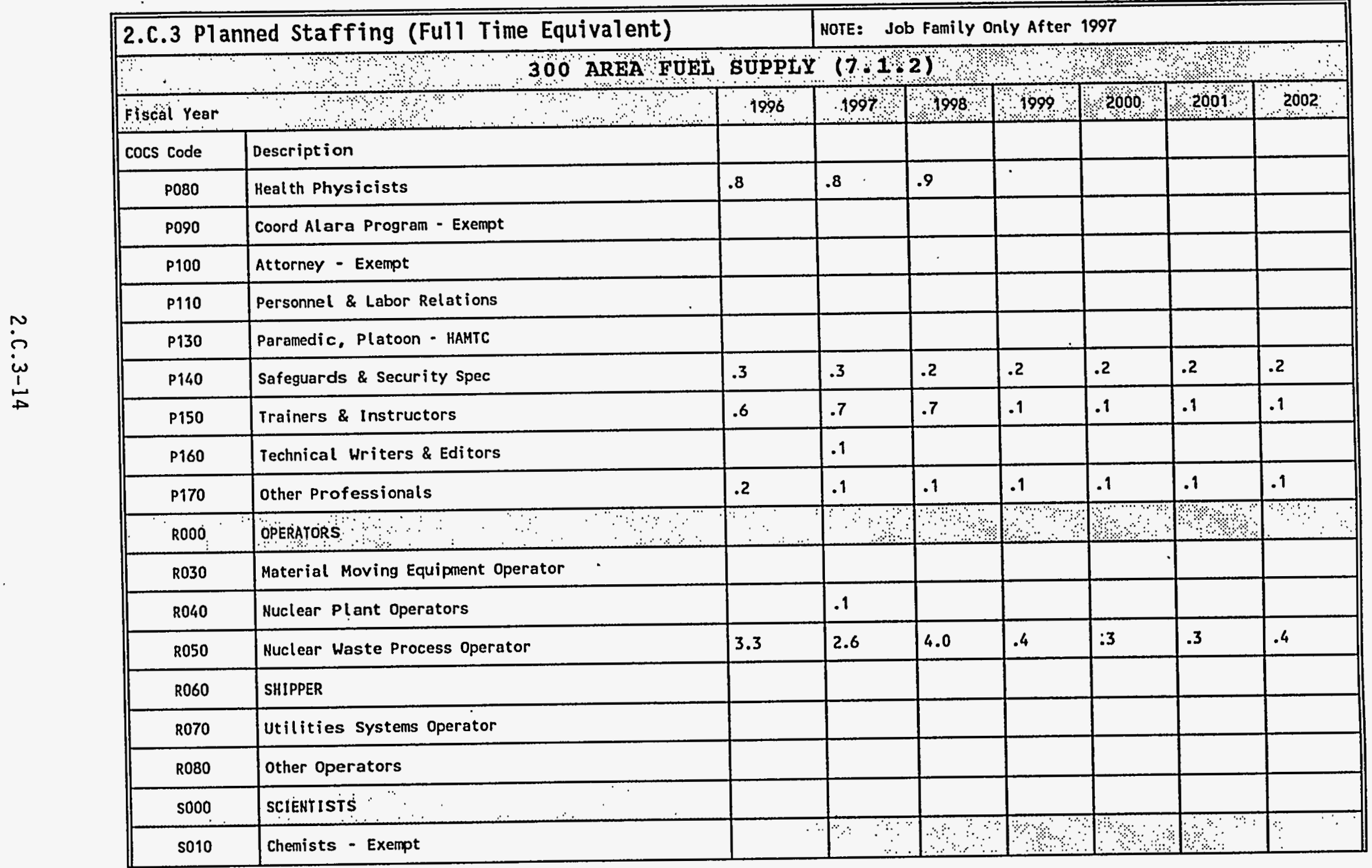


TRANSITION PROJECTS

FY 1996 MYPP

1.3.1/7.1/6.12

2.C.3 Planned Staffing Profiles

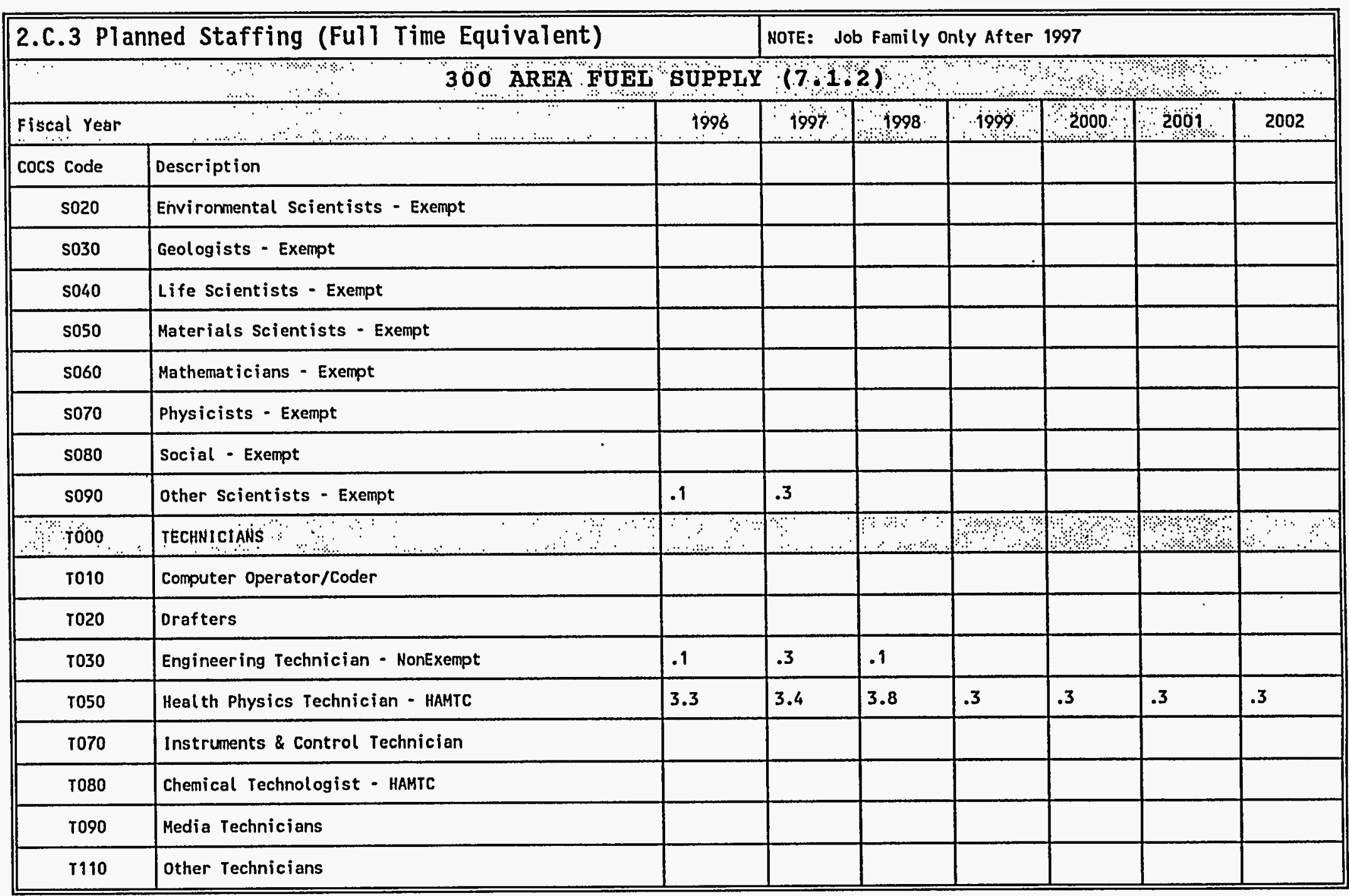


FY 1996 MYPP

TRANSITION PROJECTS

1.3.1/7.1/6.12

\section{C 3 Planned staffing profiles}

\begin{tabular}{|c|c|c|c|c|c|c|c|c|}
\hline \multicolumn{3}{|c|}{ 2.C.3 Planned Staffing (Full Time Equivalent) } & \multicolumn{6}{|c|}{ NOTE: Job Family Only After 1997} \\
\hline$\therefore$ & (a) & $7 \cdot 1 \div 3$ & की & म् & 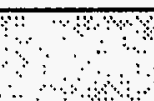 & का? & +3 & \\
\hline Fiscal Year & 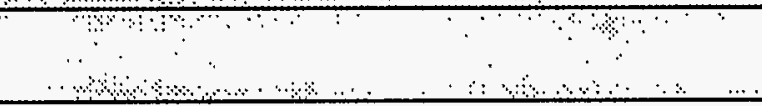 & 1996 & 1997 & 1098 & 1999 & 200 & 2001 & 2002 \\
\hline cocs code & Description & & & & & & & \\
\hline c000 & CRAFts : & 42.4 & 416 & 4103 & 30,8 & $9.8 \%$ & 393 & $\begin{array}{l}38.8 \\
\therefore 8\end{array}$ \\
\hline $\mathrm{co00}$ & Crafts & 0.9 & 0.9 & 0.9 & 0.9 & 0.9 & 0.9 & 0.8 \\
\hline $\mathrm{co10}$ & Carpenters & 0.7 & 0.7 & 0.7 & 0.7 & 0.7 & 0.7 & 0.7 \\
\hline $\mathrm{c020}$ & Electricians & 15.8 & 13.4 & 13.4 & 13.4 & 13.4 & 13.4 & 13.4 \\
\hline $\mathrm{CO} 40$ & Machinists & & & . & & & & \\
\hline $\cos 0$ & Masons & & & & & . & & \\
\hline c060 & Millwrights & 4.7 & 4.7 & 4.7 & 4.7 & 4.7 & 4.7 & 4.7 \\
\hline $\operatorname{coto}$ & Painters & 4.8 & 4.8 & 4.8 & 4.8 & 4.8 & 4.8 & 4.8 \\
\hline $\cos$ & Plumbers \& Pipefitters & 7.1 & 7.1 & 7.1 & 7.1 & 7.1 & 7.1 & 7.1 \\
\hline $\operatorname{coso}$ & Structural \& Metal Workers & & & & & & & \\
\hline c100 & Vehicle/Equipment Mechanics & & & & & & & \\
\hline
\end{tabular}


2.C. 3 Plañned staffing Profiles

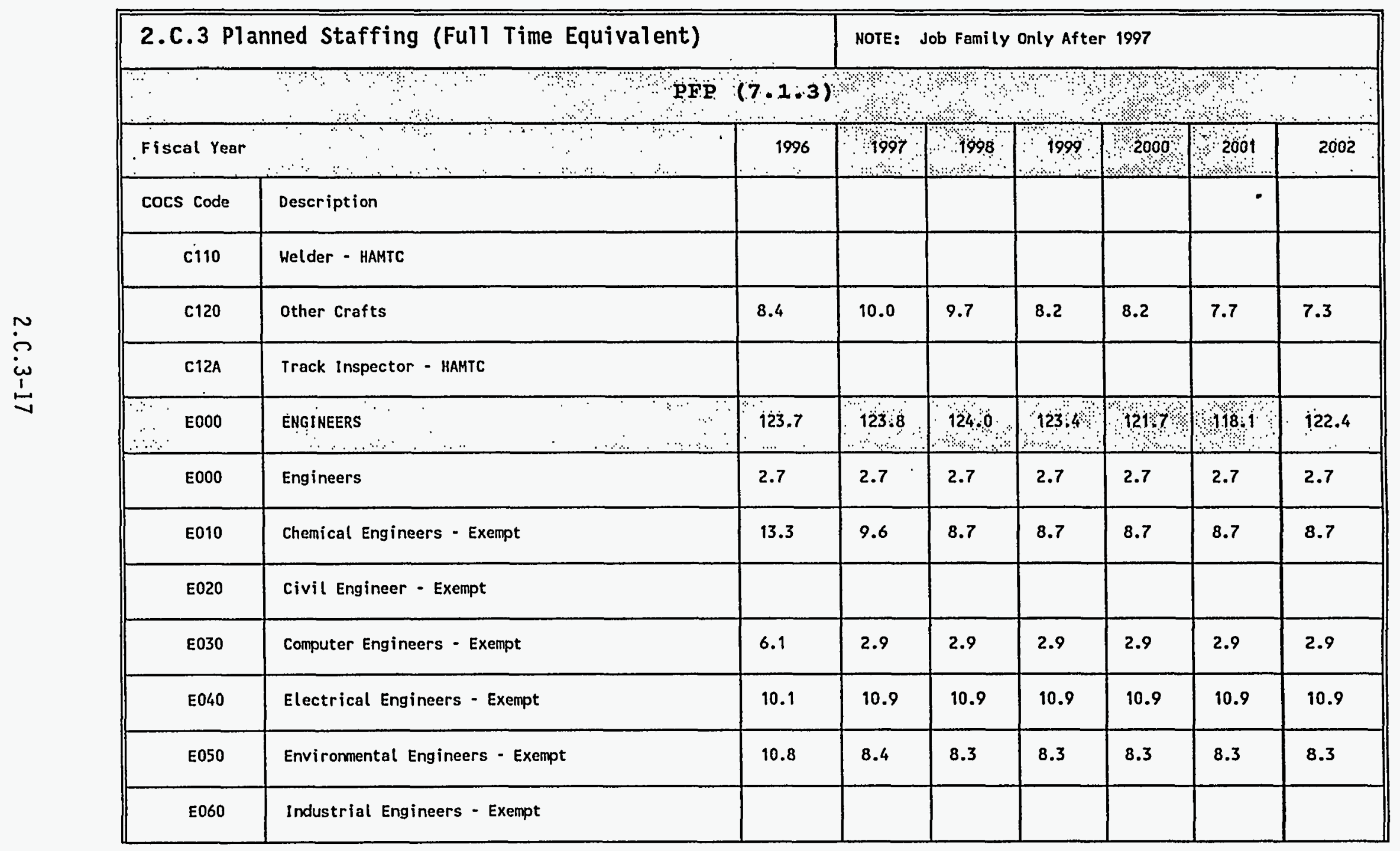


TRANSITION PROJECTS

FY 1996 MYPP

1.3.1/7.1/6.12

2.C.3 Planned staffing Profiles $\mathrm{s}$

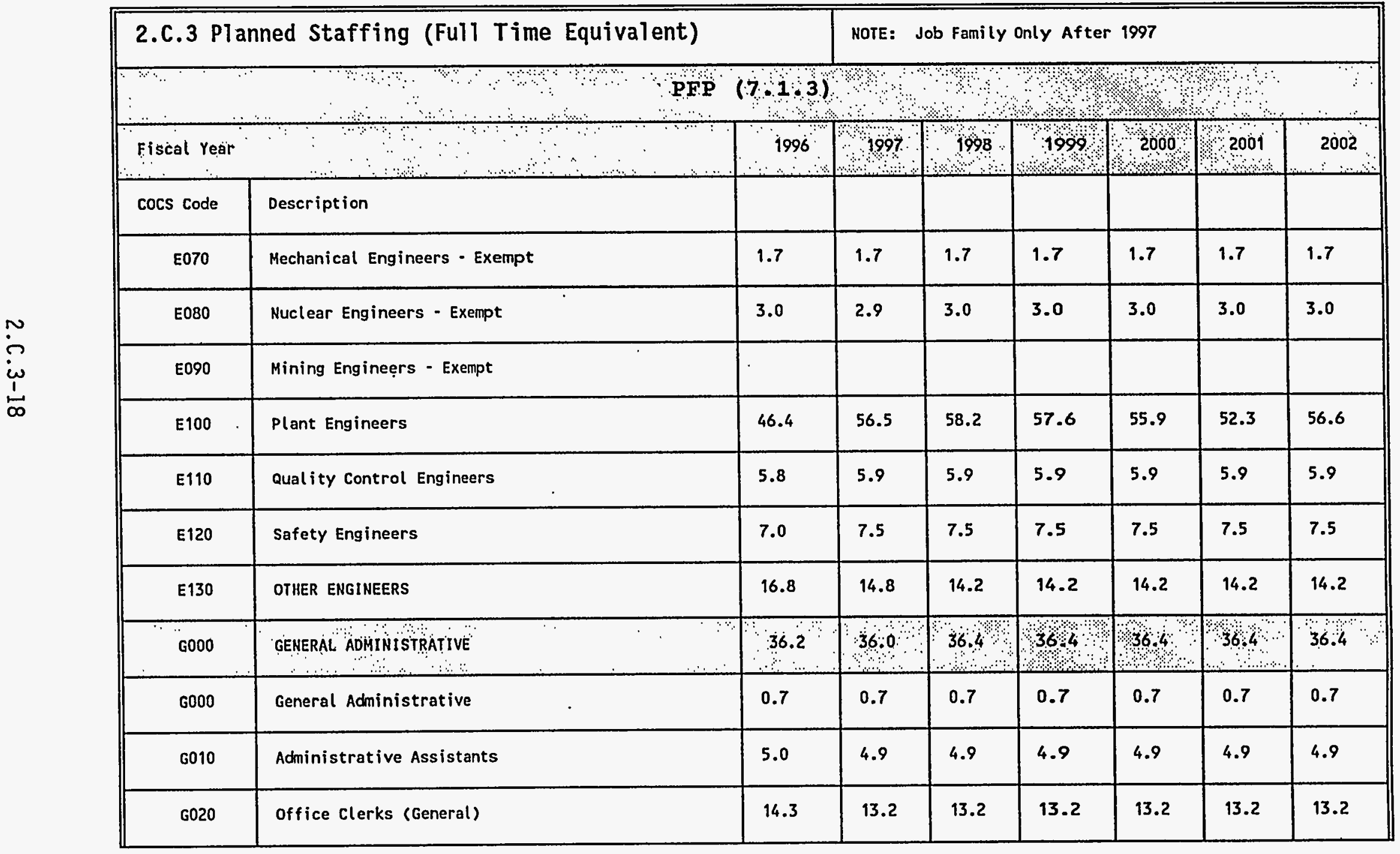


2.C.3 Planned Staffing profiles ous

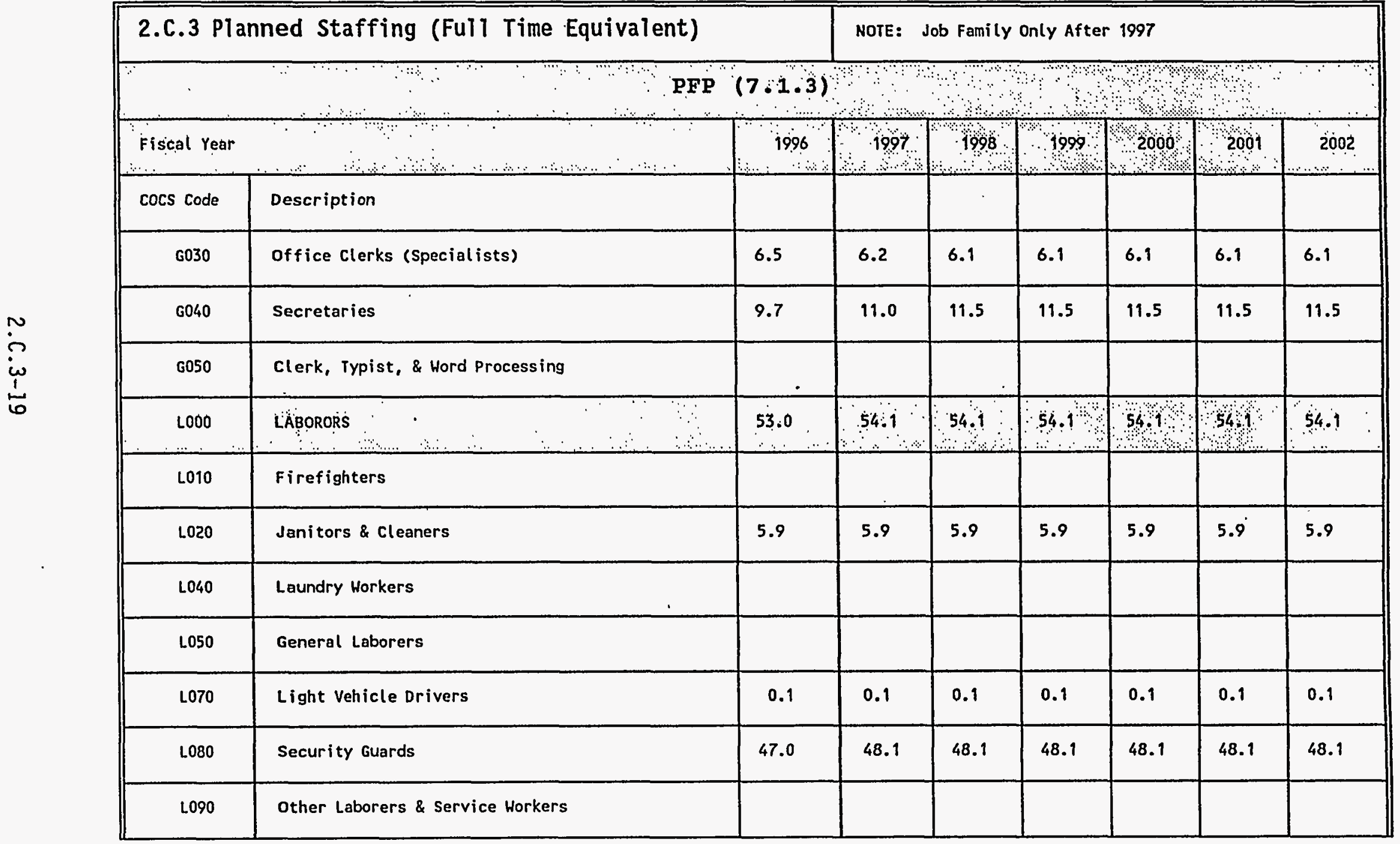




\section{TRANSITION PROJECTS}

\section{FY 1996 MYPP}

1.3.1/7.1/6.12

\section{C.3 Planned Staffing Profiles}

\begin{tabular}{|c|c|c|c|c|c|c|c|c|}
\hline \multicolumn{3}{|c|}{ 2.C.3 Planned Staffing (Full Time Equivalent) } & \multicolumn{6}{|c|}{ NOTE: Job Family Only After 1997} \\
\hline \multicolumn{9}{|c|}{ (1) $\quad$ PFP (7.1.3) } \\
\hline Fiscal Yea & & 1996 & 1997 & 1998 & 199 & 2000 & 200 & 2002 \\
\hline cocs Code & Description & & & & & & & \\
\hline$\because M 000$ & MANAGERS & 49.6 & 53.6 & $53: 2$ & 53,3 & 53.3 & 533 & 53 \\
\hline M000 & Managers & 1.0 & 1.0 & 1.0 & 1.0 & 1.0 & 1.0 & 1.0 \\
\hline M010 & First Line Supervisors & 20.0 & 18.6 & 18.2 & 18.3 & 18.2 & 18.2 & 18.2 \\
\hline M020 & Managers \& Executives & 28.6 & 28.3 & 28.3 & 28.3 & 28.3 & 28.3 & 28.3 \\
\hline M030 & Project/Program Managers & 0.0 & 5.7 & 5.7 & 5.7 & 5.7 & 5.7 & 5.7 \\
\hline$M 040$ & Project/Program Engineers & & & & & & & \\
\hline $\mathrm{P} 000$ & ADMINISTRATION \& OTHER PROFESSIONAL & $77: 0$ & 77.3 & 702 & 68.8 & 68.8 & 68.8 & $68: 8$ \\
\hline POOO & Administration \& Other Professional & 0.8 & 0.8 & 0.8 & 0.8 & 0.8 & 0.8 & 0.8 \\
\hline P010 & Accountants \& Auditors & 8.8 & 5.8 & 5.8 & 5.8 & 5.8 & 5.8 & 5.8 \\
\hline P020 & Archi tect - Exempt & & & & & & & \\
\hline P030 & Buyers/Procurement & & & & & & & \\
\hline
\end{tabular}


TRANSITION PROJECTS

FY 1996 MYPP

1.3.1/7.1/6.12

2.C. 3 Planned stäfing profiles

\begin{tabular}{|c|c|c|c|c|c|c|c|c|}
\hline \multicolumn{3}{|c|}{ 2.C.3 Planned Staffing (Full Time Equivalent) } & \multicolumn{6}{|c|}{ NOTE: Job Family Only After 1997} \\
\hline & $\therefore$ PFP & $7 \cdot 1 \cdot 3$ & $\therefore$ & $\because$ & \% & 1 & अ & \\
\hline Fiscal Year & a & 1996. & 1997 & 1998 & $\begin{array}{r}1998 \\
\end{array}$ & 2000 & 2001 & 2002 \\
\hline cocs code & Description & & & & & & & \\
\hline PO40 & Cormunications Special ists & & & & & & & \\
\hline P050 & Compliance Inspectors & 1.1 & 1.1 & 1.1 & 1.1 & 1.1 & 1.1 & 1.1 \\
\hline P060 & Computer Systems Analysts & & & & & & & \\
\hline P070 & Planner/Scheduler/Estimators & 17.6 & 15.3 & 15.1 & 15.1 & 15.1 & 15.1 & 16.1 \\
\hline P080 & Health Physicists & 0.1 & 0.1 & 0.1 & 0.1 & 0.1 & 0.1 & 0.1 \\
\hline PO90 & Coord Alara Program - Exempt & & & & & & & \\
\hline P100 & Attorney - Exempt & & & & & & & \\
\hline P110 & Personnel \& Labor Relations & & & & & & & \\
\hline P130 & Paramedic, Platoon - HAMTC & & & & . & & & \\
\hline P140 & Safeguards \& Security Spec & 7.6 & 13.7 & 6.8 & 5.4 & 5.4 & 5.4 & 5.4 \\
\hline P150 & Trainers \& Instructors & & & & & & & \\
\hline
\end{tabular}


TRANSITION PROJECTS

1.3.1/7.1/6.12

2.c 3 Planned Staffing profiles

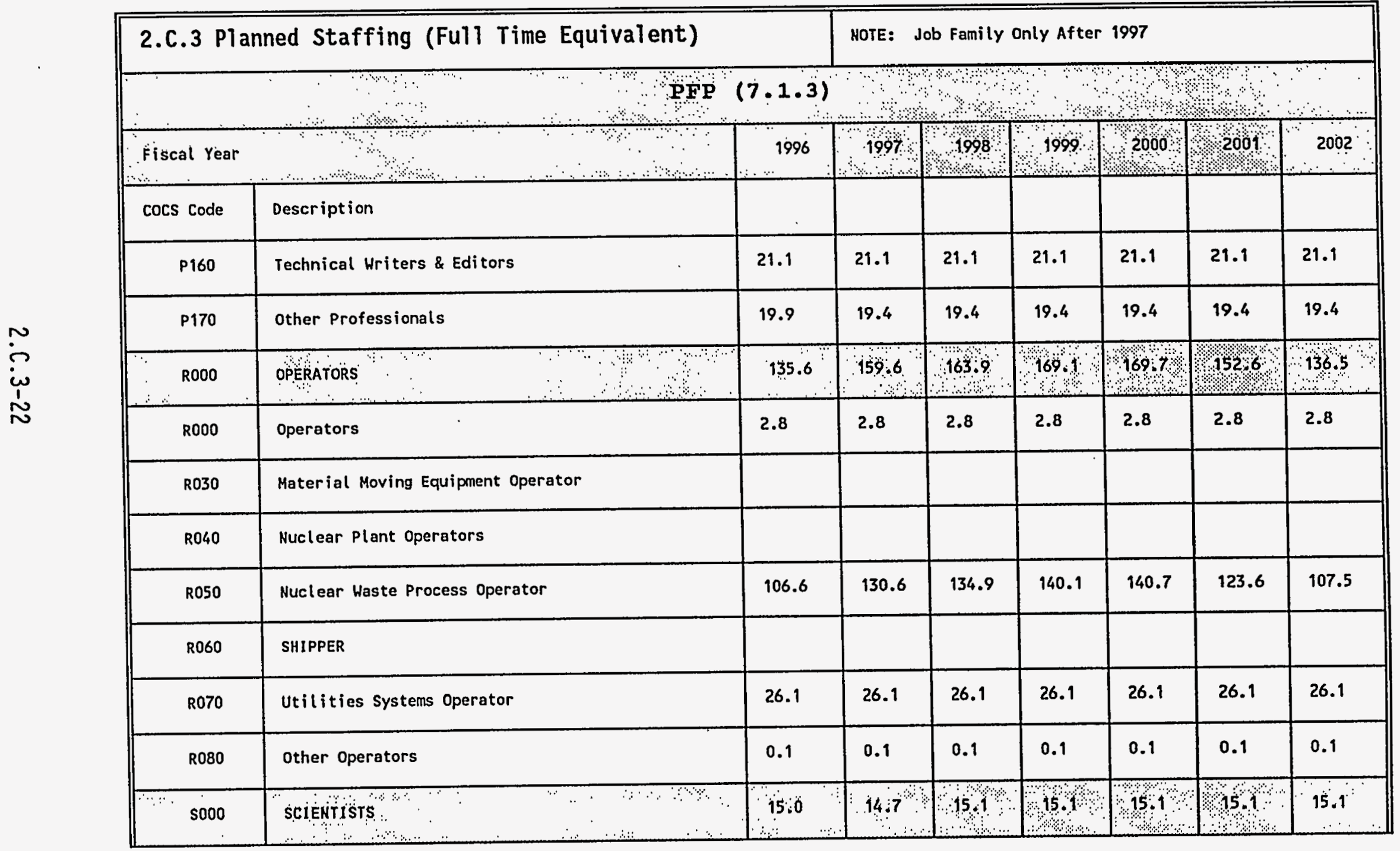


TRANSITION PROJECTS

FY 1996 MYPP

$1.3 .1 / 7.1 / 6.12$

2.C.3 Planned Staffing Profiles

\begin{tabular}{|c|c|c|c|c|c|c|c|c|}
\hline \multicolumn{3}{|c|}{ 2.C.3 Planned Staffing (Full Time Equivalent) } & \multicolumn{6}{|c|}{ NOTE: Job Family Only After 1997} \\
\hline & $\quad \mathrm{pFP}$ & $(7.1 .3$ & 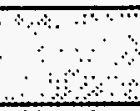 & 4 & \begin{tabular}{lll} 
& 4 \\
\hdashline & 4 \\
4 & 4 \\
\end{tabular} & में & $\begin{array}{l}8 \% \\
\end{array}$ & \\
\hline Fiscal Year & $\begin{array}{r}\quad \\
\ldots\end{array}$ & 1996. & 1997 & 1998 & $\begin{array}{r}1999 \\
\end{array}$ & 2000 & 2001 & 2002 \\
\hline cocs code & Description & & & & & & & \\
\hline s000 & Scientists & 0.4 & $0.4:$ & 0.4 & 0.4 & 04 & 0,4 & 0.4 \\
\hline s010 & Chemists - Exempt & 1.1 & 0.1 & 0.1 & 0.1 & 0.1 & 0.1 & 0.1 \\
\hline so20 & Environmental Scientists - Exempt & 0.9 & 0.9 & 0.9 & 0.9 & 0.9 & 0.9 & 0.9 \\
\hline s030 & Geologists - Exempt & & & & & & & \\
\hline s040 & Life Scientists - Exempt & & & & & & & \\
\hline s050 & Materials Scientists - Exempt & & & & & & & \\
\hline s060 & Mathematicians - Exempt & & & & & & & \\
\hline s070 & Physicists - Exempt & 1.1 & 1.9 & 1.4 & 1.1 & 1.1 & 1.1 & 1.1 \\
\hline s080 & Social - Exempt & & & & & & & \\
\hline S090 & Other Scientists - Exempt & 11.5 & 11.4 & 12.3 & 12.3 & 12.3 & 12.3 & 12.3 \\
\hline T000 & TECHNICIANS & $81: 3$ & 875 & 93 ? & $93.1+$ & 93.7, & 89,7 & 898 \\
\hline
\end{tabular}


1.3.1/7.1/6.12

2.C. 3 Planned Staffing Profiles $\quad$ -

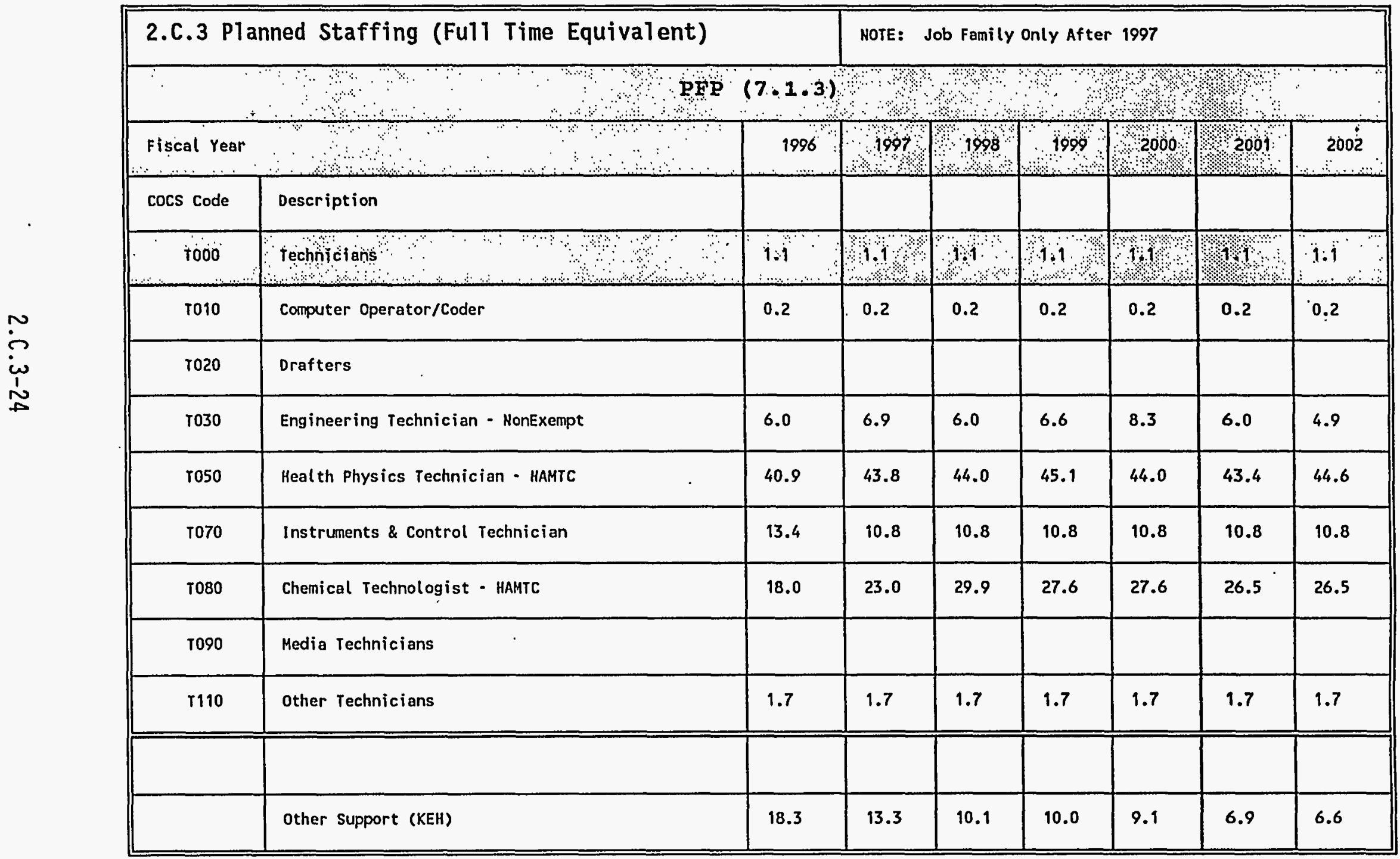




\section{C.3 Planned Staffing Profiles}

\begin{tabular}{|c|c|c|c|c|c|c|c|c|}
\hline \multicolumn{3}{|c|}{ 2.C.3 Planned Staffing (Full Time Equivalent) } & \multicolumn{6}{|c|}{ NOTE: Job Family Only After 1997} \\
\hline & $\therefore \quad$ PROGRAM AND ENVIRONMEI & NTAL M & NAGEME & NTP 17 & $16)$ & +1 & अि: & \\
\hline Fiscal Year & $\therefore$ & 1996 & $\therefore 1997$ & 1998 & 1999 & 2000 & 2001 & 2002 \\
\hline cocs Code & Description & & & & & & & \\
\hline$\because \quad c 000$ & CRAFTS & & @। & ? & 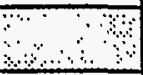 & ২০। & 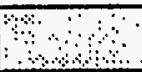 & $\cdots \because$ \\
\hline $\operatorname{co10}$ & Carpenters & & & & & & & \\
\hline $\mathrm{CO20}$ & Electricians & .4 & .4 & .4 & .4 & .4 & .4 & .4 \\
\hline $\mathrm{CO} 40$ & Machinists & & & & & & & \\
\hline $\cos 0$ & Masons & & & & & & & \\
\hline $\mathrm{cos0}$ & Millwrights & & & & & & & \\
\hline $\operatorname{coto}$ & Painters & & & & & & & \\
\hline $\cos$ & Plumbers \& Pipefitters & & & & & & & \\
\hline $\mathrm{CO90}$ & Structural \& Metal Workers & & & & & & & \\
\hline c100 & Vehicle/Equipment Mechanics & & & & & & & \\
\hline c110 & Welder - HAMTC & & & & & & & \\
\hline C120 & other Crafts & & & & & & & \\
\hline C12A & Track Inspector - HAMTC & & & & & & & \\
\hline$E 000$ & ENGINEERS & $\because \because$ & & मा & $\begin{array}{lll} & \vdots \\
\end{array}$ & & + & $\because \div$ \\
\hline E010 & Chemical Engineers - Exempt & 4.3 & 1.6 & 0.9 & 0.9 & 0.9 & 0.9 & 0.9 \\
\hline$\varepsilon 020$ & Civil Engineer - Exempt & & & & & & & \\
\hline E030 & Computer Engineers - Exempt & 2.1 & 2.1 & 1.9 & 1.9 & 1.9 & 1.9 & 1.9 \\
\hline$E 040$ & Electrical Engineers - Exempt & 2.1 & 2.9 & 2.9 & 2.9 & 2.9 & 1.9 & 1.9 \\
\hline
\end{tabular}


TRANSITION PROJECTS

FY 1996 MYPP

1.3.1/7.1/6.12

2.C.3 Planned Stäfing Profíes

\begin{tabular}{|c|c|c|c|c|c|c|c|c|}
\hline \multicolumn{3}{|c|}{ 2.C.3 Planned Staffing (Full Time Equivalent) } & \multicolumn{6}{|c|}{ NOTE: Job Family Only After 1997} \\
\hline$\because$ & PROGRAM AND ENVIRON & NAT i & NAGEME & 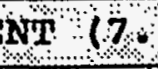 & $16)$ & +3 & - & \\
\hline Fiscal Year & $\therefore$ & 1996 & $\because 1997$ & 1998 & 1999 & 2odon: & 2001 & 2002 \\
\hline cocs Code & Description & & & & & & & \\
\hline E050 & Environmental Engineers - Exempt & & & & & & & \\
\hline E060 & Industrial Engineers - Exempt & & & & & & & \\
\hline E070 & Mechanical Engineers - Exempt & 1.0 & 1.0 & 1.0 & 1.0 & 1.0 & 1.0 & 1.0 \\
\hline E080 & Nuclear Engineers - Exempt & & & & & & & \\
\hline E090 & Mining Engịneers - Exempt & & & & & & . & \\
\hline E100 & Plant Engineers & 3.5 & 4.5 & 4.5 & 4.5 & 2.5 & 2.5 & 2.5 \\
\hline E110 & Quality Control Engineers & & & & & & & \\
\hline E120 & Safety Engineers & & & & & & & \\
\hline - E130 & OTHER ENGINEERS & 2.0 & 2.0 & 2.0 & 2.0 & 2.0 & 2.0 & 2.0 \\
\hline$\because \mathrm{GOOOO}$ & GENERAL ADHINISTRAT IVE & $\therefore$ & $\therefore$ & 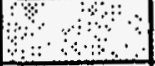 & 2 & - & अ४ & $\because \because \quad \therefore$ \\
\hline 6010 & Administrative Assistants & 1.0 & 1.0 & 1.0 & 1.0 & 1.0 & 1.0 & 1.0 \\
\hline G020 & office Clerks (General) & 3.0 & 3.0 & 3.0 & 3.0 & 3.0 & 3.0 & 3.0 \\
\hline G030 & office clerks (Specialists) & & & & & & & \\
\hline G040 & Secretaries & 2.0 & 2.0 & 2.0 & 2.0 & 2.0 & 2.0 & 2.0 \\
\hline G050 & Clerk, Typist, \& Hord Processing & & & & & & & \\
\hline LO00 & LABOROQRS & & $\because$ & $\begin{array}{lll} & \ddots \\
\end{array}$ & कि & Yै & कै & $\begin{array}{ll}\ddots \\
\therefore \\
\cdots\end{array}$ \\
\hline L.010 & Firefighters & & & & & & & \\
\hline L020 & Janitors \& Cleaners & & & & & & & \\
\hline
\end{tabular}


2. C. 3 Planned Stäffing profiles

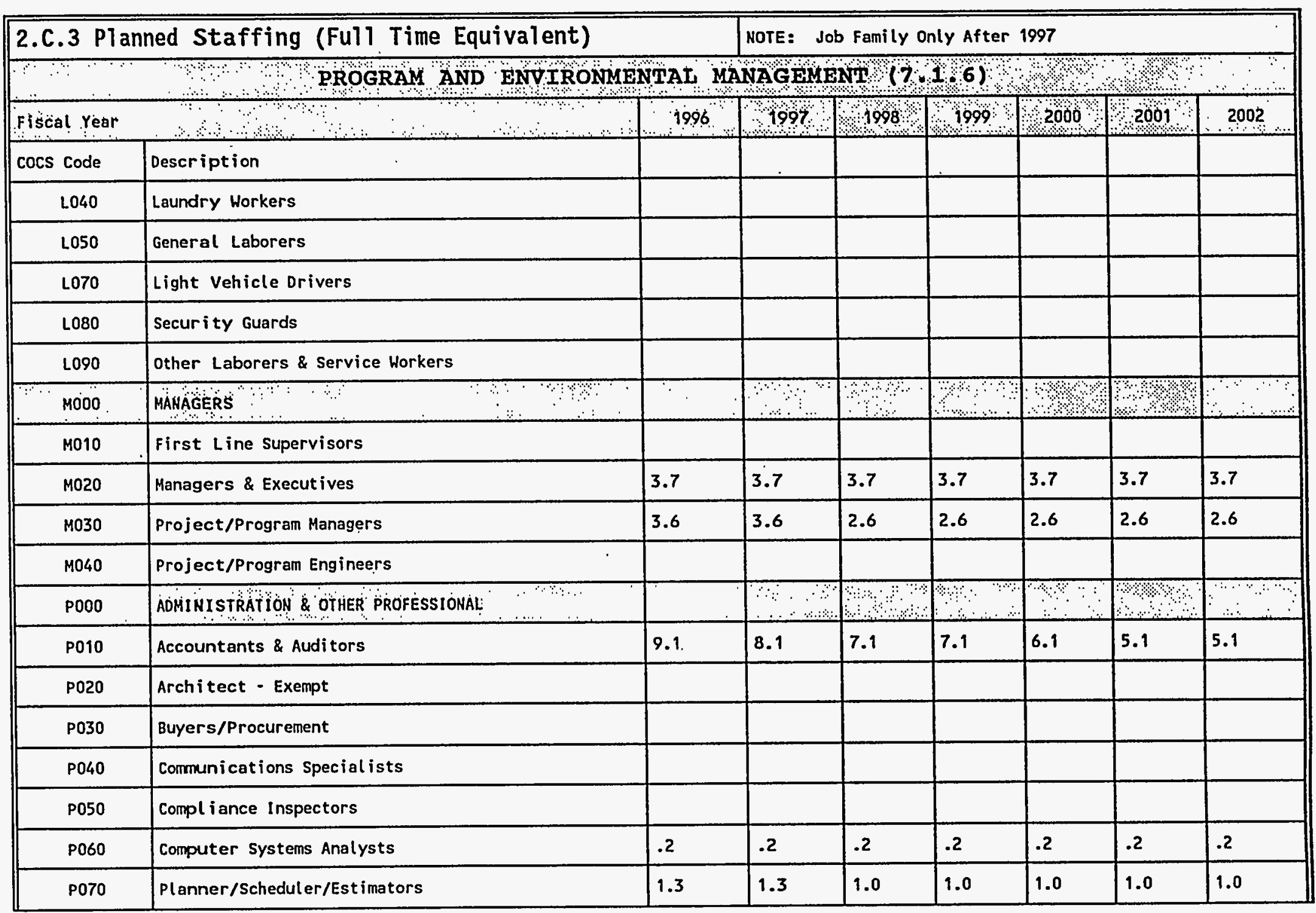


TRANSITION PROJECTS

FY 1996 MYPP

1.3.1/7.1/6.12

2.C.3 Planned Staffing Proffles $\quad$ os

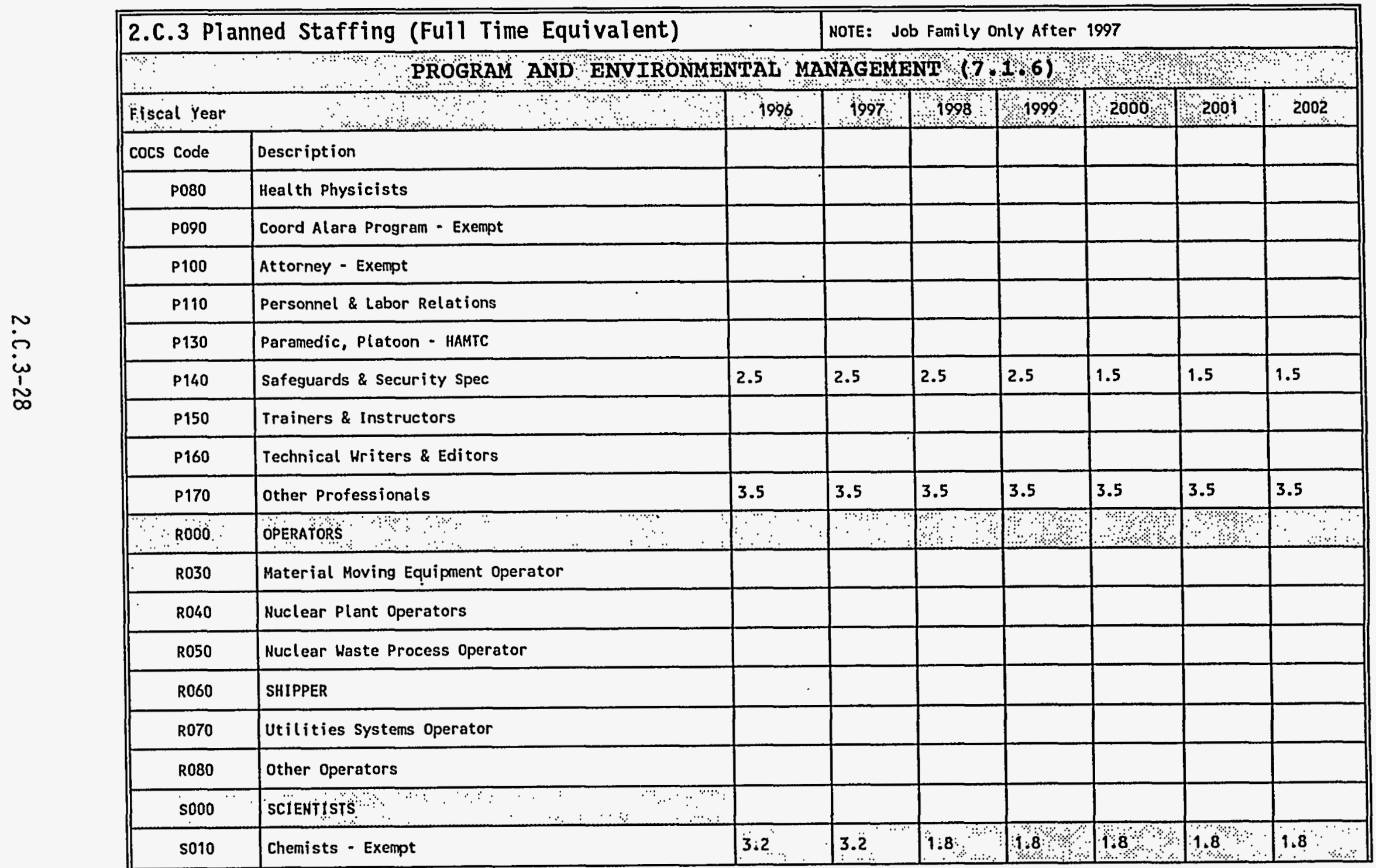




\section{3 Planned staffing Profiles}

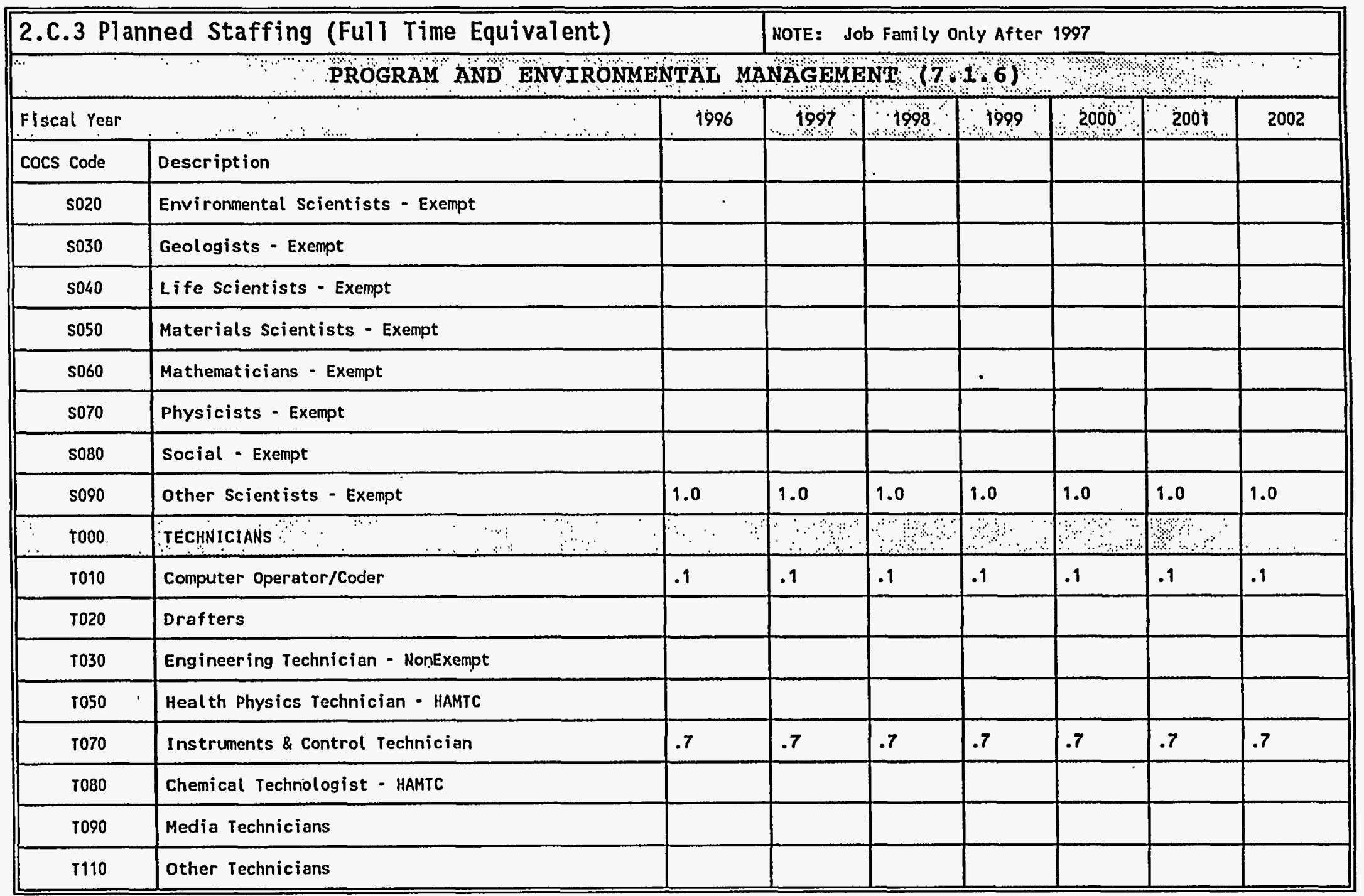




\section{A Technicaj objectives:}

\section{B Plant (1.3.1.7)}

\section{B.2 Program Objectives}

Key near term objectives have been selected that are designed to aid in the fulfillment of the top level

objectives. These objectives, organized by Work Breakdown Element, are as follows:

\section{TRANSITION}

- Complete the design, procurement and construction to allow the transfer of organics out of the canyon.

- Complete the transfer of organics out of the canyon.

- Complete Engineering Studies, preparation of Functional Design Requirements, Conceptual Design, and Definitive Design for steam replacement in B Plant.

- Prepare procedures and complete training needed to implement the Interim Safety Basis documentation.

- Complete decontamination of the areas south of the 207-B basin, the 221-BB roof, the outdoors areas near the east end of B Plant, and the B-216-59 pump pit area.

- Complete the Fire Hazard Analysis needed to deactivate the B Plant sand filter.

- Complete the compliance review and place $E$ filter in service.

- Complete the Engineering studies, Functional Design Documentation, and Conceptual Design for decoupling the WESF Low Level Waste system from B P1 ant.

- Complete the Engineering studies, Functional Design Documentation, and Conceptual Design for decoupling the WESF Solid Waste system from B Plant.

- Complete the Engineering studies, Functional Design Documentation, and Conceptual Design for decoupling the WESF Deionized water system from B Plant.

- Complete the Engineering studies, Functional Design Documentation, and Conceptual Design for decoupling the WESF 1 iquid effluents control system from B Plant.

- Complete the Project W-252 Operability Test Procedure preparation.

- Issue the Project W-252 Facility Effluent Monitoring Plan and the Operating Specification Document.

- Complete the Project W-059 Value Engineering study, the Preliminary Fire Hazard Analysis, the Site Survey, the Program Management Plan, and the Quality Assurance Project Plan.

B PLANT SURVEILLANCE AND MAINTENANCE

- Perform surveillances of systems and structures needed to maintain B Plant in a safe mode.

- Maintain the Lock and Tag program.

- Perform solid, liquid, and gaseous waste and effluent sampling.

- Provide maintenance of systems at B PTant including electrical, raw and sanitary water, steam, compressed air, HVAC, fire protection, stack monitoring and other instrumentation.

- Perform analyses of safety issues and prepare and issue safety documentation.

- Develop new and update current operational and maintenance procedures to support facility evolutions.

WESF OPERATIONS, MAINTENANCE, AND UPGRADES

- Provide surveillance of systems and structures as needed to assure the facility is maintained in a safe - mode. 


\section{A Technical Öbjectives}

- Provide maintenance of needed systems including electrical, raw and sanitary water, compressed air, HVAC, fire protection, effluent monitors, and radiation monitors.

- Develop and maintain needed surveitiance and maintenance procedures.

- Develop and maintain safety documentation including the Interim Safety Basis documentation.

- Complete upgrades to the Pool cell pump system, the weight factor loop, the pool cell temperature instrumentation, the beta monitoring system and the heat exchanger.

- Provide Project $W-452$ support for completion of definitive design.

- Provide Project $W-450$ support for NEPA, safety, and functional design. Support establishment of performance specifications and initiation of definitive design.

- Provide design, procurement, and construction of the $A$ cell hood.

REGULATORY COMPLIANCE

- Prepare an Standards and Requirements Identification Document (S/RID) for B Plant and WESF.

- Assess compliance with the B Plant / WESF S/RID.

- Prepare and submit a RCRA Part A permit application to Ecology.

- Revise and issue the TSD Inspection PIan for B PIant.

- Submit the B PI ant Tank Integrity Assessment report to DOE-RL.

$\omega$ - Complete and submit the annual Dangerous Waste report.

- Prepare and issue a Process History Standdown History document.

- Maintain and update Material Safety Data Sheets for B PI ant and WESF.

- Maintain the Carcinogen Control Plan.

- Prepare and issue a Hazards Communication Plan.

- Maintain pollution prevention documentation and issue quarterly and annual reports.

- Develop and issue pollution prevention goals.

\section{CESIUM RECOVERY}

- Provide procedures, plans, and preparations needed to complete the return of 25 cesium capsules from ARECO in Lynchburg, Virginia to WESF for storage. Complete closeout documentation and exit the facility without incident.

- Provide for the surveillance of formerly leased cesium capsules at PNL.

- Maintain the NRC and DOE Certificates of Compliance (CoC) for the Beneficial Uses Shipping System (BUSS) cask.

- Complete annual maintenance of the BUSS cask and transport trailer and maintain them in a state of readiness for use.

- Develop an uitrasonic testing process to qualify the outer welds of capsules to be reencapsulated in FY98.

- Establish the quality assurance of existing inventory of inner and outer capsule spares so they can be used for reencapsulation in FY98.

\section{PROGRAM MANAGEMENT AND ADMINISTRATION}

- Prepare and submit an annual budget request in the form of Activity Data Sheets and comprehensive MultiYear Program Plans which establish the scope, schedule, and cost baseline for the program.

- Maintain program baseline management including schedules, budgets, and reports. 


\section{A Technical Objectives}

- Provide training services. Complete updating the training packages for the Net 90 Quals, Phase I Certifications and Canyon Sampling. Initiate updating the training packages for Management Certifications, Manipulator Operations, and Inside and Outside Surveiliances.

PUREX/U0 ${ }_{3}(7.1 .1)$

\section{Surveillance and Maintenance:}

Continue S\&M of the remaining active systems at PUREX throughout the year. System deactivations will decrease S\&M requirements. Late in FY 1996, completion of canyon deactivation and PR Room deactivation will cause a significant reduction in S\&M requirements.

Provide $\$ 40 \mathrm{~K}$ of funding, within this ADS, for U03 S\&M in FY 1996 as agreed between EM-60 and EM-40 in the memorandum of agreement for $\mathrm{UO}_{3}$ turnover. Provide no funding for $\mathrm{UO}_{3}$ disposition.

\section{Deactivation:}

- Complete disposition of the spent reactor fuel at PUREX.

Complete stabilization of $\mathrm{N} \mathrm{Cell}$

- Complete deactivation of the PR Room.

- Complete flushing of the PUREX canyon vessels.

- Complete disposition of the PUREX organic.

- Complete deactivation of the tank farm waste lines.

Compliance:

$-$

Submit the PUREX Preclosure Work Plan including endpoint criteria and post-transition S\&M plan to Ecology and EPA.

\section{Area Fuel Supply (7.1.2)}

The overall objective of the Fuel Supply Shutdown project is to perform shutdown activities and transfer facilities to EM-40, while maintaining compliance with applicable DOE, state, and federal requirements.

- Provide required administrative, safeguard, maintenance, and surveitlances necessary to continue safe storage of 1900 MT of Special Nuclear Materials (SNM).

- Provide required surveillance to maintain RCRA compliance until closure, including safety, quality, environmental, and security requirements. Provide maintenance to meet IOSR, and code requirements, to maintain a safe work place. 


\section{TRANSITI : PROJECTS}

\section{Aechnical Objectives}

- Maintain the following programs for Fuel Supply: Conduct of Operations, Lessons Learned, Systems Engineering, Self-Assessment, Radiological Control, Fissile Material Facilities, Safety Documentation, Unresolved Safety Question (USQ), Priority Planning Grid (PPG) System, Occurrence Reporting, etc..

- Specific objectịves relating to RCRA permitted TSD Closure Activities for 304, 303K, and WATS are as foll lows:

-

-

Continue required closure plan reviews and support monthly Unit Manager Meetings with Ecology. Complete closure of 304 Concretion and 303K. Complete closure of WATS.

- Specific objectives relating to the isolation of the 313 South building (to decrease a serious safety hazard caused by an unsafe roof) are as follows:

- Complete 313 isolation of South Building access.

- Remove al1 313 South utilities.

- Remove 313 South roof loadings (i.e. HVAC units, etc.)

Complete NEPA, historical studies, and sampling/characterization activities.

Stabilize or remove unattached hazardous materials.

Stabilize or remove unattached radiologically contaminated materials.

- Transfer Fuel Supply to EM-40 by completing the following remaining shutdown activities:

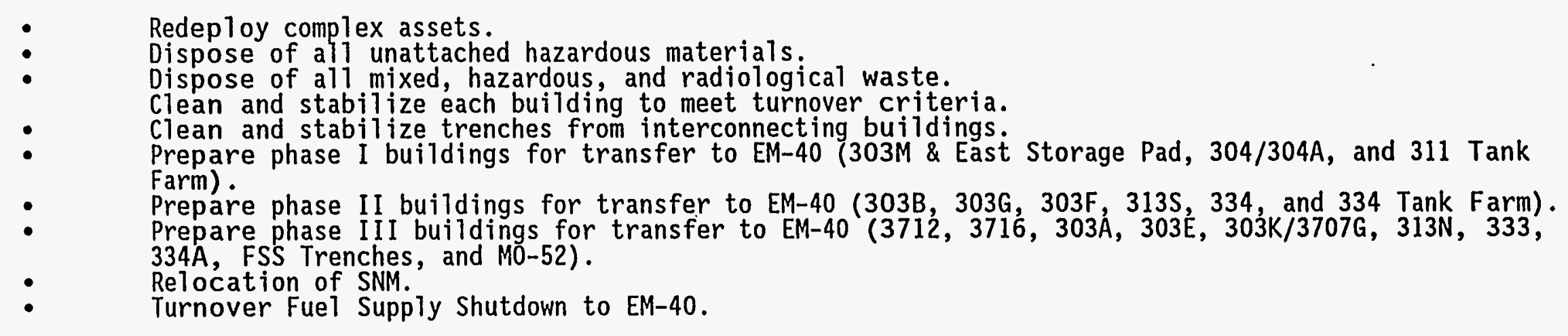

PFP (7.1.3)

The overall objective of the Plutonium Finishing Plant (PFP) Transition Project is to manage the PFP Complex to a deactivated condition while maintaining the facilities in a safe and environmentally sound condition, and 


\section{A Technical objectives}

while performing Material Management and Material Dispositioning activities as part of the deactivation process. As part of the overal1 objective plutonium vulnerability corrective actions will be undertaken. Many of the identified vulnerabilities wi 11 be corrected through the stabilization and packaging activities required by the DNFSB Recommendation 94-1 Hanford Site Integrated Stabilization Management PIan, others wil1 be corrected as a part of plutonium handing facilities transition (deactivation) to the Environmental Restoration Program and the remaining can not be fully corrected until environmental restoration is complete. It is expected that the result of the PFP EIS ROD will be consistent with these actions. Specific activities to be performed are as follows:

- Proceed with currently funded interim actions within current operational and legal requirements and complete these interim actions during $F Y$ 1996. These activities include continued stabilization of reactive ash and scrap material, continued developmental testing of vertical calcination technology (i.e., direct denitration conversion of aqueous plutonium-bearing solutions), terminal cleanout (TCO) of specific exhaust ventilation ducting, process vacuum piping, and RMA/RMC-Line gloveboxes and the installation of six (6) new thermal furnaces to support increased material stabilization following approval of the ROD. Completion of these interim actions leads to the mitigation of identified safety concerns while supporting the completion of TPA milestones on 234-5Z Ductwork Cleanout (M-83-02-T04) and 10-L Solution Downloading (M-83-02-T03).

- Complete the second annual update of the revised SAR. Various small plant capital equipment renovations and projects have been identified that would improve operations and thereby reduce future operational costs.

- Continued support to the completion of planned upgrades to the MICON Distributive Control System, Project B610 , and the Emergency Diesel Generator System, Project C-189. Completion of these projects wi1l significantly improve the operability control of HVAC and related facility support service systems and the emergency diesel generator system.

- Provide support to the implementation of a Package Steam system for the PFP Complex. This will enable the PFP Complex to generate its own steam based on need and disconnect the facility from the central Hanford steam distributive system. This is anticipated to reduce the overall costs for steam for the facility in subsequent years.

- Provide the necessary project management and engineering support to path forward planning required for full implementation of DNFSB Recommendation 94-1 at PFP. This includes project management and engineering support to thermal stabilization activities, direct denitration conversion testing on solutions, polycube stabilization forward planning \& development, TCO planning and new packaging system \& Laboratory upgrades.

- Provide support as required to the issuance of the Draft PFP Cleanup EIS and subsequent Record of Decision.

- Work to develop a Part B Permit Application, or a RCRA Closure Plan, or a Preclosure work plan for the 241-Z TSD units. 


\section{A Techinical objectives}

- Complete air emissions inventories and provide information as necessary to support the Hanford Site Air Operating Permit required by the CAAA.

- Receive Fast Flux Test Facility (FFTF) green fuel for storage at PFP. Funding for modifications and transfer are included in the Advanced Reactor program.

\section{Program and Environmental Management (7.1.6)}

The overal 1 objective of the Program and Environmental Management program element is to provide management support for resources and activities that are common to all Transition Projects plants.

The Program and Environmental Management program element consists primarily of level-of-effort tasks which are repetitive from year to year. These tasks are detailed in Section 1.B.2, Program objectives and, thus, are not repeated here. 


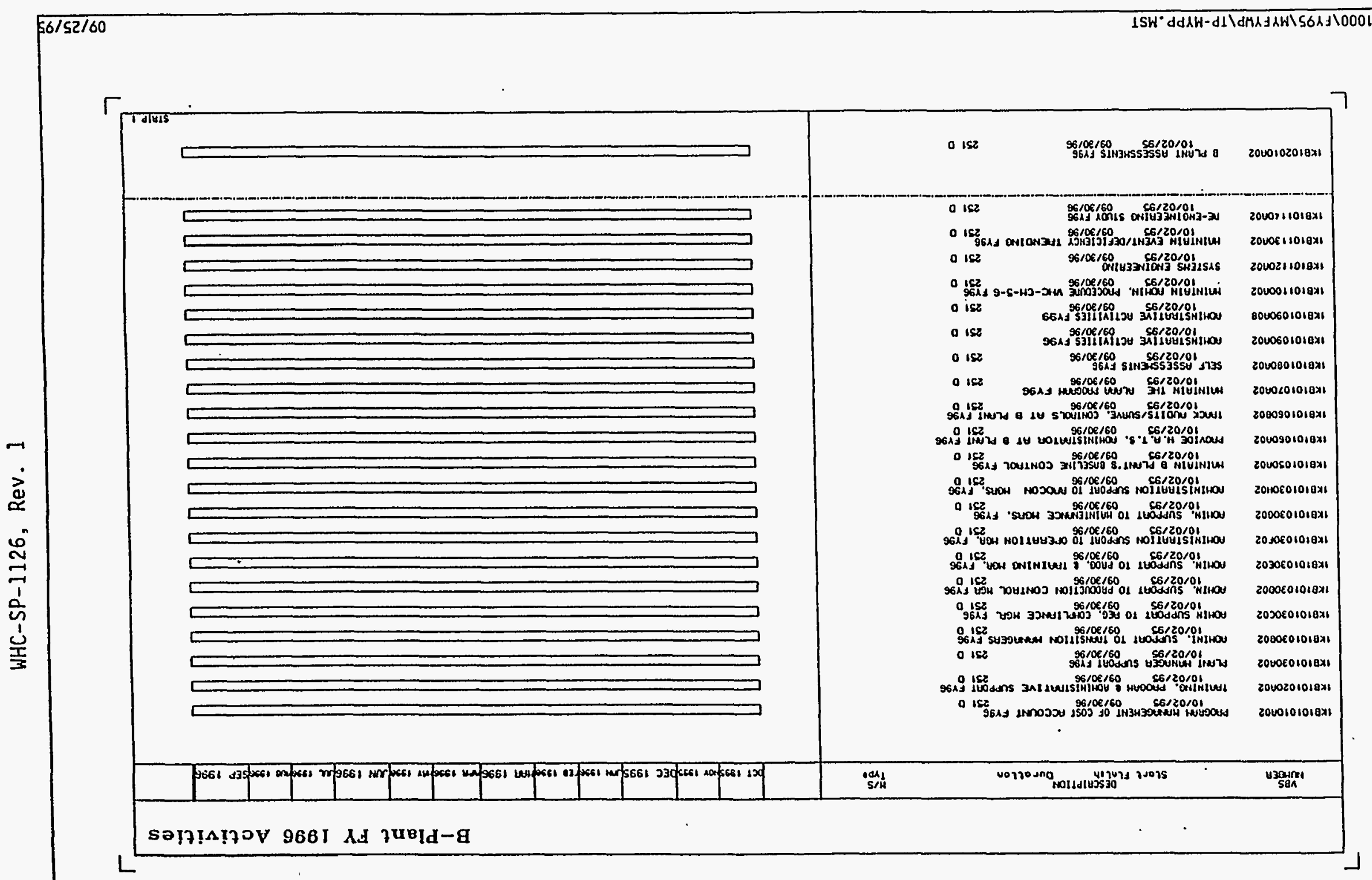




\section{TRANSITION PROJECTS}

FY 1996 MYPP

\subsection{1/7.1/6.12}

\section{B Program Performance Baseline Schedule}

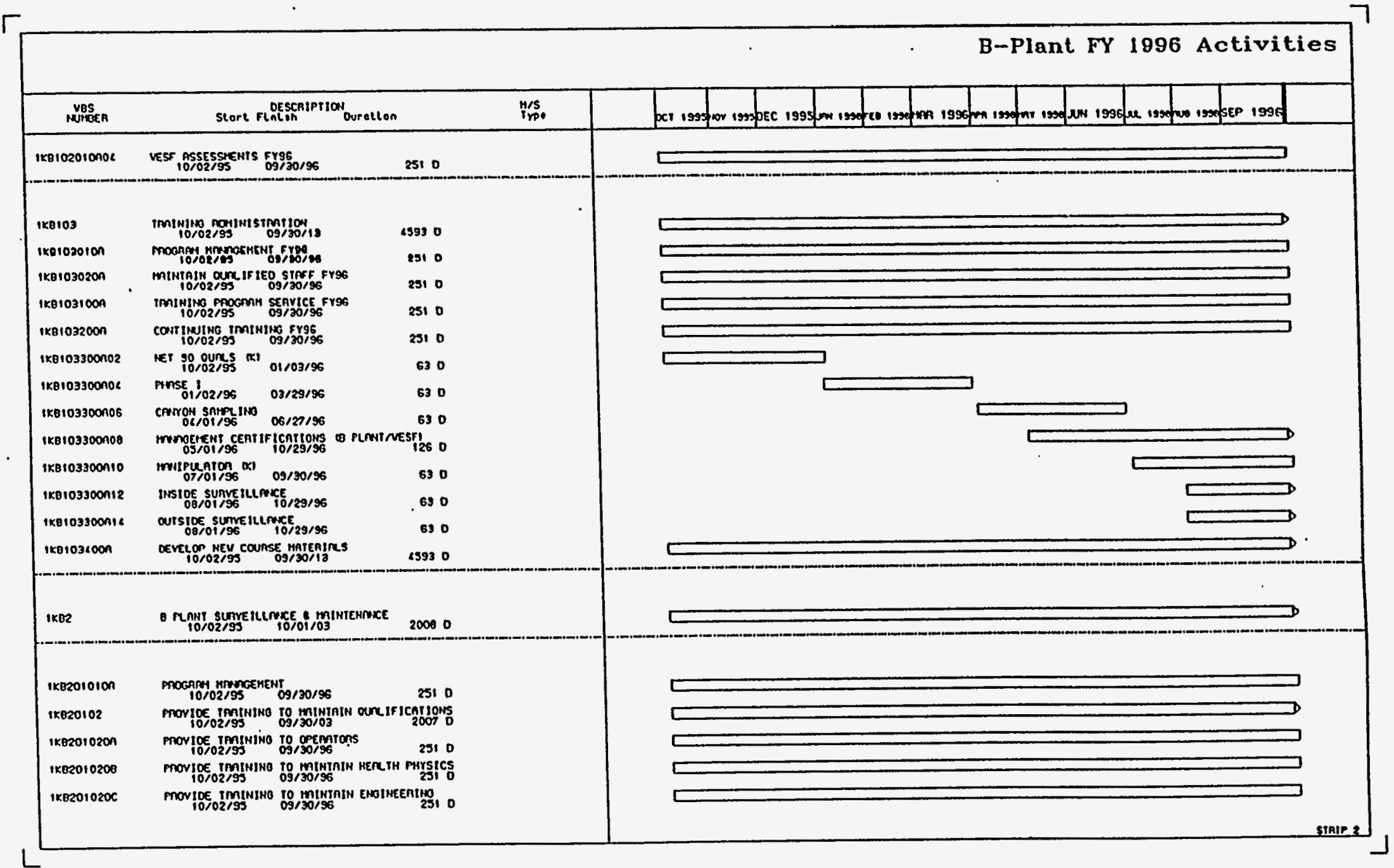


WHC-SP-1126, Rev. I

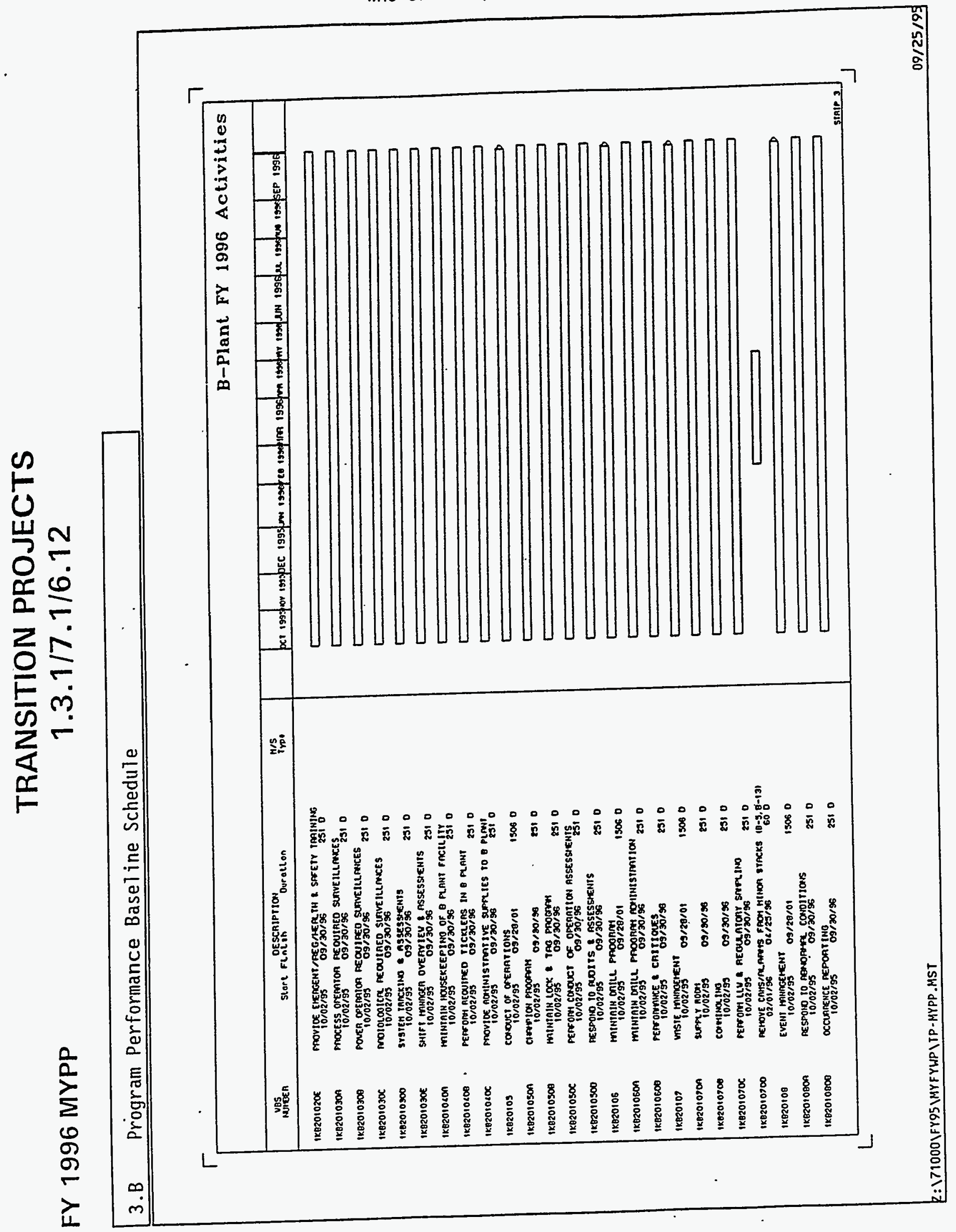


TRANSITION PROJECTS

FY 1996 MYPP

1.3.1/7.1/6.12

3.B Program Performance Baseline Schedule

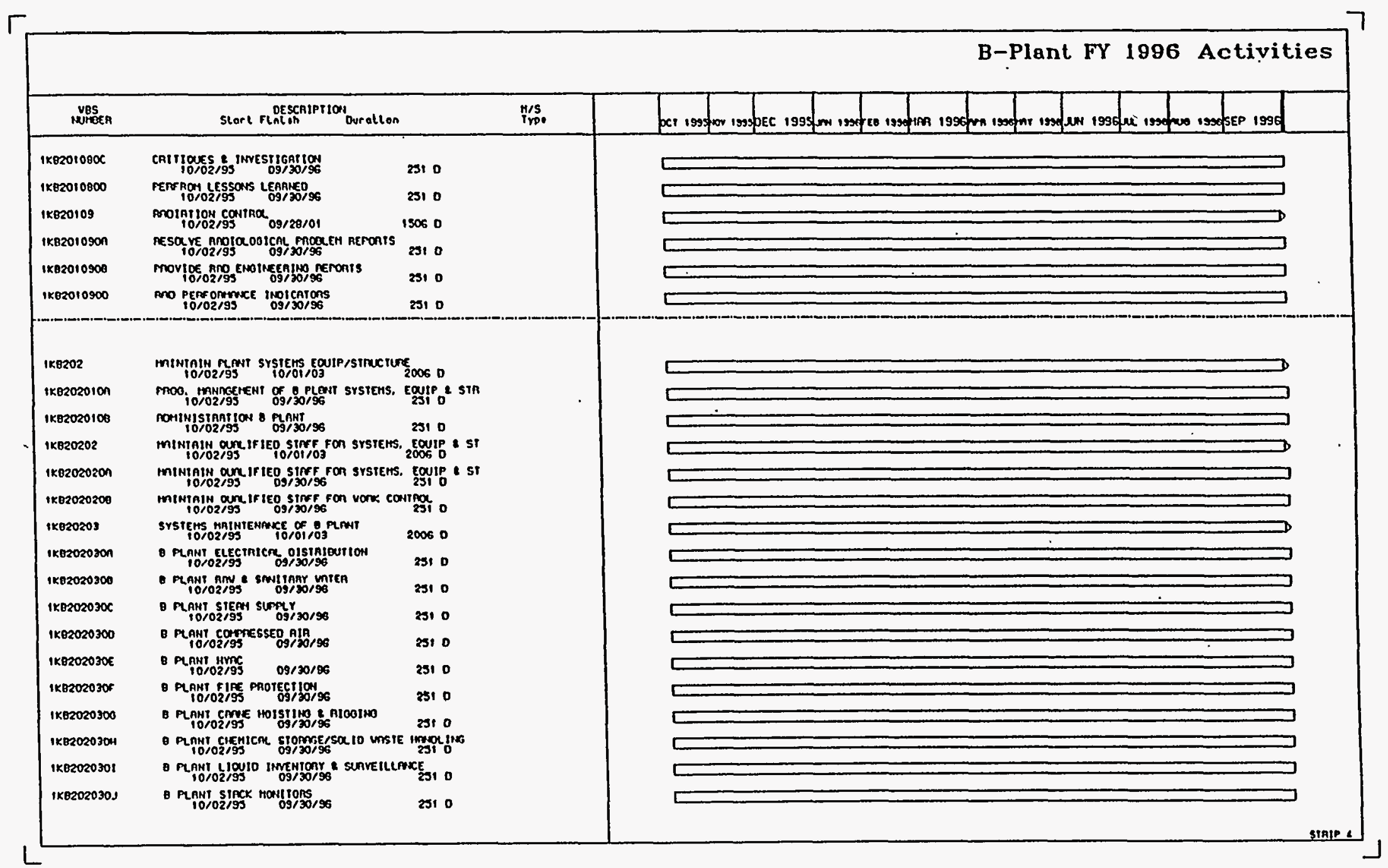




\section{FY 1996 MYPP}

\section{TRANSITION PROJECTS}

\subsection{1/7.1/6.12}

\section{B Program Performance Baseline Schedule}

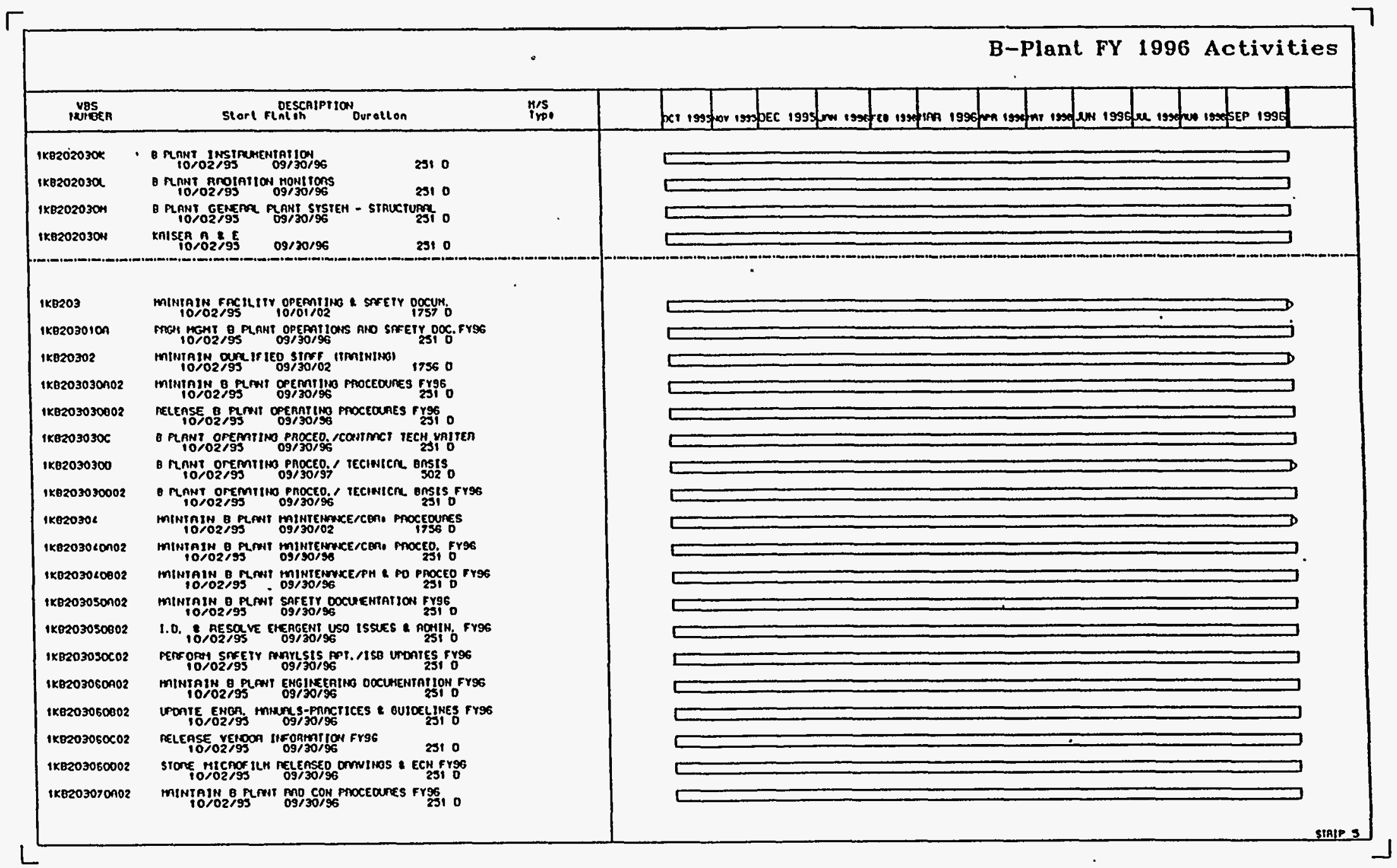


TRANSITION PROJECTS

FY 1996 MYPP

1.3.1/7.1/6.12

3.B Program Performance Baseline Schedule

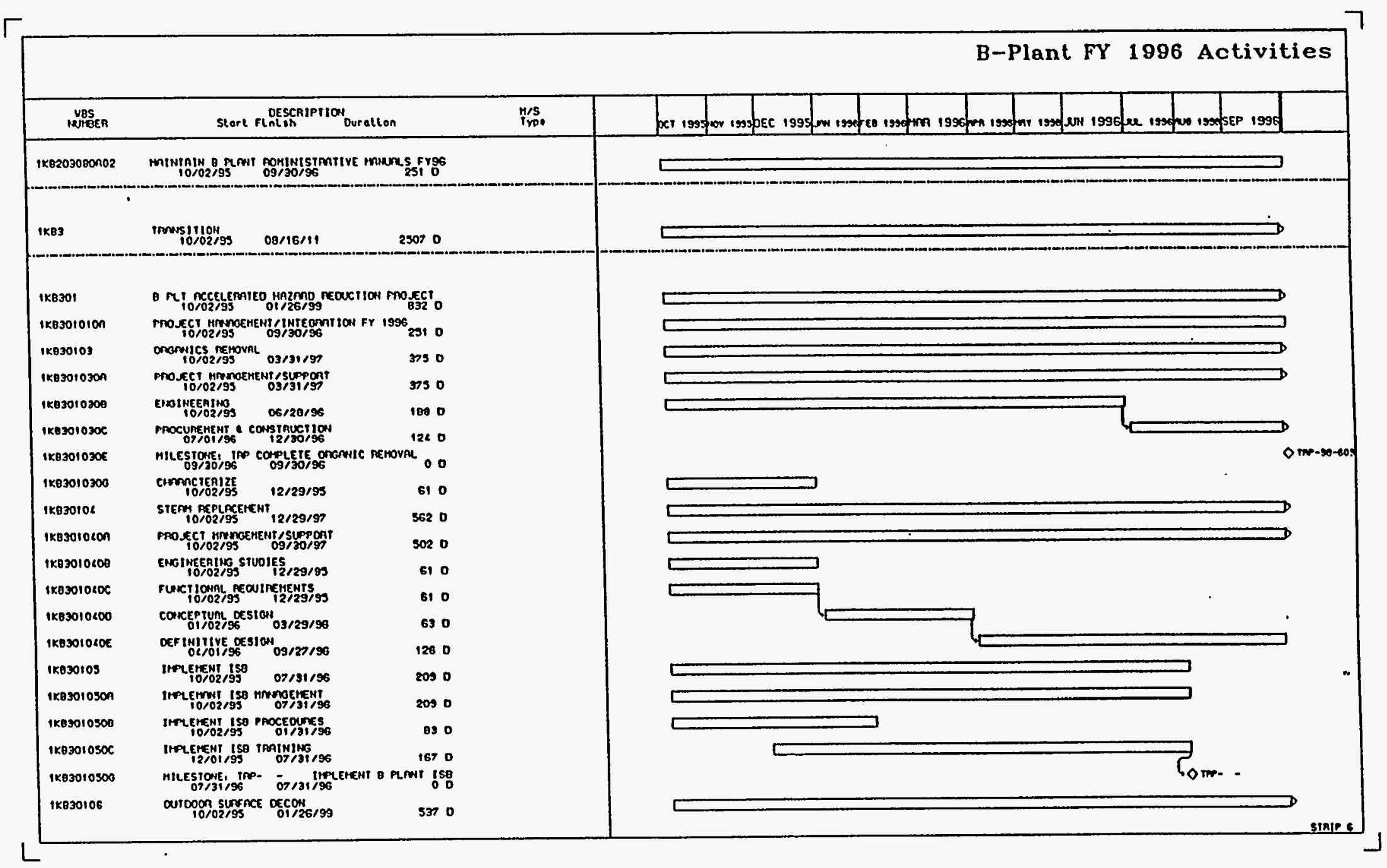




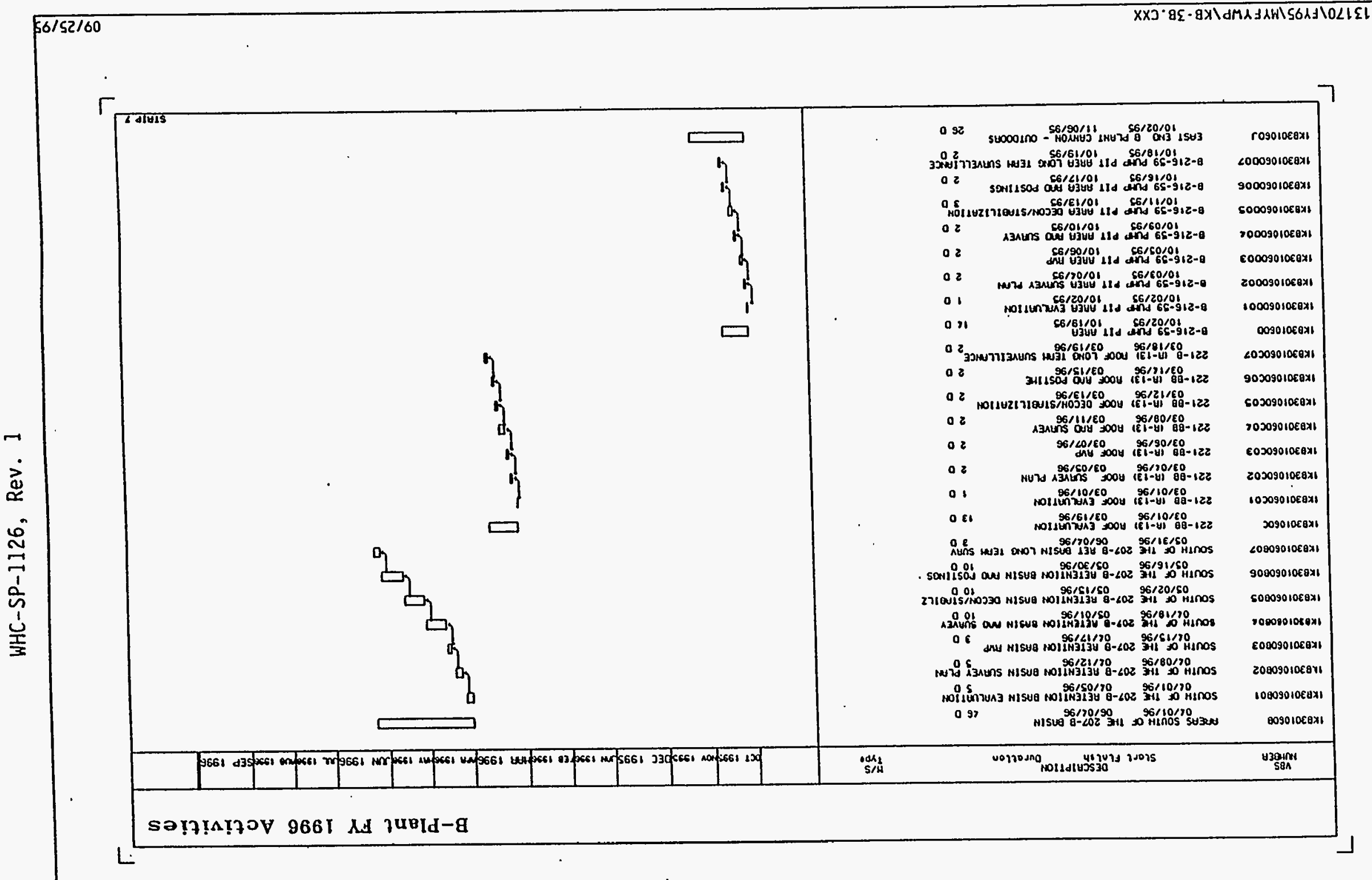

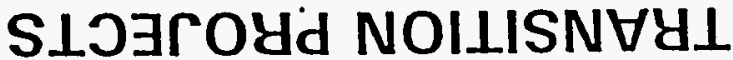




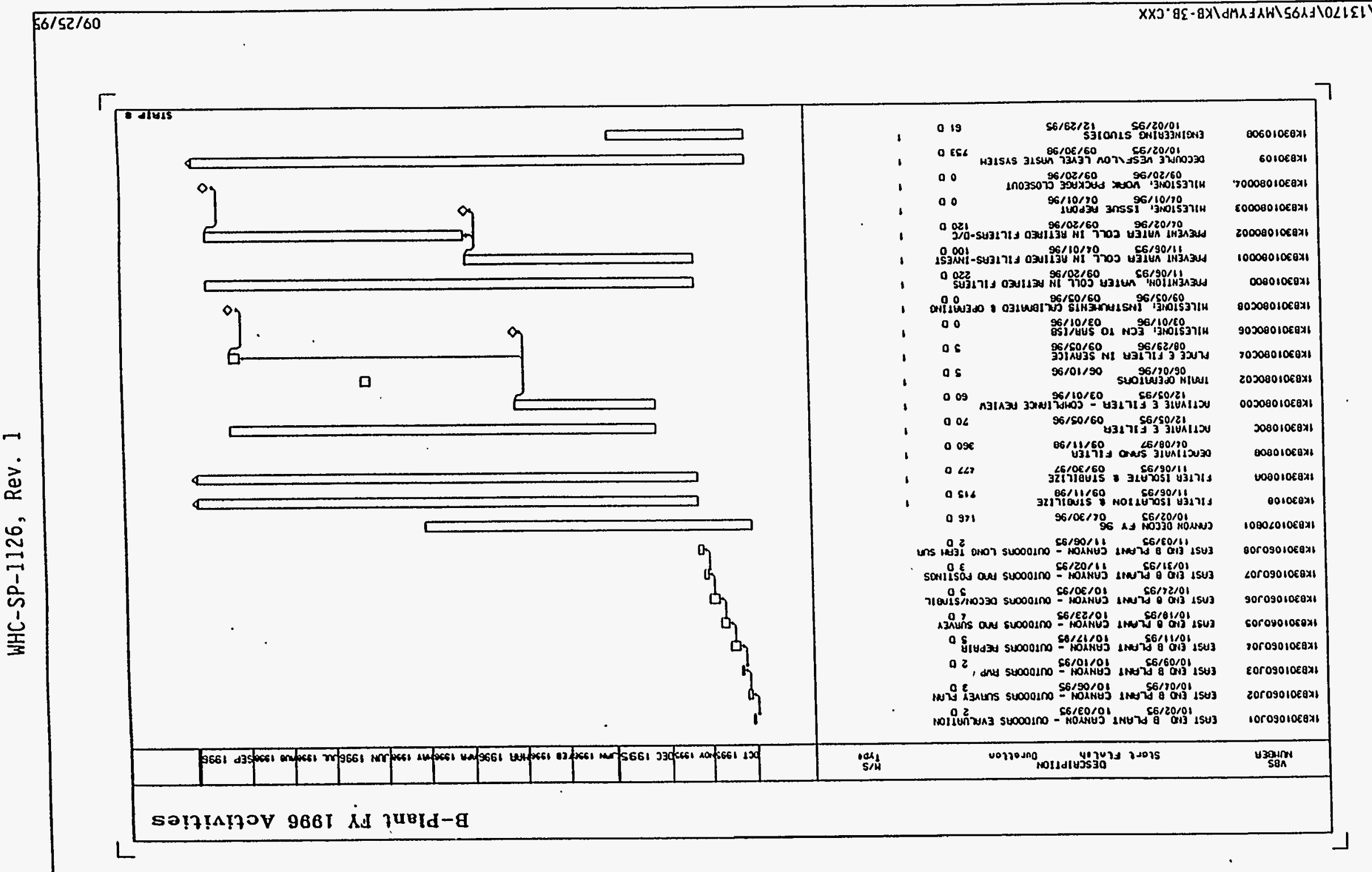

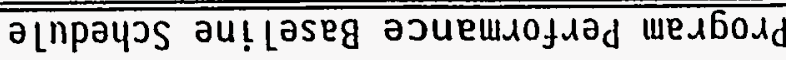


FY 1996 MYPP

\section{TRANSITIUN PROJECTS}

\subsection{1/7.1/6.12}

3.B Program Performance Baseline Schedule

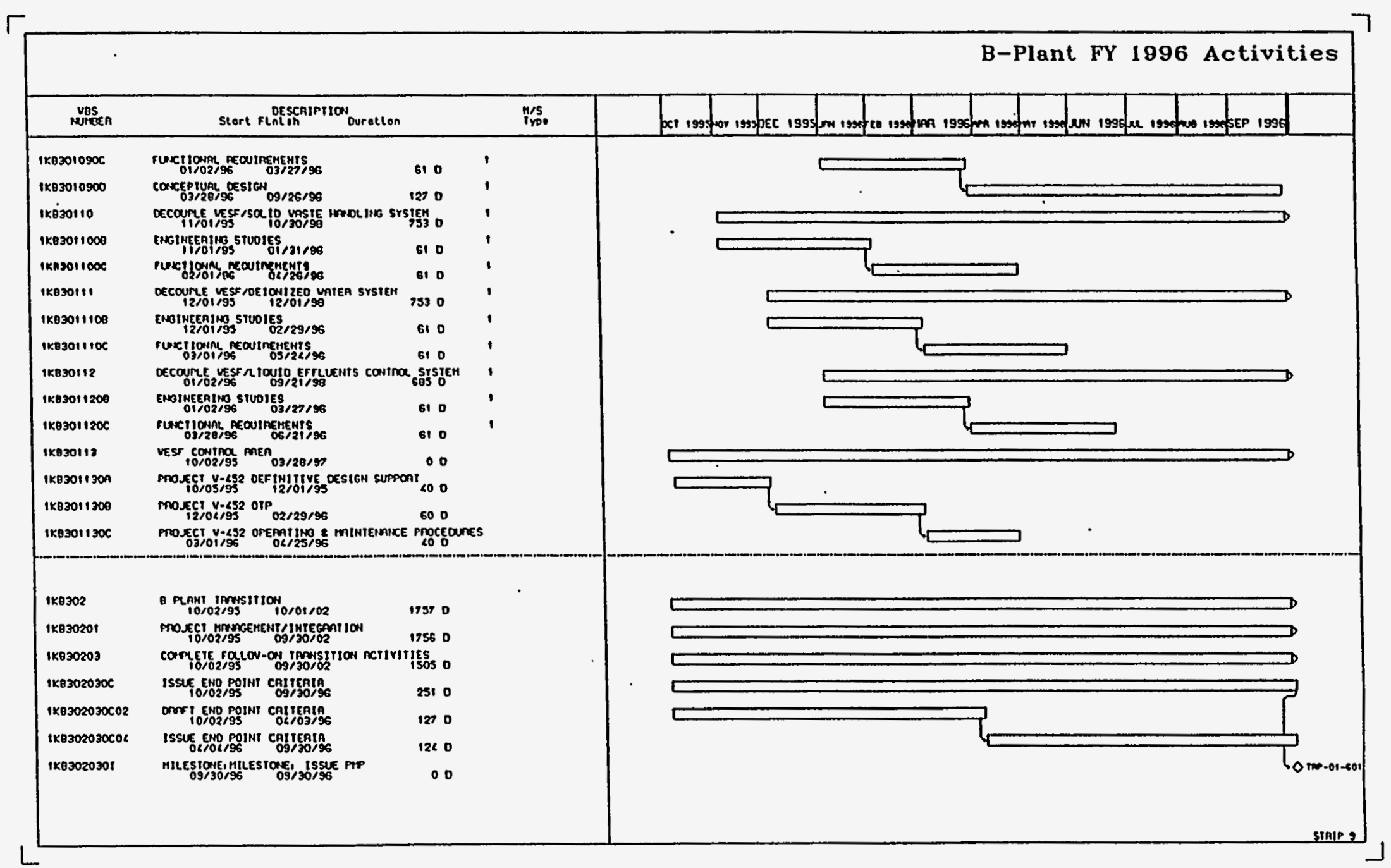




\section{FY 1996 MYPP}

\section{TRANSITIUN PROJECTS}

\subsection{1/7.1/6.12}

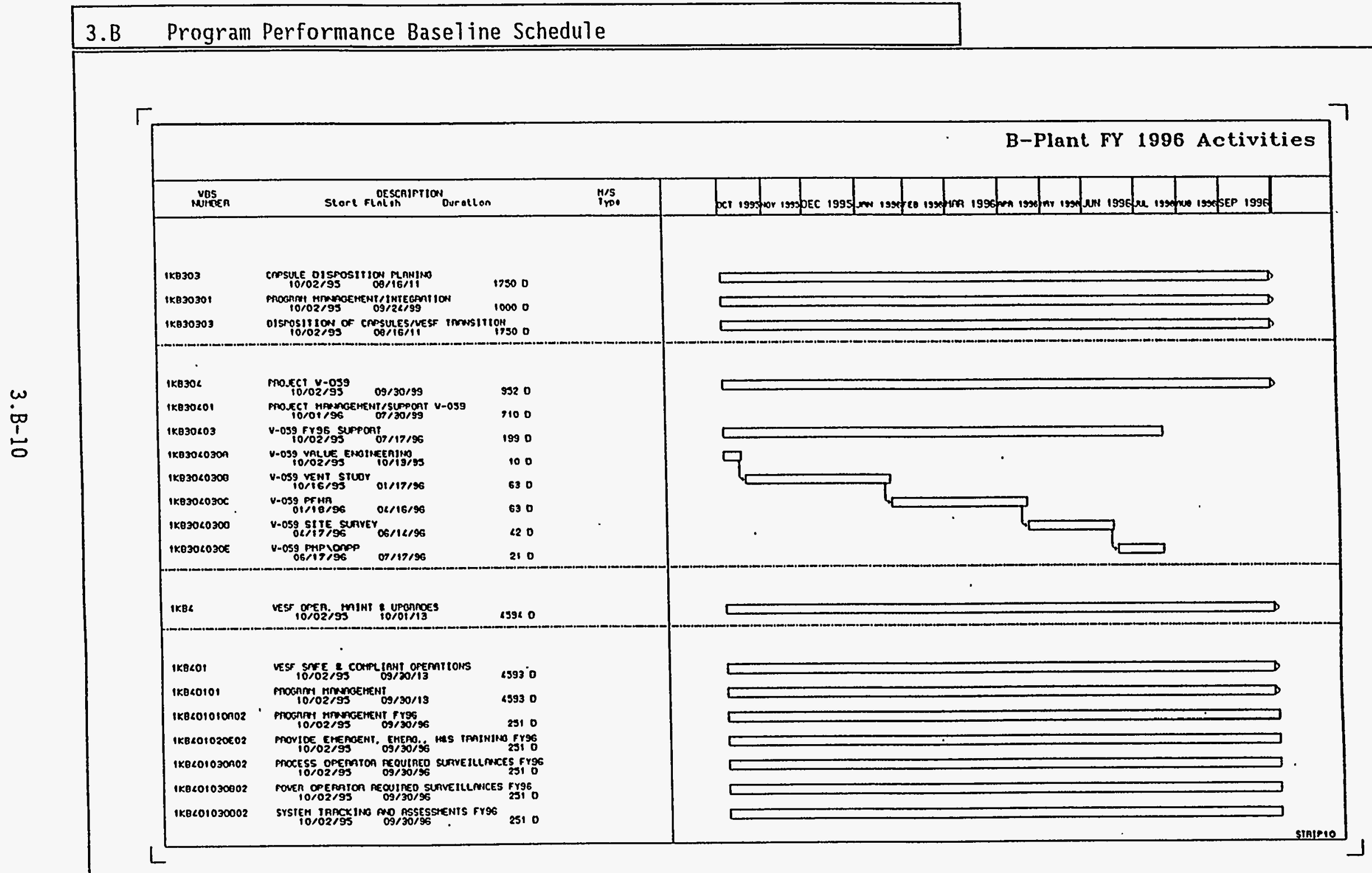


TRANSITION PROJECTS

FY 1996 MYPP

1.3.1/7.1/6.12

3.B Program Performance Basel ine Schedule

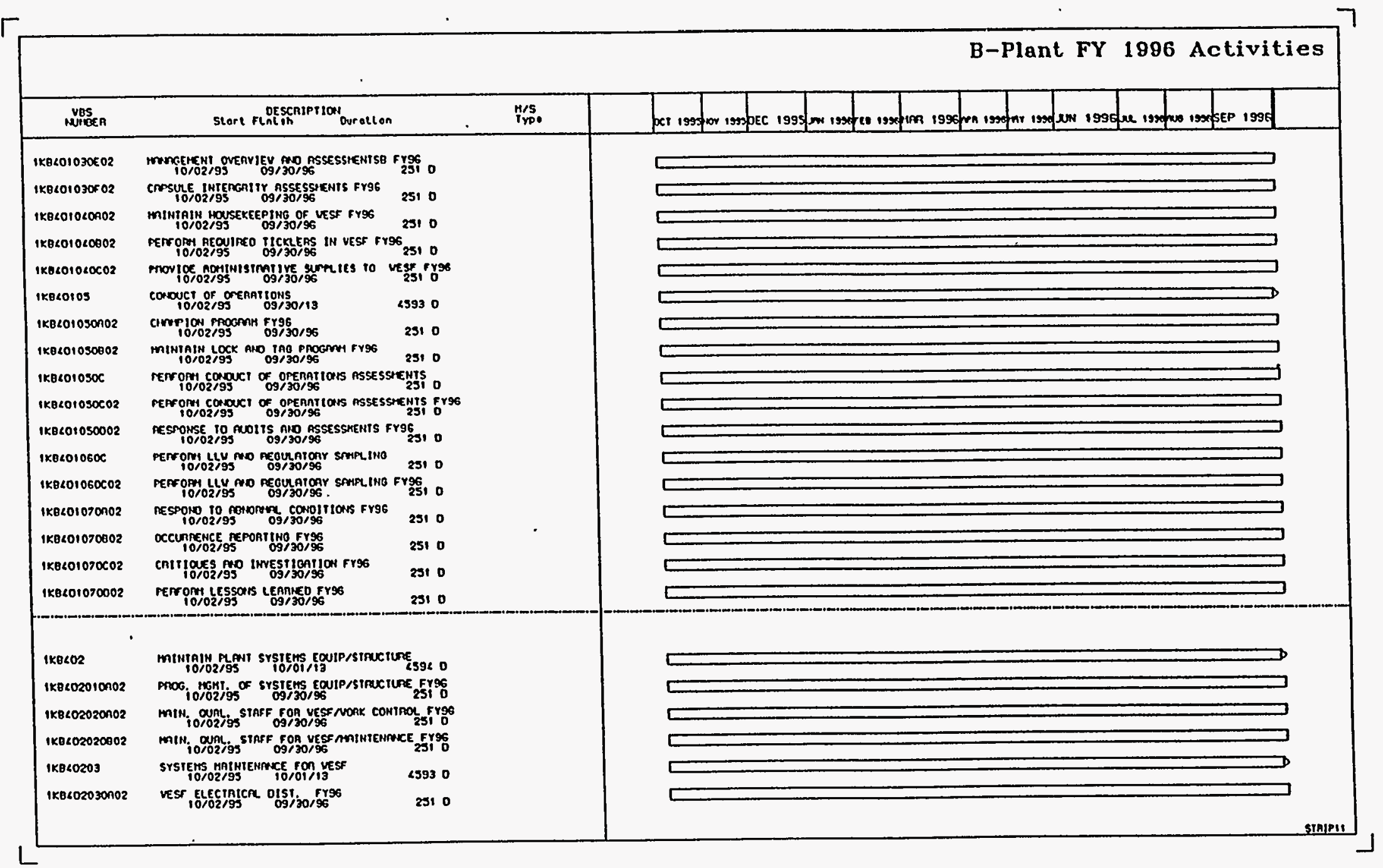




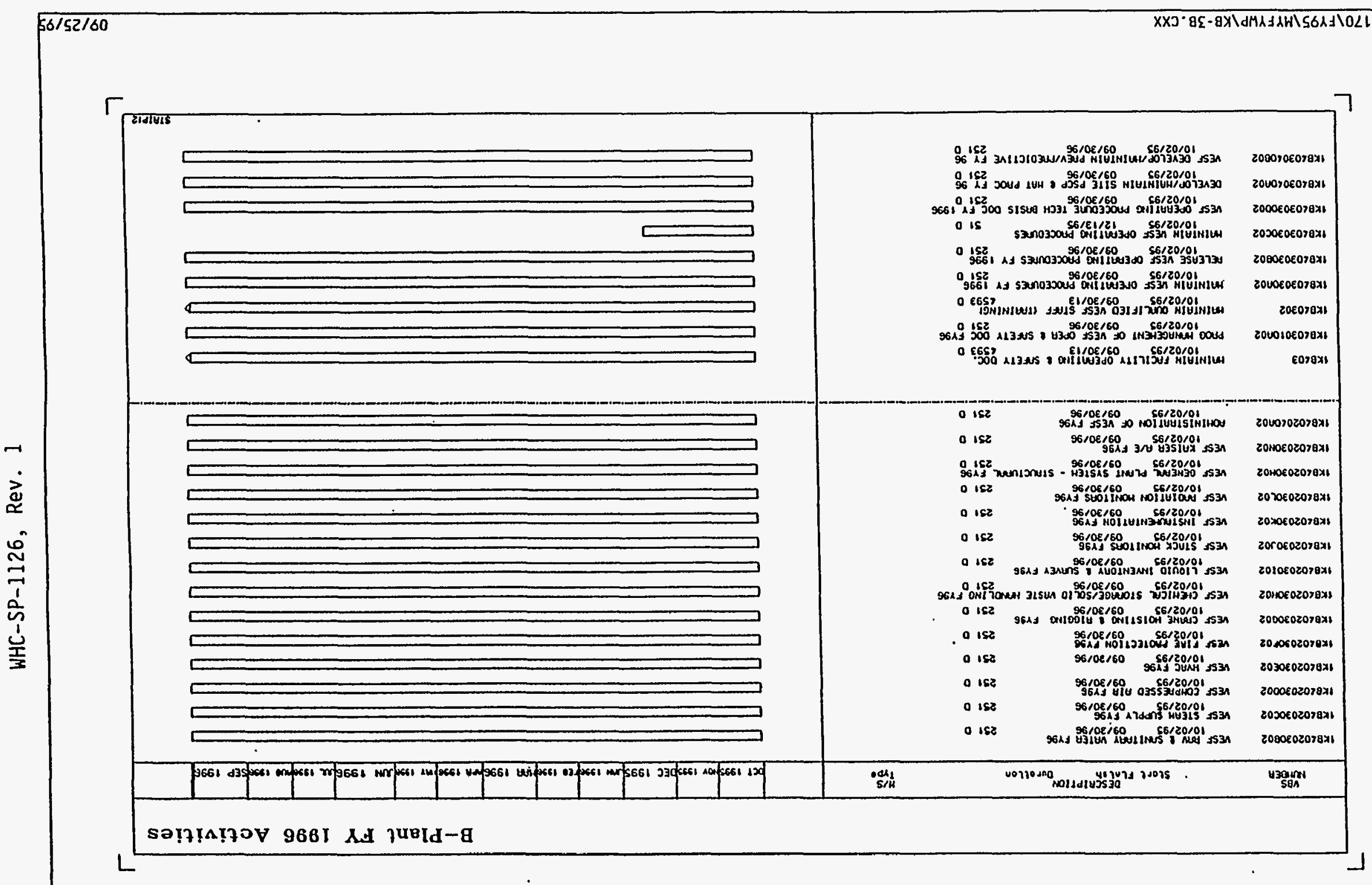




\section{B Program Performance Baseline Schedule}

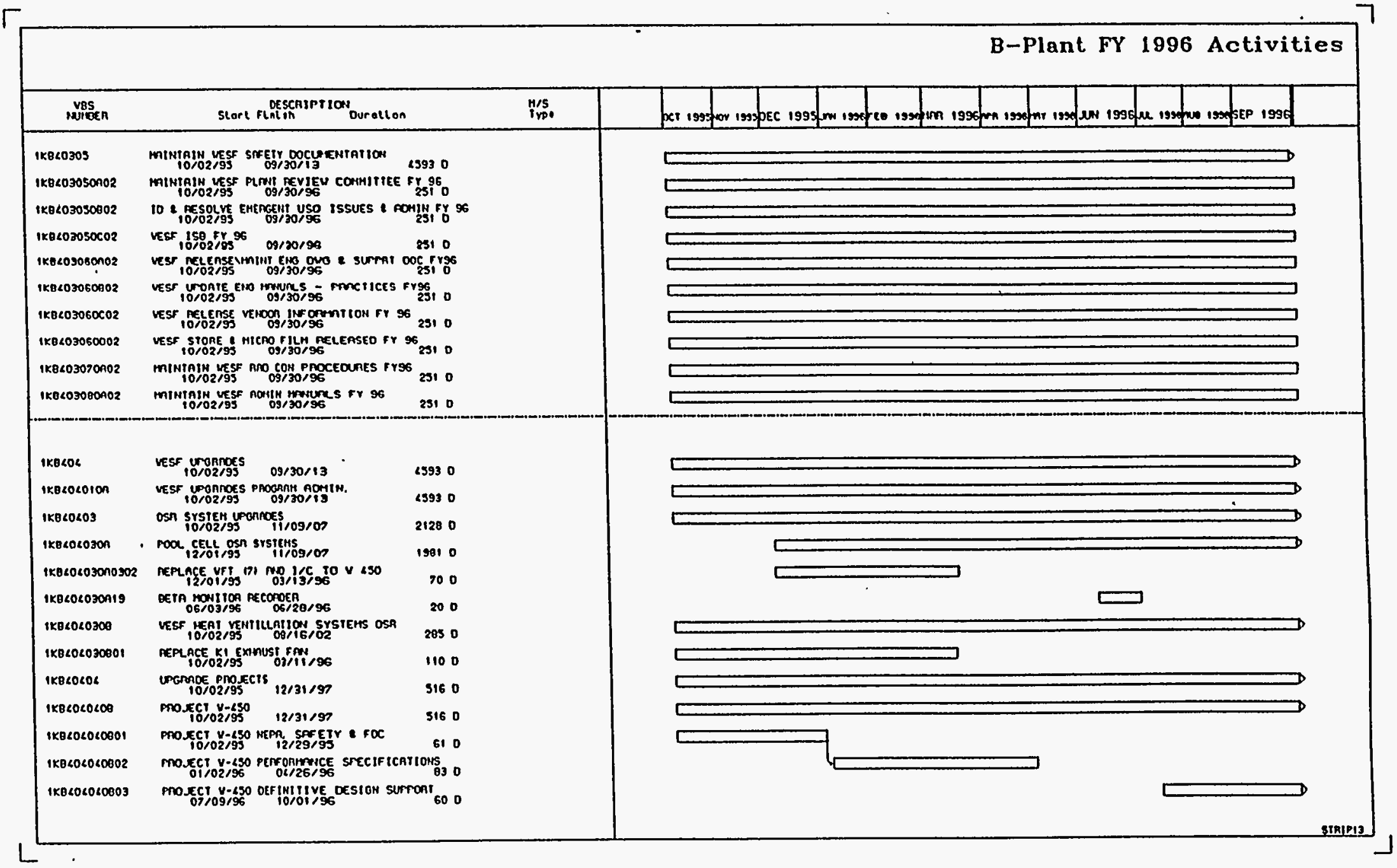




\section{FY 1996 MYPP}

\section{TRANSITION PROJECTS}

\subsection{1/7.1/6.12}

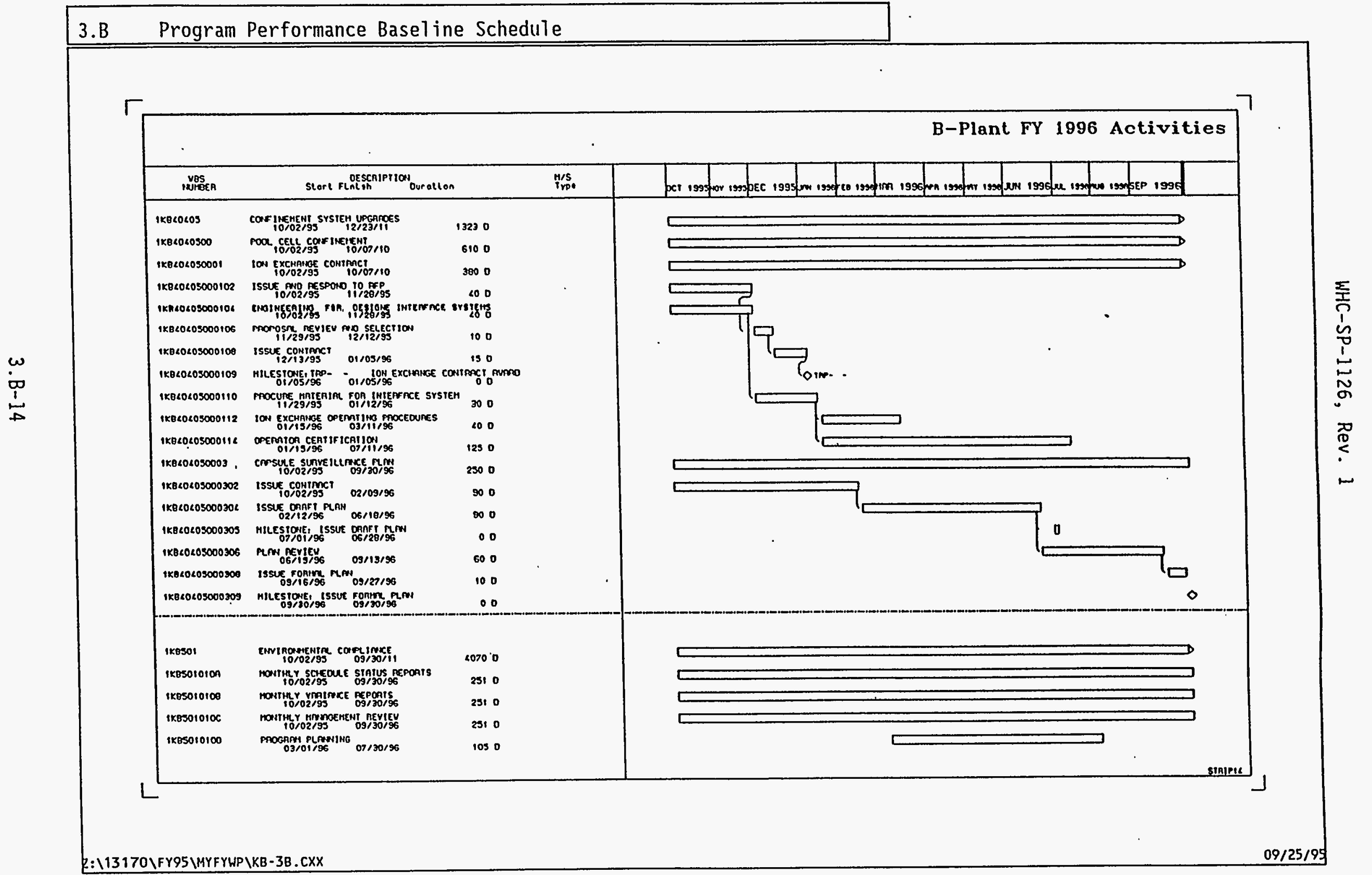




\section{TRANSITION PROJECTS}

FY 1996 MYPP

\subsection{1/7.1/6.12}

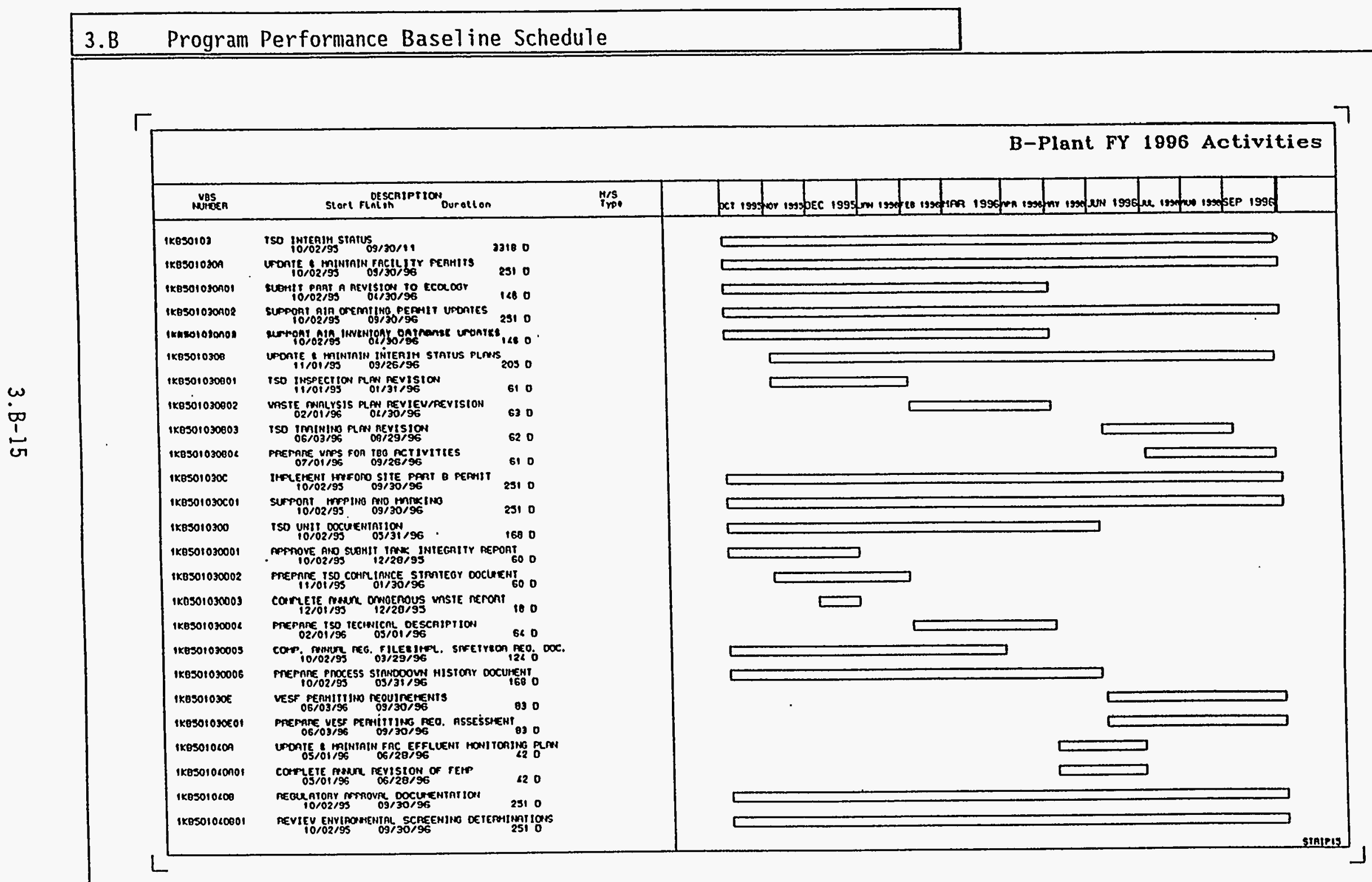




\section{TRANSITION PROJECTS \\ 1.3.1/7.1/6.12}

FY 1996 MYPP

3.B Program Performance Baseline Schedule

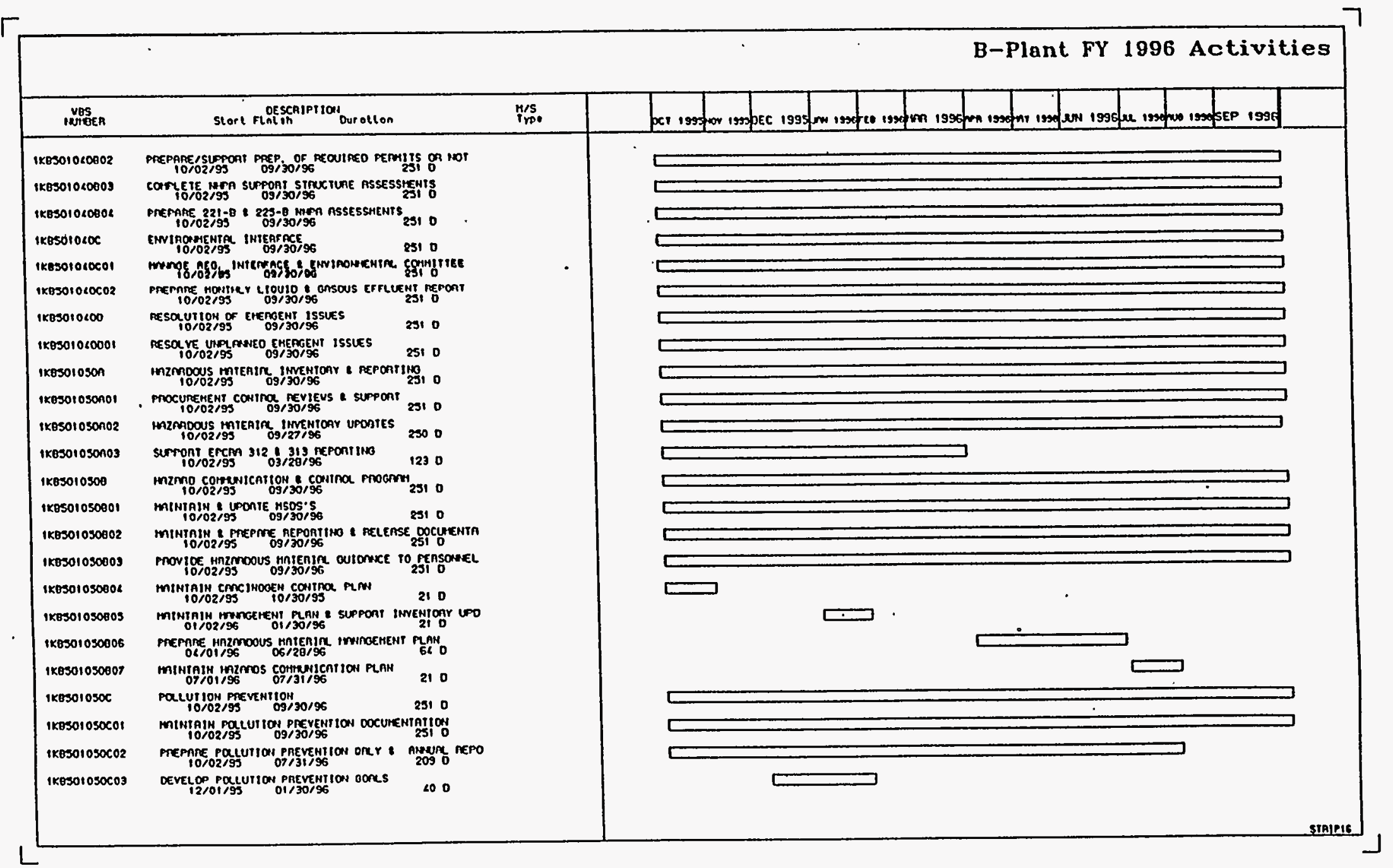




\section{TRANSITION PROJECTS}

\section{FY 1996 MYPP \\ 1.3.1/7.1/6.12}

3.B Program Performance Baseline Schedule

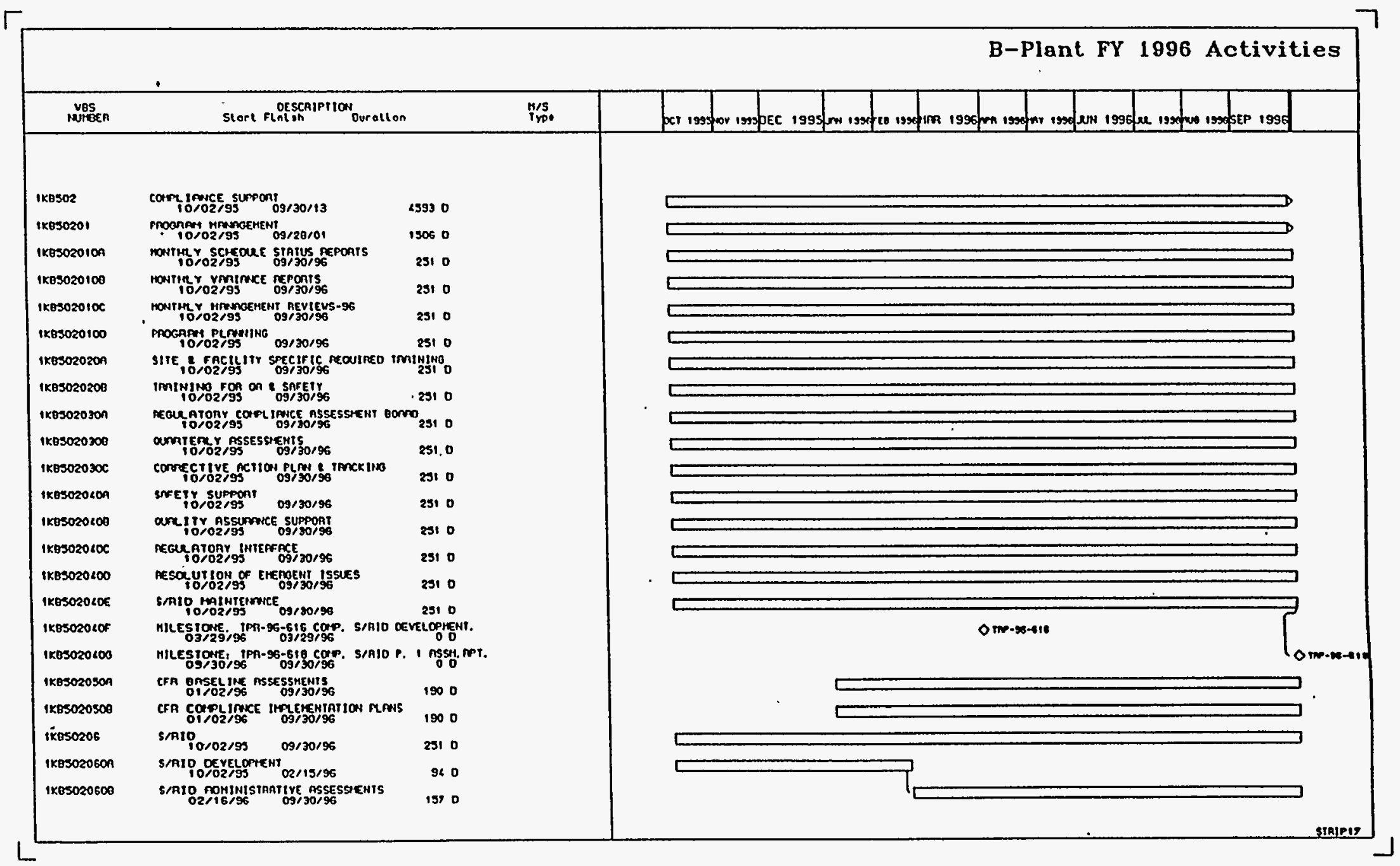




\section{FY 1996 MYPP}

\section{TRANSITION PROJECTS}

\subsection{1/7.1/6.12}

3.8 Program Performance Baseline ScheduTe

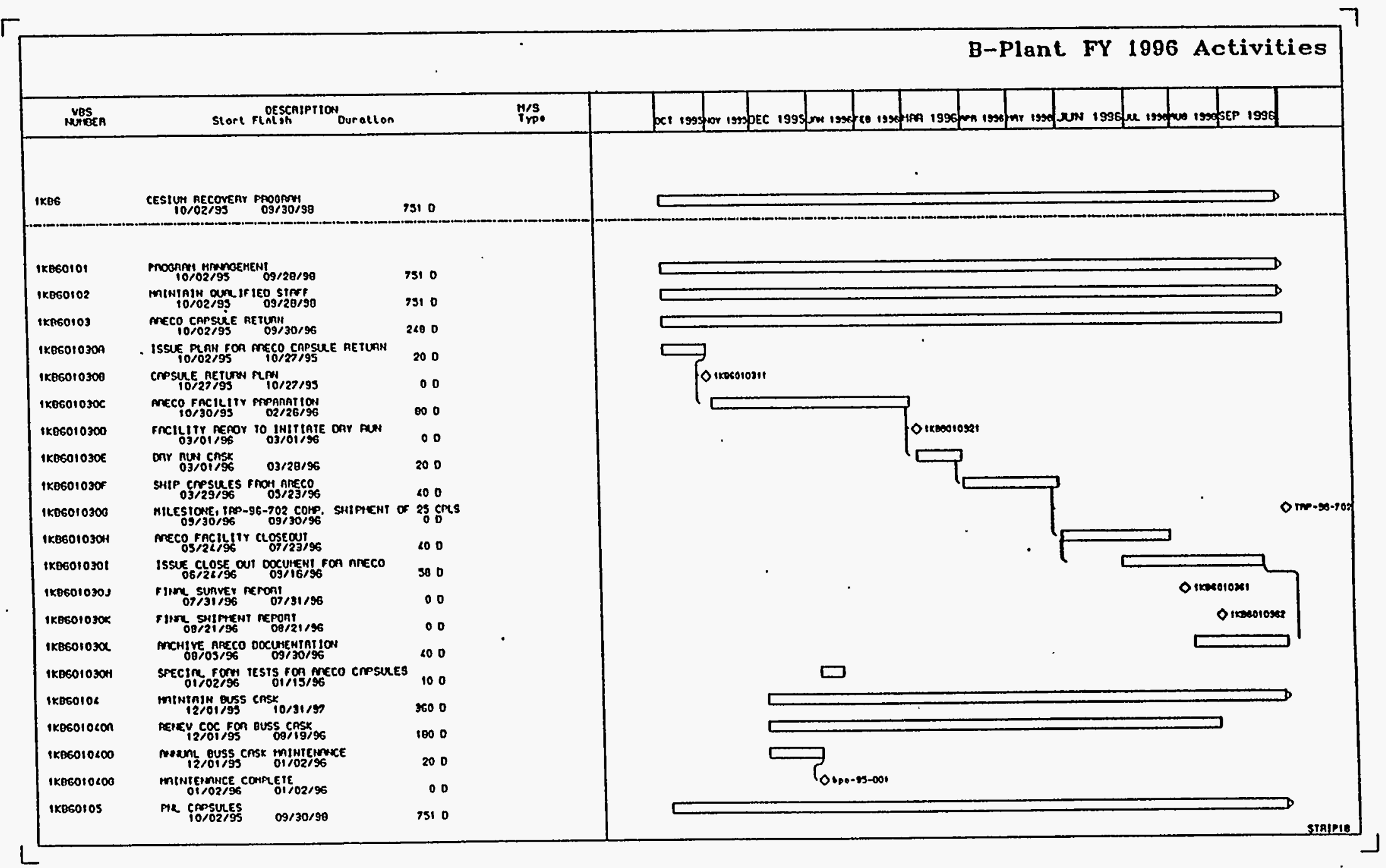


WHC-SP-1126, Rev. 1

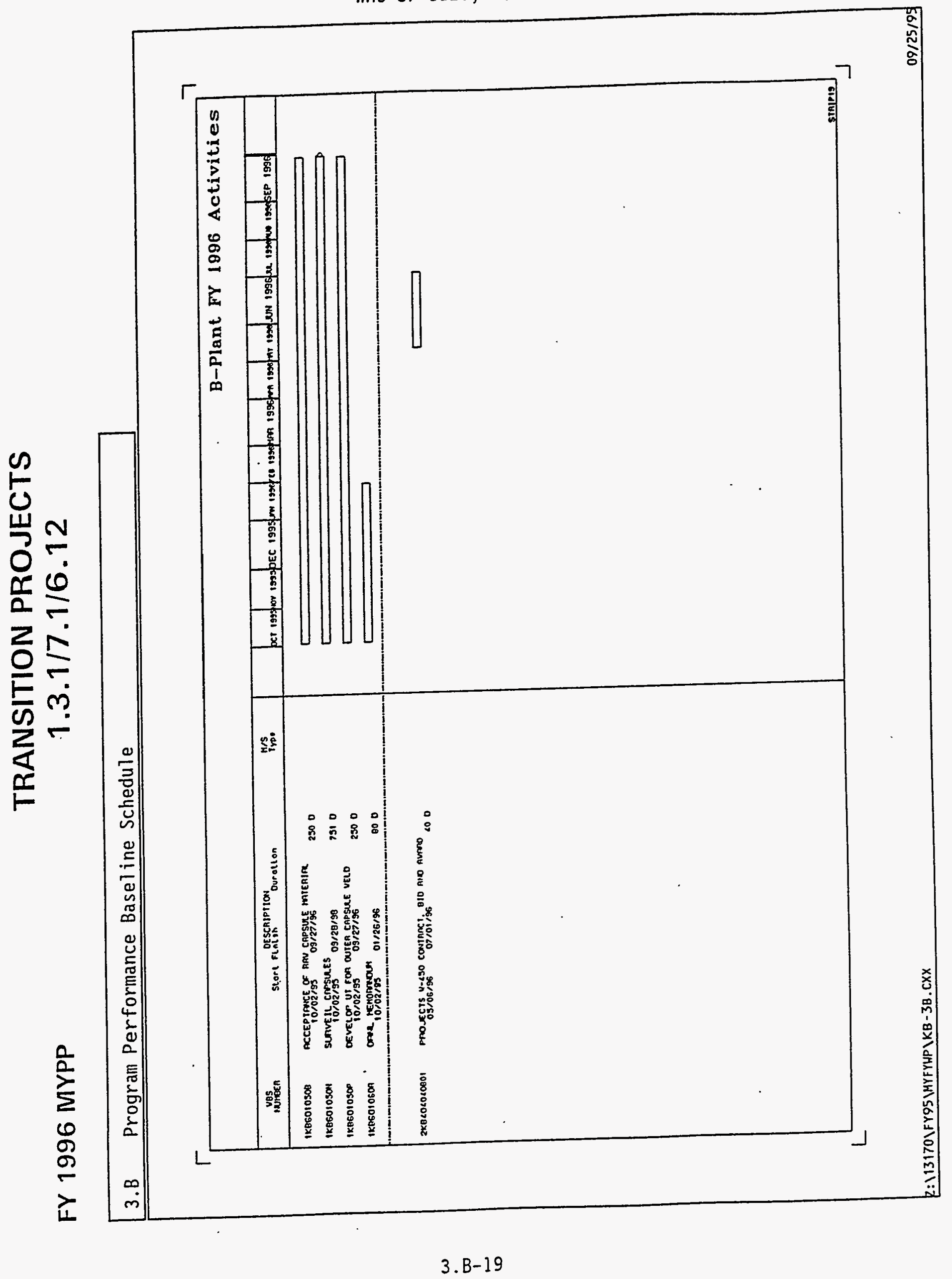




\section{TRANSITION PROJECTS}

FY 1996 MYPP

1.3.1/7.1/6.12

\section{B Program Performance Baseline Schedule}

PUREX (7.1.1)

The PUREX Transition Project's Program Performance Baseline Schedule is available upon request from the plant. 


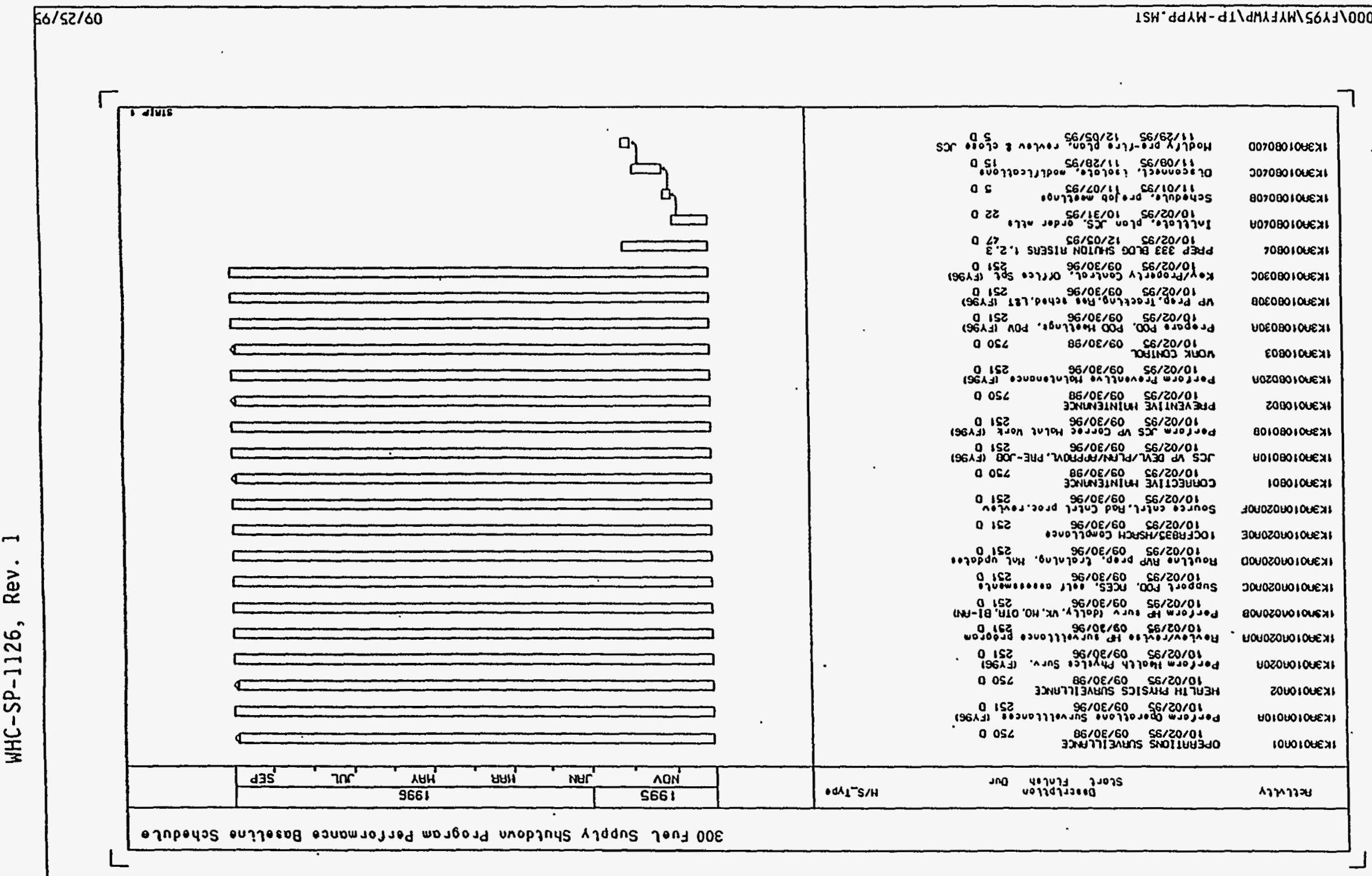




\section{TRANSITIUN PROJECTS}

\section{FY 1996 MYPP}

1.3.1/7.1/6.12

3.B Program Performance Basel ine ScheduTe

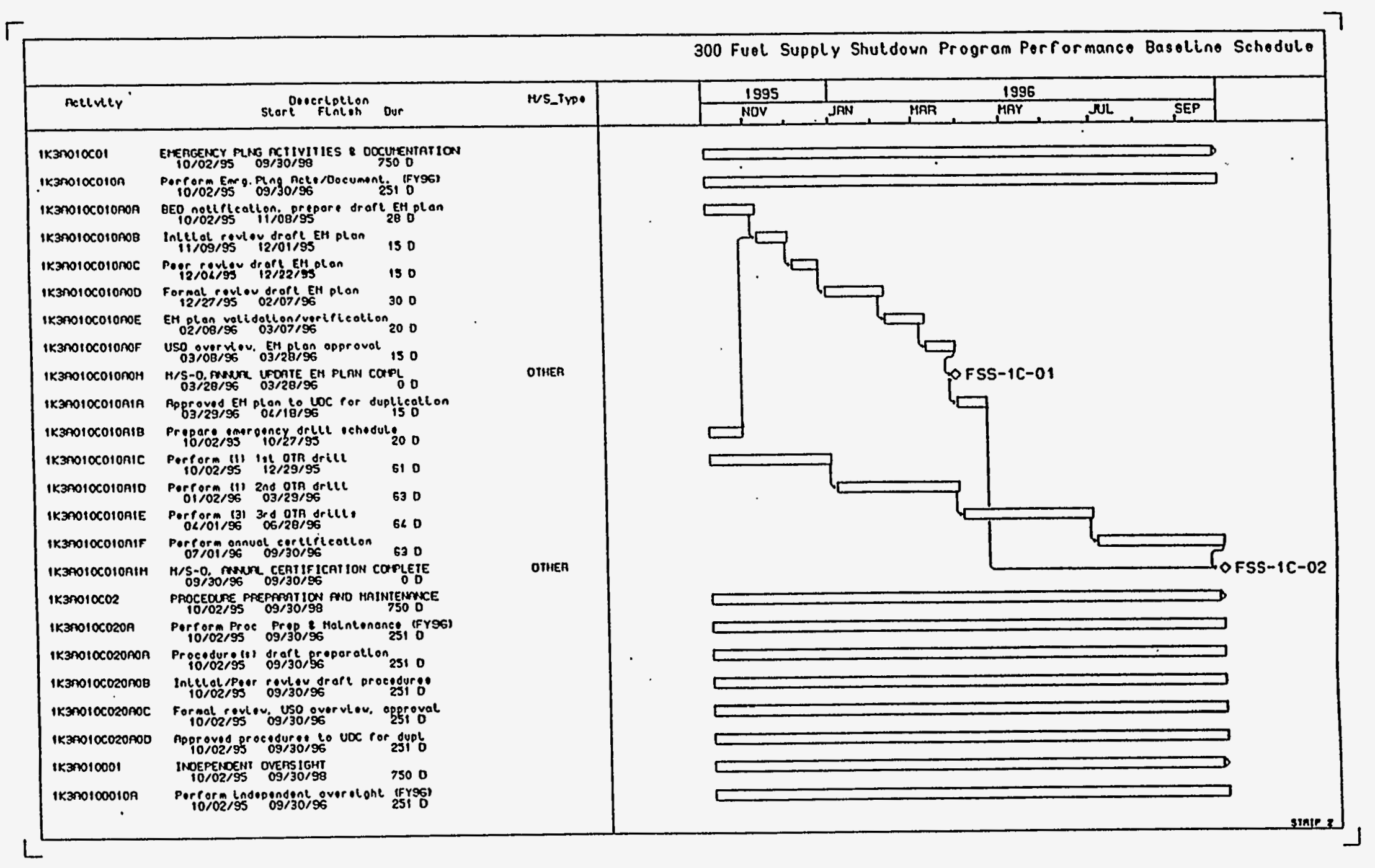




\section{TRANSITIUN PROJECTS}

\section{FY 1996 MYPP}

\subsection{1/7.1/6.12}

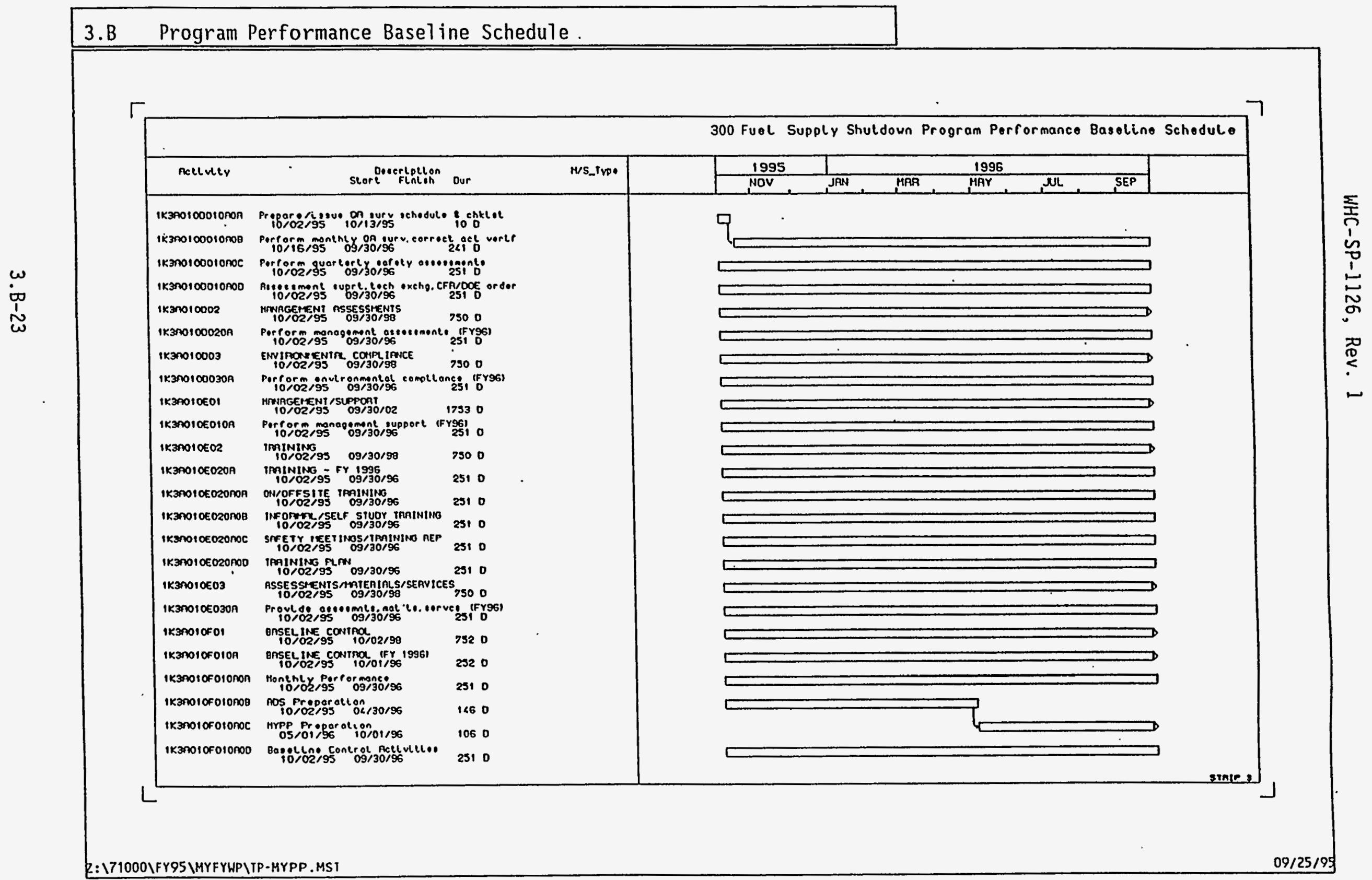




\section{- FY 1996 MYPP}

\section{TRANSITION PROJECTS}

\subsection{1/7.1/6.12}

\section{B Program Performance Baseline Schedule}

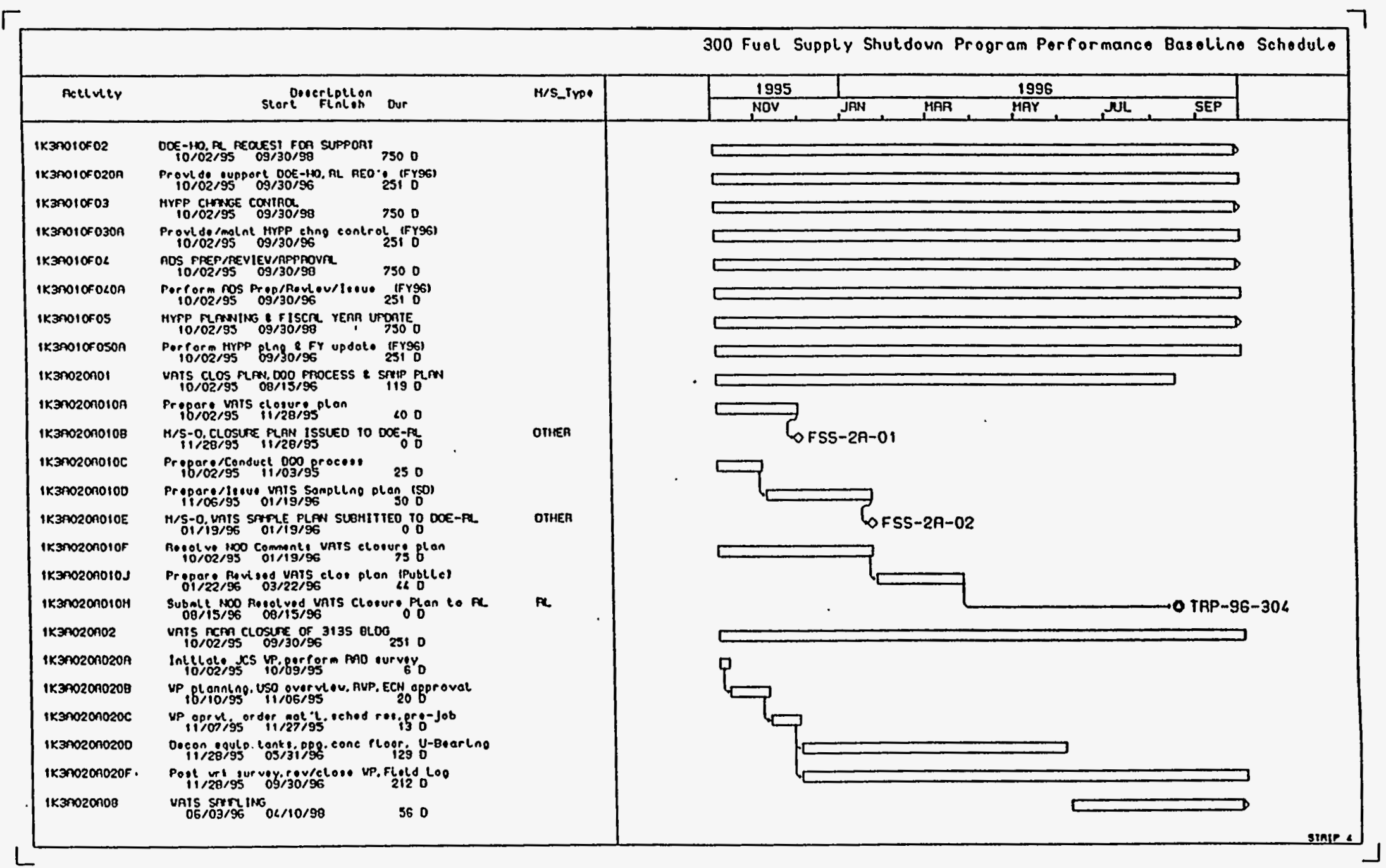




\section{TRANSITION PROJECTS}

\section{FY 1996 MYPP}

\subsection{1/7.1/6.12}

\section{B Program. Performance Baseline Schedule}

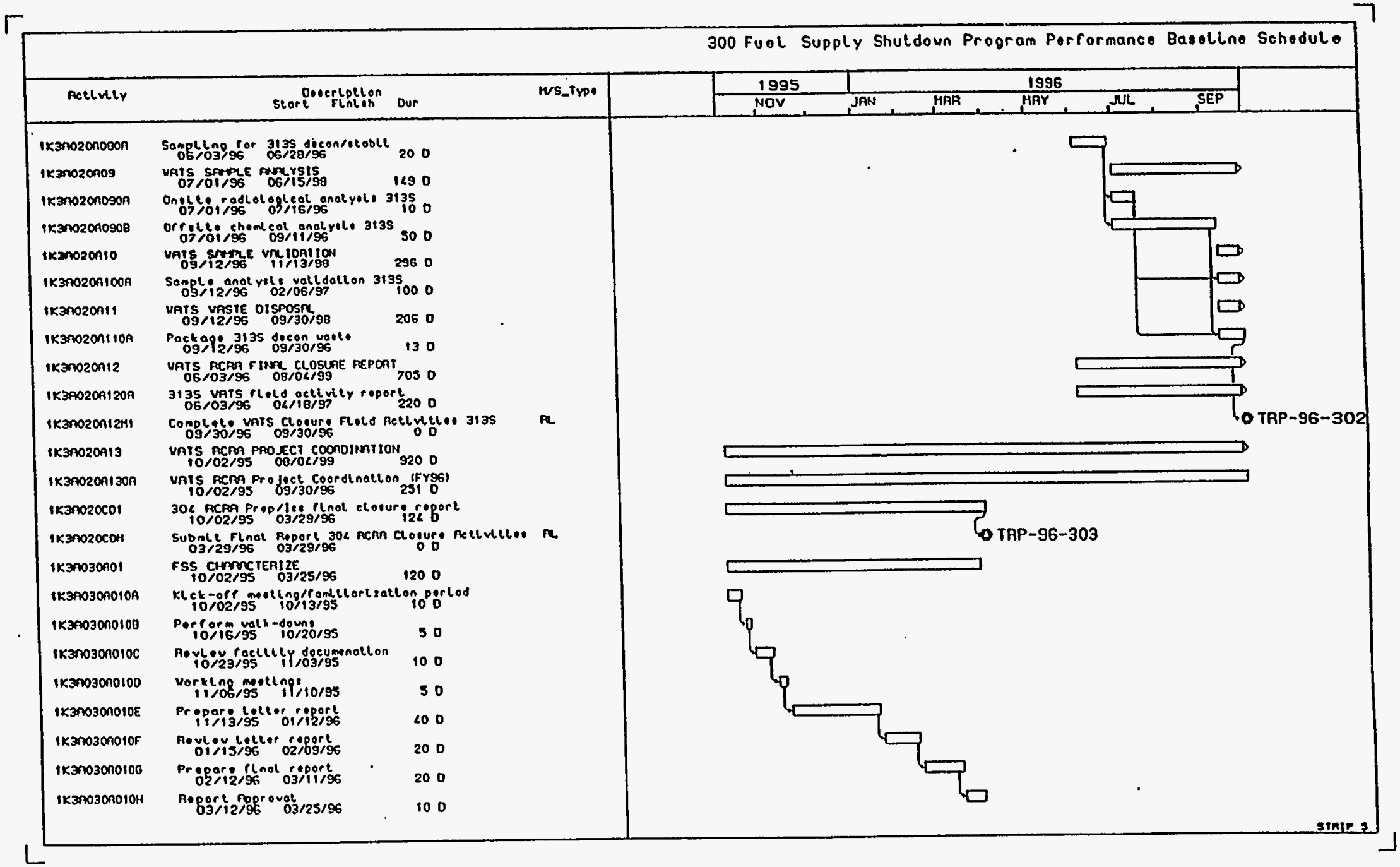




\section{TRANSITION PROJECTS}

\section{FY 1996 MYPP}

\subsection{1/7.1/6.12}

3. B Program Performance Baseline Schedule

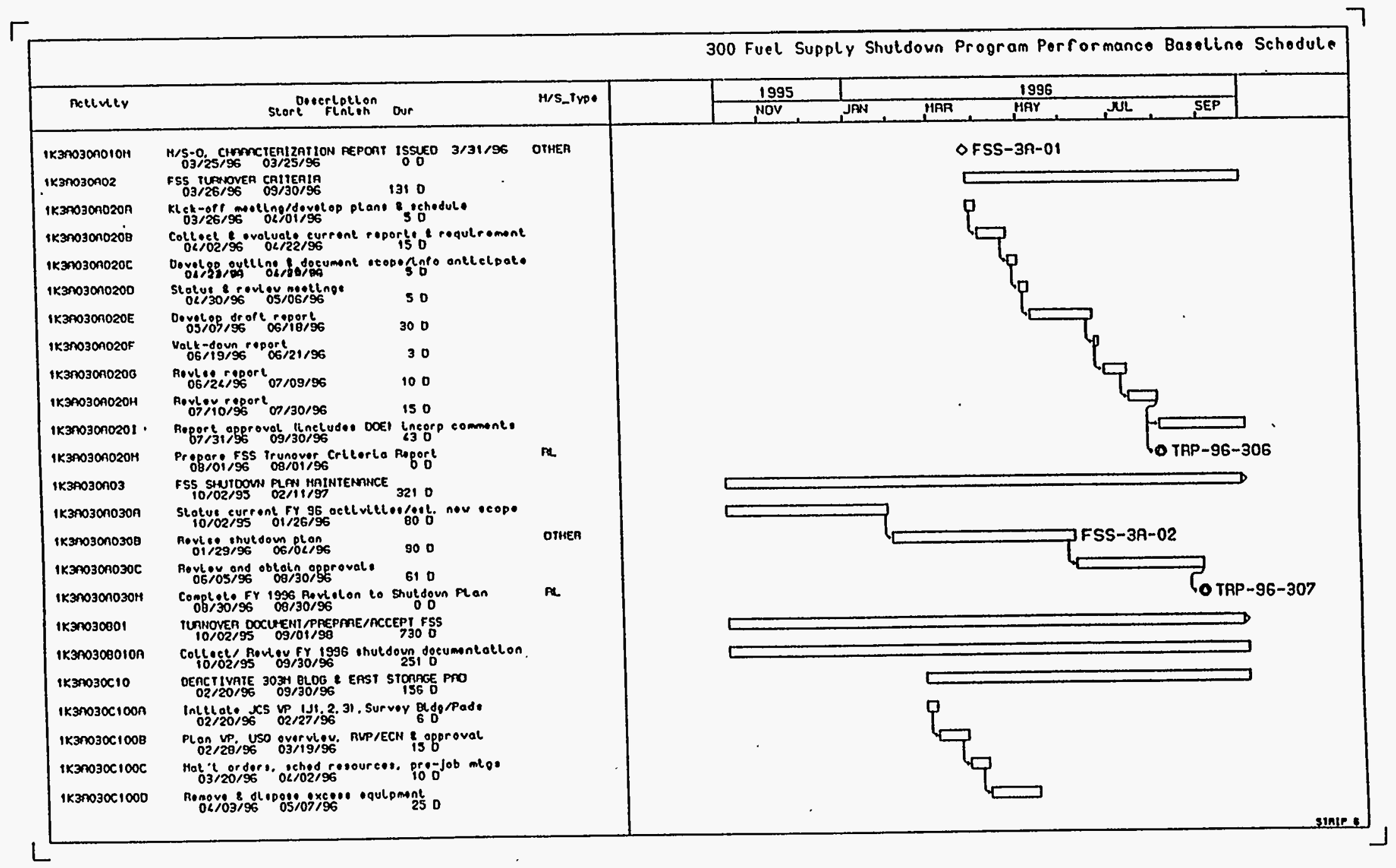




\section{TRANSITIUN PROJECTS}

FY 1996 IVYPP

\subsection{1/7.1/6.12}

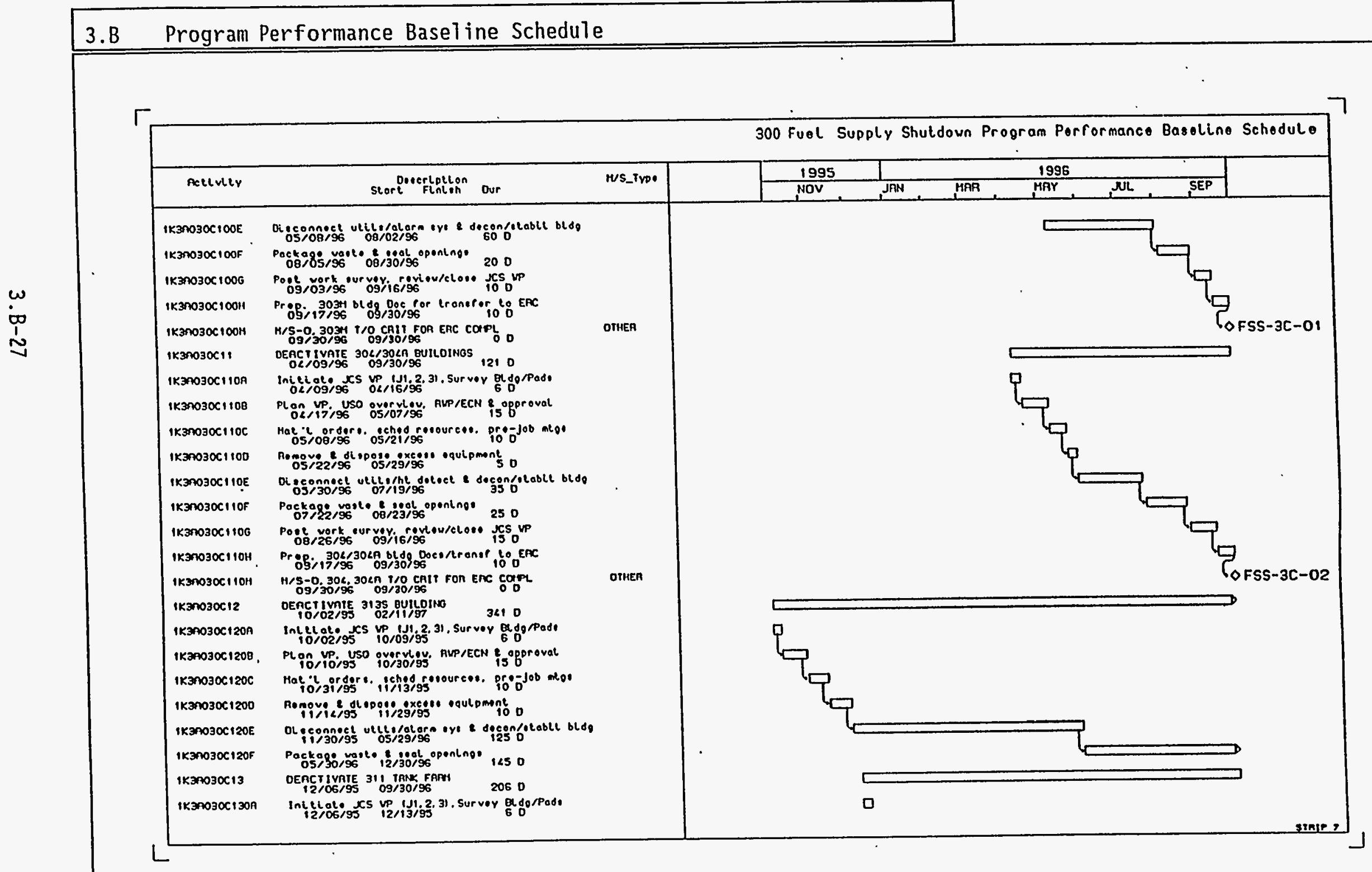




\section{TRANSITION PROJECTS}

FY 1996 MYPP

\subsection{1/7.1/6.12}

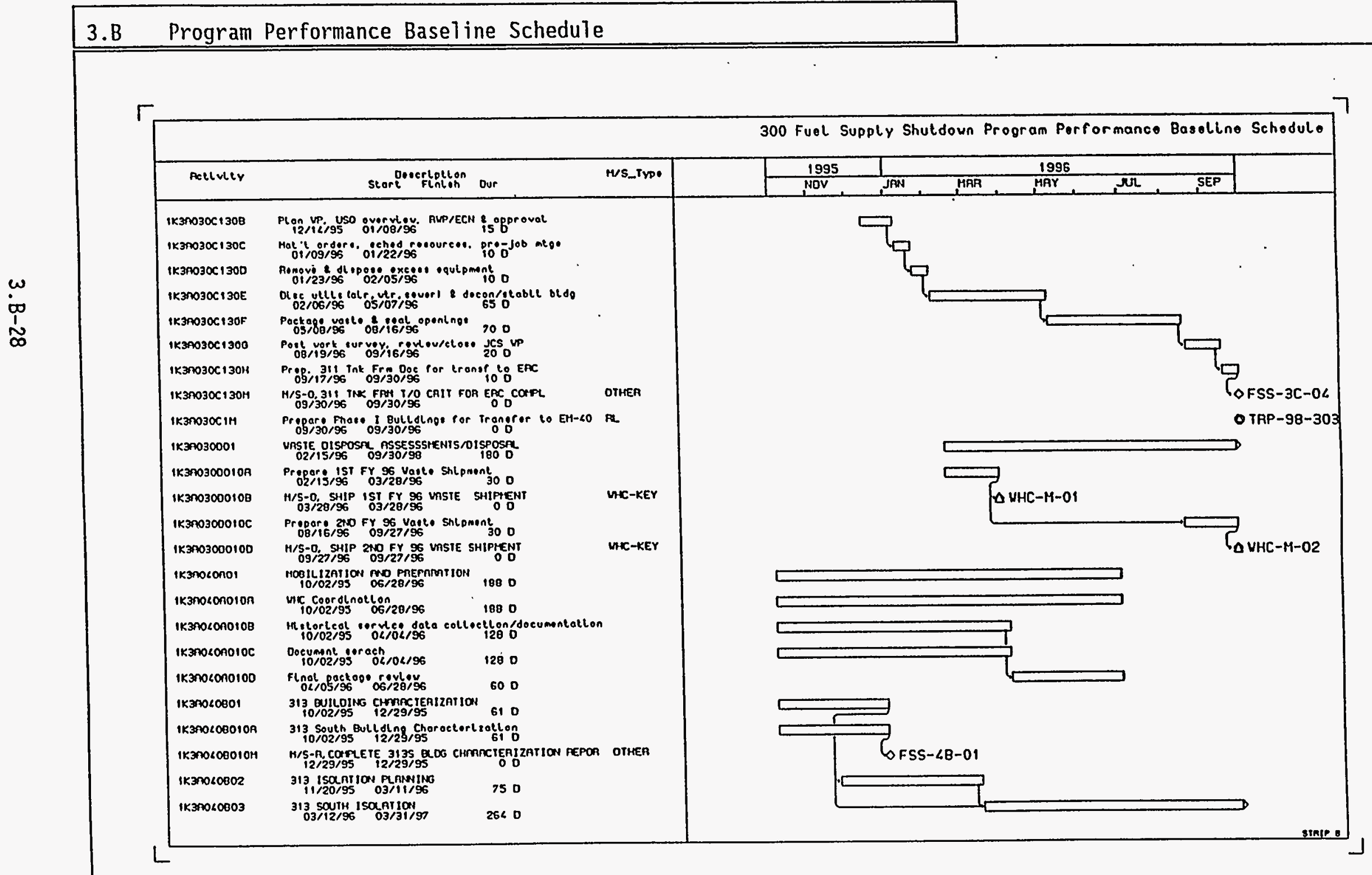




\section{TRANSITION PROJECTS}

\section{FY 1996 MYPP}

1.3.1/7.1/6.12

3.B Program Performance Baseline Schedule

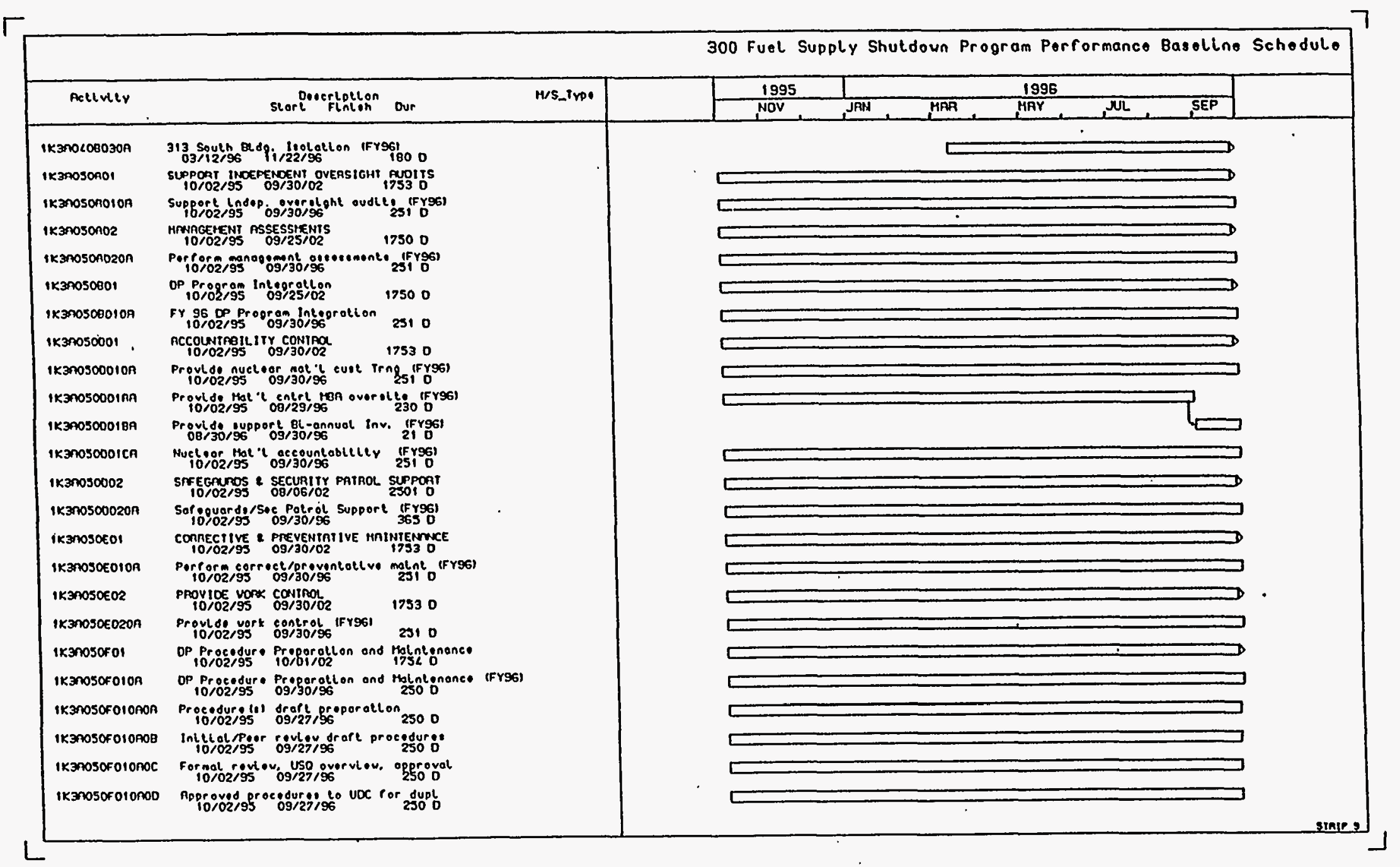




\section{FY 1996 MYPP}

\section{TRANSITIUN PROJECTS}

\subsection{1/7.1/6.12}

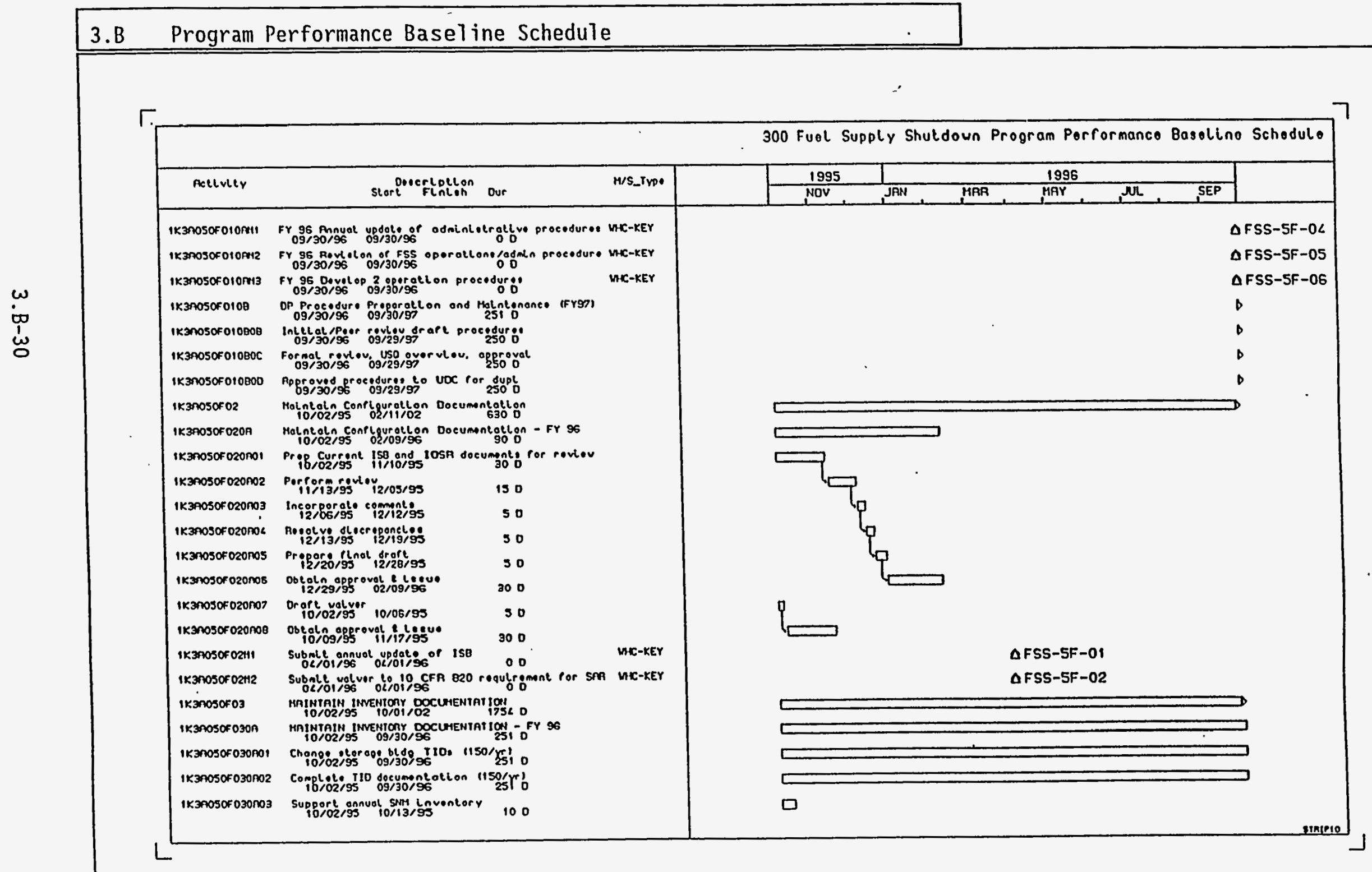


TRANSITION PROJECTS

FY 1996 MYPP

1.3.1/7.1/6.12

3.B Program Performance Baseline Schedule

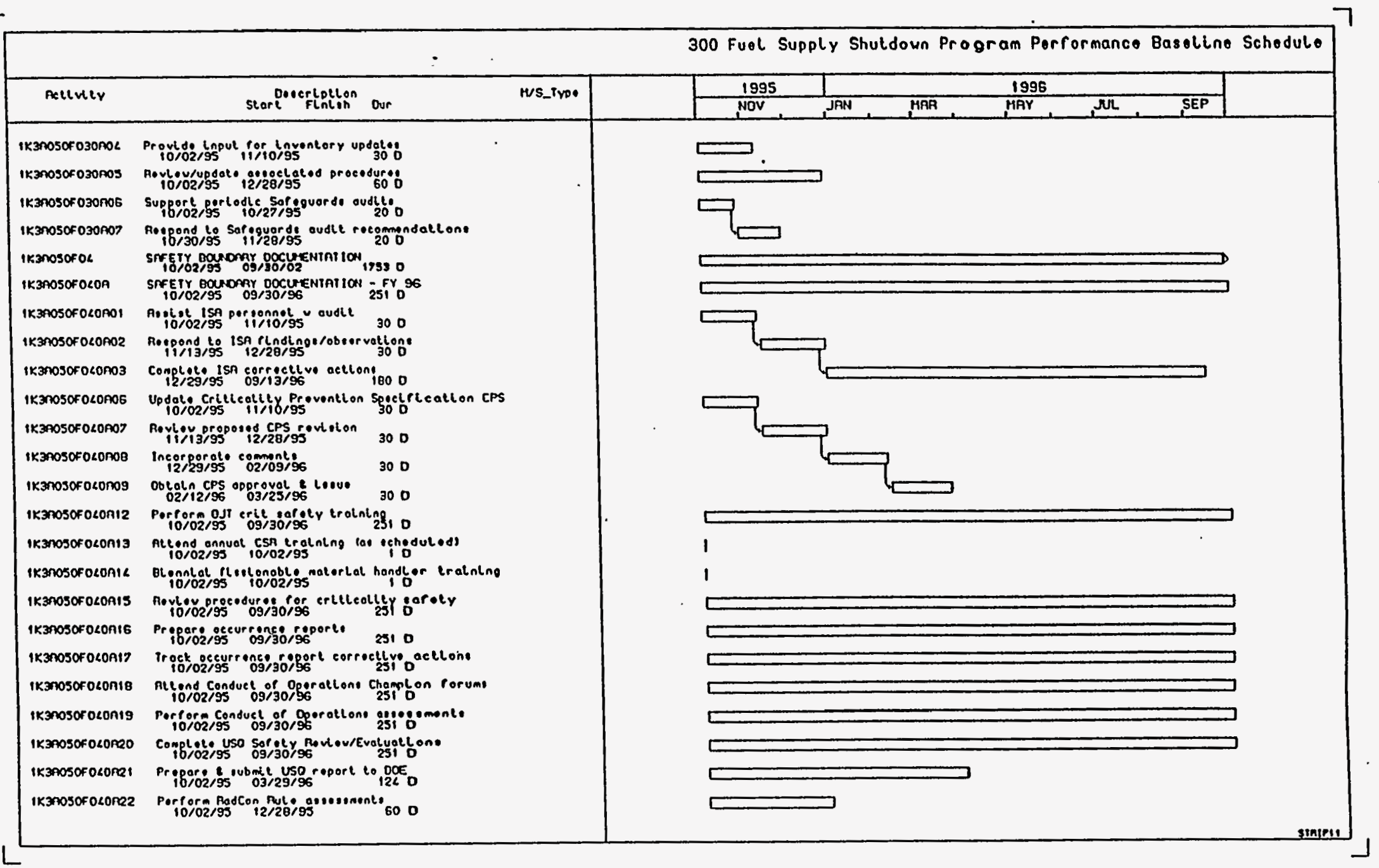


FY 1996 MYPP

TRANSITION PROJECTS

1.3.1/7.1/6.12

\section{B Program Performance Basel ine Schedule}

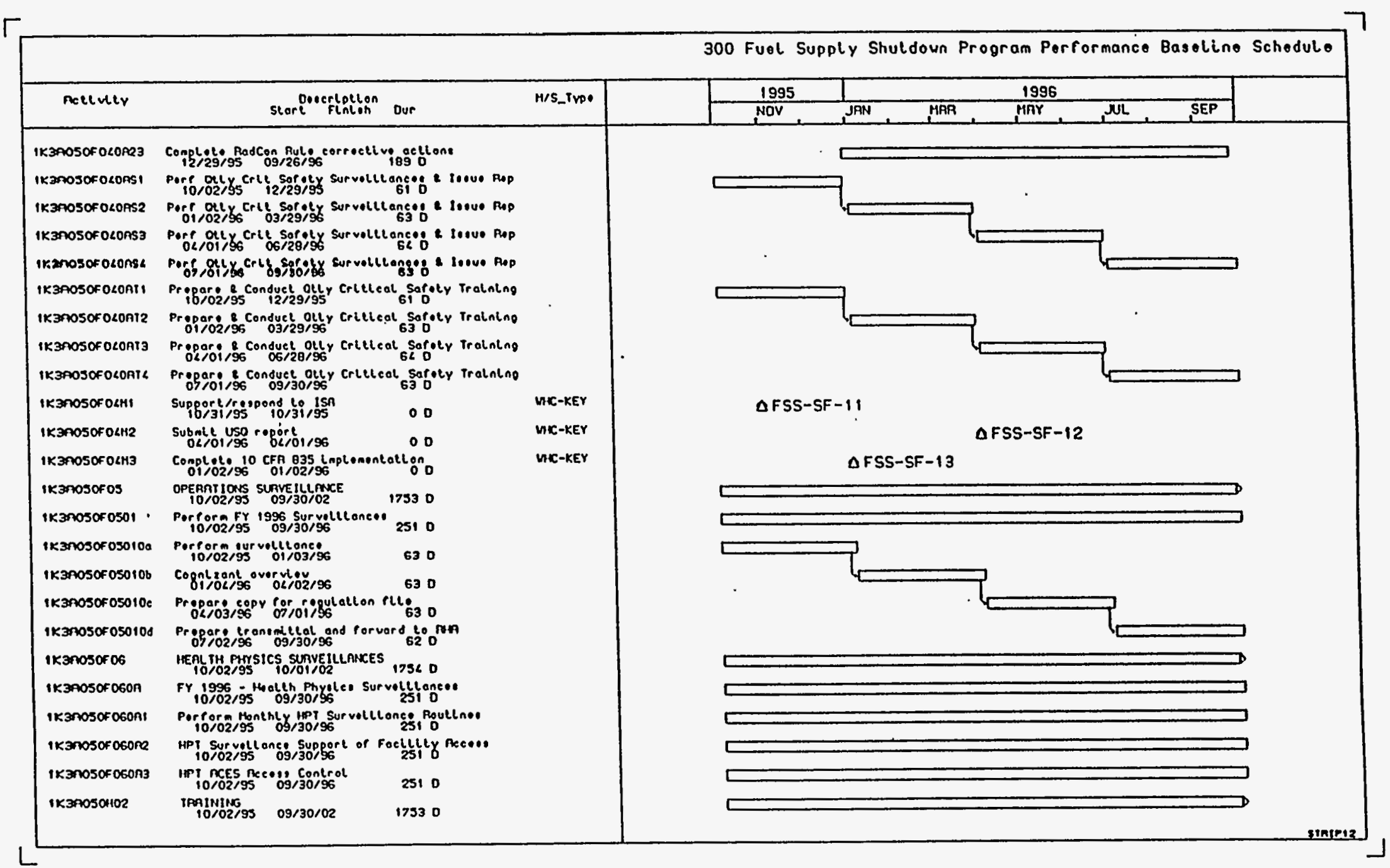




\section{TRANSITION PROJECTS}

\section{FY 1996 MYPP}

\subsection{1/7.1/6.12}

\section{B Program Performance Baseline Schedule}

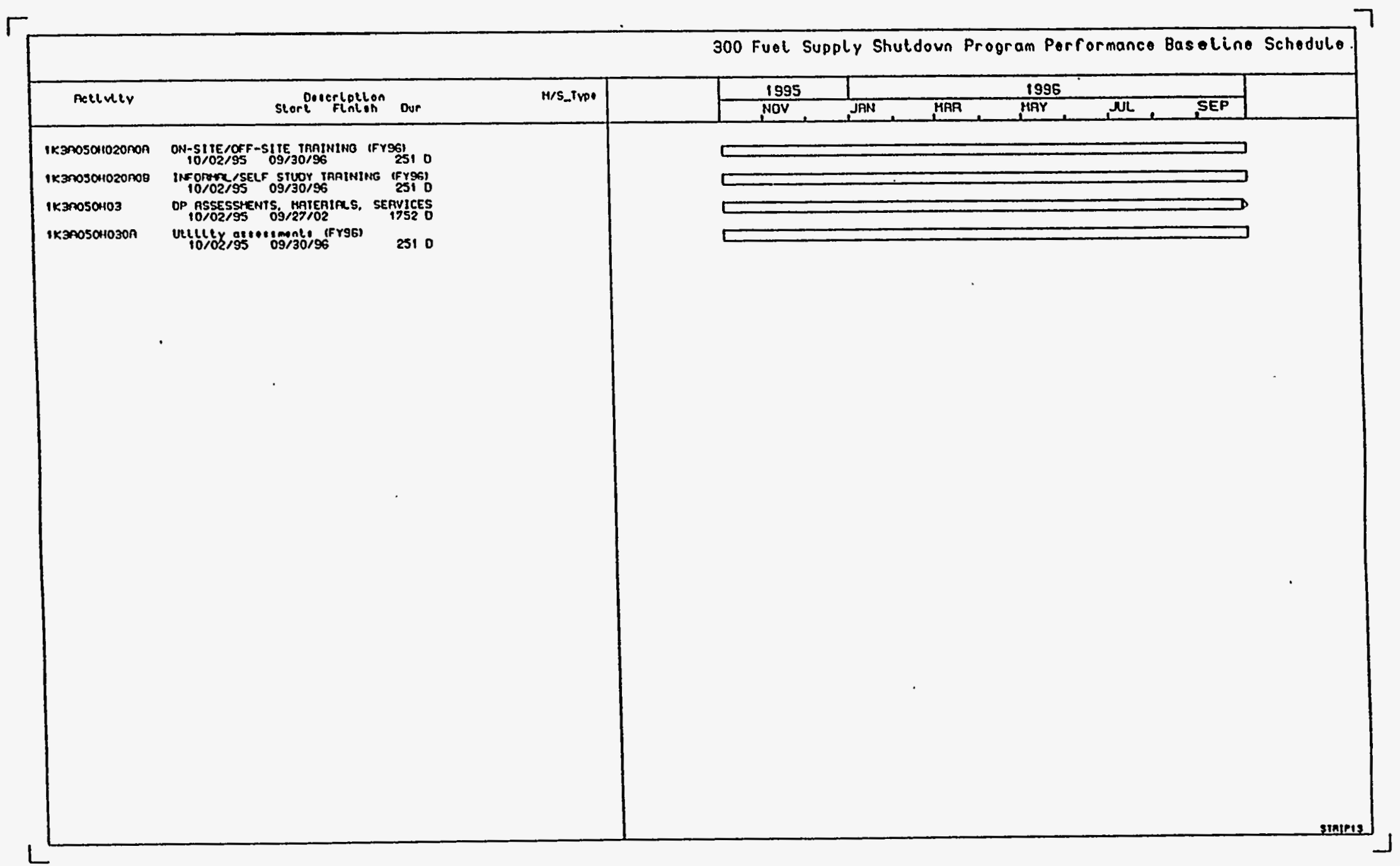


TRANSITION PROJECTS

FY 1996 MYPP

1.3.1/7.1/6.12

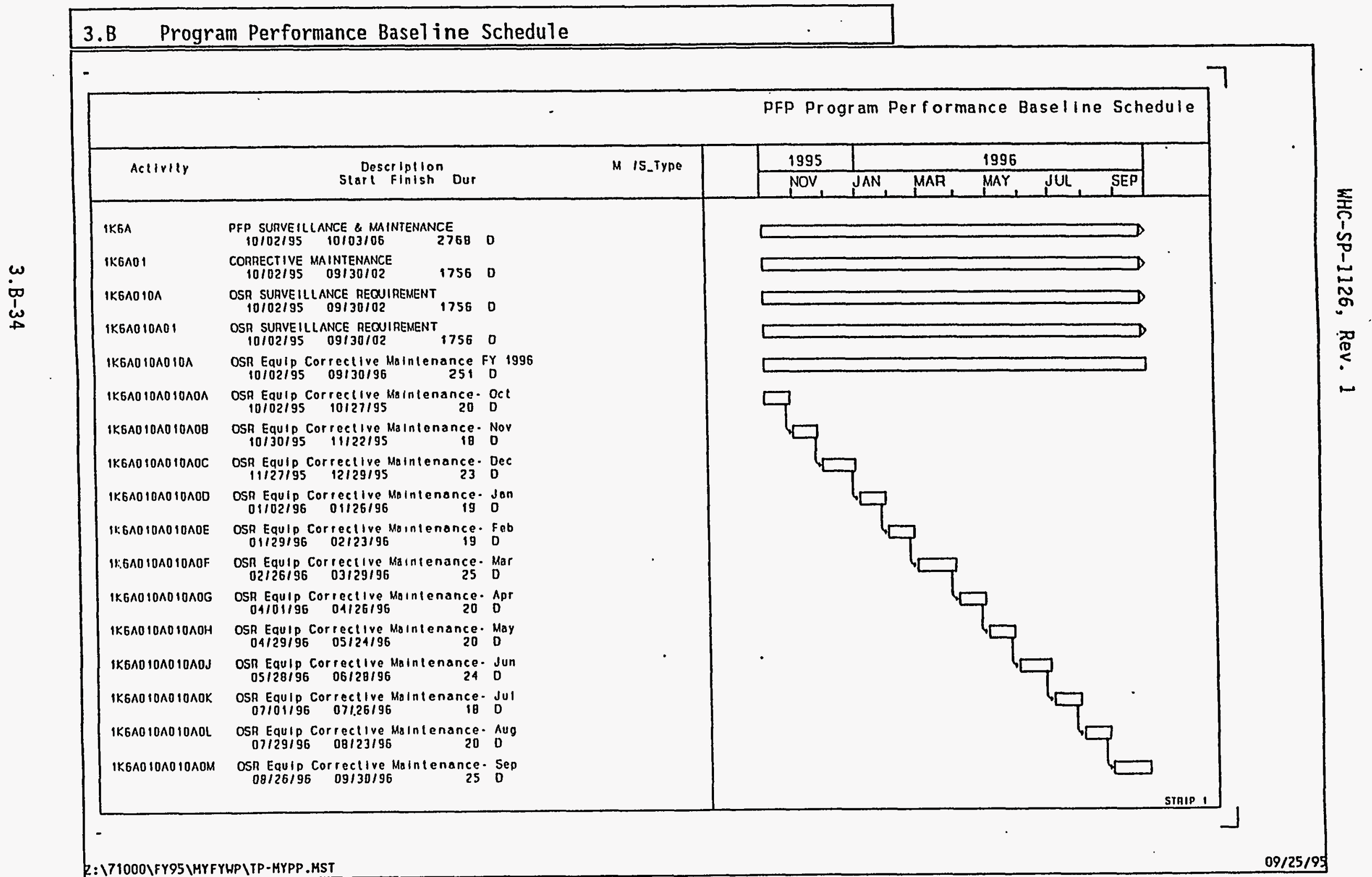




\section{TRANSITIUN PROJECTS}

\section{FY 1996 MYPP}

\subsection{1/7.1/6.12}

\section{B Program Performance Baseline Schedule}

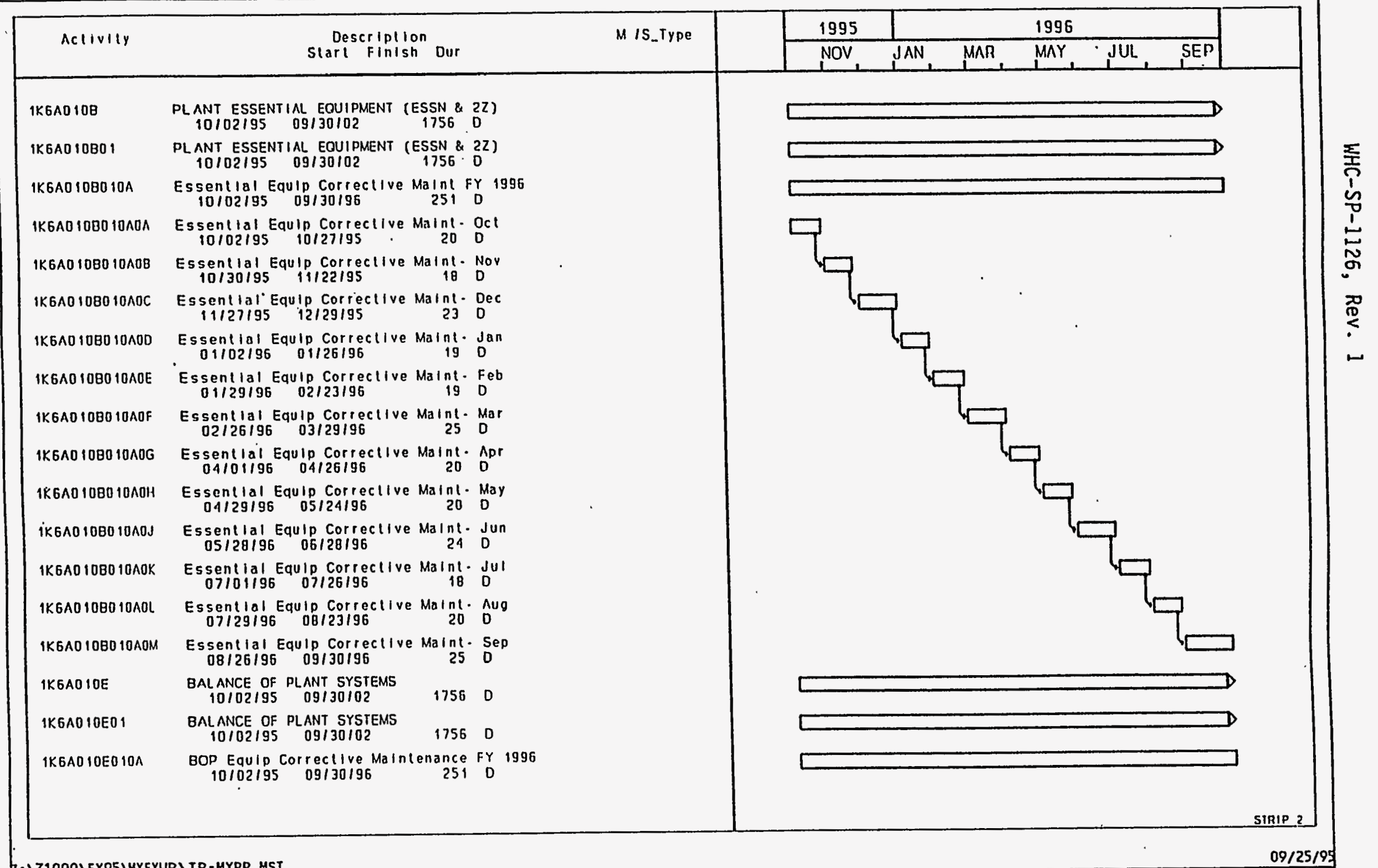




\section{TRANSITION PROJECTS}

\section{FY 1996 MYPP}

\subsection{1/7.1/6.12}

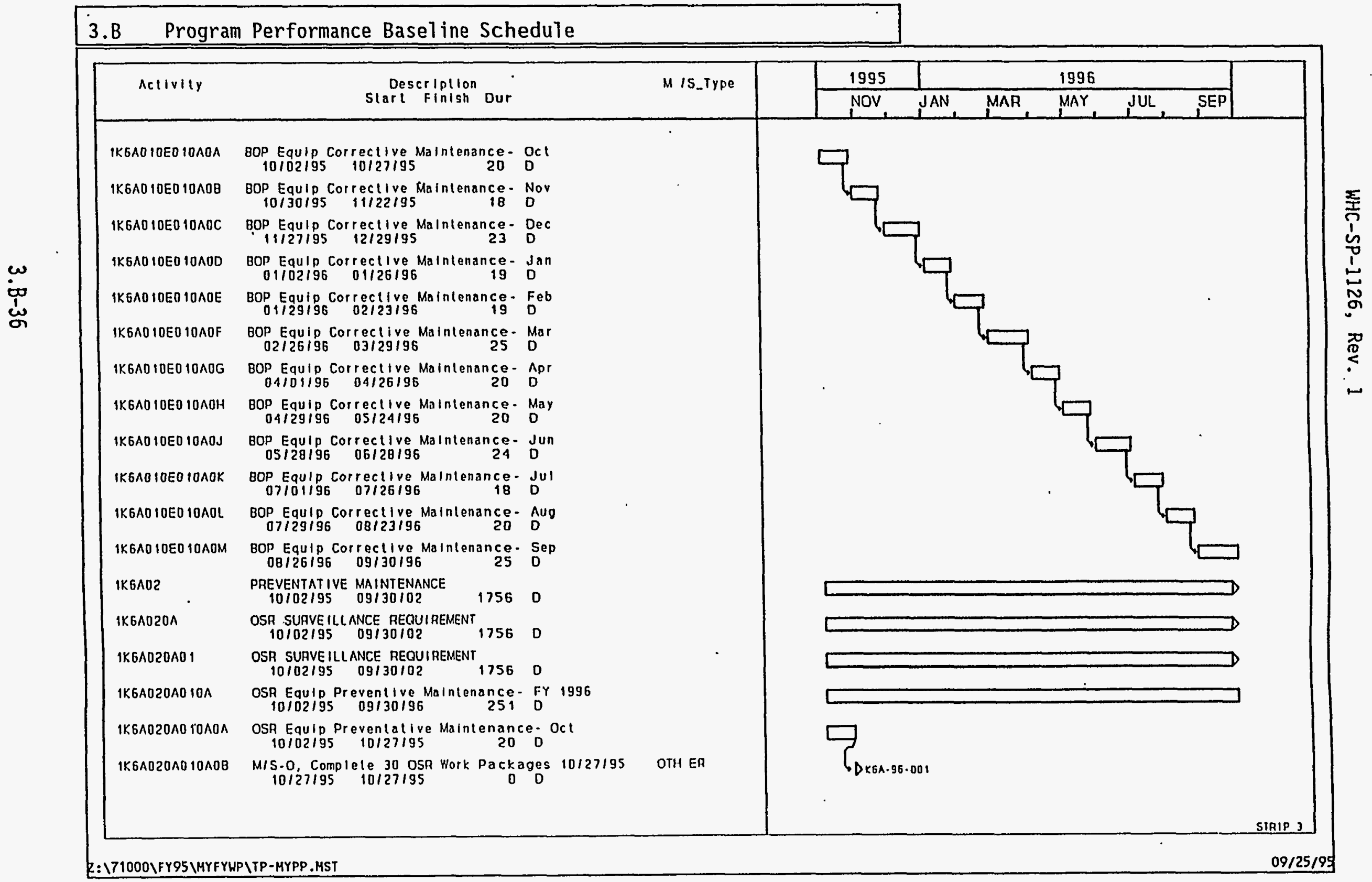




\section{B Program Performance Basel ine Schedule}

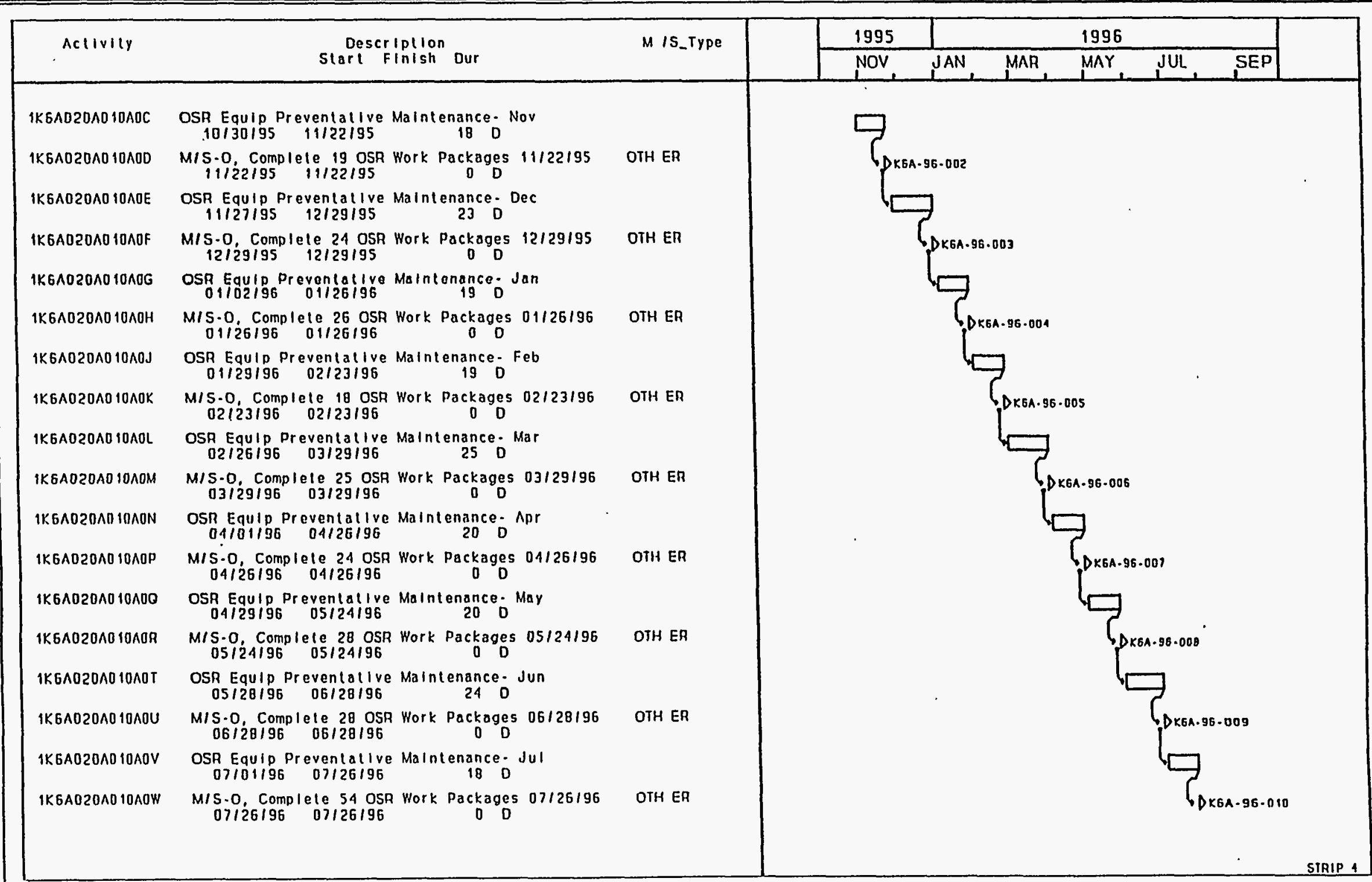


TRANSITION PROJECTS

FY 1996 MYPP 1.3.1/7.1/6.12

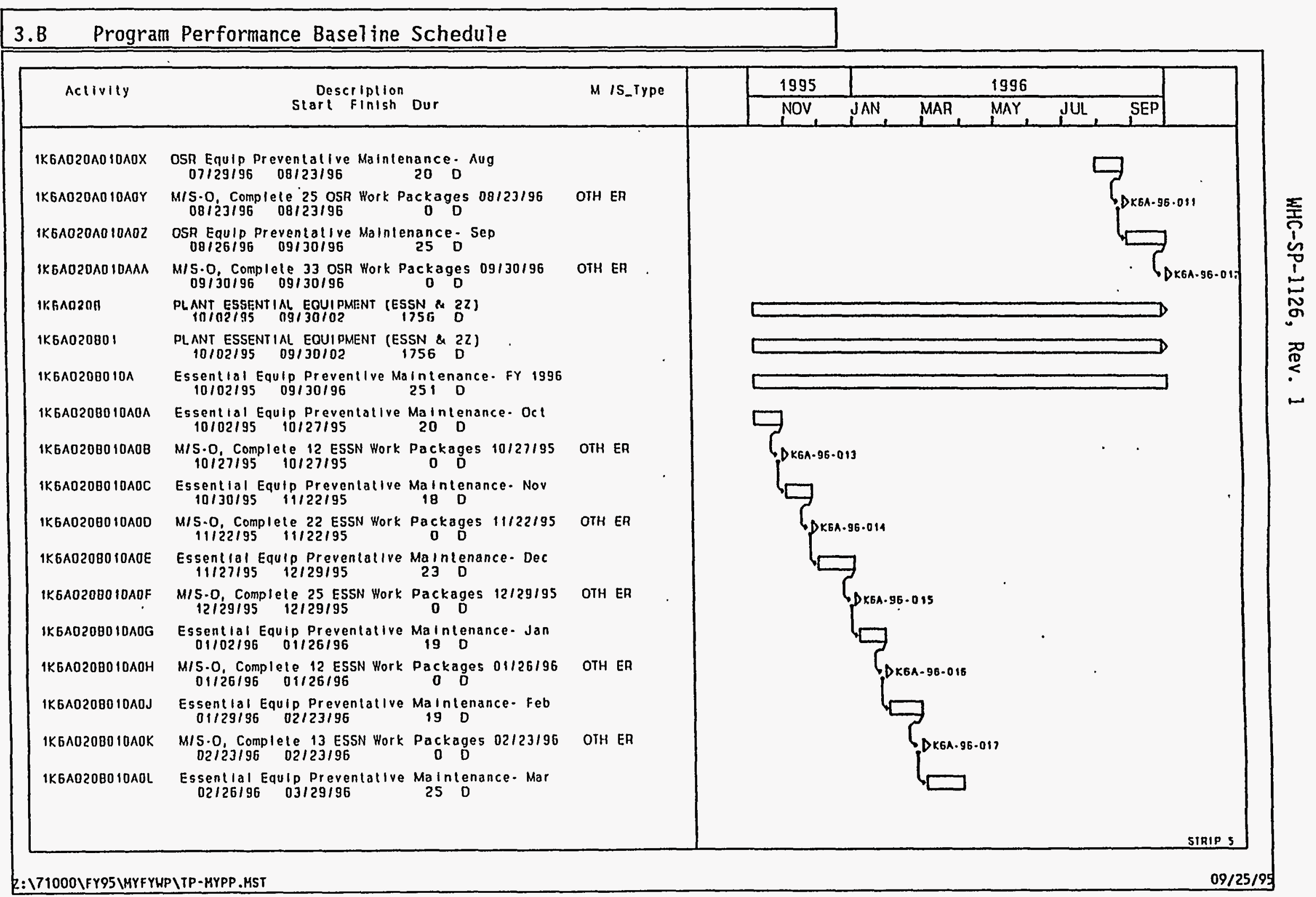


FY 1996 MYPP

\section{TRANSITION PROJECTS}

1.3.1/7.1/6.12

\section{B Program Performance Basel ine ScheduTe}

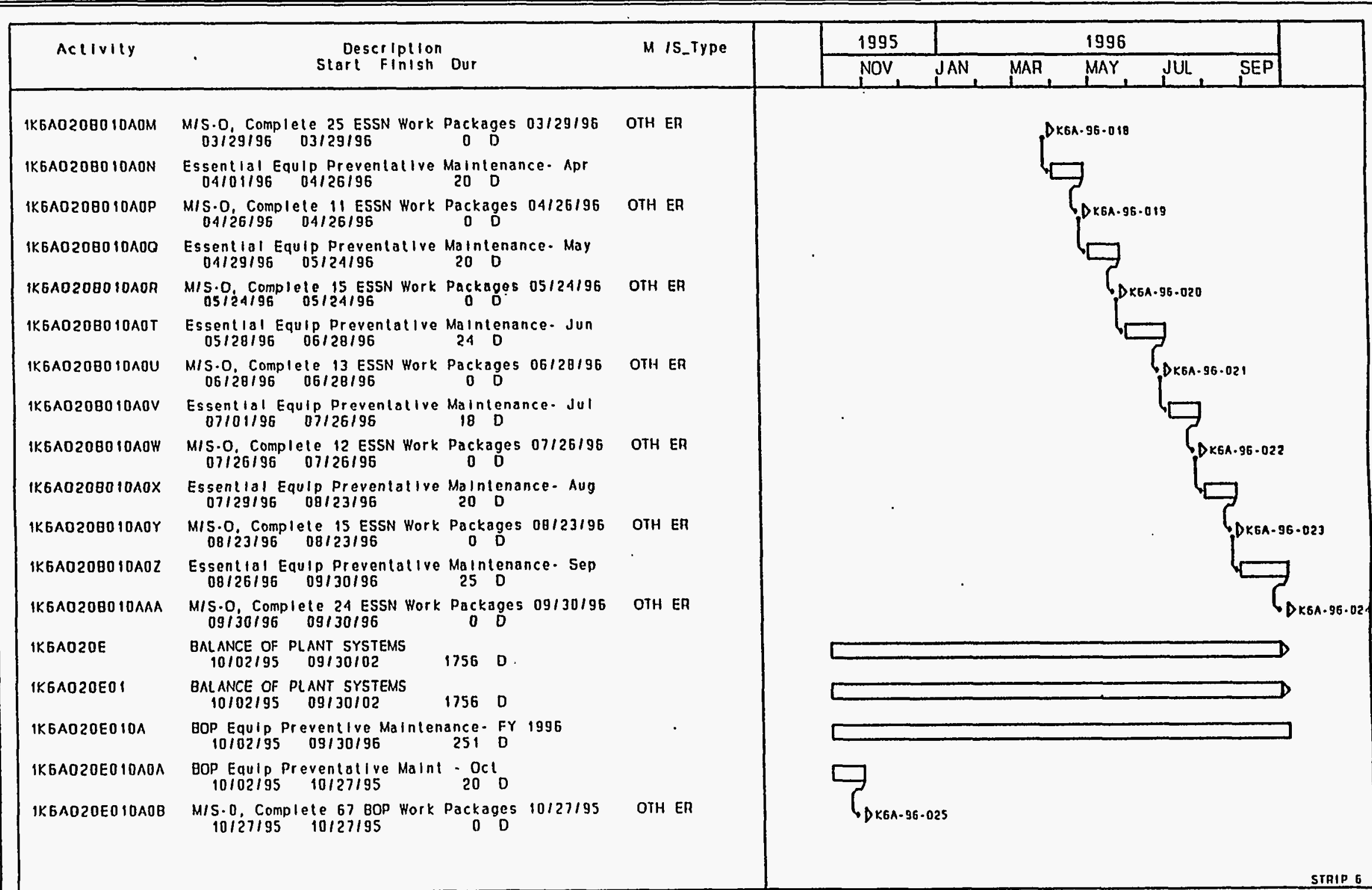




\section{TRANSITION PROJECTS}

\section{FY 1996 MYPP}

1.3.1/7.1/6.12

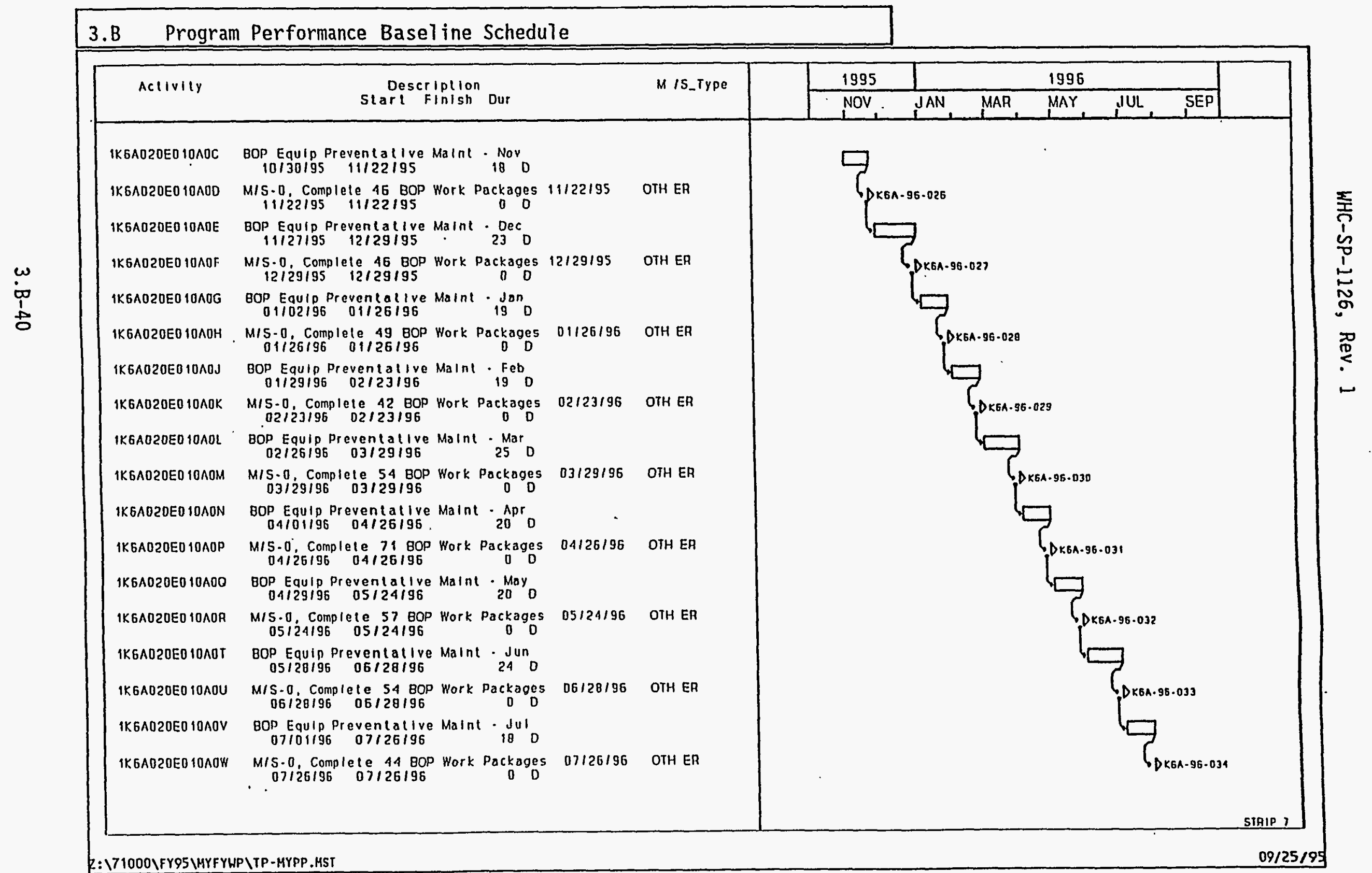




\section{TRANSITION PROJECTS}

FY 1996 MYPP

\subsection{1/7.1/6.12}

\section{B Program Performance Baseline Schedule}

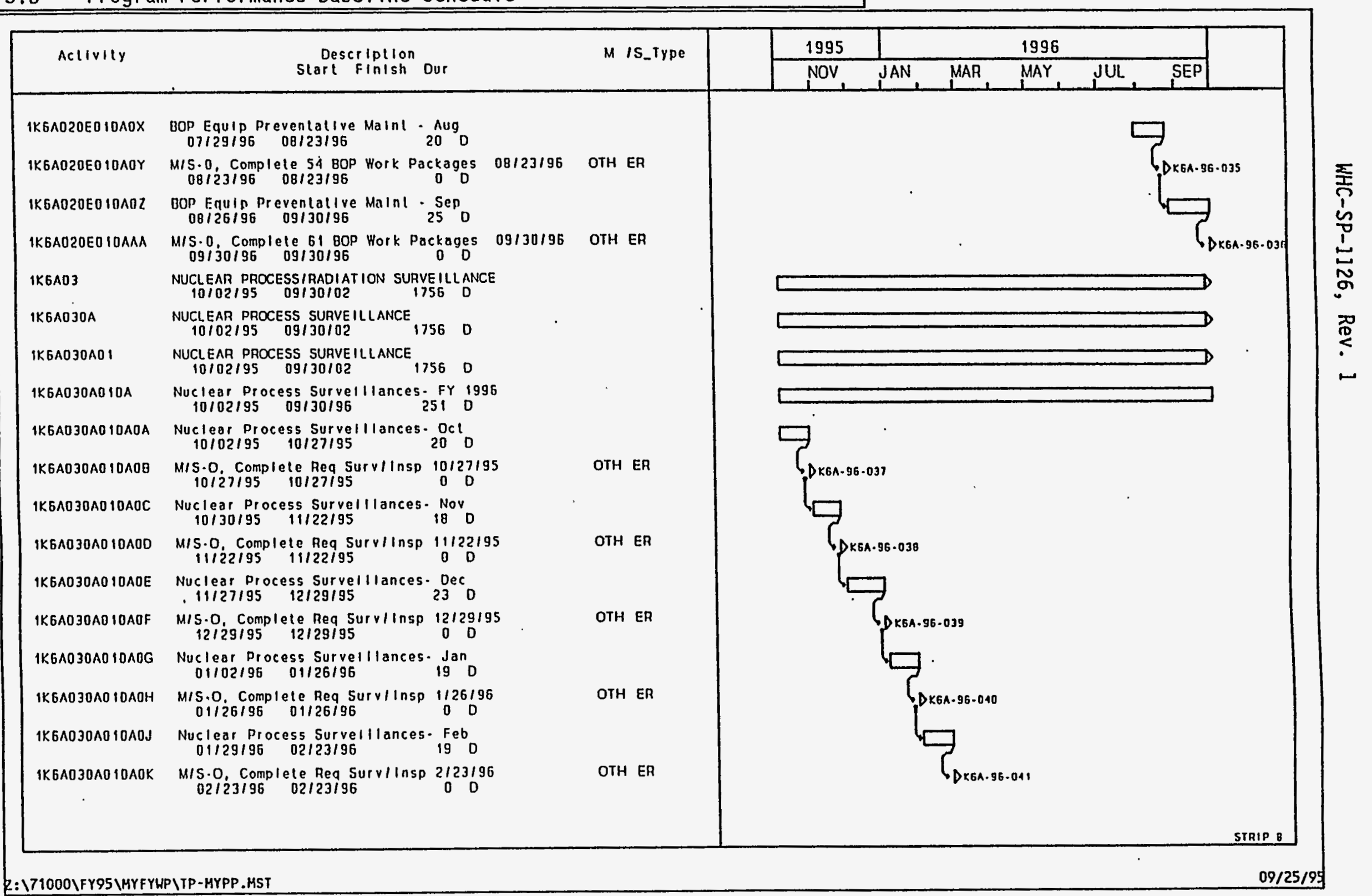


TRANSITION PROJECTS

FY 1996 MYPP

\subsection{1/7.1/6.12}

\section{B Program Performance Baseline Schedule}

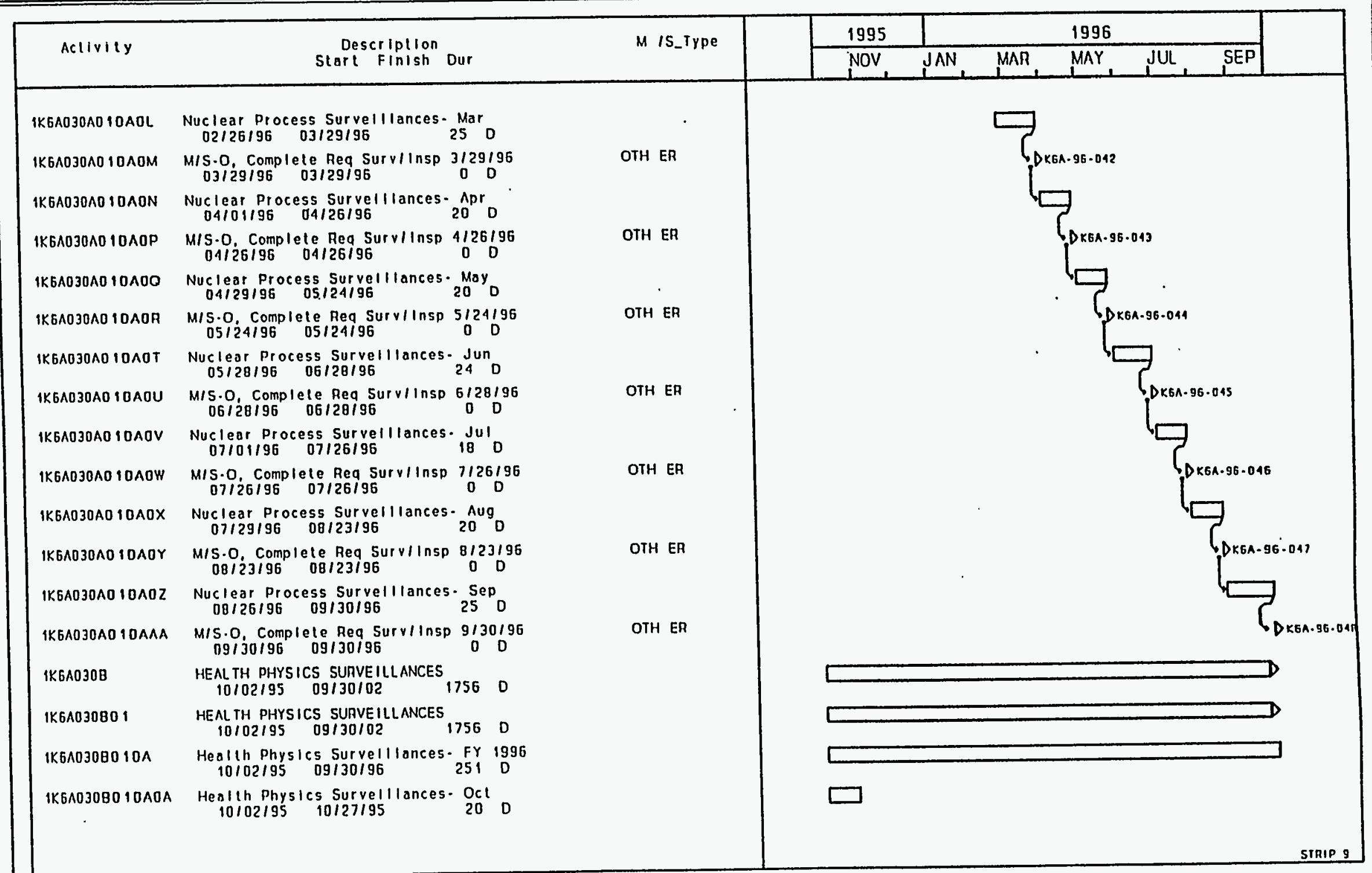




\section{TRANSITION PROJECTS}

\section{FY 1996 MYPP}

\subsection{1/7.1/6.12}

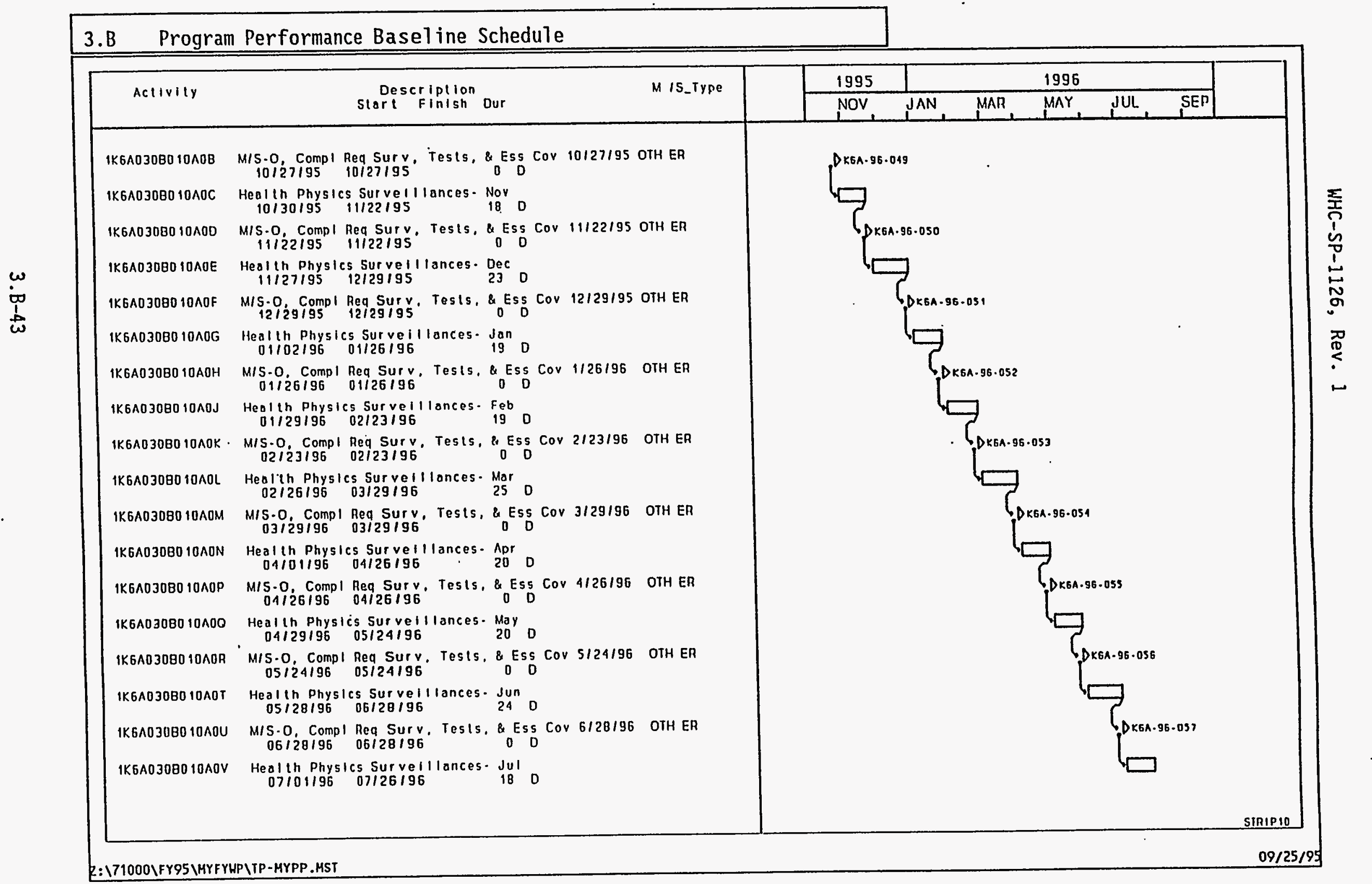


TRANSITION PROJECTS

FY 1996 MYPP

1.3.1/7.1/6.12

3. B Program Performance Basèline Schedule

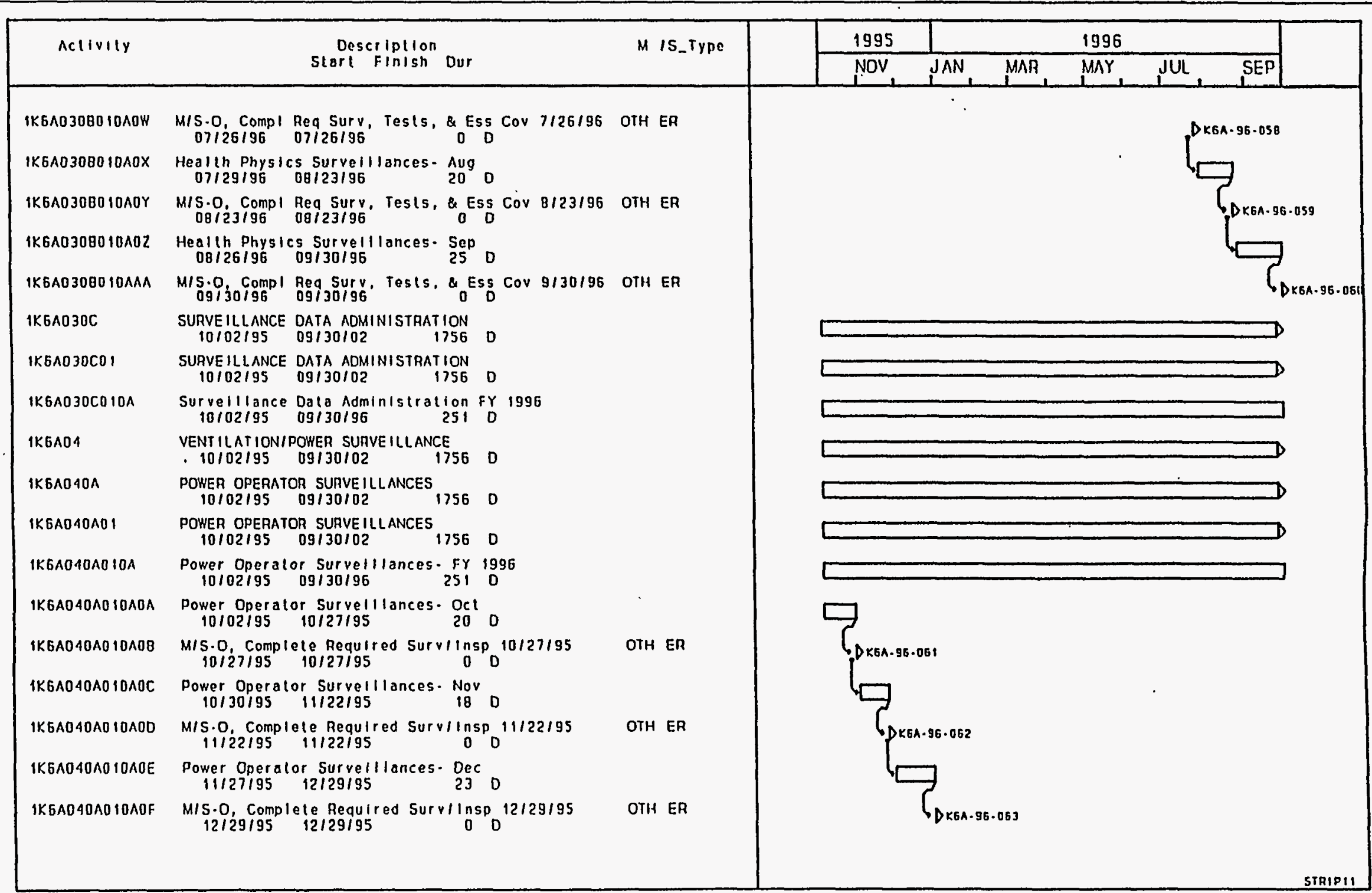




\section{FY 1996 MYPP}

\section{TRANSITION PROJECTS}

1.3.1/7.1/6.12

\section{B Program Performance Baseline Schedule}

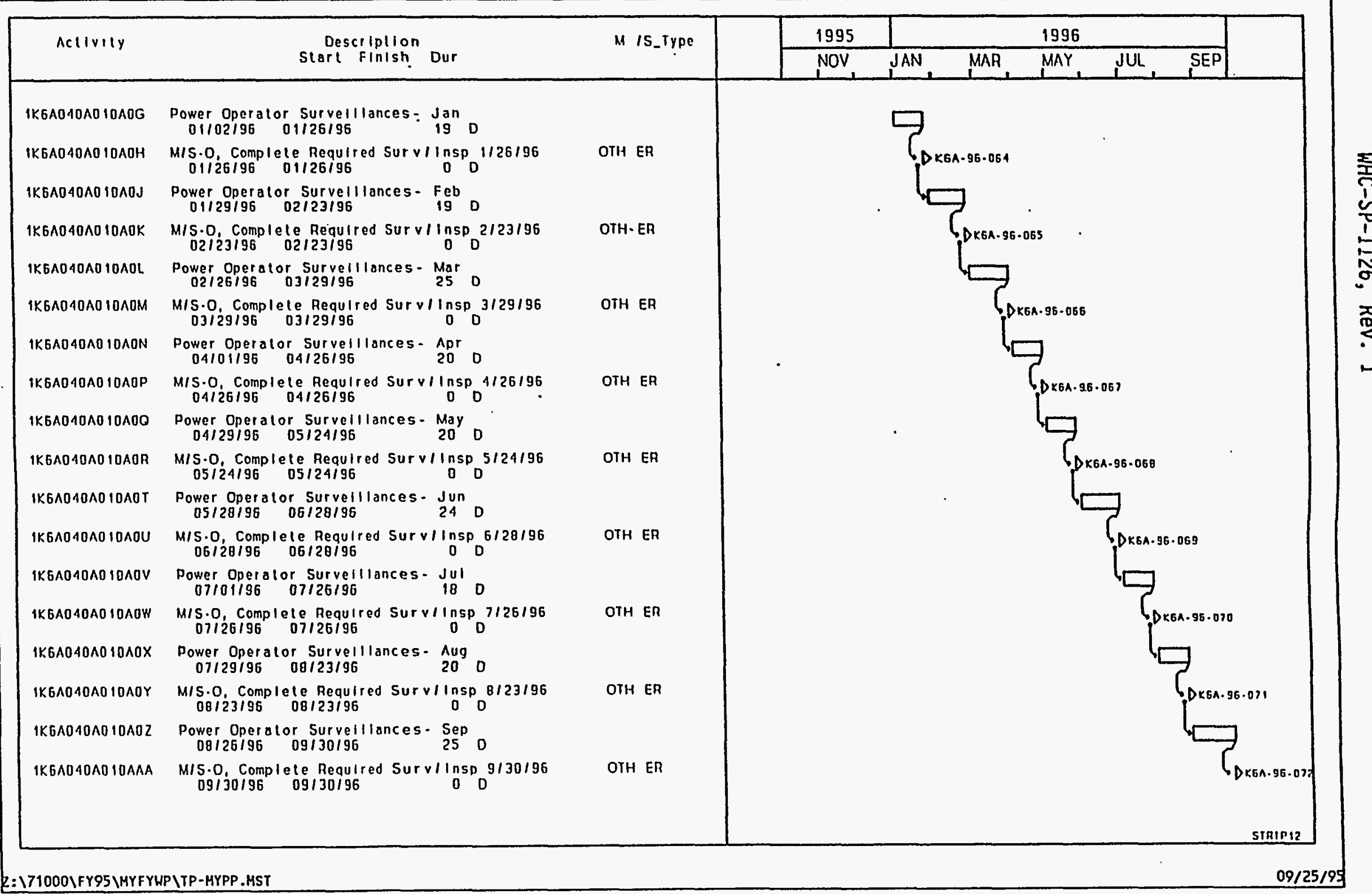




\section{FY 1996 MYPP}

\section{TRANSITION PROJECTS}

1.3.1/7.1/6.12

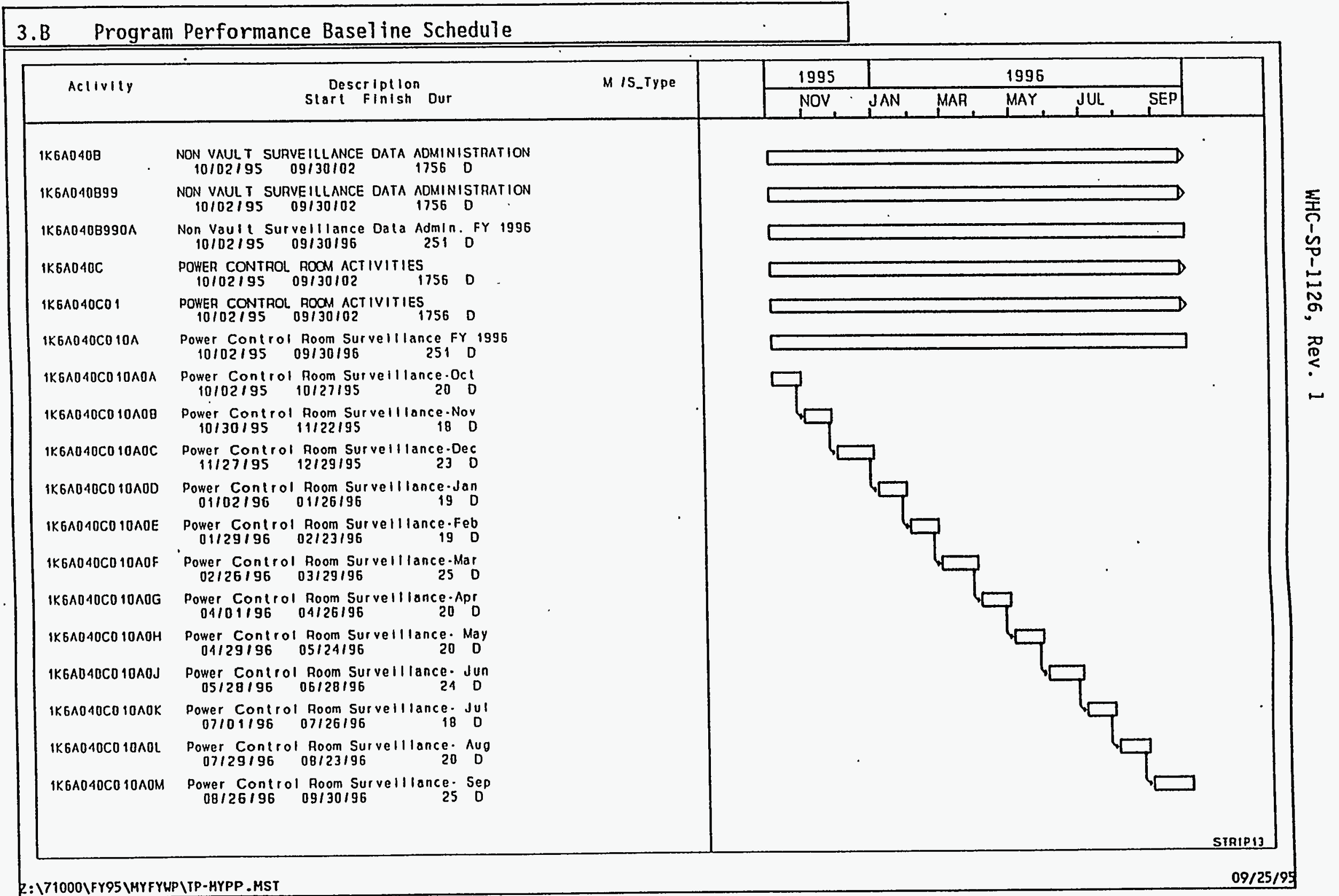


FY 1996 MYPP

TRANSITION PROJECTS

1.3.1/7.1/6.12

3.B Program Performance Basel ine Schedule

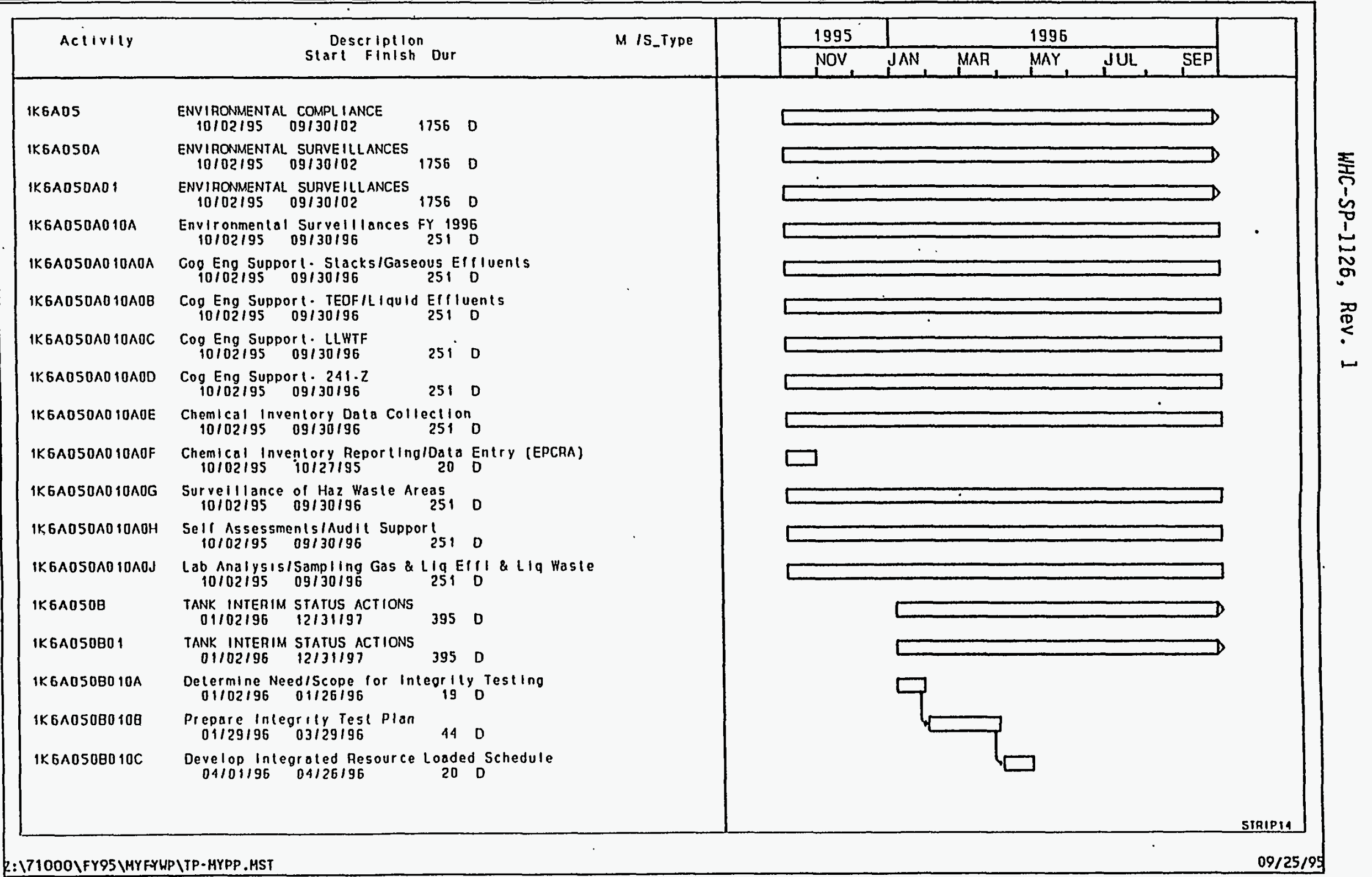




\section{TRANSITION PROJECTS}

FY 1996 MYPP

1.3.1/7.1/6.12

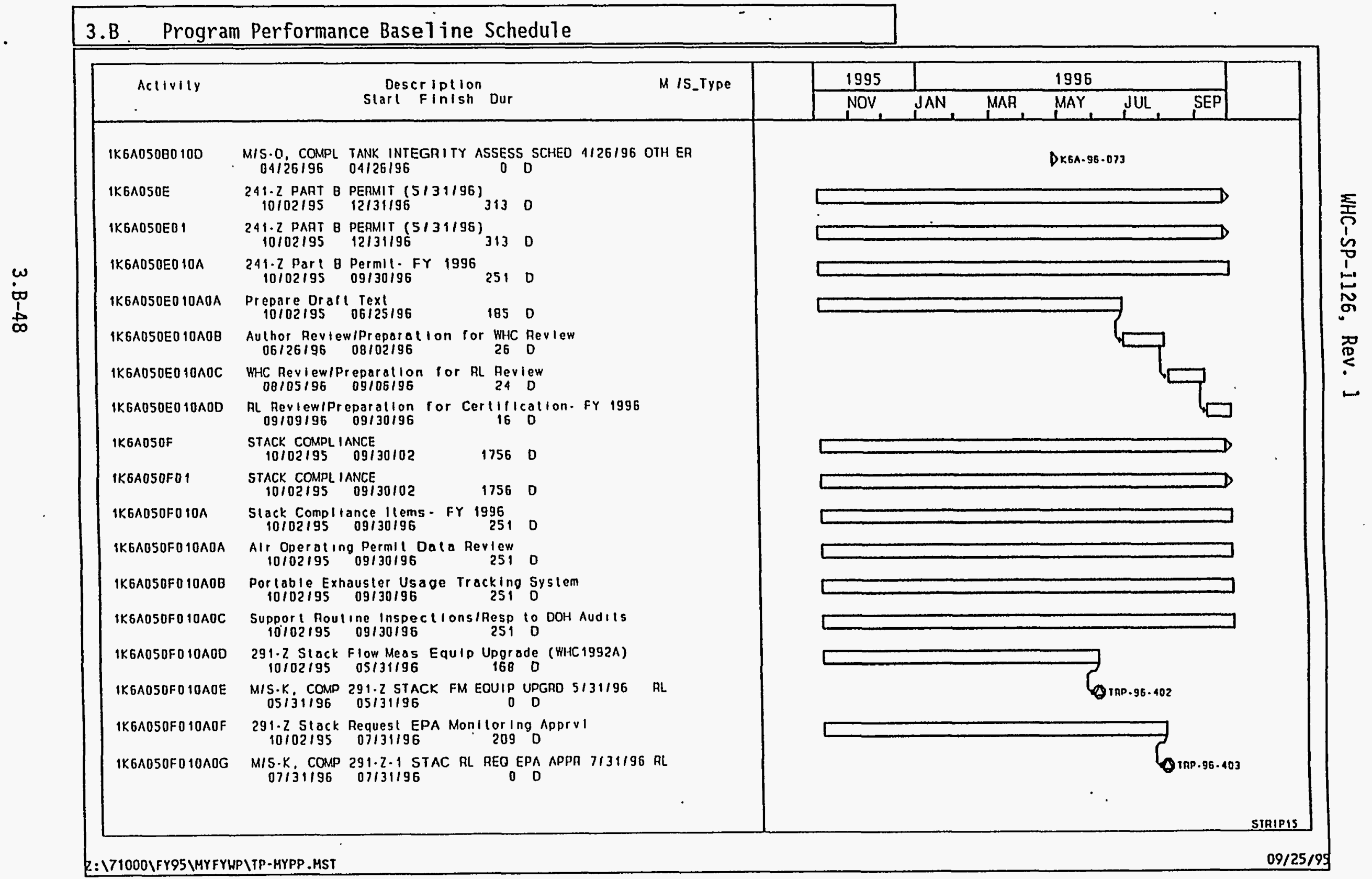


FY 1996 MYPP

TRANSITIGIN PROJECTS

1.3.1/7.1/6.12

3.B Program Performance Baseline Schedule

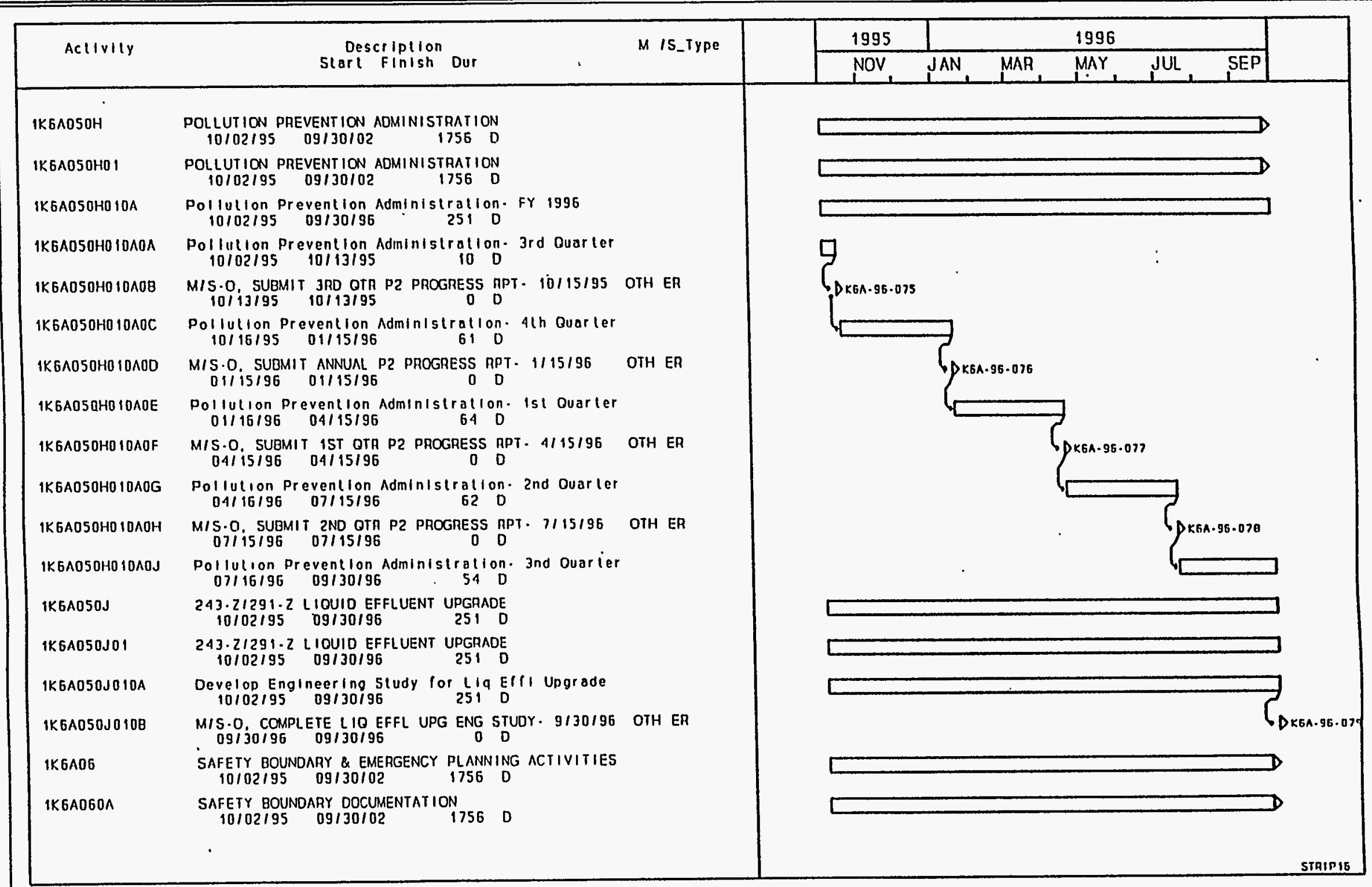




\section{TRANSITION PROJECTS}

\section{FY 1996 MYPP}

\subsection{1/7.1/6.12}

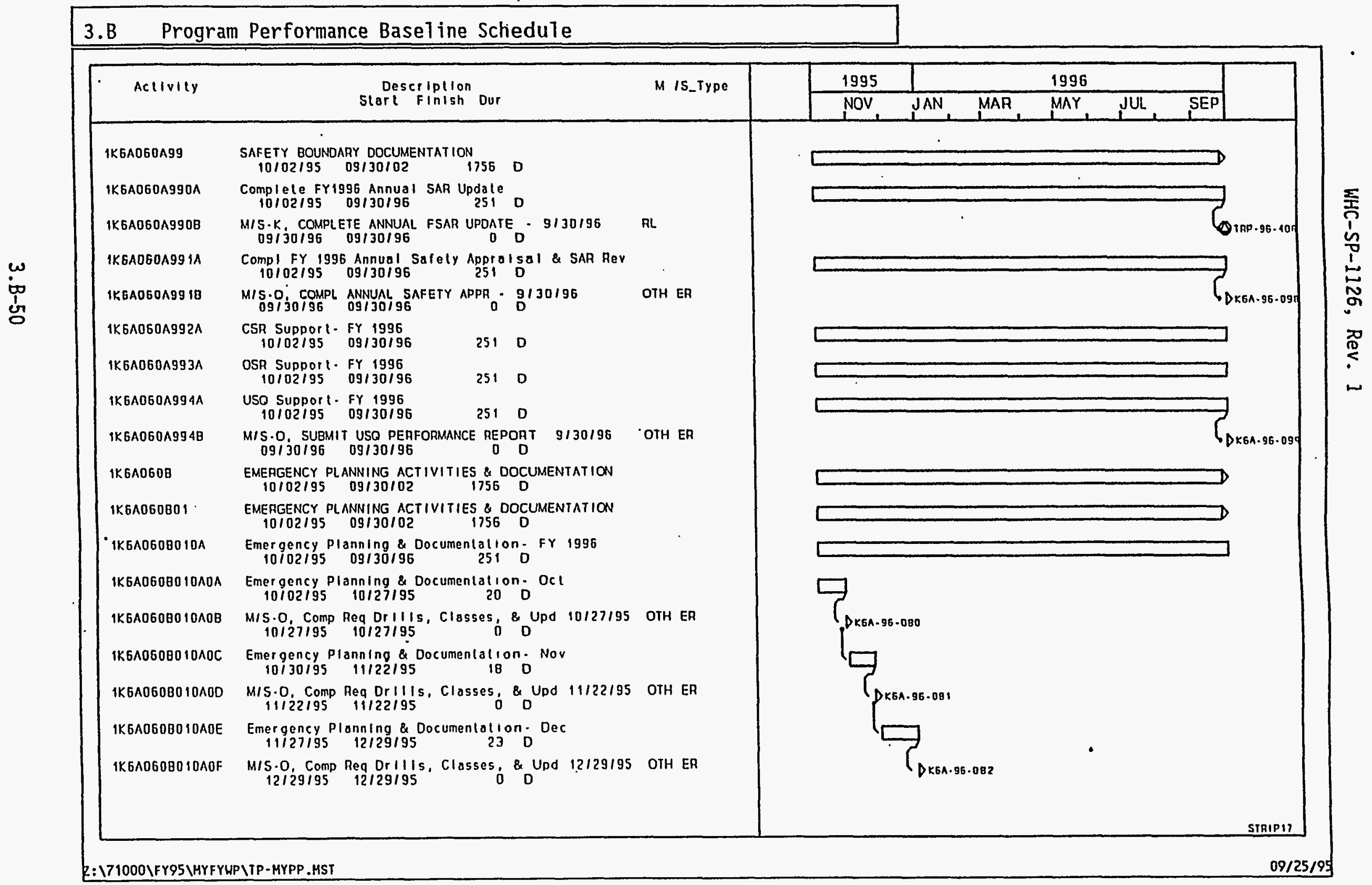




\section{TRANSITION PROJECTS}

FY 1996 MYPP

\subsection{1/7.1/6.12}

\section{B Program Performance Basel ine Schedule}

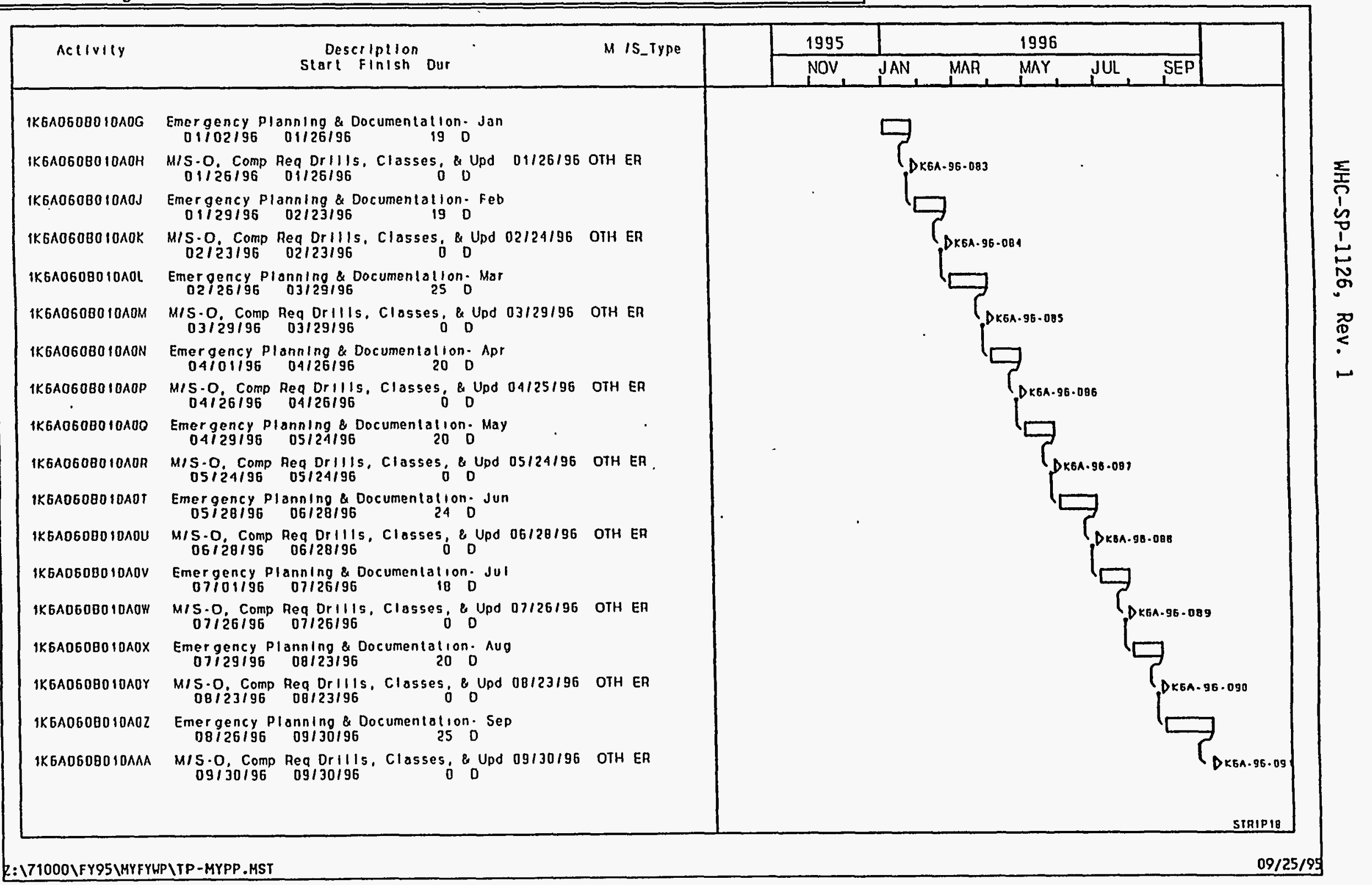




\section{TRANSITION PROJECTS}

\section{FY 1996 MYPP}

\subsection{1/7.1/6.12}

\section{B Program Performance Basel ine Schedu7e}

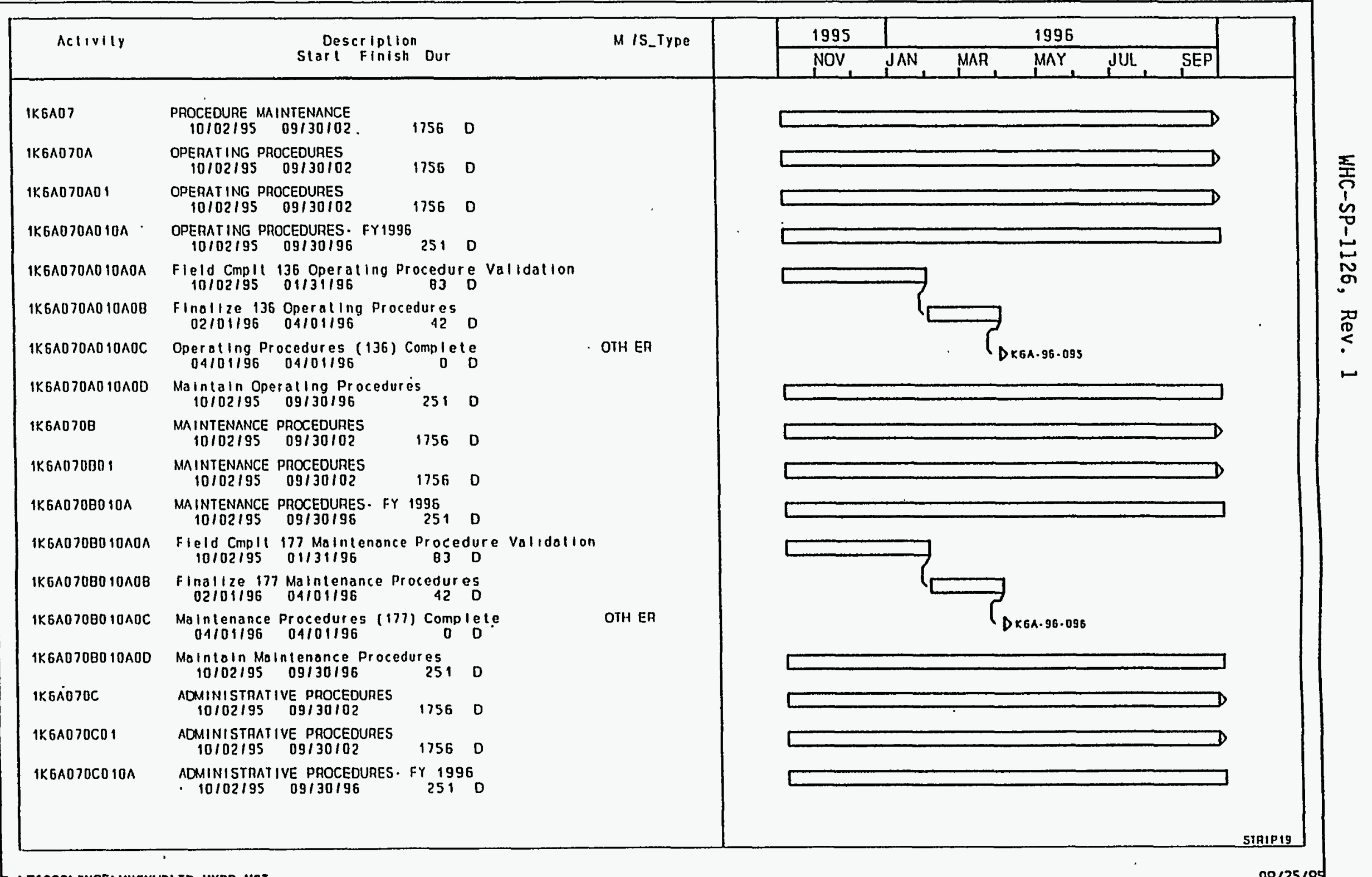




\section{FY 1996 MYPP}

\section{TRANSITION PROJECTS}

1.3.1/7.1/6.12

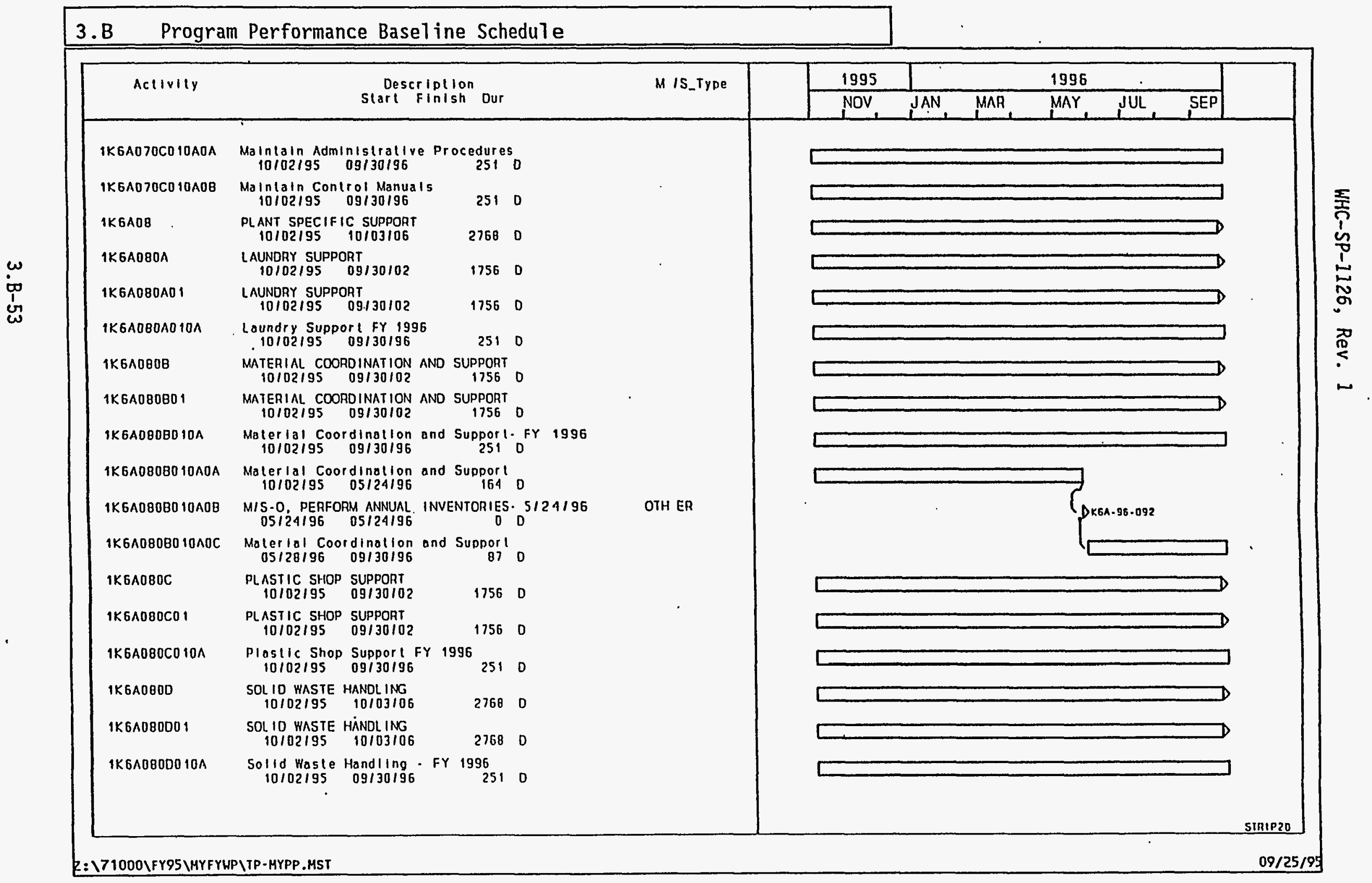




\section{TRANSITION PROJECTS}

FY 1996 MYPP

1.3.1/7.1/6.12

3.B. Program Performance Baseline Schedule

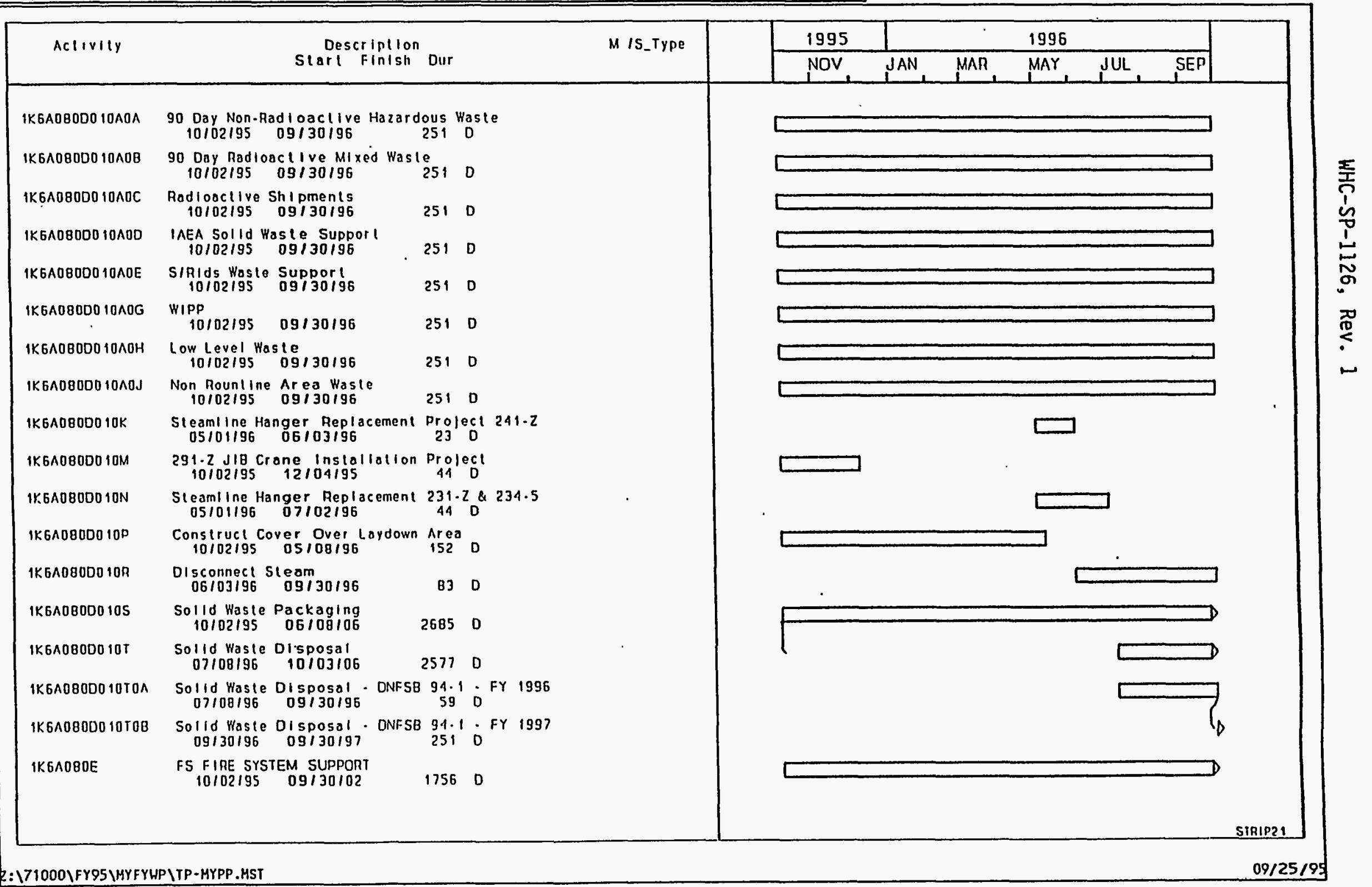




\section{TRANSITION PROJECTS}

FY 1996 MYPP

1.3.1/7.1/6.12

3.B Program Performance Baseline Schedule

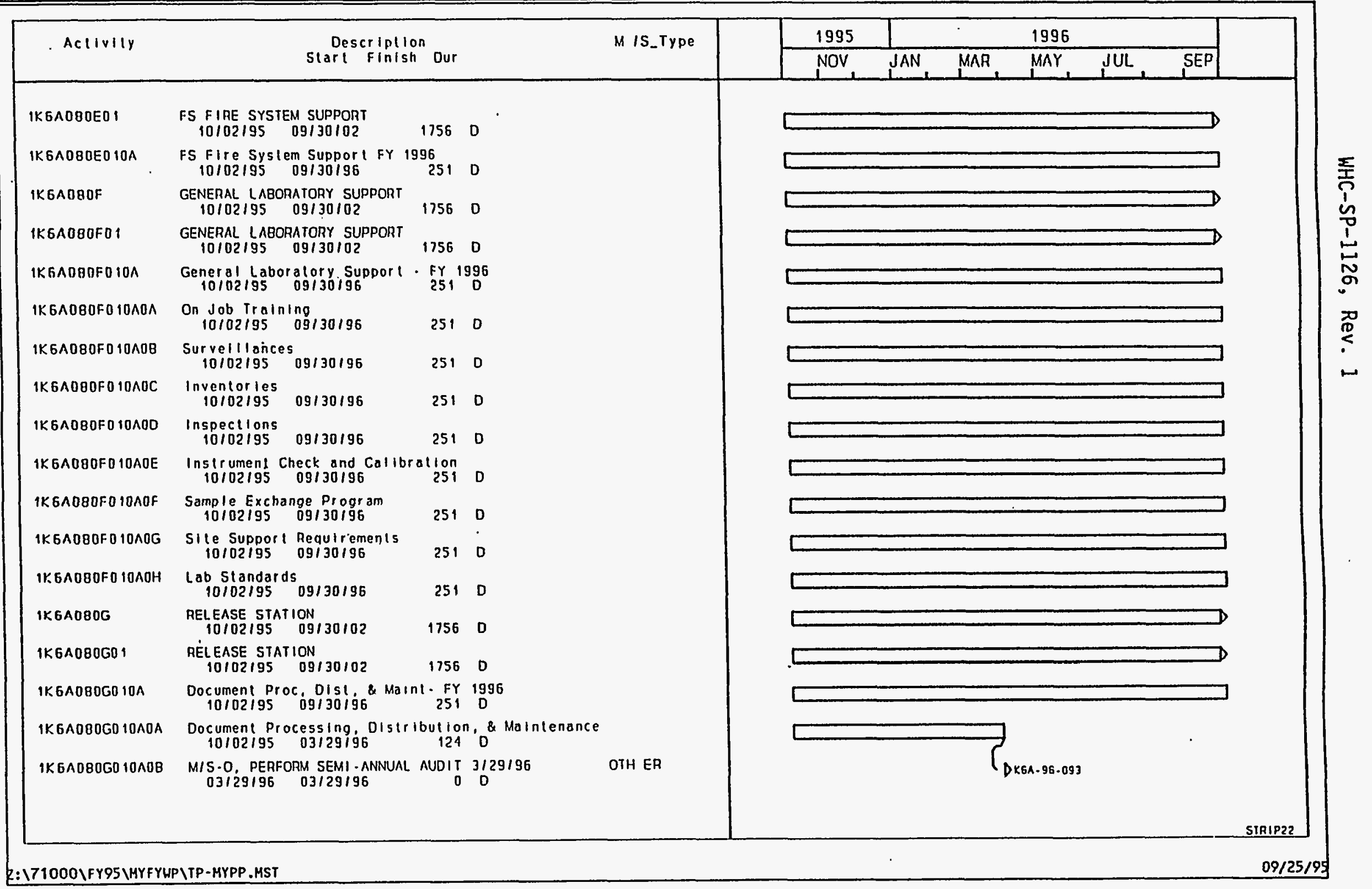


FY 1996 MYPP

\section{TRANSITION PROJECTS}

1.3.1/7.1/6.12

\section{B Program Performance Basel ine Schedule}

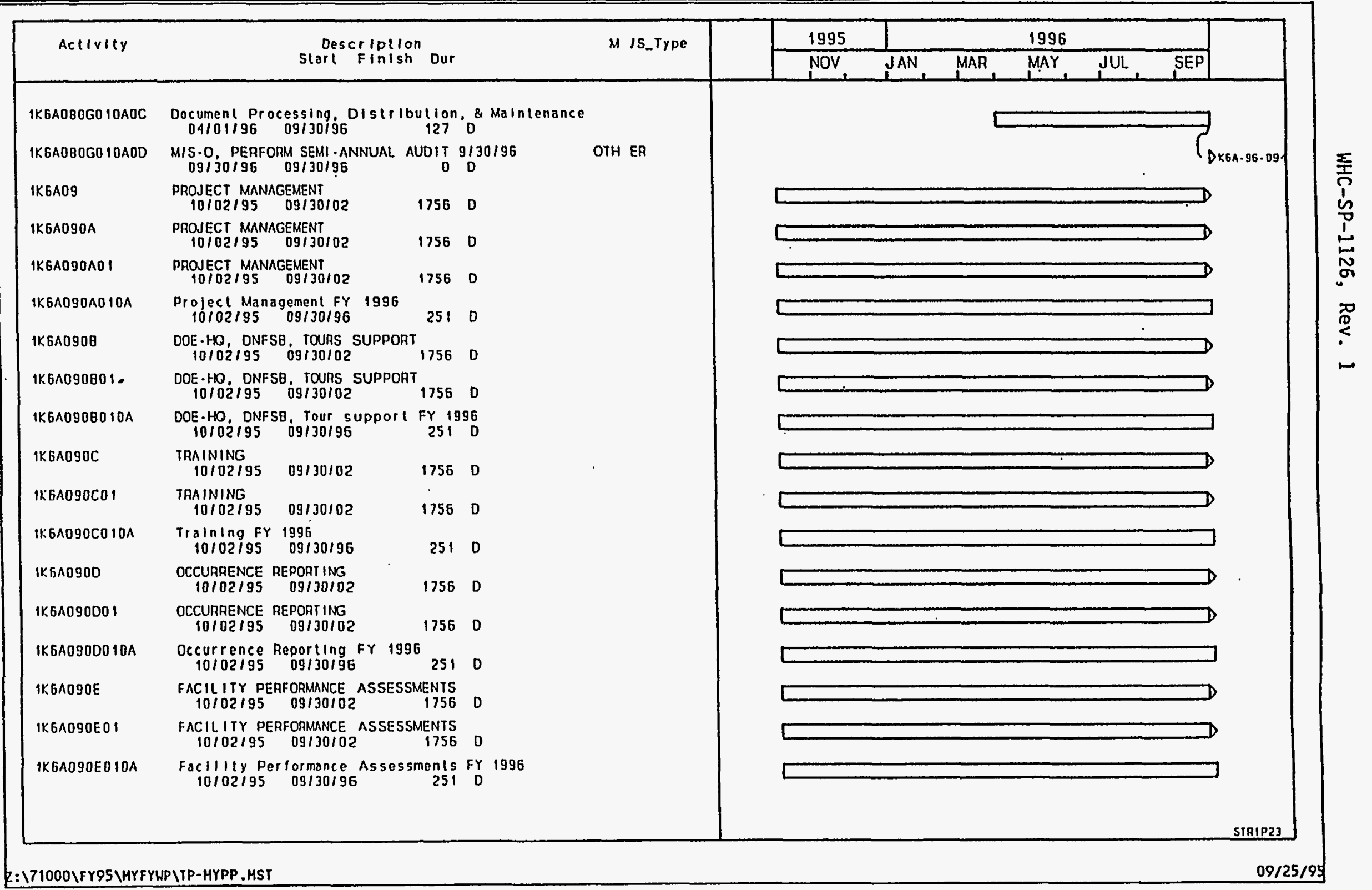




\section{TRANSITION PROJECTS}

\section{FY 1996 MYPP}

\subsection{1/7.1/6.12}

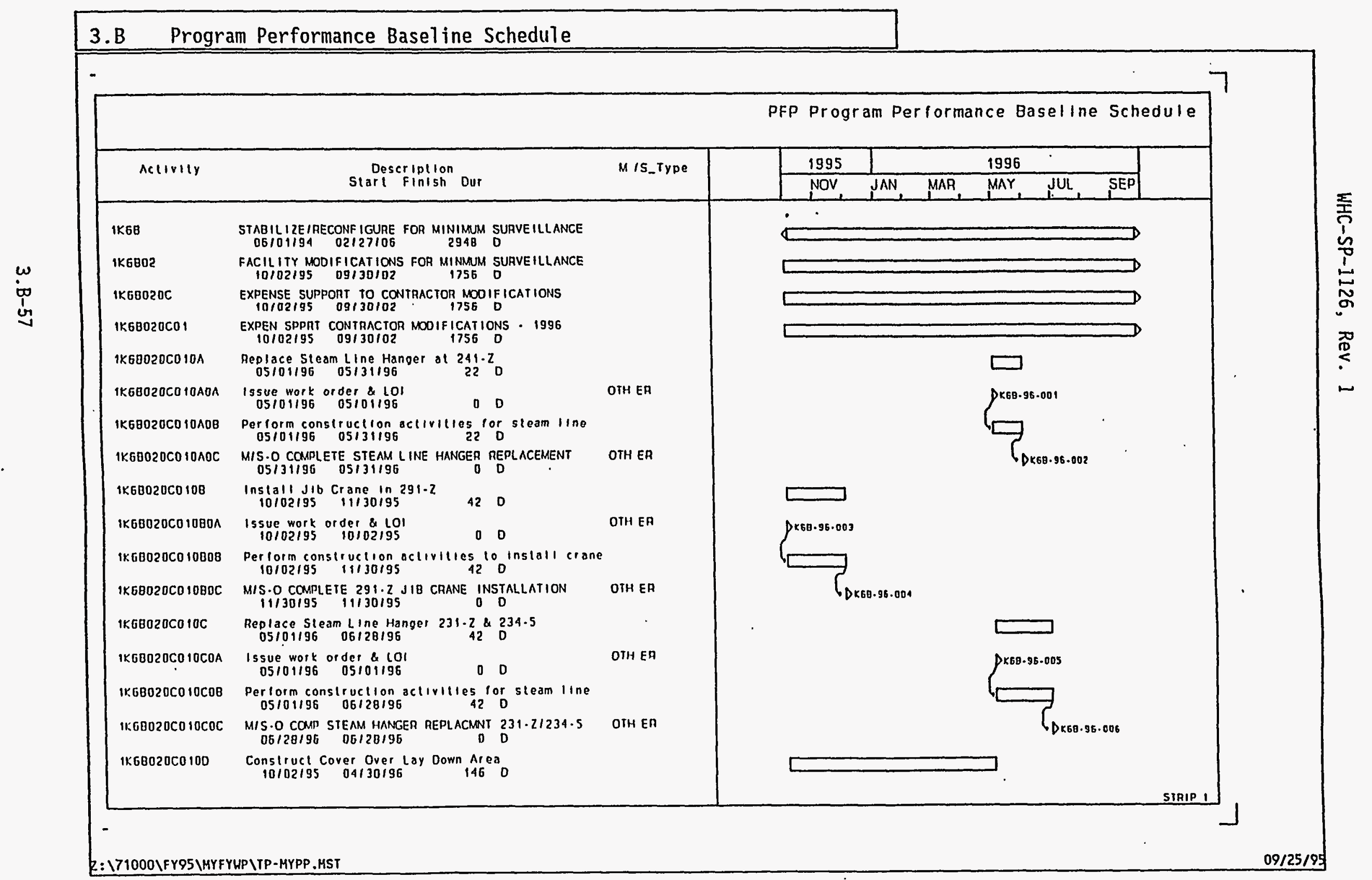


TRANSITIUN PROJECTS

FY 1996 MYPP

\subsection{1/7.1/6.12}

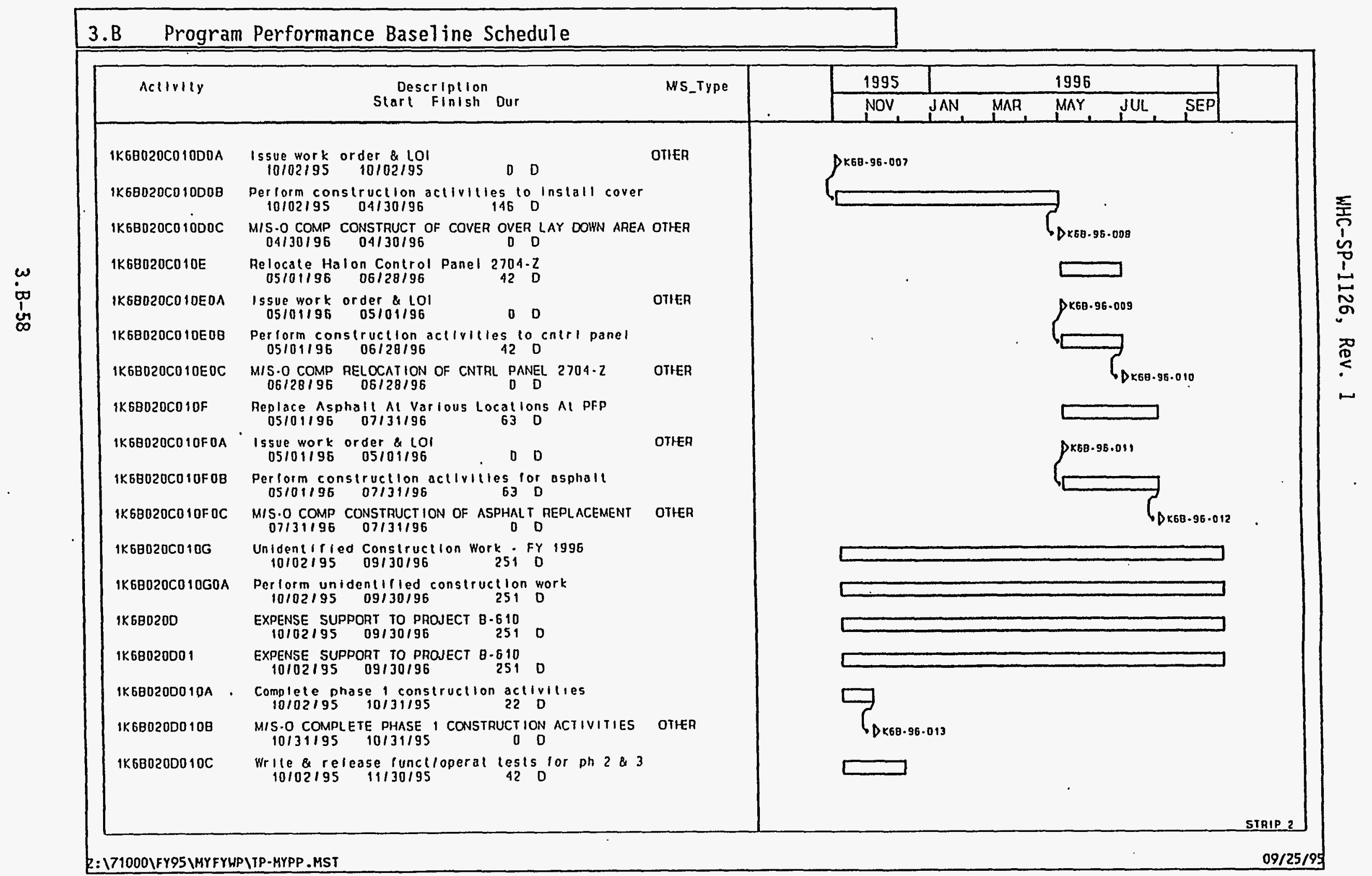




\section{TRANSITION PROJECTS}

FY 1996 MYPP

\subsection{1/7.1/6.12}

\section{B Program Performance Basel ine Schedule}

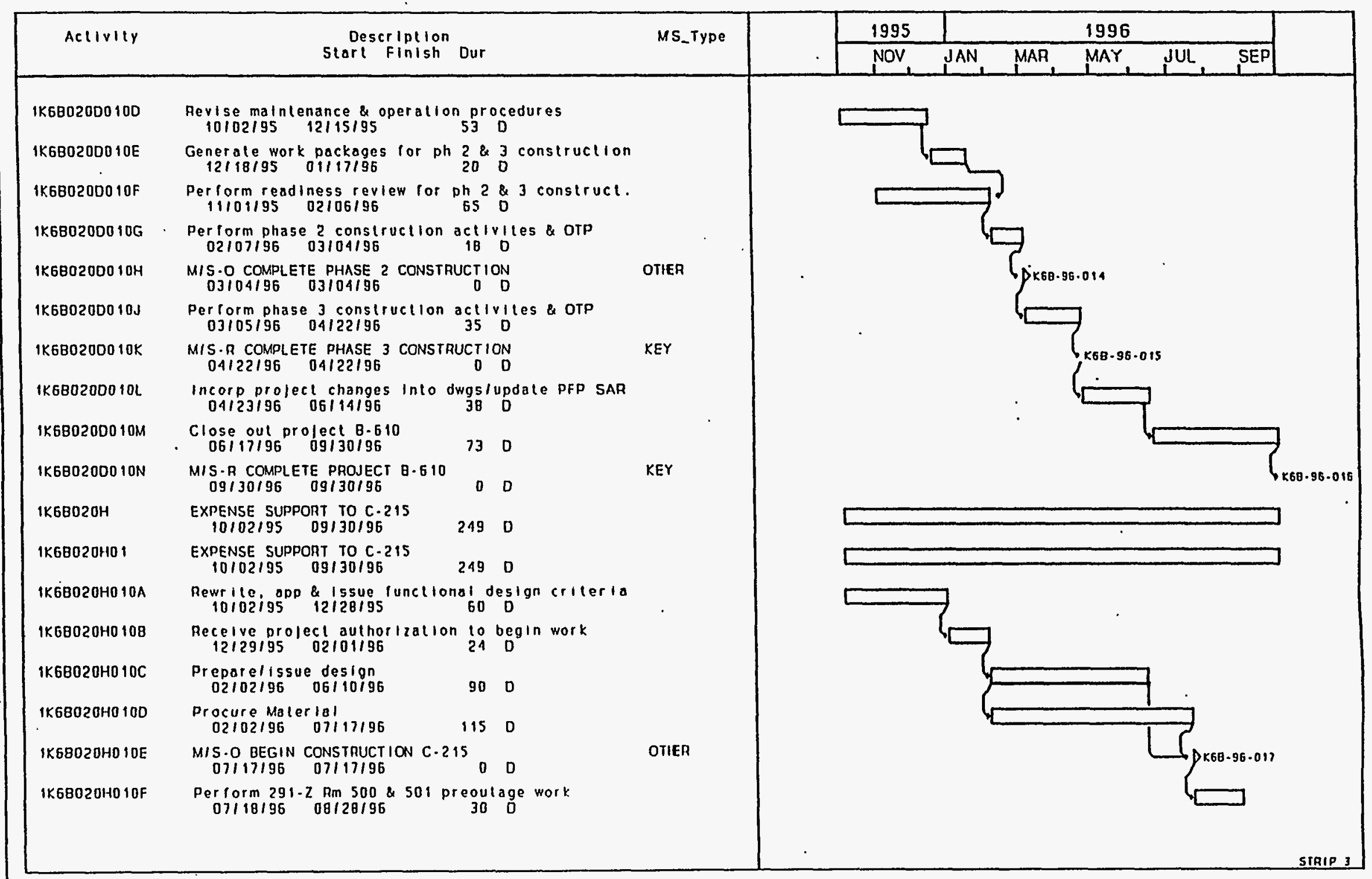




\section{TRANSITION PROJECTS}

\section{FY 1996 MYPP}

\subsection{1/7.1/6.12}

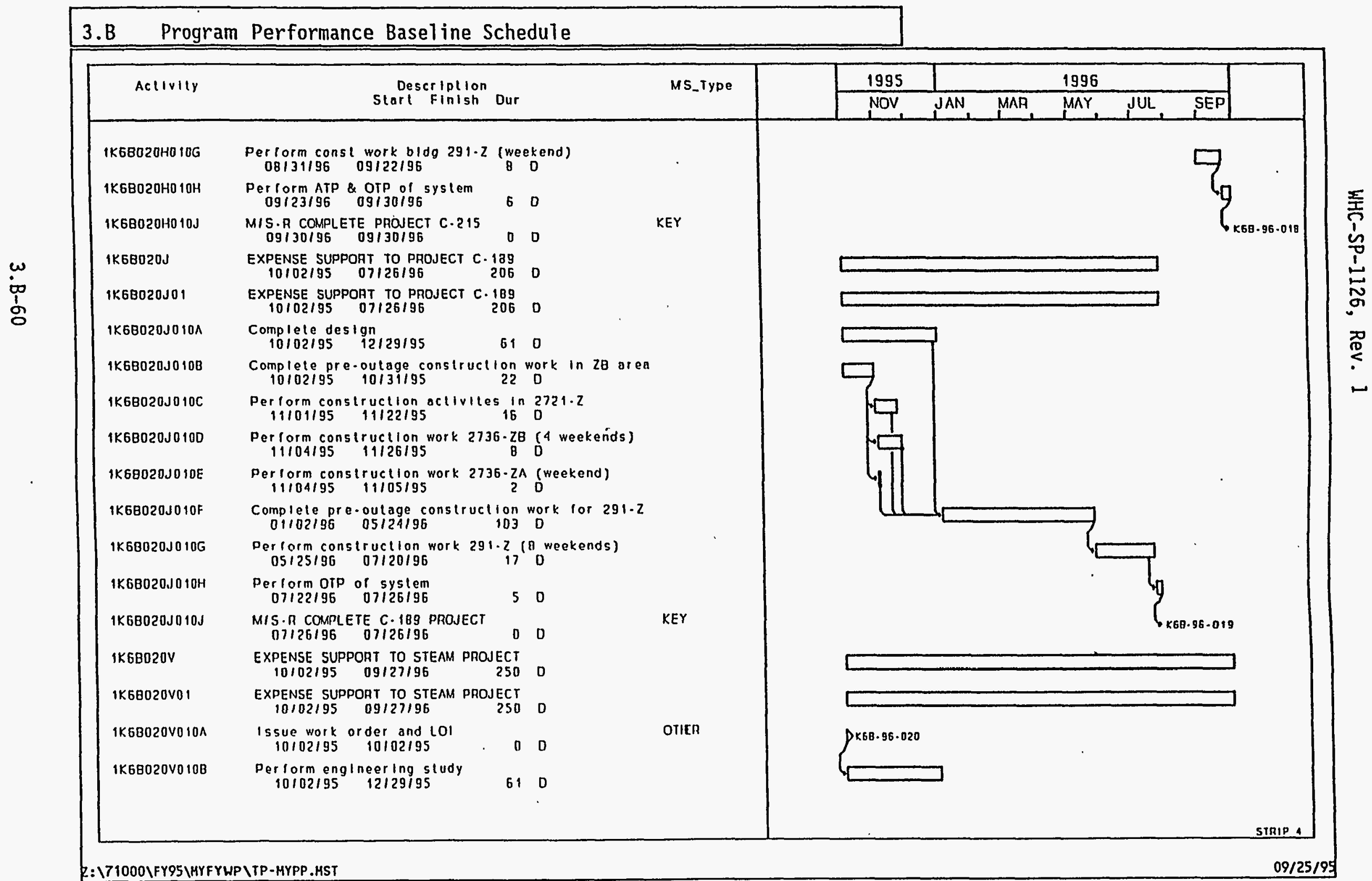




\section{TRANSITION PROJECTS}

\section{FY 1996 MYPP}

\subsection{1/7.1/6.12}

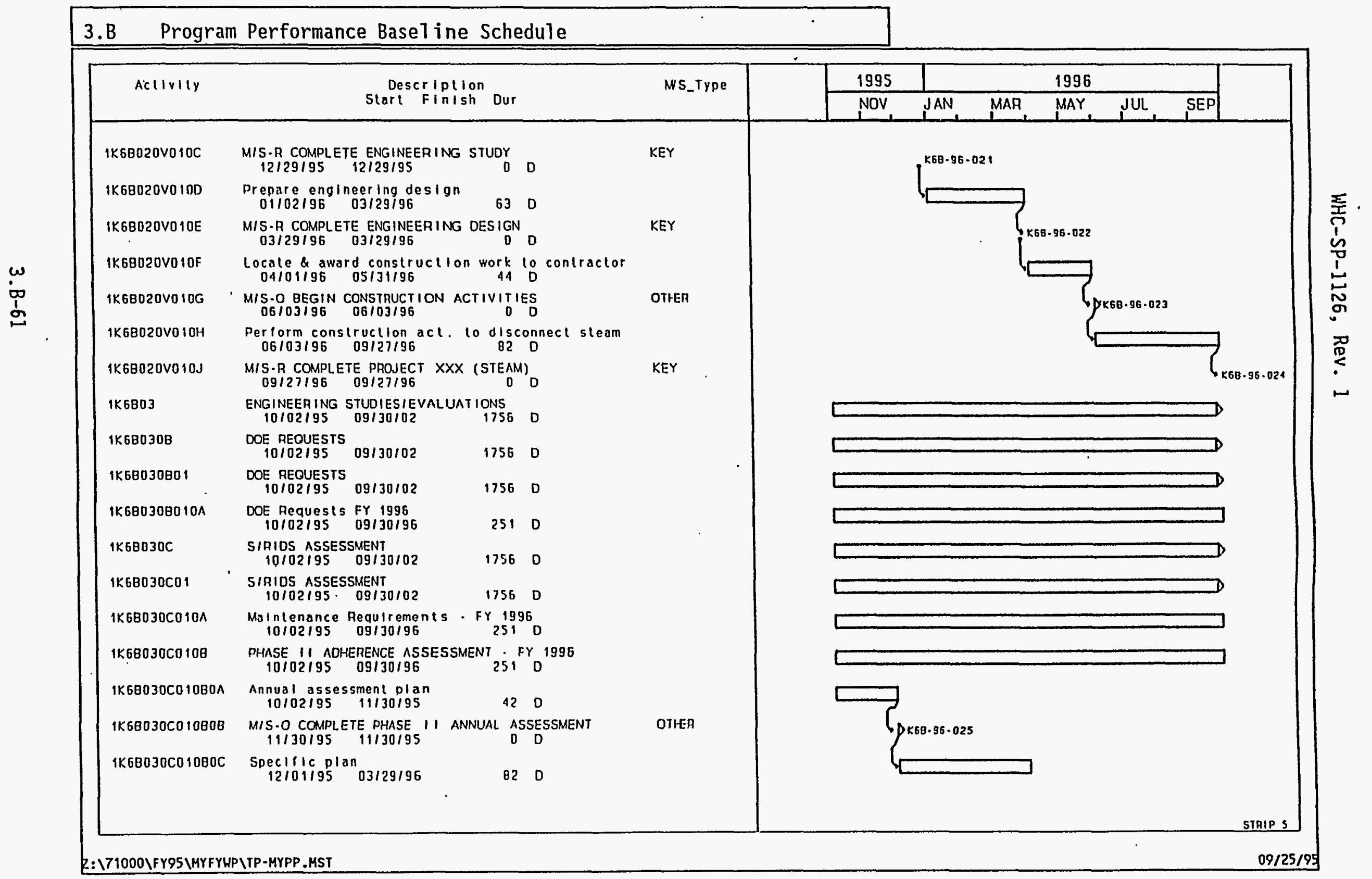




\section{TRANSITION PROJECTS}

FY 1996 MYPP

\subsection{1/7.1/6.12}

\section{B Program Performance Basel ine Schedule}

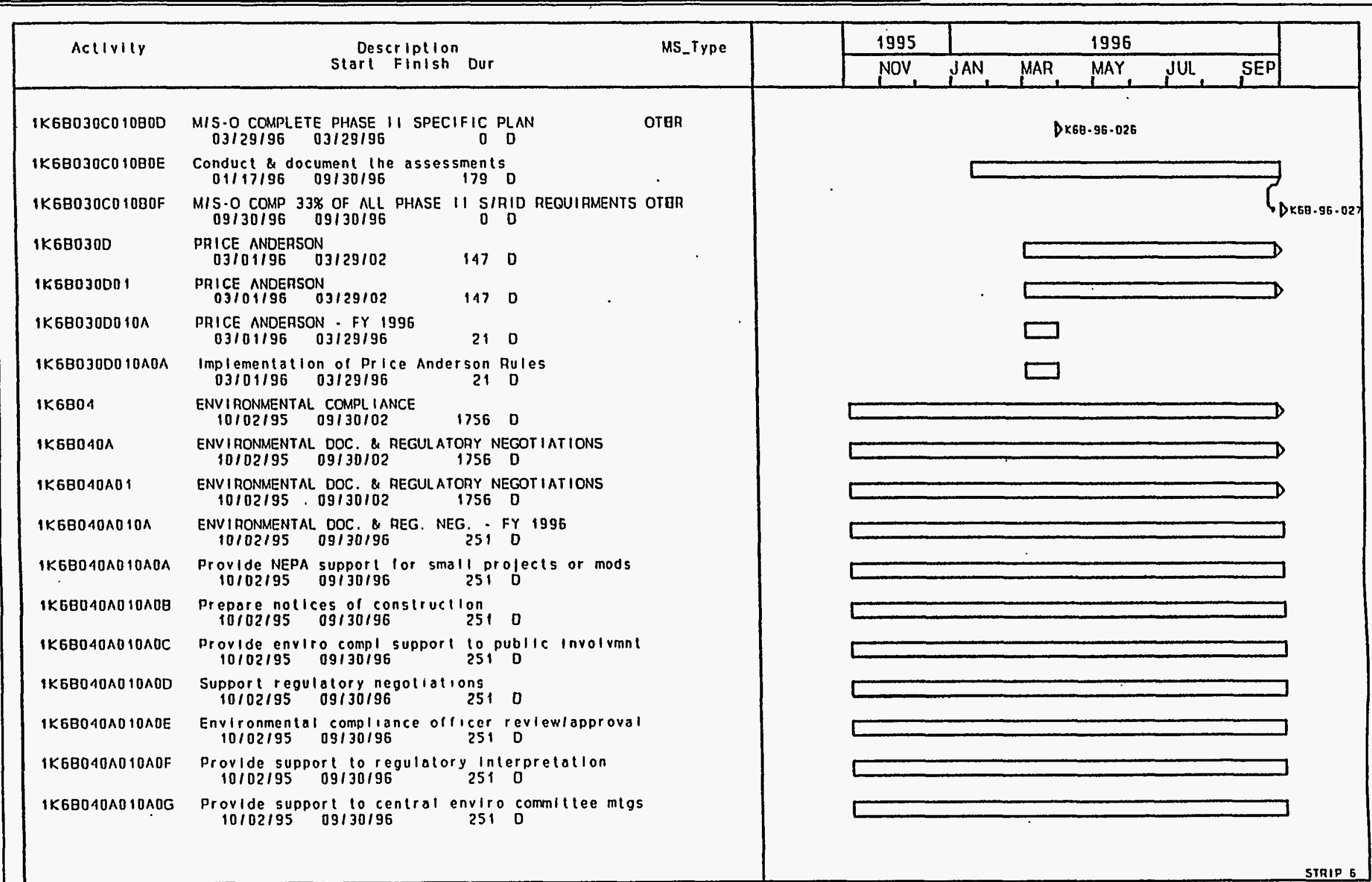




\section{TRANSITION PROJECTS}

\section{FY 1996 MYPP}

\subsection{1/7.1/6.12}

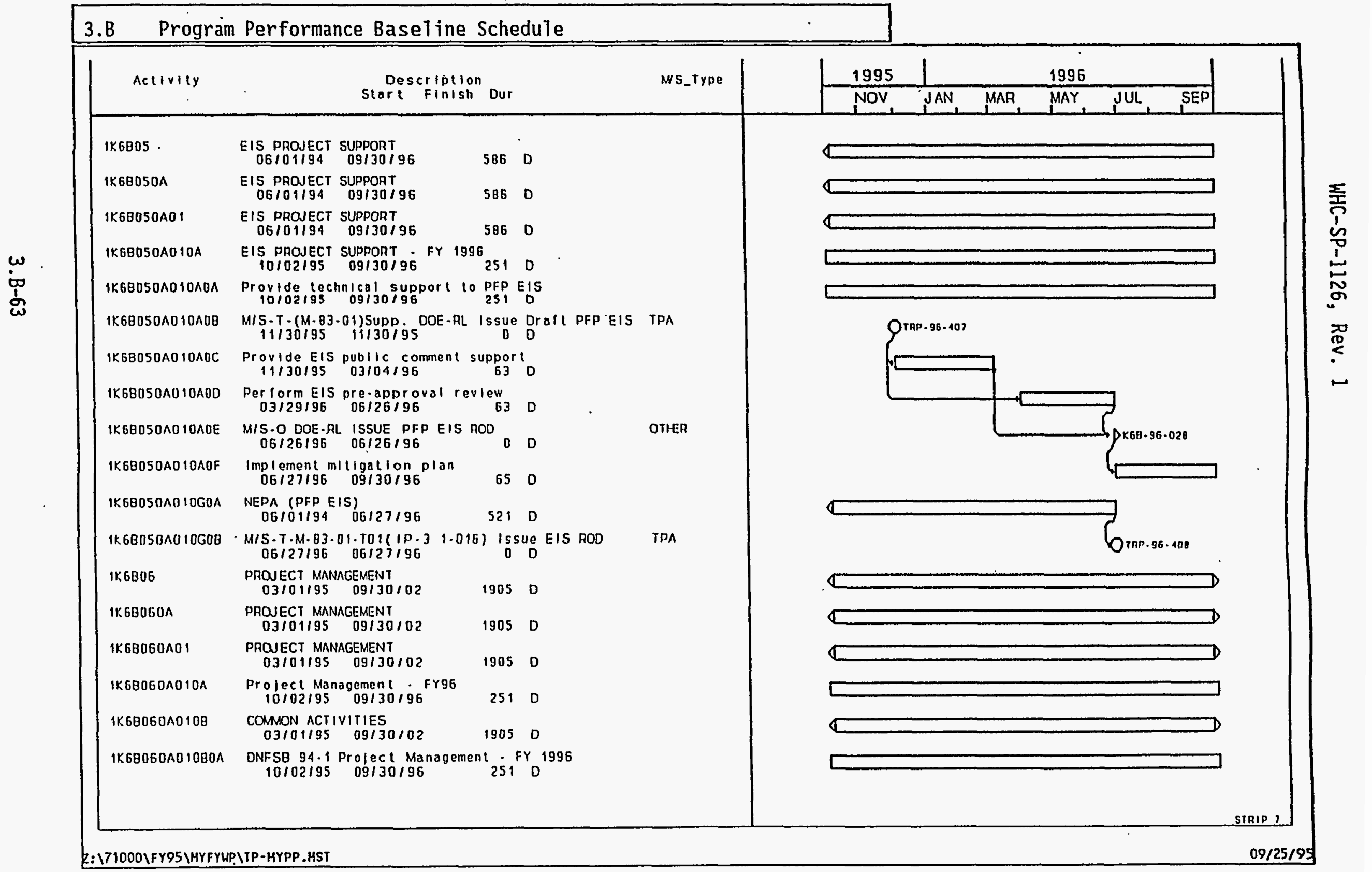




\section{TRANSITION PROJECTS}

\section{FY 1996 MYPP}

\subsection{1/7.1/6.12}

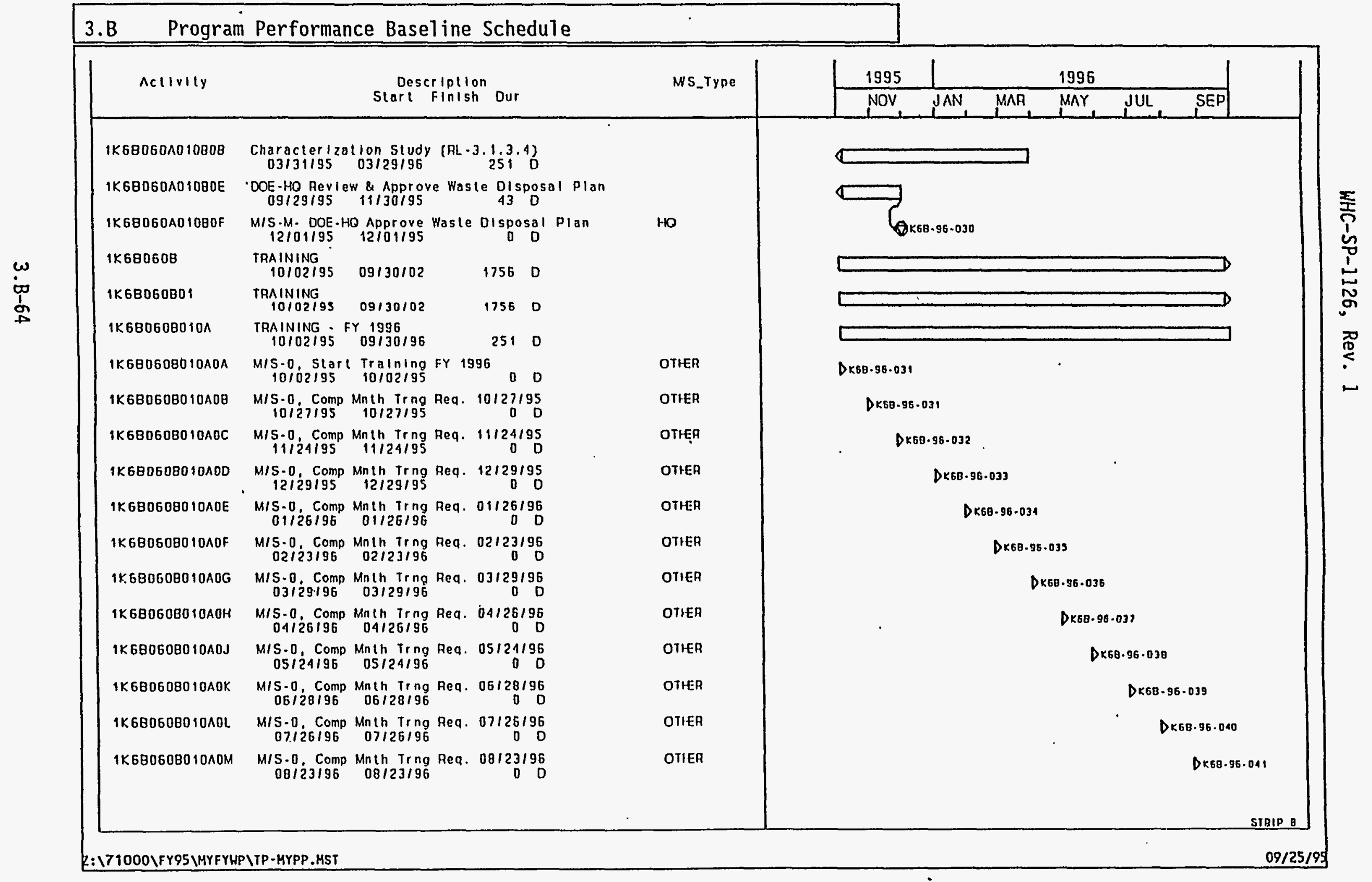




\section{TRANSITION PROJECTS}

\section{FY 1996 MYPP}

\subsection{1/7.1/6.12}

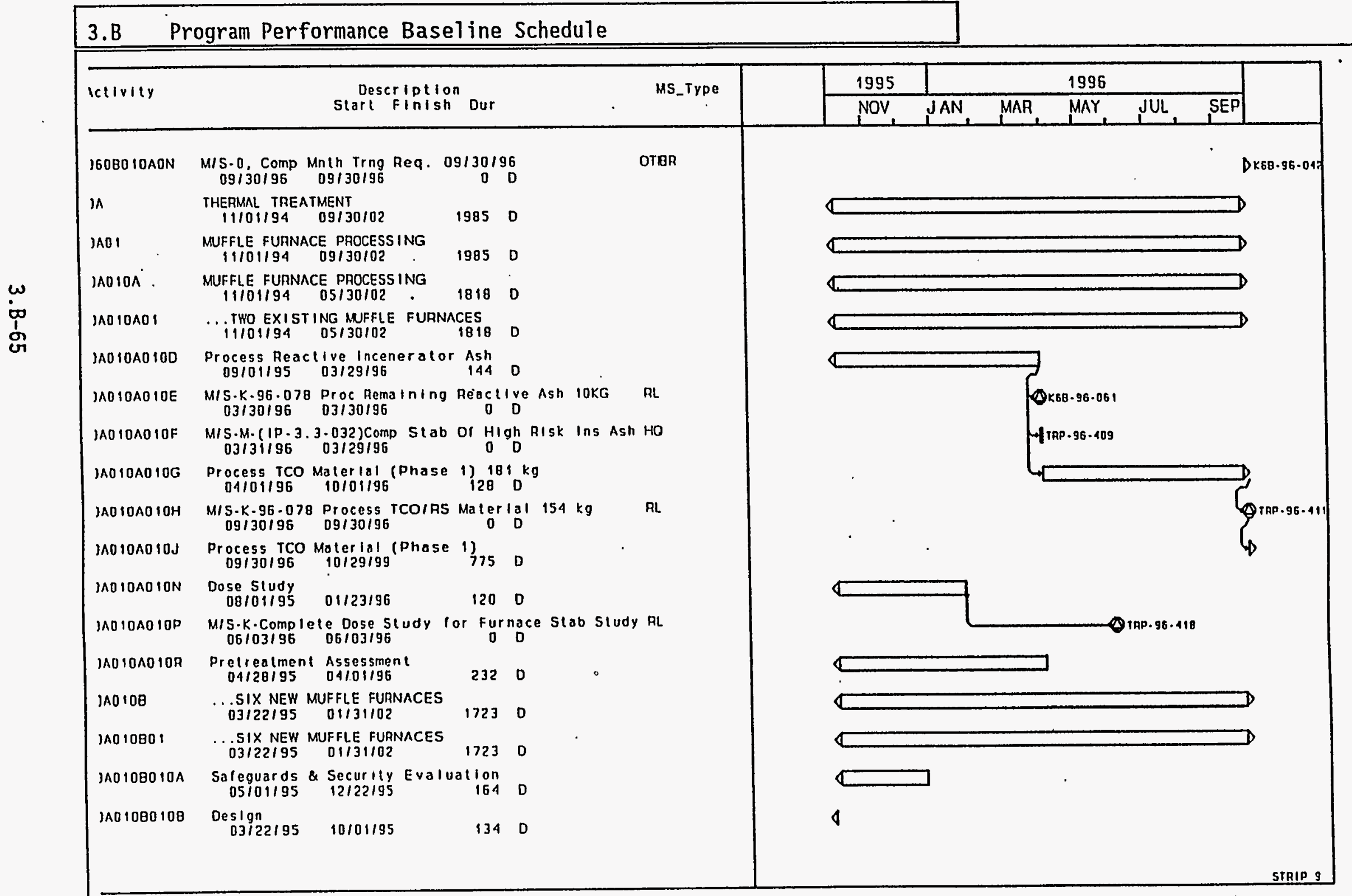




\section{TRANSITION PROJECTS}

FY 1996 MYPP

\subsection{1/7.1/6.12}

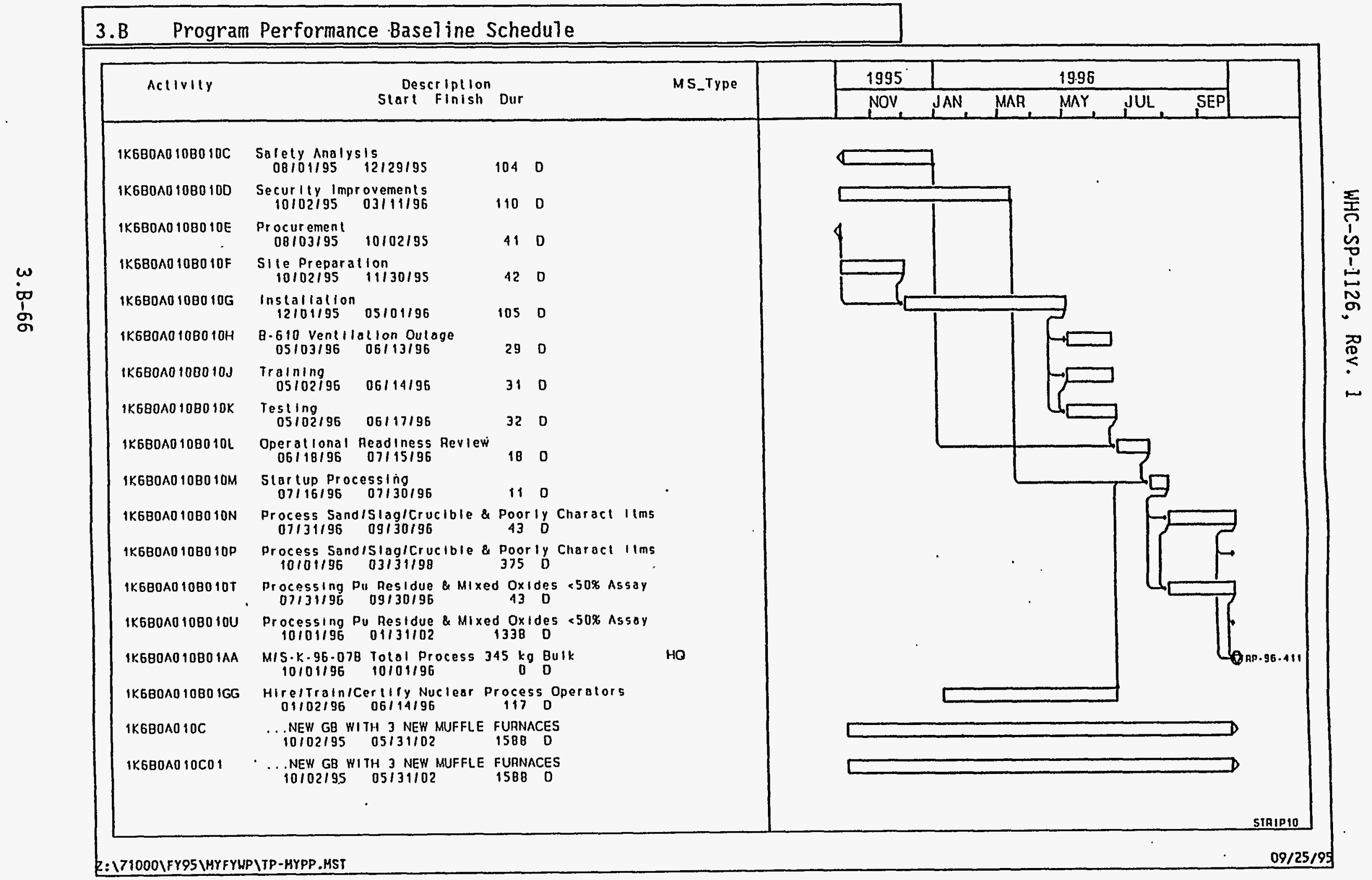




\section{TRANSITION PROJECTS}

FY 1996 MYPP

1.3.1/7.1/6.12

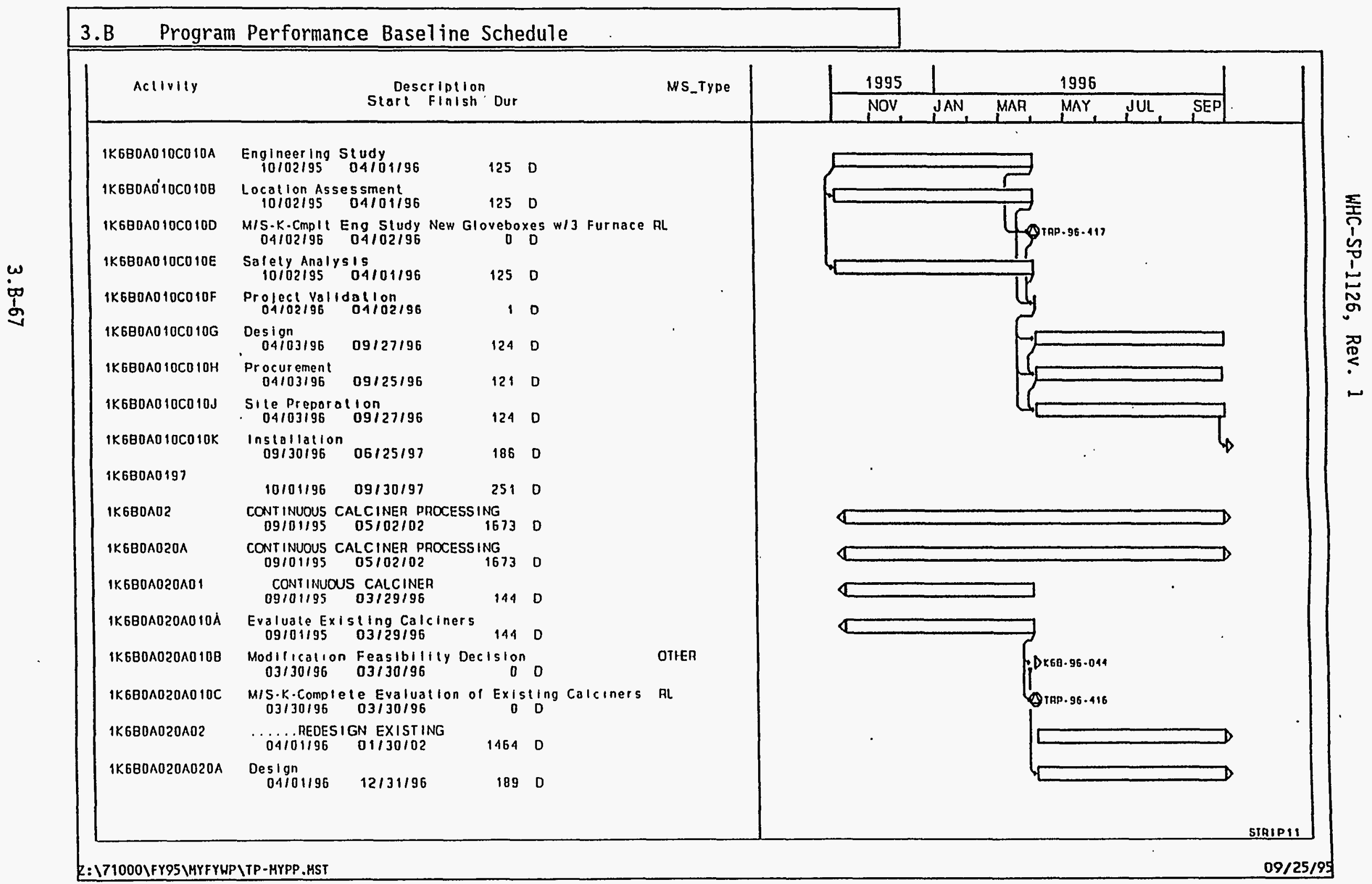




\section{TRANSITION PROJECTS}

FY 1996 MYPP

1.3.1/7.1/6.12

\section{B Program Performance Basel ine Schedule}

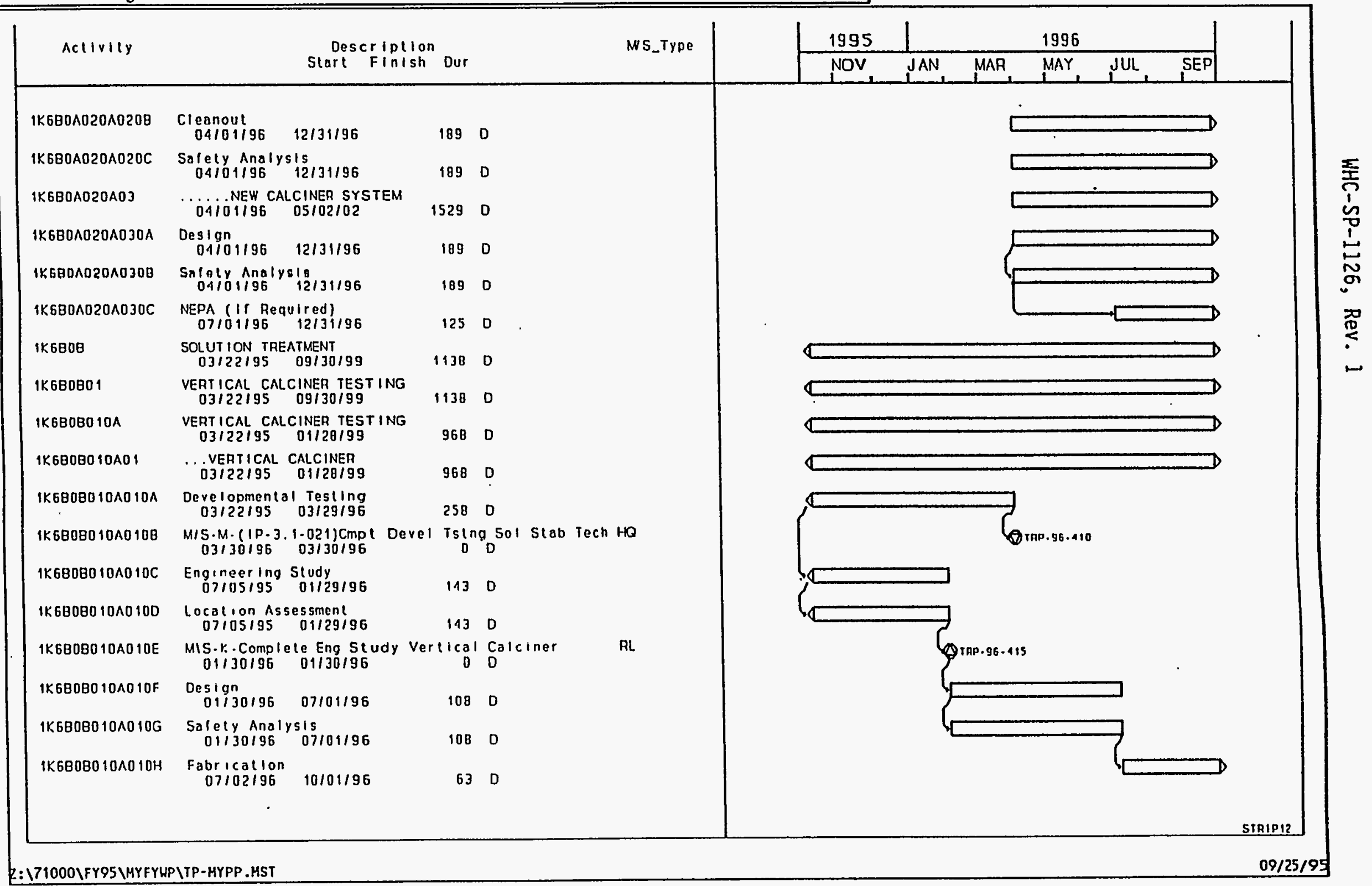




\section{TRANSITION PROJECTS}

FY 1996 MYPP

1.3.1/7.1/6.12

\section{B Program Performance Baseline Schedule}

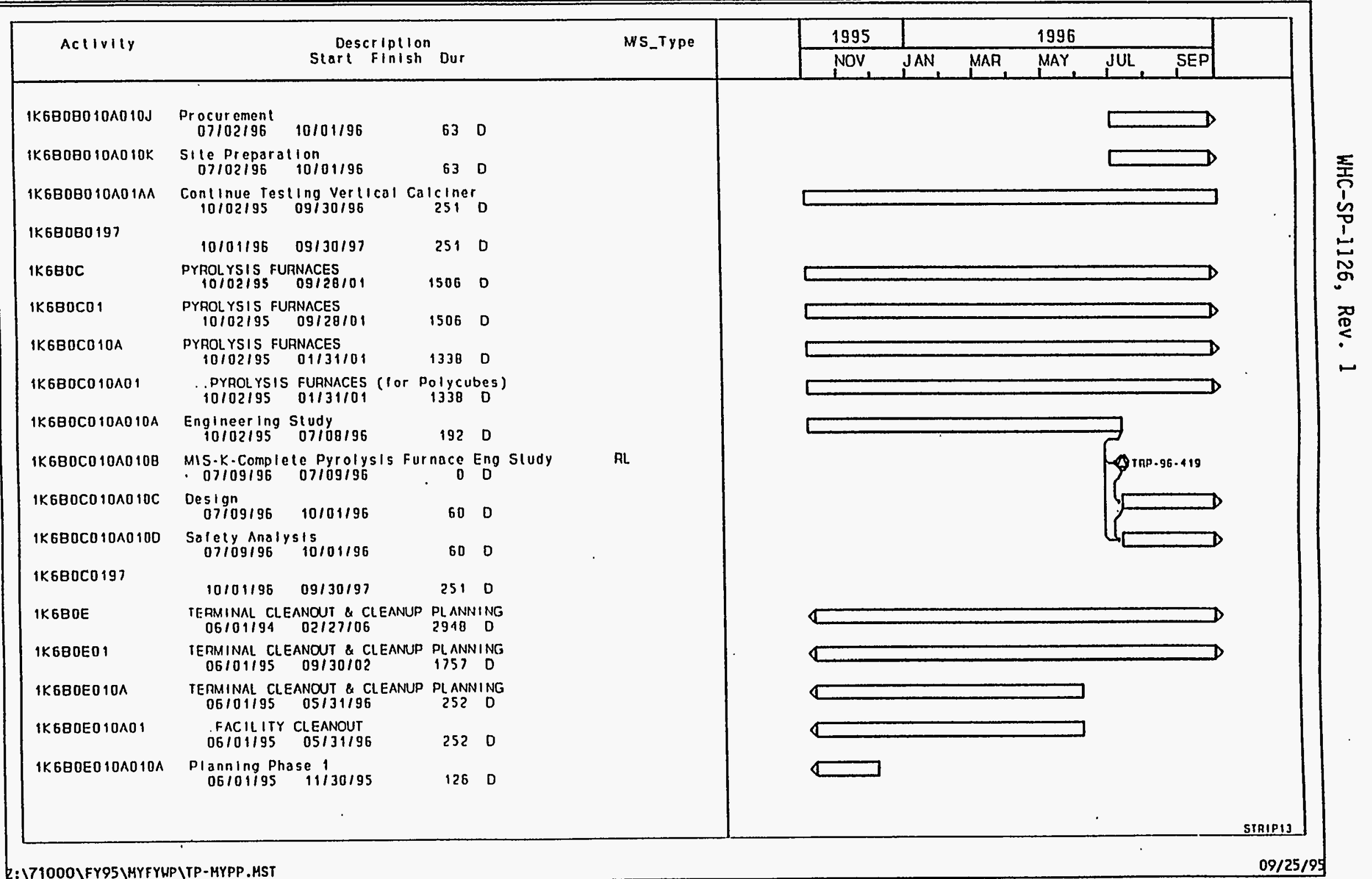




\section{TRANSITION PROJECTS}

FY 1996 MYPP

\subsection{1/7.1/6.12}

\section{B Program Performance Baseline Schedule}

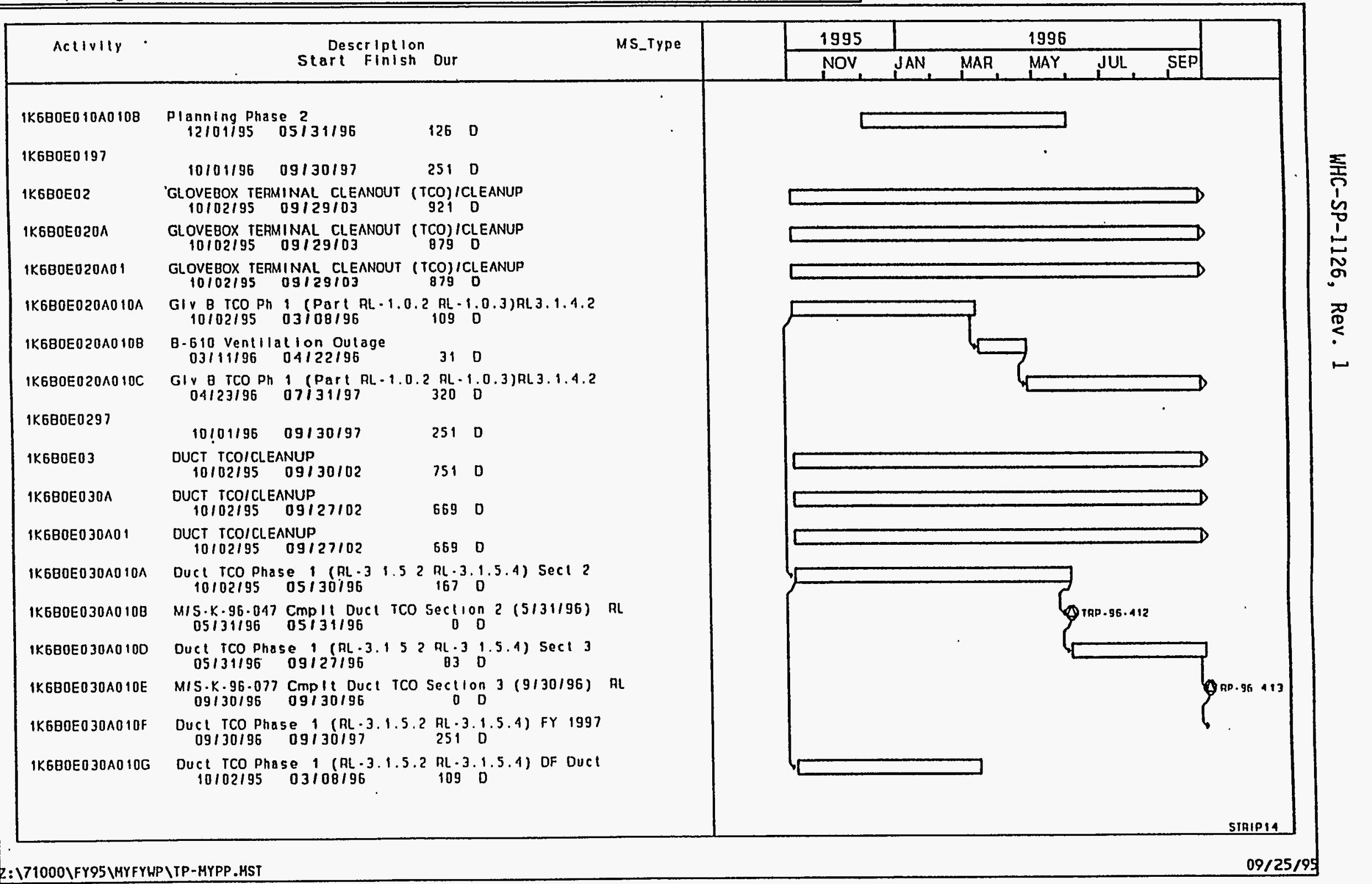




\section{TRANSITION PROJECTS}

\subsection{1/7.1/6.1.2}

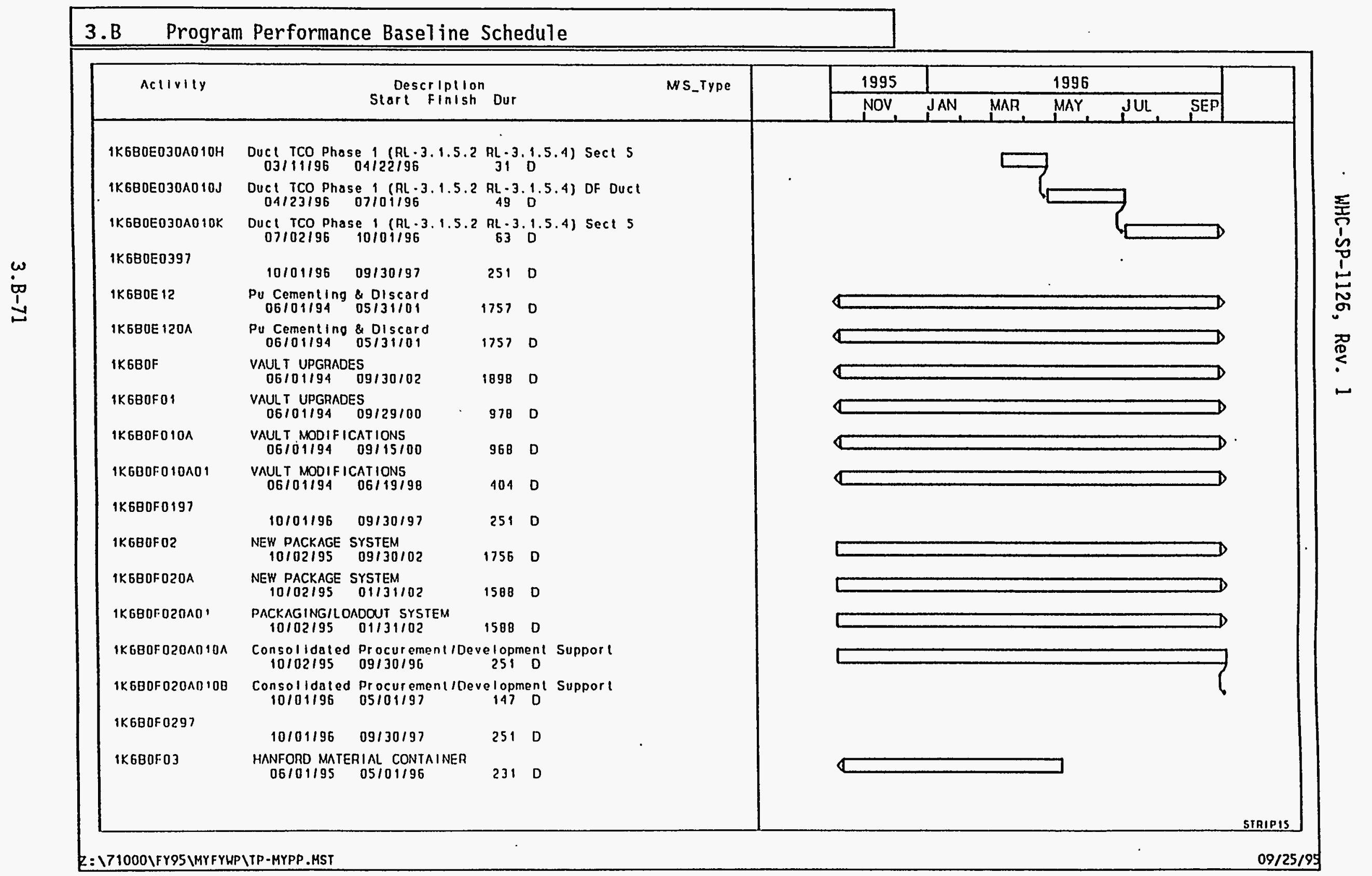




\section{TRANSITION PROJECTS}

FY 1996 MYPP

1.3.1/7.1/6.12

\section{B Program Performance Baseline Schedule}

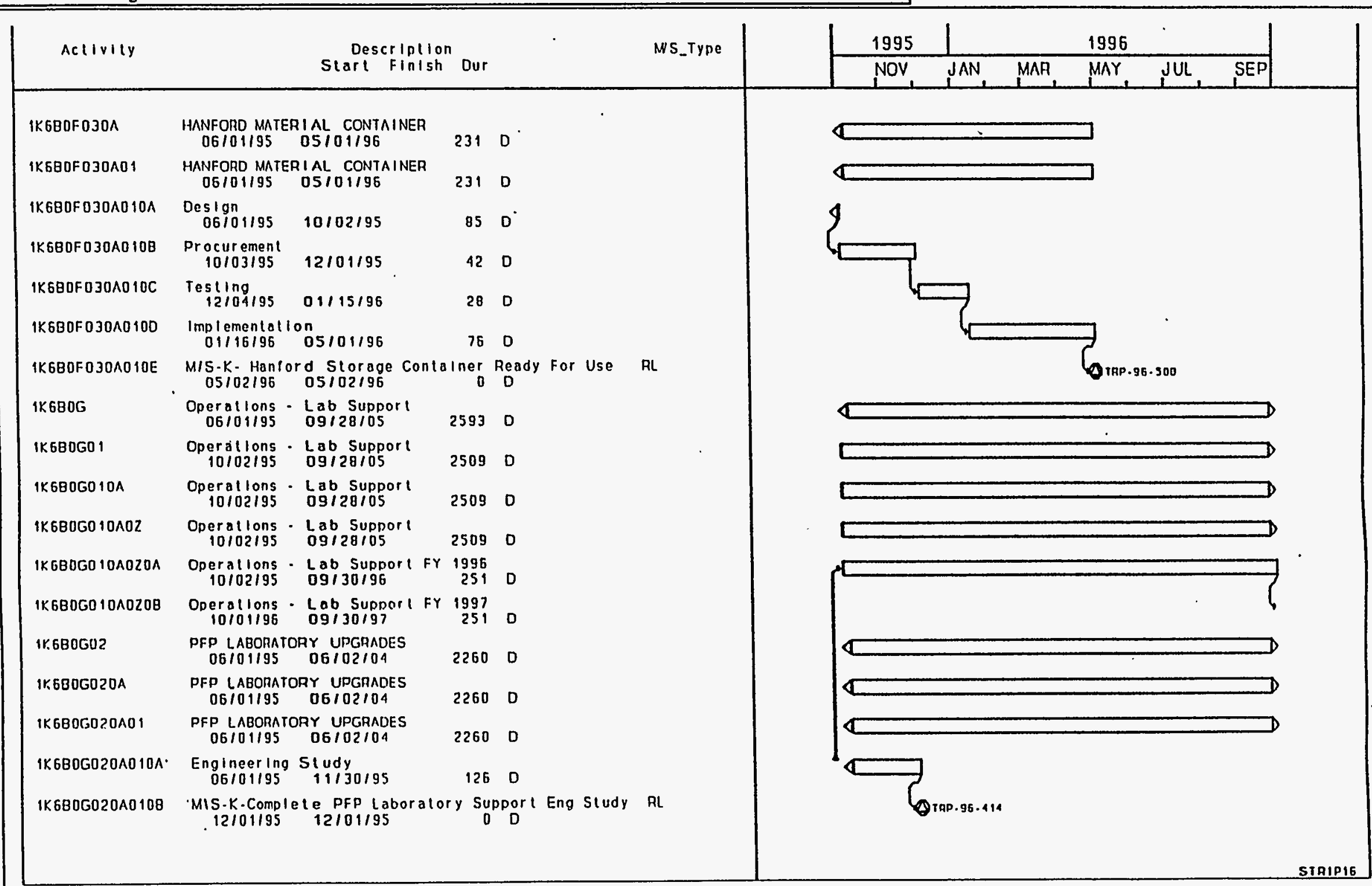




\section{TRANSITION PROJECTS}

\section{FY 1996 MYPP}

\subsection{1/7.1/6.12}

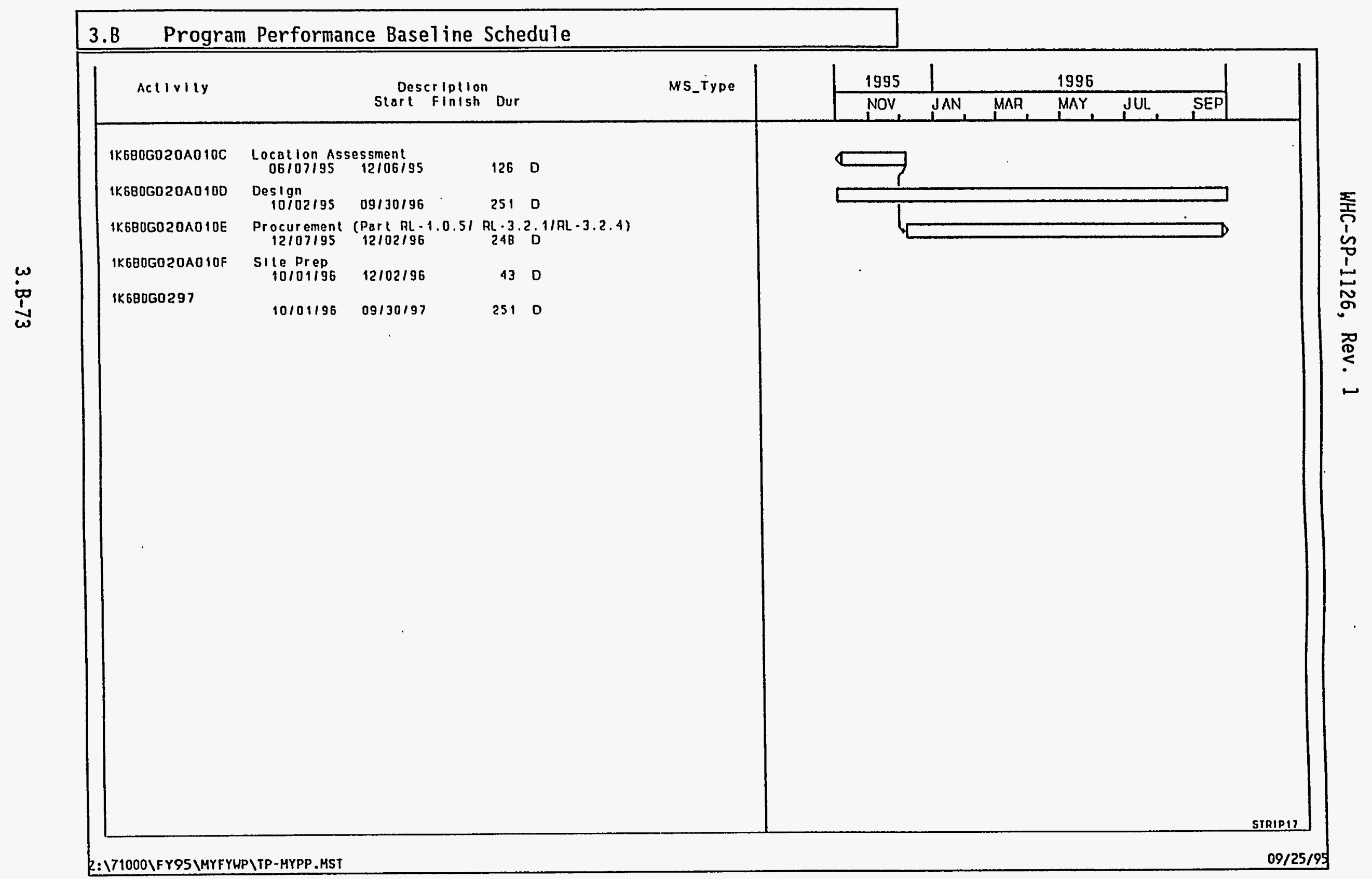




\section{FY 1996 MYPP}

TRANSITION PROJECTS

1.3.1/7.1/6.12

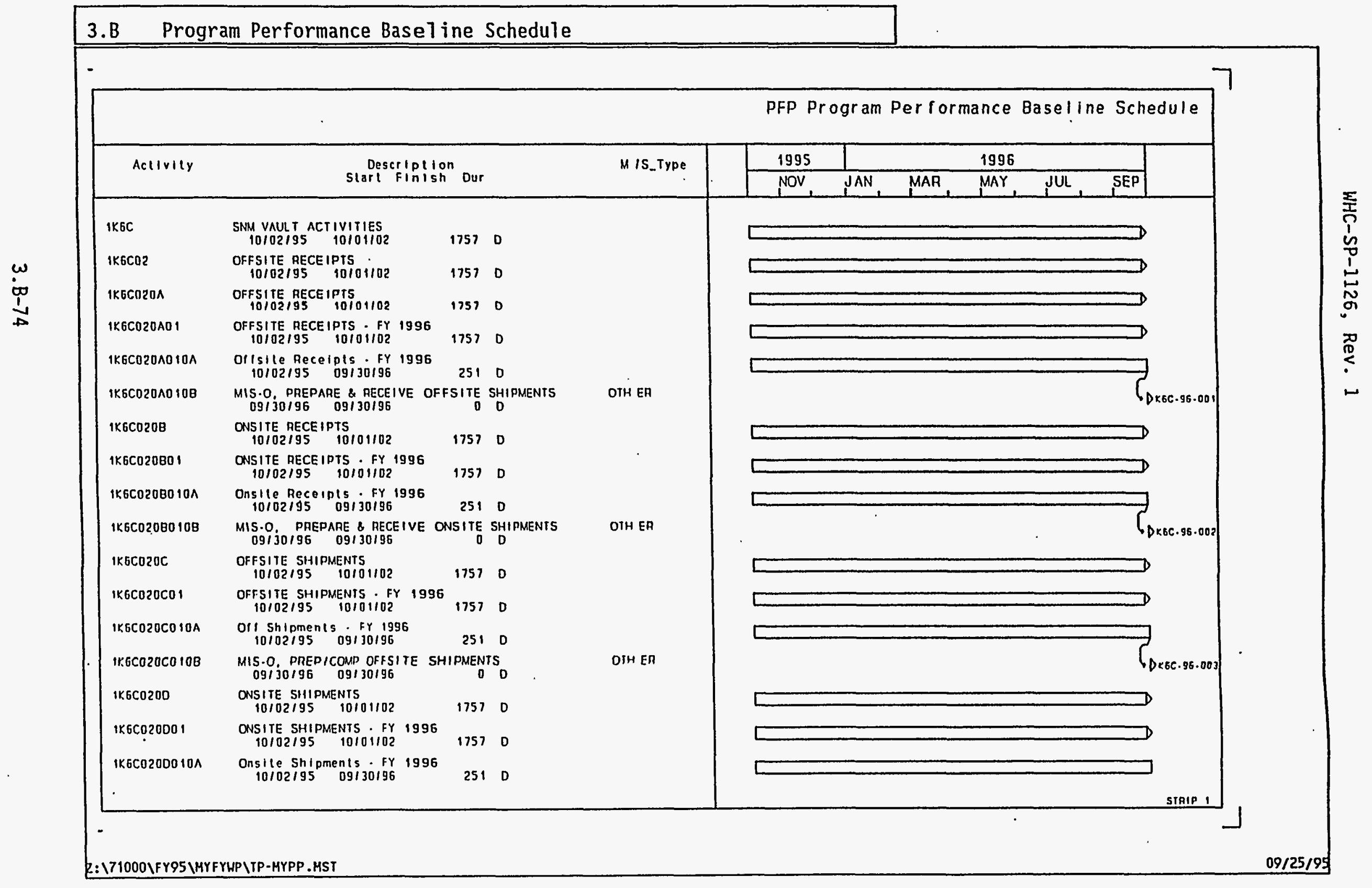


TRANSITION PROJECTS

FY 1996 MYPP

1.3.1/7.1/6.12

\section{B Program Performance Basè ine Schedule}

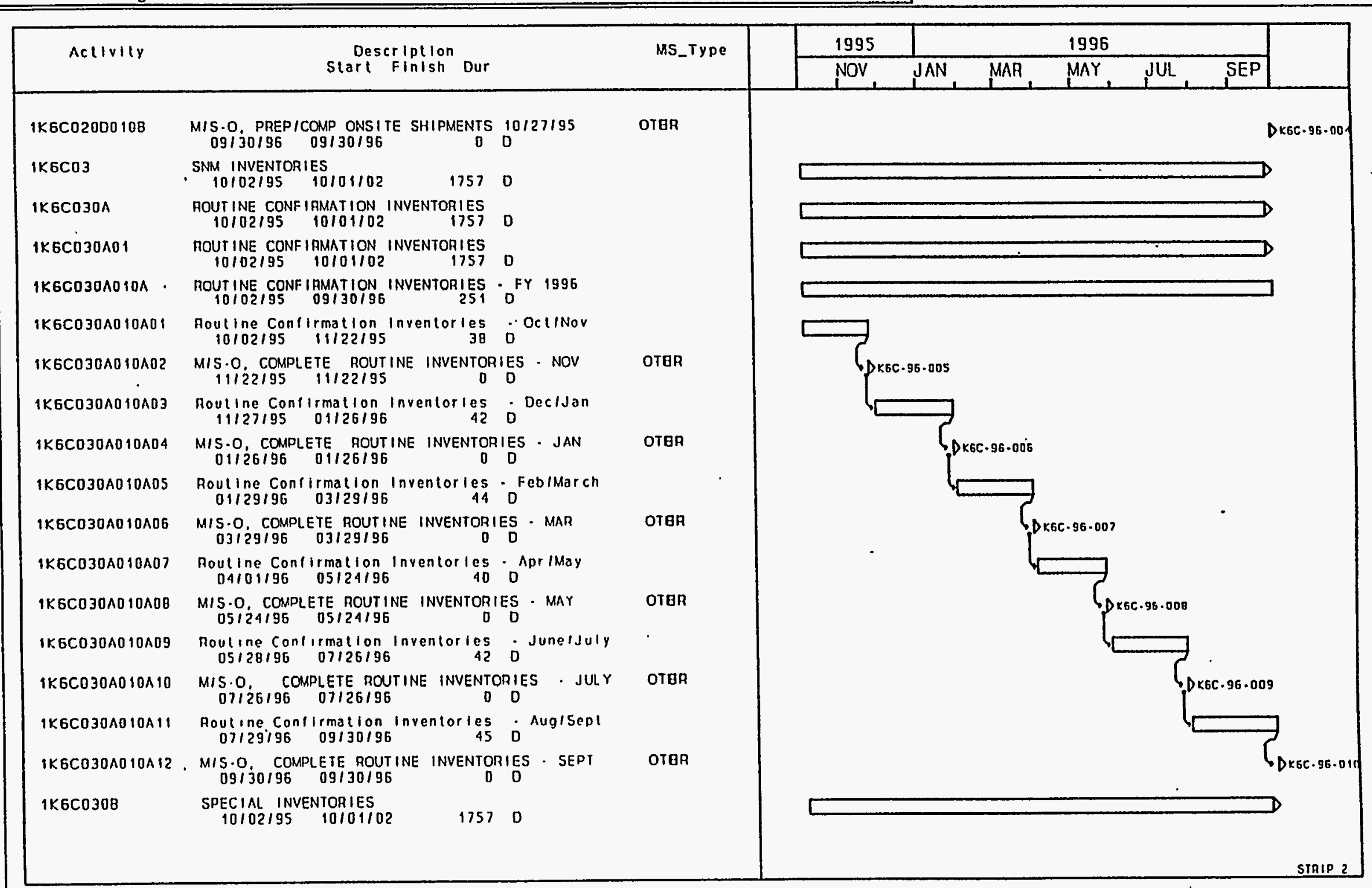




\section{FY 1996 MYPP}

\section{TRANSITION PROJECTS}

1.3.1/7.1/6.12

\section{B Program Performance Baseline Schedule}

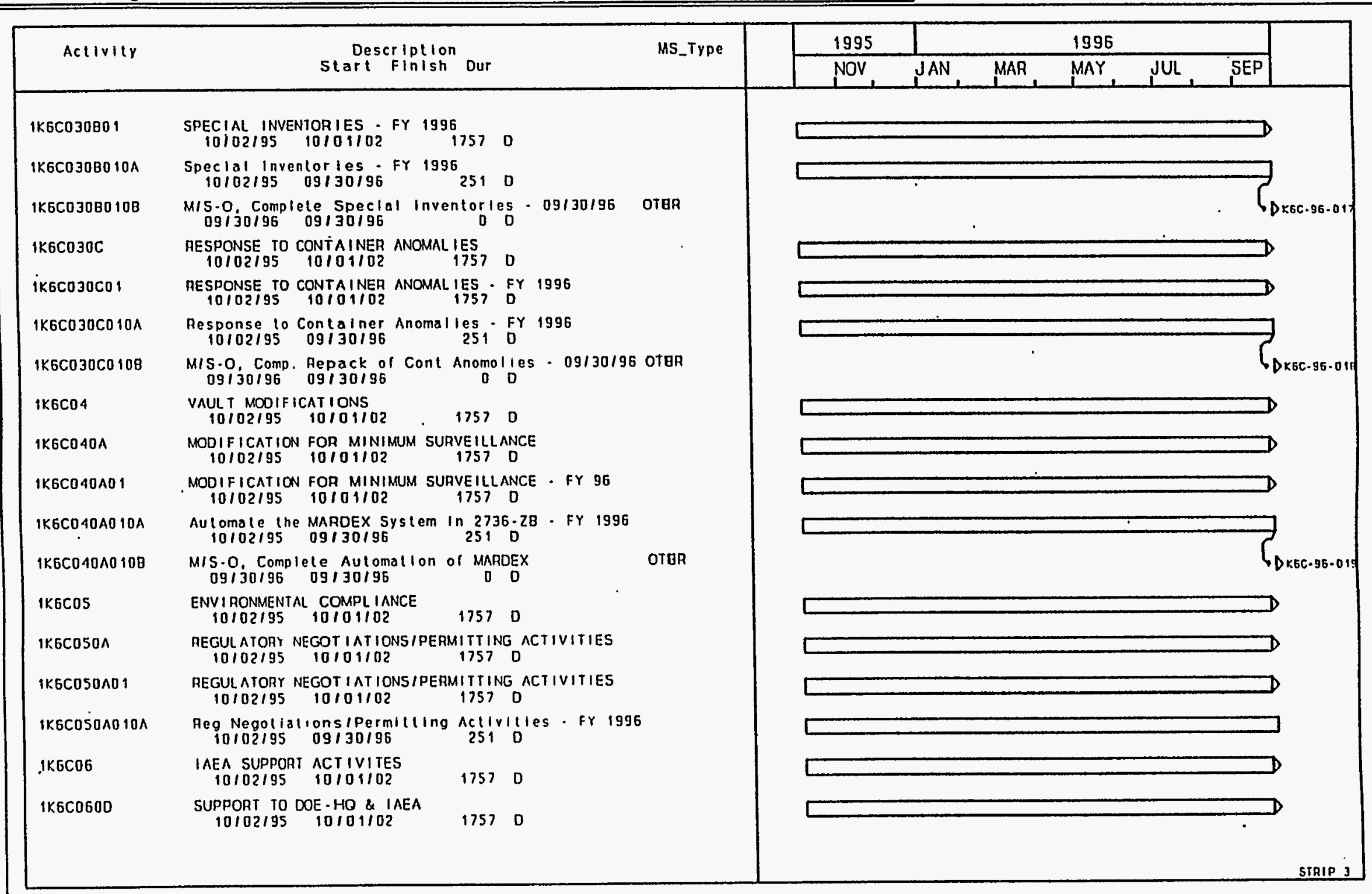




\section{TRANSITIUN PROJECTS}

\section{FY 1996 MYPP}

1.3.1/7.1/6.12

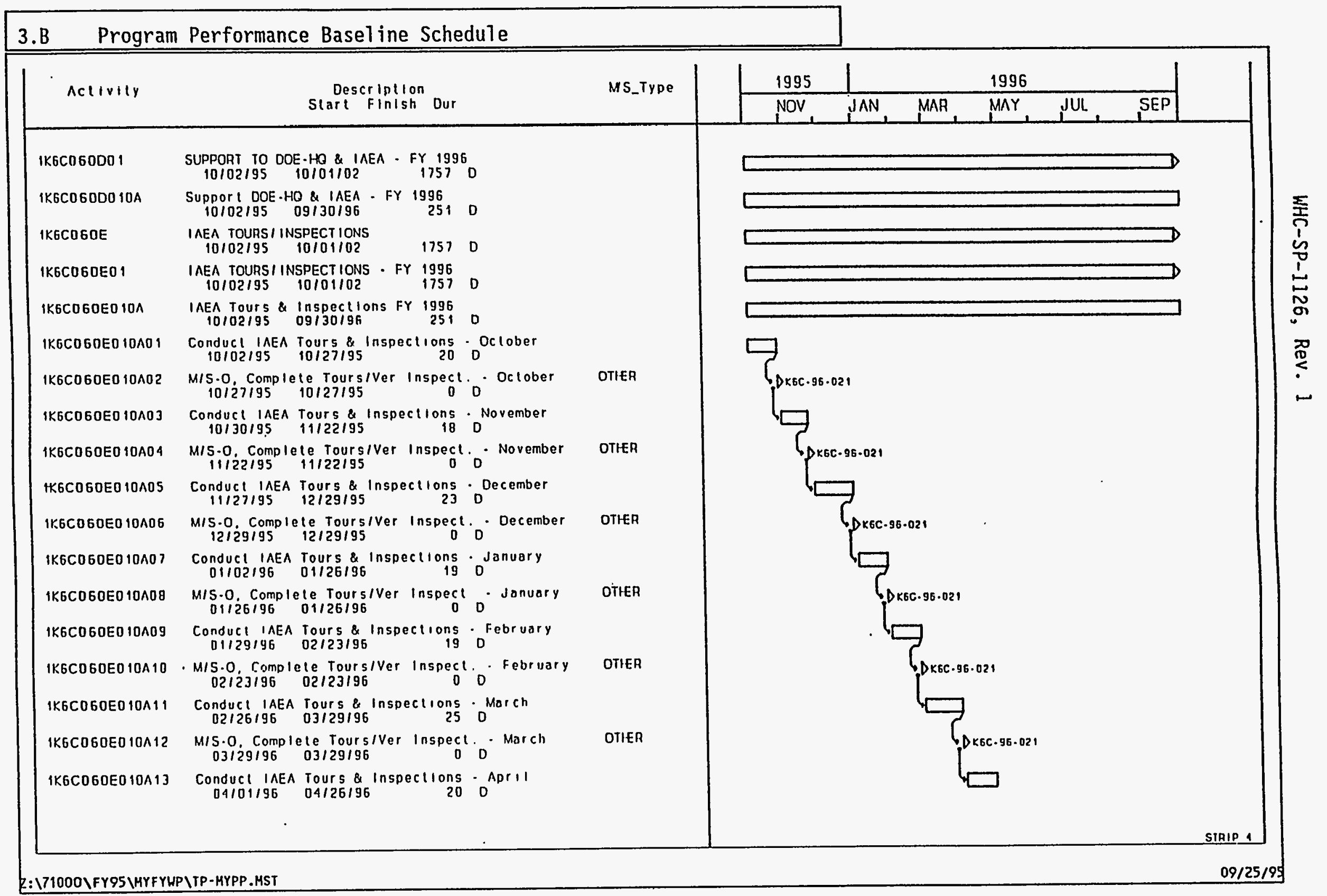


TRANSITIUN PROJECTS

FY 1996 MYPP

1.3.1/7.1/6.12

3.B Program Performance Baseline Schedule

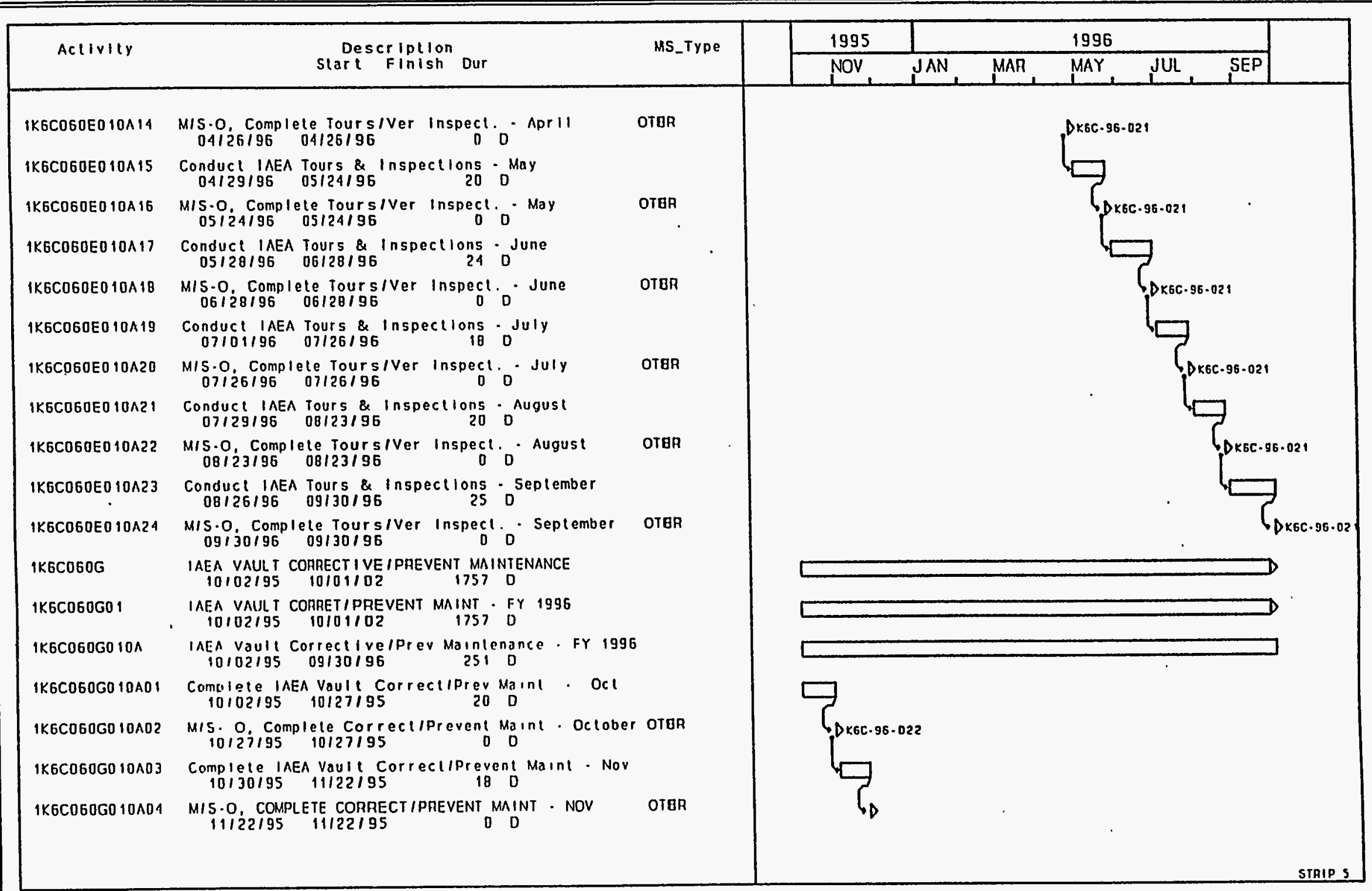


FY 1996 MYPP

\section{TRANSITION PROJECTS}

1.3.1/7.1/6.12

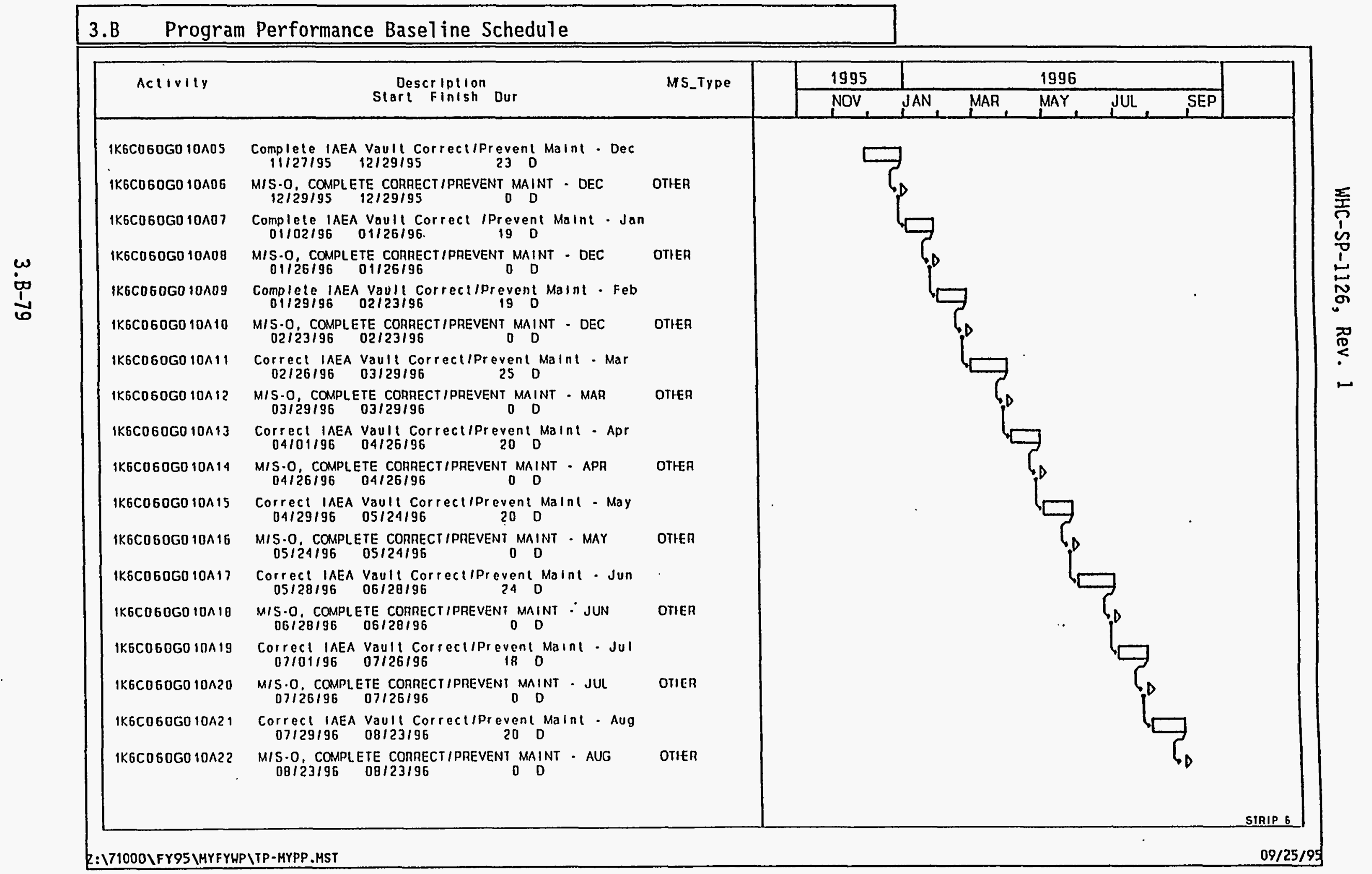




\section{TRANSITION PROJECTS \\ 1.3.1/7.1/6.12}

\section{FY 1996 MYPP}

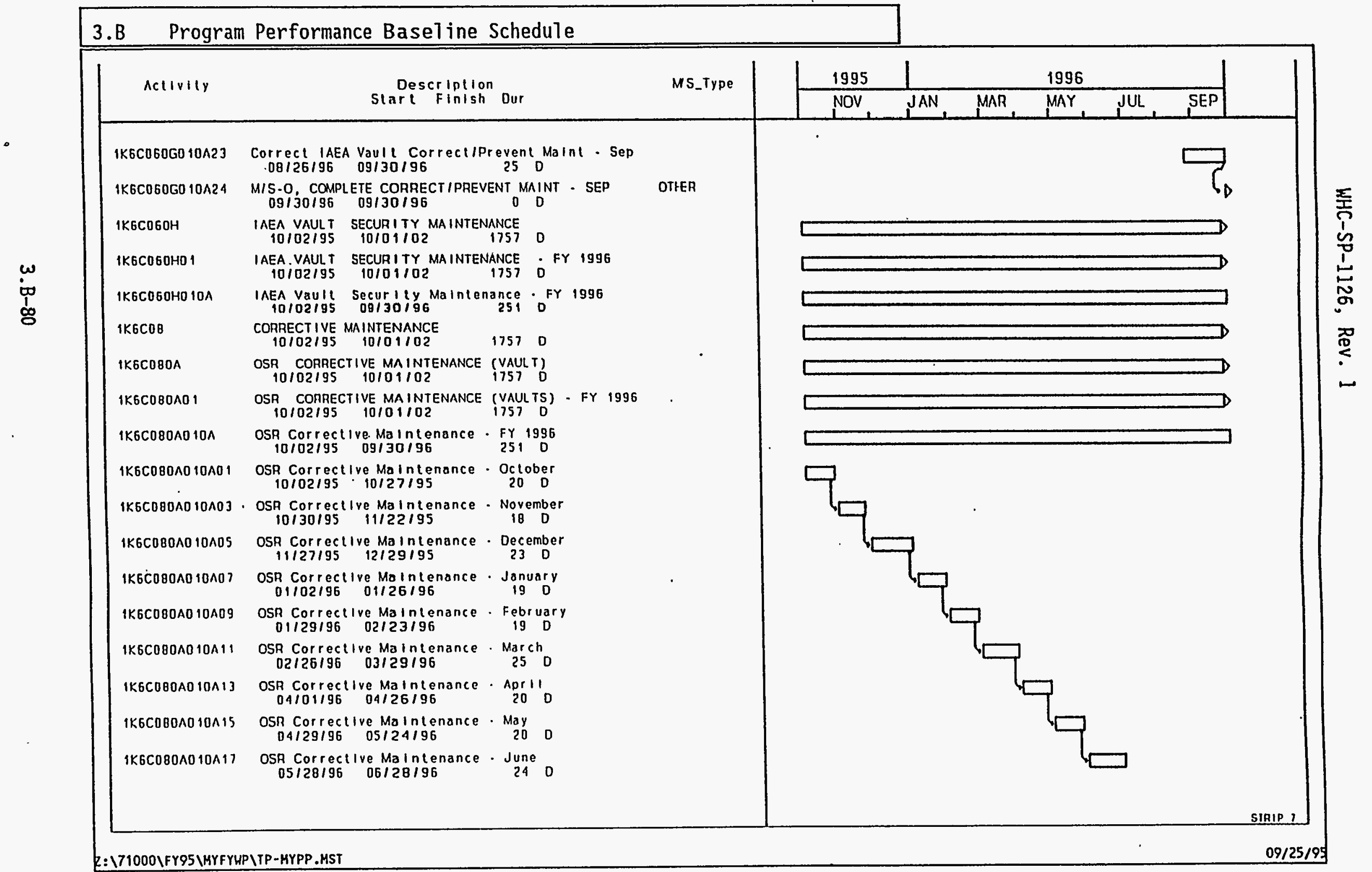




\section{TRANSITION PROJECTS}

FY 1996 MYPP

\subsection{1/7.1/6.12}

\section{B Program Performance Baseline Schedule}

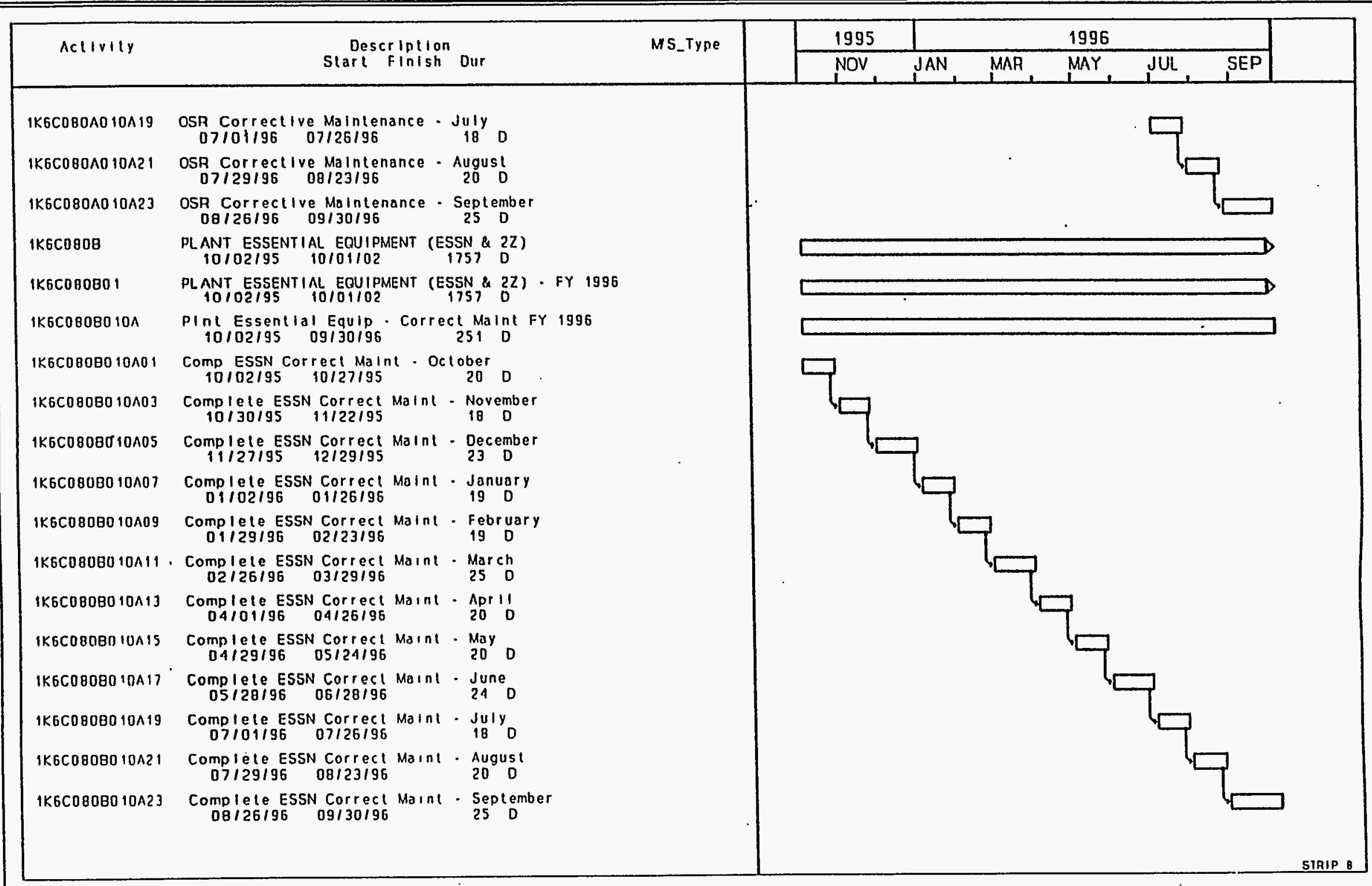




\section{TRANSITION PROJECTS}

FY 1996 MYPP

1.3.1/7.1/6.12

\section{B Program Performance Baseline Schedule}

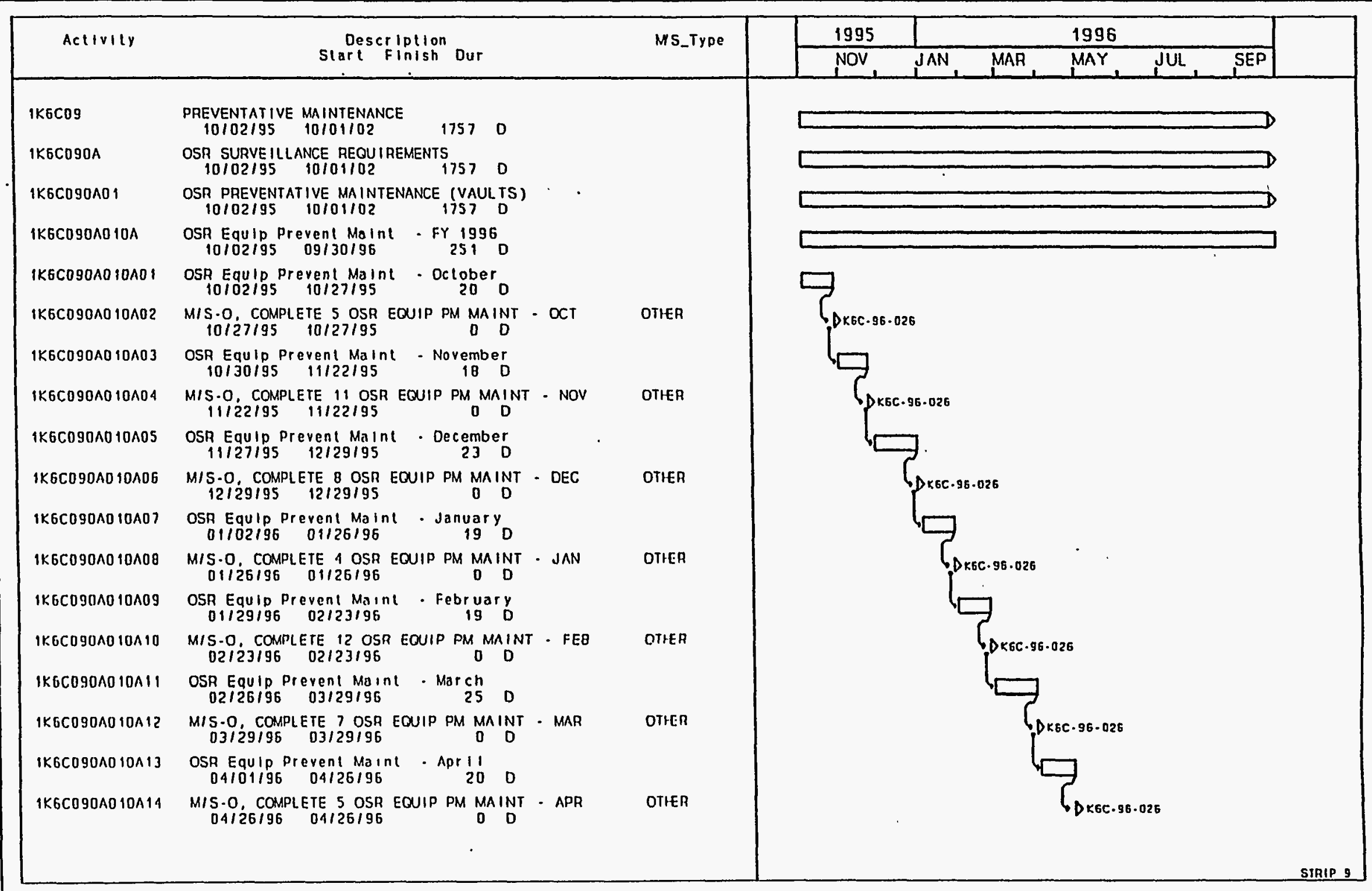




\section{TRANSITION PROJECTS \\ 1.3.1/7.1/6.12}

FY 1996 MYPP

\section{B Program Performance Baseline ScheduTe}

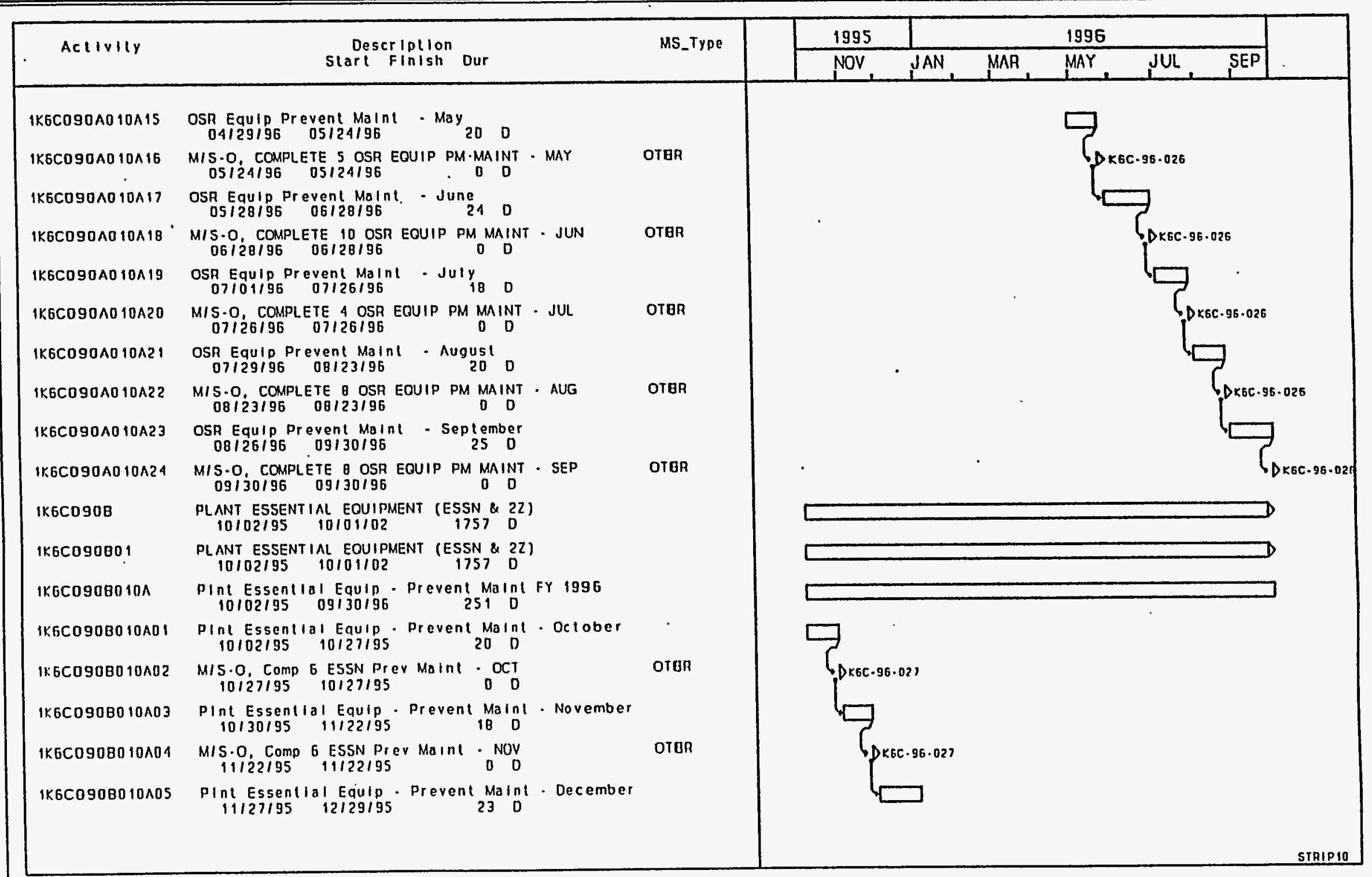




\section{TRANSITIUN PROJECTS 1.3.1/7.1/6.12}

FY 1996 MYPP

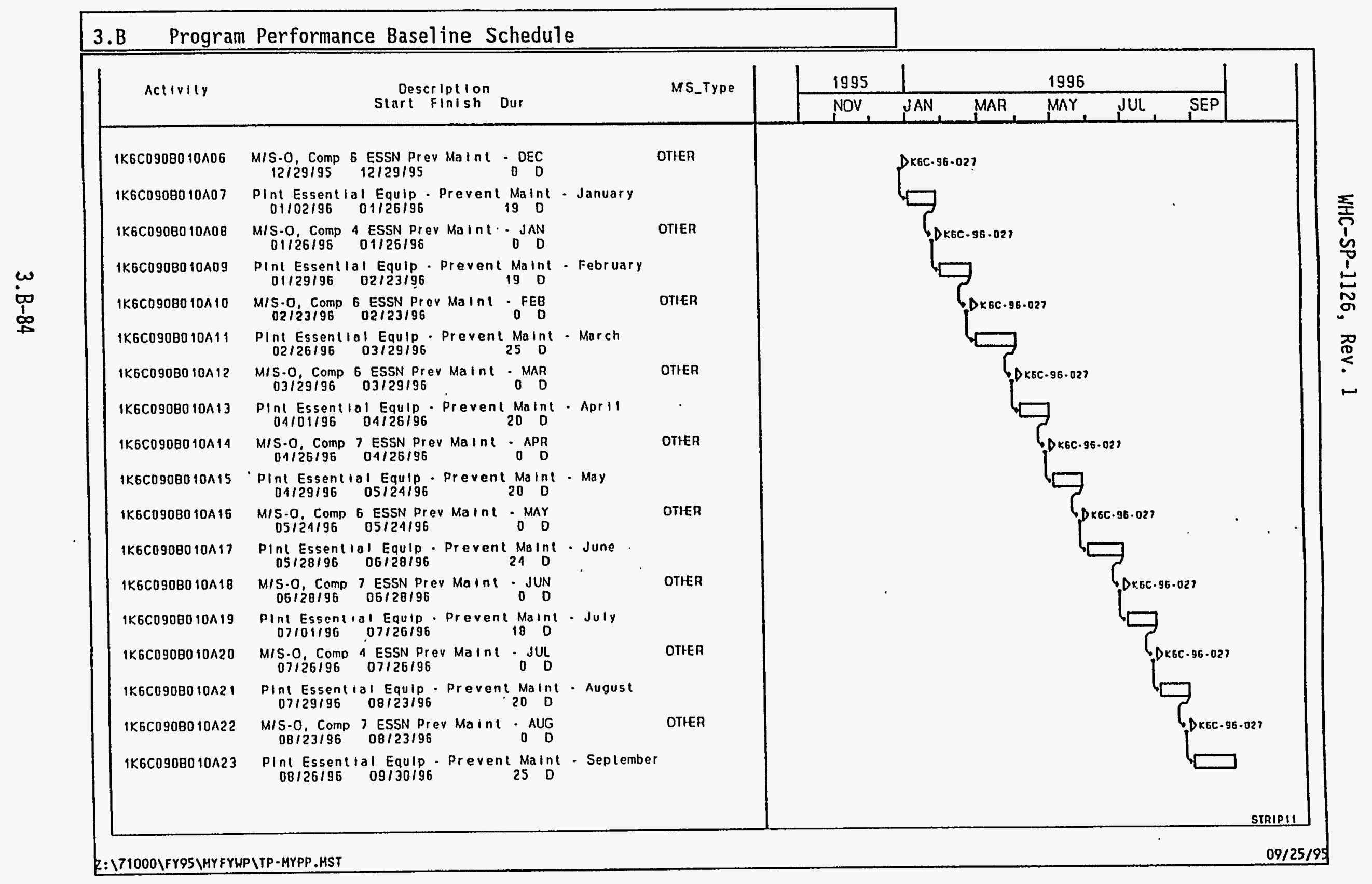




\section{FY 1996 MYPP}

\section{TRANSITION PROJECTS}

1.3.1/7.1/6.12

\section{B Program Performance Baseline Schedule}

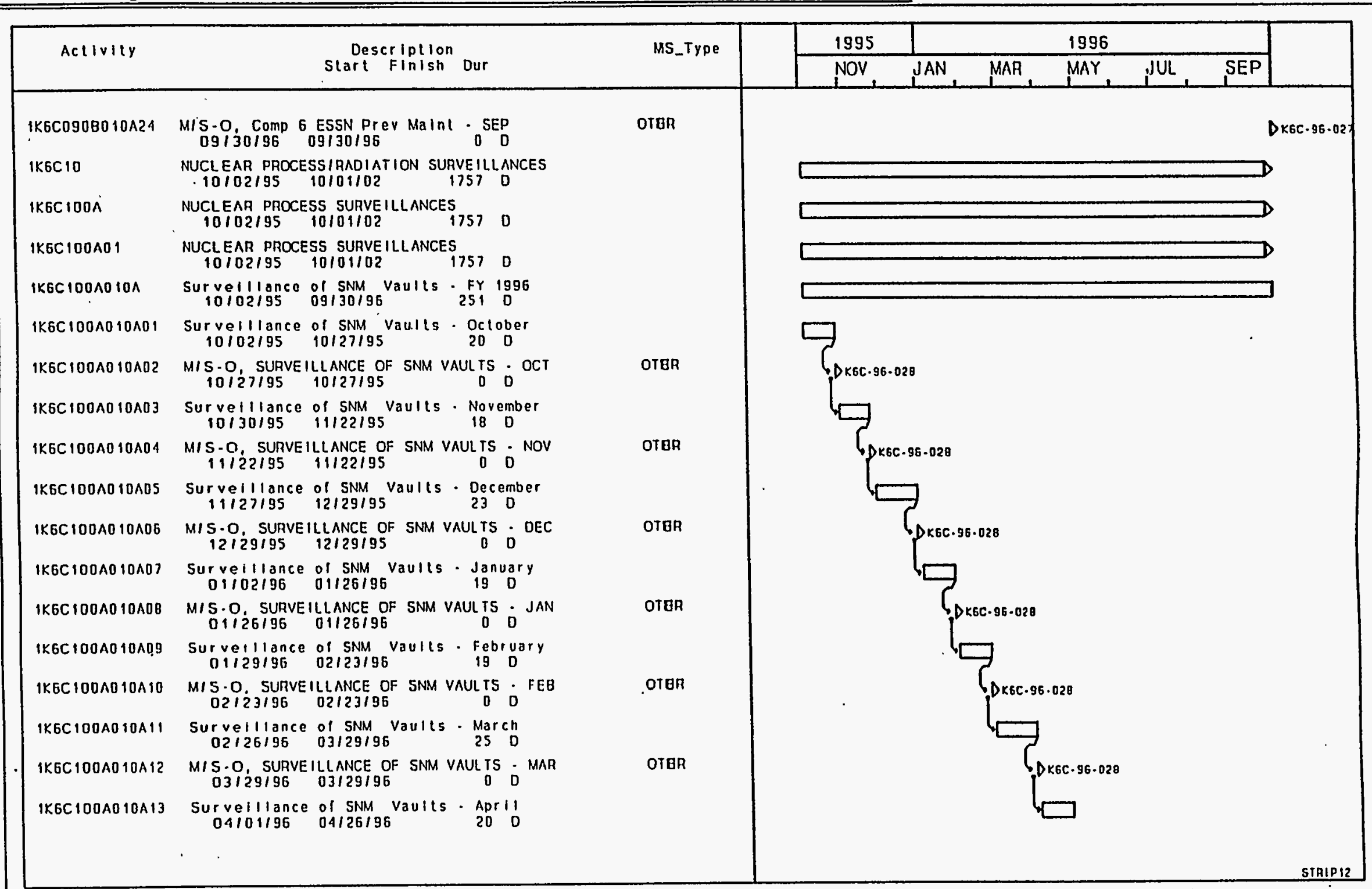




\section{TRANSITION PROJECTS}

FY 1996 MYPP

1.3.1/7.1/6.12

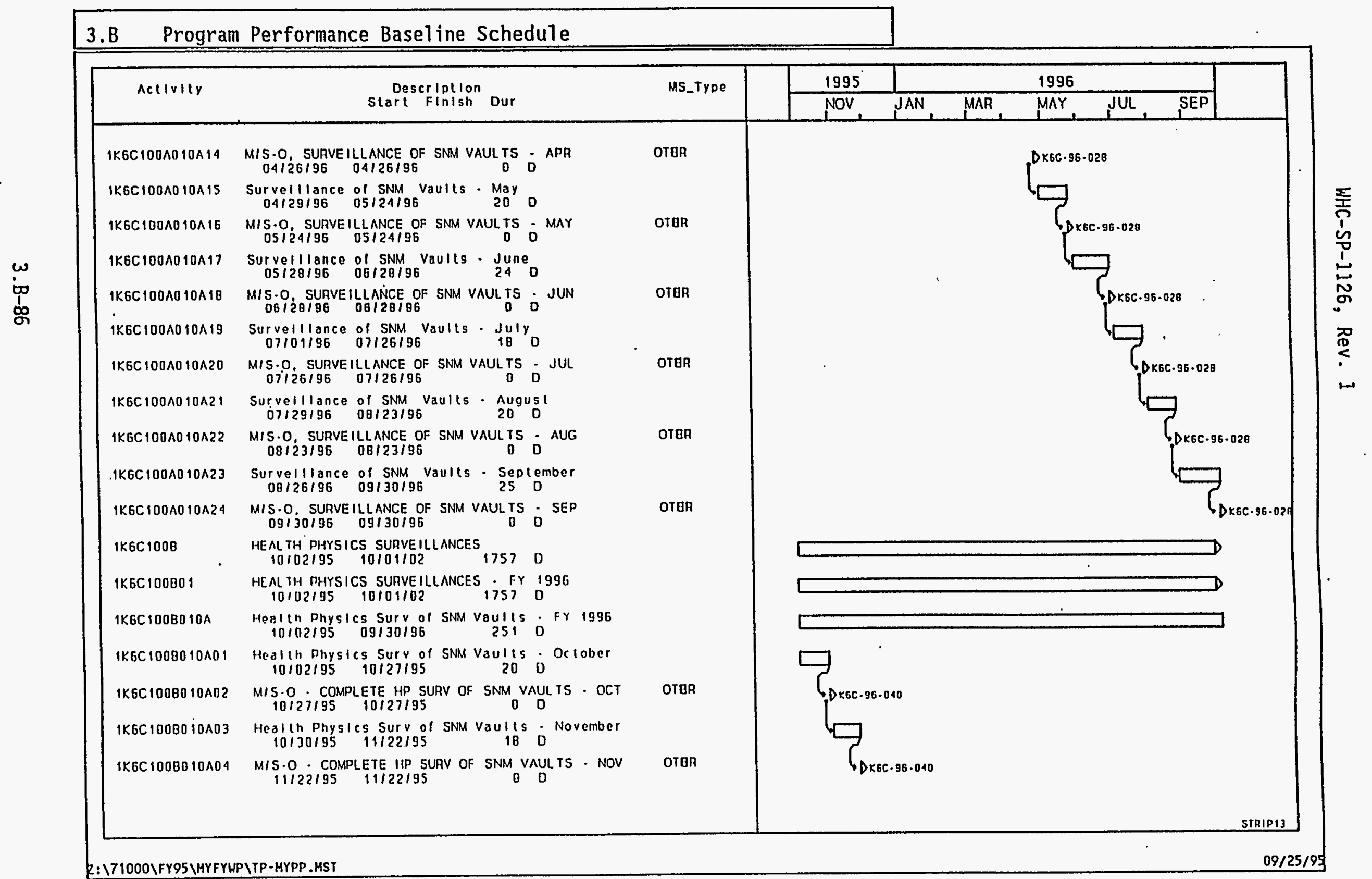


FY 1996 MYPP

\section{TRANSITION PROJECTS}

1.3.1/7.1/6.12

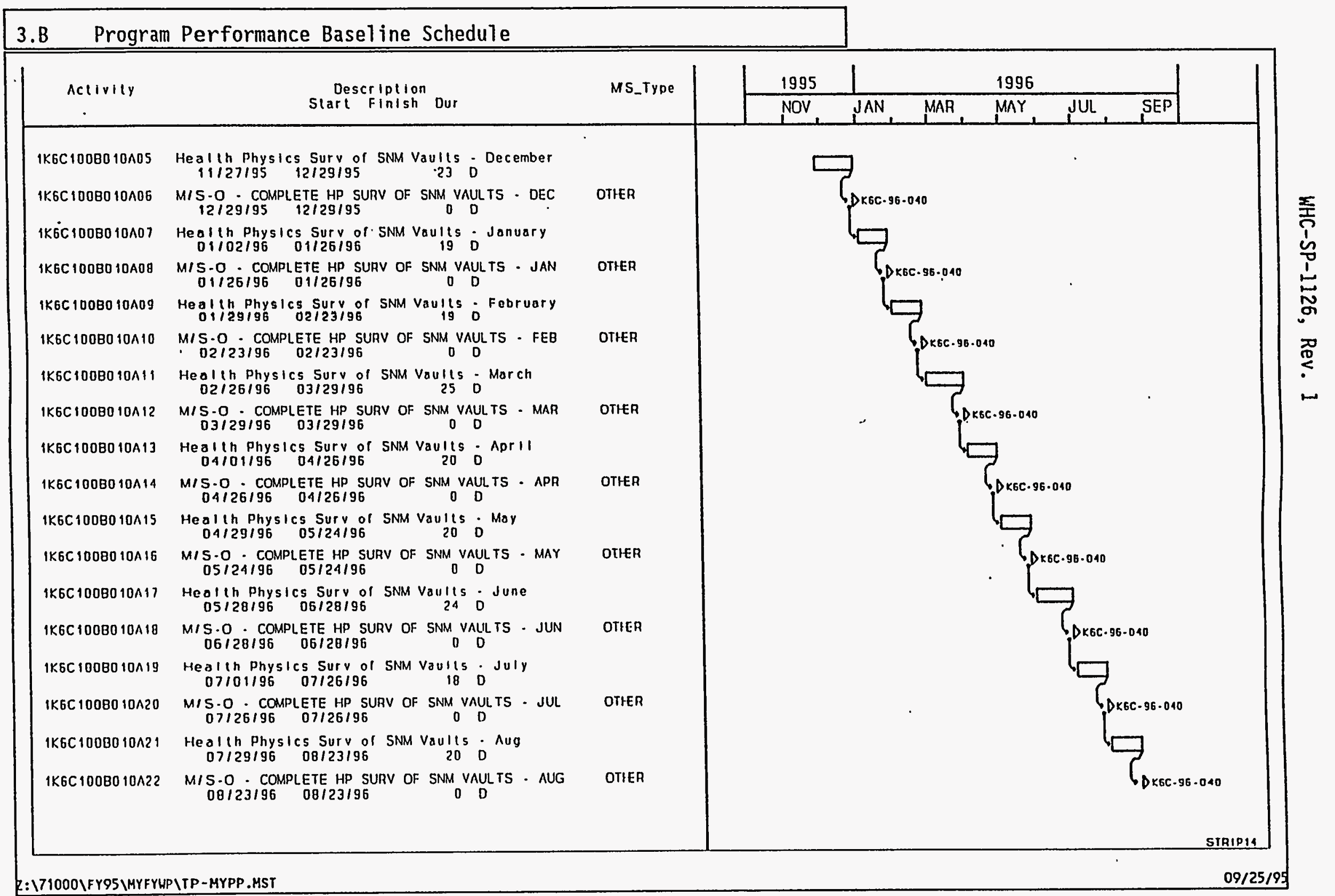




\section{TRANSITION PROJECTS}

FY 1996 MYPP

\subsection{1/7.1/6.12}

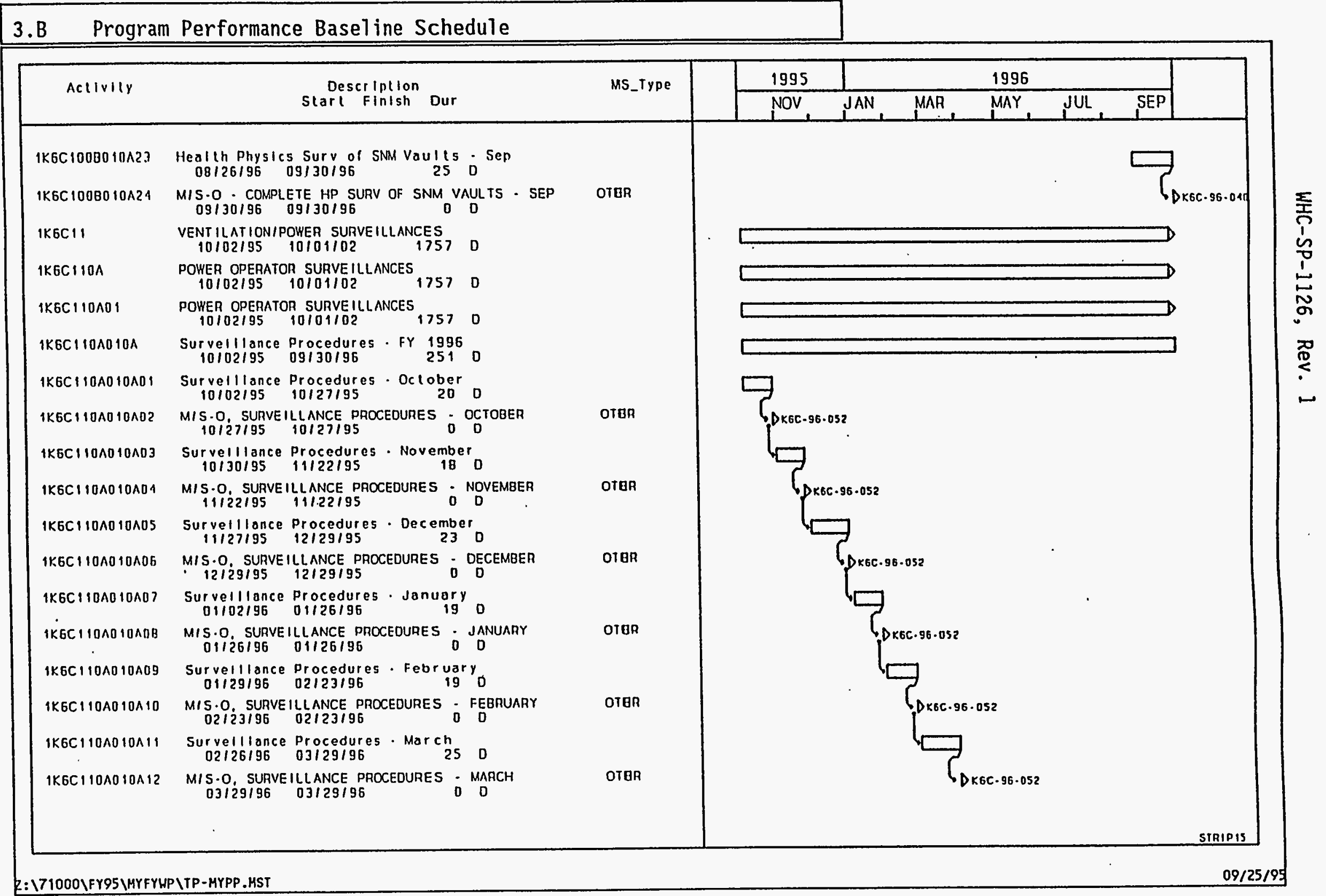




\section{B Program Performance Baseline ScheduTe}

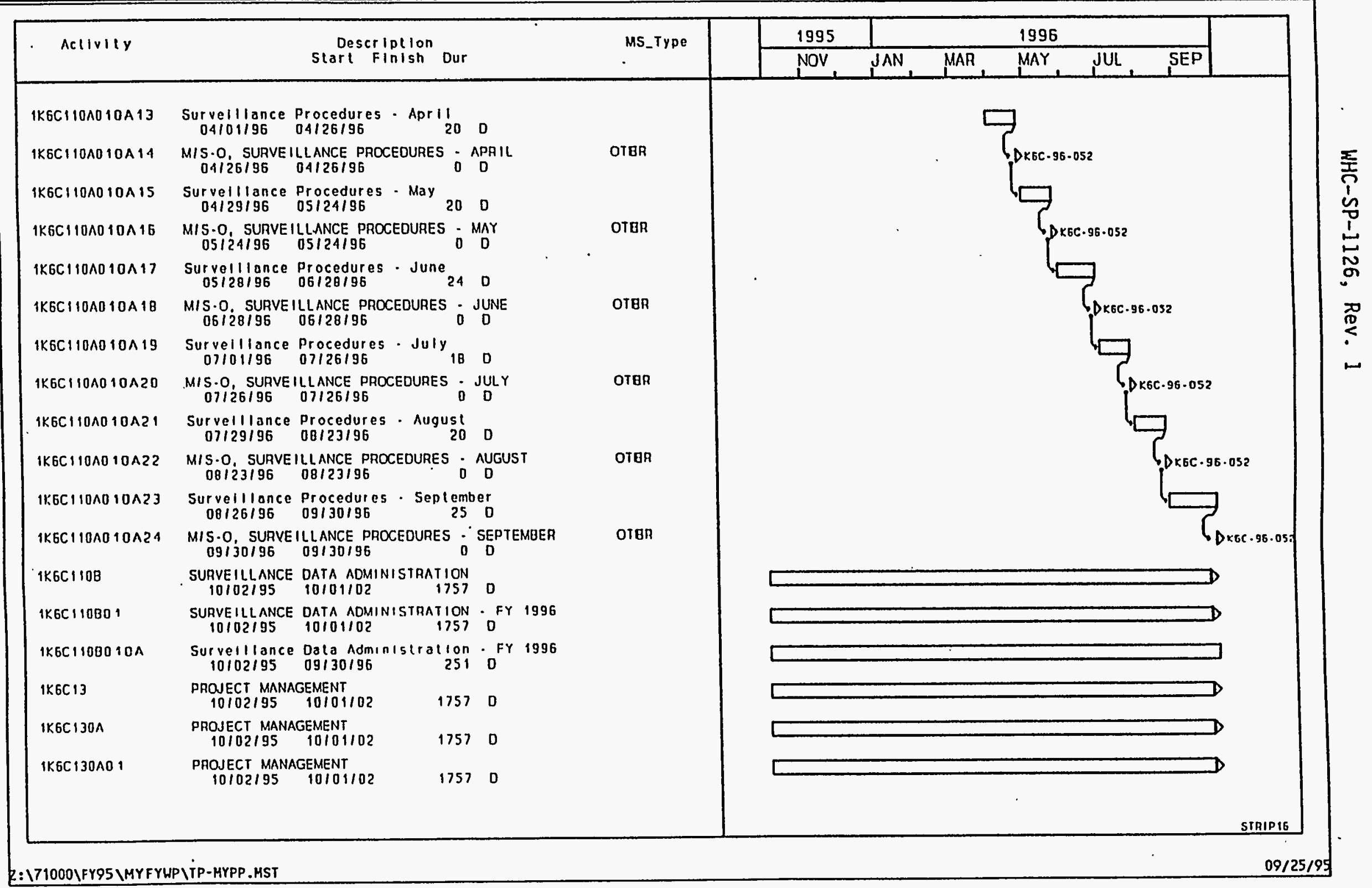




\section{TRANSITION PROJECTS}

FY 1996 MYPP

1.3.1/7.1/6.12

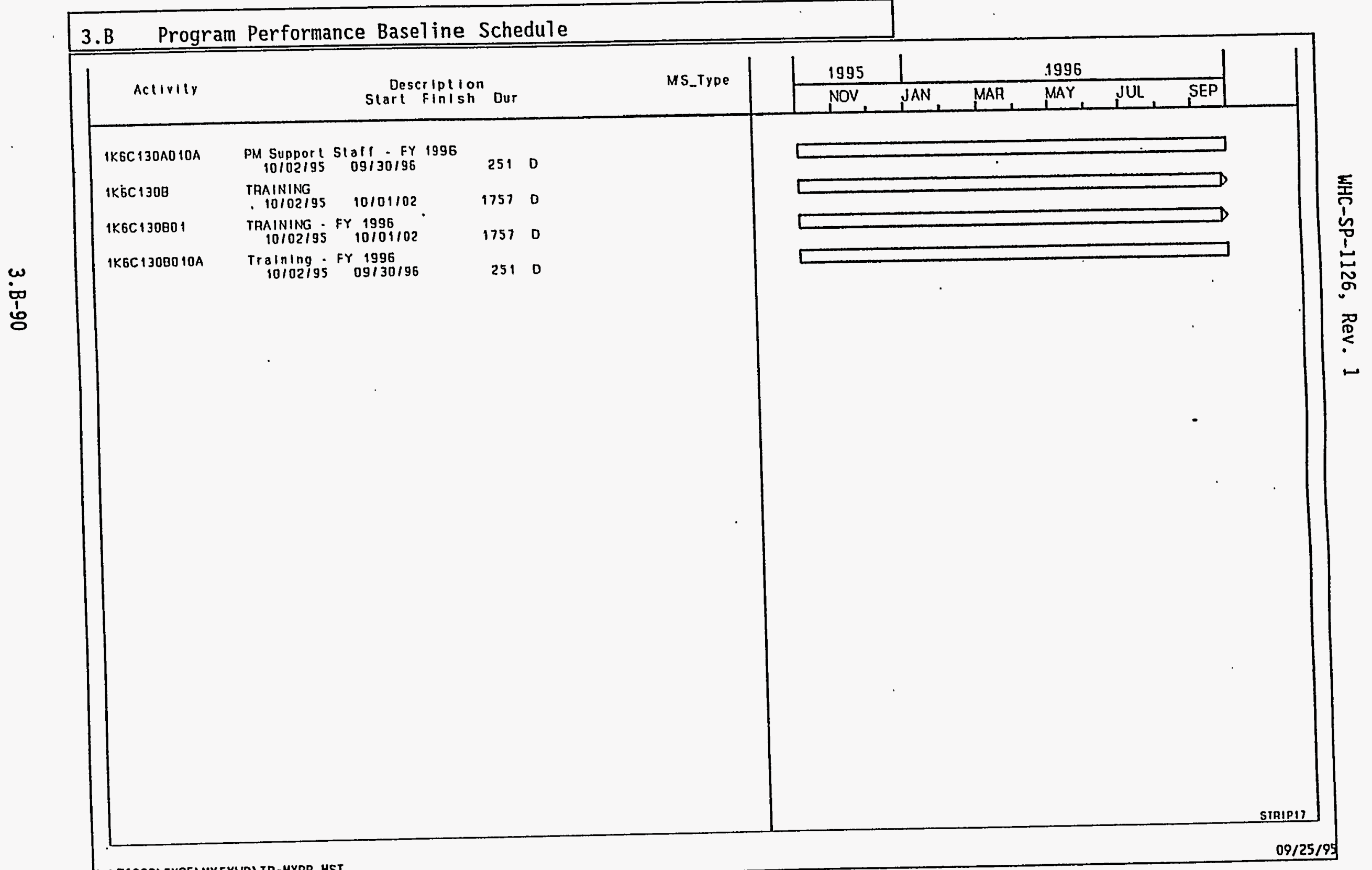




\section{TRANSITION PROJECTS}

\section{FY 1996 MYPP}

\subsection{1/7.1/6.12}

\section{B Program Performance Base 7 ine ScheduTe}

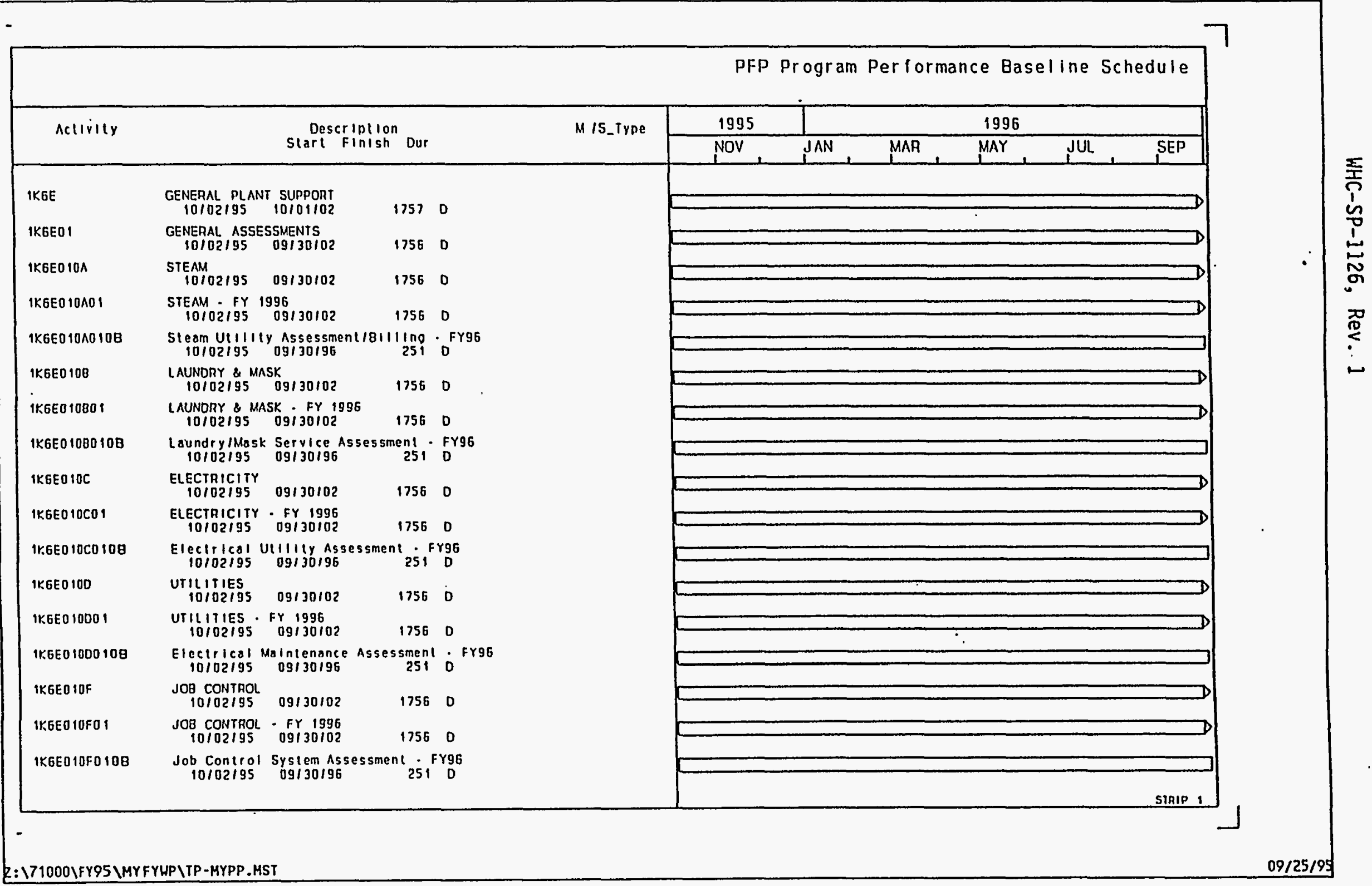


FY 1996 MYPP

\section{TRANSITION PROJECTS}

\subsection{1/7.1/6.12}

\section{B Program Performance Base 7 ine Schedule}

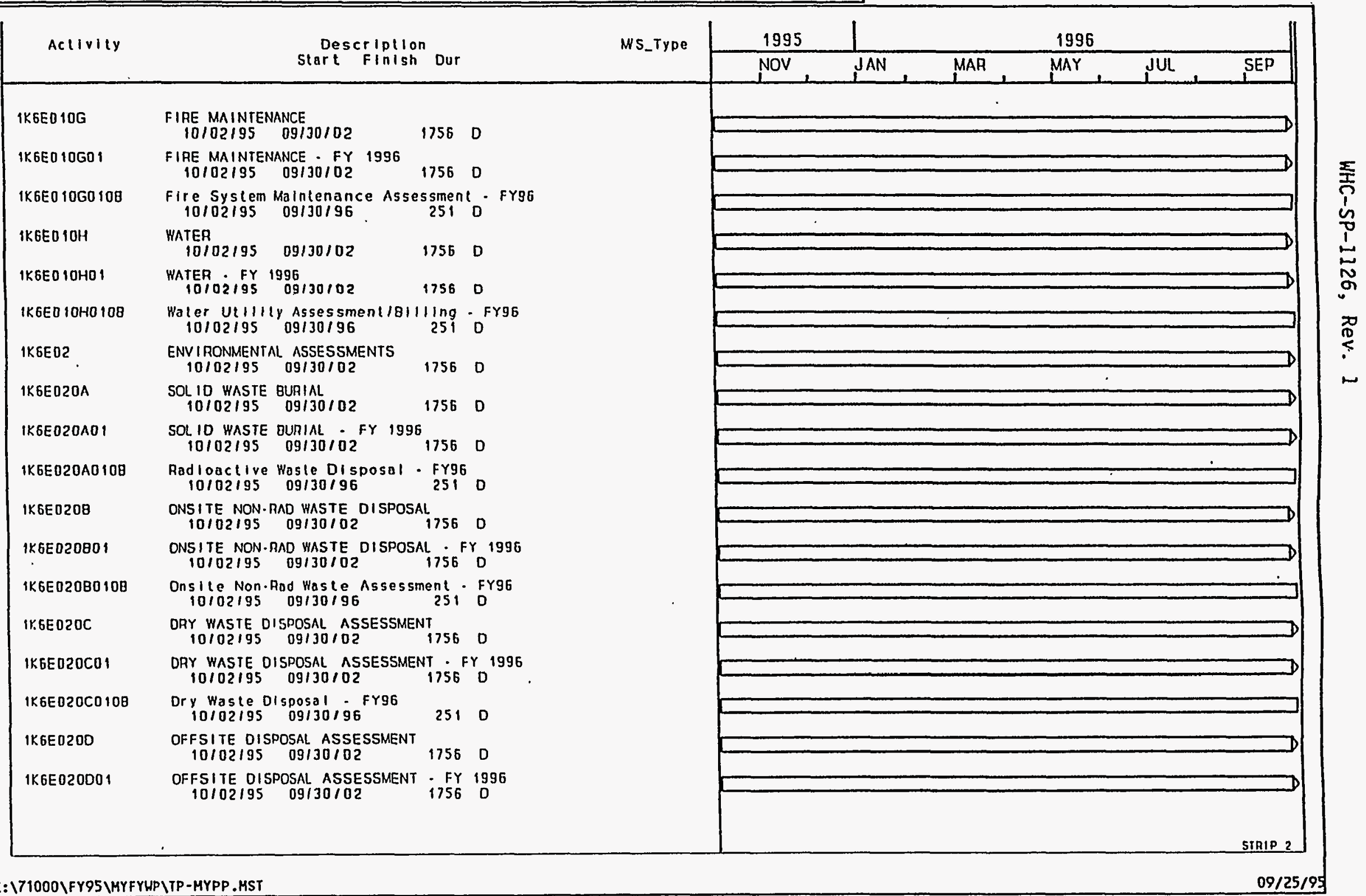




\section{TRANSITION PROJECTS}

FY 1996 MYPP

1.3.1/7.1/6.12

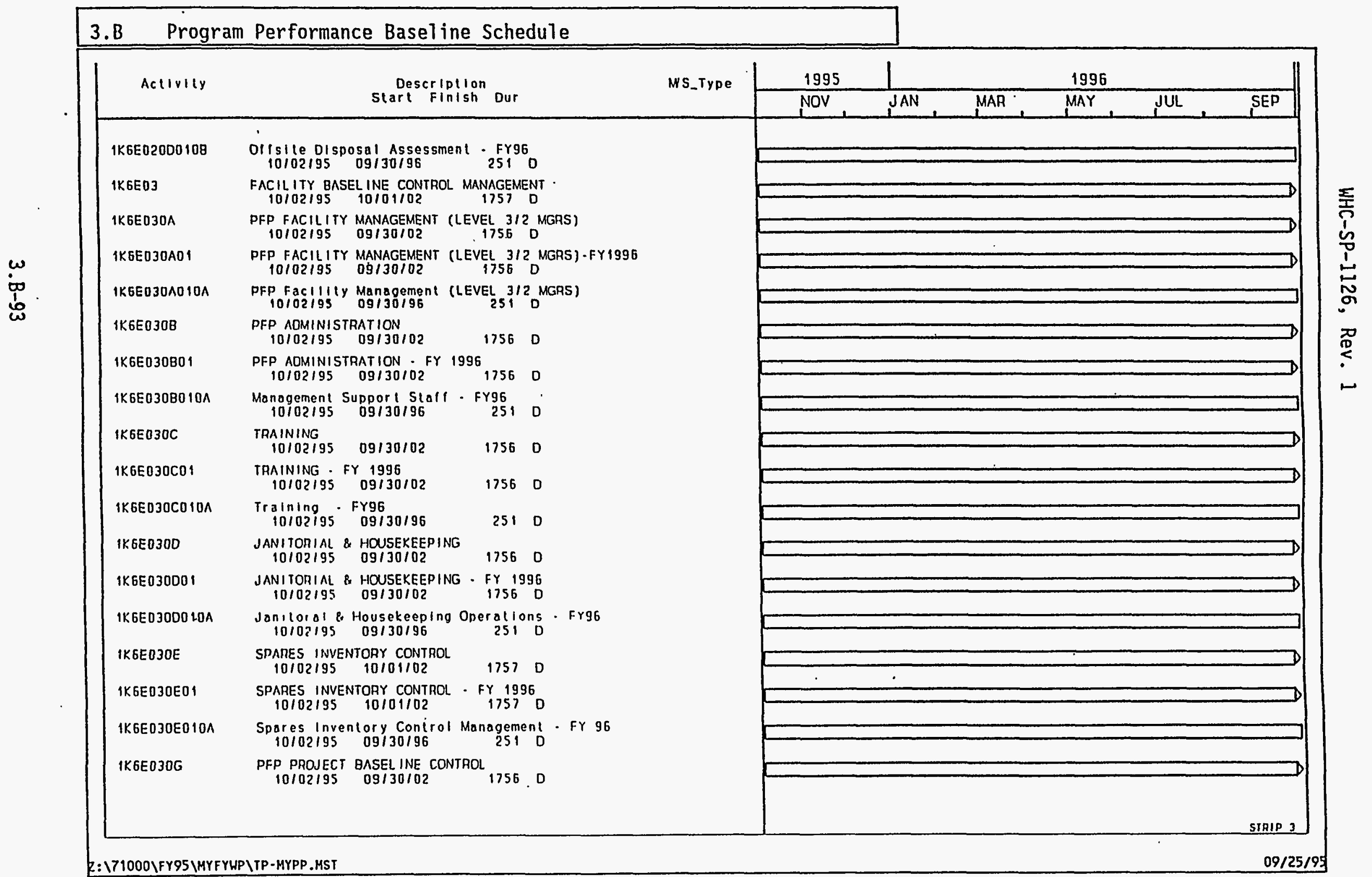




\section{TRANSITION PROJECTS}

\section{FY 1996 MYPP}

1.3.1/7.1/6.12

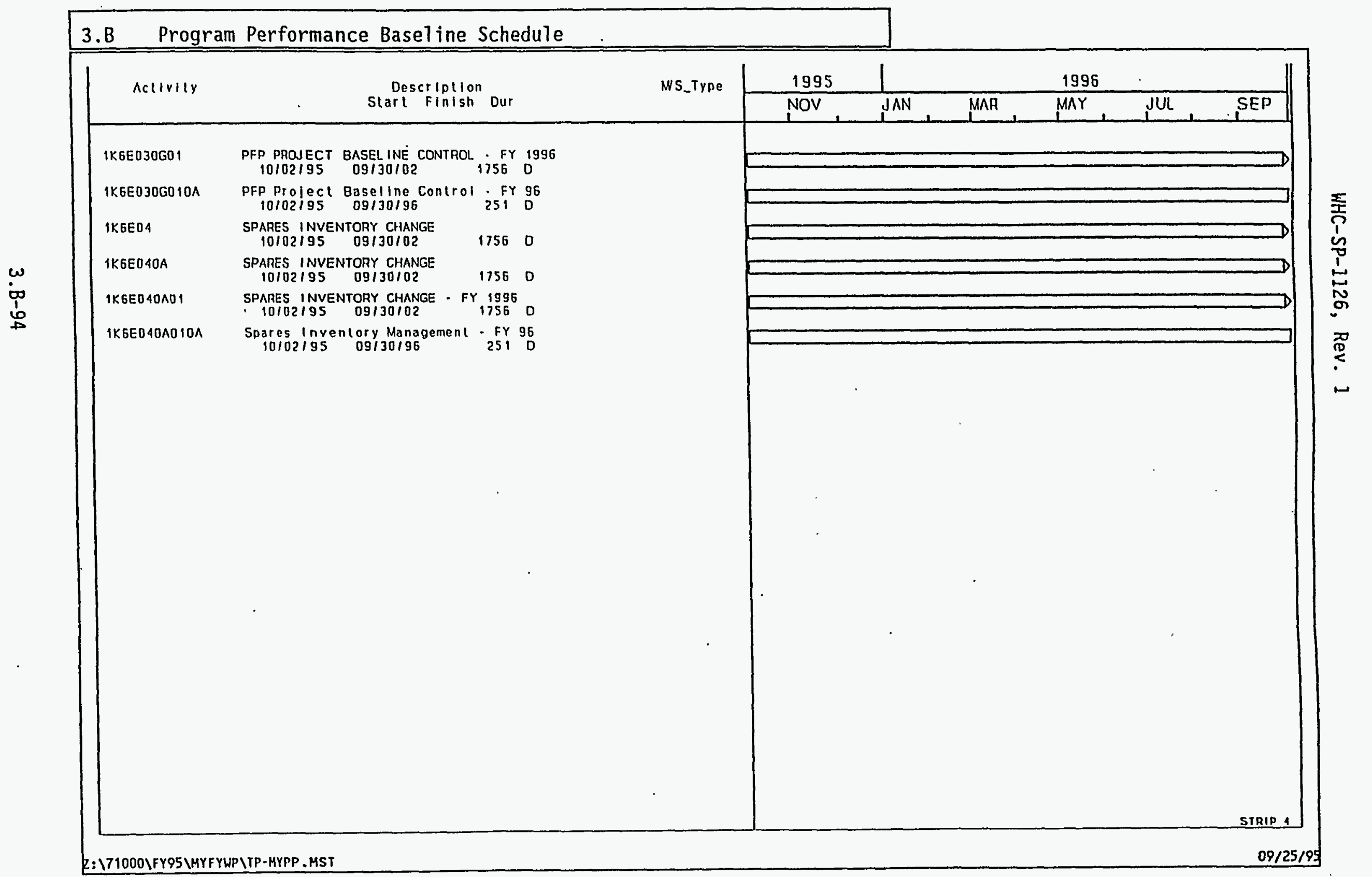




\section{FY 1996 MYPP}

\section{TRANSITION PROJECTS}

1.3.1/7.1/6.12

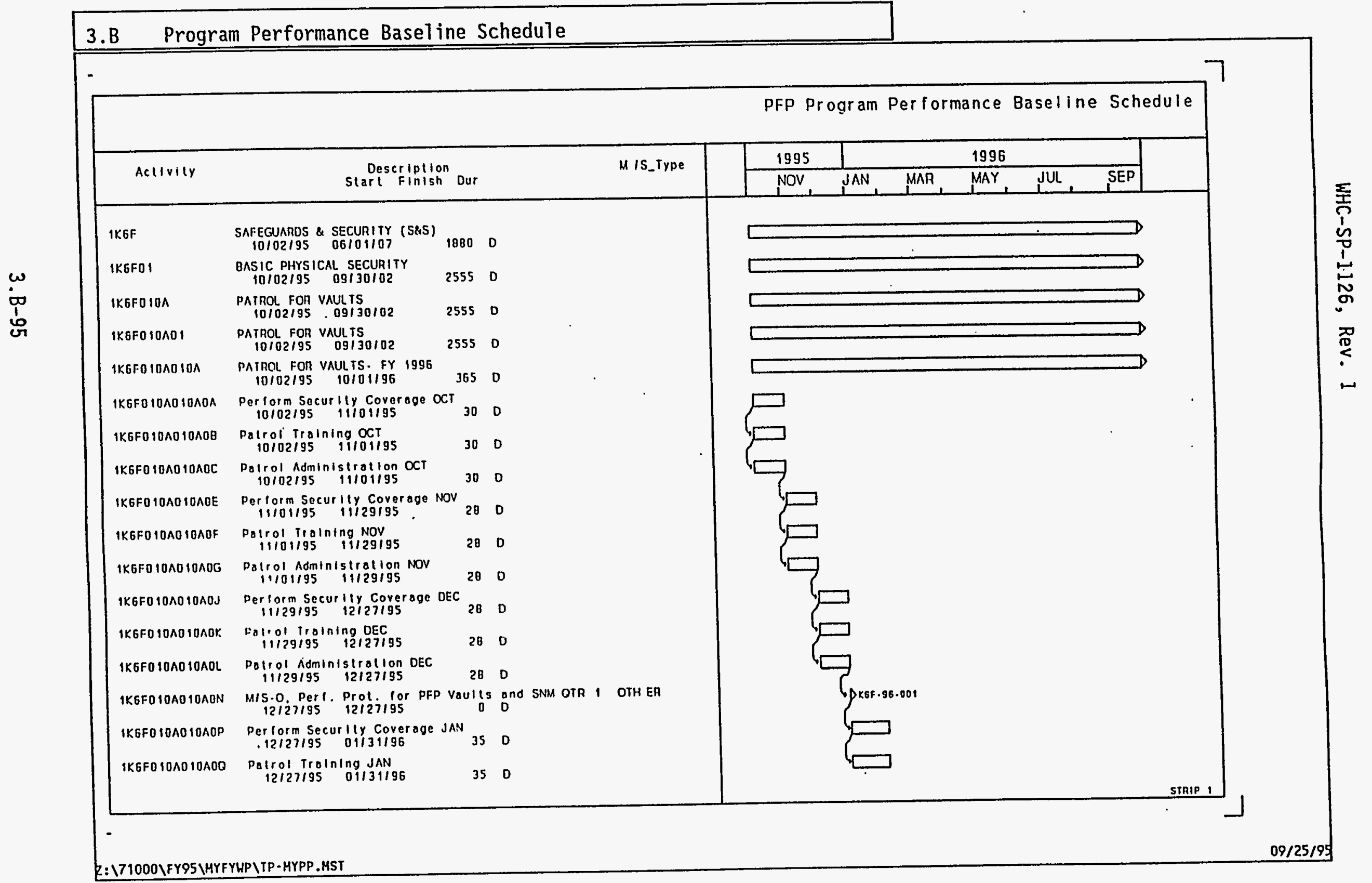




\section{TRANSITION PROJECTS}

FY 1996 MYPP

\subsection{1/7.1/6.12}

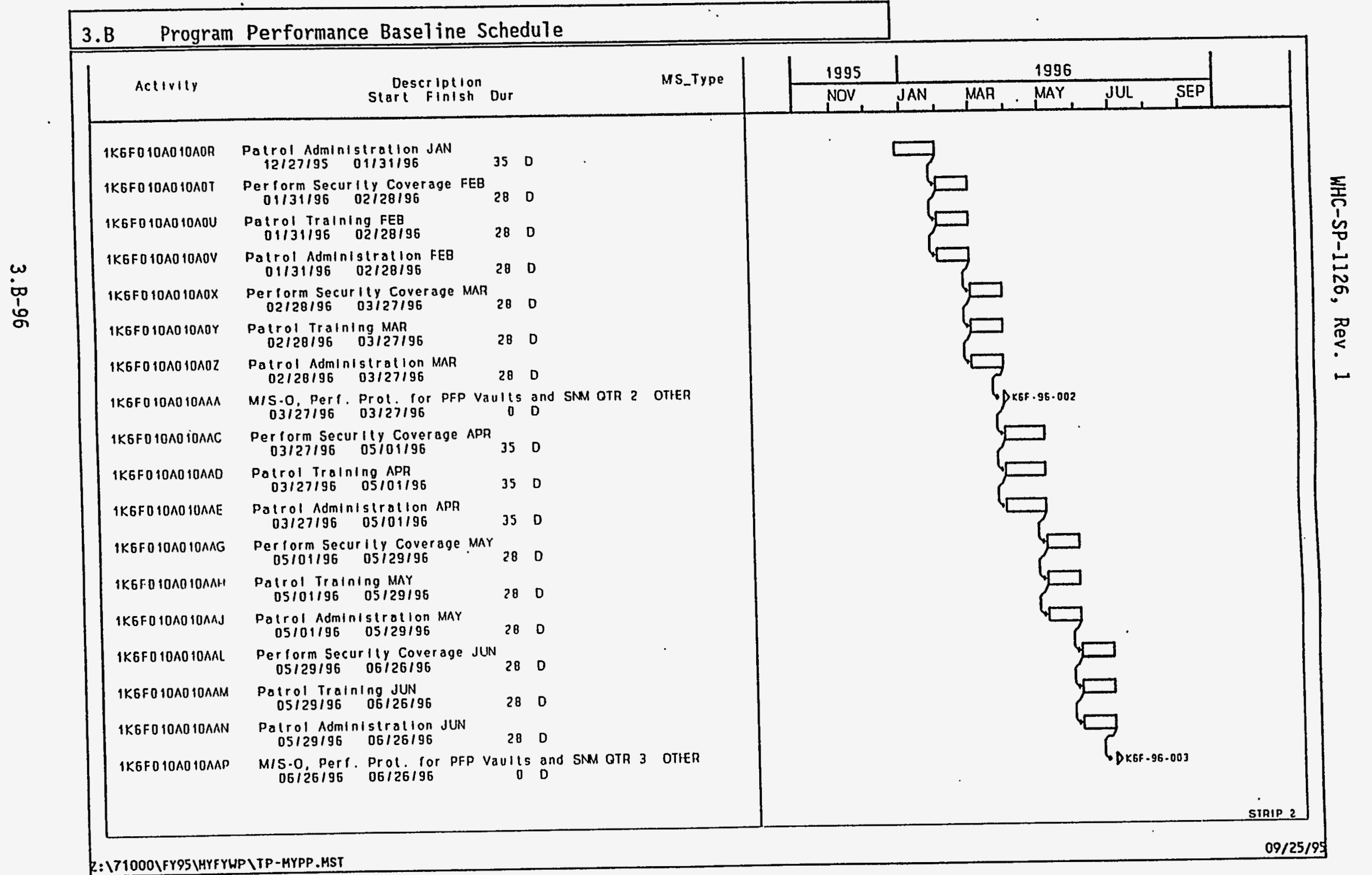




\section{TRANSITION PROJECTS}

FY 1996 MYPP

1.3.1/7.1/6.12

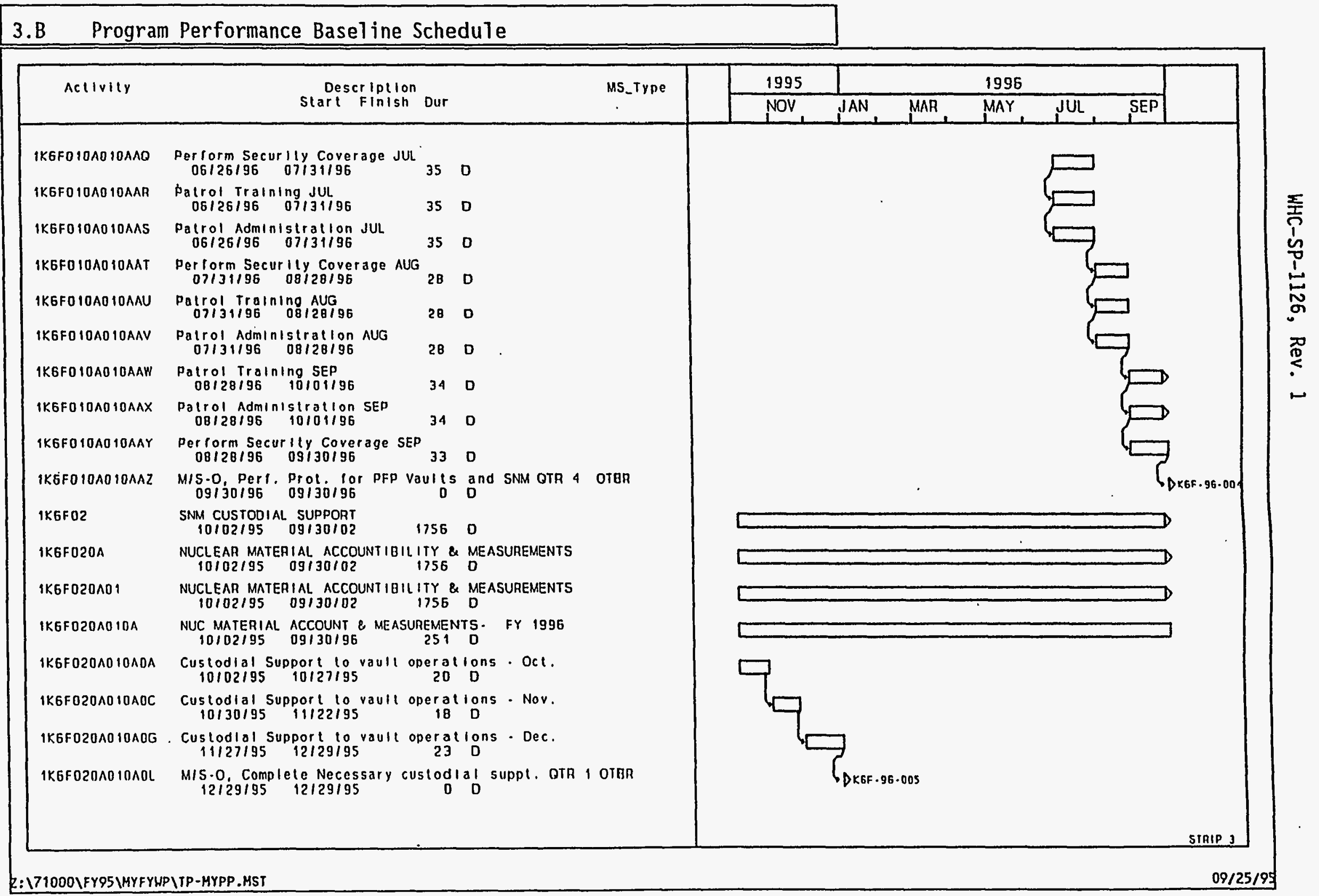




\section{TRANSITIOIN PROJECTS}

\section{FY 1996 MYPP}

1.3.1/7.1/6.12

\section{B Program Performance Baseline ScheduTe}

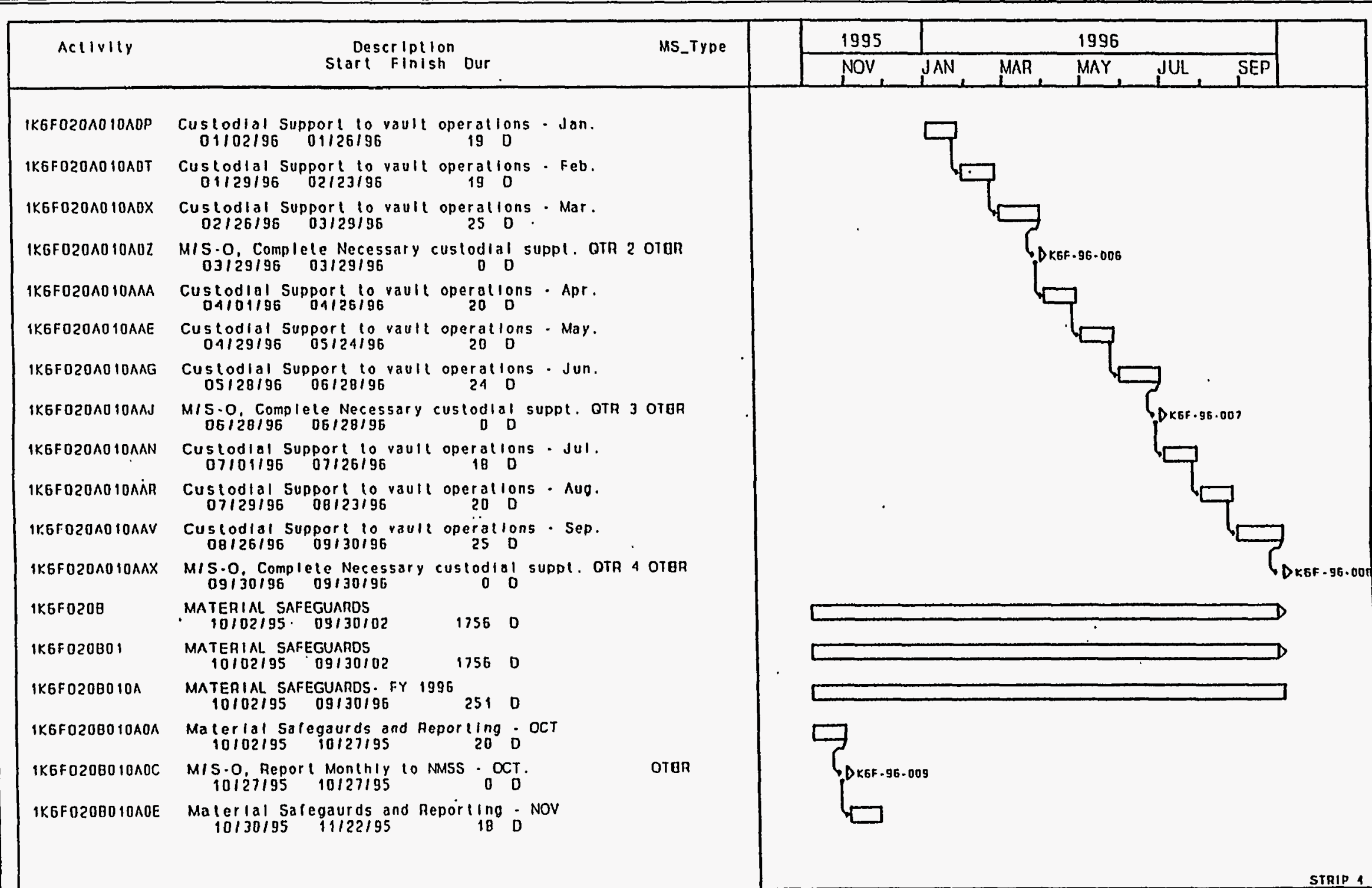


FY 1996 MYPP

TRANSITION PROJECTS

1.3.1/7.1/6.12

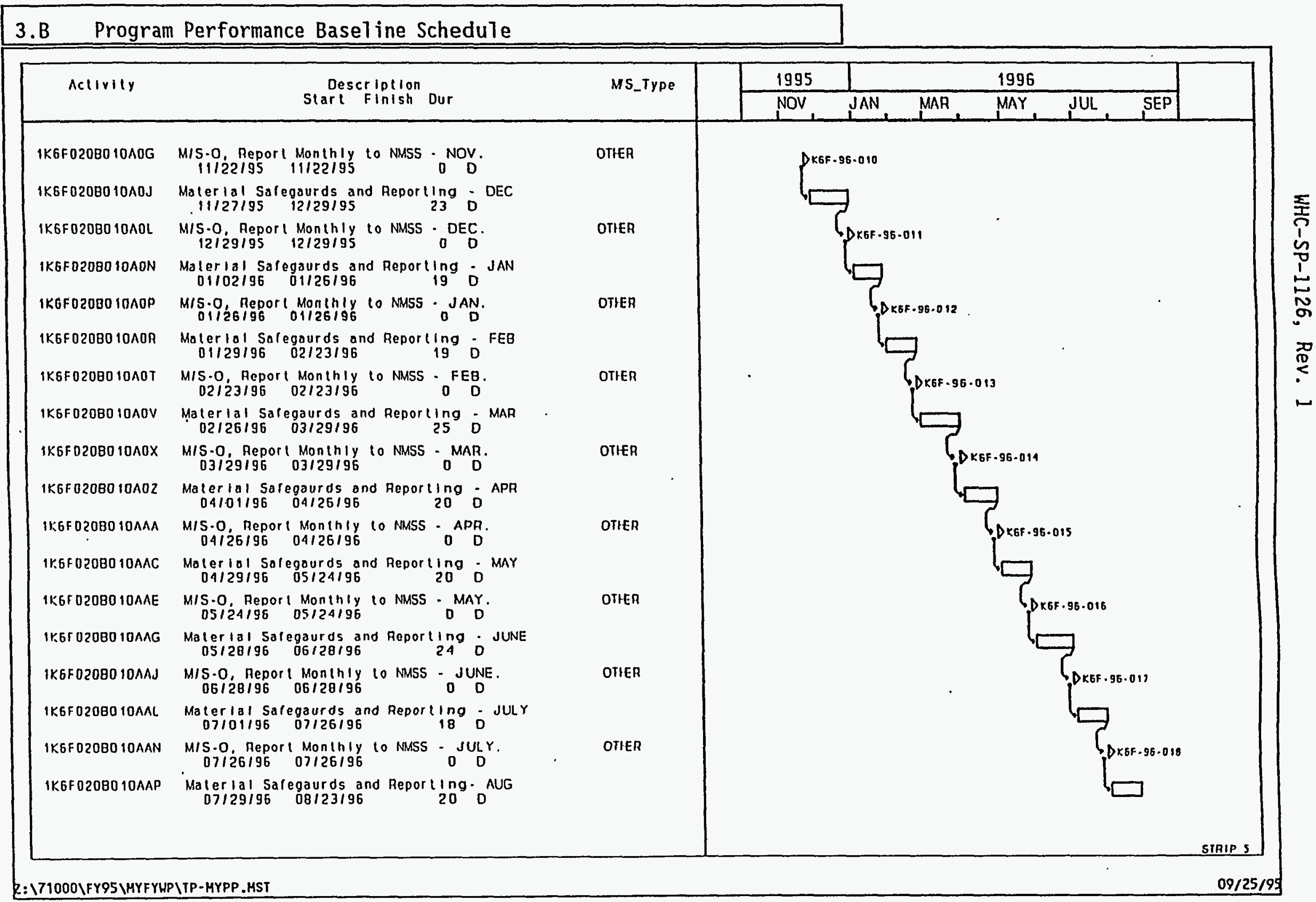




\section{FY 1996 MYPP}

\section{TRANSITION PROJECTS}

\subsection{1/7.1/6.12}

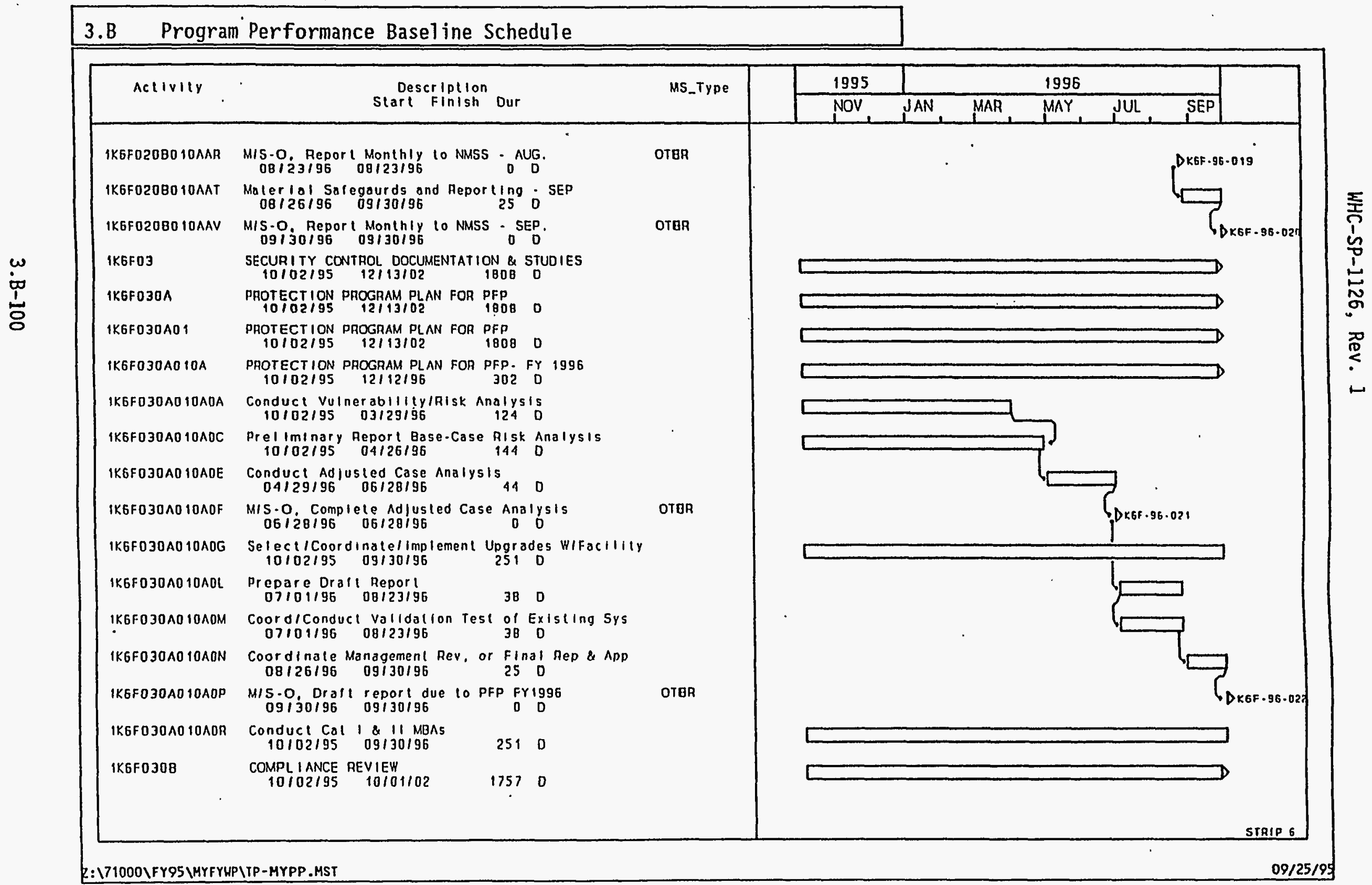


FY 1996 MYPP

TRANSITION PROJECTS

1.3.1/7.1/6.12

3.B Program Performance Baseline Schedule

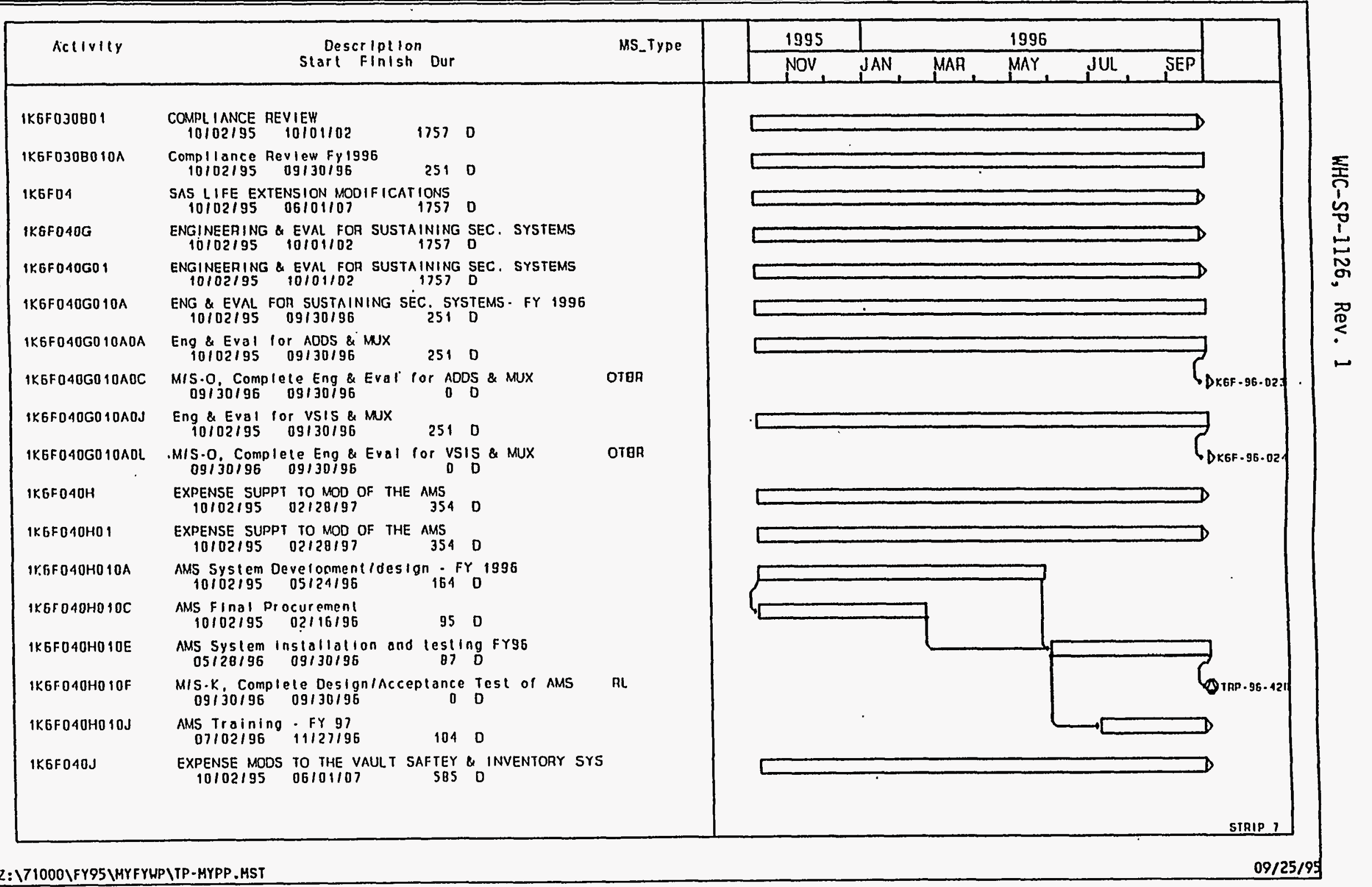




\section{TRANSITION PROJECTS}

FY 1996 MYPP

1.3.1/7.1/6.12

3.B Program Performance Baseline Schedule

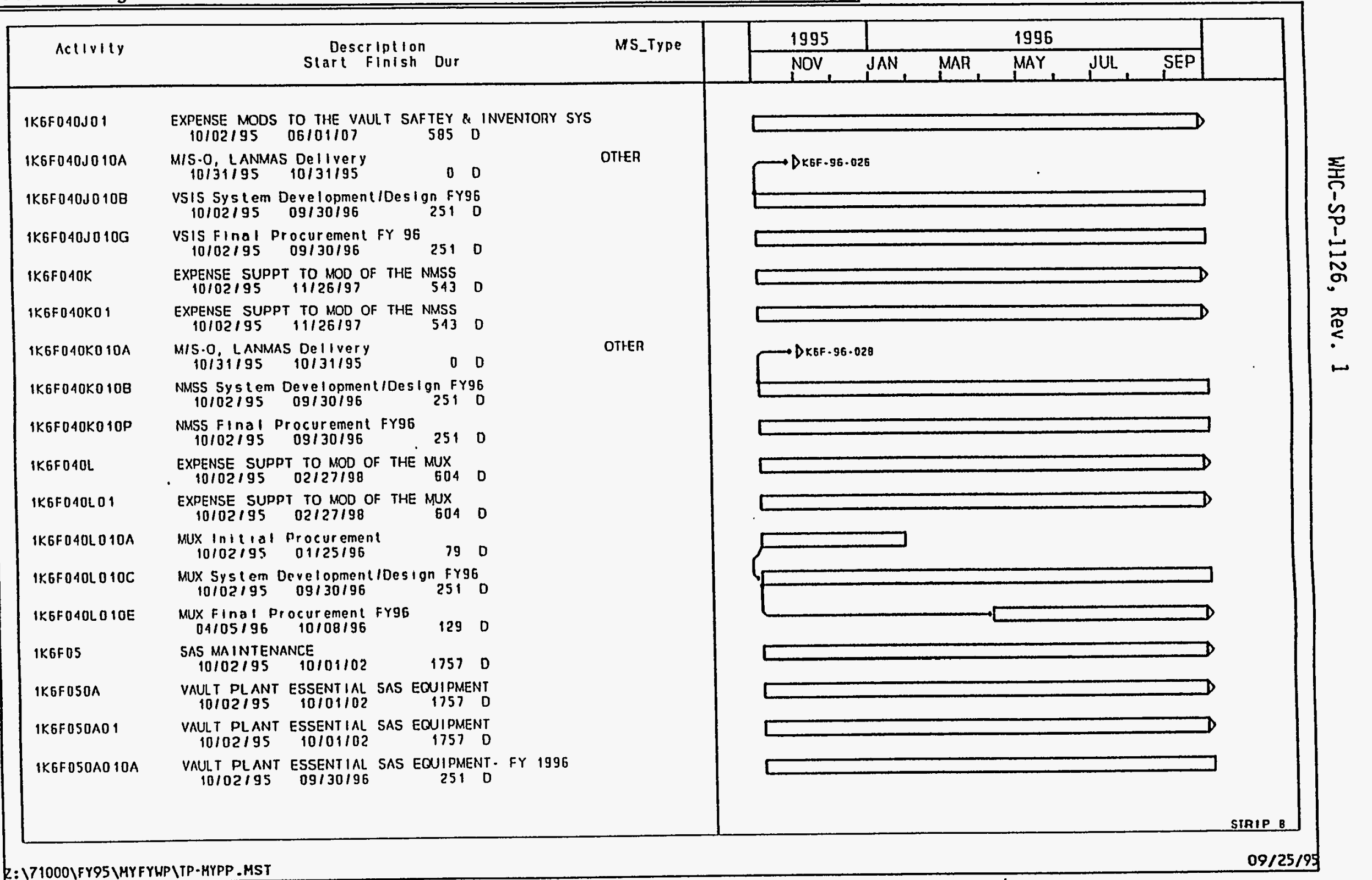




\section{TRANSITION PROJECTS}

FY 1996 MYPP 1.3.1/7.1/6.12

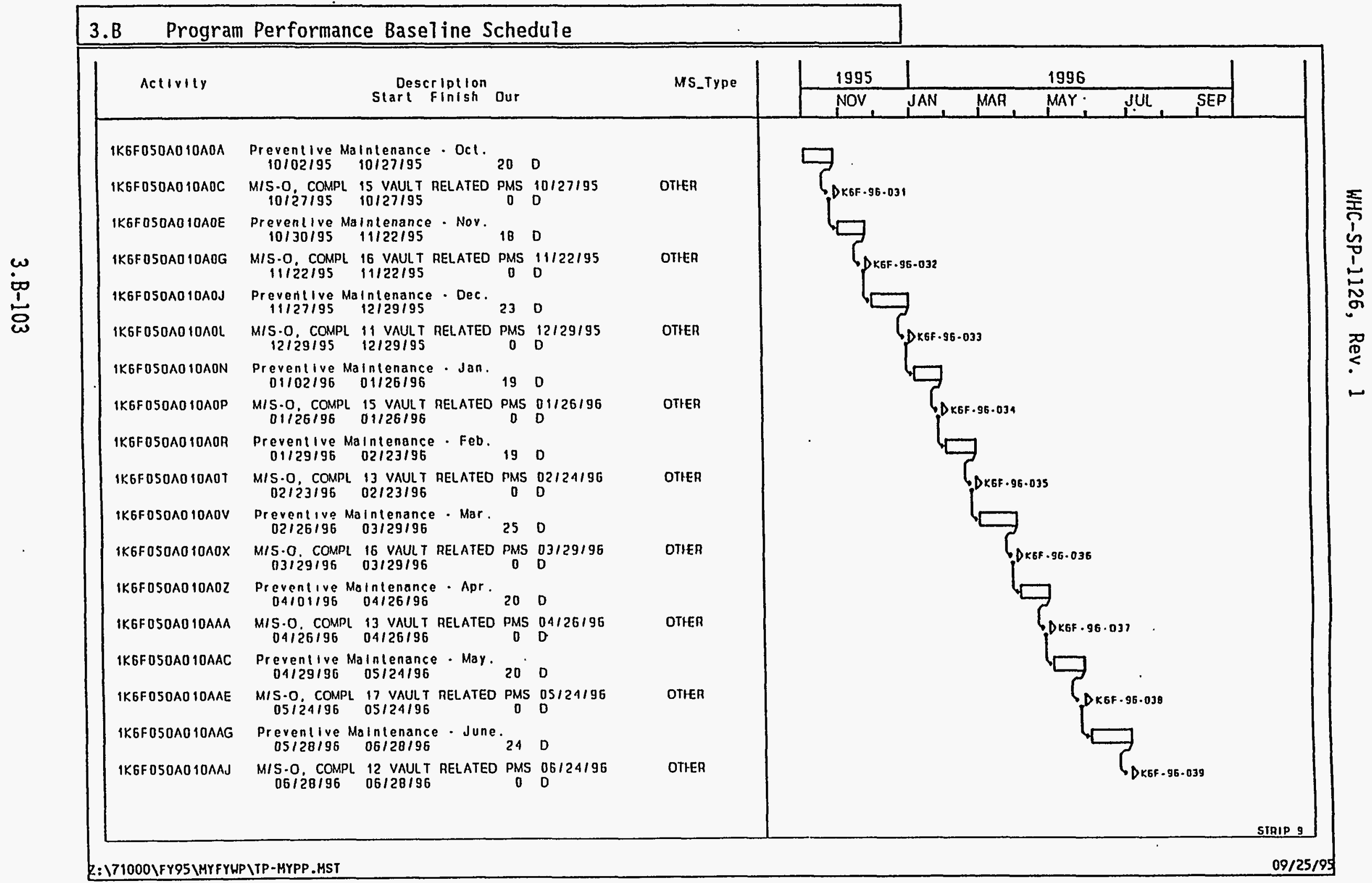


FY 1996 MYPP

TRANSITION PROJECTS

1.3.1/7.1/6.12

\section{B Program Performance Baseline ScheduTe}

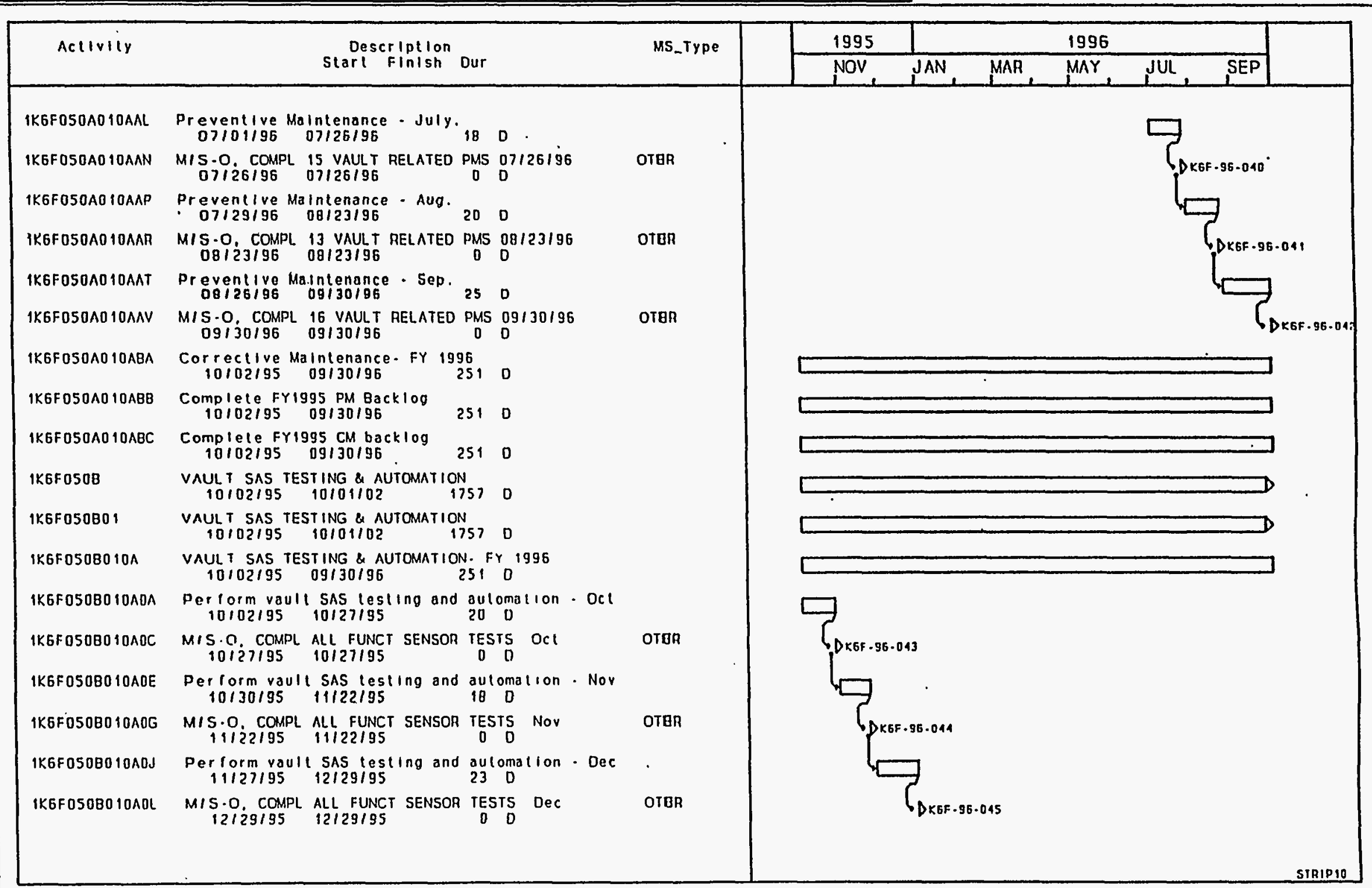




\section{TRANSITION PROJECTS}

\section{FY 1996 MYPP}

\subsection{1/7.1/6.12}

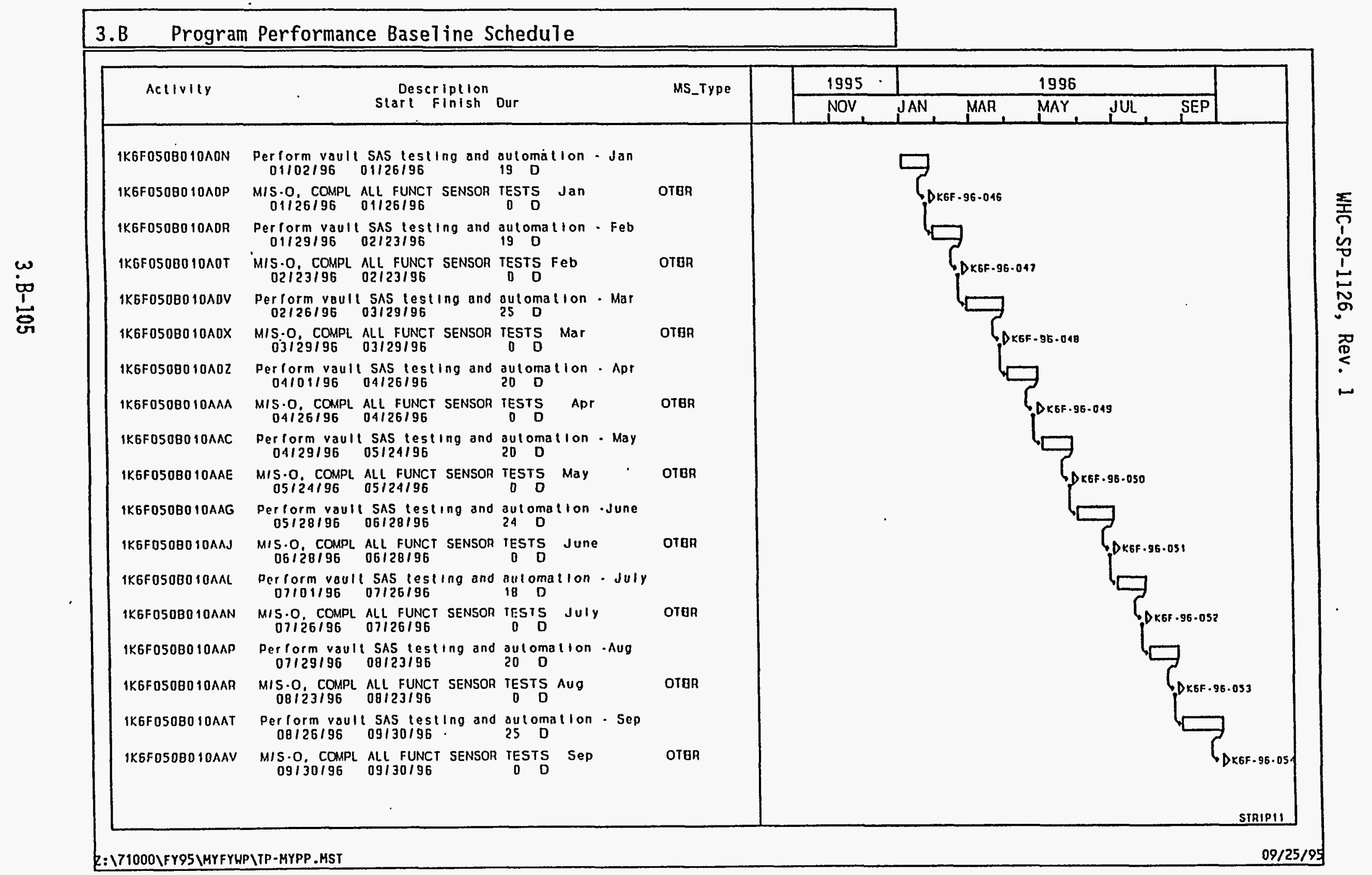




\section{TRANSITION PROJECTS}

\section{FY 1996 MYPP}

\subsection{1/7.1/6.12}

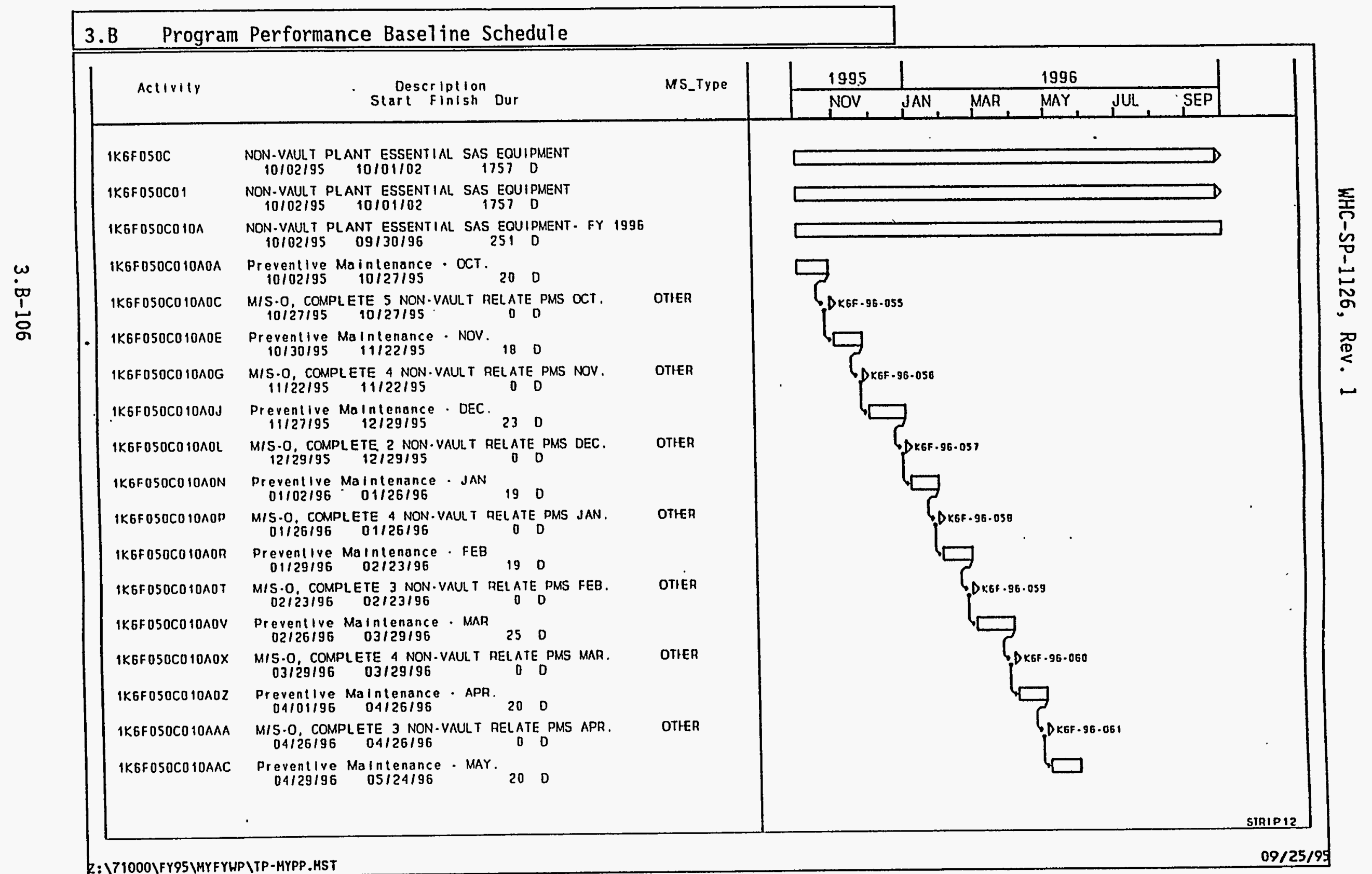




\section{TRANSITION PROJECTS}

FY 1996 MYPP

1.3.1/7.1/6.12

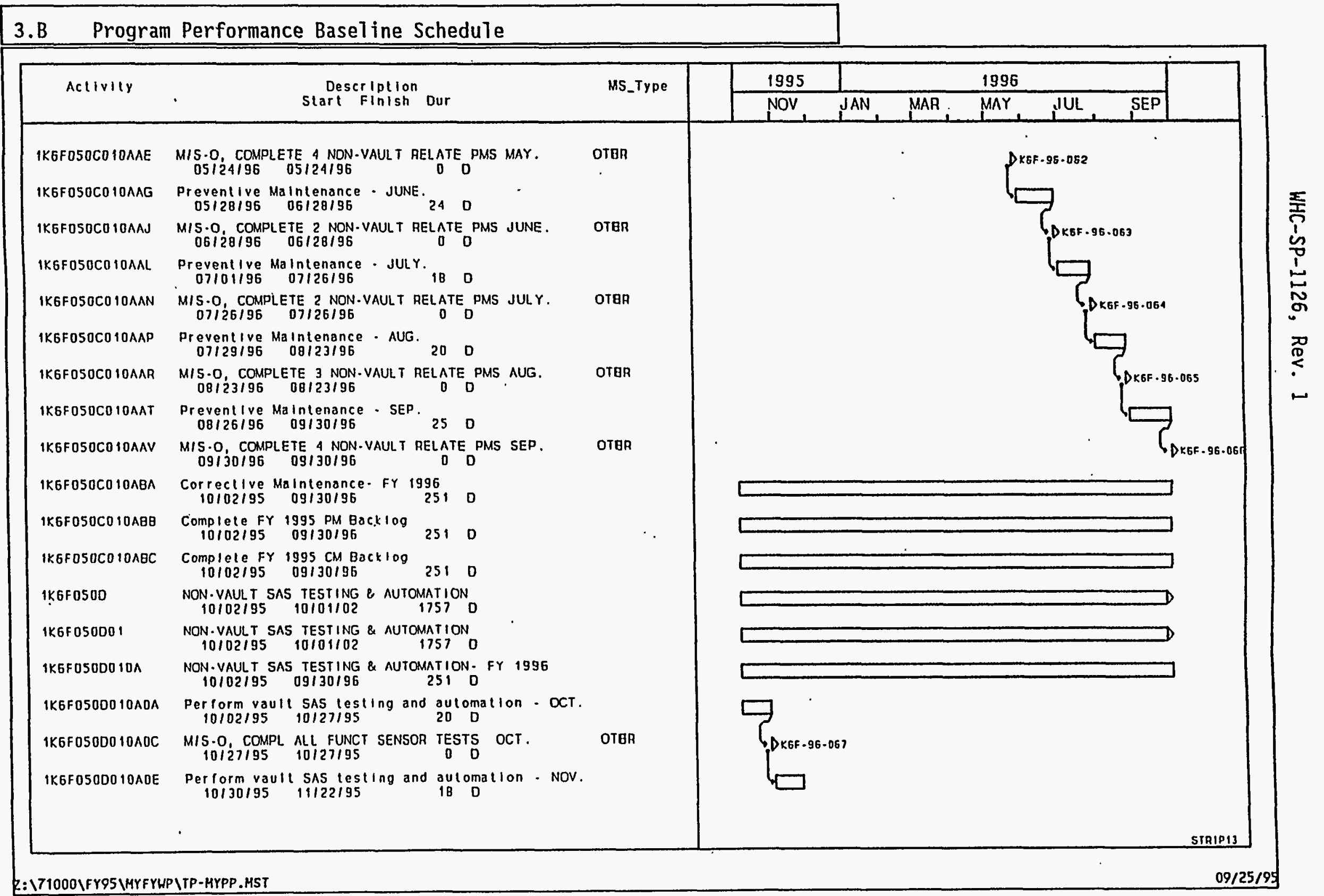




\section{TRANSITION PROJECTS}

\section{FY 1996 MYPP}

\subsection{1/7.1/6.12}

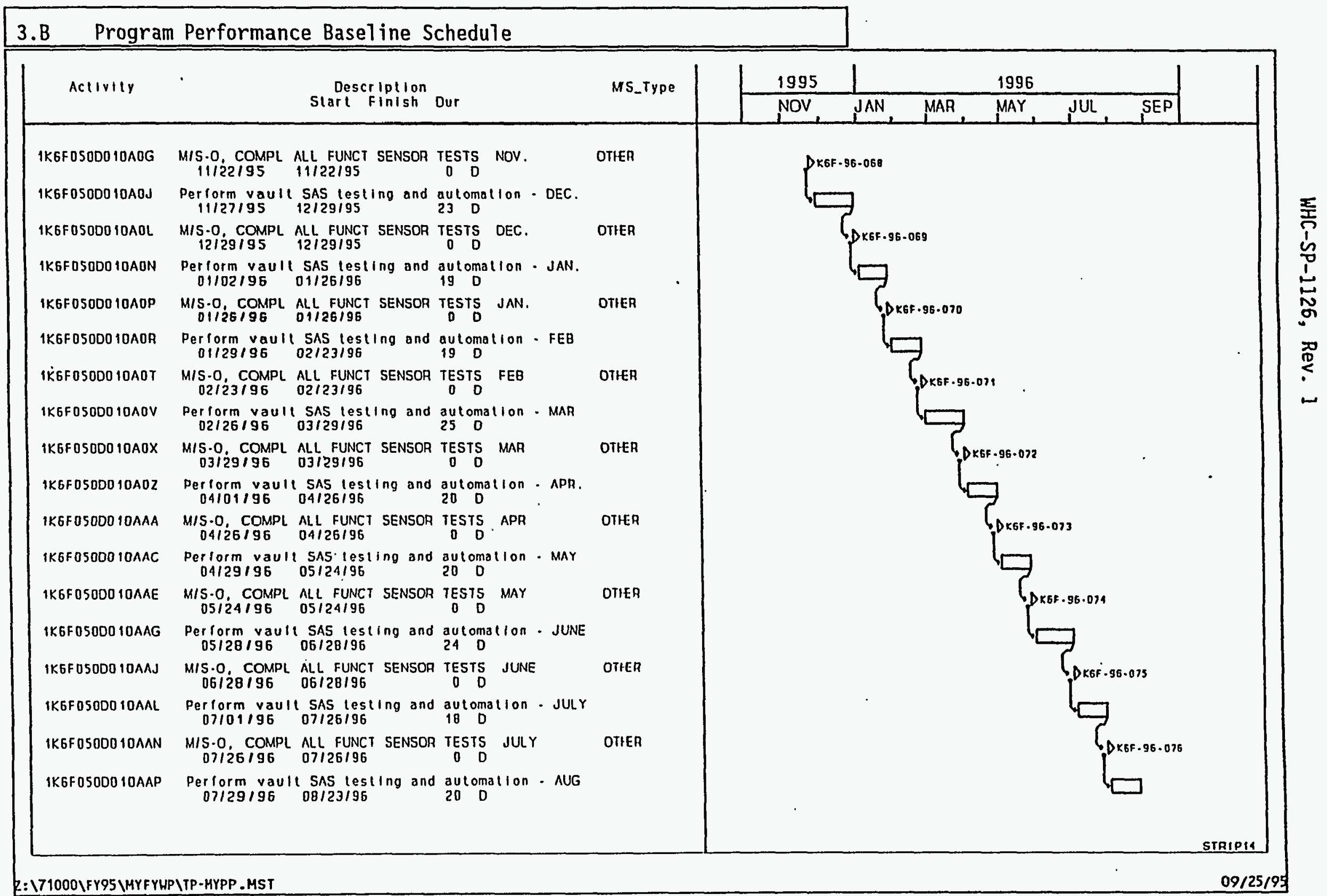


TRANSITION PROJECTS

FY 1996 MYPP

1.3.1/7.1/6.12

\section{B Program Performance Baseline Schedule}

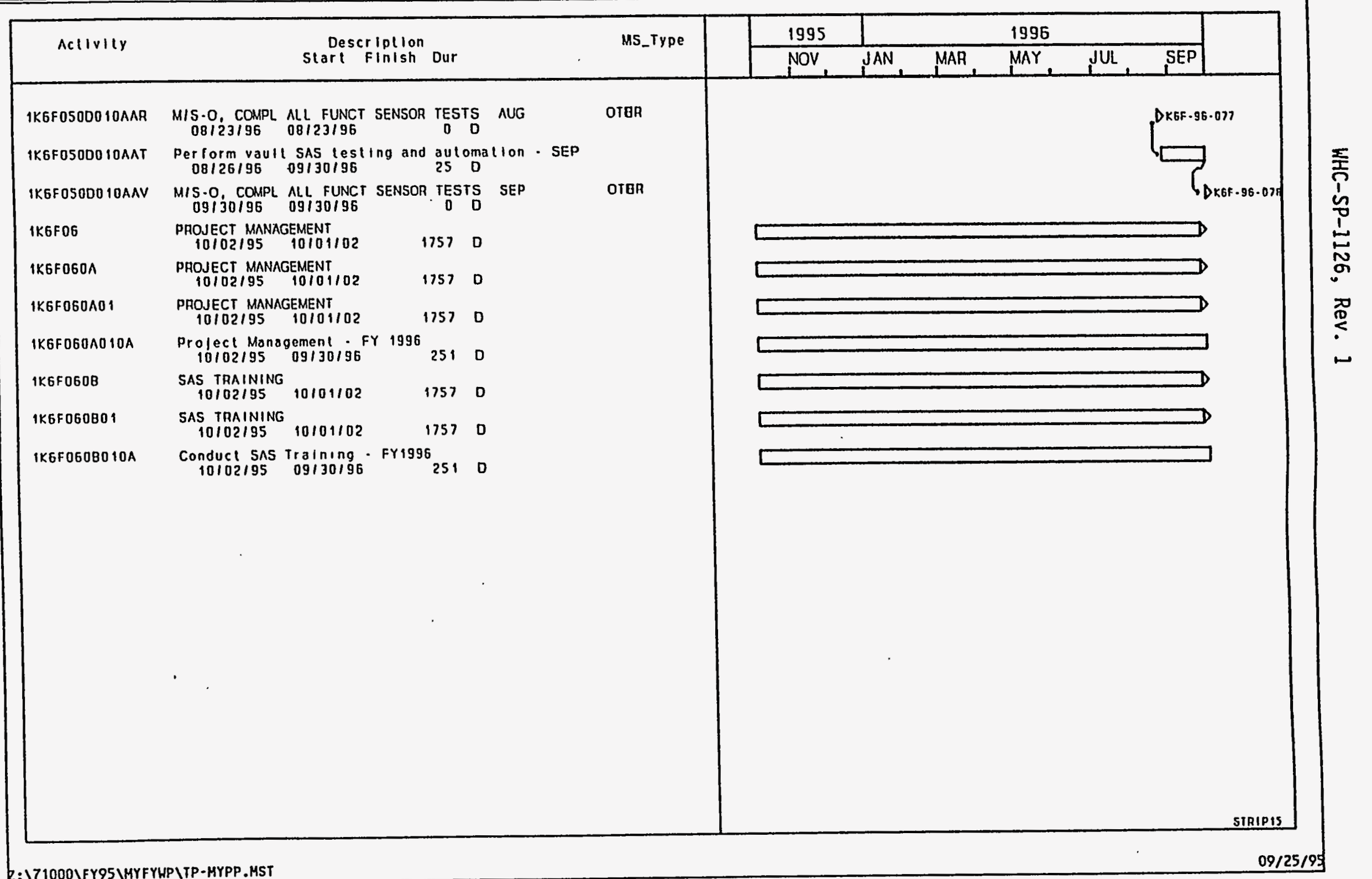


3.B Program Performance Baseline Schedule

Program and Environmental Management (7.1.6)

The Program and Environmental Management program element provides management support for resources and activities that are common to all Transition Projects plants. These activities consist

primarily of level-of-effort tasks which are repetitive from year to year and, thus, no Program

Performance Basel ine Schedule is provided here. 


\section{TRANSIT. $-N$ PROJECTS}

\section{C. 1 Cost Baseline by Month-Operating Expense}

(s in 000's)

\begin{tabular}{|c|c|c|c|c|c|c|c|c|c|c|c|c|c|c|c|c|}
\hline $\begin{array}{c}\text { AL } \\
\text { WBS } \\
\end{array}$ & $\begin{array}{l}\text { WBS } \\
\text { TITLE }\end{array}$ & & COST ELEMENT & OCT & NOV & DEC & JAN & FEB & MAR & APR & MAY & JUN & JUL & AUG & SEP & TOTAL \\
\hline 1.3.1 & Transition & 0 & LABOR & 6.907 & 6,307 & 7.683 & 6,545 & 6,566 & 8,612 & 6,873 & 6,909 & 7,981 & 6,009 & 6,869 & 8,692 & 85,953 \\
\hline and & Projects & 1 & MATERIALS & 346 & 327 & 297 & 225 & 219 & 285 & 275 & 221 & 330 & 240 & 330 & 420 & 3,515 \\
\hline \multirow[t]{8}{*}{7.1} & & 2 & $\begin{array}{l}\text { PURCHASED } \\
\text { SERVICES }\end{array}$ & 918 & 889 & 1,218 & 1.009 & 913 & 1.131 & 939 & 1.010 & 1.292 & 1.038 & 1.145 & 1.464 & 12.966 \\
\hline & & 4 & SITE SERVICES & 1,559 & 1.469 & 1,724 & 1,515 & 1,511 & 1.819 & 1,556 & 1.569 & 1.770 & 1.464 & 1.579 & 1,847 & 19,382 \\
\hline & & $\mathbf{5}$ & $\begin{array}{l}\text { INTERNAL } \\
\text { CHARGES }\end{array}$ & 557 & 538 & 633 & 555 & 602 & 774 & 552 & 550 & 636 & 544 & 636 & 757 & 7,334 \\
\hline & & 6 & IRM & 136 & 126 & 145 & 127 & 128 & 155 & 128 & 127 & 146 & 118 & 130 & 156 & 1.622 \\
\hline & & 7 & $\begin{array}{l}\text { DEPARTMENTAL } \\
\text { OVERHEAD }\end{array}$ & 2,181 & 2,042 & 2.430 & 2,052 & 2,103 & 2,673 & 2,180 & 2.198 & 2,569 & 1.981 & 2.246 & 2.719 & 27,374 \\
\hline & & 8 & G\&A/CSP & 765 & 717 & 874 & 763 & 737 & 926 & 758 & 768 & 845 & 667 & 739 & 934 & 9.494 \\
\hline & & & TOTAL BUDGET & 14.109 & 12,802 & 15,552 & 13.161 & 13,161 & 17.043 & 14.543 & 14,047 & 16,240 & 12,883 & 14,575 & 18,008 & 176,204 \\
\hline & & & $\begin{array}{l}\text { TOTAL FUNDING } \\
\text { EM-30/EM-60 }\end{array}$ & 14,109 & 12,882 & 15,552 & 13.161 & 13,161 & 17.043 & 14.543 & 14,047 & 16,240 & 12,883 & 14,575 & 18,008 & 176,204 \\
\hline
\end{tabular}


TRANSIT. $-N$ PROJECTS

FY 1996 MYPP

1.3.1/7.1/6.12

\section{C.1 Cost Baseline by Month-Operating Expense}

\section{$(\$$ in 000 's)}

RL WBS

COST ELEMENT

OCT NOV D

JAN FEB MAR APA

APR MAY JUN JUL AUG SEP TOTAL

$\begin{array}{cc}1.3 .1 .7 & \text { B PLANT I } \\ \text { WESF }\end{array}$

- LABOR

1 MATERIALS

$1410 \quad 127$

2 PURCHASED SERVICES

3 OTHER CONTRACTORS

4 SITE SERVICES

5 INTERNAL CHARGES

6 IRM

7 DEPARTMENTAL OVERHEAD

8 G\&A/CSP TOTAL BUDGET TOTAL FUNDING ADS 4190

\begin{tabular}{|c|c|c|c|c|c|c|c|c|c|c|c|c|}
\hline 1410 & $12 \pi$ & 1390 & 1302 & 1312 & 1702 & 1401 & 1402 & 1004 & 1004 & 1702 & 17.40 & 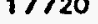 \\
\hline 62 & 56 & 85 & 66 & 61 & 71 & 50 & 50 & 64 & 48 & 52 & 65 & 730 \\
\hline 138 & 124 & 243 & 147 & 127 & 166 & 129 & 125 & 142 & 100 & 111 & 181 & 1733 \\
\hline 97 & 84 & 103 & 82 & 88 & 114 & 81 & 48 & 49 & 127 & 140 & 185 & 1198 \\
\hline 305 & 274 & 351 & 287 & 288 & 377 & 300 & 301 & 360 & 269 & 299 & 380 & 3791 \\
\hline 132 & 119 & 152 & 126 & 126 & 165 & 132 & 132 & 159 & 119 & 132 & 165 & 1659 \\
\hline 21 & 19 & 25 & 20 & 20 & 27 & 21 & 21 & 26 & 19 & 22 & 28 & 269 \\
\hline 188 & 169 & 211 & 181 & 174 & 234 & 186 & 193 & 224 & 173 & 190 & 231 & 2354 \\
\hline 271 & 244 & 320 & 263 & 253 & 336 & 266 & 271 & 318 & 244 & 269 & 336 & 3391 \\
\hline 2624 & 2360 & 3080 & 2534 & 2449 & 3252 & 2566 & 2593 & 3026 & 2403 & 2647 & 3311 & 32845 \\
\hline 2624 & 2360 & 3080 & 2534 & 2449 & 3252 & 2566 & 2593 & 3026 & 2403 & 2647 & 3311 & 32845 \\
\hline
\end{tabular}




\section{TRANSITIUN PROJECTS}

\section{C.1 Cost Baseline by Month soperating Expense}

(s in 000's)

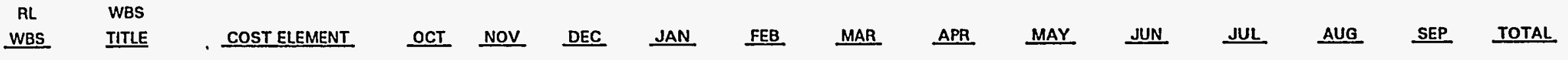

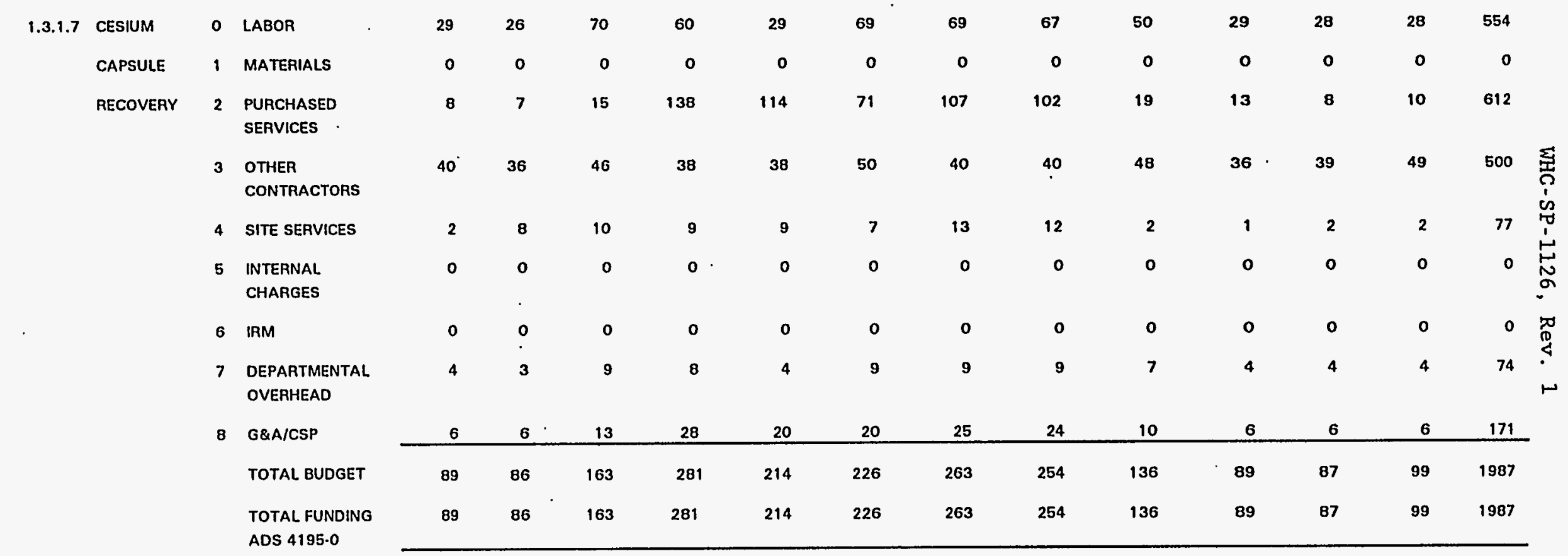




\section{TRANSITIUN PROJECTS}

\section{C.1 Cost Baseline by Month - Operating Expense}

(s in 000's)

\begin{tabular}{|c|c|c|c|c|c|c|c|c|c|c|c|c|c|c|c|c|}
\hline $\begin{array}{c}\text { RL } \\
\text { WBS } \\
\end{array}$ & $\begin{array}{l}\text { WBS } \\
\text { TITLE }\end{array}$ & & COST ELEMENT & OCT & NOV & DEC & JAN & FEB & MAR & APR & MAY & JUN & $\underline{\mathrm{JUL}}$ & AUG & SEP & TOTAL \\
\hline \multirow[t]{11}{*}{7.1 .1} & PUAEX & 0 & LABOR & 1811 & 1658 & 1978 & 1749 & 1768 & 2373 & 1799 & 1743 & 2049 & 1523 & 1783 & 2490 & 22724 \\
\hline & Transition & 1 & MATERIALS & 75 & 75 & -24 & -24 & -24 & -25 & -24 & -26 & -26 & -28 & -27 & -29 & -107 \\
\hline & Project & 2 & $\begin{array}{l}\text { PURCHASED } \\
\text { SERVICES }\end{array}$ & 341 & 365 & 427 & 299 & 220 & 225 & 221 & 222 & 225 & 221 & 217 & 213 & 3196 \\
\hline & & 3 & $\begin{array}{l}\text { OTHER } \\
\text { CONTRACTORS }\end{array}$ & 24 & 27 & 24 & 30 & 24 & 30 & 24 & 30 & 25 & 27 & 25 & 28 & 316 \\
\hline & & 4 & SITE SERVICES & 657 & 560 & 671 & 654 & 543 & 543 & 542 & 541 & 542 & 539 & 540 & 539 & 6571 \\
\hline & & 5 & $\begin{array}{l}\text { INTERNAL } \\
\text { CHARGES }\end{array}$ & 145 & 167 & 160 & 162 & 160 & 161 & 140 & 139 & 140 & 139 & 140 & 133 & 1786 \\
\hline & & 6 & IRM & 21 & 21 & 21 & 21 & 21 & 21 & 21 & 21 & 21 & 21 & 21 & 21 & 252 \\
\hline & & 7 & $\begin{array}{l}\text { DEPARTMENTAL } \\
\text { OVERHEAD }\end{array}$ & 429 & 403 & 459 & 416 & 418 & 514 & 421 & 413 & 467 & 376 & 416 & 522 & 5253 \\
\hline & & 8 & G\&A/CSP & 440 & 423 & 481 & 426 & 417 & 509 & 419 & 411 & 458 & 376 & 415 & 519 & 5294 \\
\hline & & & TOTAL BUDGET & 3843 & 3699 & 4097 & 3632 & 3547 & 4351 & 3563 & 3494 & 3901 & 3194 & 3530 & 4434 & 45285 \\
\hline & & & $\begin{array}{l}\text { TOTAL FUNDING } \\
\text { ADS } 6622-0\end{array}$ & 3843 & 3699 & 4097 & 3632 & 3547 & 4351 & 3563 & 3494 & 3901 & 3194 & 3530 & 4434 & 45285 \\
\hline
\end{tabular}




\section{TRANSITIUN PROJECTS}

\section{C.1 Cost Baseline by Month - Operating Expense}

(s in 000's)

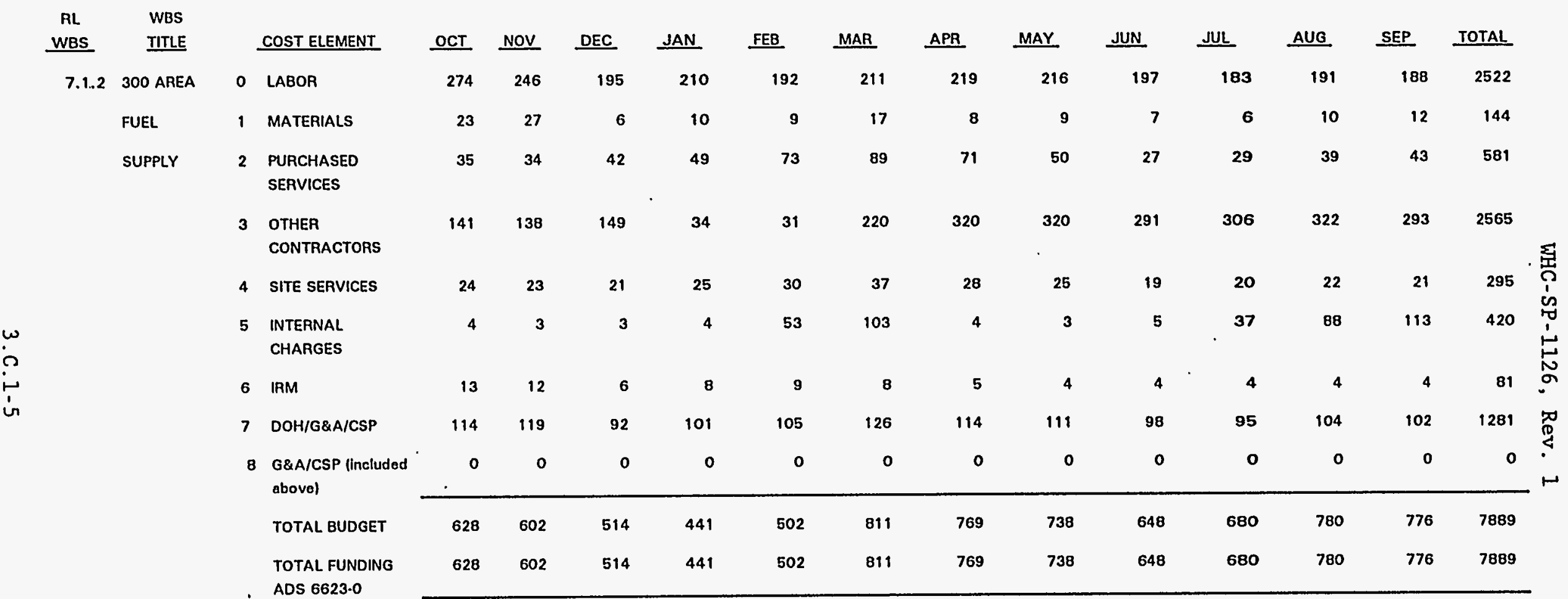


TRANSII.JN PROJECTS

\section{C. 1 Cost Baseline by Month - Operating Expense}

(s in 000 's)

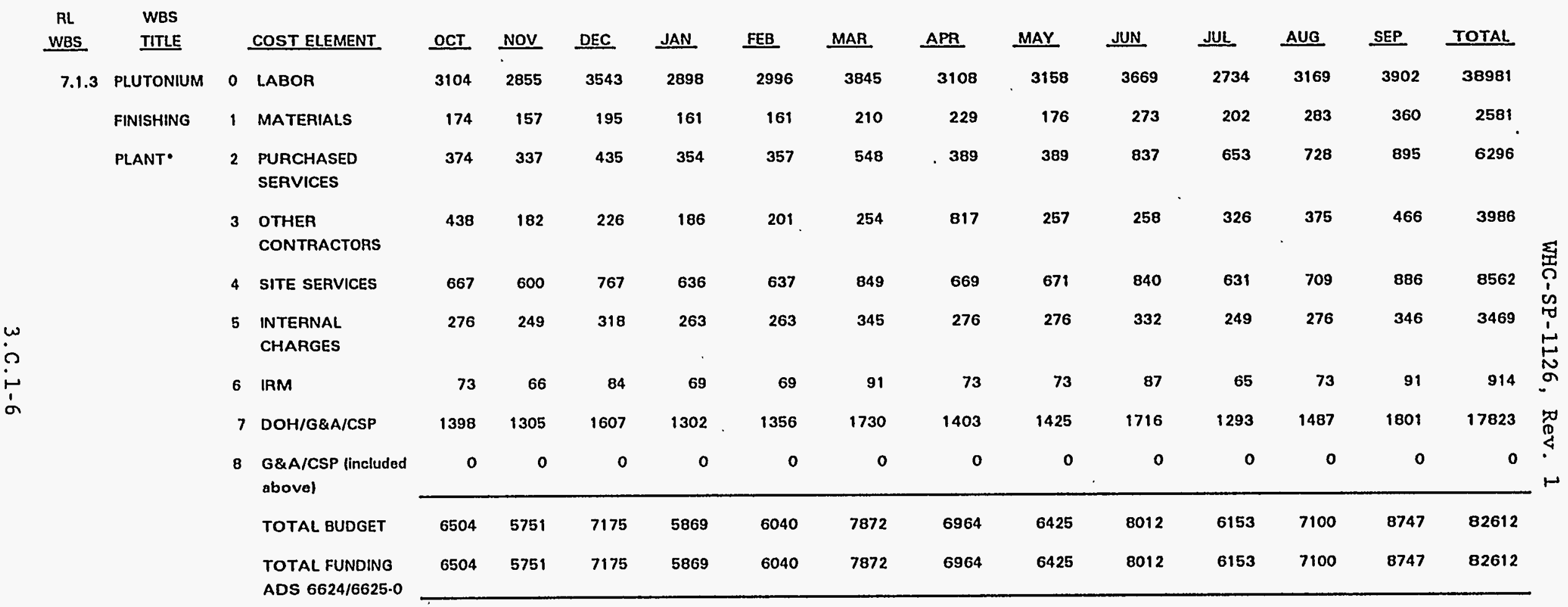

- NOTE: PFP Includes ADS 6625 - New

Facility Planning (WBS 7.1.3.6) 


\section{TRANSII.JN PROJECTS}

\section{C.1 Cost Baseline by Month operating Expense}

\begin{tabular}{|c|c|c|c|c|c|c|c|c|c|c|c|c|c|c|c|c|}
\hline $\begin{array}{c}\text { RL } \\
\text { WBS }\end{array}$ & $\begin{array}{l}\text { WBS } \\
\text { TITLE }\end{array}$ & & COST ELEMENT & OCT & NoV & $\underline{D E C}$ & JAN & FEB & MAR & APR & MAY & JUN & JUL & AUG & SEP & TOTAL \\
\hline \multirow[t]{11}{*}{7.1 .6} & PROG \& & $\mathbf{0}$ & LABOR & 279 & 251 & 307 & 266 & 269 & 352 & 277 & 273 & 332 & 236 & 266 & 344 & 3452 \\
\hline & $\begin{array}{l}\text { ENVIRON- } \\
\text { MENTAL }\end{array}$ & 1 & MATERIALS & 12 & 12 & 35 & 12 & 12 & 12 & 12 & 12 & 12 & 12 & 12 & 12 & 167 \\
\hline & MGMT & 2 & $\begin{array}{l}\text { PURCHASED } \\
\text { SERVICES }\end{array}$ & 22 & 22 & 56 & 22 & 22 & 32 & 22 & 122 & 42 & 22 & 42 & 122 & 548 \\
\hline & & 3 & $\begin{array}{l}\text { OTHEA } \\
\text { CONTRACTORS }\end{array}$ & 0 & 0 & 0 & o & 0 & 0 & 0 & 0 & 0 & o & $\mathbf{0}$ & 0 & $\mathbf{0}$ \\
\hline & & 4 & SITE SERVICES & 4 & 4 & 4 & 4 & 4 & 6 & 4 & 19 & 7 & 4 & 7 & 19 & 86 \\
\hline & & 5 & $\begin{array}{l}\text { INTERNAL } \\
\text { CHARGES }\end{array}$ & 0 & 0 & 0 & 0 & 0 & 0 & 0 & 0 & 0 & $\mathbf{0}$ & .0 & 0 & $\mathbf{0}$ \\
\hline & & 6 & IRM & 8 & 8 & 9 & 9 & 9 & 8 & 8 & 8 & 8 & 9 & 10 & 12 & 106 \\
\hline & & 7 & $\begin{array}{l}\text { DEPARTMENTAL } \\
\text { OVERHEAD }\end{array}$ & 48 & 43 & 52 & 45 & 46 & 60 & 47 & 47 & 57 & 40 & 45 & 59 & 589 \\
\hline & & 8 & G\&A/CSP & 48 & 44 & 60 & 46 & 47 & 61 & 48 & 62 & 59 & 41 & 49 & 73 & 638 \\
\hline & & & TOTAL BUDGET & 421 & 384 & 523 & 404 & 409 & 531 & 418 & 543 & 517 & 364 & 431 & 641 & 5586 \\
\hline & & & $\begin{array}{l}\text { TOTAL FUNDING } \\
\text { ADS } 6620.0\end{array}$ & 421 & 384 & 523 & 404 & 409 & 531 & 418 & 543 & 517 & 364 & 431 & 641 & 6586 \\
\hline
\end{tabular}


TRANSITION PROJECTS

FY 1996 MYPP

1.3.1/7.1/6.12

3.C.2 Cost Baseline by Month - CENRTC

Transition Projects Cost Baseline Summary by Month for CENRTC will be added to the Multi-Year Program Plan via approved change request incorporating carryover workscope. 


\section{TRANSITION PROJECTS}

FY 1996 MYPP

1.3.1/7.1/6.12

3.C.3 Cost Baseline by Month - GPp

Transition Projects Cost Baseline Summary by Month for GPP will be added to the Multi-Year Program Plan via approved change request incorporating carryover workscope. 


\section{TRANSITION PROJECTS}

FY 1996 MYPP

1.3.1/7.1/6.12

\section{C.4 Cost Basel in ne by Month - Line Items}

Transition Projects Cost Baseline Summary by Month for Line Items will be added to the Multi-Year Program Plan via approved change request incorporating carryover workscope. 
TRANSITION PROJECTS

FY 1996 MYPP

1.3.1/7.1/6.12

3.D. Program Funding Requíred

(s in $000^{\prime} s$ )

\section{RL}

WBS

1.3.1.7

$\omega$
$\vdots$
1

SAFETY VENTILATION UPGRADE

4190-01

B PLANT

WBS/ADS

TITLE

ADS

NUMBER

4190-00

4195-00

CESIUM CAPSULE RECOVERY

DOR


TRANSITION PROJECTS

FY 1996 MYPP

1.3.1/7.1/6.12

3.D Program Funding Required

$\left(\$\right.$ in $\left.000^{\prime} 8\right)$

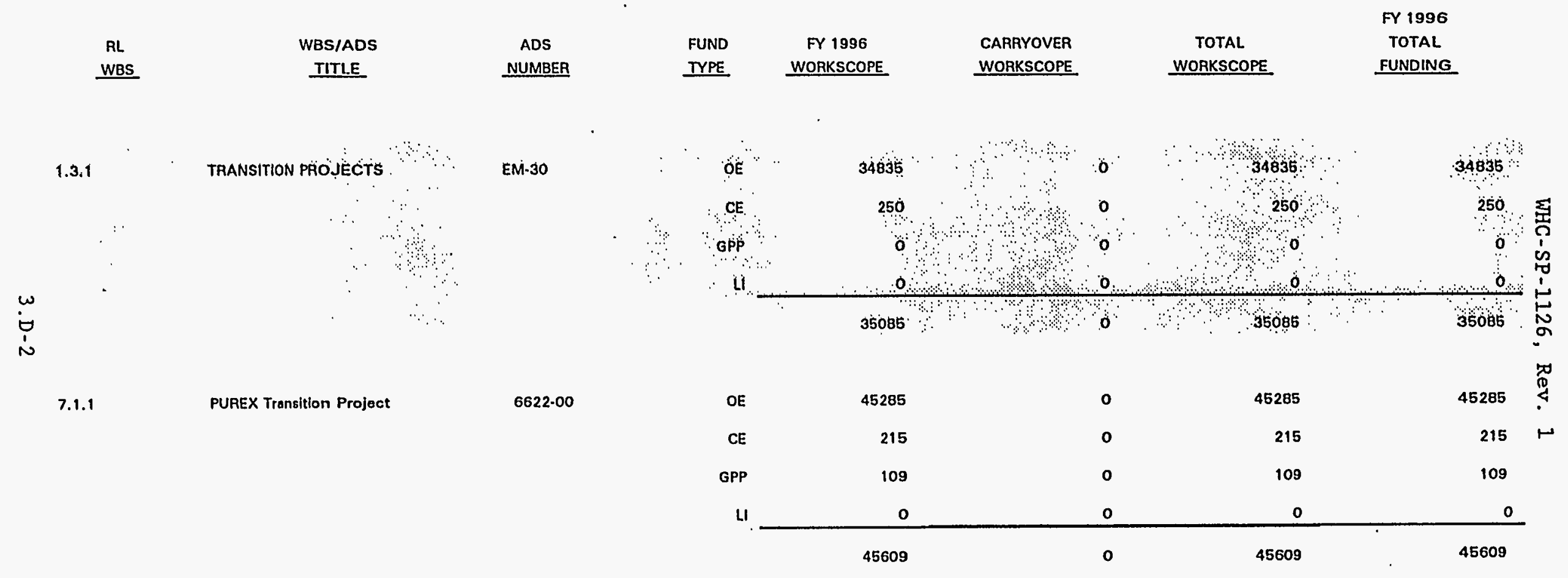


TRANSITION PROJECTS

FY 1996 MYPP

1.3.1/7.1/6.12

3.0 program Funding Required

$\left(s\right.$ in $\left.000^{\prime} s\right)$

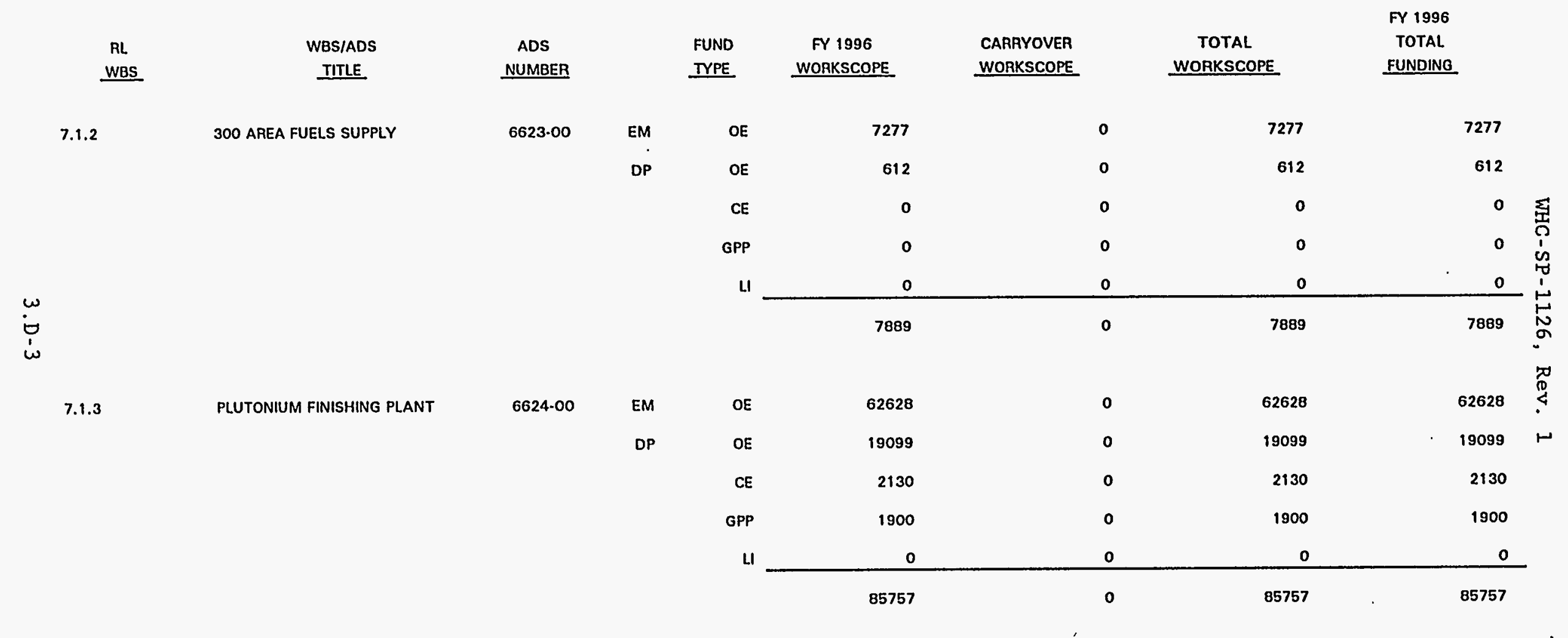


TRANSITION PROJECTS

FY 1996 MYPP

1.3.1/7.1/6.12

3.D Program Funding Required

(s in $000^{\circ} s$ )

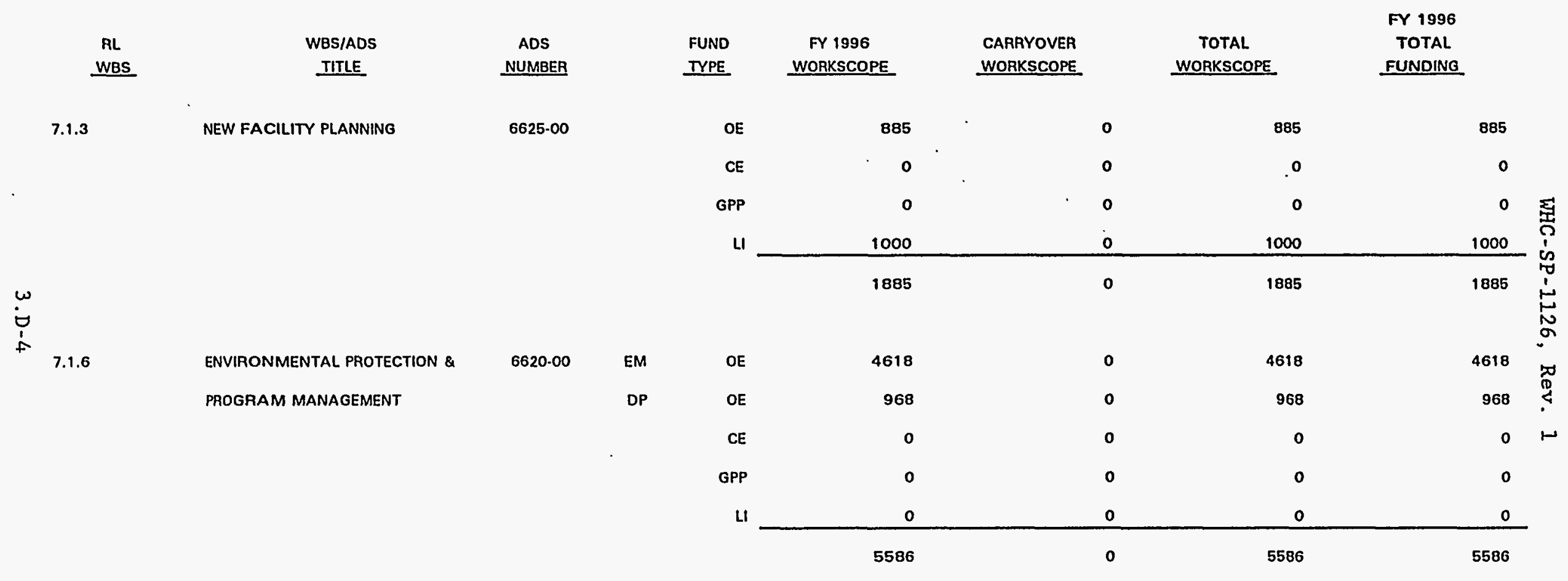


TRANSITION PROJECTS

FY 1996 MYPP

1.3.1/7.1/6.12

3.D Program Funding Required

$(s$ in 000 's)

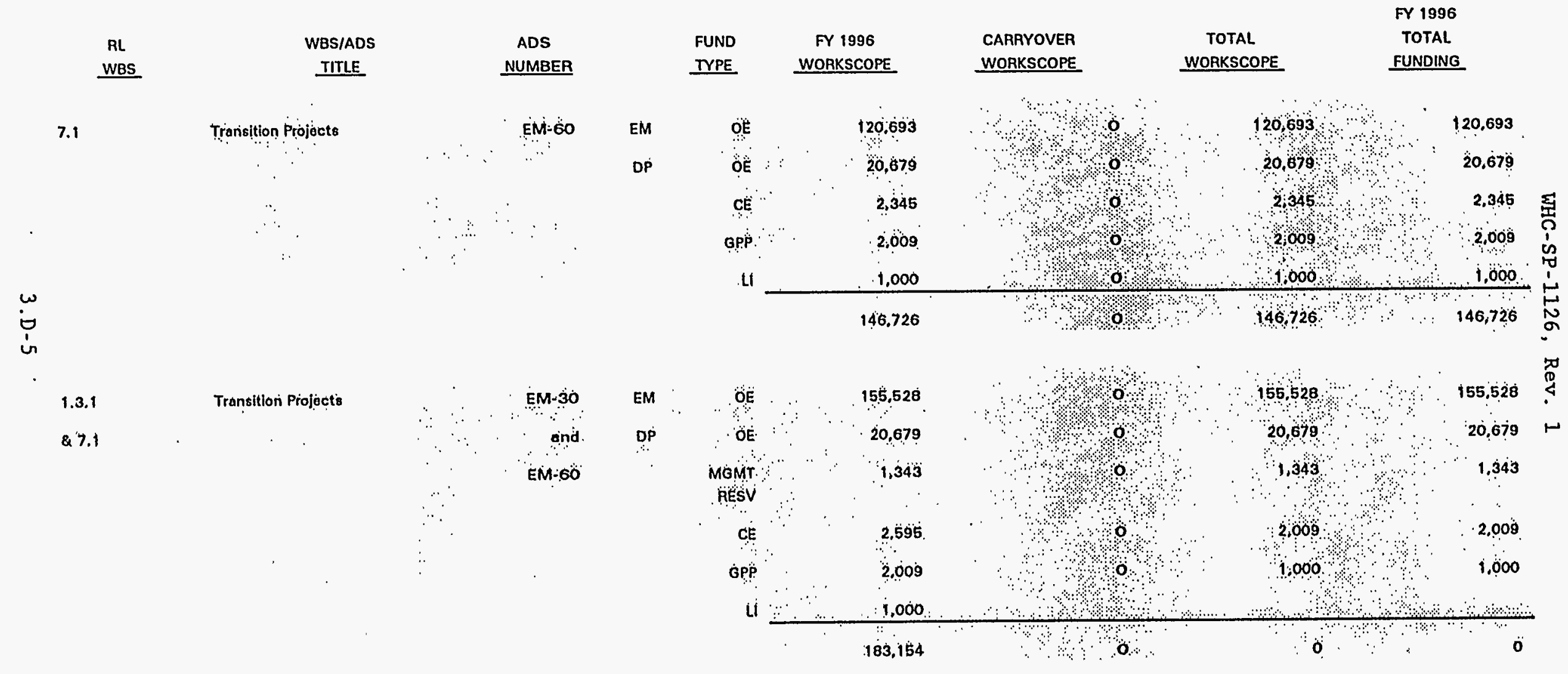




\section{TRANSITION PROJECTS}

FY 1996 MYPP

1.3.1/7.1/6.12

\section{E.1 Performance Based Initiatives}

Transition Projects Performance Based Initiatives will be added to the Multi-Year Program Plan via letter upon final approval of the initiatives. 


\section{TRANSITION PROJECTS}

3.E.2 Performance Measurement Criteria

Transition Projects Performance Measurement Criteria will be available upon receipt of RL guidance.

$\omega$
$i$
$i$
$i$ 


4.A. Doh programobjectives

\section{DEPARTMENT OVERHEAD $(6.12 .1)$}

All Transition Projects Overhead programs will be managed in a safe, efficient, and cost effective manner. All activities will be conducted to execute a high quality program that ensures that Transition Projects will achieve and maintain compliance with all applicable DOE orders and directives within the funding constraints imposed.

Specific objectives for FY 1996 are discussed in the following subsections.

$\stackrel{+}{i}$

Provide Division Senior Management to ensure minimum required staff, facilities and equipment are available to conduct all operations in a safe, environmentally sound, total quality and cost effective manner. Maintain effective communication between customer and service objectives.

- Cover site service costs; e.g., dosimetry, bus, occupancy, telephone, required to support staff and safe, secure, facility transition.

- Initiate independent budget reviews

- Provide resources for employee training and development

- Coordinate staff planning and career development

- Coordinate annual division salary planning, action tracking, and great ideas

- Coordinate the efforts at all plants and facilities associated with the transition from production or standby for production to turnover to the surplus facilities (decontamination and decommissioning) program. This includes implementation of a sound and consistent systems engineering approach at all facilities, prioritization of tasks to reduce operating costs as rapidly as possible, consistent with ensuring safety, and introduction of commercial standards to this work. 


\section{B DOH Program Planning Assumptions}

\section{General Assumptions}

- DOH will be consolidated to one rate per WHC division.

- $\quad 3.0 \%$ escalation for labor, materials.

- DOH costs have been planned at existing staff levels.

- Internal assessments same as FY 1995 with appropriate escalation.

- Labor priced out per 1996 rate table.

- IRM, and outside services same as FY 1995 with appropriate escalation, until guidance becomes available.

- Staffing assumptions consistent with FY 1995 programmatic staffing ceiling.

- Transition Projects Senior Management and related cost will be prorated to the other Transition Projects subprogram elements. Proration factor is based on number of employees per each sub-program. 
FY 1996 MYPP

$\stackrel{f}{i}$
TRANSITION PROJECTS

1.3.1/7.1/6.12

$\mathrm{DOH}$

WORK BREAKDOWN STRUCTURE

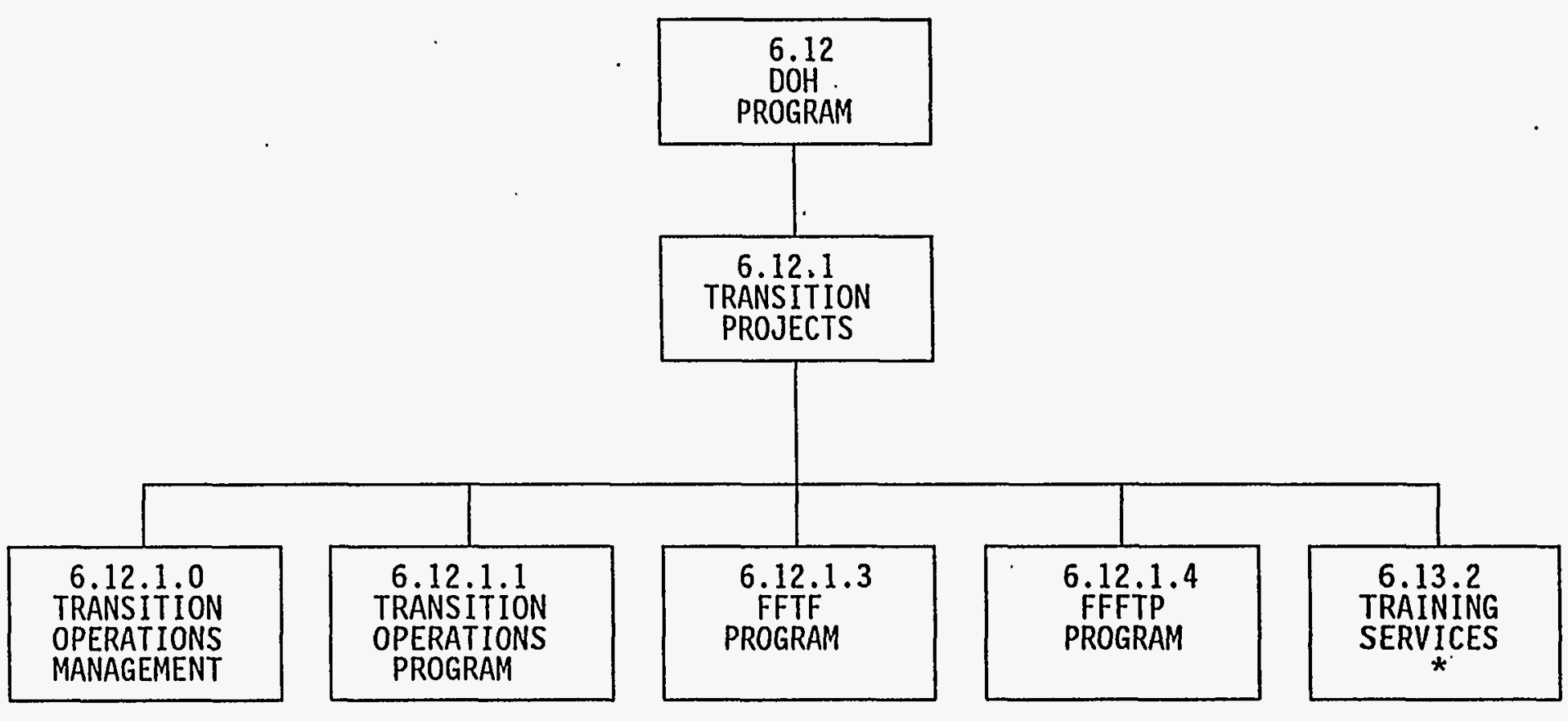

* Denotes Training Services DOH WBS Dictionary is included in the Site Support Program Plan (SSPP) for 6.13 
FY 1996 MYPP

TRANSITION PROJECTS

1.3.1/7.1/6.12

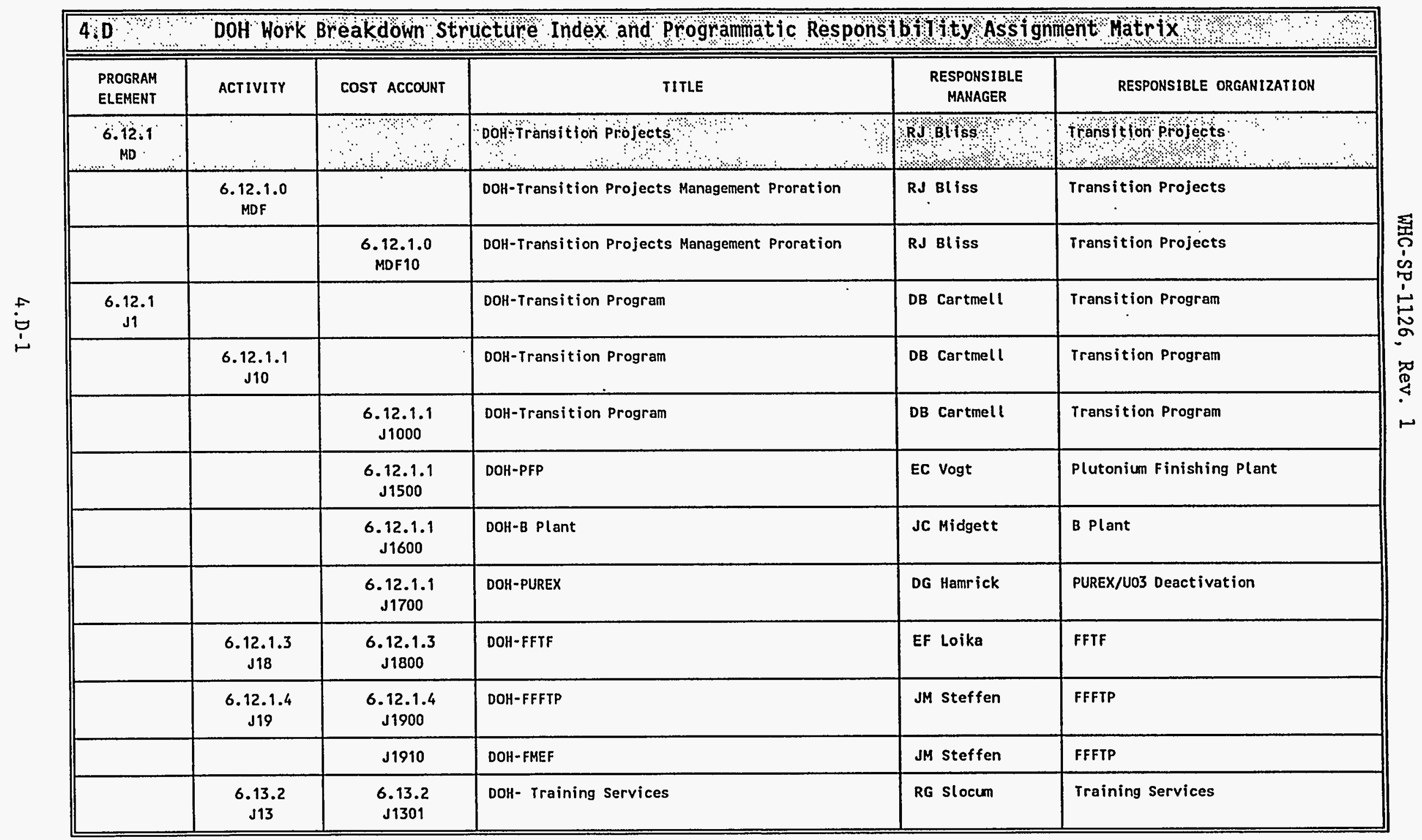




\begin{tabular}{|c|c|c|c|c|c|c|c|}
\hline $\begin{array}{l}\text { Work } \\
\text { Breakdown } \\
\text { Structure } \\
\text { Dictionary }\end{array}$ & \multicolumn{4}{|c|}{$\begin{array}{c}\text { Westinghouse Hanforu Company } \\
\text { _FUELS \& MATERIALS EXAMINATION FACILITY (FMEF) } \\
\text { Part I - Sum mary } \\
\text { (Dollars in 000's) }\end{array}$} & \multicolumn{3}{|c|}{$\begin{array}{l}\text { FY } 1996 \\
\text { Indirect } \\
\text { Program Plan } \\
\text { Rev. \# } 0 \\
25-\text { Sep-95 } \\
\end{array}$} \\
\hline $\begin{array}{r}\text { Cost Account Number } \\
\text { 1J1910 } \\
\end{array}$ & \multicolumn{4}{|c|}{$\begin{array}{l}\text { Cost Account Title } \\
\text { FUELS \& MATERIALS EXAMINATION FACILITY (FMEF) }\end{array}$} & \multicolumn{3}{|c|}{$\begin{array}{l}\text { Proposed Rate: } 13.3 \% \\
\text { (Rated Service Pool Only) }\end{array}$} \\
\hline $\begin{array}{r}\text { SMS WBS Number } \\
6.12 .1 .1 \\
\end{array}$ & \multicolumn{4}{|c|}{$\begin{array}{l}\text { SMS TItll } \\
\text { TRANSITION PROJECTS PROGRAM }\end{array}$} & \multicolumn{3}{|c|}{$\begin{array}{l}\text { Funding Source: } \\
\end{array}$} \\
\hline RL SMS Program Manger & \multicolumn{4}{|c|}{ ND MOORER } & \multicolumn{3}{|l|}{ sws } \\
\hline Cost Account Manager & \multicolumn{4}{|l|}{ JM STEFFEN } & \\
\hline \multicolumn{5}{|c|}{ WHC SMS Program Manager JM STEFFEN } & \multicolumn{3}{|l|}{ DOH XX } \\
\hline \multirow{3}{*}{$\begin{array}{l}\text { Financial Manager } \\
\text { Responsible Analyst }\end{array}$} & \multirow{2}{*}{\multicolumn{4}{|c|}{$\begin{array}{l}\text { TL MAIN } \\
\text { JG SALAZAR }\end{array}$}} & MGT PRO & & \\
\hline & & & & & \multirow{2}{*}{\multicolumn{3}{|c|}{$\begin{array}{c}\text { POOL } \\
\text { FY } 1996 \text { Baseline } \\
\text { Full-Time Equivalents (FTEs) }\end{array}$}} \\
\hline & \multicolumn{2}{|c|}{$\begin{array}{l}\text { JG SALAZAR } \\
\text { FY } 1996 \text { Approved Funding } \\
\text { Full-Time Equivalents (FTEs) }\end{array}$} & \multicolumn{2}{|c|}{$\begin{array}{c}\text { FY } 1996 \text { Unfunded } \\
\text { Full-Time Equivalents (FTEs) }\end{array}$} & & & \\
\hline TYPE OFFTE & \begin{tabular}{|l|l|} 
Exempt & Non-Exempt \\
\end{tabular} & Bargaining & Exempt Non-Exempt & Bargaining & Exempt & Non-Exempt & Bargaining \\
\hline Organizational & \begin{tabular}{r|r|}
5.6 & 2.8 \\
\end{tabular} & 0 & \begin{tabular}{l|l|}
0 & 0 \\
\end{tabular} & 0 & 5.6 & 2.8 & 0 \\
\hline Support & 0.1 & 6.8 & 0 & 0 & 1.2 & 0.1 & 6.8 \\
\hline TOTALFTES & 6.8 & 6.8 & 0 & 0 & 6.8 & 2.9 & 6.8 \\
\hline COST ELEMENTS & FY 1996 Approved Fund & ling Budget & FY 1996 Unfunded BL & udget & & 1996 Basoline $B$ & Budget \\
\hline . Labor-Regular & $17.21 \%$ & 958 & (17.2. & $\underline{0}$ & 19.2 & 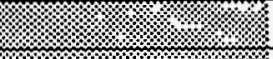 & 958 \\
\hline . Labor - Overtime & 1 & 0 & স. & 0 & & & \\
\hline o Total Labor & 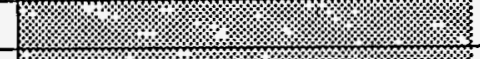 & 958 & 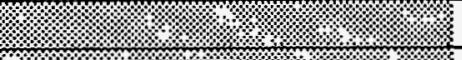 & 0 & 4 & ? & 958 \\
\hline 1 Materials & " l- & 54 & ":. & 0 & 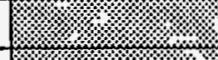 & 2 & 54 \\
\hline 2 Purchased Services & 19 & 2 & & & 12.28 & 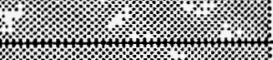 & 2 \\
\hline 3 Other Hanford & 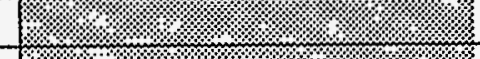 & 0 & & 0 & 12 & 1.2 .20 & \\
\hline Subbtotal Originated Cösis & 18 & 1,014 & & $\$$ & & & $1 2 \longdiv { 1 0 0 1 4 }$ \\
\hline 4 Site Senvices & 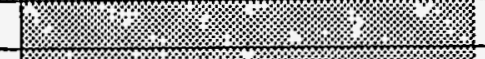 & 68 & 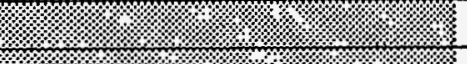 & 0 & & $\sqrt{2}$ & 68 \\
\hline 5 Internal Charges & 14 & 250 & 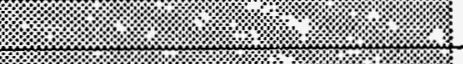 & $\underline{0}$ & & 2.2 & 250 \\
\hline 6 IRM Support & 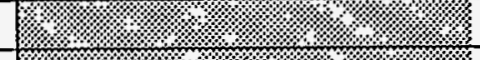 & 0 & 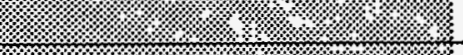 & 0 & & & 0 \\
\hline 7 Overheads & $18=19$ & 123 & 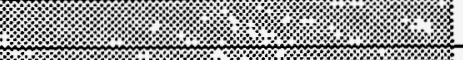 & $\underline{0}$ & & 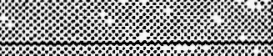 & 123 \\
\hline 8 Revenue & 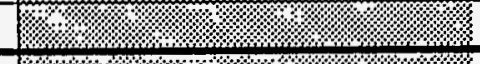 & $(1,455)$ & 4 & & 12 & 18 & $(1,455$ \\
\hline TOTAL DOLLARS & शः & 0 & (1) & & 13 & (4) & \\
\hline $\begin{array}{l}\text { SIGNATURES } \\
\text { Financial Analyst: } \\
\text { CAM: }\end{array}$ & +2 & $\begin{array}{l}\text { Date: } \\
\text { Date: }\end{array}$ & BASELINE API & PROVAL & & & Date: \\
\hline
\end{tabular}


Provide for the maintenance and operation of FMEF, including configuration control, building maintenance, modification/upgrades, excess material handling and general support to building occupants. Occupancy revenue offsets the building operating costs by $90 \%$. However, building modifications currently underway will increase the space available to rent in FY 96 which will increase the revenues to fully offset the total costs.

\section{APPROVED FUNDING BUDGET:}

\section{Activity Detailed Description - FMEF OPERATIONS}

To provide for operation of FMEF in a safe and efflcient manner in accordance with approved WHC procedures, DOE orders and Federal/State environmental documents. This includes funds for operating personnel, craft support, property management support, work order for special equipment (elevators, chillers, cranes, etc, ) and utilities to operate this 250,000 square foot building.

\section{Total Activity Cost}

\section{Activity Detailed Description - FMEF ENGINEERING}

To provide facility cognizant engineering resources to support current FMEF operations, revenue generating functions, and possible new programs/projects for future facility operations. This includes funds for engineers, drafting/drawing configuration support and QA/safety support.

Total Activity Cost

\section{Activity Detailed Description - FMEF BUILDING OCCUPANCY REVENUE}

Revenue is generated by leasing office space to other Hanford organizations. Current building occupants include site maintenance training, TWRS survellance systems engineering, light duty utility arm testing, well water sampling program, Rust Geotech, spent nuclear support and others.

Activity Detailed Description

$$
\text { Total Activity Cost }
$$




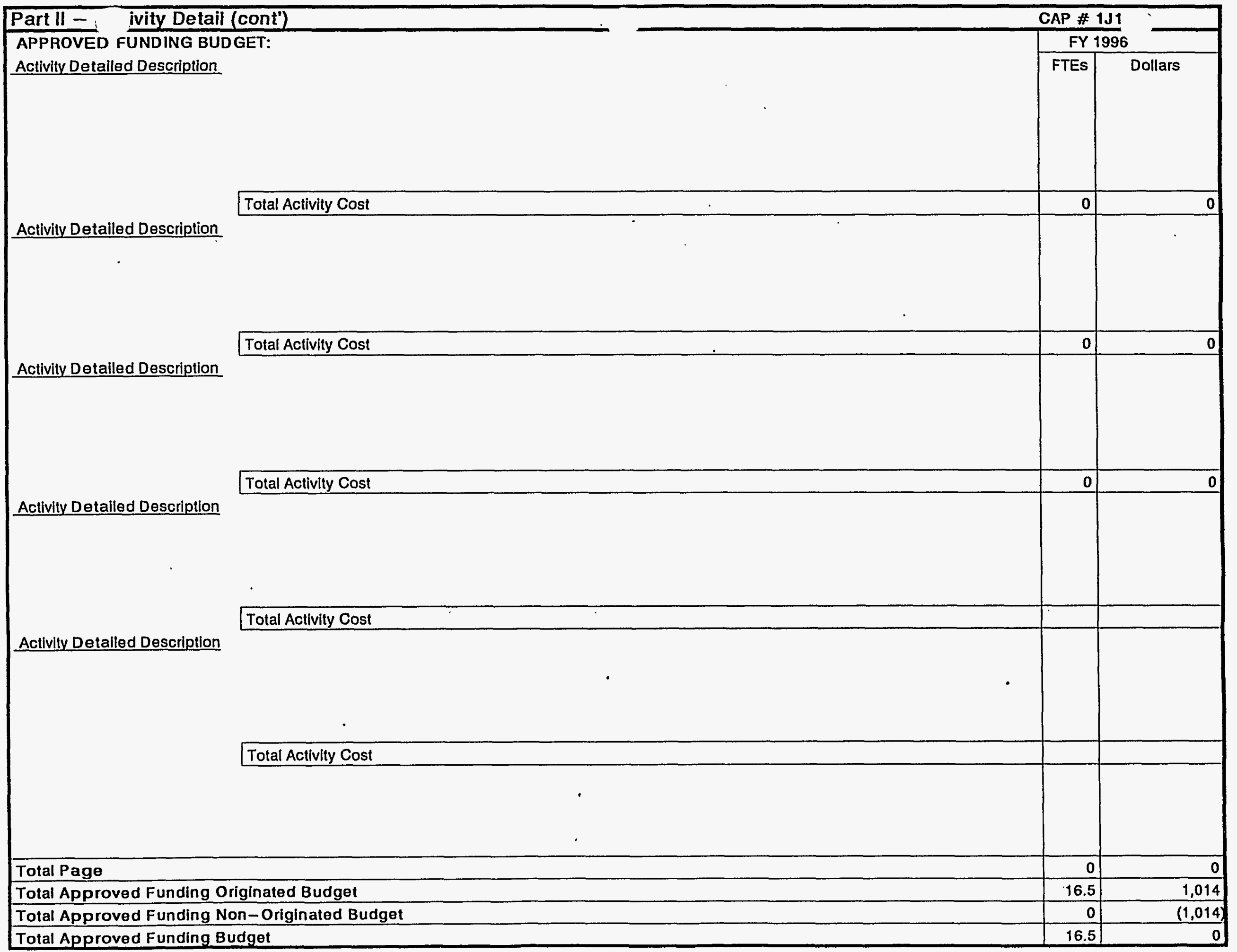




\section{Part II -}

UNFUNDED BUDGET:

Activity Detailed Description

Activity Detalled Description

Activity Detailed Description

$$
\text { Total Activity Cost }
$$

Total Activity Cost

Total Activity Cost

Activity Detailed Description

Activity Detailed Description

$$
\text { Total Activity Cost }
$$

Total Activity Cost
CAP \#1J1'

FY 1996

FTEs Dollars

0

0

Total Page

Total Unfunded Originated Budget

Total Unfunded Non-Originated Budget

Total Unfunded Budget

Total Approved Funding \& Unfunded Budgets 
TRANSITION PROJECTS

FY 1996 MYPP

1.3.1/7.1/6.12

\section{F DOH PIanned staffing Profiles}

\begin{tabular}{|c|c|c|c|c|c|c|c|c|}
\hline \multicolumn{3}{|c|}{ 4.F DOH Planned Staffing (Full Time Equivalent) } & \multicolumn{6}{|c|}{ NOTE: Job Family Only After 1997} \\
\hline & PROGRAM AND ENVIRONM & NTAL & NAGEM & N2 7. & $1.6) \%$ & ४ै। & & 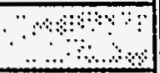 \\
\hline \multicolumn{2}{|r|}{ ? } & \multirow[t]{2}{*}{1996} & \multirow[t]{2}{*}{$1997:$} & \multirow[t]{2}{*}{998} & \multirow[t]{2}{*}{1999,} & \multirow[t]{2}{*}{2000} & \multirow[t]{2}{*}{2001} & \multirow[t]{2}{*}{2002} \\
\hline cocs code & Description & & & & & & & \\
\hline coón & CBAETS & 2.8 & ? & s. & ४ै। & अै. : & 3 & ४ \\
\hline c010 & Carpenters & & & & & & & \\
\hline co20 & Electricians & 1.0 & & & & & & \\
\hline C040 & Machinists & & & & & & & . \\
\hline $\cos 0$ & Masons & & & & & & & \\
\hline 0060 & Millwrights & & & & & & & \\
\hline $\mathrm{co70}$ & Painters & & & & & & & \\
\hline $\cos 0$ & Plumbers \& Pipefitters & & & & & & & \\
\hline $\cos 0$ & structural \& Metal Horkers & & & & & & & \\
\hline $\mathrm{c100}$ & Vehicle/Equipment Mechanics & & & & & & & \\
\hline$c 110$ & Helder - HAMTC & & & & & & & \\
\hline $\mathrm{c120}$ & other crafts & & & & & & & \\
\hline C12A & Track Inspector - HAMTC & & & & & & & \\
\hline$E 000$ & ENGINEERS & 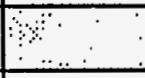 & 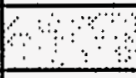 & «. & अे & 184 & एक & ओ \\
\hline$E 010$ & Chemical Engineers - Exempt & & & & & & & \\
\hline$E 020$ & Civil Engineer - Exempt & & & & & & & \\
\hline E030 & Computer Engineers - Exempt & & & & & & & \\
\hline
\end{tabular}


TRANSITION PROJECTS

$1: 3.1 / 7.1 / 6.12$

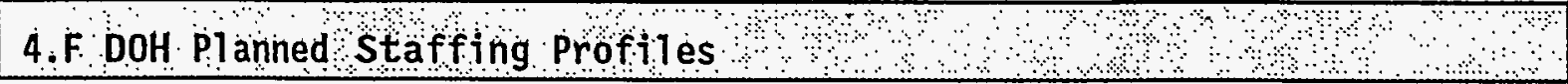

\begin{tabular}{|c|c|c|c|c|c|c|c|c|}
\hline \multicolumn{3}{|c|}{ 4.F DOH Planned Staffing (Full Time Equivalent) } & \multicolumn{6}{|c|}{ NOTE: Job Family Only After 1997} \\
\hline \multicolumn{9}{|c|}{ PROGRAM AND ENVIRONMENTAL MANAGEMENT $(7,1,6)$} \\
\hline Fiscal Yëar & $\therefore \quad: \quad-\quad$ & 1096 & 1997 & 1998 & 1999. & 2000 & 2001 & 2002 \\
\hline cocs Code & Description & & & & & & & \\
\hline E040 & Electrical Engineers - Exempt & 1.0 & & & & & & \\
\hline E050 & Environmental Engineers - Exempt & .3 & & & & & & \\
\hline E060 & Industrial Engineers - Exempt & & & & & & & \\
\hline EO70 & Mechanical Engineers - Exempt & 2.5 & & & & & & \\
\hline E080 & Nucl ear Engineers - Exempt & & & & & & & \\
\hline E090 & Mining Engineers - Exempt & & & & & & & \\
\hline E100 & Plant Engineers & & & & & & & \\
\hline E110 & Qual ity Control Engineers & & & & & & & \\
\hline E120 & Safety Engineers & .1 & & & & & & \\
\hline E130 & OTHER ENGINEERS & .8 & & & & & & \\
\hline, 6000 & GENERAL AOMINISTRAT IVE & $\therefore \therefore$ & $\because$ & & ४ & i: & & \\
\hline G010 & Administrative Assistants & 4.5 & & & & & & \\
\hline G020 & Office Clerks (General) & 1.0 & & & & & & \\
\hline G030 & Office Clerks (Specialists) & & & & & & & \\
\hline 6040 & Secretaries & .3 & & & & & & \\
\hline 6050 & Clerk, Typist, \& Word Processing & & & & & & & \\
\hline Loo & LABORORS $\bigcirc \mathrm{S}$ & +1: & (1) & +. & + & ४ै.े & 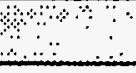 & ॥ \\
\hline Lo10 & Firefighters & & & . & & & & \\
\hline
\end{tabular}


4.F DOH Planned Staffing Profiles

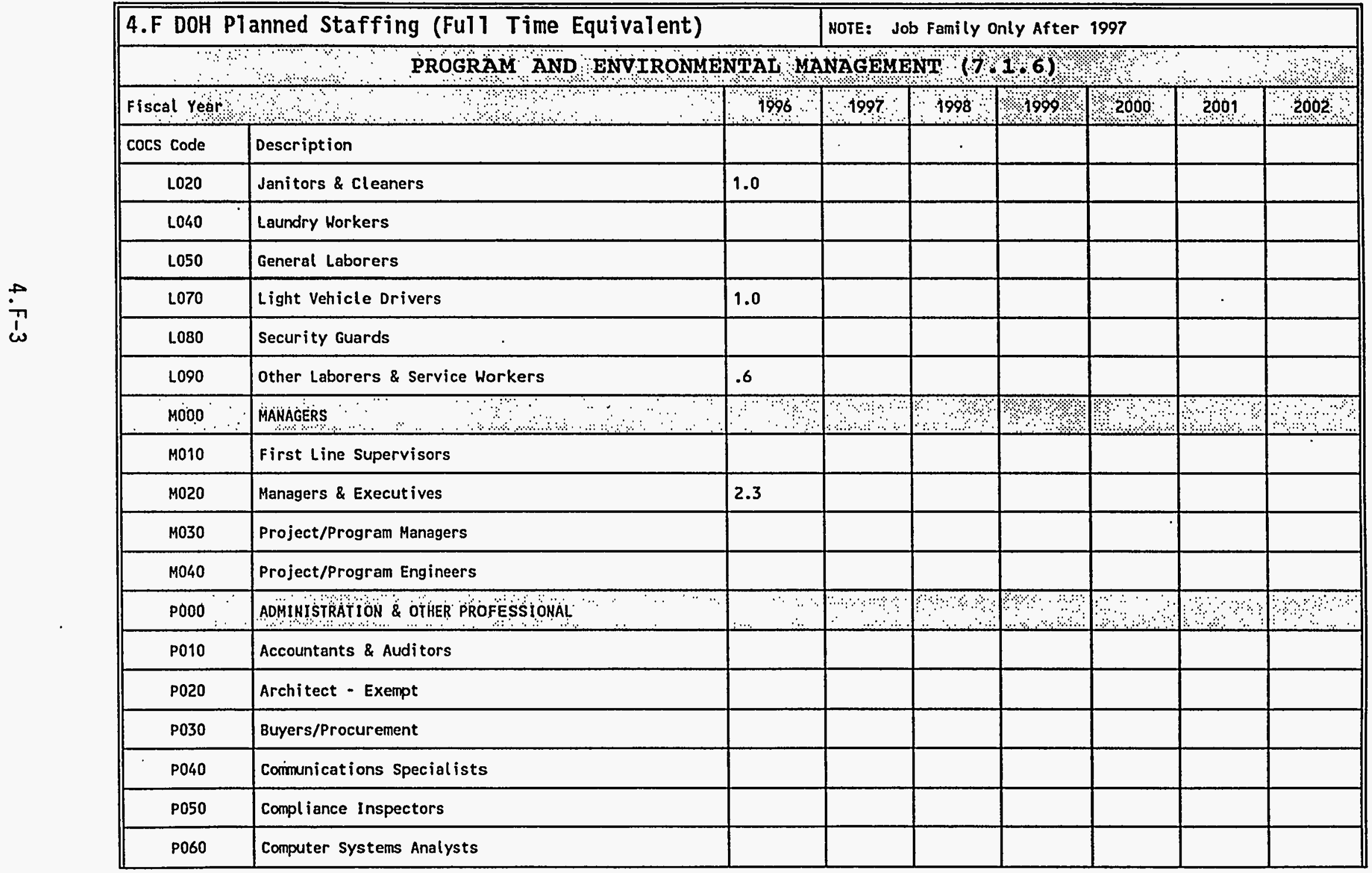


4.F DoH Planned staffing proflles

\begin{tabular}{|c|c|c|c|c|c|c|c|c|}
\hline \multicolumn{3}{|c|}{ 4.F DOH Planned Staffing (Full Time Equivalent) } & \multicolumn{6}{|c|}{ NOTE: Job Family Only After 1997} \\
\hline & PROGRAM AND ENVIRONM & NTAt & NAGEY & $r 8$ & 1.69) & अ & & \\
\hline \multicolumn{2}{|l|}{ Fiscal Year } & 1996 & 1997 & 1998 & 1999 & 2000 & 2001 & 2002 \\
\hline cocs code & Description & & & & & & & \\
\hline PO70 & Planner/Scheduler/Estimators & & & & & & & \\
\hline p080 & Heal th Physicists & & & & & & & \\
\hline PO90 & Alara Program - Exempt & & & & & & & \\
\hline P100 & Attorney - Exempt & & & & & & & \\
\hline P110 & Personnel \& Labor Relations & & & & & & & \\
\hline P130 & Paramedic, Platoon - HAMTC & & & & & & & \\
\hline P140 & Safeguards \& Security Spec & & & & & & & . \\
\hline P150 & Trainers \& Instructors & & & . & & & & \\
\hline P160 & Technical Writers \& Editors & & & & & & & \\
\hline P170 & Other Professionals & & & & & & & \\
\hline R000 & OPERATORS & 1.0 & & & मा & , & & \\
\hline R030 & Material Moving Equipment Operator & & & & & & & \\
\hline R040 & Nuclear Plant Operators & & & & & & & \\
\hline R050 & Nuclear Waste Process Operator & & & & & & & \\
\hline R060 & SHIPPER & & & & & & & \\
\hline R070 & Utilities Systems Operator & & & & & & & \\
\hline ROBO & Other Operators & & & & & & & \\
\hline 5000 & Scientists & ए: & $\because$ & & खे & ৪ি & ४্ & \\
\hline
\end{tabular}




\section{F DOH Planned Stafflng Prof 1 1 es 2 s}

\begin{tabular}{|c|c|c|c|c|c|c|c|c|}
\hline \multicolumn{3}{|c|}{ 4.F DOH Planned Staffing (Ful1 Time Equivalent) } & \multicolumn{6}{|c|}{ NOTE: Job Family Only After 1997} \\
\hline \multicolumn{9}{|c|}{ PROGRAM AND ENVIRONMENTAL MANAGEMENT $(7,1.6)$} \\
\hline \multicolumn{2}{|l|}{ Frscal Year } & 1996 & 1997 & 1998 & 10990 & 2000 & 2001 & 2002 \\
\hline cocs code & Description & & & & & & & \\
\hline s010 & Chemists - Exempt & & & & & & & \\
\hline s020 & Environmental Scientists - Exempt & & & & & & & \\
\hline s030 & Geologists - Exempt & & & & & & & \\
\hline 5040 & Life Scientists - Exempt & & & & & & & \\
\hline s050 & Materials Scientists - Exempt & & & & & & & \\
\hline s060 & Mathematicians - Exempt & & & & & & & \\
\hline s070 & Physicists - Exempt & & & & & & & \\
\hline s080 & Social - Exempt & & & & & & & \\
\hline S090 & Other Scientists - Exempt & & & & & & & \\
\hline To00 & 4 & & $\therefore \because \because$ & & ४ & ख!: & $\therefore$ & अ? \\
\hline T010 & Computer Operator/Coder & & & & & & & \\
\hline T020 & Drafters & .5 & & & & & & \\
\hline T030 & Engineering Technician - NonExempt & 1.5 & & & & & & \\
\hline T050 & Heal th Physics Technician - HAMTC & & & & & & & \\
\hline T070 & Instruments \& Control Technician & & & & & & & \\
\hline T080 & Chemical Technologist - HAMTC & & & & & & & \\
\hline T090 & Media Technicians & & & & & & & \\
\hline$T 110$ & other Technicians & .3 & & & & & & \\
\hline
\end{tabular}




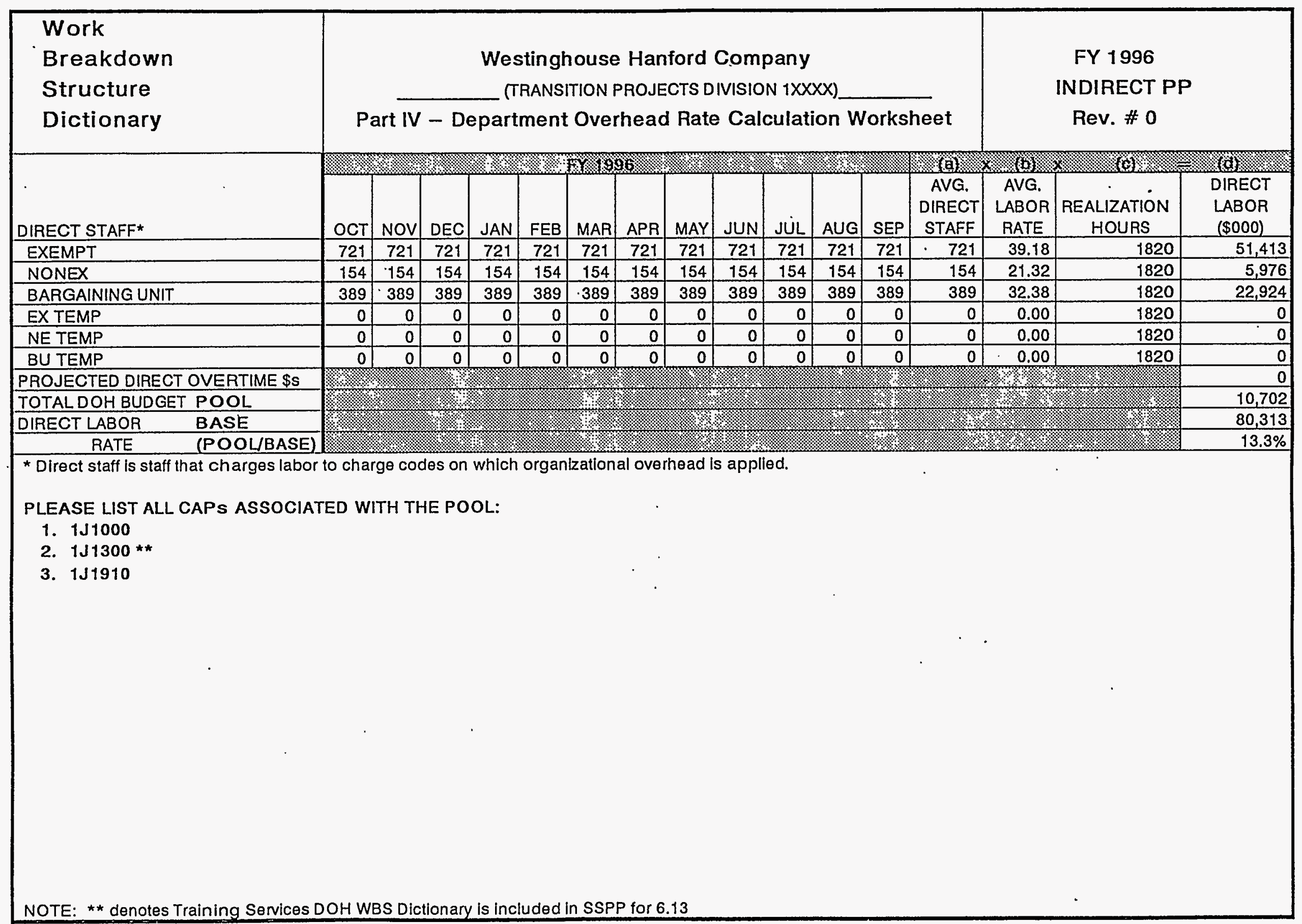




\section{WHC-SP-1126, Rev. 1 \\ TRANSITION PROJECTS \\ FY 1996 MYPP \\ 1.3.1/7.1/6.12 \\ DISTRIBUTION LIST}

\section{NAME \\ ORGANIZATION \\ ADDRESS (if applicable)}

Kerry D. Cameron

RL, PID

Rick Martinez

DOE-HQ, EM-65

19901 Germantown Road

Germantown, MD 20874

G. Tom Tebb

Environmental Protection Agency

Dan Josue

Ecology

300 Desmond Dr. SE

Lacey, WA 98503

Norman D. Moorer

$\mathrm{RL}$, SOD

Norman D. Moorer

$\mathrm{RL}$, SOD

Daniel B. Cartmell

WHC, TrP Program

Ronald J. Bliss

WHC, TrP

\section{Central Files}

WHC

Office of Science and Technical Information (OSTI)

P. 0. Box 62

Oak Ridge, TN 37831

National Technical Information Service (NTIS)

5285 Port Royal Road

Springfield, VA 22161

Marcella A. Baumann

WHC, TrP Financial Integration

Kathrine A. Davis

WHC, TrP Financial Integration
MSIN PHONE

A5-58 (509) 376-8035

(301) 903-4484

B5-18 (509) 736-3020

R3-78 (509) 376-9447

R3-78 (509) 376-9447

R3-50 (509) $372-3982$

B3-04 (509) 376-6427

A3-88

A3-36 (615) 576-8401

A3-36 (703) $487-4650$

R3-61 (509) 372-3082

R3-61 (509) 372-3082 